\title{
NUCLEAR-POWERED \\ GPS SPACECRAFT \\ DESIGN STUDY
}

Prepared for

OFFICE OF SPACE APPLICATIONS

DIVISION OF NUCLEAR RESEARCH AND APPLICATIONS

ENERGY RESEARCH AND DEVELOPMENT ADMINISTRATION

Contract No. AT(49-15)-3063

MAY, 1977

APPROVED FOR RELEASE:

Prepared by:

Cont michael maneneci 4 aug 77 GPS STUDY MANAGER SAMSO/YE

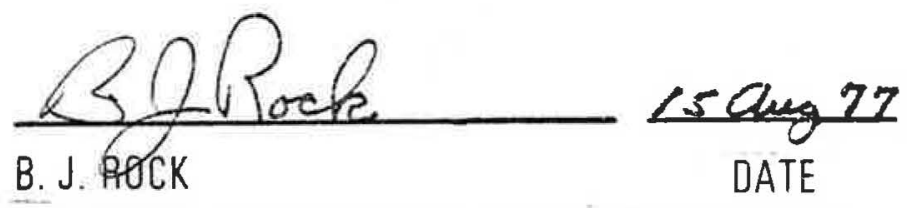

ASSISTANT DIRECTOR FOR SPACE APPLICATIONS ERDA/NRA

DATE
Bernard Raab, Program Manager

Robert J. Eby

Mark A. Frieder

Joseph M. Gonzalez

Robert D. Karam

Hugh A. Raymond

Robert R. Schreit, Jr.

Adam Shostak 


\section{NOTICE}

This report was prepared as an account of work sponsored by the United States Government. Neither the United States nor the United States Energy Research and Development Administration, nor any of their employees, nor any of their contractors, subcontractors, or their employees, makes any warrantee, express or implied, or assumes any legal liability or responsibility for the accuracy, completeness or usefulness of any information, apparatus, product or process disclosed, or represents that its use would not infringe privately-owned rights. 



\section{TABLE OF CONTENTS}

Para. No.

$\underline{\text { Title }}$

Page

Section I

Introduction

1-1

1.1

Objectives

1-1

1.2

Ground Rules

1-2

1.3

Summary and Conclustions

1-3

Section II

Critical Parameters and Program Goals

2-1

2.1

Power-To-User

2-1

2.2

Mean-Mission-Duration

2-5

2.3

Orbital Predictability

2-8

2.4

Thermal Control of On-Board Clocks

2-14

2.5

Survivability

2-15

Section III

Approach to the Reference Design

$3-1$

3.1

Selection of Attitude Control Approach

$3-1$

3.2

Thermal Control Approach

3-12

3.3

Mechanical Design Approach

$3-16$

3.4

Approximate Size Considerations

$3-17$

Section IV

Power Systems Input Data

4-1

4.1

Candidate Power Systems

4-1

4.2

Organic Rankine System

4-2

4.3

Brayton-Cycle System

4-5

Section V

Reference Design Details

5-1

5.1

Organic-Rankine-Powered Spacecraft

5-1

5.2

Brayton-Powered Spacecraft

5-8

5.3

Overall Dimensions

5-13

5.4

Four Spacecraft Cluster 
TABLE OF CONTENTS (Cont'd.)

Para. No.

Title

$\underline{\text { Page }}$

Section VI

Structural Design and Analysis

6-1

6.1

Summary of Analysis

6-1

6.2

Basic Spacecraft Model

6-2

6.2 .1

Structural Concept

6-2

6.2 .2

Analytical Model

6-4

6.2 .3

Design Conditions

6-5

6.2 .4

Static Analysis

6-7

6.2 .5

Dynamic Analysis

6-11

6.3

Spacecraft-To-IUS- Adapter

6-12

6. 3.1

Analytical Approach

6-12

6.3 .2

Adapter Design and Analytical Model

6-12

6.3 .3

Design Conditions

6-14

6. 3.4

Static Analysis

6-14

6.3 .5

Dynamic Analysis

6-17

6.4

CG Requirements in Shuttle Bay

6-20

6.5

Power Conversion Unit (PCU)

6-20

Section VII

Attitude and Reaction Control Systems

7-1

7.1

Equipment Complement Summary

7-1

7.2

Requirements

7-1

7.2. 1

Earth Acquisition

7-1

7.2 .2

Spin-Rate Control

7-2

7.2. 3

Velocity Control

7-2

7. 2.4

On-Orbit Control

7-2 
TABLE OF CONTENTS (Cont'd.)

Para. No.

$\underline{\text { Title }}$

Page

7.3

Description

$7-4$

7.3 .1

Initial Acquisition

$7-4$

7.3 .2

Positioning SV's in the Constellation

7-6

7.3 .3

Normal On-Orbit Operation

$7-8$

7.3 .4

Orbit Maintenance

7-9

7.4

ACS Components

$7-10$

7.4 .1

Combined Earth Sensor (CES Barnes 13-169

$7-10$

7.4 .2

Reaction Wheels (RW) - Bendix

$7-10$

7.4 .3

Spin Sun Sensor - Adcole 18980

$7-10$

7.4 .4

Control Electronics Assembly

$7-11$

7.5

RCS Description

$7-14$

7.6

Changes Caused by Use of the Brayton Power System

$7-18$

Section VIII

Thermal Design and Analysis

$8-1$

8.1

Problems Addressed

8-1

8.2

Performance of Radial Heat Pipe in Spinning Mode

8-1

8.3

Equipment Shelf Temperature Control

8-2

8.4

Spacecraft Orbital Temperature Variations

$8-14$

8.5

Active Thermal Control of Frequency Standards

Section IX

Higher Altitude Version

$9-1$

9.1

Higher Altitude Version

$9-1$

Section X

Weight and Cost Summary

10-1

10.1

Weight and Cost Summary

10-1 
TABLE OF CONTENTS (Cont'd.)

Para. No.

$\underline{\text { Title }}$

$\underline{\text { Page }}$

Section XI

Critical Parameters -- Results

$11-1$

11.1

Power to User

11-1

11.2

Mean Mission Duration (MMD)

11-4

11.3

Orbit Predictability

$11-5$

11.4

Temperature Control of Frequency Standards

11-7

11.5

Vulnerability

$11-7$

Appendix A

Summary of GPS Program and Phase I Space Vehicle

A-1

Appendix B

Calculation of Maximum Allowable User Power

$\mathrm{B}-1$

Appendix C

Effect of Solar Pressure on GPS Orbit Predictability

$\mathrm{C}-1$

Appendix D

An Example of the Effect of Ephemeris Errors on User Position Determination

D-1

Appendix E

Orbit Prediction Uncertainity of Dual-Spin and Bias-Momentum Spacecraft

$\mathrm{E}-1$

Appendix F

ACS Elements for Bias-Momentum-Controlled GPS Spacecraft

F-1

Appendix G

Organic Rankine Cycle Input Data

G-1

Appendix $\mathrm{H}$

Control System Design for a Yaw Rotator

GPS Spacecraft

$\mathrm{H}-1$

Appendix $J \quad$ Estimate of Position Rate Uncertainty for the Reference Nuclear GPS Spacecraft

$\mathrm{J}-1$

Appendix K

Retrofit Designs for a Nuclear GPS

$\mathrm{K}-1$ 


\section{LIST OF ILLUSTRATIONS}

Fig. No.

Title

$\underline{\text { Page }}$

1. $2-1$

Shuttle/IUS Performance

1-4

$2.1-5$

Power-to-User Growth Options Suggested by SAMSO/YE

$2-4$

2. 2-1

MMS Sensitivity Characteristics

2-6

2.3-3

System Accuracy Degradation with Time from

Last Upload

$2-10$

$2.3-4$

Relative Displacement of GPS/NDS Spacecraft due to $1 \%$ Increment of Total Solar Pressure Force

(Case $\mathrm{i}=0$; Sun Vector in Orbit Plane)

$2.3-5$

Effect of Radial and Lateral Errors of User

Position Error

$2-14$

$3.2-1$

Thermally Integrated Design Approach

$3-13$

$3.2-2$

Thermally-Integrated Design Approach

$3-14$

$3.2-3$

Brayton Radiator Temperature Profile

$3-15$

$3.3-1$

Mechanical -Design Approach

$3-16$

$3.4-1$

Ground Rule: Four Spacecraft per Shuttle/IUS Launch

$3-17$

4. $1-1$

Dynamic Power System Development Program

4-1

4. 2-1

Organic-Rankine Cycle Schematic

$4-2$

4. 3-2

C103 Brayton System with 2400 Wt Heat Source

4-6

$4.3-3$

Superalloy Brayton System with 3200 Wt Heat Source

4-7

$4 \cdot 3-4$

C103 Brayton System with 3200 Wt Heat Source

4-7

5. 1-1

N/GPS Organic Rankine Cycle Spacecraft Profile

5-2

5. 1-2

N/GPS Spacecraft Exploded View (Organic Rankine Cycle Version) 


\section{LIST OF ILLUSTRATIONS (Cont'd.)}

Fig. No.

$\underline{\text { Title }}$

$\underline{\text { Page }}$

$5.1-3$

Organic-Rankine Power System Module Inboard Profile

$5-4$

5. 1-4 Lower Closure Bulkhead -Radiator Extension

(Both Spacecraft)

$5-6$

$5.1-5$

Equipment Module; Organic Rankine Spacecraft

$5-7$

$5.1-6$

Lower Equipment Shelf Reference Design

(Both Spacecraft)

$5-9$

$5.2-1$

N/GPS Brayton Cycle Spacecraft Profile

5-10

$5.2-2$

Brayton Power System Module; Inboard Profile

$5-11$

$5.2-3$

Equipment Module - Brayton Spacecraft

5-12

$5.2-4$

Working Fluid Loop; Brayton Spacecraft

$5-13$

$5.2-5$

Alternate Equipment Shelf Design

$5-14$

$5.3-1$

Major Dimensions

$5-15$

$5.3-2$

N/GPS External Views

$5-16$

$5.4-2$

Spacecraft Mounted in Shuttle

$5-17$

$6.2-1$

Analytical Beam Model -- Organic Rankine Spacecraft

$6-3$

$6.2-2$

Analytical Beam Model -- Brayton Spacecraft

6-3

$6.2-7$

Shuttle Installation Alternatives

6-8

$6.2-8$

Design Load Factors for Translation and Rotation

6-9

6. 3-1

Four-Spacecraft Adapter Structure

$6-13$

$6.3-3$

Four-Spacecraft Analytical Model

$6-15$

$6.3-5$

Maximum Spacecraft Deflections in Shuttle

Emergency Landing

6-17

$6 \cdot 3-7$

IUS Structure System, 1st Mode

6-18

$6.3-8$

IUS Structural System, 2nd Mode

$6-19$ 


\section{LIST OF ILLUSTRATIONS 'Cont'd.)}

Fig. No.

$\underline{\text { Title }}$

Page

$6 \cdot 5-1$

Power Conversion Unit

$6-21$

6.5-2

3-D View KIPS Dynamic Model

$6-22$

7.1-2

AVCS Component Locations

7-3

7. 3-1

Orbit Insertion Sequence

7-5

7. 5-2

Jet Orientation/Utilization

7-16

7.5-3

Reaction Control Fluid Schematic

$7-17$

8. 2-1

Pumping Capacity of ATS-6-Type Heat Pipe (Ammonia in Aluminum) Rotating About an Axis Normal to its Axis

8-2

8. 3-1

Lower Equipment Shelf Reference Design (Both

Spacecraft)

8-3

8. 3-2

Equipment Module - Brayton Spacecraft

$8-4$

8. 3-3

Heat Pipe/Header Interface

8-6

8. 3-5

Lower Equipment Shelf Component Dissipation (Watts $\mathrm{T}$ )

8-7

8. 3-6

Annular Header - Equipment Shelf (Organic-Rankine)

$8-9$

8. 3-7

Annular Header - Equipment Shelf (Organic-Rankine)

$8-9$

8. 3-8

Organic - Rankine Spacecraft Lower Equipment Shelf

Temperature Map

$8-10$

8. 3-9

Equipment Shelf Header Design Parameters (Brayton Spacecraft)

$8-11$

$8.3-10$

Brayton Spacecraft Lower Equipment Shelf

Temperature Map

Brayton Spacecraft Lower Equipment Shelf

8. 3-11

Temperature Map

$8-12$

Organic Rankine Spacecraft

$8-13$

$8.3-12 a$

Brayton Spacecraft

$8-13$

$8.3-12 b$

Overall Spacecraft Temperature Transient in

8. 4-1

Maximum Eclipse Orbit

$8-15$ 
LIST OF ILLUSTRATIONS (Cont'd.)

Fig. No.

Title

Page

$8.4-2$

N/GPS Orbital Temperature Variation

$8-16$

8. 5-1

CS Frequency Standard Temperature Variations with Heater/Thermostat Control

9.3

Spacecraft Design for 120-Hour Orbit

9-2

11.3

High Power Amplifier Efficiency Variation with

Temperature

11-3

11. $2-1$

Variation of Spacecraft MMD with Reliability of

Power System

11-5

\section{LIST OF TABLES}

Table No.

- Title

$\underline{\text { Page }}$

$1.3-1$

Summary of Improvements in Critical Parameters

1-5

2.1-1

NAV Subsystem Link Budgets

2-1

2. 1-2

Power-to-User Improvement Options

2-2

2.1-3

NAV Subsystem Link Budgets - Option (2)

2-3

2. 1-4

NAV Subsystem Link Budgets - Options (3) \& (4)

2-3

2. 1-6

Power to User Options Versus D. C. Loads*

$2-4$

2.2-2

GPS-NDS Phase I Reliability

2-7

2.3-1

One Sigma Error Budget, Meters

2-8

2. 3-2

On-Board Clock Frequency Stability

$2-9$

3. 1-1

Candidate Attitude Orientation and Control Approaches

3-5

3. 4-2

Approximate Radiator Areas - Integrated Design

$3-18$

$4 \cdot 2-2$

Organic-Rankine System Baseline Power System

4-3

4. 2-3

Organic-Rankine System Radiator Design Parameters

4-4

$4.3-4$

Brayton-Cycle Systems Alternatives

4-6

$6.2-3$

Mass Properties About Spacecraft CG

$6-4$ 
LIST OF TABLES (Cont ${ }^{\prime} \mathrm{d}$.)

Table No.

$\underline{\text { Title }}$

$\underline{\text { Page }}$

$6.2-4$

Titan IIIC Design Load Criteria

$6-5$

$6.2-5$

Space Shuttle Ascent/Descent Payload Design

Load Factors *

$6-6$

$6.2-6$

Emergency Landing Ultimate Load Factors

$6-7$

6.2-9

Summary of S/C Interface Loads - Analytical

Beam Model $\star$

$6-10$

$6.2-10$

Summary of S/C Frequencies Analytical Meam Model

6-11

$6.3-2$

Model Frequency Comparisons

$6-12$

$6.3-4$

Summary of Adapter - IUS Interface Analytical Model

$6-16$

$6.3-6$

Summary of $4 \mathrm{~S} / \mathrm{C}+$ Adapter to IUS Frequencies

Analytical Model

$6-17$

$6.5-3$

Organic-Rankine Converter System Summary of Model Analysis

$6-23$

AVCS Weight and Power

$7-2$

7.1-1

Propellant Budget

$7-14$

RCS Weight and Power Summary $7-19$

7. 5-4

Properties of Dowtherm.- A

8-6

8. 3-4

Higher Altitude Versions

9-1

9. 1-1

Fixed Antenna Design for 120-Hour Orbit

$9-2$

9. 1-2

Weight Breakdown-Organic-Rankine Spacecraft

10-2

10. 1-1

Weight Breakdown Brayton Powered Spacecraft

10-3

10. 1-3

Recurring Costs -- Organic-Rankine Spacecraft

$10-4$

10. 1-4

Recurring Costs -- Brayton Spacecraft

10-4

10. 1-5

Brayton and Organid-Rankine; Non-Recurring Dosts *

$10-5$ 


\section{LIST OF TABLES (Cont'd.)}

Table No.

11. 1-1

11. 1-3

11. 3-1

11. 5-1 $\underline{\text { Title }}$

$\underline{\text { Page }}$

Fixed Power Budget

Total Power Budget (Watts)

11-2

11-3

Maximum Orbit Ephemerides Errors Due to

Unpredicted Spacecraft Conditions

11-6

Nuclear Effects on Surfaces *
11-8 


\section{SECTION I}

\section{INTRODUCTION}

\section{$1.1 \quad$ OBJECTIVES}

This is the final report of a study to investigate the potential benefits of a nuclear (radioisotope) -powered satellite for advanced phases of the Global Positioning System (GPS) program. The study extended over approximately 11 months and 6000 hours of technical effort, and was jointly sponsored and managed by the SAMSO GPS Joint Program Office (YE) and ERDA/NRA Office of Space Applications.

A number of "critical parameters" were suggested by the SAMSO study manager (Capt. M. Marcucci) as parameters in which improvement is desired in advanced phases of the program. These parameters are not all comparable in terms of importance, scope, or relevance to nuclear-powered designs. Nevertheless, as ultimately elaborated and modified, they formed the bases for most of the design decisions which were made in the study. The critical parameters were defined as follows:

1. Power to user

2. Mean mission duration of spacecraft

3. Spacecraft orbital predictability

4. Thermal control of on-board frequency standards

5. Spacecraft vulnerability

The first four of these provided the major influences to the study, with parameters 1,3 and 4 becoming most relevant to the question of nuclear power. The study was dedicated solely to an elaboration of the capability and consequences of the nuclear-powered approach. 
The first phase of the effort was devoted to a study of the capability of a retrofit design approach, i.e., where nuclear power replaces solar power in the phase 1 navigation development satellite (NDS) of the GPS program, without major changes to the remainder of the spacecraft. This exercise enabled a detailed evaluation to be made of the factors which influence the critical parameters in the NDS satellite as well as in the nuclear-retrofit cases. This led to certain modifications in the study ground rules cond suggested the design approaches which would be most fruitful in the second phase of the study. The second phase was devoted to the preziminary design of alz-new reference nuclearpowered GPS spacecraft. The nuclear-powered spacecraft which were so evolved would be totally compatible with the existing NDS spacecraft, so that a mixed system of solar-and nuclear-powered spacecraft can exist within the program simultoneously.

The bulk of this report is devoted to the all-new nuclear-powered version. The retrofit designs are described in the Appendix (Appendix K). $1.2 \quad$ GROUND RULES

The ground rules which were established at the start of the study are as follows:

1. Spacecraft weight limit of $1200 \mathrm{lbs}$. without AKM.

2. Spacecraft volume limit based on Atlas-F capability.

3. Orbit is 12-hour GPS orbit; modifications for operation to 120-hour orbit to be described, if possible.

4. Study to emphasize improvements in critical parameters, as defined.

5. Spacecraft vulnerability considerations limited to $x$-rays; survival level $=$ JCS standard.

6. Weight analysis to be based on $\phi 1$ NDS where applicable. 
The ground rules were added to and modified following the first phase briefing on 17 November 1976, as follows:

7. All new reference designs to be based on both Organic-Rankine and Brayton-cycle power systems, with no further consideration to be given to thermoelectric systems for the advanced (nonretrofit) designs.

8. Power system heat sources to be based on $2400 \mathrm{Watt}_{\mathrm{T}}$ and 3200 Watt $_{\mathrm{T}}$ versions of $\mathrm{MHW}$ heat source.

9. Configuration and weight ground rules to be compatible with Shuttle/IUS launch with four spacecraft per launch.

10. Retain individual spacecraft interface ring at 36-inch diameter.

11. Retain possible back-up launch vehicle capability of Titan III/IUS.

Converting to the Shuttle/IUS as the baseline launch vehicle has the effect of extending the weight cutoff above 1200 pounds per spacecraft. However, a lower weight is beneficial since larger plane changes can be accomplished by the IUS as a continuous inverse function of payload weight (Figure 1.2-1). This, in turn, allows a greater number of missions to share the launch, and presumably the cost of the shuttle flight, with the GPS spacecraft on a two-stage IUS.

\section{$1.3 \quad$ SUMMARY AND CONCLUSIONS}

A nuclear-powered retrofit version of the phase 1 NDS appears feasible, using either advanced selenide radioisotope thermoelectric generators, an Organic-Rankine-cycle generator, or a Brayton-cycle generator. For each case, a custom-designed generator configuration is required which differs in varying levels of detail from the generators currently in development. The following benefits would result, however, from the retrofit approach:

1. Increased power-to-user: enables achievement of high-power mode throughout satellite design life of 7 years.

2. Improves frequency standard temperature stability. 


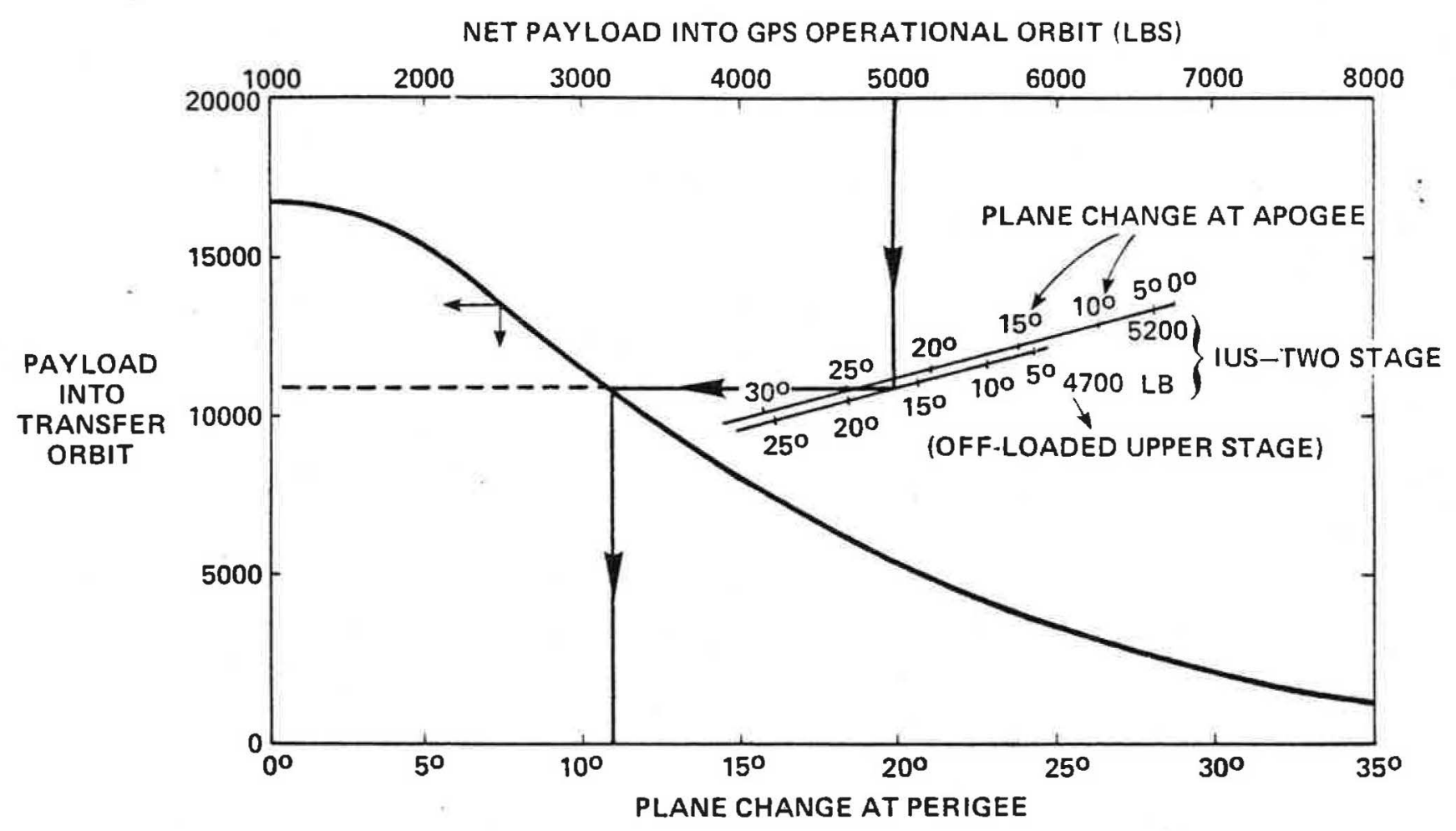

Figure 1.2-1. Shuttle/IUS Performance

3. Improves spacecraft orbital predictability somewhat.

4. Results in extended mean-mission-duration, exceeding 5.5 years.

5. Minimizes development costs of new spacecraft if nuclear power is desired

The limited improvements in critical parameters which would result from the retrofit approach, and the requirement for custom-designed power systems with the increased development costs for these, led to consideration of new spacecraft designs specifically tailored to make use of the best features of the advanced nuclear power systems, with such systems configured as closely as possible to those in current development.

Reference designs were developed for both Organic-Rankine and Brayton-cycle generators. Both spacecraft are similar in external features and overall design approach, differing only in various internal details of the power systems modules. To maximize orbit predictability, the spacecraft were designed to 
approach the ideal uniform sphere as closely as possible in a practical cylindrical shape. All external surfaces are covered with the same material with highly predictable thermal and reflective optical properties. To further enhance ephemerides predictability, the spacecraft slowly rotate in orbit, thereby minimizing the effects of solar-force variations.

To assure thermal uniformity and minimum temperature variation in orbit, all internal spacecraft equipment is cooled via the power system fluid loop. Despite the close thermal and mechanical coupling of spacecraft electronic equipment with the power system, the two may be fabricated, assembled, and tested completely separately, and joined after the separate assembly and test processes are completed. Further testing can be accomplished with electrical heater(s) in place of nuclear heat source(s), which need not be mated to the spacecraft until both heat sources and spacecraft are delivered to the launch site.

The resulting values for the critical parameters which are predicted for the reference nuclear GPS (N/GPS) spacecraft are compared to those of the NDS spacecraft in Table 1.3-1. Substantial improvements in power-to-user and in orbit predictability are achieved, and significant improvements in each of the other critical parameters are predicted.

Table 1.3-1. Summary of Improvements in Critical Parameters

\begin{tabular}{|c|c|c|c|}
\hline PARAMETER & N/GPS & $\Phi 1$ NDS & 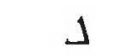 \\
\hline 1. POWER TO USER, dBW & $\begin{array}{l}\text { L1:-154 } \\
\text { L2:-163 }\end{array}$ & $\begin{array}{l}-160 \\
-166\end{array}$ & $\begin{array}{l}+6 \\
+3\end{array}$ \\
\hline 2. MEAN MISSION DURATION, YRS. & $5.5+$ & 4.5 & $+1+$ \\
\hline 3. ORBIT PEDICTABILITY, M/DAY & $0.03-0.3$ & 2.5 & $\div \times 10+$ \\
\hline 4. MAXIMUM CLOCK TEMPERATURE VARIATION, ${ }^{\circ} \mathrm{C} / O R B I T$ & & & \\
\hline WITHOUT CONTROL & 2 & 2 & - \\
\hline WITH HEATER CONTROL & 0.2 & - & $+\times 10$ \\
\hline 5. VULNERABILITY, JCS & $1+*$ & 0.01 & N/A \\
\hline
\end{tabular}

*POWER SYSTEM ONLY 
A spacecraft weight of slightly over 1000 pounds is calculated. The IUS payload weight for four spacecraft plus adapter is approximately 4400 pounds.

These results apply to both Brayton and Organic Rankine -powered versions of the reference design. A significant conclusion of the study is that neither design differs substantially from the other in any of the major parameters. However, if the superalloy Brayton system is used instead of the baselined columbium system, a reduction of some $5 \mathrm{lb}$ in the $\mathrm{L} 1(\mathrm{P})$ link results. This may be partially or fully recovered by an increase in the weight and volume of the Brayton system reference design.

Significant legacy from earlier phases of the GPS program is achieved in the navigation, attitude control, reaction control and telemetry subsystems. The reference design may be readily adapted to higher orbit operation with no apparent penalty, requiring only modifications to the antennas and to the Earth sensor.

Other than the power systems themselves no advanced or non-flightqualified technology is required or postulated for the reference nuclear-powered GPS spacecraft. One of the two radioisotope dynamic power systems is now planned to be space qualified in the Air Force Space Test Program in calendar year 1981-2. 
SECTION II

CRITICAL PARAMETERS AND PROGRAM GOALS

\section{$2.1 \quad$ POWER-TO-USER}

The current Phase I GPS navigation development satellite ( $\phi 1$ NDS) is designed for a "normal" and a "high-power" mode. The link power calculations for these modes are shown in Table 2.1-1. The $\phi 1$ NDS cannot maintain the "high-power" mode throughout its 5-year design life due to degradation of the solar arrays.

Table 2.1-1. NAV Subsystem Link Budgets, $\phi 1$ NDS

\begin{tabular}{|c|c|c|c|c|c|c|}
\hline & \multicolumn{2}{|c|}{ L-1 Normal Mode } & \multicolumn{2}{|c|}{ L-1 High Power Mode } & \multicolumn{2}{|c|}{ L-2 Nermal Mede } \\
\hline & $\mathbf{P}$ & $\mathrm{C} / \mathrm{A}$ & $\mathrm{P}$ & $\mathrm{C} / \mathrm{A}$ & $P$ & $\mathrm{C} / \mathrm{A}$ \\
\hline HPA Total RF Output & & & & & & \\
\hline Circuit Losses & & & & & & \\
\hline 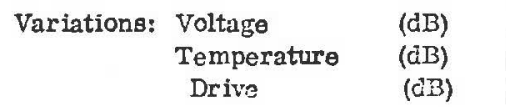 & & & & & & \\
\hline VSWR Uncertainty & & & & & & \\
\hline Measurement Error & & & & & & \\
\hline FRNSA Output & & & & & & \\
\hline P Signal Ratio, Wor st Case (dS) & -4.65 & & -6.00 & & 0.0 & \\
\hline C/A Signal Ratio, Worst Case (dB) & & -2.09 & & -1.4 & & $\div 0.44$ \\
\hline Antenna Gain & & & & & & \\
\hline Net Link Losse日 & & & & & & \\
\hline User Signal & -162.41 & -159.85 & -162.44 & -157.84 & -165.56 & -165.12 \\
\hline Required Signal & -163.00 & -160.00 & -163.00 & -158.00 & -166.00 & -165.00 \\
\hline Margin & +.59 & +.15 & +.56 & $\div 16$ & +.44 & +.88 \\
\hline
\end{tabular}

Improvements in this critical parameter can be made by distributing excess power equally among the four signals in the two links, or can be made 
preferentially in whatever order is desired by the user community. As a guideline for this study, the following sequence of improvements (Table 2.1-2) was suggested by the SAMSO program manager.

Table 2.1-2. Power-to-User Improvement Options

\begin{tabular}{|c|l|}
\hline Option Nos. & \\
\hline 1. & Description \\
2. & Increase of L-1 C/A link power to maximum allowable flux \\
& density limits. \\
3. & Increase of L-1 P link power to $3 \mathrm{~dB}$ below revised C/A link \\
& $\begin{array}{l}\text { level } \\
\text { Use of any remaining power to increase the L-2 links by } 3 \mathrm{~dB} .\end{array}$ \\
\hline
\end{tabular}

As indicated in Appendix B, maximum allowable user power for the L-1 $\mathrm{C} / \mathrm{A}$ link is computed to be $-154 \mathrm{dBW}$ based on the current allowable flux density limit of $-152 \mathrm{dBW} / \mathrm{m}^{2} / 4 \mathrm{kHz}$, established by international convention. The $-154 \mathrm{dBW}$ value takes into account the spectrum spreading factor of the L-1 C/A link and also considers atmospheric attenuation to be zero, whereas the worst case link calculations allow $2 \mathrm{~dB}$ for atmospheric losses.

Table 2.1-3 shows the link power budget which would be required for option 2. Similarly Table 2.1-4 projects the power budgets of options 3 and 4. The four increased power options and the baseline normal mode are summarized graphically in Figure 2. 1-5.

For initial estimating purposes, DC-RF conversion efficiencies, and housekeeping power requirements similar to those of the $\phi 1$ NDS were used to calculate the approximate required spacecraft DC power for the four options, shown in Table 2. 1-6. 
Table 2.1-3. NAV Subsystem Link Budgets - Option (2)

\begin{tabular}{|c|c|c|c|c|c|}
\hline & & \multicolumn{2}{|c|}{$\begin{array}{c}\text { MAX. LEGAL } \\
\text { L-1 POWER }\end{array}$} & \multicolumn{2}{|c|}{$\begin{array}{l}\text { UNCHANGED } \\
\text { L-2 MODE }\end{array}$} \\
\hline & & (P) & $(C / A)$ & (P) & $(C / A)$ \\
\hline HPA TOTAL RF OUTPUT & $(\mathrm{dBW})$ & & & & \\
\hline FILTER AND FEED LOSSES & $(d B)$ & & & & \\
\hline PRNSA OUTPUT & (dBW) & & & & \\
\hline $\begin{array}{l}\text { P SIGNAL RATIO (WORST } \\
\text { CASE) }\end{array}$ & $(d B)$ & -9.5 & - & 0.0 & \\
\hline $\begin{array}{l}\text { C/A SIGNAL RATIO } \\
\text { (WORST CASE) }\end{array}$ & $(d B)$ & - & -0.5 & - & +0.4 \\
\hline ANTENNA GAIN & $(d B)$ & & & & \\
\hline NET LINK LOSSES & $(d B)$ & & & & \\
\hline USER SIGNAL & (dBW) & -163.0 & .154 .0 & -165.6 & -165.6 \\
\hline
\end{tabular}

Table 2.1-4. NAV Subsystem Link Budgets - Options (3) \& (4)

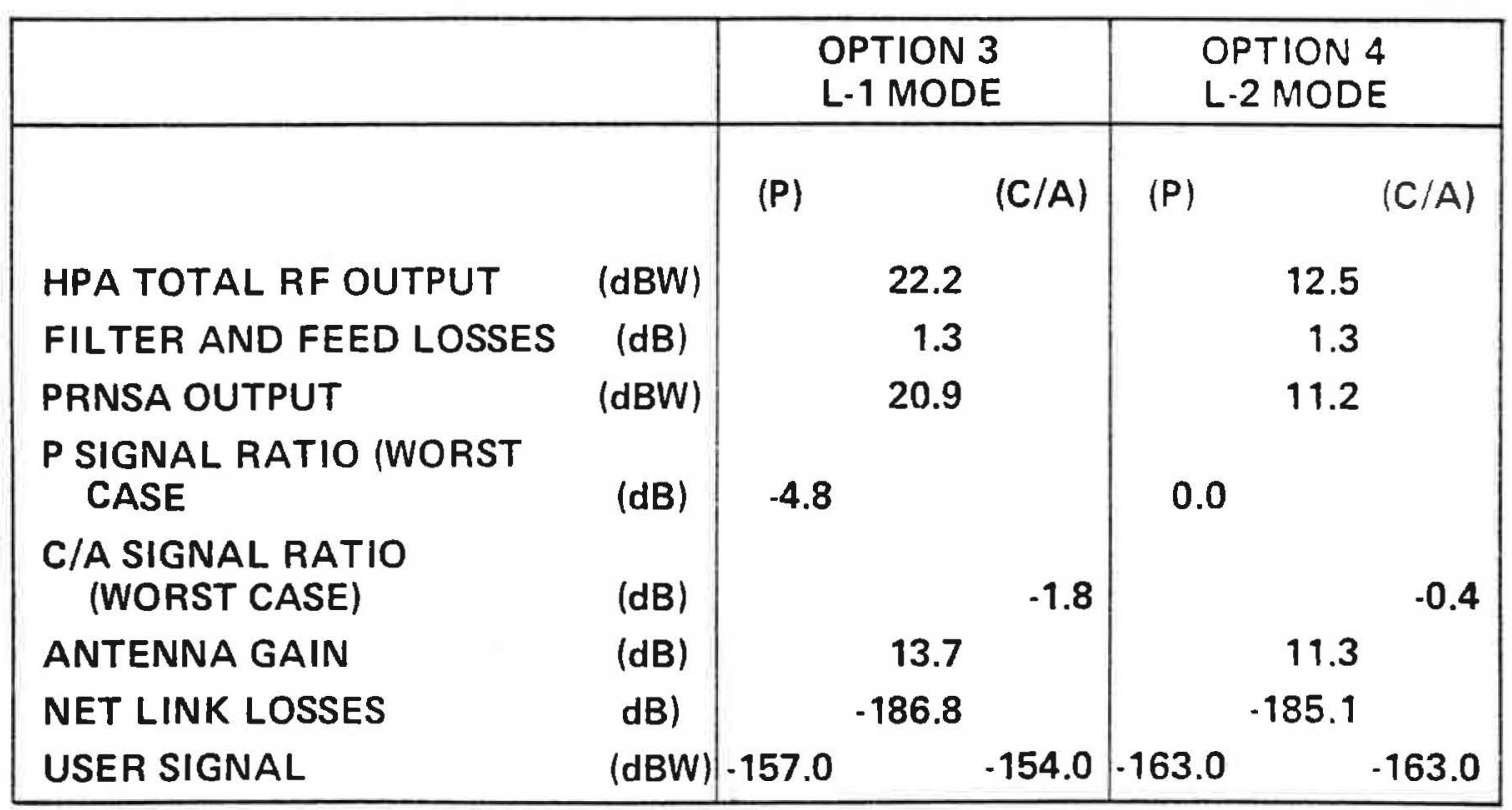




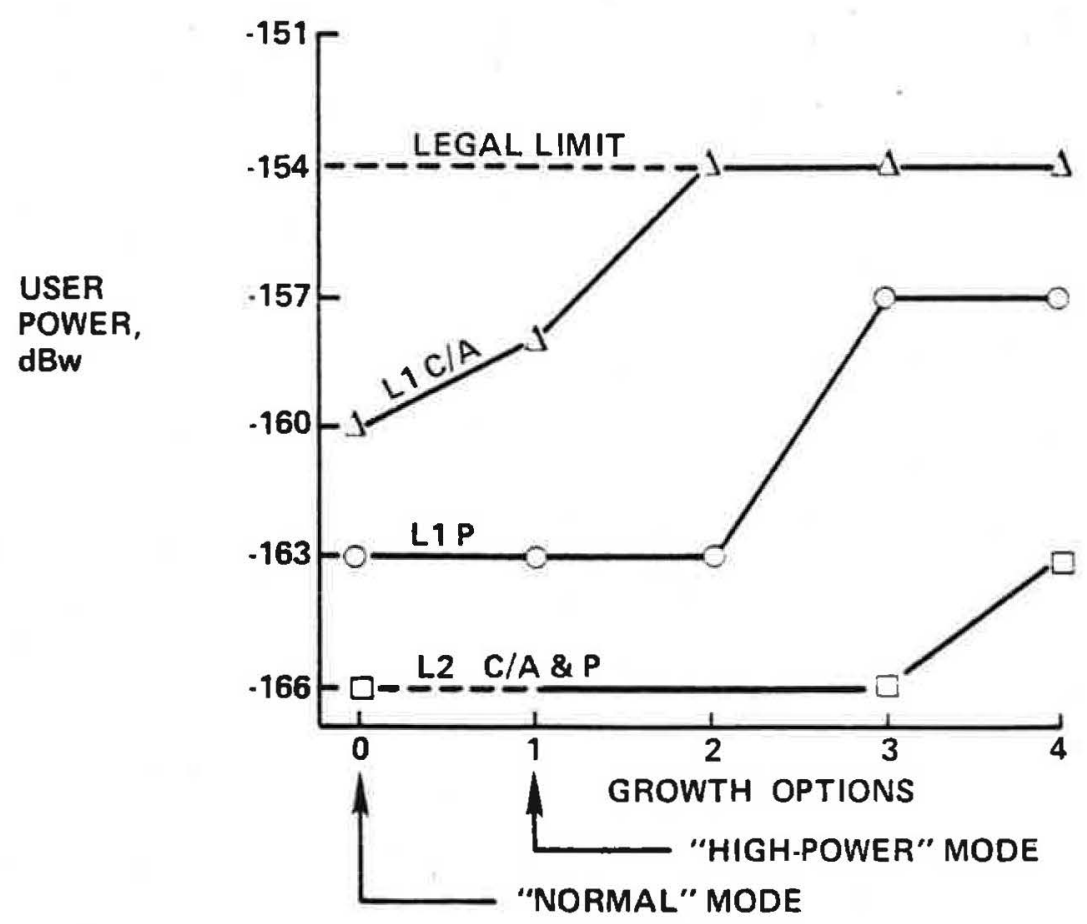

Figure 2.1-5. Power-to-User Growth Options Suggested by SAMSO/YE

Table 2.1-6. Power to User Options Versus D.C. Loads*

\begin{tabular}{|c|c|c|c|}
\hline & OPTION & $\begin{array}{c}\text { NAV SUBSYS. } \\
\begin{array}{c}\text { D.C. POWER } \\
\text { (Watts) }\end{array} \\
\end{array}$ & $\begin{array}{l}\text { SPACECRAFT } \\
\text { D.C. POWER } \\
\text { (Watts) } \\
\end{array}$ \\
\hline 1. & $\begin{array}{l}\text { MAINTAIN HIGH POWER } \\
\text { MODE THROUGHOUT } \\
\text { SPACECRAFT LIFE }\end{array}$ & 306 & 378 \\
\hline 2. & $\begin{array}{l}\text { INCREASE L-1 C/A LINK } \\
\text { POWER TO ALLOWABLE } \\
\text { POWER FLUX DENSITY } \\
\text { LIMITS }\end{array}$ & 498 & 565 \\
\hline 3. & $\begin{array}{l}\text { INCREASE L-1 P LINK } \\
\text { POWER TO } 3 \text { dB BELOW } \\
\text { REVISED C/A LINK }\end{array}$ & 637 & 704 \\
\hline & $\begin{array}{l}\text { INCREASE L-2 LINKS BY } \\
3 \mathrm{~dB}\end{array}$ & 664 & 731 \\
\hline
\end{tabular}

* Numerical Values are Approximate; Used for Scoping.Purposes 
The requirement for some $700 \mathrm{~W}_{\mathrm{E}}$ of spacecraft prime power led to the specification of nuclear-dynamic power systems for the nonretrofit nuclear spacecraft designs. Although these systems are scheduled to be ground-demonstrated at the $1300 \mathrm{~W}_{\mathrm{E}}$ output power level, they are projected to be capable of operating under derated conditions with good efficiency, by off-loading nuclear-isotope fuel. The characteristics of the power systems used in the new-design phase of the study are discussed in Section IV.

\subsection{MEAN-MISSION-DURATION}

The mean mission duration (MMD) of a satellite is a function of both its reliability $R$ and its truncated lifetime or wear-out limit $T_{L}$, as given by the relationship:

$$
M M D=\int_{0}^{T} \quad R d t=\frac{1}{\lambda}\left[1-e^{-\left(\Sigma_{i} \lambda_{i}\right) T_{L}}\right]
$$

Typically, $R$ is derived from the failure rates, $\lambda_{i}$, of the various components of the spacecraft:

$$
R=e^{-\left(\lambda_{1}+\lambda_{2}+\ldots\right) t}
$$

Over the range of values expected in spacecraft programs, the meanmission-duration is much more sensitive to truncated lifetime than it is to failure rate. An example of these sensitivities is given by Figure 2.2-1 which shows that for a constant truncated lifetime of 5 year's, and failure rates ranging from 0.05 failures per year ( 5 year reliability of 0.779 ) to 0.010 failures per year ( 5 year reliability of 0.951 ), the MMD improves by only $11 \%$ while for a constant failure rate of 0.05 and truncated lifetimes ranging from 5 years to 8 years, the MMD improves by $49 \%$.

The $\phi 1$ NDS has an estimated mission duration of approximately 4.5 years, based on the reliability values shown in Table 2.2-2. Design lifetime is given as 6 years, governed by solar array degradation. 


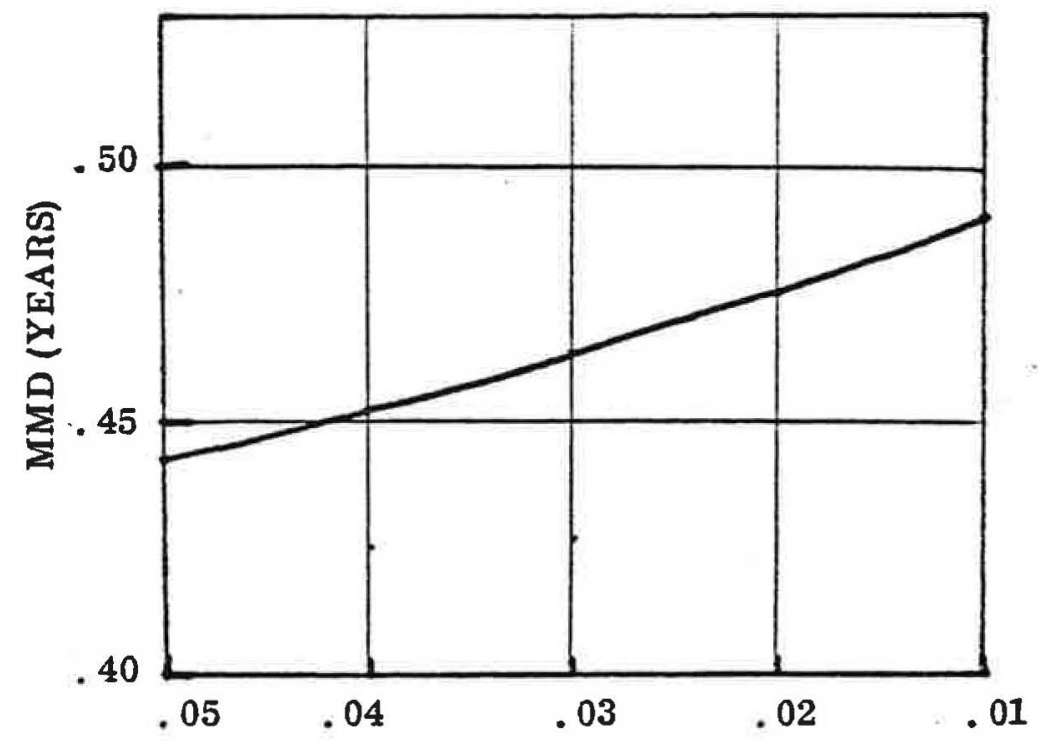

Spacecraft failure rate $(\lambda)$-failures/year

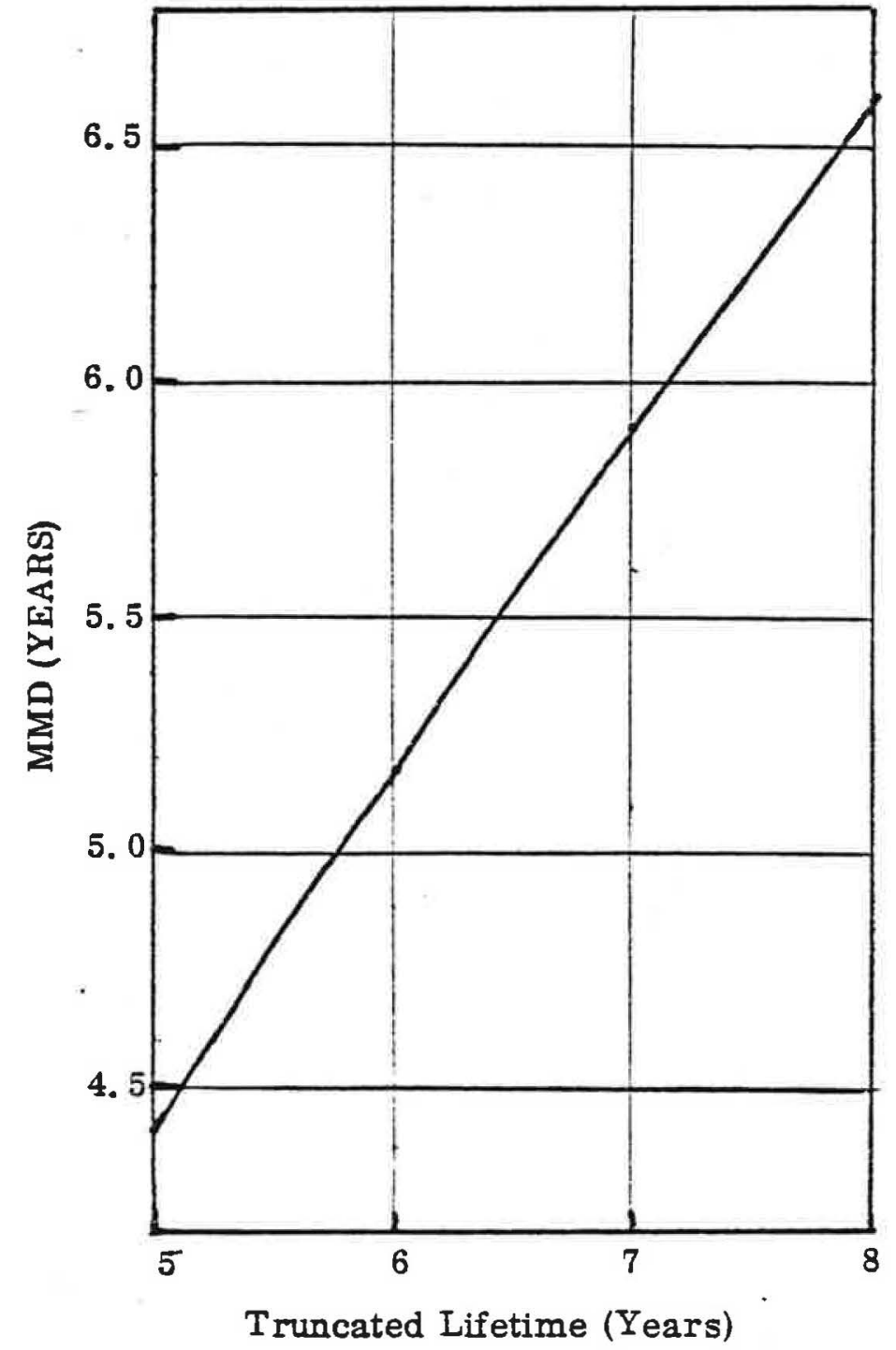

Figure 2.2-1. MMS Sensitivity Characteristics 
Table 2.2-2. GPS-NDS Phase I Reliability

\section{SUBSYSTEM}

$\mathrm{T}, \mathrm{T} \& \mathrm{C}$

AVCS

EPS

RCS

THERMAL

STRUCTURE

NAV

OIS

NDS SPACECRAFT
5-YEAR RELIABIIITY*

.8927

.9847

.9628

.9945

.9999

.9992

.9407

9968

.7885

\section{* July 1976 predictions}

The MMD is a critical parameter of the entire GPS program because of the large number of operational satellites eventually required. Ultimately, the satellite replacement rate and hence the logistics costs of the space segment, will be determined by the MMD of the satellites in orbit. Over a period of tens of years, hundreds of millions of dollars can be expended in this replacement program, so that even small changes in satellite MMD can have substanial economic impact.

A nuclear spacecraft may extend the design lifetime and hence MMD by virtue of the lower predicted degradation rate of the power subsystem, and by the elimination of the battery failure mode as a limiting factor. Reliability values currently assigned to the nuclear power systems are based on subsystem and com:ponent test histories of similar, if not identical, components. Ground and space testing now planned as part of the development programs and follow-on test programs will, it is expected, generate such numbers with adequate confidence levels. At the present time, no wearout or degradation modes for the dynamic generators have been identified, other than degradation due to radioisotope decay.

For the purpose of this study, a range of possible reliability values is considered for the nuclear power systems. Design lifetime is assumed to be at least 7 years. 
The question of orbital stability relates to the accuracy of the ranging calculations done by the user equipment. Table 2.3-1 below shows the one-sigma user equivalent range error (UERE) budget in meters for the Phase I and Phase III systems.

Table 2.3-1. One Sigma Error Budget, Meters

\begin{tabular}{|l|c|c|}
\hline & Phase I & Phase III \\
\hline Space Vehicle Ephemeris & 3.9 & 1.6 \\
Atmospheric Delay & 2.6 to $5.6^{*}$ & 2.6 to $5.6^{*}$ \\
Space Vehicle Group Delay & 2.8 & 1.0 \\
Receiver Noise & 1.6 & 1.6 \\
Multipati & 1.3 to $3.0^{*}$ & 1.3 to $3.0^{*}$ \\
& & \\
Total, Root-Sum-Squared & 5.9 to 8.2 & 3.9 to 6.9 \\
\hline
\end{tabular}

*varies with propagation path

These are initiation errors, valid for 12 hours following satellite

upload with fresh data. For later times, accuracy will degrade at a rate determined by the combination of 1) clock drift, and 2) satellite drift.

The space vehicle group delay in Table 2.3-1 includes the contribution of clock drift. Table 2.3-2 summarizes, to an order of magnitude, the expected stability of the three frequency standards which are loosely associated with the three phases of the GPS program. Clearly, achievement of the phase I error budget will require the introduction of cesium clocks in phase $\mathrm{I}$, as is now planned for the later spacecraft in this phase.

With the successful introduction of cesium clocks the errors due to clock drift and to unpredictable ephemeris drift will be of the same order of magnitude. Subsequent introduction of a more stable hydrogen-master frequency standard will not improve system degradation rate significantly without the simultaneous introduction of a spacecraft with a more accurately predictable vehicle ephemeris. This is illustrated 
Table 2.3-2. On-Board Clock Frequency Stability

\begin{tabular}{|c|c|c|c|c|}
\hline \multirow{2}{*}{$\begin{array}{c}\text { GPS } \\
\text { PHASE }\end{array}$} & \multirow{2}{*}{$\begin{array}{l}\text { FREQUJENCY } \\
\text { STANDARD }\end{array}$} & \multirow{2}{*}{$\begin{array}{l}\text { FREQUENCY } \\
\text { STABILITY } \\
\frac{\Delta f}{I} / \text { day }\end{array}$} & \multicolumn{2}{|c|}{ Range Error Buildup } \\
\hline & & & $\mathrm{m} / \mathrm{hr}$ & m/day \\
\hline I & $\mathrm{Rb}$ & $10^{-12}$ & 1.1 & 26 \\
\hline II & Cs & $10^{-13}$ & 0.1 & 2.6 \\
\hline III & $\mathrm{H}_{2}$-maser & $10^{-14}$ & 0.01 & 0.26 \\
\hline
\end{tabular}

in Figure 2.3-3, where the user-calculated position error is shown as a function of time from last update, assuming the geometric arrangement of a complete $3 \times 8$ satellite constellation. The upper pair of curves assumes a spacecraft of the NDS type, with an unpredicted ephemeris drift of $1.25 \mathrm{~m} /$ day, as currently claimed by NSWC*. The lower curve assumes the introduction of a spacecraft whose ephemeris can be predicted to within $0.25 \mathrm{~m} /$ day.

The factors which generally influence ephemeris predictability are discussed below, with mathematical detail in Appendix C. Those which apply to the reference nuclear-powered spacecraft have been calculated -- and will be discussed in Section $\mathrm{X}$, following the description of the reference designs.

To the extent that vehicle ephemeris drift can be modelled and predicted, the ground user will be able to compensate for this drift by the application of perturbation coefficients which will be transmitted by the satellite along with the Keplerian orbit elements. Such important disturbances as geodetic variations in the gravitational field of the Earth, and the major solar-pressure effects, can be modelled and predicted with good accuracy. However, there are significant disturbances which

*Naval Surface Weapons Center, the program element charged with software development for GPS upload. 

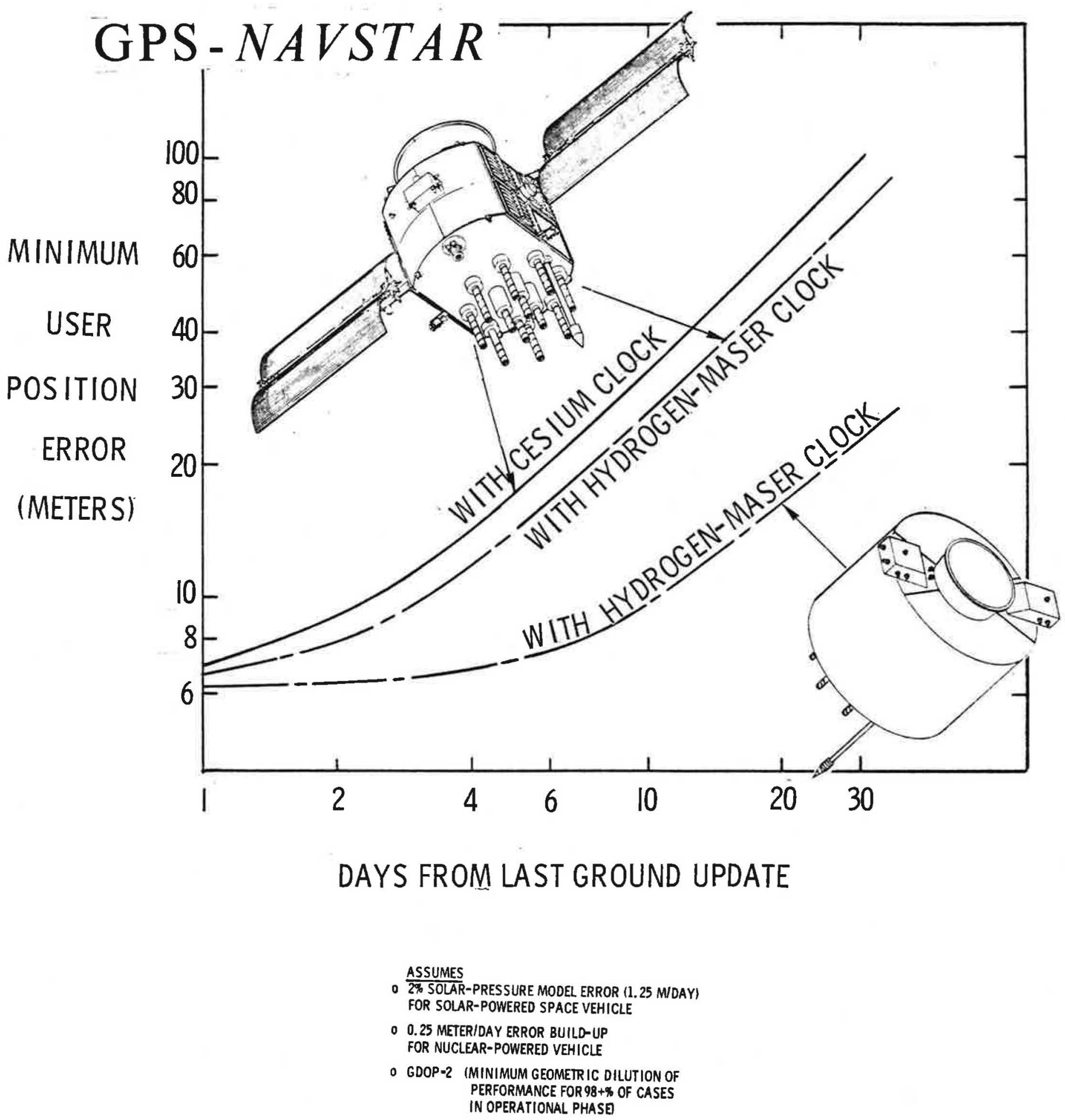

Figure 2.3-3. System Accuracy Degradation with Time from Last Upload 
cannot be modelled or which vary so much or so unpredictably from day to day that they will result in unpredictable changes in satellite ephemerides, and hence timedependent degradation in system accuracy. Among such unpredictable disturbances are:

1. Solar pressure variations due to non-repetitive orientation of spacecraft surfaces with different $\alpha$ and $\epsilon$ values to the sun.

2. Solar pressure variations due to non-repetitive shadowing of spacecraft surfaces by the antennas, thrust cone, etc.

3. Solar pressure variations due to apparently random changes in thermal-control louver blade angle (i.e. not synchronized with orbital position).

4. Impulse variations due to thermal radiation variations controlled by the louvers (or due to misestimated temperatures on various surfaces).

5. Impulses due to gas leakage from the reaction control system or outgassing from any surface.

The phenomena noted in items 1 . and 2. above do, of course, repeat on an annual basis and only change slowly from day to day. However, the extent to which they repeat is governed by the accuracy of the control system, and the extent to which the satellite can be physically modelled. For example, Figure 2.3-4 shows the error build-up in both radial and lateral components of the predicted orbit due to a $1 \%$ error in modelling the solar pressure force on a spacecraft of NDS size for the worst case (noon) orbit (Appendix C). If this "error" is fixed in time, it will eventually be corrected by the ground-based computer. However, if it varies from day to day, the computer model will not identify it and correct it. Clearly, errors of a few meters/ day are possible from this source.

The significance of item 3 arises from the fact that the louvers are mounted on the sun-parallel faces in the NDS satellites. Consequently, when they open they almost always reflect the sun and when closed they are essentially shadowed. 


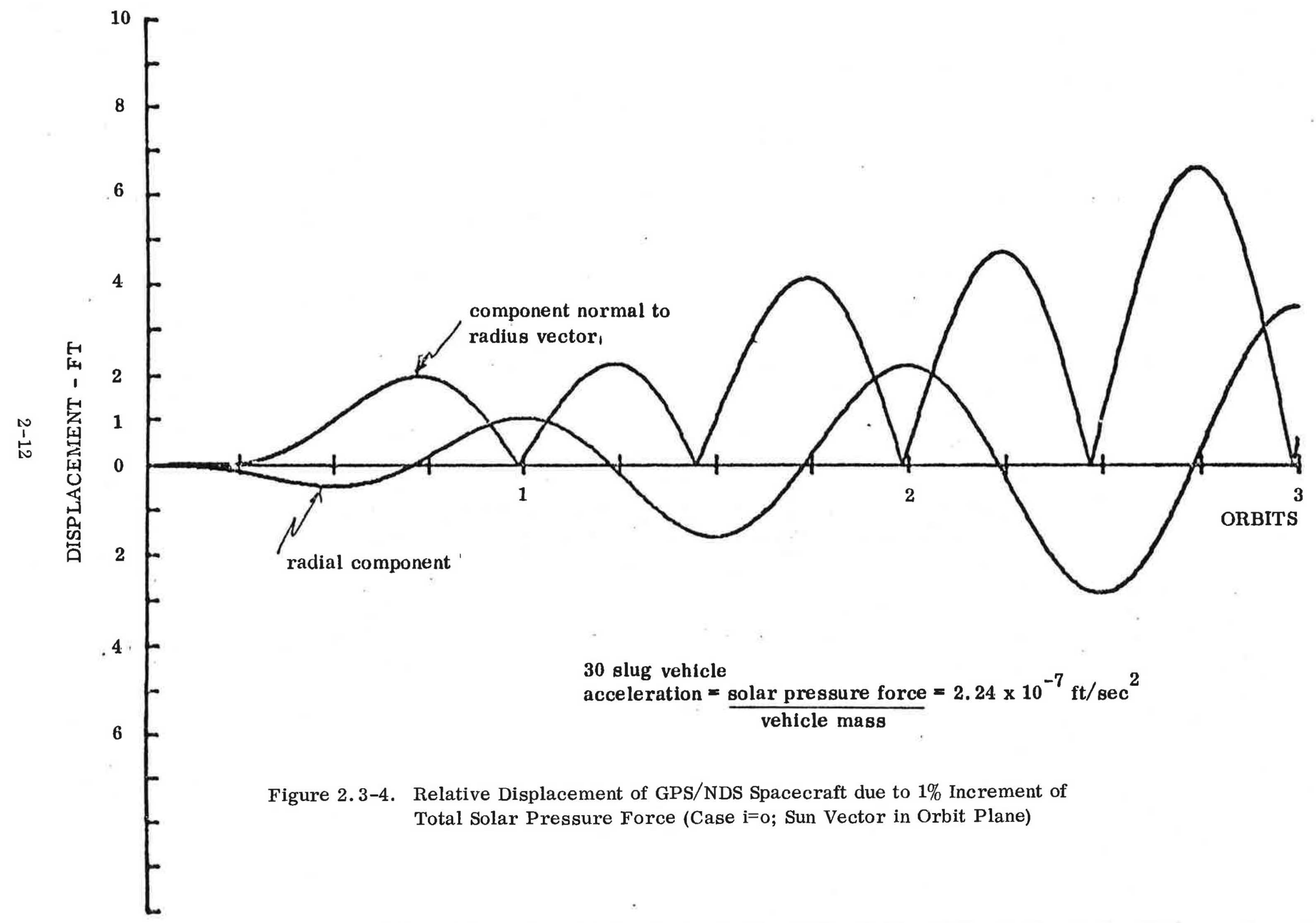


Item 4 can be significant because the louvers on both sides of the spacecraft operate independently. A certain amount of skepticism that thermal radiation from within the spacecraft can result in significant orbital offset is inevitable. However, it is noted that for radiant energy, the ratio of energy to momentum is the constant $c$ (speed of light in vacuum). Therefore, if the energy radiated is $Q$, then the momentum imparted by this radiation is $Q / c$, and the total force applied is simply $(1 / \mathrm{c}) \mathrm{dQ} / \mathrm{dt}=$ radiated power $/ \mathrm{c}$.

An unbalanced radiation of 500 watts can, therefore, result in a force of 1. 7 micronewtons, which if applied in the same direction for 12 hours to a 1000 pound spacecraft, can result in a displacement of 3.5 meters, clearly within the range of interest. The fact that the louvers operate independently and in response to baseplate temperature would cause this force to be applied at various portions of the orbit, and hence, since spacecraft yaw angle is constantly changing, in unpredictable directions.

The simple calculation illustrates by inference the care in design and assembly which is necessary to minimize the effects of item 5.

It is obvious that by eliminating the solar arrays, a nuclear spacecraft would have a positive impact on item 1 above simply by reducing the projected area and hence the absolute magnitude of the total solar force. Perhaps not as obviously, however, a nuclear spacecraft can have a substantial impact on all of the above disturbances. Elimination of the sun-pointing requirement of the solar arrays introduces a number of design options which can substantially improve or eliminate most or all of the listed disturbances. This requirement strongly influenced the design approaches which were explored and that which was ultimately selected, as discussed in Section III.

The relative value of radial and transverse errors in satellite position to ground user position errors is illustrated in Figure 2.3-5 (Appendix D). It is assumed that the positions of satellites 1 and 2 are known exactly and that the position of satellite 3 is known in error. The error is either radial, $\delta_{R}$, or transverse, $\delta_{\perp}$. The user position error resulting from this simplified analysis is shown on the figure 
as a function of satellite position above the horizon. Clearly, for all useful satellite positions, the radial error is more important, by a factor of 4 or more, than the transverse error in satellite position.

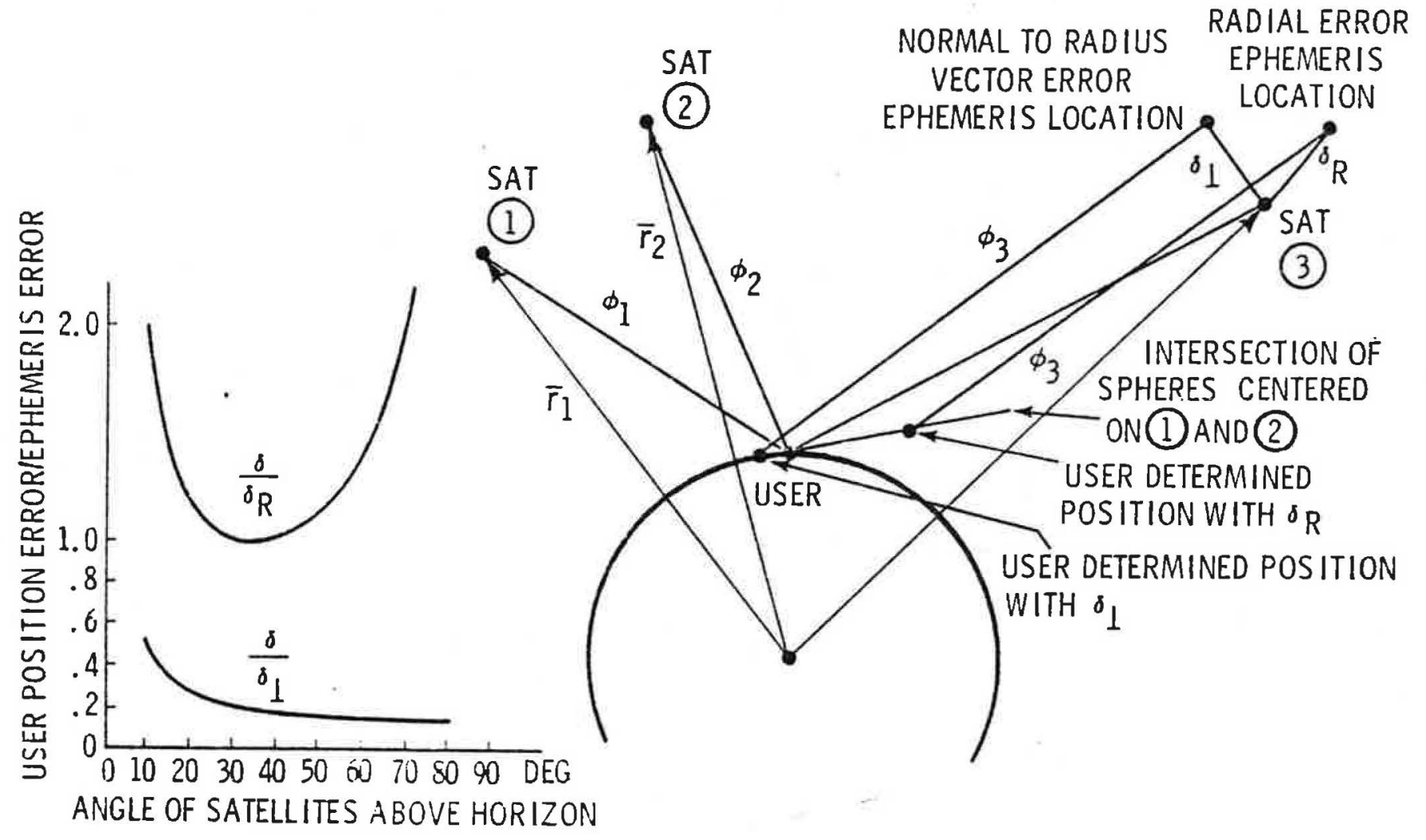

Figure 2.3-5. Effect of Radial and Lateral Errors of User Position Error 2.4 THERMAL CONTROL OF ON-BOARD CLOCKS

The stability of the on-board frequency standard is closely allied to the question of ephemeris drift, as was discussed above. Unpredictable clock drift from the time of last upload will cause an error in the ranging calculation, given by $\delta R=c(\Delta f / f)$, where $c$ is the speed of light, equivalent to a radial error in satellite position.

The order-of-magnitude stability characteristics of the various frequency standards is given in Table 2.3-2, based on various random noise factors controlling the standards. In addition, these are all temperature sensitive to one degree or another. Although they typically are temperature-controlled by internal heaters, the achievable control is a function of the temperature stability of the heat sink. 
A rule-of-thumb which was applied in this study is that the baseplate temperature coefficient of frequency stability, $\Delta \mathrm{f} / \mathrm{f}$ per ${ }^{\circ} \mathrm{C}$, is the same order of magnitude as the random frequency drift per day as given in Table 2.3-2. Therefore, baseplate temperature control of $1^{\circ} \mathrm{C} /$ day for the worst-case orbit was set as a design goal for the reference spacecraft.

\section{$2.5 \quad$ SURVIVABILITY}

Because Phase III of the GPS program is specified to meet JCS survivability standards, the study ground rules were that the power systems themselves were to be evaluated for JCS-level survivability. Two areas of possible concern in the nuclear power systems are the electronic control systems and the external thermal-control surfaces. These were to be evaluated for vulnerability insofar as possible. However, it became apparent in the course of the study that, although spacecraft survivability is a critical parameter to the GPS program, it is not primarily a power systems issue at the present time. 



\section{SECTION III}

\section{APPROACH TO THE REFERENCE DESIGN}

\subsection{SELECTION OF ATTITUDE CONTROL APPROACH}

The attitude control approach has a substantial impact on many of the spacecraft subsystems and on the method of operating the spacecraft in space, as well as on weight, power, reliability, and orbit predictability.

With regard to the important orbit predictability problem, the reference design strives to approximate the ideal spherical satellite. Because this can only be approximated by an exterior profile with a minimum number of variations in surface reflectivity/absorptivity, the selected attitude control approach will have a significant modifying effect on the orbit predictability. This comes about through two influences:

1. The attitude control approach determines which spacecraft surfaces face the sun for how long and in which sequence.

2. The attitude control approach and system design determines the magnitude, frequency, and direction of jet firings required to unload stored angular momentum. The magnitude and possible methods of applying the jet impulses will determine the errors which this action can introduce into the predicted ephemeris.

Three different attitude-control approaches were considered, with two versions of one of them forming a total set of four:

1. Dual-Spin - In this approach, the rotation of the spacecraft body is used to provide a relatively large angular momentum normal to the orbit plane, thus making the spacecraft very insensitive to torques about the other two axes. This system has been highly 
developed and is in wide use for synchronous-orbit communications spacecraft. With nuclear power, it can be used in other orbits as well, since power is not generated by body-mounted solar cells. It requires that directional Earth-oriented antennas be despun via a bearing and power-transfer assembly. The antenna assembly rotates at orbit frequency, while the spacecraft typically rotates $30-60 \mathrm{rpm}$. This configuration is shown below.

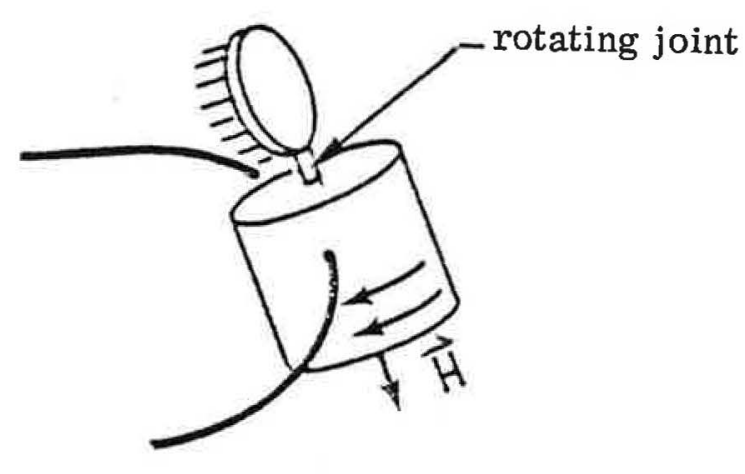

2. Bias Momentum - In this approach, some of the benefits of the spinner are retained by providing the biasing angular momentum via an internal wheel in constant rotation. The major advantage is elimination of the rotating bearing and RF joint to the antennas. Because of practical limitations on size, weight, and rotation speed of the wheel, however, the biasing momentum is typically much less than that of the spinner. Two choices for orientation of the momentum vector are possible:

2a. $\mathrm{H}$ along spacecraft $\mathrm{Z}$ axis -- This essentially reproduces the spinner attitude orientation. Antenna array must be oriented in the $\mathrm{x}$ or $\mathrm{y}$ direction, as in the spinner, but without the rotational bearing. The following illustration shows this configuration. 


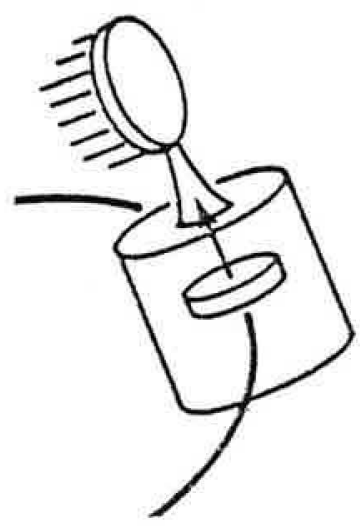

2b. H normal to spacecraft $\mathrm{Z}$ axis -- This retains the attitude orientation of the phase I space vehicle, although without the possibility for yaw steering. This is shown in the drawing below.

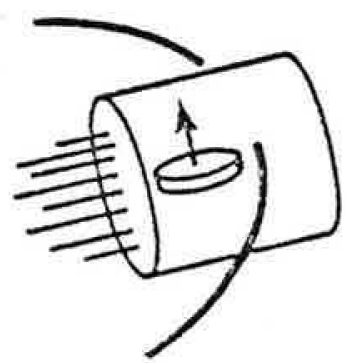

3. Zero-Momentum Yaw Rotator - In this approach the spacecraft is fully stabilized in pitch and roll by a redundant set of momentum wheels, but allowed to rotate slowly in yaw (about the spacecraft axis). This is shown in the drawing below.

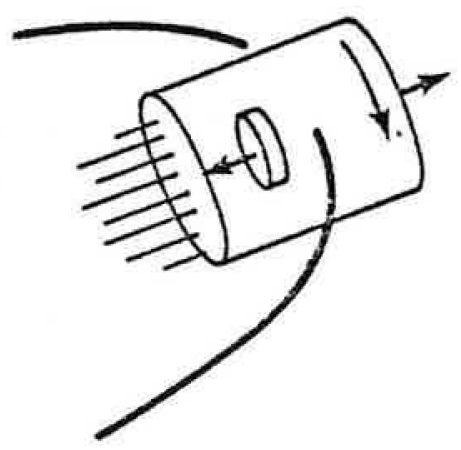

An internal momentum wheel is used to counterbalance the yaw momentum so that there is no net momentum to precess in orbit. In the case of the Organic Rankine power system the angular 
momentum of the rotating unit alone is adequate (and ideal) to provide the counterbalancing momentum. The yaw rotation rate is normally uncontrolled, but can be corrected by jets if necessary. Since the antenna pattern is axially symmetric about the Earthpointing axis, no harm is done to the transmitted signal by the slow (1/2-to-1 $\mathrm{rpm})$ rotation.

The remaining obvious candidate for attitude control consideration is the fully 3-axis-controlled vehicle. This was believed to offer no significant advantage over a bias momentum system unless yaw steering was to be performed in order to assist in thermal control. In that case, the spacecraft would resemble the retrofit nuclear-powered spacecraft already considered in the first phase of the study.

The various factors which entered into the decision on attitude control approach are listed in Table 3.1-1, with factors rated or estimated for the four approaches which were considered. The zero-momentum yaw rotator (no. 4) was ultimately selected, with the classical dual-spinner (no. 1) a close second. Although in early estimates the latter produced the least orbit prediction error, as well as a low weight, low power, and space-proven approach, the former scored quite acceptibly in orbit predictability and was judged to be equal or superior to the dual spinner in every other respect, with the possible exception of the peak power dissipation of the attitude control system. This peak power difference (ultimately it vanished) was not considered significant compared to most of the more basic factors of operation and performance on the list. In addition, it was suspected that the orbit predictability error could be made very much smaller for the yaw rotator than this early estimate. This ultimately proved to be the case.

The various factors are each described below, and the bases for the estimates and judgments are discussed.

\section{Estimated Orbit-Prediction Error}

The dual spin and bias-momentum systems with spacecraft $\mathrm{Z}$-axis normal to the orbit plane (cases 1 and 2) both share a common advantage in the significant orbit-predictability area: they maintain a fixed body orientation to the sun as they orbit the earth. Over a period of time, therefore, the prediction error with regard to 
Table 3.1-1. Candidate Attitude Orientation and Control Approaches

\begin{tabular}{|c|c|c|c|c|}
\hline CHARACTERISTIC & $\begin{array}{l}1 \\
\text { DUAL SPIN, } \\
\text { Z NORMALTO } \\
\text { ORBIT PLANS }\end{array}$ & $\begin{array}{l}2 \\
\text { BIAS MOMENTUM, } \\
\text { Z NORMAL TO }\end{array}$ & $\begin{array}{l}3 \\
\text { BIAS MOMENTUM, } \\
\text { Z TO NADIR }\end{array}$ & $\begin{array}{c}4 \\
\text { ZERO MOMENTUM, } \\
\text { Z TO NADIR. } \\
\text { YAW ROTATOR }\end{array}$ \\
\hline $\begin{array}{l}\text { 1. ESTIMATED ORBIT PREDICTION } \\
\text { ERROR, M/DAY } \\
\text { RADIAL } \\
\text { TRANSVERSE }\end{array}$ & $\begin{array}{l}0.1 \\
0.3\end{array}$ & $\begin{array}{l}0.6 \\
1.5\end{array}$ & $\begin{array}{l}>0.6 \\
>15\end{array}$ & 0.2 \\
\hline $\begin{array}{l}\text { ATTITUDE CONTROL SYSTEM WEIGHT, } \\
\text { LBS. (5 DAY/10 DAY UNLOAD PERIOD) }\end{array}$ & $39 / 39$ & $140 / 200$ & $74 / 99$ & $61 / 71$ \\
\hline RADOME REQUIRED & YES & YES & YES & NO \\
\hline WEIGHT OF ACS WITH RADOME, LBS. & $82 / 82$ & $183 / 243$ & $112 / 137$ & $61 / 71$ \\
\hline PEAK ACS POWER, WATTS (E) & 20 & 15 & 20 & $20-40$ \\
\hline $\begin{array}{l}\text { 3. RF POWER LOSS (RADOME \& ROT. } \\
\text { JOINT), dB }\end{array}$ & 0.2 & 0.1 & 0.1 & $\mathbf{0}$ \\
\hline $\begin{array}{l}\text { 4. RELIABILITY AND SPACE EXPERIENCE } \\
\text { RATING }\end{array}$ & A & B & B & A \\
\hline 5. THERMAL CONTROL RATING & A & B & B & A \\
\hline 6. VEHICLE SHAPE CONSTRAINT RATING & B & C & C & A \\
\hline 7. GPS LEGACY RATING & C & C & A & A \\
\hline $\begin{array}{l}\text { 8. EASE OF AUTONOMOUS UNLOADING } \\
\text { OF WHEELS }\end{array}$ & c & B & B & A \\
\hline $\begin{array}{l}\text { 9. HEAT PIPE APPLICABILITY FOR } \\
\text { THERMAL CONTROL }\end{array}$ & D & A & A & B \\
\hline $\begin{array}{l}\text { 10. COMPATIBLE WITH DYNAMIC POWER } \\
\text { SYSTEMS }\end{array}$ & B & B & A & A \\
\hline
\end{tabular}


solar pressure effects on the vehicle body approaches a very low value, estimated at some $0.1 \mathrm{~m} /$ day in the radial direction and $0.2 \mathrm{~m} /$ day in the normal-to-radial direction (Appendix $\mathrm{E}$ ).

The remaining error is that associated with reaction jet firings. Because of its large angular momentum, the spinner is particularly insensitive to external torques; therefore, internal momentum wheels are not provided. However, because it maintains a fixed orientation in inertial space, the spacecraft orientation to the Earth changes a little each day as the vehicle orbit plane precesses with respect to Earth. The orbit precession rate due to Earth oblateness has been calculated to be 0.0272 degrees/day (Appendix E). Therefore, if an antenna pointing error of \pm 0.5 degree is specified, and if half this error is assigned to the results of orbital precession, then this correction must be made at approximately 9-day intervals, by jet firing.

- A matched pair of jets are fired to precess the vehicle angular momentum vector by the required 0.25 degree. However, imperfections in jet orientation and thrust matching can lead to a net translation of the vehicle, thus upsetting its orbit, and hence obviously its predictability.

The major part of this error can be cancelled out by only applying half the correction at each of two opposite positions in the vehicle orbit, 6 hours apart. In this way, total errors of only 0.1 meter/ day in the radial direction and 0.3 meter/day in the transverse direction are predicted (Appendix E).

The bias momentum systems are both more easily precessed by solar-pressure torques since the biasing angular momenta which can be achieved by internal wheels are much smaller than those which can be achieved by a rotating spacecraft. Consequently more frequent thrusting is required, estimated at a 1.5-day 
interval rather than the 9.2 days for the spinner. Because the solar pressure uncertainties are also somewhat greater for the non-rotating spacecraft, the estimated orbit prediction error is as shown for the Z-normal-to-orbit-plane, case 2 in Table 3.1-1. Case 3 in Table 3.1-1 involves the additional uncertainty of a spacecraft which changes its body angle to the sun as it orbits the Earth, so that its uncertainty would be greater than that of case 2, and was not estimated separately.

The zero-momentum case is entirely different from the other three. Because it includes reaction wheels to compensate for imposed torques about two axes, with the third $(Z)$ axis essentially free-spinning, jet thrusting intervals are tradeable against momentum wheel storage capacity. It has been estimated (Section VII) that a very modest wheel size of $0.5 \mathrm{ft}-\mathrm{lb}-\mathrm{sec}$ would extend this interval to 10 days. Moreover, jet unloading requires only very small thrust levels, thus significantly reducing the orbit ephemeris disturbance which this can introduce. In this case, jet thrusting may be the limiting uncertainty. As in the phase 1 (solar-powered) spacecraft the modelability of the spacecraft was initially thought to be limiting. However, because of the factors of reduced size, simplified shape, simplified and uniform surface finish, passive and uniform thermal radiation, and continuous rotation in orbit, the solar force uncertainty was initially estimated to be at least an order of magnitude less than that of the existing phase 1 NDS design. Following completion of the reference design, this factor was re-estimated in more detail, and the initial estimate was found to be conservative by a significant factor (see Section $\mathrm{X}$ ).

2. Attitude Control System Weight and Power These weight figures have been estimated (Appendix F) for both 5-day and 10-day unload periods. Case 1 (spinner) exhibits the 
lowest weight of this subsystem. Because of the fixed high angular momentum of the satellite, the ACS weight does not depend on the unload period. As explained earlier, the jet firing period depends only on the orbit precession rate.

In the spinner case, as well as in cases 2 and 3, the antenna array is fixed with respect to the Earth and exhibits a continually changing view to the sun. Projected areas, angles, and shadows change continuously over each orbit, but also from day to day. This provides an element of error in estimating solar pressure forces which would be undesirable in a system designed for high predictability. Therefore cases 1, 2, and 3 would very likely require a radome-type dielectric cover over the antennas, thus substantially reducing this uncertainty factor. The weight of such a cover has been estimated for the three cases and added to that of the ACS in Table 3. 1-1.

The zero-momentum yaw rotator, case 4 , continually rotates about the $\mathrm{Z}$-axis, so that projected area, shadows, and other variations are reproduced many times and averaged out over each orbit. This case, therefore, is judged not to require a dielectric cover, so that in total weight of ACS plus antenna cover, it is the lightest system in the group.

In principle, case 4 may be expected to exhibit the largest peak power consumption. This comes about because the actuators of the momentum wheels are continually transferring momentum from one wheel to another as the vehicle rotates. During a one-quarter $\left(90^{\circ}\right)$ rotation about the spacecraft $\mathrm{Z}$-axis, the yaw and roll axes are completely interchanged, together with momentum wheels that are fixed to the body of the spacecraft. Any momentum stored in one wheel, therefore, must be transferred to the other and viceversa. During the 5-to-10-day period between unloading of the 
wheels, the power consumed by the actuators gradually rises until the peak power is reached. Following unload this falls abruptly to the low initial value and begins the cycle again.

In a more detailed study of this control approach, it was determined that a peak power consumption of ten watts per wheel is adequate, leading to a total peak power of less than 20 watts for a 10-day unload period (Section VII). This could be reduced still further by unloading more frequently, or by pre-loading the wheels in the opposite direction, or by some combination of the two approaches.

\section{RF Power Loss}

This line illustrates the fact that cases 1, 2, and 3 all suffer some RF power loss through the radome, while case 1 suffers an additional loss in the rotating joint. Values are order-of-magnitude estimates.

4. Reliability and Space Experience

Spin stabilization historically has been the most commonly applied attitude control technique, leading to a high experience rating and good expectation for high reliability for this system. Bias momentum systems have been rarely flown, probably because the limited angular momenta which can be practically achieved lead to a preference for a fully 3 -axis controlled vehicle.

A zero-momentum yaw-rotator satellite is not very common, but considerable practical space experience has been accumulated on such a system over the past several years by an Air Force satellite program with classified details.

5. Thermal Control

In general, rotating spacecraft may be said to enjoy a thermal control advantage because the solar input is averaged over the 
entire surface of the spacecraft. Bias momentum systems in nonequatorial or non-sum synchronous orbits have more restrictive geometric conditions leading to more complex thermal control solutions, in general. (In the selected reference design, with its forced-convection cooling of equipment, this difference would not be applicable. )

6. Vehicle Shape Factor

This factor relates to the ease of approximating an ideal shape for orbit predictability: a sphere with a uniform surface coating. The two bias-momentum approaches would be significantly penalized because the thermal control requirements would introduce a variety of surface coatings and treatments. The spinner is constrained mainly by the need for an "erected" antenna system. The yaw rotator, however, offers the maximum freedom of selection of external shape because of the benefits of the rotating action on thermal control, while retaining zero net angular momentum and the more favorable antenna orientation. (Again, in the reference design, this difference would be minimized because of the selected thermalcontrol approach.)

\section{GPS Program Legacy}

Cases 1 and 2 would benefit least from the phase 1 GPS satellite program since they involve a completely different attitude orientation in space. Cases 3 and 4 retain the same navigation system/ antenna arrangement, and similar attitude and reaction control system.

\section{Ease of Autonomous Unloading of Wheels}

Although each of the attitude control approaches can, in principle, be mechanized for completely autonomous operation (i. e., without ground command), this item rates the ease or degree of complication involved in doing so, The spinner is given the poorest rating in this category because not only is a relatively high level of thrusting involved, but it must be applied at opposing positions of the same 
orbit, $180^{\circ}$ apart. Also, the input information, which determines the proper time to initiate thrusting corrections, must be derived from the scanning Earth sensor, as must be the feedback measurement on which the control of the thrusters is based.

The bias momentum systems involve moderately high thrust levels to precess the spin vector and are also dependent on information from the scanning Earth sensor. By contrast, the zero-momentum yaw-rotator has the lowest thrust levels, and derives its control information from a tachometer which measures the speed of the momentum wheels. When these reach their maximum preset values, the thrusters may be programmed to fire until the speed reduces to its preset value. This is judged to be the simplest of the possible autonomous control schemes. (In addition, the low correction torques which are required here make the use of magnetic unloading most feasible. This would eliminate the possibility of any orbit perturbation from this source.)

9. Heat Pipe Applicability for Thermal Control This item merely identifies the fact that a spinner satellite would find it generally impossible to use heat pipes to assist in thermal control. This is due to the centrifugal gravity of the satellite which would severly restrict the permissible orientation and direction of heat flow of the heat pipes. The yaw rotator could have a similar potential problem, if its rotation rate were such as to create a troublesome magnitude of centrifugal force. This is not the case in the reference design (Section VII).

10. Compatible with Dynamic Power Systems

This item expresses the fact that the rotating units of the power systems would prefer to be oriented in the spacecraft $\mathrm{Z}$-direction for launch (or IUS) operation. For case 3 , this 
would result in an angular momentum in the wrong direction on orbit, thus requiring either a less-preferred orientation during launch or a counterbalancing momentum wheel on orbit. In any case, the problem is not controlling, and more important for the higher-angular-momentum Organic Rankine power system than for the Brayton power system.

As indicated previously, these considerations led to the selection of the two-axis-controlled, zero-net-mementum yaw rotator as the reference design approach.

\subsection{THERMAL CONTROL APPROACH}

In their most common conceptions, nuclear power systems, like solarpower systems, are treated as appendages to the main body of the spacecraft. This is the so-called "separated" design approach in which the power system is interfaced electrically and mechanically, but isolated thermally from the remainder of the spacecraft. With the preferred attitude-control approach the slow axial rotation of the spacecraft would simplify thermal control of the spacecraft, but excursions of $10-20^{\circ} \mathrm{C}$ during eclipse orbits may be expected, even with the application of louvers and heaters.

The desire for closer thermal control of the frequency standards, and the knowledge that the power system radiator requirements result in a largely "empty" radiator internally, led to the consideration of the so-called "integrated" design. In this approach, the spacecraft equipment is contained within the power system radiator and all (or most) of the equipment thermal dissipation is rejected through the radiator.

In earlier studies of an integrated design *, the spacecraft dissipative equipment was mounted to the internal surfaces of a flat sided, isothermal, powersystem radiator of low overall temperature. However, the power systems now under development do not have isothermal or low-average-temperature (relative to required

*AEC SNSO 3063-2, Standardization and Economics of Nuclear Spacecraft, Fairchild Report No. 217-053-2, March 1973 
equipment temperature) radiators. Radiator temperatures typically vary from approximately $100^{\circ} \mathrm{C}$ at the inlet to approximately 5 -to-50 ${ }^{\circ} \mathrm{C}$ at the outlet, depending on the power system, for the Brayton and Organic-Rankine systems respectively. In addition, it was known from the earlier studies that mounting equipment to the radiator panels directly is mechanically intricate and presents accessibility and testability problems.

The thermally-integrated design approach which was finally selected is shown in Figure 3.2-1. An oversized radiator is used to overcool the radiator fluid, in the Organic Rankine case, to approximately $30^{\circ} \mathrm{C}$. From the radiator outlet the liquid Dowtherm-A working fluid is routed to an equipment cooling section internally. Spacecraft equipment is cooled by conduction to the fluid loop, so as not to penetrate the hermetic integrity of the loop. This should raise the loop temperature by some $10^{\circ} \mathrm{C}$, from whence it is returned to the power system.

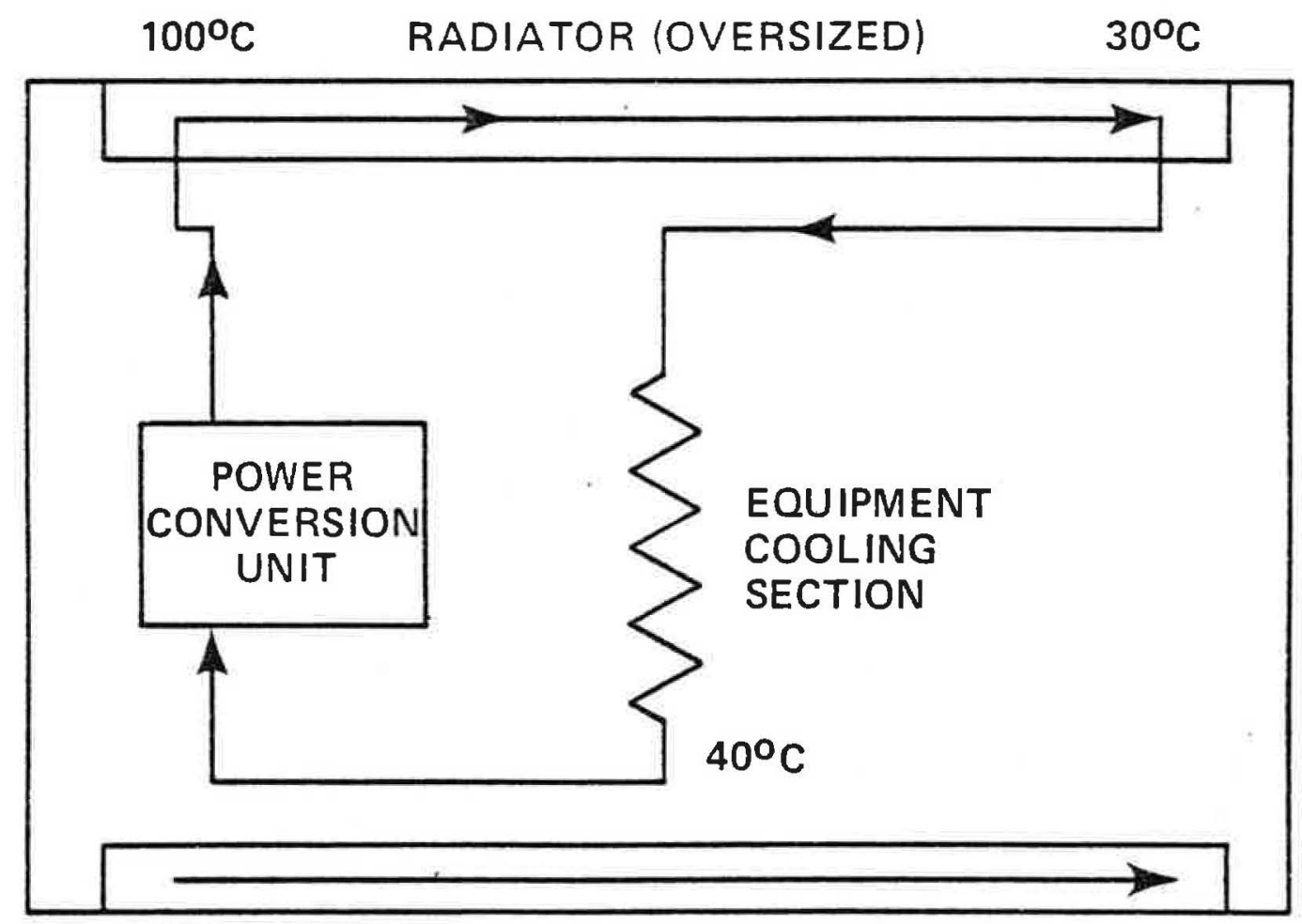

Figure 3.2-1. Thermally Integrated Design Approach 
The Organic Rankine system is not affected by the temperature rise introduced by the equipment since it normally requires approximately $70^{\circ} \mathrm{C}$ as the inlet to the jet condensor, and will provide a radiator by-pass loop to control this temperature by mixing the radiator flow with uncooled by-pass flow. The Brayton system, however, requires a lower inlet temperature to its compressor, and so a modification of this approach is required. This is shown in Figure 3.2-2. In this case, the He/Xe working fluid is removed from the radiator at what would be its normal outlet temperature and routed through the equipment section. Its temperature rise through the equipment, however, is approximately $35^{\circ} \mathrm{C}$. Therefore, it is then returned to a separate section of the radiator and allowed to recool to 5 -to $-10^{\circ} \mathrm{C}$ before returning to the Brayton rotating unit. If this is implemented as shown in Figure 3.2-2, the radiator temperature will show a variation as illustrated in Figure 3.2-3. The skin can be continuous, therefore, and no insulator section need be interspersed between the two radiator sections.

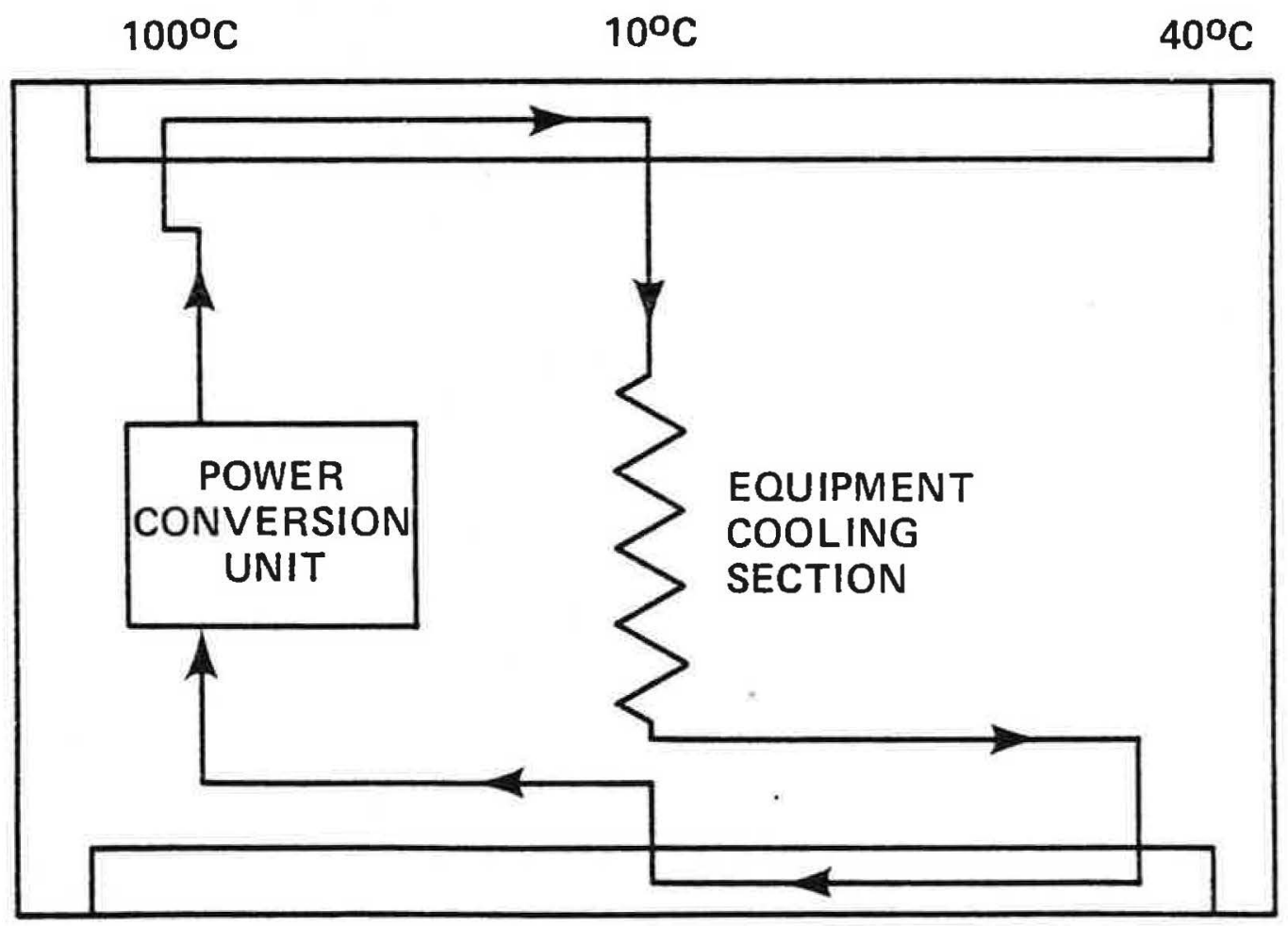

Figure 3.2-2. Thermally-Integrated Design Approach 


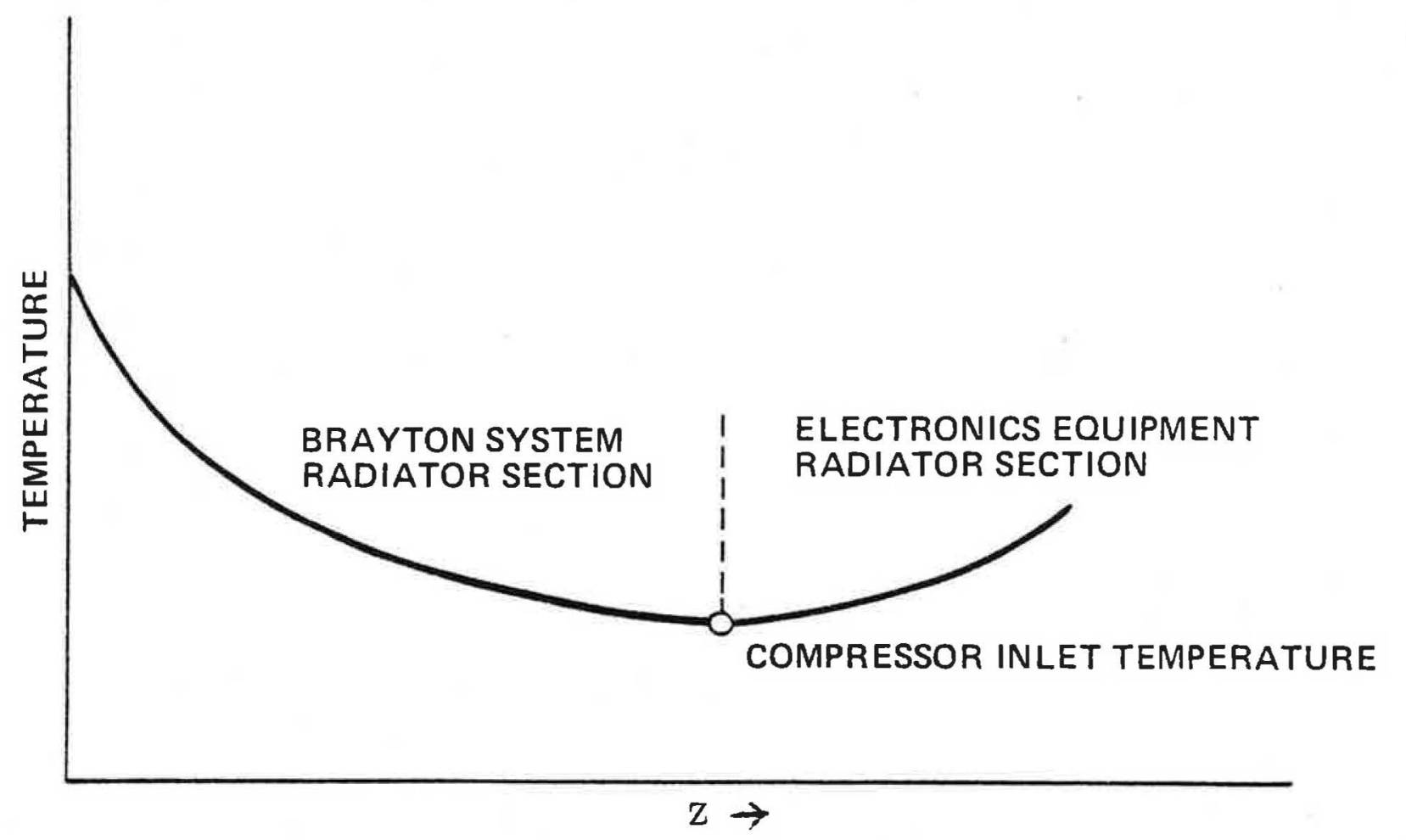

Figure 3.2-3. Brayton Radiator Temperature Profile

In this design approach, the entire spacecraft, with minor exceptions, is enclosed by the power system radiator and is cooled by the power system fluid loop. It is a good approximation, therefore, to say that the spacecraft is thermally interconnected during eclipse, and will cool down as a single body. Internal gradients must be kept small because of the relatively high sink temperature (i. e., of the fluid loop) into which the equipment must reject heat.

Therefore, a good approximation of the cool-down rate during eclipse can be gotten by calculating

$$
\frac{d T}{d t}=\frac{\Delta Q}{M C_{p}}
$$

For $\Delta Q=500$ watts as the approximate heat loss during eclipse, $M=1200$ pounds as the overall spacecraft weight and $\mathrm{C}_{\mathrm{p}}=0.2 \mathrm{cal} / \mathrm{gm}^{\circ} \mathrm{C}$ as a representative specific heat for solid structure,

$$
\frac{\mathrm{dT}}{\mathrm{dt}}=4^{\circ} \mathrm{C} / \mathrm{hr}
$$


It will be seen that this is a good approximation for the spacecraft as a whole (Section VIII) and is achieved without any active thermal control action whatsoever. Internal equipment, e.g. , the frequency standards, will tend naturally to vary less during eclipse, and can be finely controlled by active means because of the small base variation of the heat sink. This verifies the main advantage of the thermally integrated design approach which was adopted.

\section{3 MECHANICAL DESIGN APPROACH}

A mechanical design which would allow the spacecraft equipment to be assembled and tested separately from the power system, despite their eventual close integration, was desired. In concept, this is shown in Figure 3.3-1. The two major subassemblies would be manufactured and assembled separately, but with mechanical and thermal interfaces provided for their eventual mating. As may be anticipated, the thermal interfaces provide the major design problems in this approach, but are shown to be amendable to solution for both candidate power systems.

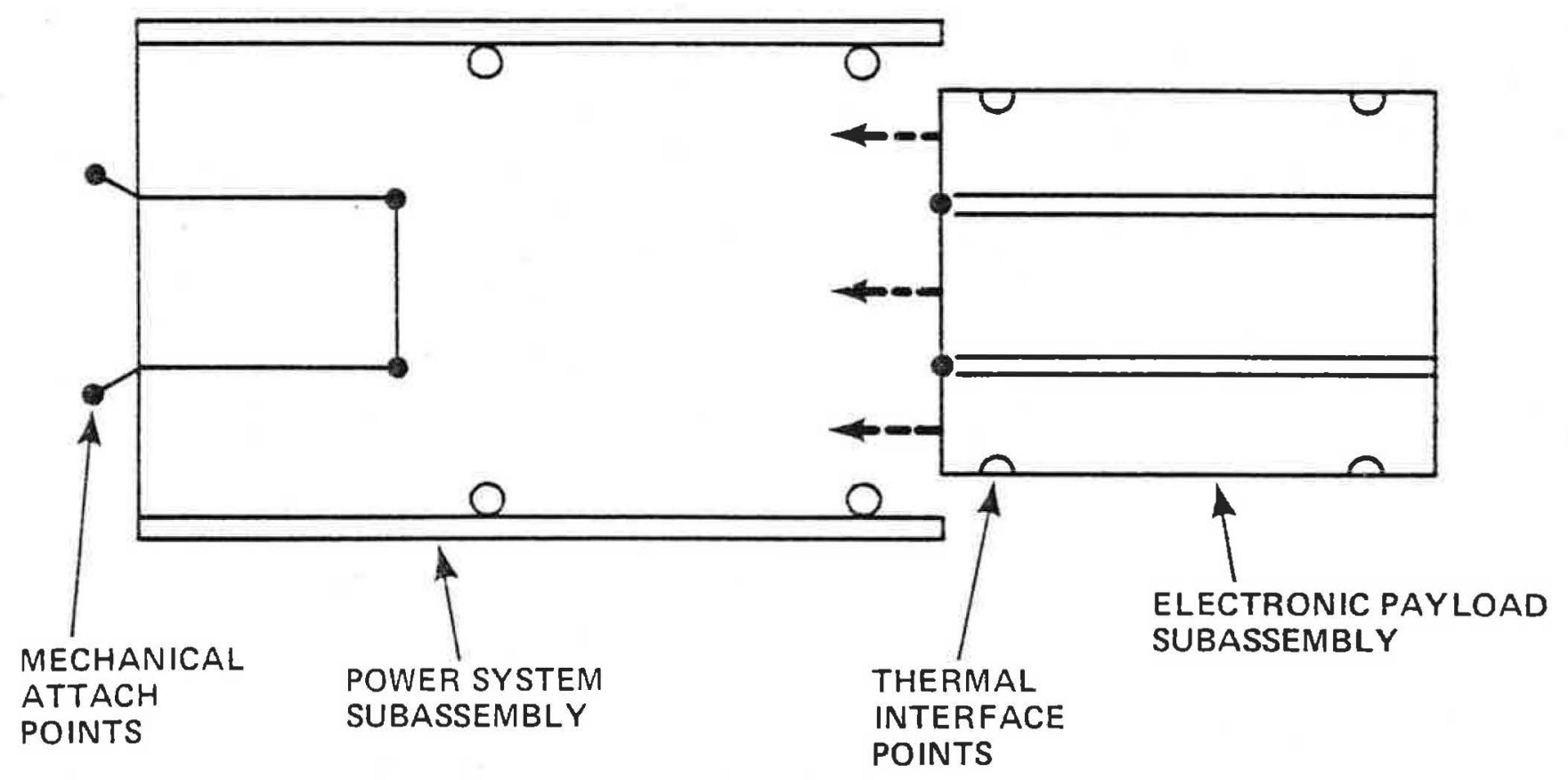

Figure 3.3-1. Mechanical-Design Approach 
Before embarking on detailed design efforts, it is necessary to verify that the overall size of the power system radiator is in keeping with the general desired spacecraft size, and can be accommodated with the desire to mount four spacecraft atop the IUS.

Figure 3.4-1 shows three alternative cross sections for clustering four spacecraft within the shuttle bay. Flat-sided spacecraft might be preferred from the viewpoints of fabricability and the mounting of louvers, equipment radiators, etc., in the typical spacecraft. However, in this case, the use of louvers or directly-radiating equipment is not anticipated. Some small advantage in external surface area per unit length is also evident for flat-sided spacecraft, but from the viewpoint of simplicity and orbit predictability, the cylindrical shape was selected. For a shuttle bay diameter of 15 feet, a spacecraft diameter of 72 inches allows 2 inches of clearance between adjacent spacecraft and 3 inches between spacecraft and shuttle bay wall, in the four spacecraft cluster.

\section{ALTERNATIVE APPROACHES:}
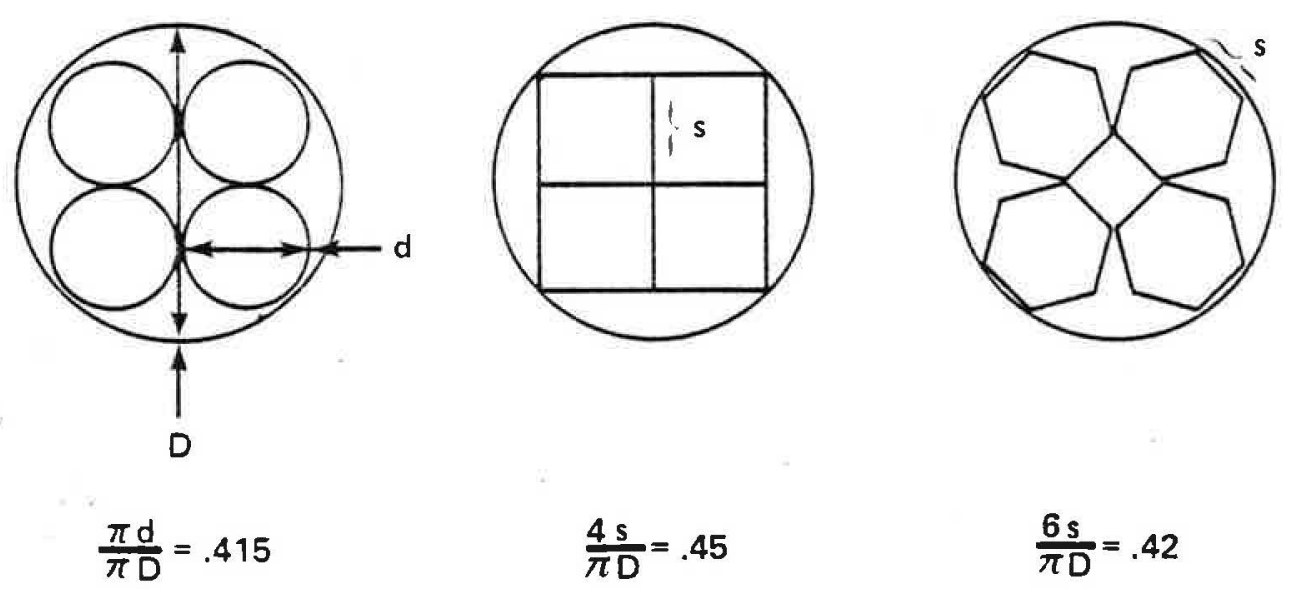

DECISION: RETAIN CYLINDER FOR SIMPLICITY AND ORBIT PREDICTABILITY; PAY SLIGHT PENALTY IN LENGTH OF RADIATOR

Figure 3.4-1. Ground Rule: Four Spacecraft per Shuttle/IUS Launch 
The nominal spacecraft length, governed mainly by required radiated area, is calculated approximately in Table 3.4-2 for the two power systems. Although the two systems have substantially differing thermal power outputs to reject (3050 and 4600 watts $_{t}$ ) the effective radiating temperatures differ in the right direction to compensate for this difference, resulting in a required radiator area, and spacecraft length, which is remarkably similar for both spacecraft. After considerations of realistic radiator fin effectiveness factors and fluid-to-wall heat transfer coefficients, the radiator sizes increased to approximately 130 square feet in both cases. However, radiator length only increased to approximately 6 feet, as a result of including a portion of one end face as part of the radiator (Section V).

Table 3.4-2. Approximate Radiator Areas - Integrated Design

\begin{tabular}{|c|c|c|c|}
\hline 1 & BRAYTON & $\begin{array}{l}\text { ORGANIC } \\
\text { RANKINE }\end{array}$ & UNIT \\
\hline TOTAL THERMAL POWER, $\mathrm{P}_{T}$ & 3200 & 4800 & WATTS $_{T}$ \\
\hline APPROX. ELECTRICAL POWER, PE & 600 & 750 & WATTE \\
\hline APPROX. RADIATED RF PWR, PRF & 150 & 200 & WATTRF \\
\hline RADIATED THERMAL PWR, PT-PRF & 3050 & 4600 & WATT $_{T}$ \\
\hline NOMINAL RADIATOR INLET TEMP. & 235 & 212 & OF \\
\hline NOMINAL RADIATOR OUTLET TEMP. & 10 & 100 & OF \\
\hline EFFECTIVE RADIATOR TEMP, TEFF & 100 & 152 & OF \\
\hline$P_{T} / A @ T_{E F F}(\epsilon=0.8)$ & 39.3 & 56.0 & WATTST/FT'2 \\
\hline MAX. SOLAR INPUT $(c=0.2)$ & 8.3 & 8.3 & WATTS $_{T} / \mathrm{FT}^{2}$ \\
\hline NOMINAL RADIATOR $\mathrm{P}_{\mathrm{T}} / \mathrm{A}$ & 31.0 & 47.7 & WATTS $T / F_{T}^{2}$ \\
\hline NOMINAL RADIATOR AREA & 98.4 & 96.4 & $\mathrm{FT}^{2}$ \\
\hline NOMINAL RADIATOR LENGTH $(\mathrm{D}=6 \mathrm{FT})$ & 5.2 & 5.1 & $\mathrm{FT}$ \\
\hline
\end{tabular}




\section{SECTION IV}

\section{POWER SYSTEMS INPUT DATA}

\section{1 CANDIDATE POWER SYSTEMS}

Two dynamic turbine-alternator power systems are currently under development by ERDA/NRA: the Organic Rankine Kilowatt Isotope Power System (KIPS), and the Brayton Isotope Power System (BIPS). Both systems are following the same basic development schedule, shown in Figure 4.1-1. Following ground demonstration of both systems and the system selection process scheduled for FY 78 , one of the two power systems will be continued on to flight system design, qualification and production.

\section{DEVELOPMENT SCHEDULE}

\begin{tabular}{|l|c|c|c|c|c|c|c|}
\hline \multicolumn{1}{|c|}{ MILESTONES } & FY75 & FY76 & FY77 & FY78 & FY79 & FY80 & FY81 \\
\hline DEV. FLT. CONCEPT & & & & & & \\
\hline SYS. GROUND DEMO. & \multicolumn{3}{|c|}{} & & & & \\
\hline SYS. SELECTION & & & & & \\
\hline FLT. SYS. DESIGN AND QUAL & & & & & \\
\hline FLT. SYS. PRODUCTION & & & & & $\Delta$ \\
\hline DELIVERY FOR FLT. TEST & & & & & & \\
\hline
\end{tabular}

Figure 4.1-1. Dynamic Power System Development Program

In the present ground demonstration phase, both power systems are de-

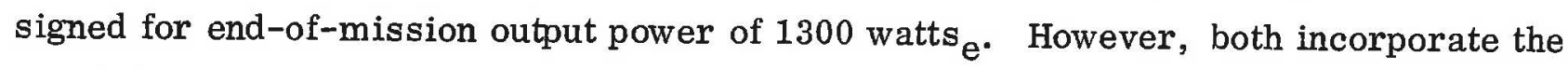

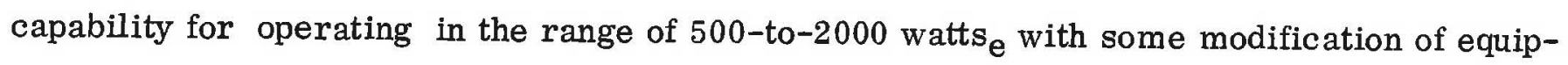
ment and adjustment of fuel loading and cycle conditions. 
Equipment modifications were to be kept to a minimum for the present study; however, the designations "KIPS" and "BIPS" were reserved for the $1300 \mathrm{w}_{\mathrm{e}}$ versions. The generic "Rankine" or "Brayton" designations are employed for the power systems used in this study.

\section{$4.2 \quad$ ORGANIC RANKINE SYSTEM}

The Organic Rankine cycle system is under development by Sundstrand Energy Systems of Rockford, Ill. The basic system schematic is shown in Figure 4.2-1.

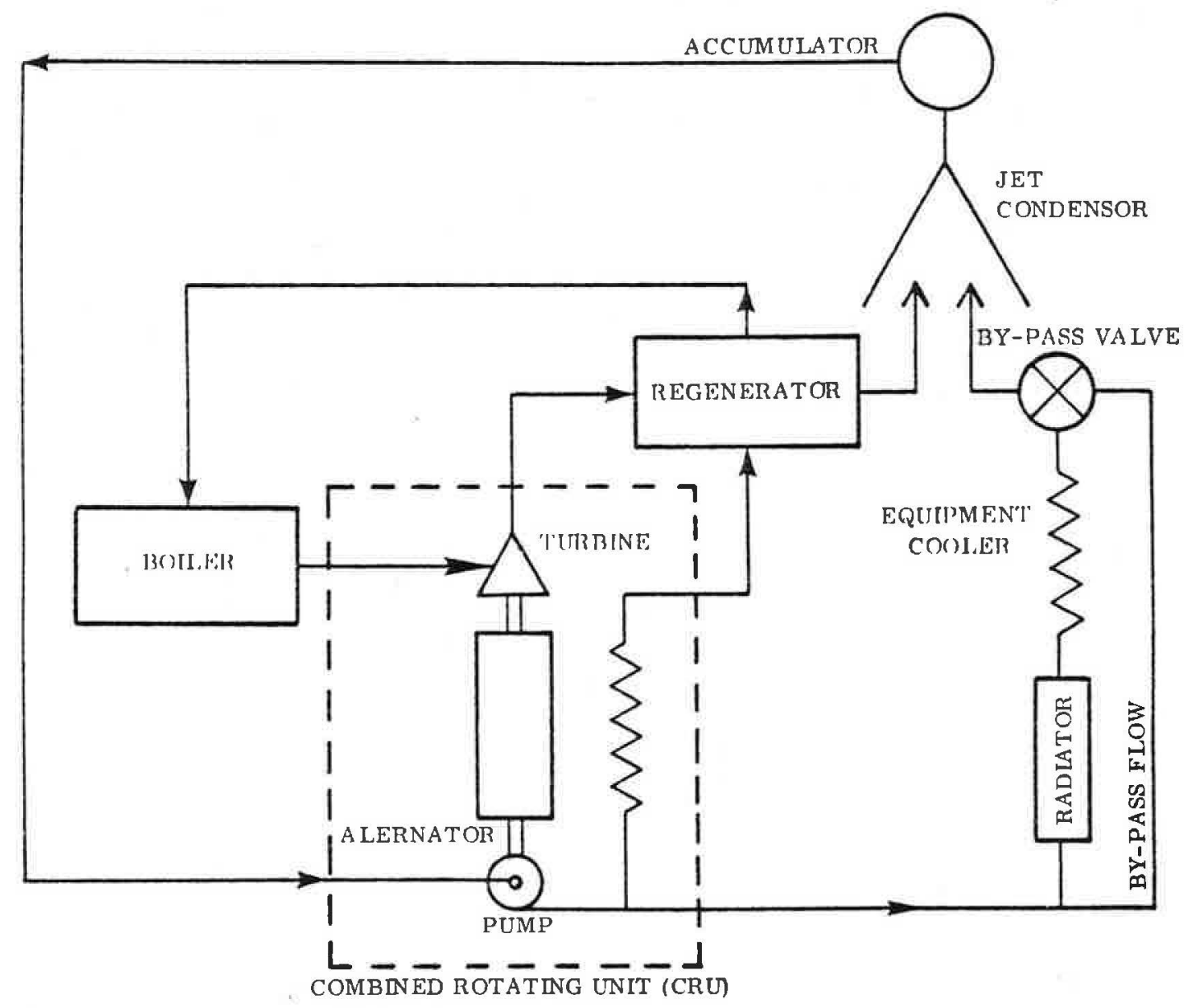

Figure 4.2-1. Organic-Rankine Cycle Schematic 
The radiator by-pass valve, normally used to control jet condensor inlet temperature, can be used here to control radiator outlet temperature, so as to assure a constant-temperature heat sink for the spacecraft equipment. However, as will be seen in Section VIII, the total maximum variation of the liquid temperature in this application will barely exceed the normal control range of this valve $\left(\sim 3^{\circ} \mathrm{C}\right)$; therefore, day-to-day control will be exercised in the normal manner. The flow control valve will serve to handle long term changes due to isotope decay or changes in radiator surface properties.

In order to achieve a radiator outlet temperature of $30^{\circ} \mathrm{C}$, a radiator area of 129 square feet is required. The other major system parameters are shown in Table 4.2-2, based on the use of two standard MHW heat sources. The radiator design parameters are shown in Table 4.2-3.

Table 4.2-2. Organic-Rankine System Baseline Power System

(RADIATOR AREA = 129 SQUARE FEET, INCLUDING EQUIPMENT HEAT LOAD)

\begin{tabular}{|l|r|r|}
\hline & BoL & 7 YR \\
\hline THERMAL INPUT, WATTT & 4800 & 4558 \\
HEAT ABSORBED, WATTT & 4684 & 4442 \\
OUTPUT POWER, WATTE & 820 & 768 \\
CONVERSION EFFICIENCY (SYSTEM) & .17 & .17 \\
TOTAL WEIGHT (INCL. RADIATOR), LBS. & & 390 \\
RADIATOR LIQUID OUTLET TEMP., OC & & 30 \\
TURBINE INLET TEMP, OC & 73 & \\
SYSTEM MASS FLOW RATE, LB/HR & & 343 \\
RADIATOR MASS FLOW RATE, LB/HR & & 322 \\
JET CONDENSER FLOW, RATE, LB/HR & & 629 \\
\hline
\end{tabular}


Table 4.2-3. Organic-Rankine System Radiator Design Parameters

\begin{tabular}{|l|c|}
\hline TOTAL HEAT REJECTED, WATT T & 4242 \\
LIQUID INLET-OUTLET TEMPERATURE, OC & $93-30$ \\
LIQUID PRESSURE DROP, PSI & 21 \\
ABSORBITIVITY/EMISSIVITY & $0.2 / 0.8$ \\
TOTAL AREA, SO. FT. & 129 \\
NUMBER OF TUBES & 1 \\
TUBE LENGTH, FT. & 174 \\
TUBE INTERNAL DIAMETER, IN. & .281 \\
TUBE PITCH, IN. & 8.8 \\
TUBE EXIT REYNOLDS NO. & 2300 \\
FIN THICKNESS, IN. & .025 \\
FIN EFFECTIVENESS & .837 \\
TUBE \& FIN MATERIAL & 6061 ALUMINUM \\
TUBE \& FIN CONDUCTIVITY, BTU/HR-FTOF & 100 \\
& \\
\hline
\end{tabular}

The requirement for very cold outlet temperature from the radiator for cooling purposes leads to certain heat transfer problems because of the high resultant liquid viscosity and tendency to laminar flow. In order to minimize temperature drop from liquid to radiator fin, high heat transfer coefficients are needed while a low pressure drop is required to minimize system pump work.

In order to meet these constraints, a dimpled tube configuration has been selected using heat transfer and friction factor data based upon Kays and London* data table FTD-1 (Appendix G, Figure G-7). The dimpled surface would be formed into a thin walled stainless steel tube and then a thick walled aluminum tube would be cast around or shrunk onto this inner liner. The single radiator tube would be wound spirally around the cylindrical radiator fin the given pitch. The use of a single tube would preclude potential problems of flow maldistribution or freezing associated with the cold outlet temperature. Freezing point of the DowTherm A working fluid is approximately $13^{\circ} \mathrm{C}$.

* Kays and London, Compact Heat Exchangers, McGraw Hill) 
End of mission (EOM) electrical output power is based on the decayed isotope output and the given radiator coating properties. The beginning of mission (BOM) output power is estimated based on the initial Heat Source Assembly (HSA) thermal output without using BOM radiator coating properties. The excess power will need to be dissipated while the higher heat input at BOM will result in higher radiator inlet temperature in order to reject the higher heat load. However, the control valve will maintain the required outlet temperature.

It is assumed that the working fluid will re-absorb all the electrical output power except for 200 watts of radiated electromagnetic (RF) power.

System weights are based upon a KIPS flight conceptual design using two heat sources and a radiator weight scaled from the KIPS current design. However, radiator weight was recalculated in the reference design.

The methods used to optimize the cycle for the conditions given is summarized in Appendix G, along with computer print-outs of the major cycle parameters.

\section{4. $3 \quad$ BRAYTON-CYCLE SYSTEM}

Initial scoping analysis established that the Brayton cycle system would require a stretched version of the MHW heat source, with four trays of fuel sphere assemblies totalling 3200 watts $_{t}$, instead of the usual three. In addition, a 2400 watt $_{t}$ case was derived. Two versions of the $3200 \mathrm{w}_{\mathrm{t}}$ Brayton system were carried through to the cost analysis phase in order to achieve a cost/benefits comparison between them: a C-103 columbium alloy system and a superalloy system. The different materials appear only in the heat source heat exchanger, the turbine inlet scroll, and the connecting ducting. The superalloy system has lower output power due to the lower turbine inlet temperature capability of this material, but benefits from reduced hardware, assembly, and test costs.

The major system parameters for the three cases are shown in Table 4. 3-4 based on a total radiator area including the equipment radiator section of 130 sq. ft. The cycle conditions for the three cases are shown in Figures 4. 3-2, 4. 3-3, and 4.3-4, with temperatures in ${ }^{\circ} R$, pressures in psia, and flow rates in $1 b / s e c$. 
Table 4.3-4. Brayton-Cycle Systems Alternatives

(RADIATOR AREA = 130 SOUARE FEET, INCLUDING EQUIPMENT RADIATOR)

\begin{tabular}{|c|c|c|c|}
\hline THERMAL INPUT, WATT $T$ & 3200 & 3200 & 2400 \\
\hline MATERIAL & SUPERALLOY & C103 & C103 \\
\hline BOL OUT PUT POWER $(3 \varphi A C)$, WATTE & 665 & 735 & 490 \\
\hline BOL CONVERSION EFFICIENCY & 0.21 & 0.23 & 0.20 \\
\hline 7 YR OUTPUT POWER (3QAC), WATTE & 614 & 678 & 452 \\
\hline $\begin{array}{l}\text { TOTAL WEIGHT (INCL. RADIATOR, } \\
\text { ENCLOSURE), LBS }\end{array}$ & 413 & 396 & 397 \\
\hline EQUIPMENT RADIATOR INLET TEMP., ${ }^{\circ} \mathrm{C}$ & 32 & 38 & 27 \\
\hline OUTLET TEMP., ${ }^{\circ} \mathrm{C}$ & 7.2 & 4.5 & -9.5 \\
\hline
\end{tabular}

(NOTE: Output Power Includes Penalty for Pressure Drops in Equipment Headers and Radiator of $\Delta P / P=.006$ )

$\epsilon=.80$

$Q=.20$

12 HR. CIRCULAR ORBIT

RADIATOR AREA:

BIPS 90

ELECTRONICS 40

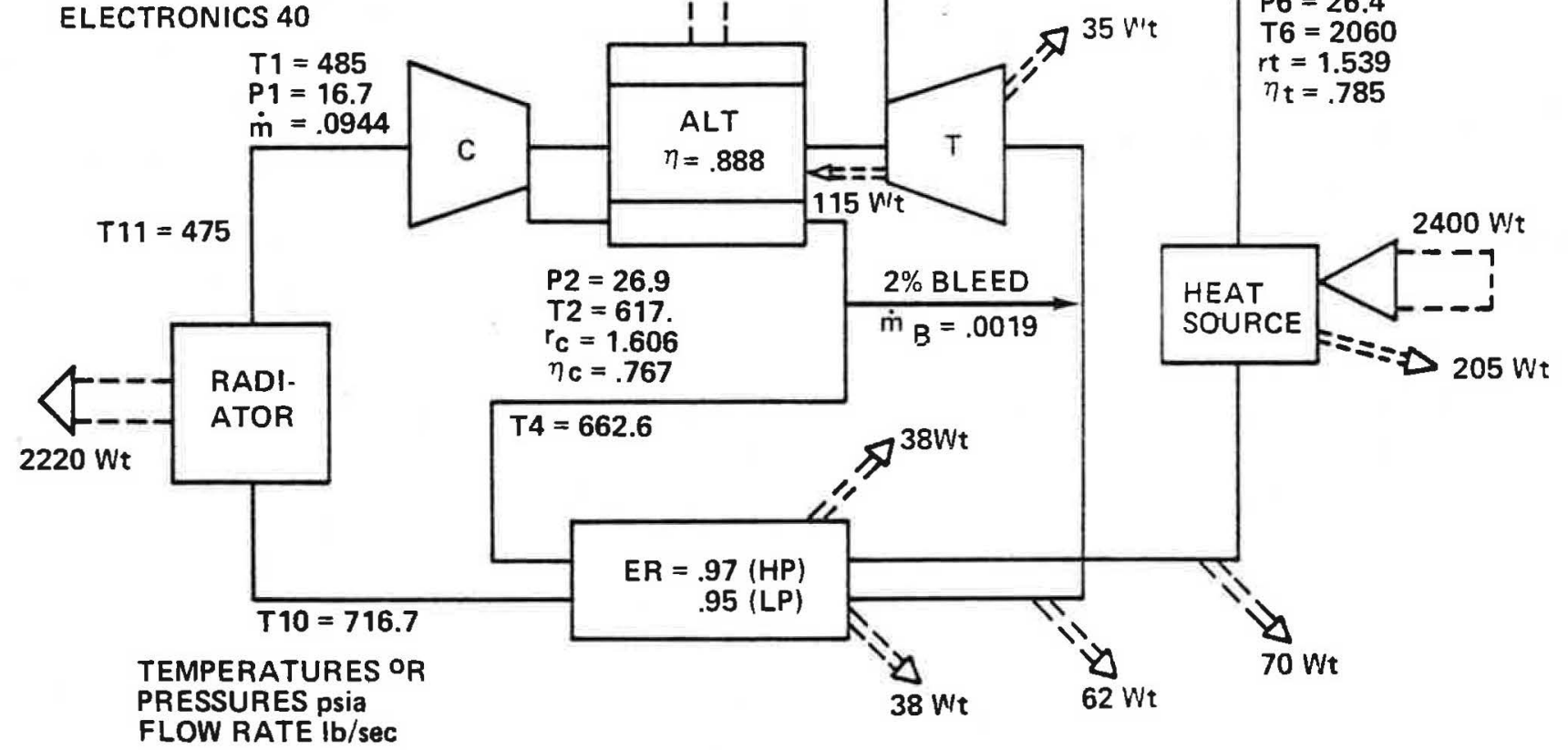

Figure 4. 3-2. C103 Brayton System with 2400 Wt Heat Source 


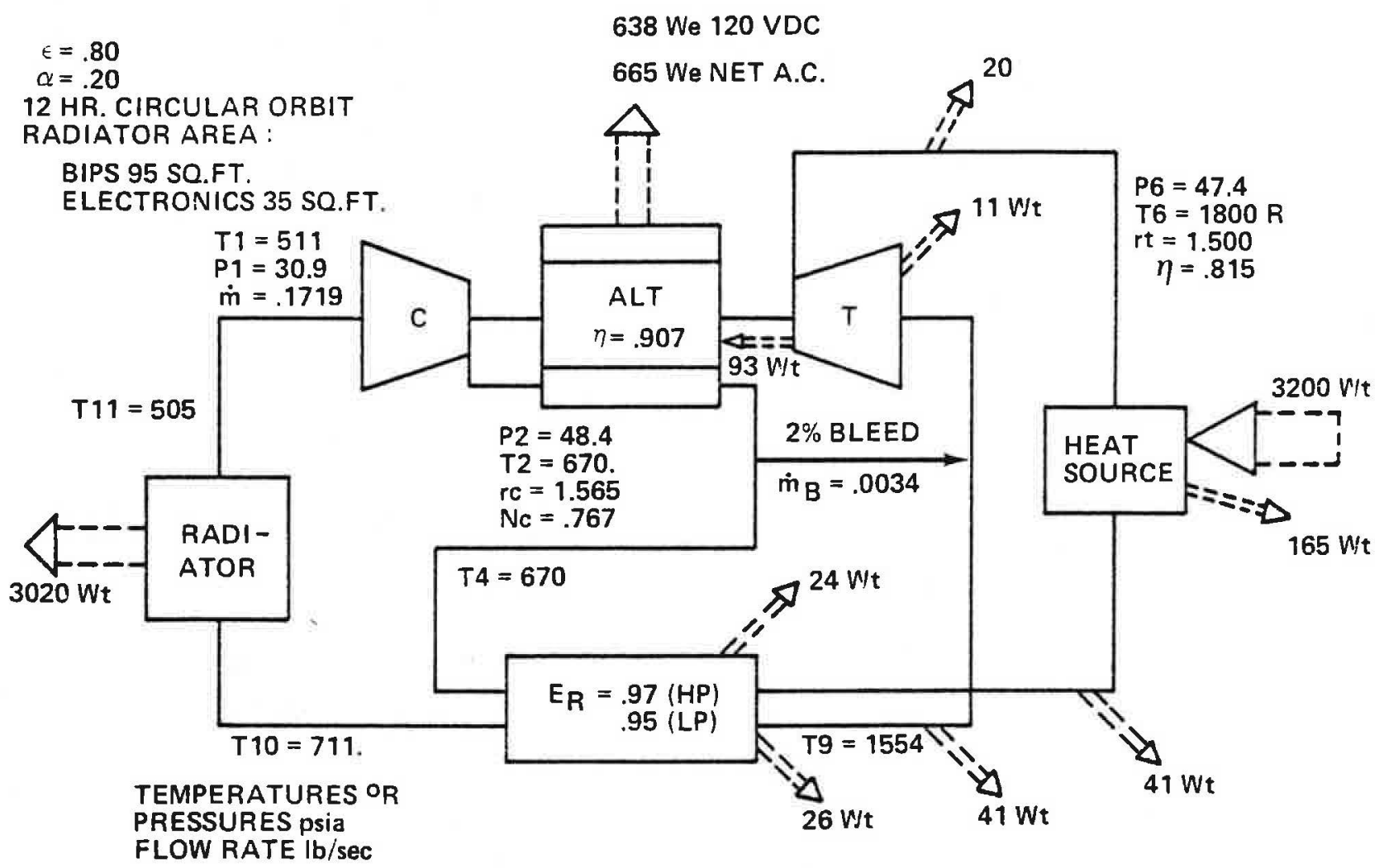

Figure 4.3-3. Superalloy Brayton System with 3200 Wt Heat Source

$$
\begin{aligned}
& \epsilon=.80 \\
& \alpha=.20
\end{aligned}
$$

12 HR. CIRCULAR ORBIT

RADIATOR AREA:

BIPS 90 SQ. FT.

ELECTRONICS 40 SO. FT.

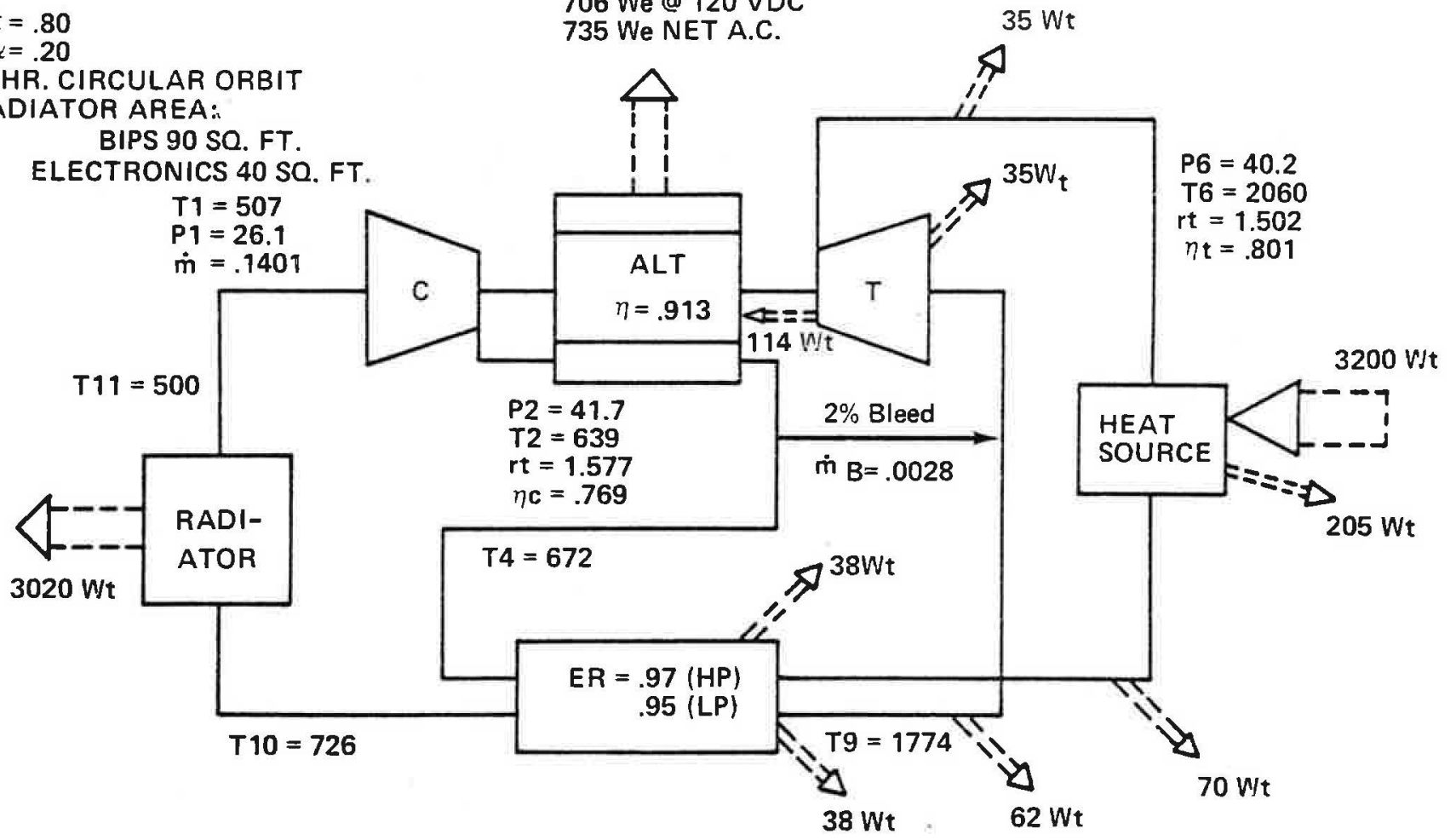

706 We @ 120 VDC

735 We NET A.C.

$35 \mathrm{Wt}$

Figure 4. 3-4. C103 Brayton System with 3200 Wt Heat Source 



\section{SECTION $\vee$}

\section{REFERENCE DESIGN DETAILS}

\subsection{ORGANIC-RANKINE-POWERED SPACECRAFT}

The external profile and major dimensions of the Rankine-cycle space vehicle (RSV) are shown in Figure 5.1-1 as assembled. The spacecraft disassembles into two major subassemblies: The Power System Module (PSM) and the Equipment Module (EM), as shown in Figure 5.1-2.

Each module can be assembled and tested fully independently of the other. System-level tests can be performed following final assembly, but with electrical heat sources in place of the nuclear heat sources. The nuclear heat sources need not be inserted until the spacecraft is delivered to the launch site. Provision is made for access to the heat source container (heat exchanger or boiler) after final assembly of the spacecraft in both reference designs. In the RSV the radiator extensions which form part of the lower bulkhead would be attached following heat source insertion.

The inboard profile of the RSV/PSM is shown in Figure 5.1-3. In addition to the power system components and interfaces, the PSM carries the reaction control modules, consisting of two identical sets of hydrazine tanks and a thruster assembly, as shown.

The major support structure is the central conic section. The power conversion unit is mounted to the upper ring of this cone, with the heat sources, RCS modules and radiator supported from various points of this cone by ribs and spanners, as shown. 


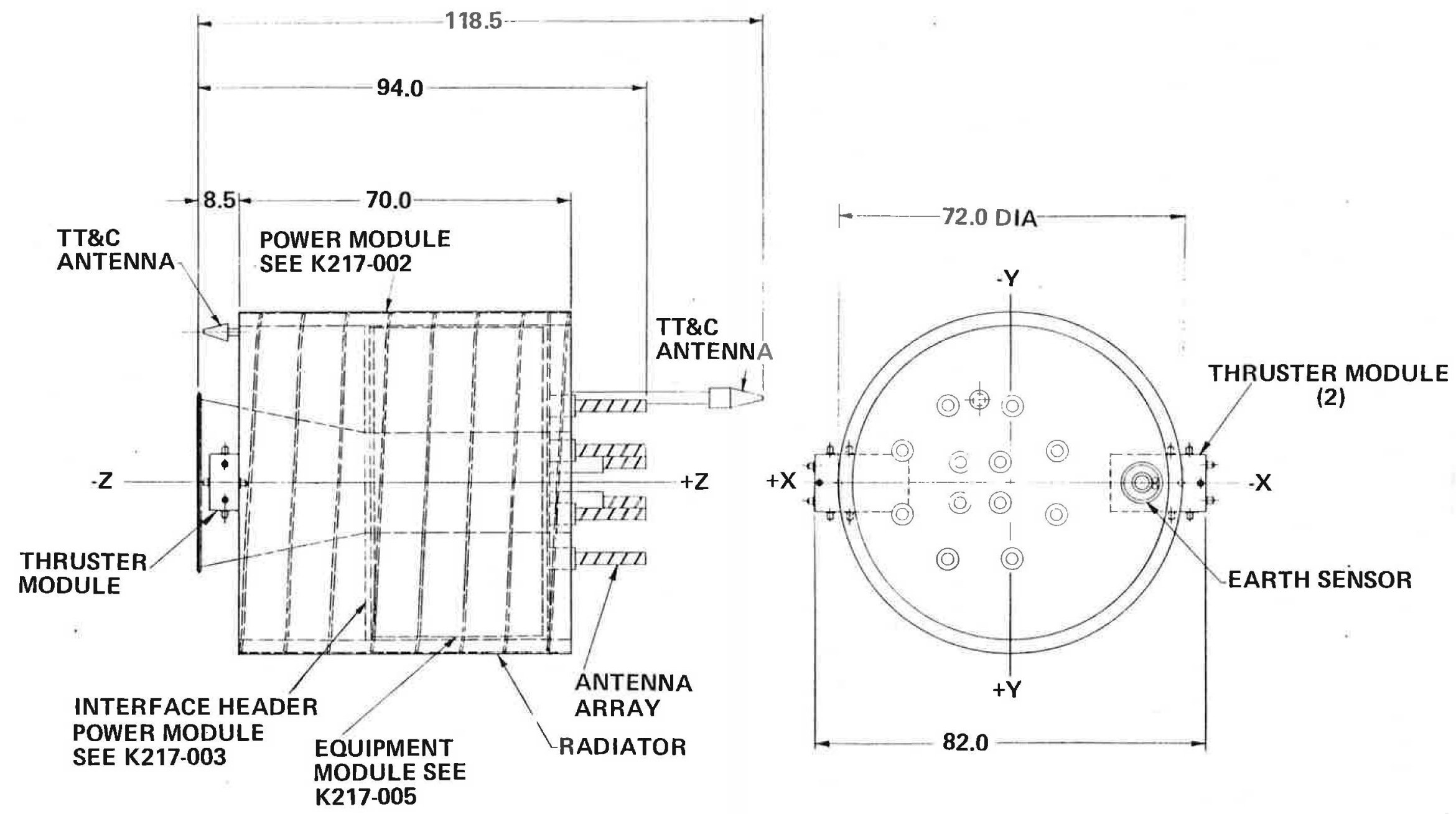

Figure 5.1-1. N/GPS Organic Rankine Cycle Spacecraft Profile 


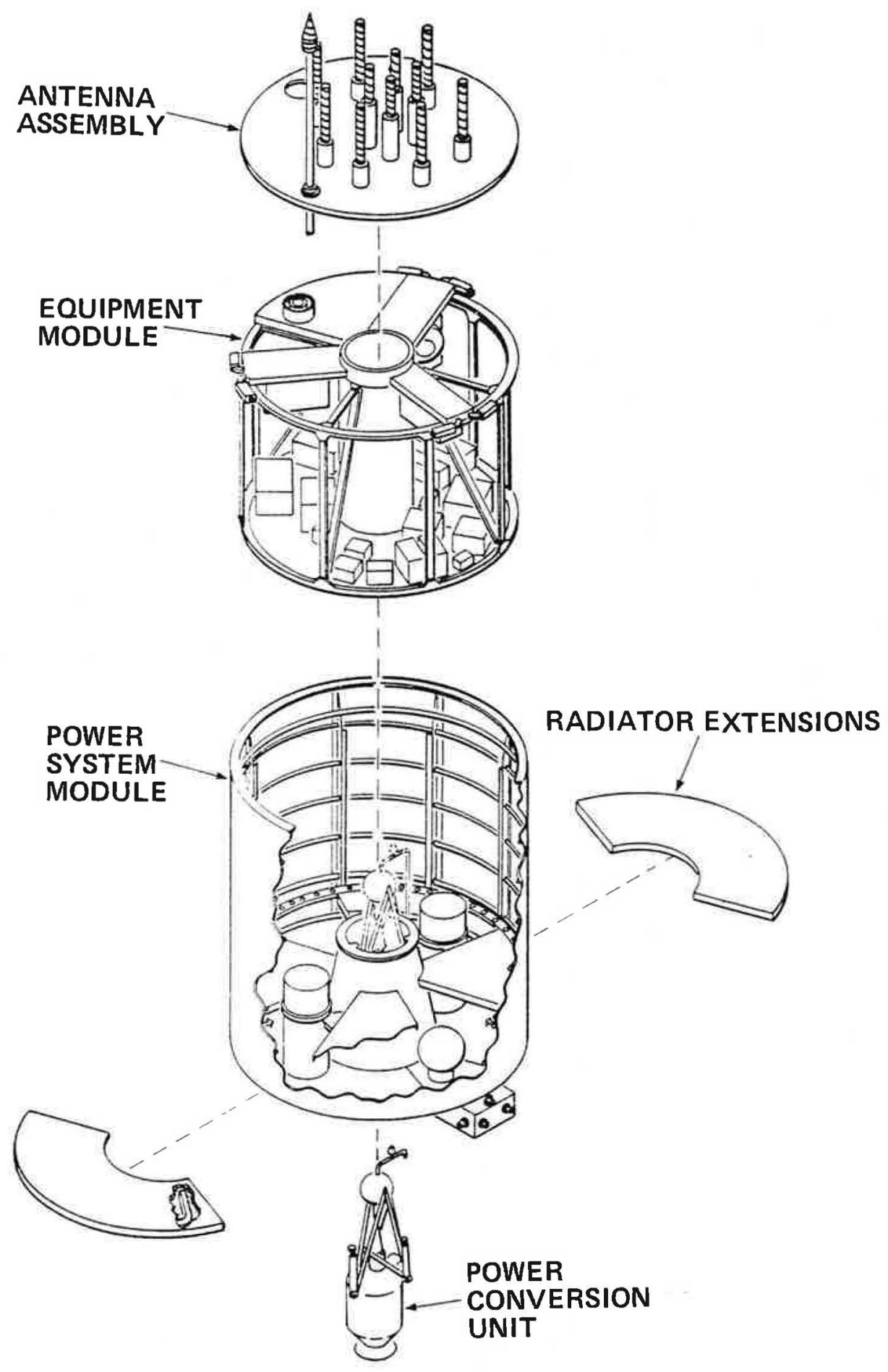

Figure 5.1-2. N/GPS Spacecraft Exploded View (Organic Rankine Cycle Version) 


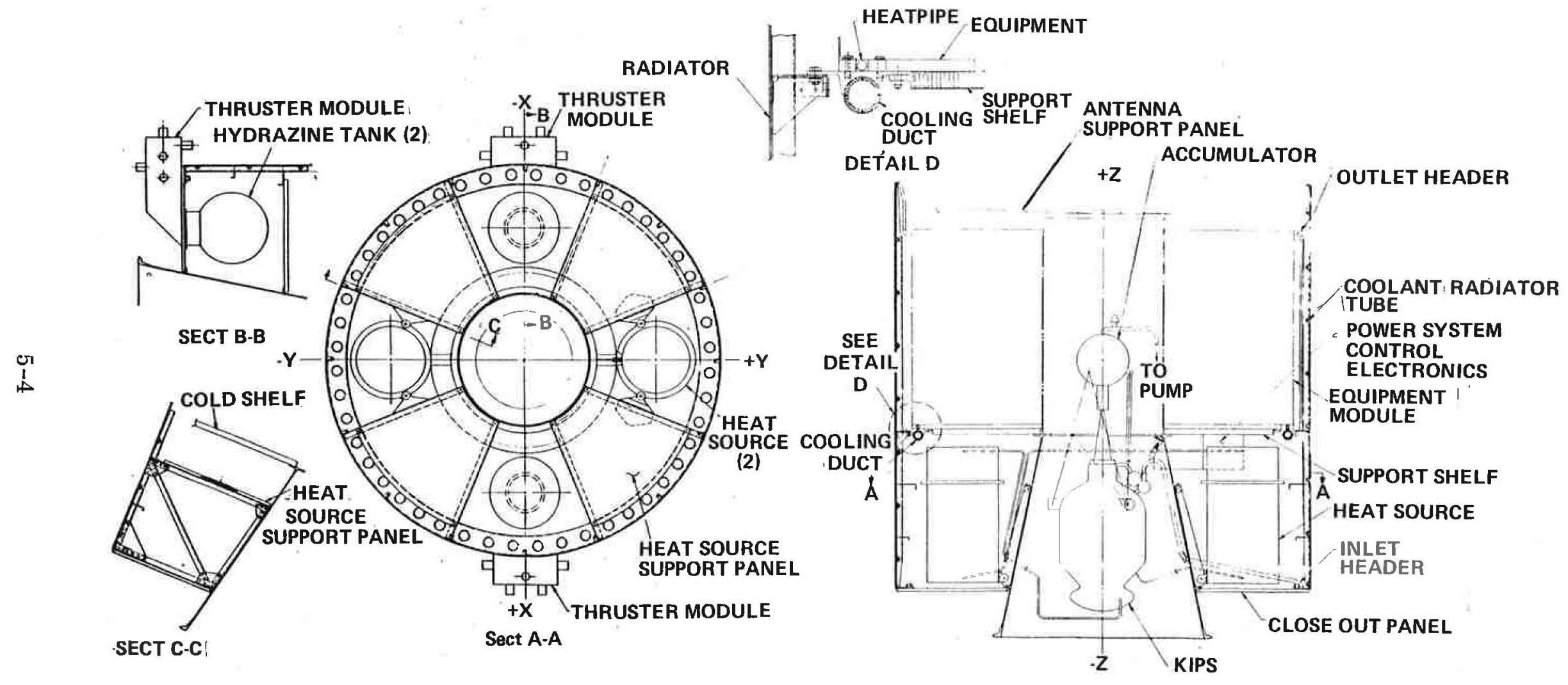

Figure 5.1-3. Organic-Rankine Power System Module Inboard Profile 
The thermal interfaces are provided by three sections of radiator tubing, called "headers" although these are not plenums for radiator tubes in this single-tube radiator design. They are simple series extensions of the single radiator tube which are provided for thermal interface purposes. They are wound in flat circles in three different locations in the PSM. The lower or inlet header provides thermal contact for the radiator extension panels. The upper two headers provide similar heat-transfer surfaces for the upper and lower equipment shelves of the EM.

The method of heat transfer is similar for all three locations, and is best illustrated by reference to the radiator extensions which form part of the lower bulkhead. (Figure 5.1-4).

The panels are constructed of aluminum honeycomb with embedded ammoniain-aluminum heat pipes. The boiler section of each heat pipe is bonded within the panel, within channels cut in the honeycomb. The condenser section is bent at right angles and fitted with a conducting saddle. The saddles are then bolted to a mating saddle on the header tube, with thermal grease applied to the smoothly fitting interface. This entire technology, including honeycomb-embedded heat pipes with right-angle bends mated via conduction contact to the heat sink, is taken directly from Fairchild ATS-6 experience. A total of 54 such heat pipes operating at similar temperature and heat flow, have performed for three years in space aboard the ATS-6 without failure.

The equipment module for the RSV is shown in Figure 5.1-5. The upper shelf, which consists of three sections (see Figure 5.1-2), contains the two cesium standards on each of two sections, and the momentum wheels and their control box on the third section. 


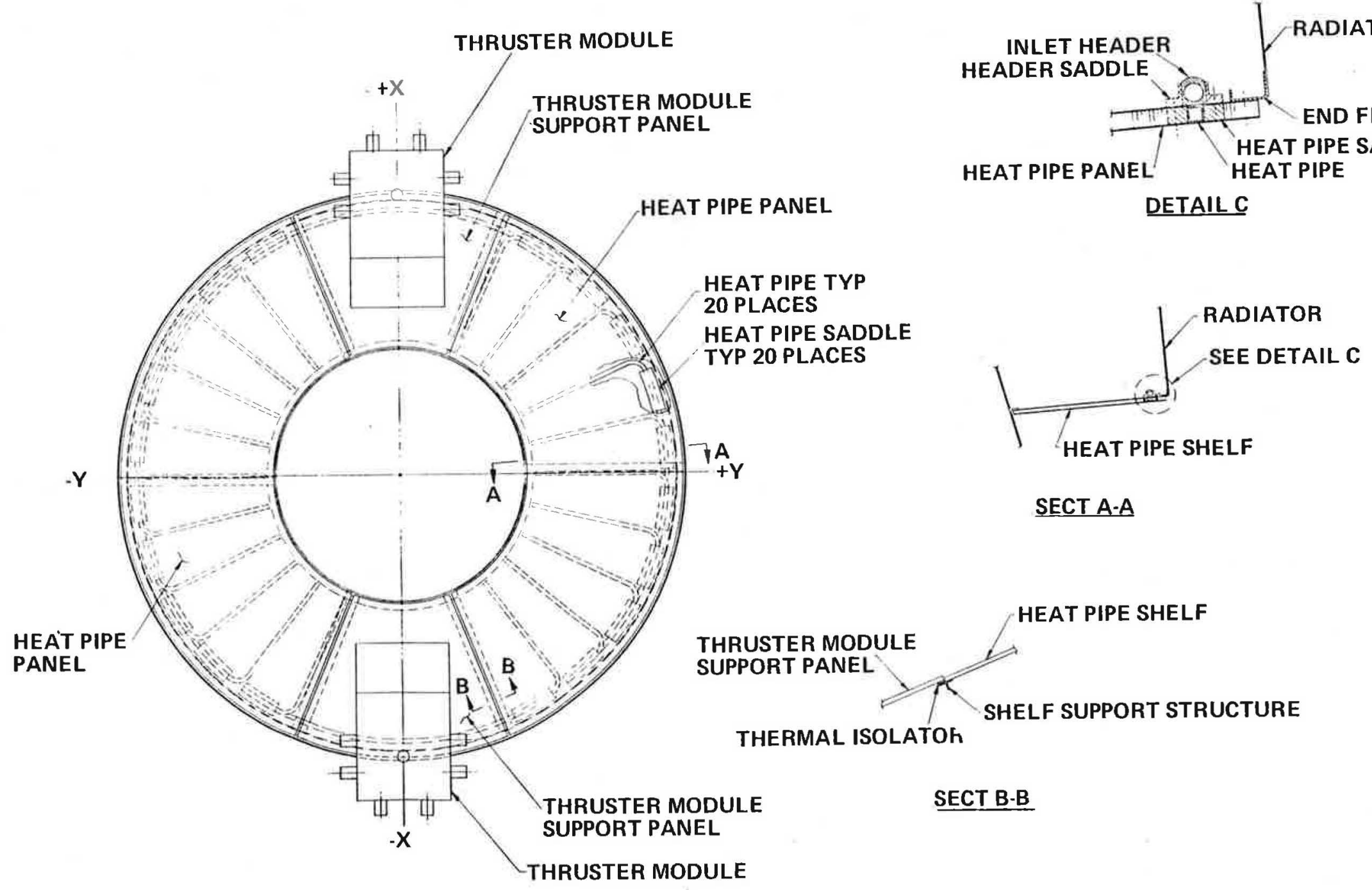

Figure 5.1-4. Lower Closure Bulkhead-Radiator Extension (Both Spacecraft) 


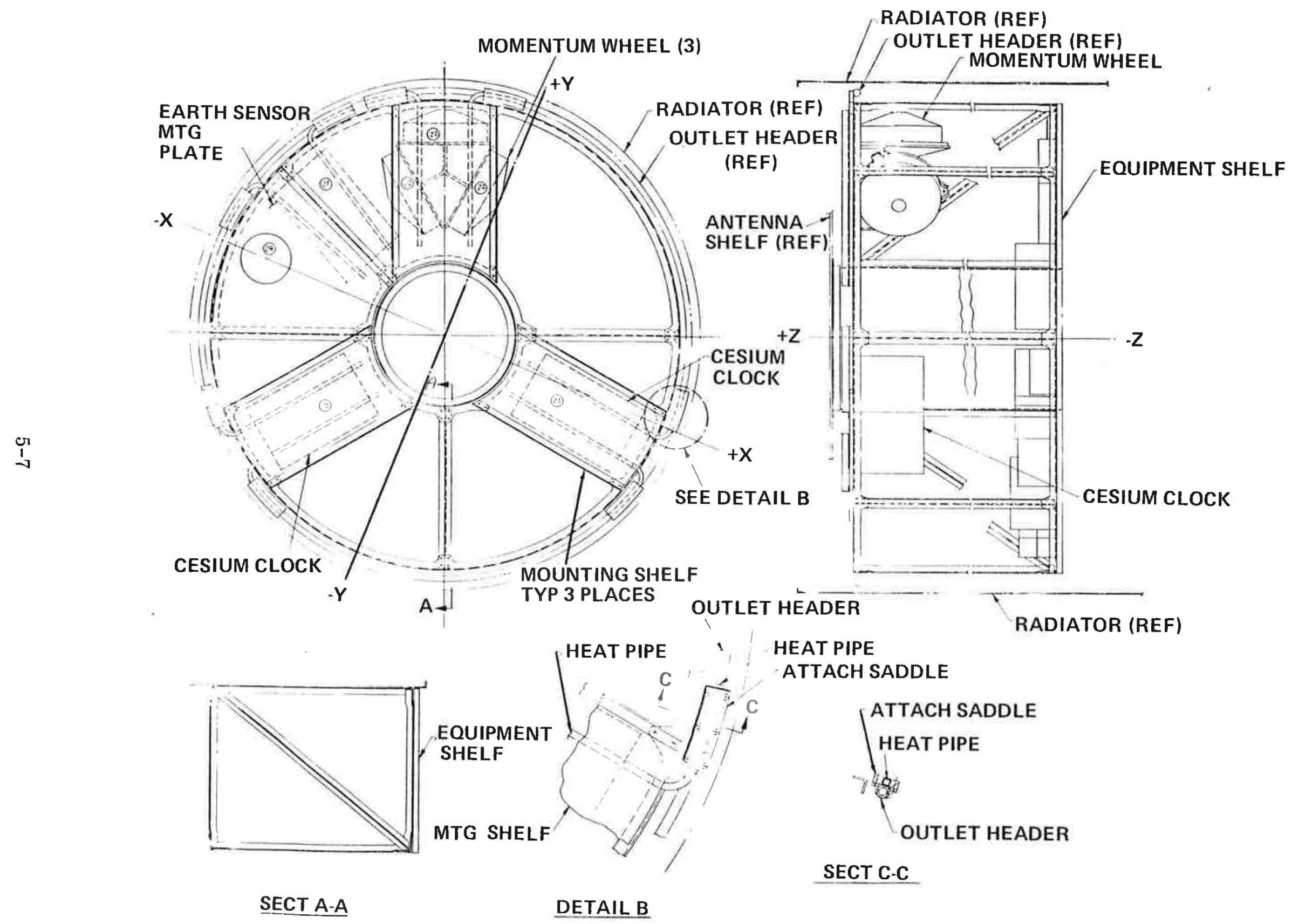

Figure 5.1-5. Equipment Module; Organic Rankine Spacecraft 
The lower shelf contains the remainder of the electronic equipment complement, as shown in Figure 5.1-6. Half of this shelf contains the $\mathrm{L}_{1}$ highpower amplifiers, designed to dissipate a total of 400 watts $_{t}$ in the two sections. The only item not included in the equipment complement which is part of the $\varnothing 1$ NDS is the load control unit. The remaining functions of this unit, which has many fewer functions to perform in this battery-less and essentially constant load spacecraft, was incorporated into the power system control box which is mounted to the underside of the support shelf of the PSM, and remains as part of the PSM.

\subsection{BRAYTON-POWERED SPACECRAFT}

The external profile and major dimensions of the Brayton-cycle space vehicle (BSV) are shown in Figure 5.2.1. The additional spacecraft height as compared to the RSV results from some added height required to accommodate the mid-radiator headers which delineate the two radiator sections, and from the desire to retain the same dimensions for the EM in both designs. In the RSV the antenna mounting plate is recessed some 5-inches below the lip of the radiator shell. In the BSV this plate is essentially flush with the top.

The inboard profile of the BSV/PSM is shown in Figure 5.2.2. In this case the entire power converter is contained within the central cone; now configured almost cylindrical. Upper and lower domes serve to hermetically seal the converter. This allows complete evacuation of the converter for ground testing of the C-103 version of the Brayton-cycle system. External access to the heat source container is still provided via a flange welded to the lower dome.

Mechanical attachment of the EM has been modified somewhat to account for the increased diameter of the mating ring. The thermal interface headers are similar in principle but differ in size and internal detail from those of the RSV (see Section VIII).

The BSV/EM (Figure 5.2-3) is identical to that of the RSV, except for the modification of the cross-braces to pick up the new attachment points, and for the inclusion of the fourth momentum wheel in the axial direction to provide the momentum cancellation of the spacecraft yaw rotation. This wheel is mounted at a very slight angle to the spacecraft $\mathrm{z}$-axis to also cancel out the angular momentum of the horizontallymounted Brayton rotating unit. 


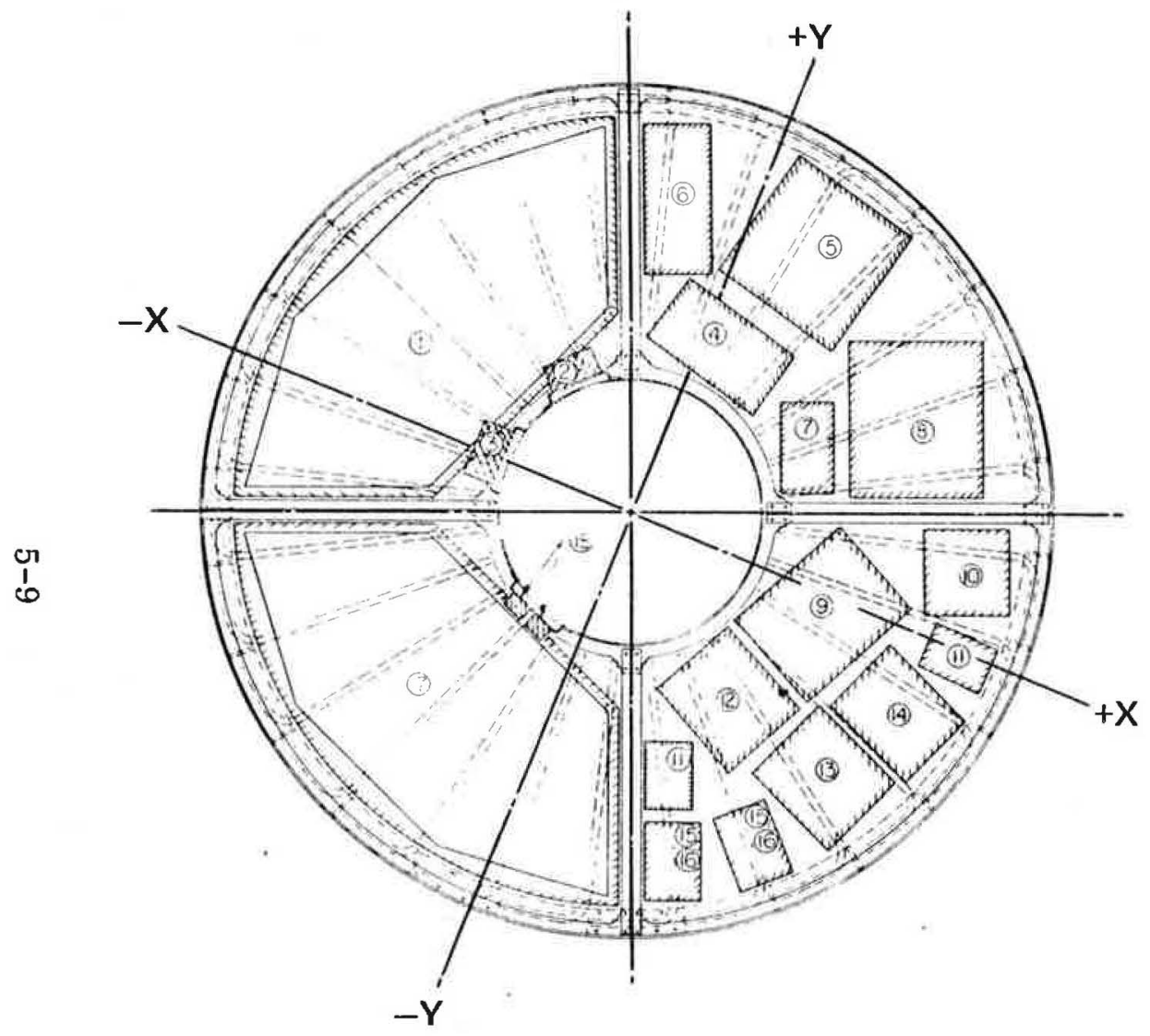

\begin{tabular}{|c|c|c|c|c|c|}
\hline ITEM & NAME & $\begin{array}{c}\text { SIZE } \\
\text { LENGTH } \times \text { WIDTH } \times \text { HEIGHT }\end{array}$ & $\begin{array}{l}\text { WEIGHT } \\
\text { LBS (EA) }\end{array}$ & $\begin{array}{l}\text { DISSIP } \\
\text { WATTS }\end{array}$ & $\begin{array}{l}\text { OTY } \\
\text { USED }\end{array}$ \\
\hline 1 & NAV SYS $L_{1} H P A$ & $16.0 \times 40.0 \times 2.0$ & 21.5 & 200 & 1 \\
\hline 2 & $L_{1} H P A$ FILTER & $5.3 \times 4.3 \times 2.2$ & 2.5 & - & 1 \\
\hline 3 & L ${ }_{2}$ HPA FILTER & $4.8 \times 4.0 \times 2.0$ & 1.6 & - & 1 \\
\hline 4 & NAV SYS L $L_{2} H P A$ & $9.6 \times 6.0 \times 1.3$ & 4.34 & 14.7 & 1 \\
\hline 5 & NAV SYS $L_{1} / L_{2} S Y N$ & $10.4 \times 10.3 \times 2.5$ & 7.43 & 10.6 & 1 \\
\hline 6 & NAV SYS $L_{1}$ MOD IPA & $11.25 \times 4.68 \times 1.65$ & 3.08 & 3.4 & $i$ \\
\hline 7 & NAV SYS $L_{2}$ MOD IPA & $6.7 \times 3.6 \times 1.65$ & 1.7 & 1.5 & 1 \\
\hline 8 & NAV SYS DÍG PROC/BB & $11.7 \times 10.2 \times 5.8$ & 20.8 & 21.1 & 1 \\
\hline 9 & PCM/TLM UNIT & $10.65 \times 8.28 \times 5.75$ & 12.0 & 8.0 & $i$ \\
\hline 10 & NAV DC/DC CONVERTER & $6.0 \times 6.0 \times 3.75$ & 7.6 & 18.1 & 1 \\
\hline 11 & TI\&C KIR-23 & $5.02 \times 3.6 \times 5.8$ & 4.3 & 2.2 & 2 \\
\hline 12 & TT\&C CMD DECODER & $8.8 \times 7.41 \times 5.75$ & 8.0 & 0.8 & 1 \\
\hline 13 & TT\&C SIG COND & $8.28 \times 7.05 \times 5.45$ & 7.0 & 2.0 & 1 \\
\hline 14 & TT\&C RF ASSY & $8.0 \times 6.5 \times 6.5$ & 5.3 & 2.7 & 1 \\
\hline 15 & TT\&C TRANSMITTER & $5.82 \times 4.25 \times 2.7$ & 5.0 & 20.0 & 2 \\
\hline 16 & TT\&C RCVR DEMOD & $5.82 \times 4.25 \times 2.7$ & 5.0 & 1.8 & 2 \\
\hline 17 & $L_{1}$ HPA FINAL AMPLIFIER & $16.0 \times 40.0 \times 2.0$ & 15.0 & 200 & 1 \\
\hline 18 & CUURRENT SENSOR & $2.5 \times 1.7 \times 1.3$ & 0.5 & 1.3 & 2 \\
\hline $\begin{array}{r}\star 19 \\
20\end{array}$ & PWR SYS CONT ELEC & & & & \\
\hline 21 & AVCS CONTROL ELECT & $16.0 \times 10.0 \times 6.0$ & 17.0 & 8.5 & 1 \\
\hline 22 & MOMENTUM WHEEL & 7.50 DIA x 4.15 & 11.0 & 10.0 & 3 \\
\hline 23 & CESIUM CLOCK & $16.0 \times 10.0 \times 10.0$ & 34.0 & 24.0 & 2 \\
\hline 24 & EARTH SENSOR & $7.44 \mathrm{DIA} \times 122$ & 13.5 & 3.0 & 1 \\
\hline
\end{tabular}

Figure 5.1-6. Lower Equipment Shelf Reference Design (Both Spacecraft) 


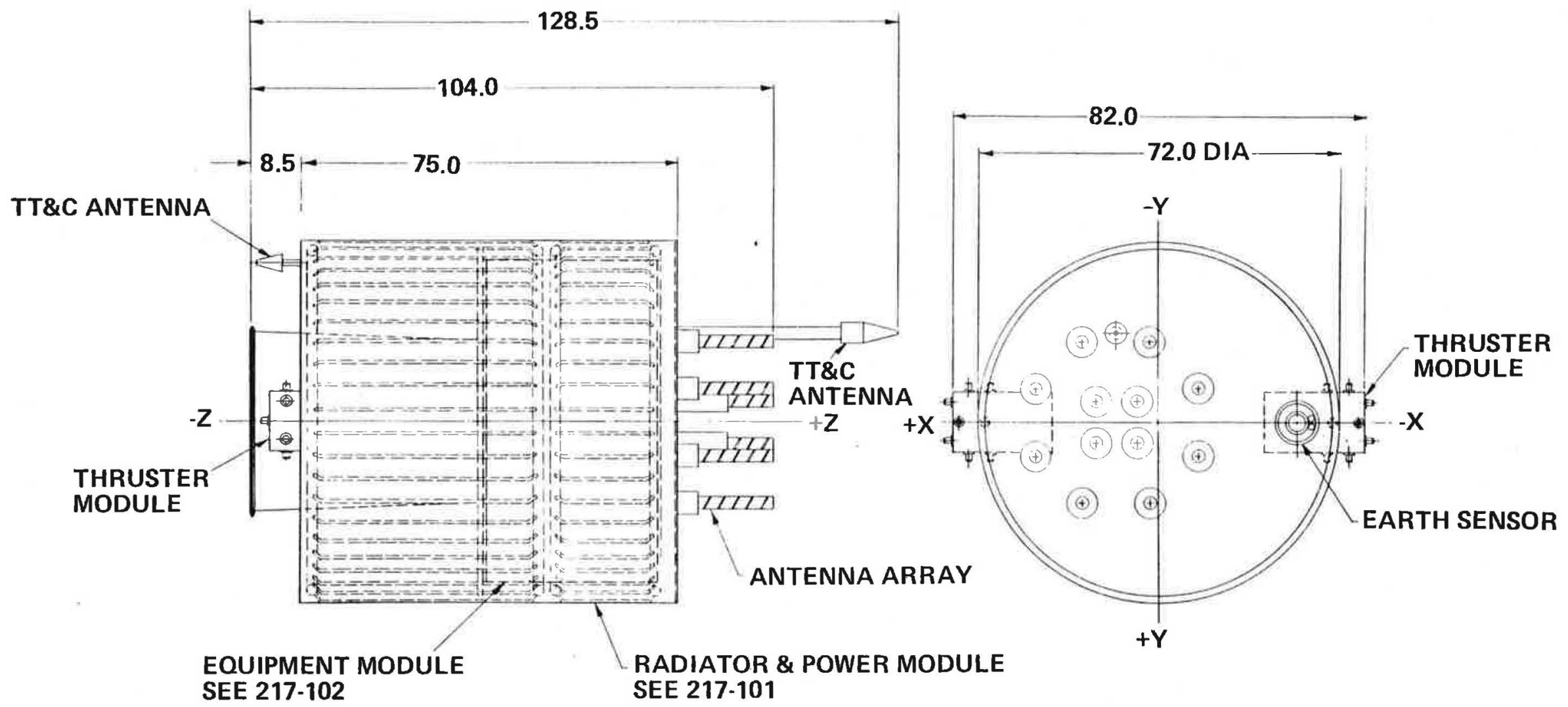

Figure 5.2-1. N/GPS Brayton Cycle Spacecraft Profile 


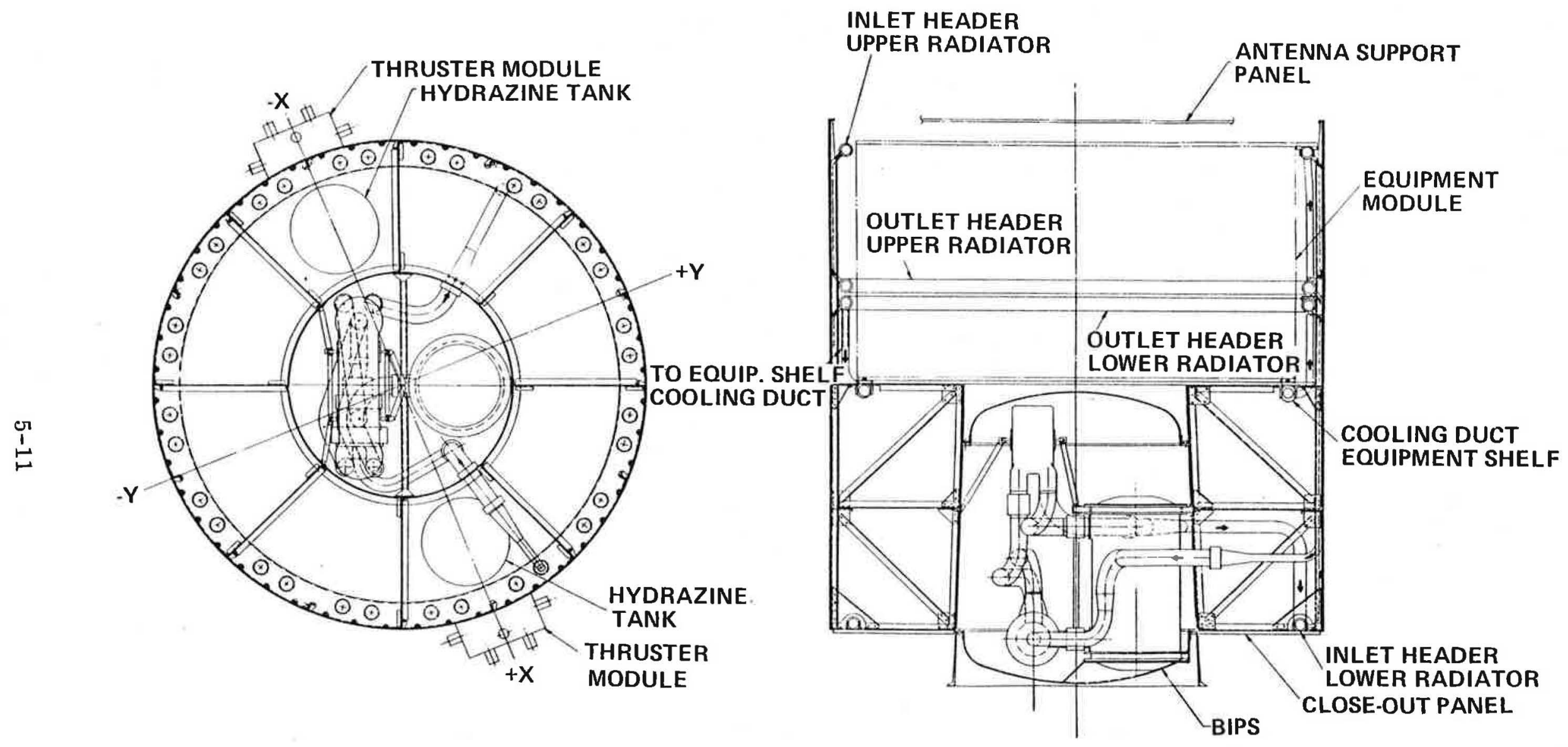

Figure 5.2-2. Brayton Power System Module; Inboard Profile 


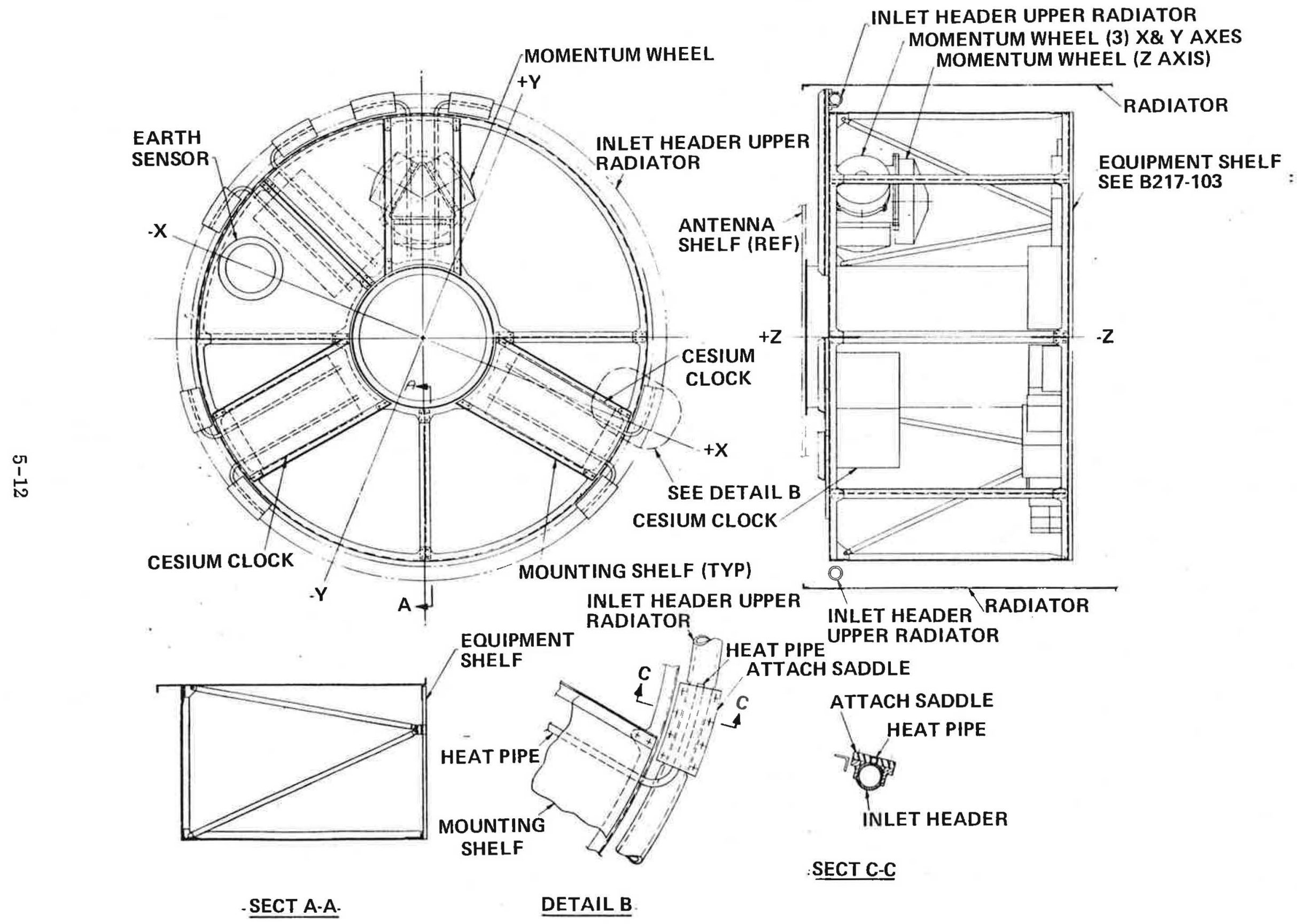

Figure 5.2-3. Equipment Module - Brayton Spacecraft 
The fluid-flow pattern in the BSV is shown in Figure 5.2-4.

An alternative equipment shelf heat transfer system is illustrated in Figure 5.2-5. This ducted shelf with coolant flowing through it was designed to be part of the RSV/PSM. The lower equipment shelf would bolt to this cooled shelf at the indicated locations between ducts. This design was found to be thermally superior to the reference design, resulting in a smaller total $\Delta \mathrm{T}$ from equipment baseplate to coolant. However, it was ultimately discarded because the large number of welds required to fabricate the ducts and the duct-to-plenum connections were judged to result in a design of questionable reliability, particularly as the coolant fluid at this point is at a pressure of 76 psia. (Appendix G).

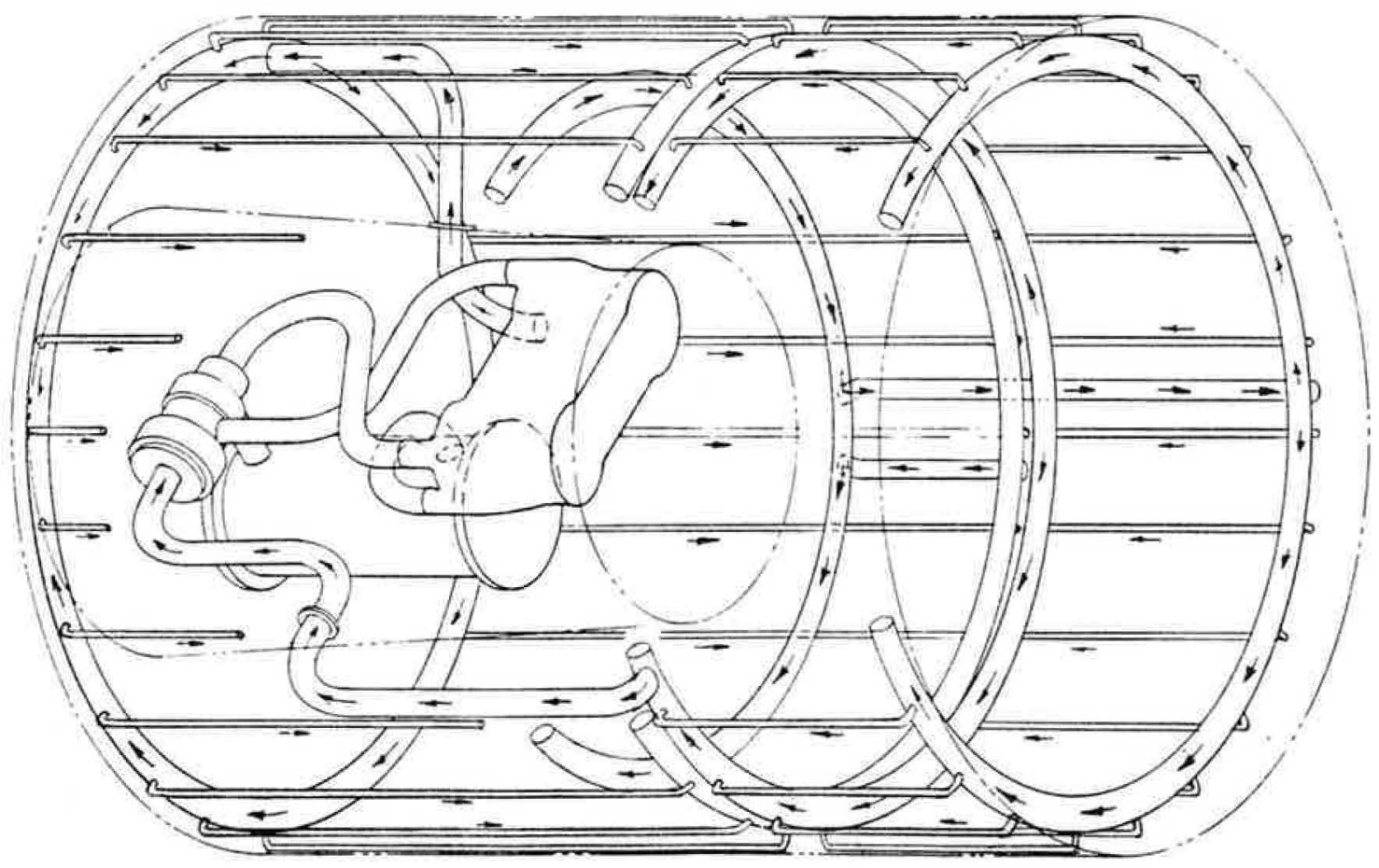

Figure 5.2-4. Working Fluid Loop; Brayton Spacecraft

5.3

OVERALL DIMENSIONS

Figure 5.3-1 shows the major dimensions and subsystems locations for the two reference designs. The BSV is shown in three-dimensional view in Figure 5.3-2. 


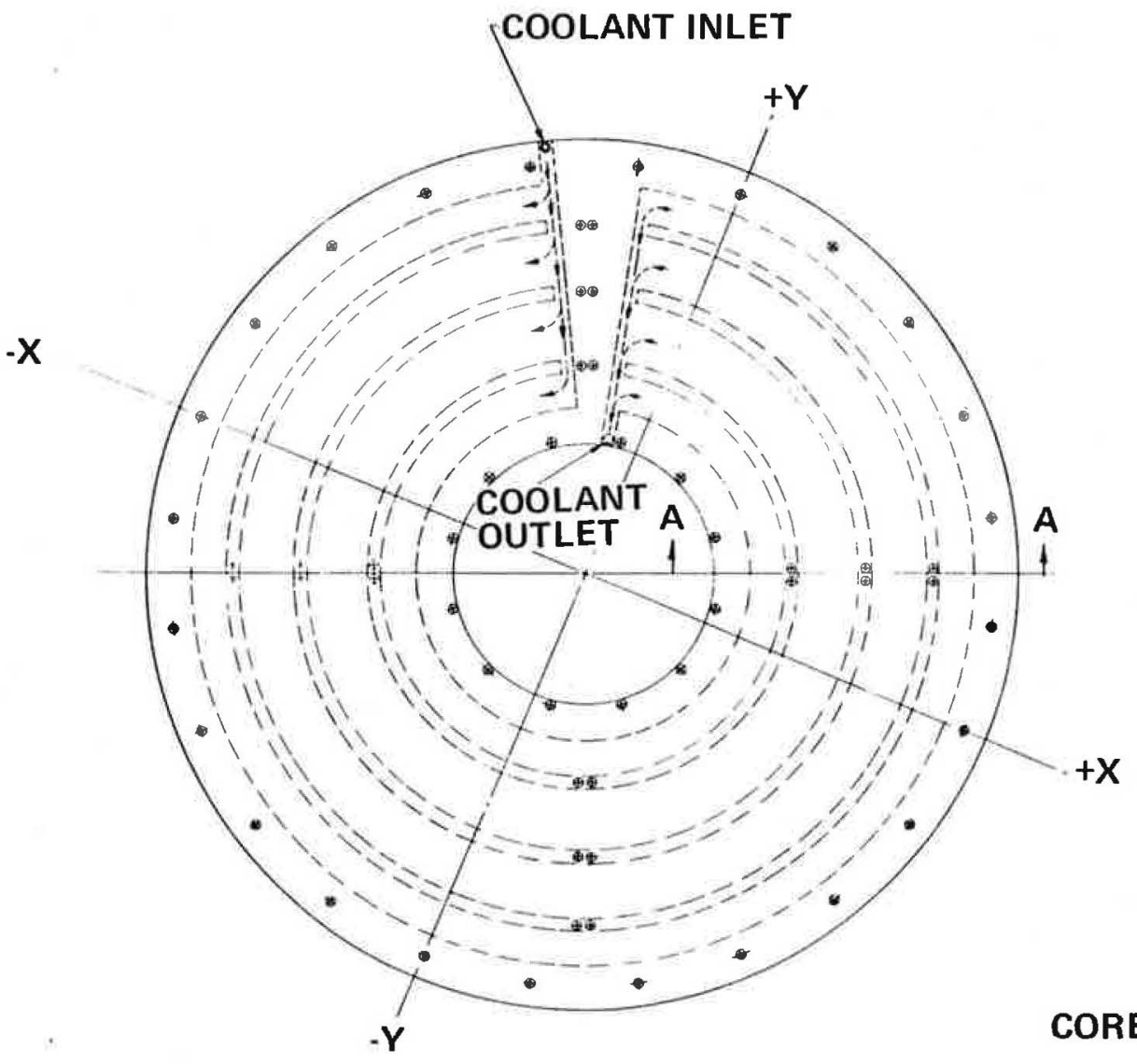

COLD SHELF
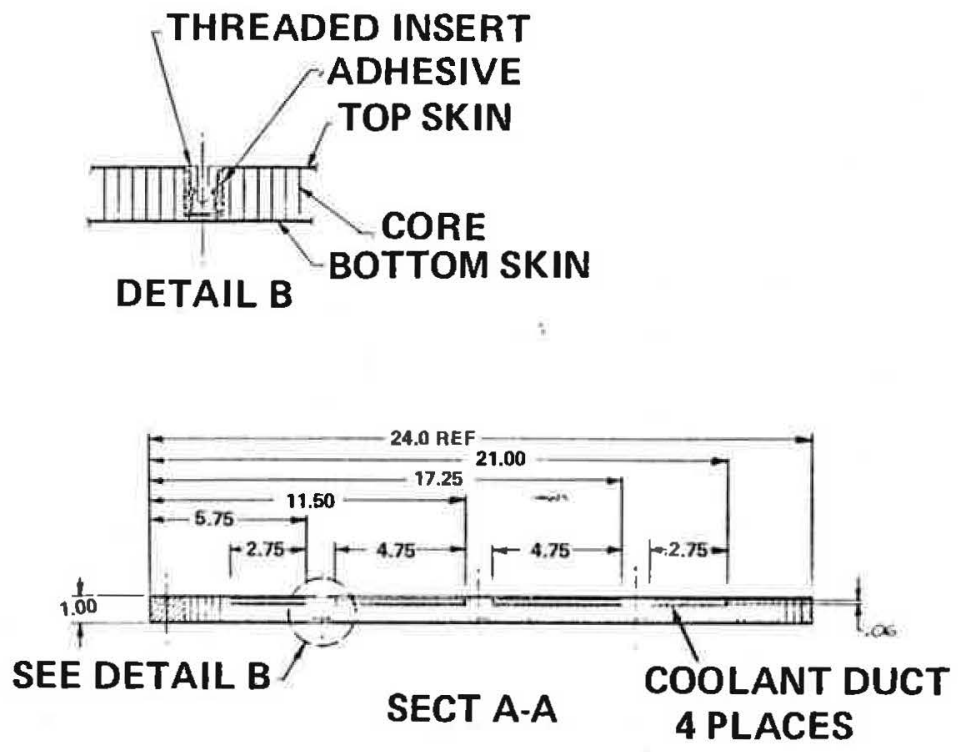

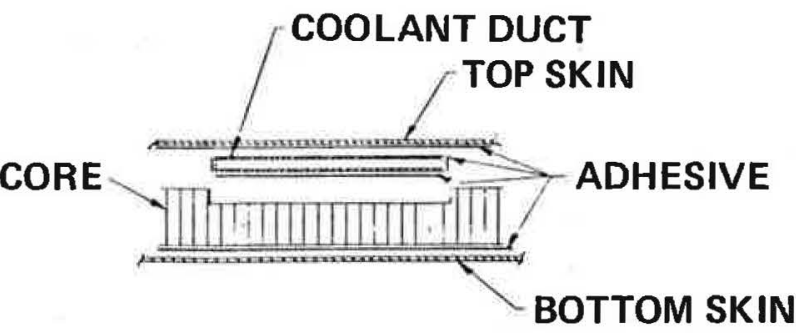

EXPLODED VIEW

NO SCALE

CONCLUSION: THERMALLY SUPERIOR; MECHANICALLY UNRELIABLE

Figure 5.2-5. Alternate Equipment Shelf Design 
ORGANIC RANKINE SPACECRAFT

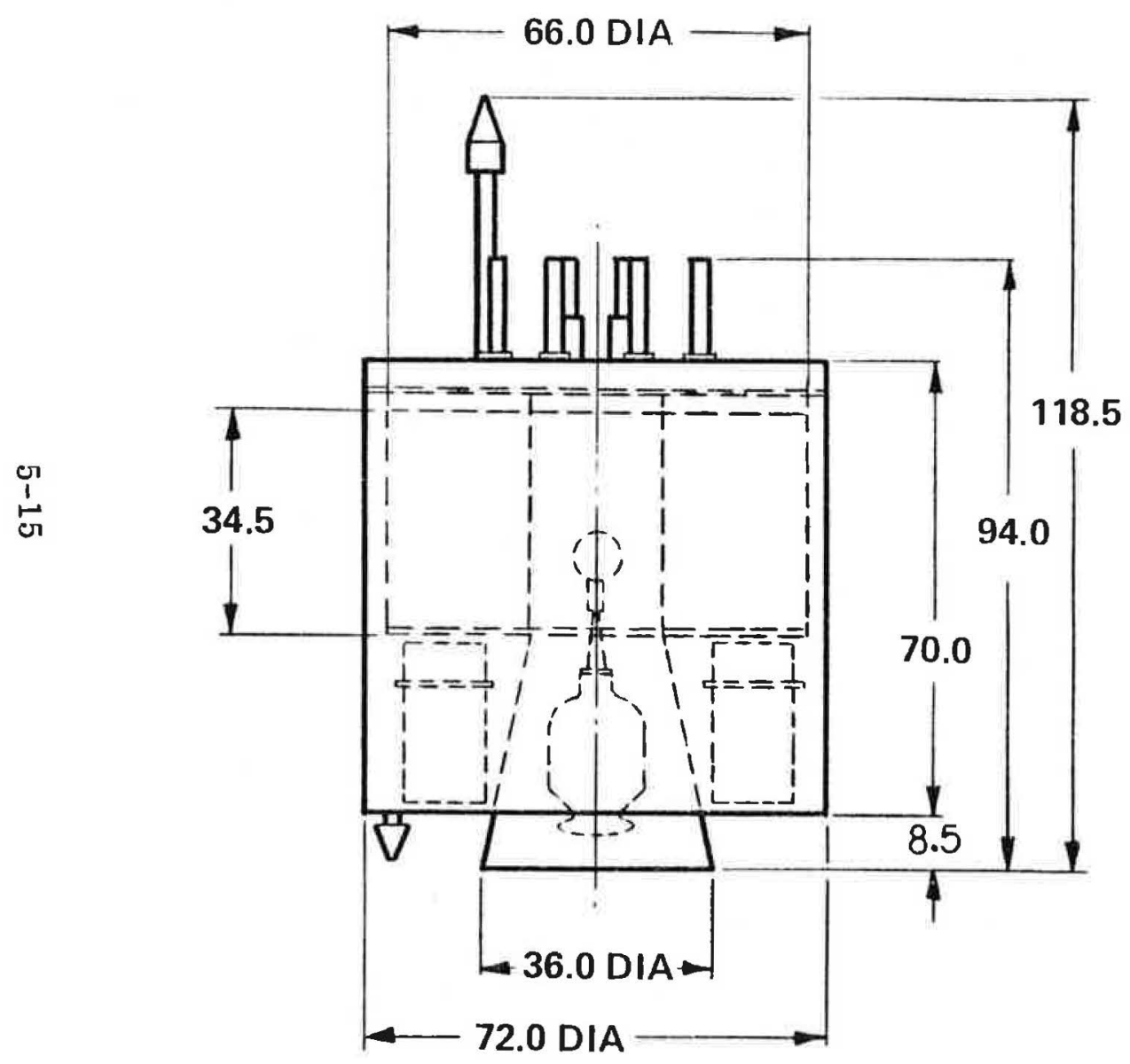

\section{BRAYTON SPACECRAFT}

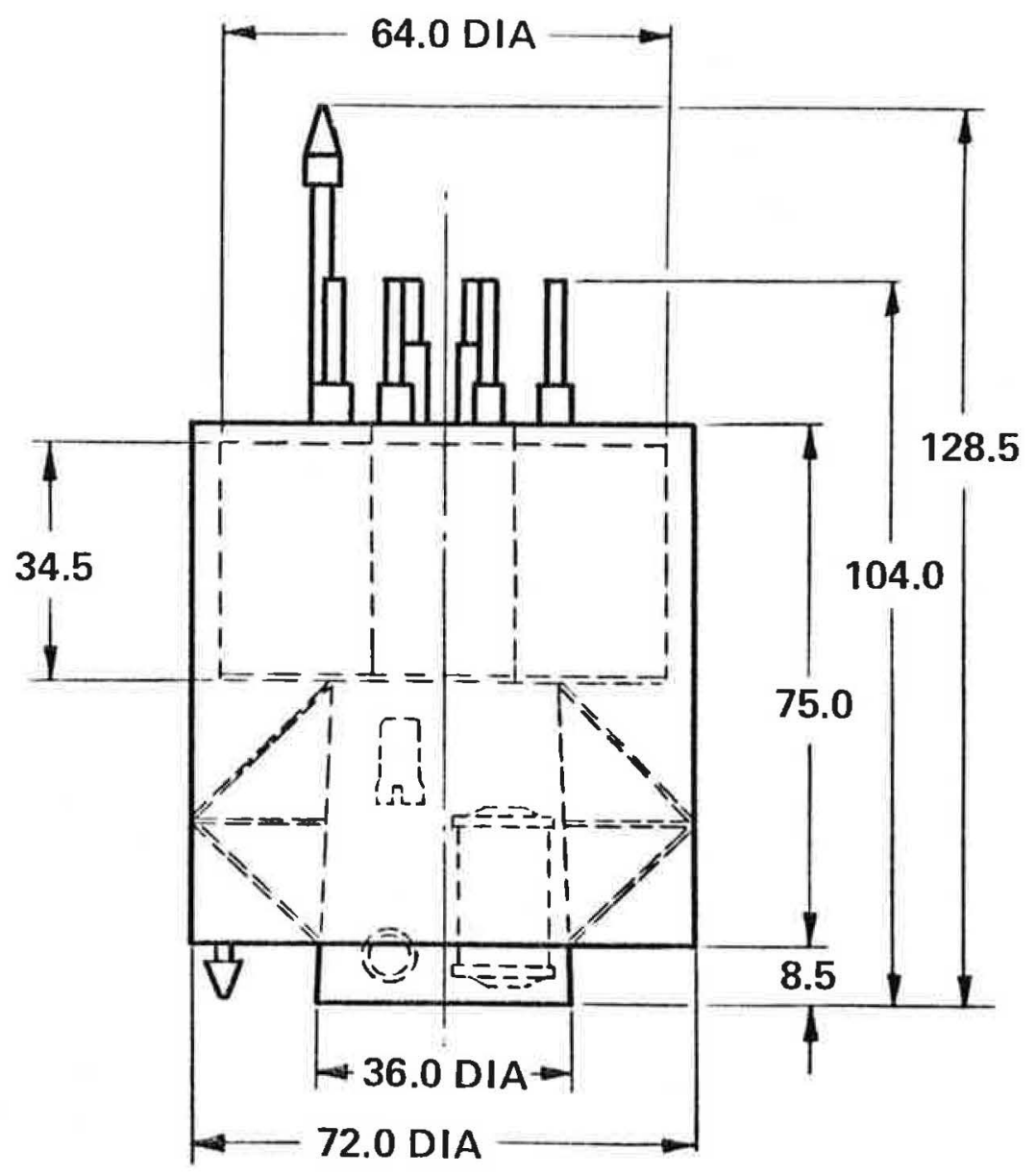



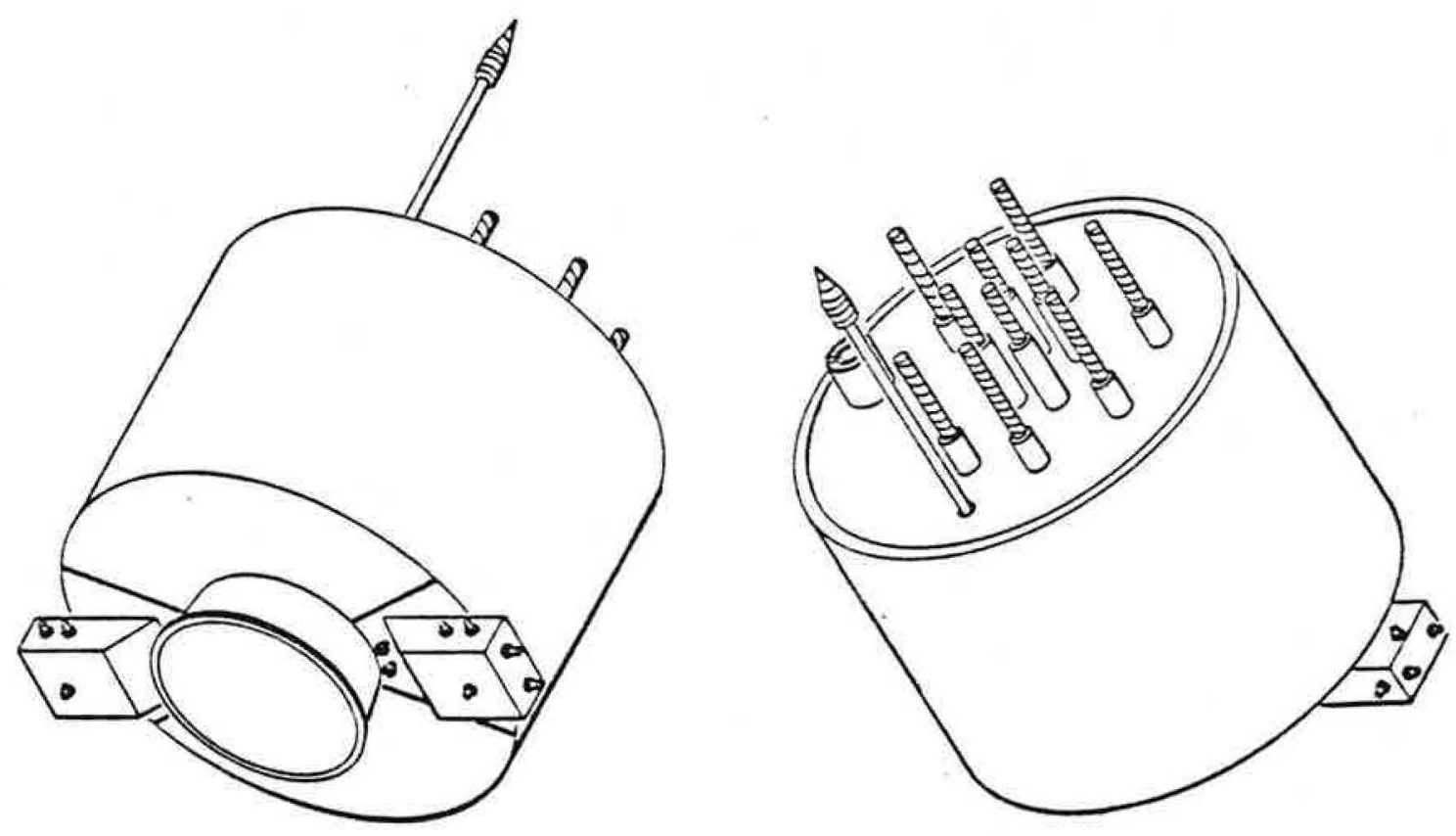

Figure 5.3-2. N/GPS External Views

5.4 FOUR SPACECRAFT CLUSTER

In order to enable a complete preliminary evaluation of the structural requirements for the spacecraft, an interface structure was designed to hold the four spacecraft cluster and mate with the IUS interface ring. This structure, shown in Figure 5.4-1, consists of welded channel beams which pick up the IUS ring at eight tie points. At the opposite face of the beams four cylindrical sections are attached which connect to the four SV thrust cones by marmon clamps. Beams and cylinders are each 8 -inches high, and beam widths were adjusted for maximum combined loads as discussed in Section VI. The interface structure weighs 360 lbs.

The overall configuration of the four spacecraft cluster on the adapter and mounted on the two-stage IUS within the Shuttle cargo bay is shown in Figure 5.4-2. Overall length in the cargo bay is less than half the available length. 


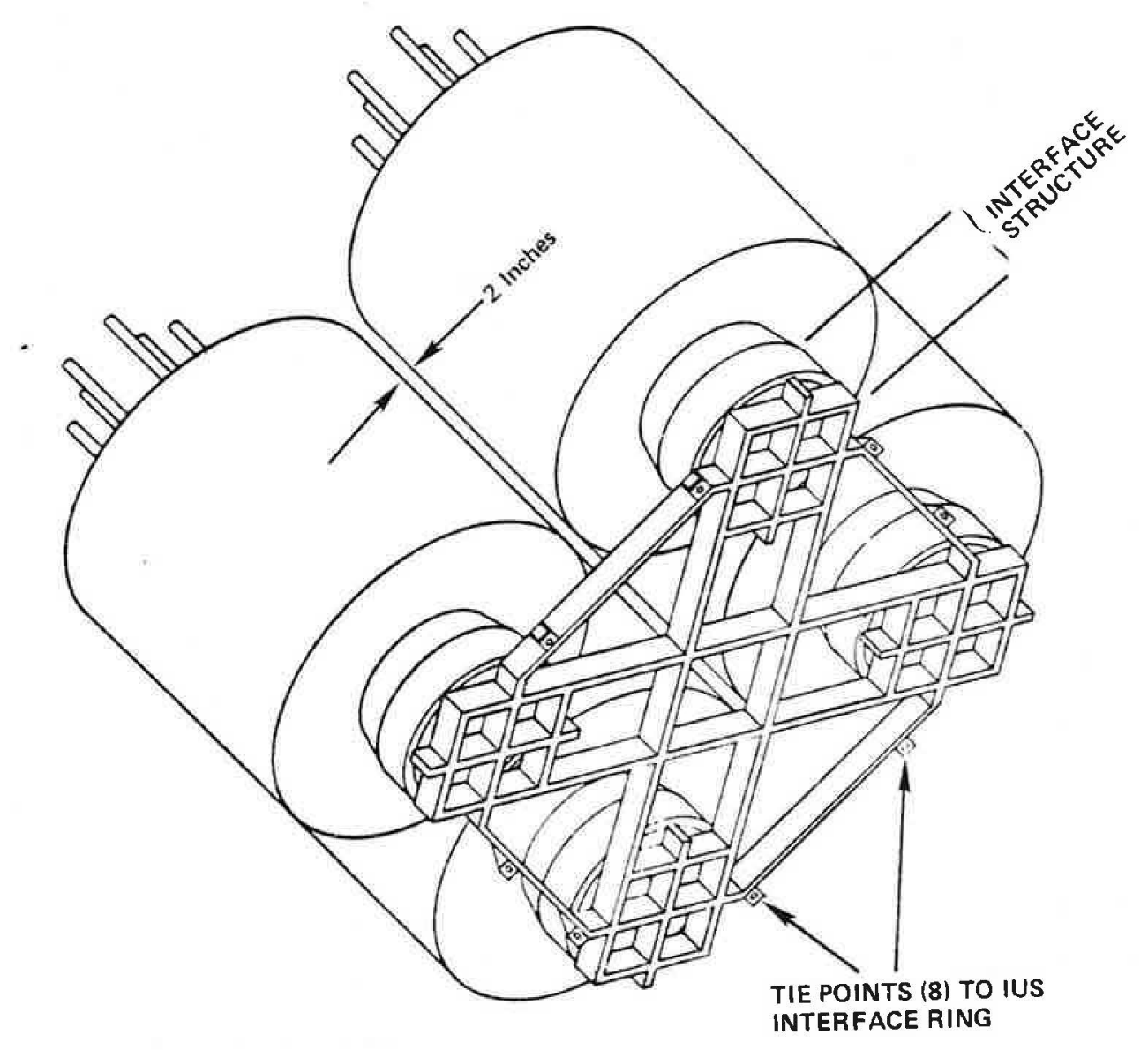

Figure 5.4-1. Four-Spacecraft Interface Structure
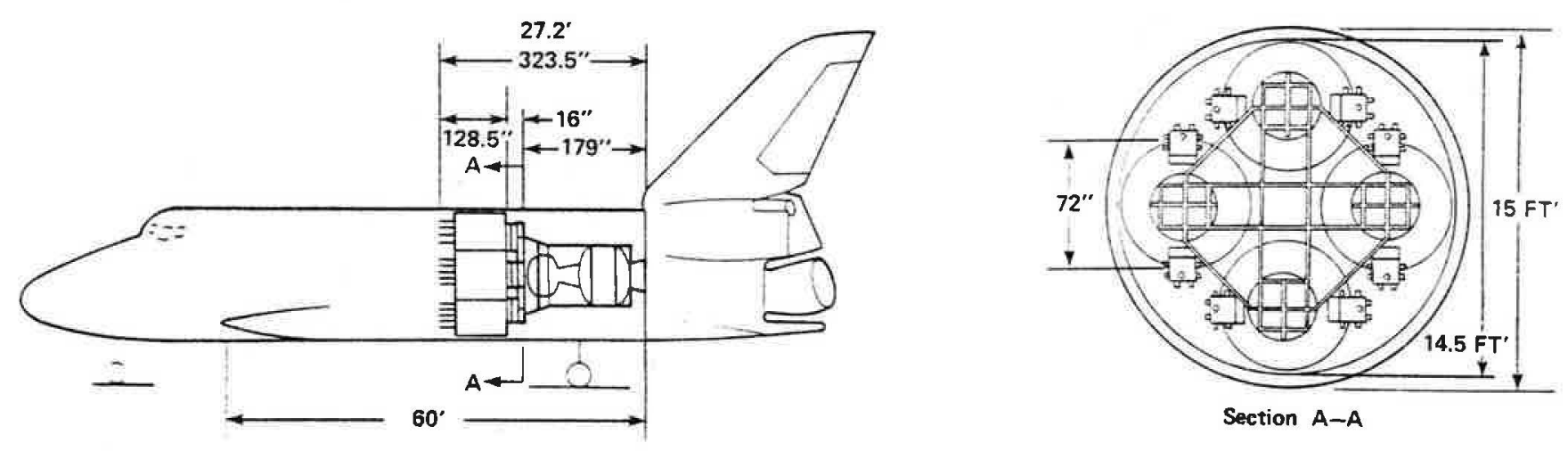

Figure 5.4-2. Spacecraft Mounted in Shuttle 
SECTION VI

STRUCTURAL DESIGN AND ANALYSIS

\section{$6.1 \quad$ SUMMARY OF ANALYSIS}

A structural and dynamic study of the proposed nuclear-powered global position system satellite has been accomplished to assure its structural integrity for both the space shuttle and Titan IIIC environments.

The basic spacecraft concept is to provide a central cylinder (or truncated cone) to support the power system, with radial ribs and shelves to support the payload equipment. The central cylinder with the power conversion unit (PCU), heat source, and radiator is an independent lower structural system. The upper payload section is designed as a separate structural system which is inserted into the radiator and onto the central cylinder. When tied together, the combined system acts as one structural unit.

The structural integrity of this spacecraft is based on a strong central cylinder/cone structure. A marmon clamp interface connection to the IUS adapter structure assures the full load carrying capability of the cylindrical structure and also provides a simple and well-proven separation technique.

In determining the design criteria for this configuration, both the space shuttle and Titan IIIC are evaluated. The load factors for the shuttle crash conditions and Titan IIIC launch are applied here to a four-spacecraft grouping mounted to the IUS interface ring via a specially designed adapter structure.

The ascent and landing load factors for the shuttle require that the exact location of the spacecraft in the cargo compartment is determined. This is required because the specified angular accelerations would increase or decrease 
the translational load factors on the spacecraft depending on its location. Therefore, for analytical purposes two cases were evaluated in which the spacecraft were located at the extreme opposite ends of the shuttle payload compartment. All design loads include a 1.5 safety factor to cover potential design and material changes.

Three mathematical models were developed to accomplish this study. The first one was a bar model of the spacecraft cantilevered at its interface. This model considered the translational and rotational mass inertias for loads and frequencies. This seven-mass bar model was synthesized to a one-mass model that exhibited the same frequencies. Finally, four synthesized spacecraft models were added to a beam/grid adapter structure which in turn was attached to the IUS interface points.

A separate calculation of the frequency modes of the power conversion unit as mounted in the reference-design spacecraft was also performed. It is concluded that the inclusion of shock-mounting isolators in this case is unnecessary and counterproductive.

\subsection{BASIC SPACECRAFT MODEL}

\subsubsection{STRUCTURAL CONCEPT}

Figures 6.2-1 and 6.2-2 depict the basic dimensions of the RSV and BSV configurations and the basic location of the equipment. An evaluation of the required structural members and the equipment weights resulted in a preliminary spacecraft weight estimate of 1120.0 pounds. The approximate distribution of this weight is shown in the figures. The IUS adapter weight of 360.0 pounds is used in the third analytical model which combines four spacecraft with the adapter and in turn is tied to the IUS interface.

The central cylinder of the power system modules is the prime structural element. It supports the PCU and heat source directly. The radiator is supported via radial ribs from the conical section. The equipment module is an independent structural system which has an upper and a lower shelf supporting equipment, frequency standards (clocks); inertia wheels, and antennas. Tied 


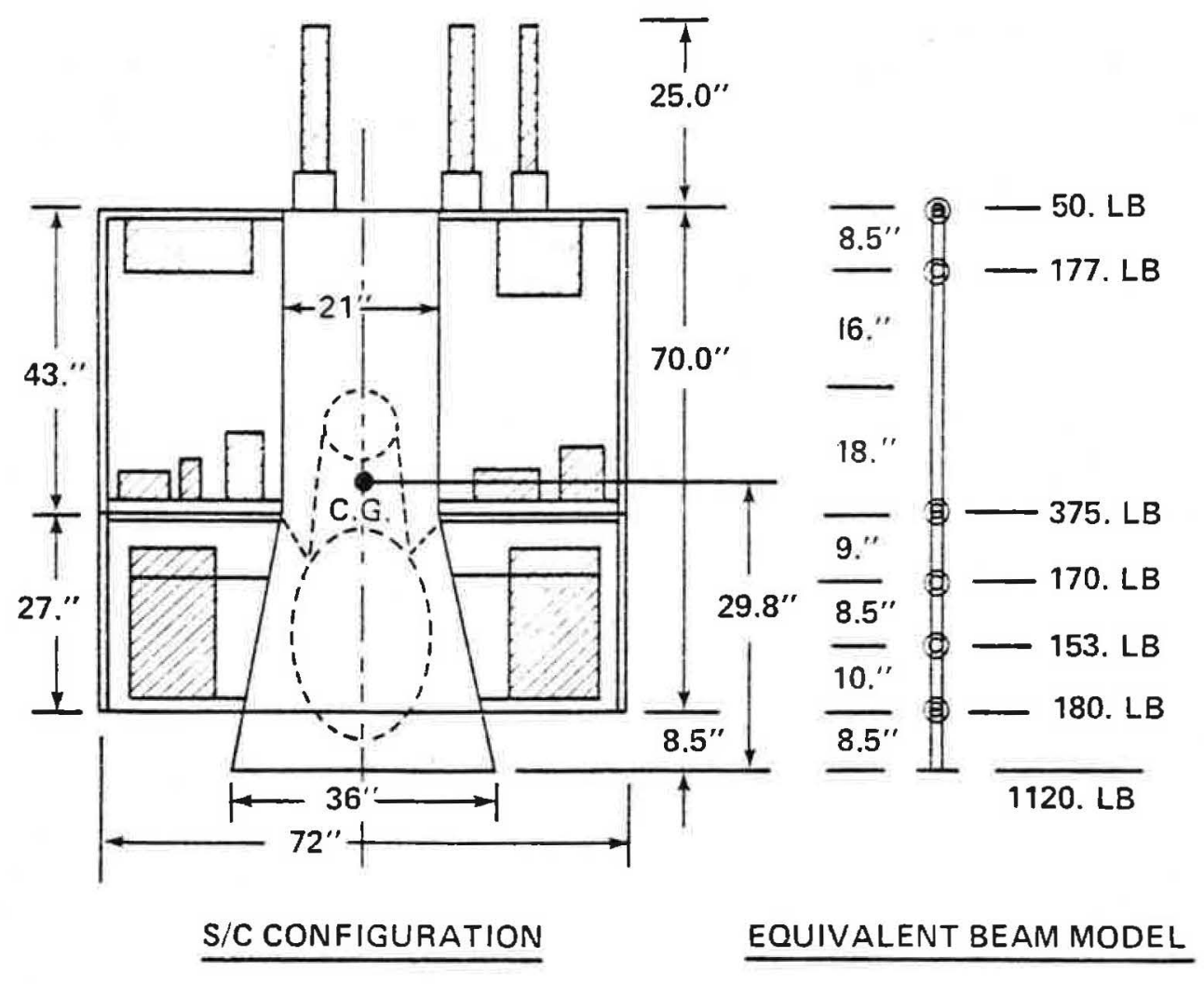

Figure 6.2-1. Analytical Beam Model -- Organic Rankine Spacecraft
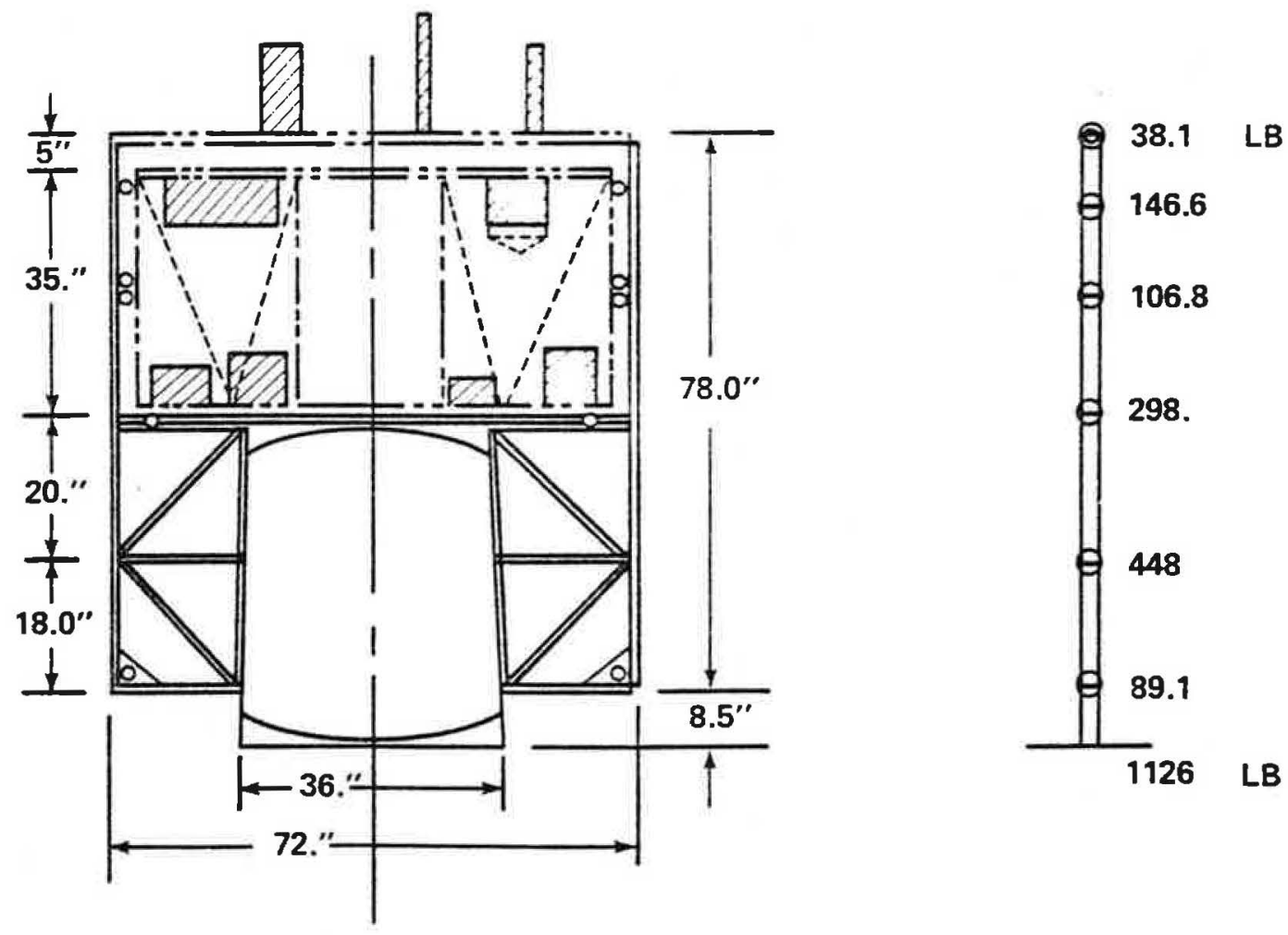

Figure 6.2-2. Analytical Beam Model -- Brayton Spacecraft 
together by rings and radial ribs it forms a toroidal cylinder. During final spacecraft assembly it is lowered onto the upper central cylinder and is fastened to it as well as to the radiator structure.

Providing a central cylinder with high strength and resonant frequency allows changes in weight distribution and radial rib design. The assurance of good frequency separation of the shelves and ribs from the central cylinder is of primary importance. In obtaining this structural frequency separation, it is estimated that a central cylinder frequency of approximately $40.0 \mathrm{~Hz}$ would be satisfactory. The mass properties used for this study are given in Table 6.2-3.

Table 6.2-3. Mass Properties About Spacecraft CG

$$
\begin{gathered}
I_{x o}=134.98 \text { slugs } \\
I_{y o}=149.64 \text { slugs } \\
I_{z 0}=164.00 \text { slugs } \\
\text { Inertia Ratio }=\frac{I_{z 0}}{I_{y 0}}=1.096
\end{gathered}
$$

\subsubsection{ANALYTICAL MODEL}

Using the dimensions depicted in Figures 6.2-1 and 6.2-2, in conjunction with the weight table, a simple equivalent beam model was generated. In addition to transferring the weight laterally to the center line, the inertias about $x, y$, and $z$ were also considered. Each of the six mass points shown in the model are analyzed for six degrees of freedom. The characteristics of a conical section 
which basically increases the stiffness over that of an equivalent cylinder has been incorporated in the model. The primary objective of this model is to define a structurally sound central cylinder to carry all the static and dynamic loadings.

\subsubsection{DESIGN CONDITIONS}

The preliminary analysis of the N/GPS utilizes the design load criteria of both the Titan IIIC launch vehicle and the space shuttle. Table 6.2-4 shows the conditions and load factors used for the Titan IUC criteria. Table 6.2-5 provides the translational and rotation factors for the space shuttle. Table 6.2-6 shows the emergency-landing condition. However, it will be seen that the "normal" landing conditions provide the worst-case quasi-state loads in the critical lateral directions.

Table 6.2-4. Titan IIIC Design Load Criteria

\begin{tabular}{|c|c|}
\hline \multicolumn{1}{|c|}{ PHASE } & ACCELERATION, $g$ \\
\hline LIFTOFF & \\
AXIAL & $3.0^{*}$ \\
LATERAL & $1.5^{*}$ \\
MAXIMUM AIRLOADS & \pm 5.0 \\
AXIAL & \\
LATERAL & $3.0^{*}$ \\
TORSIONAL & $1.5 \#$ \\
STAGE I DEPLETION SHUTDOWN & \pm 2.5 \\
AXIAL & 2.5 @ 50-INCH RADIUS \\
LATERAL & $8.0^{*}$ \\
STAGE II DEPLETION SHUTDOWN & $6.5 \#$ \\
AXIAL & \pm 3.0 \\
LATERAL & \\
\hline
\end{tabular}

* COMPRESSION

\# TENSION 
Table 6.2-5. Space Shuttle Ascent/Descent Payload Design Load Factors $\star$

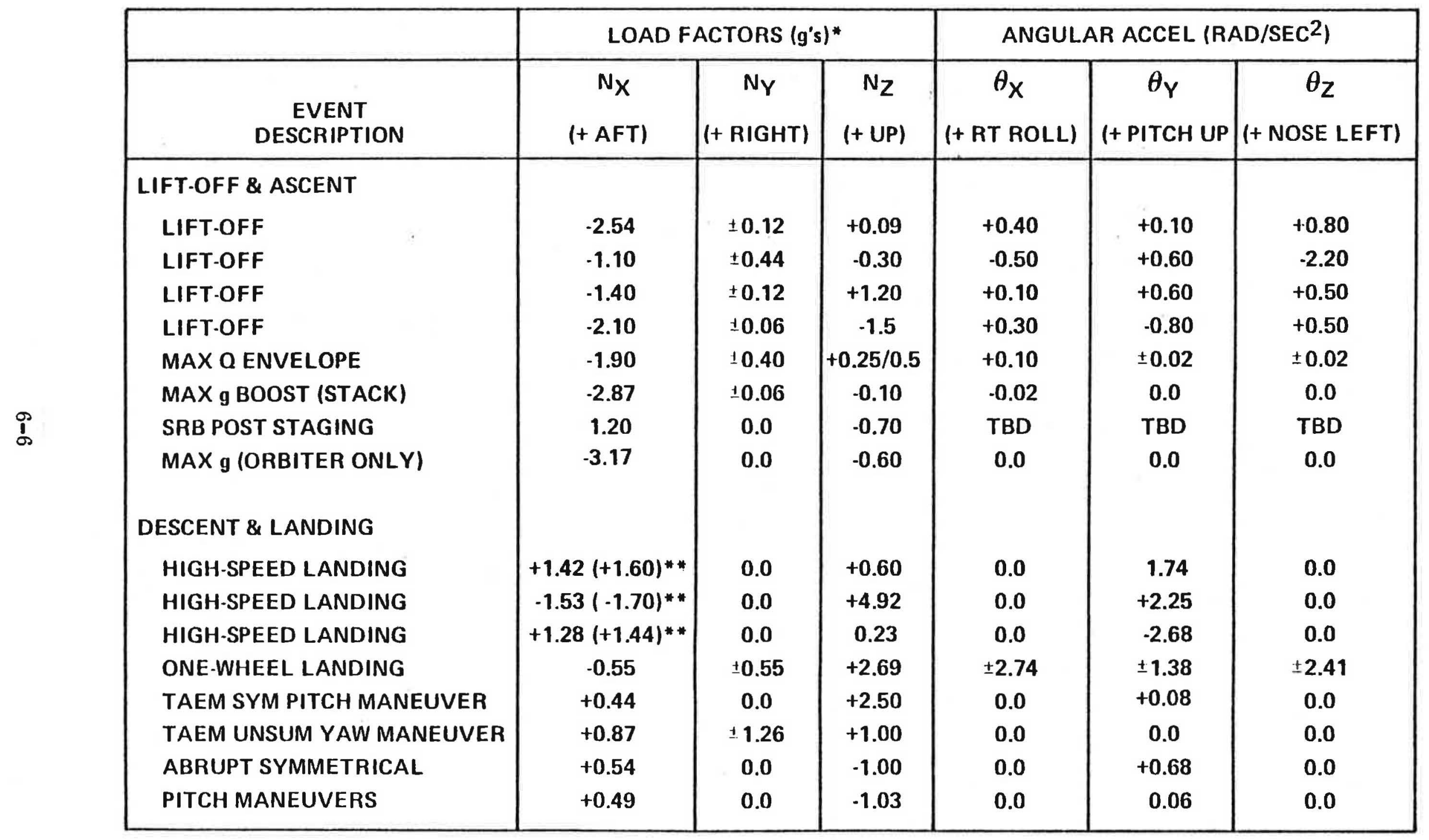

$\star$ LOAD FACTOR IS DEFINED AS THE TOTAL EXTERNALLY APPLIED LOAD ON A PAYLOAD OR COMPONENT DIVIDED BY THE PAYLOAD OR COMPONENT WEIGHT, \& CARRIES THE SIGN OF THAT APPLIED LOAD

* REFERENCED TO ORBITER STATION $X=1078, Y=0, Z=400$

* first value for total payload; Parenthetical value for payload components 
Table 6.2-6. Emergency Landing Ultimate Load Factors

\begin{tabular}{|c|c|c|}
\hline $\begin{array}{c}\text { LONGITUDINAL } \\
\text { (+ AFT) }\end{array}$ & $\begin{array}{c}\text { LATERAL } \\
\text { (+ RIGHT) }\end{array}$ & $\begin{array}{c}\text { VERTICAL } \\
\text { (+ UP) }\end{array}$ \\
\hline+9.0 & \pm 1.5 & +4.5 \\
-1.5 & & -2.0 \\
\hline
\end{tabular}

The Titan IIIC load factors only considered combined axial and lateral translational effects. The space shuttle conditions are more complicated. Since the shuttle can launch and land payloads, the payloads will now be subjected to angular accelerations in addition to the translational accelerations as shown in Table 6.2-5. Therefore, the location of the spacecraft in the shuttle payload compartment is significant in determining the design load factors. All of the translational and angular accelerations are referenced to fuselage station 1078, the standard load and CG reference point in the shuttle cargo bay. A payload can be as far forward as 496 inches or as far aft as 224 inches from the reference station (See Figure 6.2-7.)

An example of the effect of location is gotten by considering a high speed landing condition with a spacecraft CG located 174. inches aft of station 1078, the $\mathrm{N}_{\mathrm{Z}}=+4.92$ and $\theta \mathrm{y}=+2.25$. The load factors at station 1252 are shown in Figure 6.2-8.

The structural investigation for the shuttle covered the emergency landing condition, the high speed landing condition and a one-wheel landing condition. The lift-off and ascent criteria of the shuttle were less critical than that of the Titan IIIC.

\subsubsection{STATIC ANALYSIS}

With the design conditions discussed in Section 6.2.3 and the analytical model discussed in Section 6.2.2, the NASA-NASTRAN program was used to determine the central cylinder loads as well as the interface loads. Table 6.2-9 provides the summary of the interface loads for the shuttle and Titan IIIC. 


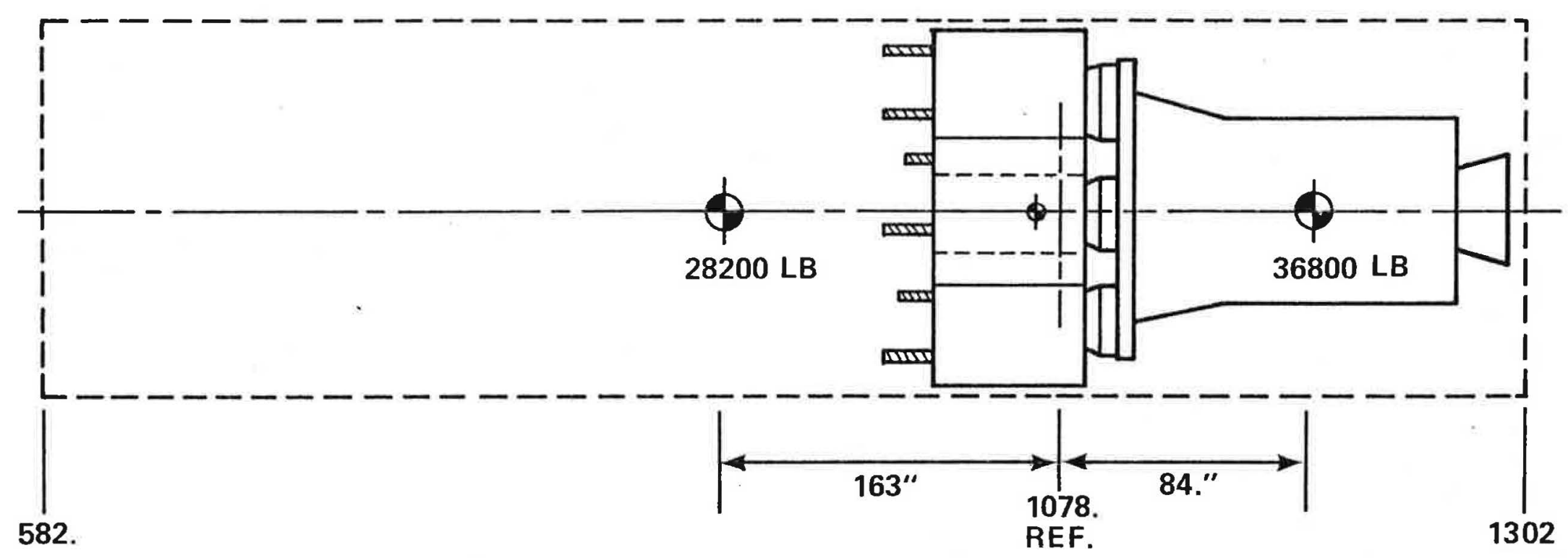

D

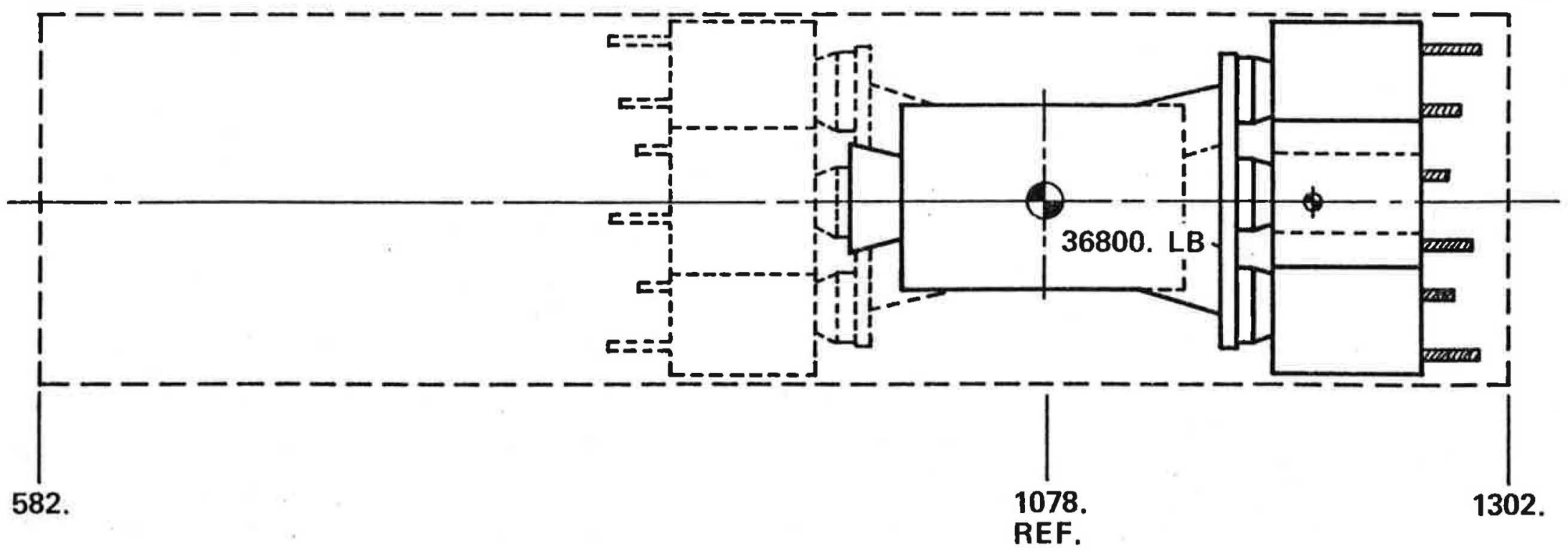

Figure 6.2-7. Shuttle Installation Alternatives 


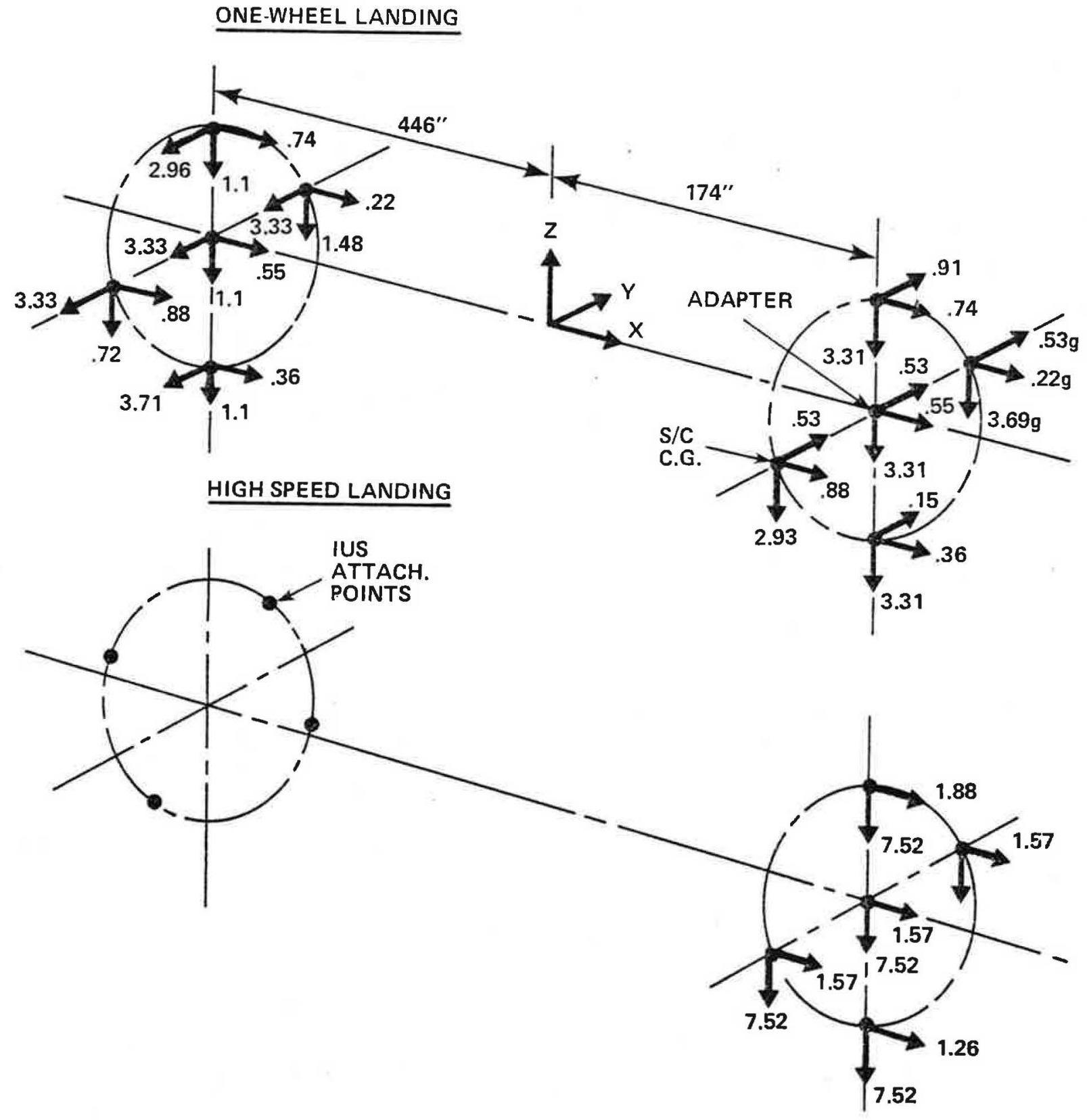

Figure 6.2-8. Design Load Factors for Translation and Rotation 
Table 6.2-9. Summary of S/C Interface Loads - Analytical Beam Model $\star$

\begin{tabular}{|c|c|c|c|c|c|c|c|c|c|}
\hline \multirow[b]{4}{*}{$\begin{array}{l}\text { LBS } \\
\text { IN.-LBS }\end{array}$} & \multicolumn{6}{|c|}{ SPACE SHUTTLE ORBITER } & \multirow{3}{*}{\multicolumn{3}{|c|}{$\begin{array}{l}\text { TITAN IIIC } \\
\text { LAUNCH }\end{array}$}} \\
\hline & \multicolumn{3}{|c|}{ EMERGENCY LANDING } & \multicolumn{3}{|c|}{ DESCENT \& LANDING } & & & \\
\hline & \multirow[t]{2}{*}{$4.5 \mathrm{~g}$ VERT } & \multirow[t]{2}{*}{$4.5 \mathrm{~g}$ LAT. } & \multirow[t]{2}{*}{$-9.0 \mathrm{AXIAL}$} & \multicolumn{2}{|c|}{ ONE-WHEEL LDG. } & \multirow{2}{*}{\begin{tabular}{|c} 
HIGH \\
SPD. LDG. \\
AFT \\
PAYLOAD
\end{tabular}} & & & \\
\hline & & & & $\begin{array}{c}\text { FWD. } \\
\text { PAYLOAD }\end{array}$ & $\begin{array}{c}\text { AFT } \\
\text { PAYLOAD }\end{array}$ & & $\begin{array}{l}y=5.0 \mathrm{~g} \\
z=3.0 \mathrm{~g}\end{array}$ & $\begin{array}{c}y=5.09 \\
z=-10.9\end{array}$ & $\begin{array}{l}y=5.0 \mathrm{~g} \\
z=8.0 \mathrm{~g}\end{array}$ \\
\hline$P_{x}$, Ibs. & -7476. & - & - & 2456. & 6123. & 12478. & - & - & - \\
\hline$P_{y}$ & - & -7467. & - & 6156. & -1510. & - & .8297. & -8297. & -8297. \\
\hline $\mathbf{P}_{\mathbf{Z}}$ & - & - & -14935. & 1460. & -1460. & -2605. & 4978. & 16594. & -13275. \\
\hline$M_{x}$, in-lbs. & - & 222420. & - & -183375. & 44978. & - & 247133. & 247134. & 247134. \\
\hline $\mathrm{M}_{\mathrm{Y}}$ & -222420 & - & - & 73151. & 182385. & 371689. & - & - & - \\
\hline$M_{\mathbf{z}}$ & - & - & - & - & - & - & - & - & - \\
\hline$\delta$, in. & .028 & .028 & - & .023 & .024 & .047 & .032 & .032 & .032 \\
\hline
\end{tabular}

${ }^{\star}$ SEVEN BAR ELEMENTS

SEVEN MASS POINTS - 6 DOF S $/ C$ WEIGHT $=1120.0 \mathrm{lbs}$.

$C G=29.78$ in.

SAFETY FACTOR $=1.5$

$\delta$ - SPACECRAFT CG DEFLECTION 
From Table 6.2-9, the high-speed landing of the shuttle is seen to result in the maximum load and bending moment. This provides the design condition for the central cylinder as the primary structural element. The conical section is . 080 inches thick with an allowable bending moment of 484137 in. lbs. and an allowable axial load of 74223.0 pounds. This results in a margin of safety for the high speed landing of:

$$
\text { M.S. }=\frac{1}{R_{b}+R_{a}}-1=\frac{1}{.77+.17}-1=.064
$$

over and above the safety factor of 1.5 applied in the calculation.

Table 6.2-9 also shows the maximum lateral deflection of the spacecraft under the calculated bending moment. When combined with the deflections of the entire spacecraft mounted on the IUS adapter (Section 6.3) this will determine the clearance requirements between adjacent spacecraft and between spacecraft and shuttle cargo bay walls.

\subsubsection{DYNAMIC ANALYSIS}

Using the same analytical model described above, a modal analysis was accomplished with the six concentrated masses, each having six degrees of freedom. The results of this analysis are given in Table 6.2-10 with the eigenvalues and mode characteristics as shown.

Table 6.2-10. Summary of S/C Frequencies Analytical Beam Model

\begin{tabular}{|l|l|}
\hline MODE & MODE CHARACTERISTIC \\
\hline$f_{1}=41.9 \mathrm{~Hz}$ & TORSION \\
$f_{2}=46.8 \mathrm{~Hz}$ & BENDING IN X DIRECTION \\
$f_{3}=46.84 \mathrm{~Hz}$ & BENDING IN Y DIRECTION \\
$f_{4}=93.9 \mathrm{~Hz}$ & 2ND BENDING MODE IN X DIRECTION \\
$f_{5}=94.0 \mathrm{~Hz}$ & 2ND BENDING MODE IN Y DIRECTION \\
$f_{6}=103.1 \mathrm{~Hz}$ & 2ND TORSIONAL MODE \\
$f_{7}=152.8 \mathrm{~Hz}$ & 1ST AXIAL MODE \\
& \\
\hline
\end{tabular}


This phase of the study evaluates the capability and requirements of the adapter which holds four spacecraft on to the interface ring of the IUS.

\section{3.1}

ANALYTICAL APPROACH

The primary effort is to define an adapter to support four spacecraft that will be supported by an IUS and fit into the space shuttle. The size of the analytical model will be dominated by the adapter. To keep the overall size of the model to a minimum, the six-mass bar model was synthesized to a one-mass system with an eight-bar structure, shown in Figure 6.3-1, that is equivalent to the bar model. This concept is valid for this purpose since the one-mass model will satisfy the load introduction into the adapter and provide the proper dynamic coupling. The comparison of resonant frequencies for the three primary modes of the two models is shown in Table 6.3-2.

Table 6.3-2. Model Frequency Comparisons

\begin{tabular}{|c|c|c|c|}
\hline Mode & Bending & Torsion & Axial \\
\hline Bar Model & 47. & 42. & 152. \\
One-Mass Model & 45. & 48. & 144. \\
\hline
\end{tabular}

\subsection{2}

\section{ADAPTER DESIGN AND ANALYTICAL MODEL}

The adapter shown in Figure 6.3-1 is composed of six-inch channels around the periphery and spacecraft support areas and eight-inch channel cross beams tying the four spacecraft together.

Four cylindrical transition sections are attached to the adapter at eight points each. These provide an even load transfer to the spacecraft via a marman clamp at the other end. The eight spacecraft attachment points are shown in Figure 6.3-1, as are the attach fittings to the IUS interface ring. Clearly, the eight bar spacecraft model has been synthesized to properly introduce loads into the adapter structure. 

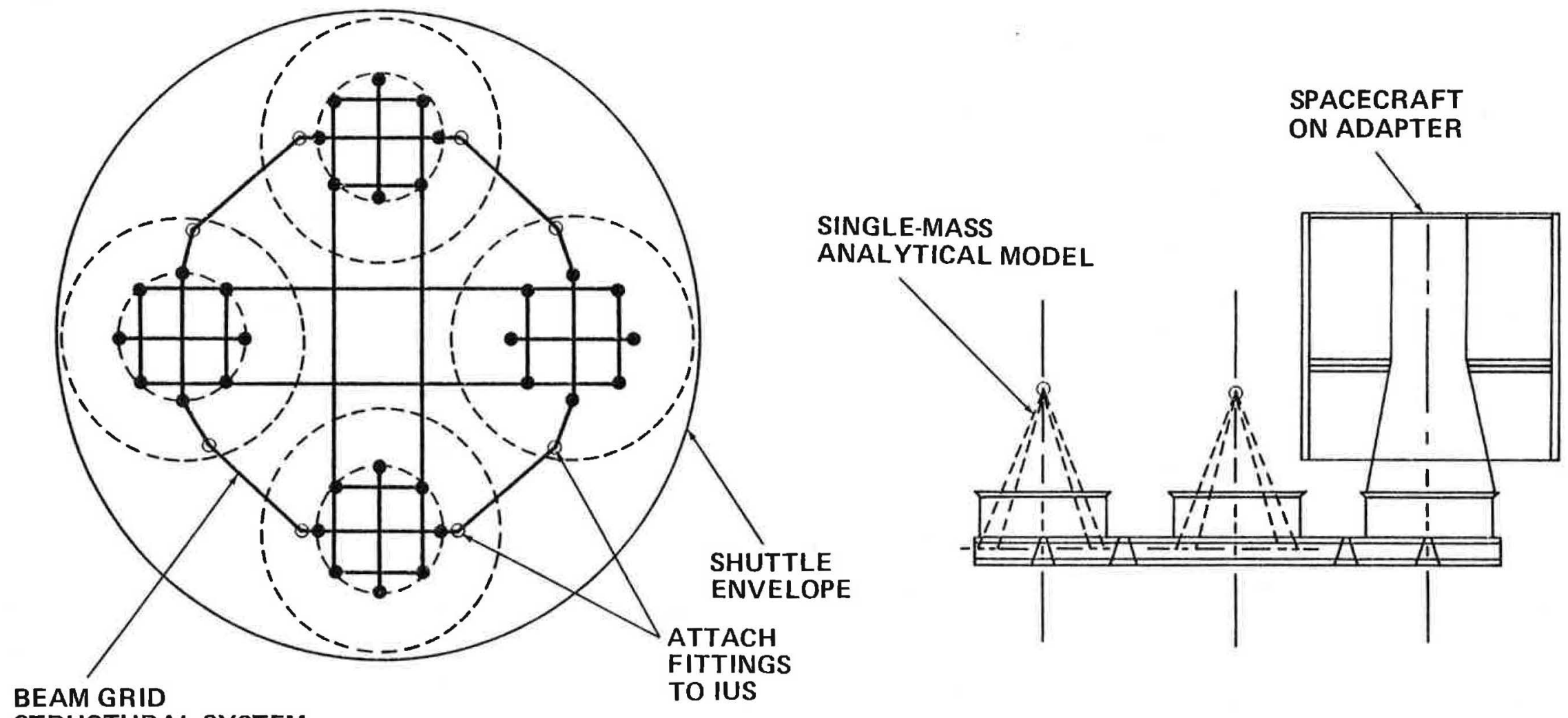

STRUCTURAL SYSTEM 
Figure 6.3-3 depicts the computer drawing of the analytical model. The spacecraft mass allows translational and rotational degrees of freedom while the eight adapter masses only consider translational degrees of freedom.

\subsubsection{DESIGN CONDITIONS}

The design conditions are the same as those used in Section 6.2 for the individual spacecraft. Since the four spacecraft are located off the shuttle longitudinal (x) axis, the landing conditions will produce different design load factors on each spacecraft. Figure 6.2-8 shows the design load factors used in analyzing the adapter.

\subsubsection{STATIC ANALYSIS}

Table 6.3-4 gives the maximum interface loads between the adapter and IUS. It was assumed that the eight interface attachment points were fully fixed. The interface bolt pattern is identical to that of the Titan IIIC interface. These interface loads will vary depending on the type of attachments and the flexural characteristics of the IUS interface ring with its supports. For the purpose of this analysis the interface ring is assumed to be rigidly mounted to the IUS. Figure 6.3-5 shows the CG displacement and satellite top displacement for the worst shuttle case (emergency landing).

With the current reference design clearances between adjacent spacecraft and between spacecraft and shuttle cargo bay wall are approximately two inches each way. Since the calculated deflections are undirectional under the quasi-static loads applied, this clearance is adequate. Deflections under dynamic (vibration) loading can be in opposite directions, and hence additive between adjacent spacecraft, but these will be seen to be much lower in magnitude (next section).

It should be recognized that the Titan IIIC cases are included for reference only. The same four-spacecraft adapter structure would not be applicable to the Titan because of the diametral limitations of the Titan shroud. Preliminary indications are that this may be enlarged to a 147-inch dynamic diameter by 30-foot length in which case a two-over two type of adapter structure may be applicable. 


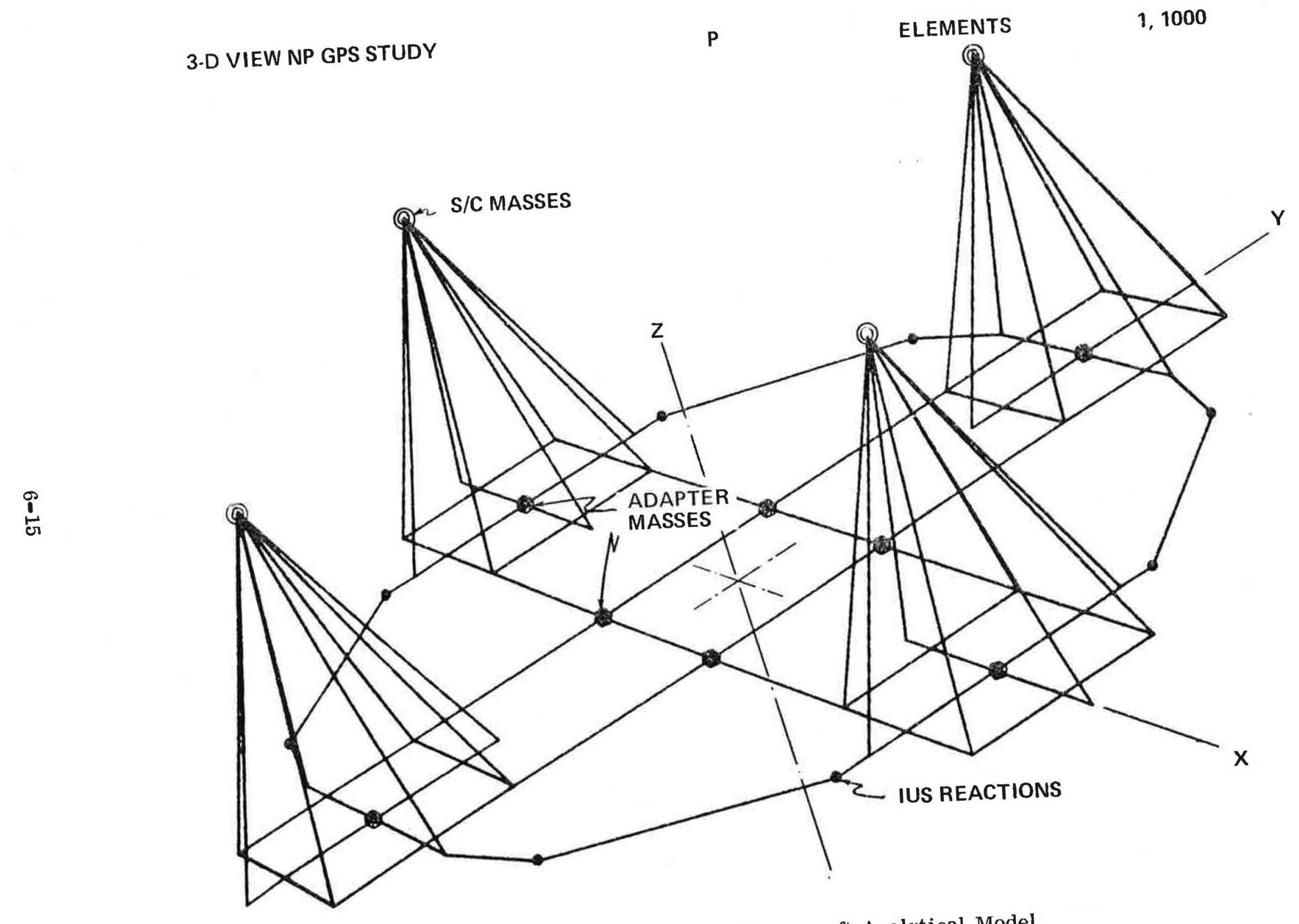

Figure 6.3-3. Four-Spacecraft Analytical Model 
Table 6.3-4. Summary of Adapter - IUS Interface Analytical Model

\begin{tabular}{|l||c|c|c||c|c|c||c||c||}
\hline \multicolumn{1}{|c||}{} & \multicolumn{9}{c||}{ SPACE SHUTTLE ORBITER } & \multicolumn{3}{c|}{ TITAN IIC } \\
LAUNCH
\end{tabular}

RESULT: ADAPTER STRENGTHENED FOR MAXIMUM BENDING MOMENT 


\section{MAXIMUM SPACECRAFT DEFLECTIONS \\ IN SHUTTLE EMERGENCY LANDING}
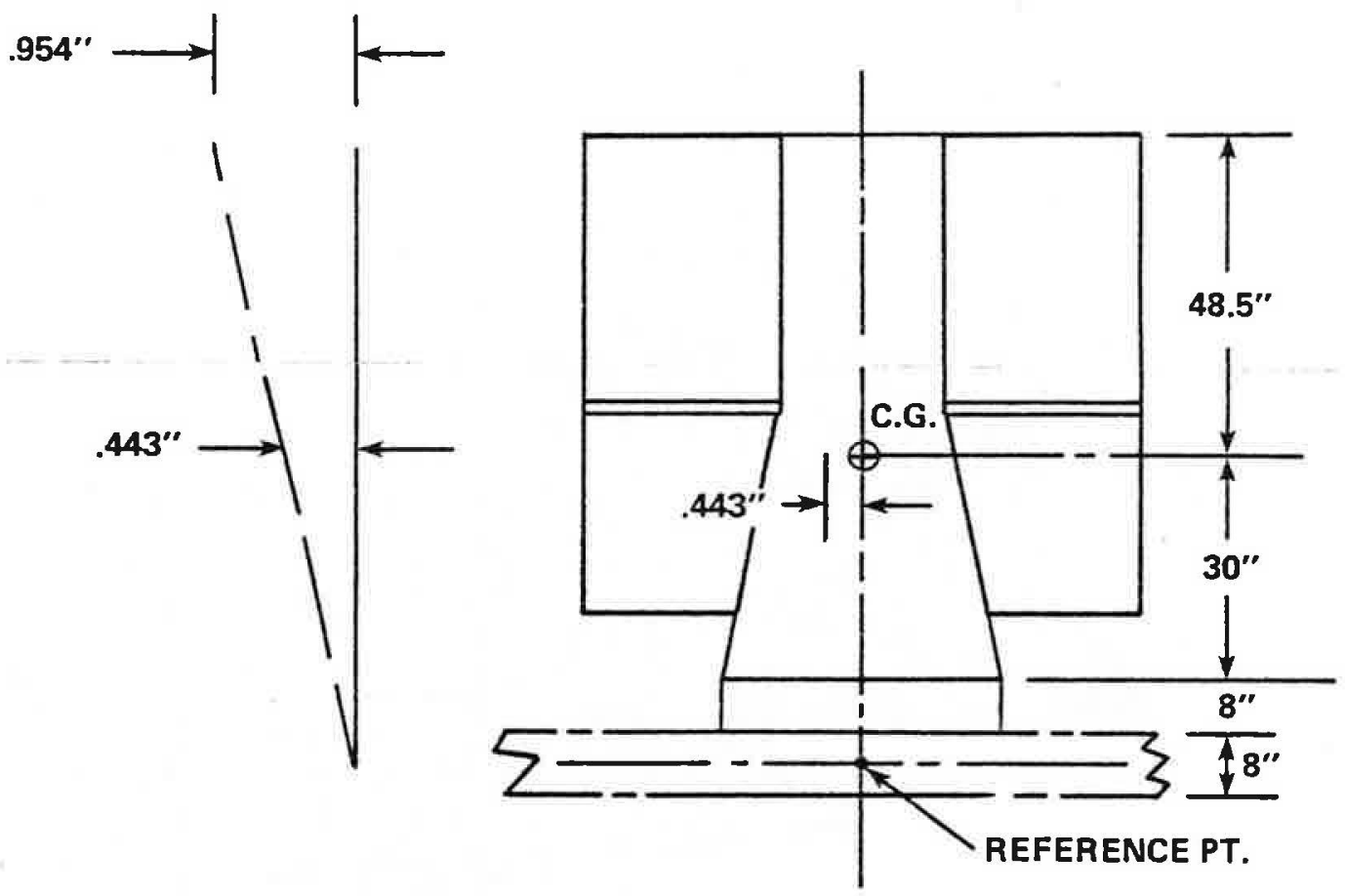

Figure 6.3-5. Maximum Spacecraft Deflections in Shuttle Emergency Landing $6.3-5$ DYNAMIC ANALYSIS

The same analytical model described above was used for the modal analysis of this integrated system. Again the IUS back-up structure was considered rigid for this analysis. The results of this modal analysis are given in Table 6.3-6 which gives the frequency values and the mode characteristics. Figures 6.3-7 and 6.3-8 depict the mode shapes of the two lowest frequency vibration modes. The displacements shown are amplified by a scale factor of 20 .

Table 6.3-6. Summary of $4 \mathrm{~S} / \mathrm{C}+$ Adapter to IUS Frequencies Analytical Model

\begin{tabular}{|c|l|}
\hline$f_{1}=9.97 \mathrm{~Hz}$ & 2 S/C BENDING TOWARDS EACH OTHER Y-DIRECTION \\
$f_{2}=15.73 \mathrm{~Hz}$ & 2 S/C BENDING IN SAME DIRECTION Y-DIRECTION \\
$f_{3}=17.46 \mathrm{~Hz}$ & 2 S/C BENDING IN SAME DIRECTION X-DIRECTION \\
$f_{4}=20.03 \mathrm{~Hz}$ & 2 S/C BENDING TOWARDS EACH OTHER X-DIRECTION \\
$f_{5}=24.60 \mathrm{~Hz}$ & 2 S/C BENDING OPPOSITE TO EACH OTHER X-DIRECTION \\
$f_{6}=27.29 \mathrm{~Hz}$ & 2 Y AXIS S/C BENDING TOGETHER IN X-DIRECTION \\
$f_{7}=30.19 \mathrm{~Hz}$ & 2 Y AXIS S/C BENDING OPPOSITE IN X-DIRECTION \\
\hline
\end{tabular}





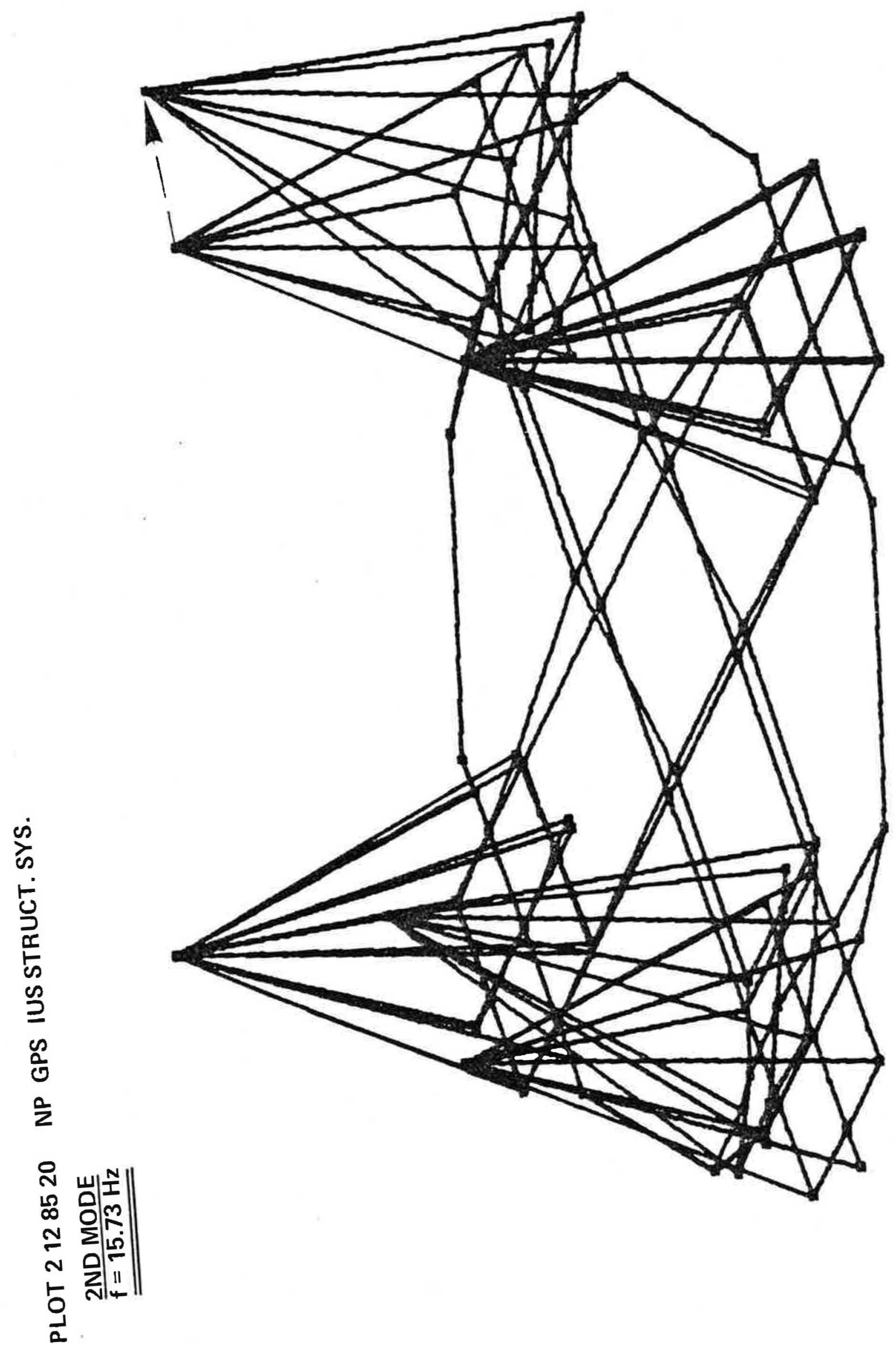


The combined CG of the four spacecraft and its adapter when joined to the IUS was estimated.

If mounted in the cargo bay as shown in Figure 6.2-7 another payload of approximately 28,000 pounds must be mounted with its CG within a few feet of the location shown $(x=915)$, if a full 65,000 -pound load is to be launched.

If it is to be launched alone, it can be mounted with its CG at any location between $\mathrm{x}=942$ and $\mathrm{x}=1133$. Two orientation options are shown in Figure 6.2-7 with the combined CG at $x=1078$.

6.5 POWER CONVERSION UNIT (PCU)

The RSV/PCU is an independent mass system tied to the upper ring of the conical structure at three points. To evaluate the dynamic characteristics of the PCU, Sundstrand's mathematical model of the PCU was used. The support legs were modified so that the regenerator supports were tied to the cone ring by " $V$ " members. Identical shock mount characteristics were also used. Figure 6.5-1 depicts the analytical model while Figure 6.5-2 depicts the computer plot of this model.

The analytical model was evaluated with and without shock mounts and compared to the fundamental structural frequencies of the spacecraft and stowed shuttle system.

The results of the analysis is given in Table 6.5-3. With the shock mounts, the first frequency of $8.6 \mathrm{~Hz}$ is relatively close to that of the stowed shuttle system of $10 \mathrm{~Hz}$ (Table 6.3-6). This would be an undesirable condition in which the PCU mass would be highly amplified. On the other hand, the fundamental frequency of the PCU without the shock mounts is $33 \mathrm{~Hz}$. This is sufficiently isolated from the spacecraft and stowed shuttle system frequencies that the chance of dynamic coupling is remote. This will assure that the loads applied to the PCU will be very close to the loads at the spacecraft interface. Hence, no shock mounts are recommended for this case, where the PCU is mounted to the rigid central cone-cylinder. 
ANALYTICAL MODEL FOR MODAL ANALYSIS; ORGANIC RANKINE CONVERTER SYSTEM

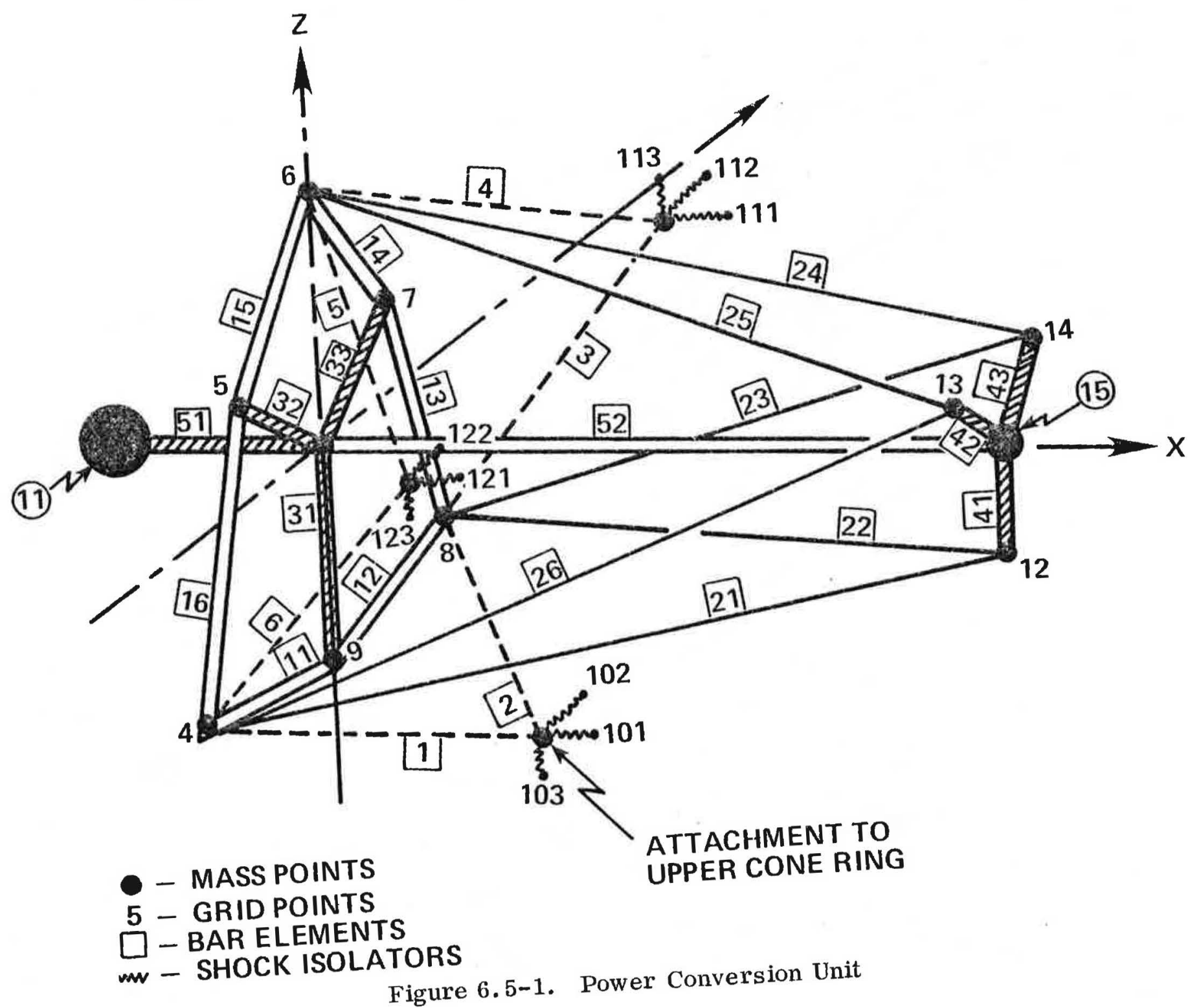




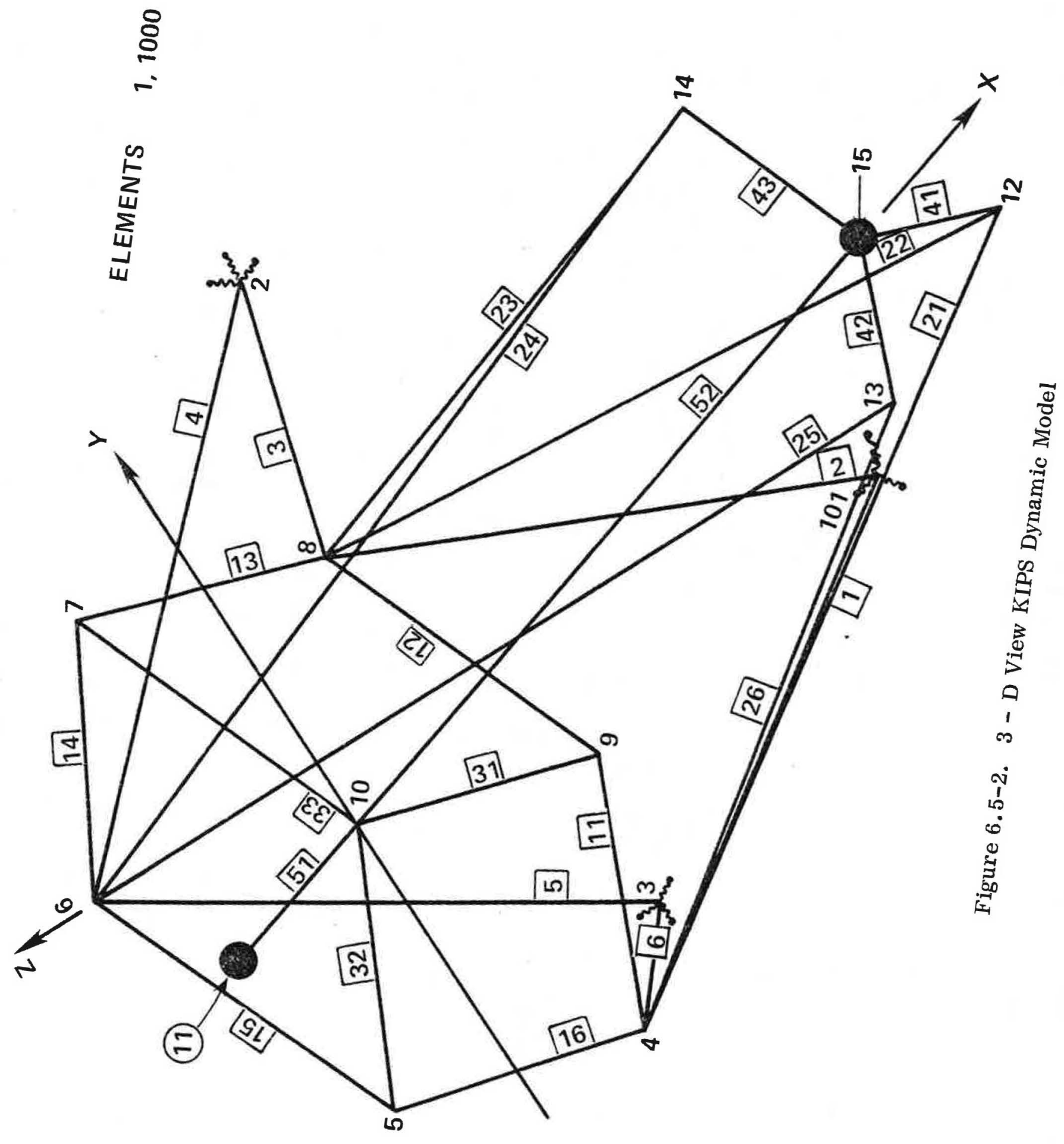


Table 6.5-3. Organic-Rankine Converter System Summary of Model Analysis

\begin{tabular}{|c|c|c|c|}
\hline \multirow[b]{2}{*}{ MODE CHARACTERISTIC } & \multirow[b]{2}{*}{$\begin{array}{c}\text { MODE } \\
\text { NO. }\end{array}$} & \multicolumn{2}{|c|}{ FREQUENCY, $\mathrm{Hz}$} \\
\hline & & $\begin{array}{c}\text { WITH } \\
\text { ISOLATORS }\end{array}$ & $\begin{array}{l}\text { WITHOUT } \\
\text { ISOLATORS }\end{array}$ \\
\hline LATERAL MOTION (REGENERATOR) & $1-2$ & 8.66 & 33.7 \\
\hline LATERAL MOTION (ACCUMULATOR) & $3-4$ & 21.70 & 69.9 \\
\hline THRUST MODE & 5 & 22.40 & 88.6 \\
\hline TORSIONAL MODE & 6 & 44.30 & 119.8 \\
\hline
\end{tabular}





\section{SECTION VII}

\section{ATTITUDE AND REACTION CONTROL SYSTEMS}

\section{1}

\section{EQUIPMENT COMPLEMENT SUMMARY}

The Attitude and Velocity Control Subsystem, AVCS, comprised of the Attitude Control Subsystem (ACS) and Reaction Control Subsystem (RCS), serves to stabilize the nuclear-powered space vehicle (SV) following separation from the launch vehicle. The AVCS consists of sensors, controllers and torquers as required to satisfy the various mission operation requirements. These operations include initial stabili zation and Earth acquisition after separation, orbit position control, on-orbit attitude control, momentum unload, and orbit maintenance.

The AVCS operates autonomously, with ground command override, during initial acquisition and normal on-orbit operations including unload. Orbit position control and orbit maintenance thrustings are ground initiated and ended while the concurrent attitude control is autonomous. Status and monitoring signals, for both ACS and RCS, are telemetered to the ground.

Performance is not impared by any single point failure. The ACS is completely redundant and the RCS thrust capability is multiply redundant.

AVCS component characteristics for an Organic-Rankine-Cycle-powered SV are given in Table 7.1-1. Modifications caused by use of a Brayton cycle power plant are considered in Section 7.6. Component locations are shown in Figure 7.1-2. 7.2 $\quad$ REQUIREMENTS

\subsubsection{EARTH ACQUISITION}

After separation from the IUS launch vehicle with its concomitant rate $( \pm 0.5 \%$ sec $)$ and attitude errors $\left( \pm 2^{\mathrm{O}}\right)$, the AVCS shall bring the SV to Earth pointing within 1.0 degrees and $.05 \%$ sec, using roll and pitch jet control. 
Table 7.1-1. AVCS Weight and Power

\begin{tabular}{|l|l|c|c|c|}
\hline \multicolumn{2}{|c|}{ COMPONENT } & $\begin{array}{c}\text { WUANTITY } \\
\text { QUER SV }\end{array}$ & $\begin{array}{c}\text { WEIGHT } \\
\text { PER SV } \\
\text { (LB.) }\end{array}$ & $\begin{array}{c}\text { MAXIMUM } \\
\text { POWER } \\
\text { (WATTS) }\end{array}$ \\
\hline \multirow{2}{*}{ ACS } & CONTROL ELECTRONICS ASSY & $1^{*}$ & 18.5 & 7.5 \\
& CONBINED EARTH SENSOR & $1^{*}$ & 13.5 & 4 \\
& REACTION WHEEL & 3 & 33.0 & 20 \\
& SPINNING SUN SENSOR & 2 & 2.6 & 4 \\
\hline \multirow{2}{*}{ RCS } & O.2 LB. THRUSTER & 12 & 10.6 & - \\
& TANKS & 2 & 10.4 & - \\
& REMAINDER OF COMPONENTS & - & 13.1 & 0.75 \\
& PROPELLANT & - & 55.0 & - \\
\hline
\end{tabular}

*INTERNALLY REDUNDANT

7.2 .2

SPIN-RATE CONTROL

With the SV pointing at the nadir, yaw torquing jets shall bring the SV to its nominal yaw spin speed within $0.02 \mathrm{rpm}$.

7.2.3 VELOCITY CONTROL

The system must accept ground commands to initiate thruster firing during a) drift orbit circularization (4\%/degree drift), b) correction of IUS launch velocity errors (80 ft/sec, $3 \sigma)$, c) orbit position, and d) two orbit corrections $(\epsilon=.0 .1)$. Total delta $-\mathrm{V}$ impulse shall be oriented within TBD degree of nominal.

7.2.4 ON-ORBIT CONTROL

The SV $\mathrm{Z}$ axis shall point toward the Earth within \pm 0.5 degrees and the SV shall rotate about the yaw axis at 0.5-1 $\mathrm{rpm}$ nominal with stored momentum control using reaction wheels controlling about pitch and roll axes. Momentum unload may be accomplished by spin-rate control or using wheel speed information to drive the appropriate jets. 


\section{COMBINED EARTH SENSOR}
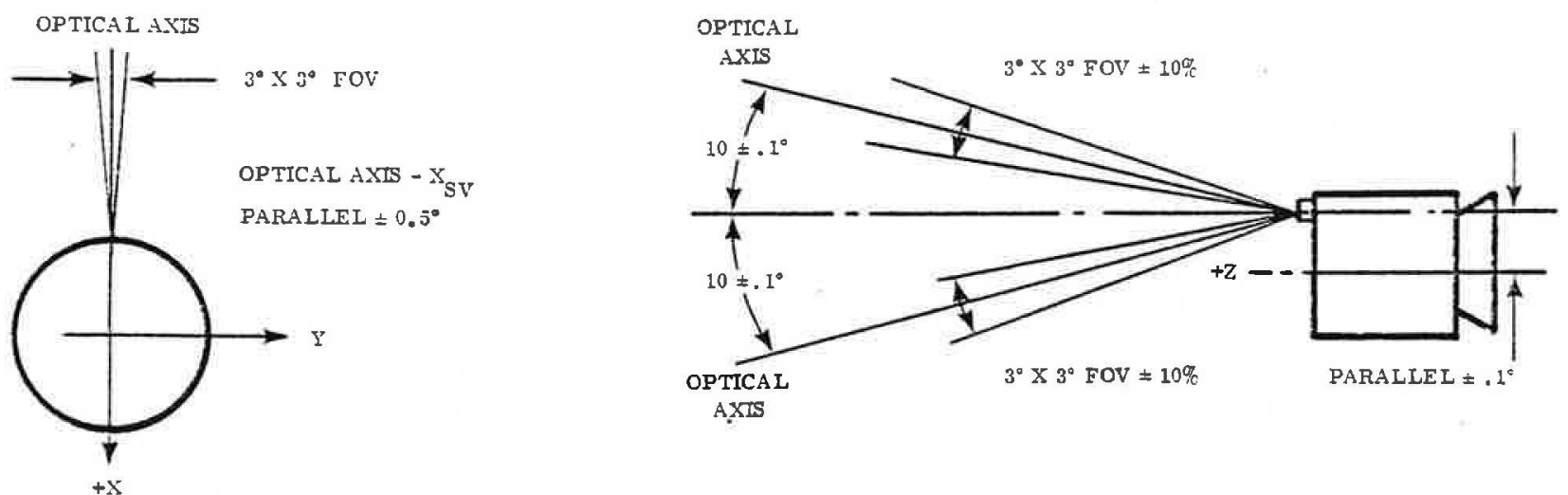

SPIN EARTH SENSOR

STATIC EARTH SENSOR
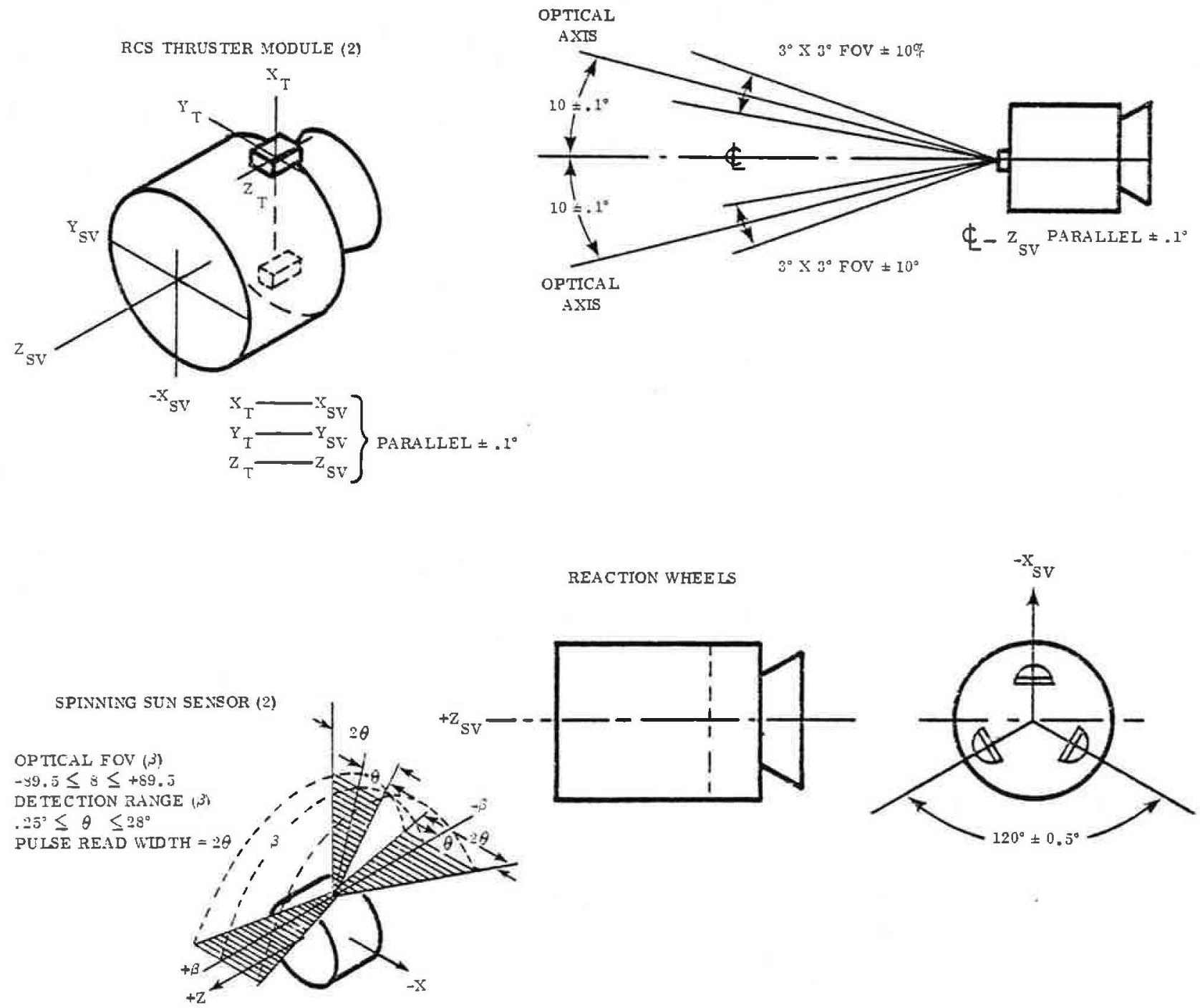

Figure 7.1-2. AVCS Component Locations 


\subsection{DESCRIPTION}

7.3.1 INITIAL ACQUISITION

Four GPS satellite vehicles are simultaneously lifted by an IUS into a nominal 12-hour orbit, inclined at $63^{\circ}$. These vehicles are eventually to be positioned at prescribed locations in precise circular 12-hour orbits; the locations can be anywhere in the orbit. To minimize fuel consumption, the SV's are dispensed from the IUS at the location which minimizes angular travel rate to the final orbital locations consistent with maximum allowable constellation deployment time.

Prior to separation of the SV's from the IUS, the IUS is positioned so that the on-board SV's look directly down at the Earth. One SV is dispensed, the IUS backs off, another is dispensed, and the process continues until all four SV's are dispensed and the IUS deploys to prevent impact with the SV's. (Alternately, each SV after separation can acquire the Earth and move itself relative to the IUS by firing its longitudinally directed thrusters.) (See Figure 7.3-1.)

At separation, each SV is pointing to the Earth within $2^{\circ}$ (Ref. 1) and has $3 \sigma$ inertial rates of about $\pm 0.5 \%$ sec. due to the combination of IUS errors and separation maneuvers. The $0.2-\mathrm{lb}$. pitch and roll attitude control jets with $3-\mathrm{ft}$. moment arms can null these rates of the vehicle, with lateral moment of inertia of 150 slug ft. ${ }^{2}$, in about 1.1 seconds. Filtering of the SV's Earth sensor output is used to obtain pseudo-rate control. During this rate nulling period, the vehicle may rotate an additional 0.6 degrees from nadir. Because the Earth subtends an angle of about $27 \mathrm{deg}$ from orbit altitude and because the Earth sensor has monotonically increasing outputs over a range of about 7 degrees and correct sense of attitude error over about 12 degrees, there is little likelihood that the described sequence of events will not occur. A backup acquisition sequence is described in Appendix H.

1. Attachment 2 to Contract F04701-76-C

System Specification, Performance and Design Requirements for the

Department of Defense Space Transportation System, Volume 3 Interim

Upper Stage System Segment, Basic Requirements for, 76 January 15

SS-STS-100, Volume 3, Code Identification 07868. 


\section{ORBIT INSERTION SEQUENCE}

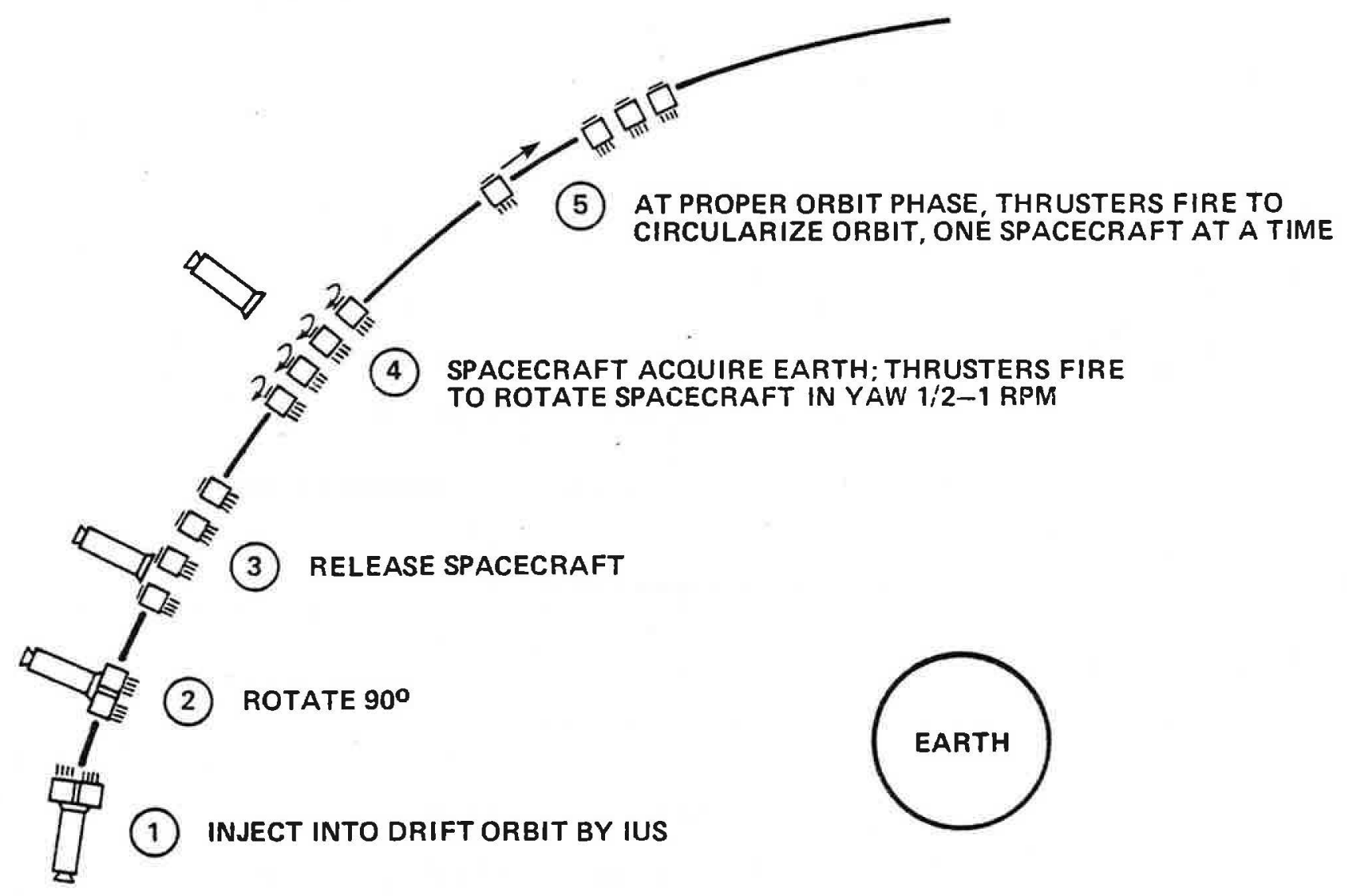

Figure 7.3-1. Orbit Insertion Sequence

When the spacecraft $\mathrm{Z}$ axis is centered on the Earth within 1 degree, and vehicle rates are below $.05 \mathrm{deg} / \mathrm{sec}$, normal on-orbit control with reaction wheels is activated. In the normal operation the SV spins about the satellite-earth line with closed loop reaction wheel control about axes normal to that line. Vehicle spin rate is uncontrolled between limiting rates equivalent to wheel unload momentum. When attitude and rate control have improved to 0.5 degrees and .01 degrees/sec., the yaw torquing jets are pulsed to bring vehicle rate to $1.03 \mathrm{rpm}$. (At this rate, RSV angular momentum is just the negative of the nuclear engine's $17.6 \mathrm{ft} .-\mathrm{lb} .-\mathrm{sec}$. spin momentum.) Vehicle yaw rate is determined either by monitoring the vehicle's spin sun sensor and/or by 
monitoring the time variation of the reaction wheel's speed. The vehicle is now in its normal on-orbit operating mode and orientation but probably in the wrong position in orbit.

The figure below illustrates the jet control mode when the Earth is in the field-of-view of the CES, after separation from the IUS. Pseudo-rate control is obtained by a deadband plus hysteresis circuit with lag feedback to drive the appropriate jets.

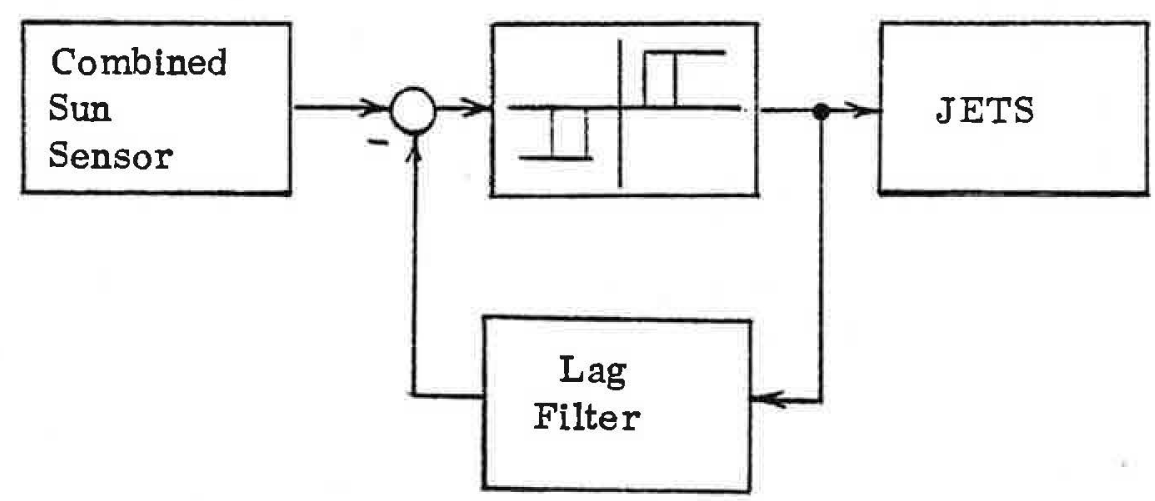

Yaw spin rate control is based on the time between successive transits of the sun by the spin sun sensor looking normal to the yaw axis. When, as shown below that time exceeds (or is less than) the nominal time the appropriate yaw jets are fired (as couples) for a fixed time (approx. 0.13 sec.) so that the spin speed is changed in increments of about $0.01 \mathrm{rpm}$.

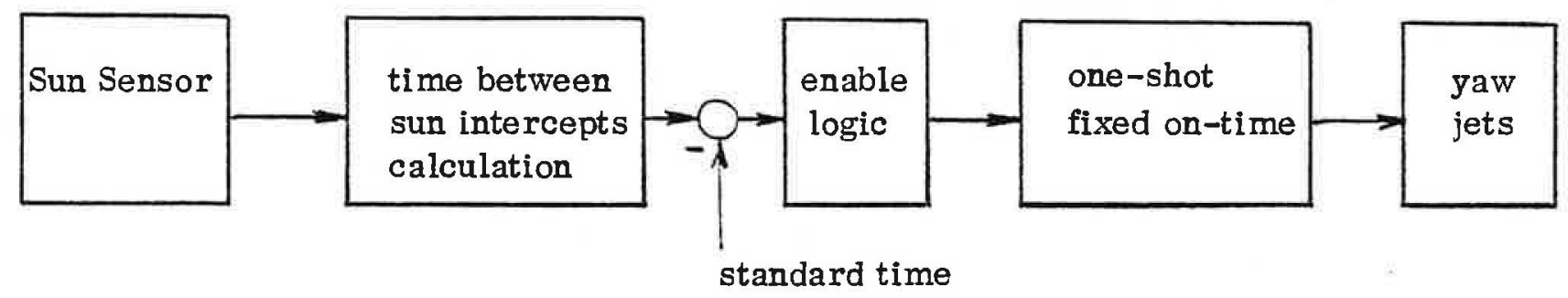

\subsubsection{POSITIONING SV'S IN THE CONSTELLATION}

After separation from IUS, the SV's will be in nominal drift orbits which provide TBD day/day longitudinal drift. This nominal drift rate is selected based on considerations of SV spread in the constellation, delta- $V$ available for the maneuver, 
allowable time for final positioning, and ground coverage during thrusting periods. After drift movement to the desired constellation position, the vehicle is brought to circular orbit speed by jet thrustings by the along-velocity change technique described below.

Deviations between nominal and actual drift orbits are determined after ground tracking. Corrections of IUS induced errors may be commanded after only a few orbits or may be delayed for each SV until the SV is at its proper position in the constellation and final orbit circularization is commenced.

Along-orbit velocity changes are achieved by firing the $\mathrm{X}$ axis thrusters (see Section 7.5) as the SV is rotating at about $1 \mathrm{rpm}$ about the local vertical. Each pulse is started about 15 degrees before the $\mathrm{X}$ axis is in the orbit plane and ends the same number degrees after; less than $1 \%$ inefficiency, due to mis-directed thrusting, is incurred. Pulse on-times are about 5 seconds so that a high specific impulse (200 sec) is achieved. Body-mounted sun sensors are used to initiate and stop the thrusting. (As shown in Section 7.5, $\Delta \mathrm{V}$ thrusting along the orbit is triply redundant, i. e., 3 jet failures can be tolerated without performance degradation.) Y torquing thrusters are used to counter the $\mathrm{Y}$ torquing effects of the $\mathrm{X}$ pointing thrusters. Total propellant requirements are given in Section 7.5 .

IUS radial velocity errors are correctable by firing the $\mathrm{Z}$ pointing thrusters at the 12-hour orbit radius or by firing to achieve the necessary along- orbit velocity changes at drift orbit apogee or perigee.

All delta-V changes are achieved by ground command firing of the SV plus or minus $\mathrm{X}$ axis pointing jets. These are pulsed when the $\mathrm{X}$ axis is within $15^{\circ}$ of the orbit plane. Pulse enables are obtained by using the spin sun sensor. The sensor looks in a plane containing the $\mathrm{Y}$ and $\mathrm{Z}$ axes.

Pulsing starts a ground-commanded delay time after the detector sees the sun and stops after that delay time plus the time for SV $30^{\circ}$ rotation. Concurrent roll and pitch control by jets uses lead-lag compensation. The delay time varies as a function of the angle between the Earth-sun line and the orbit plane. 

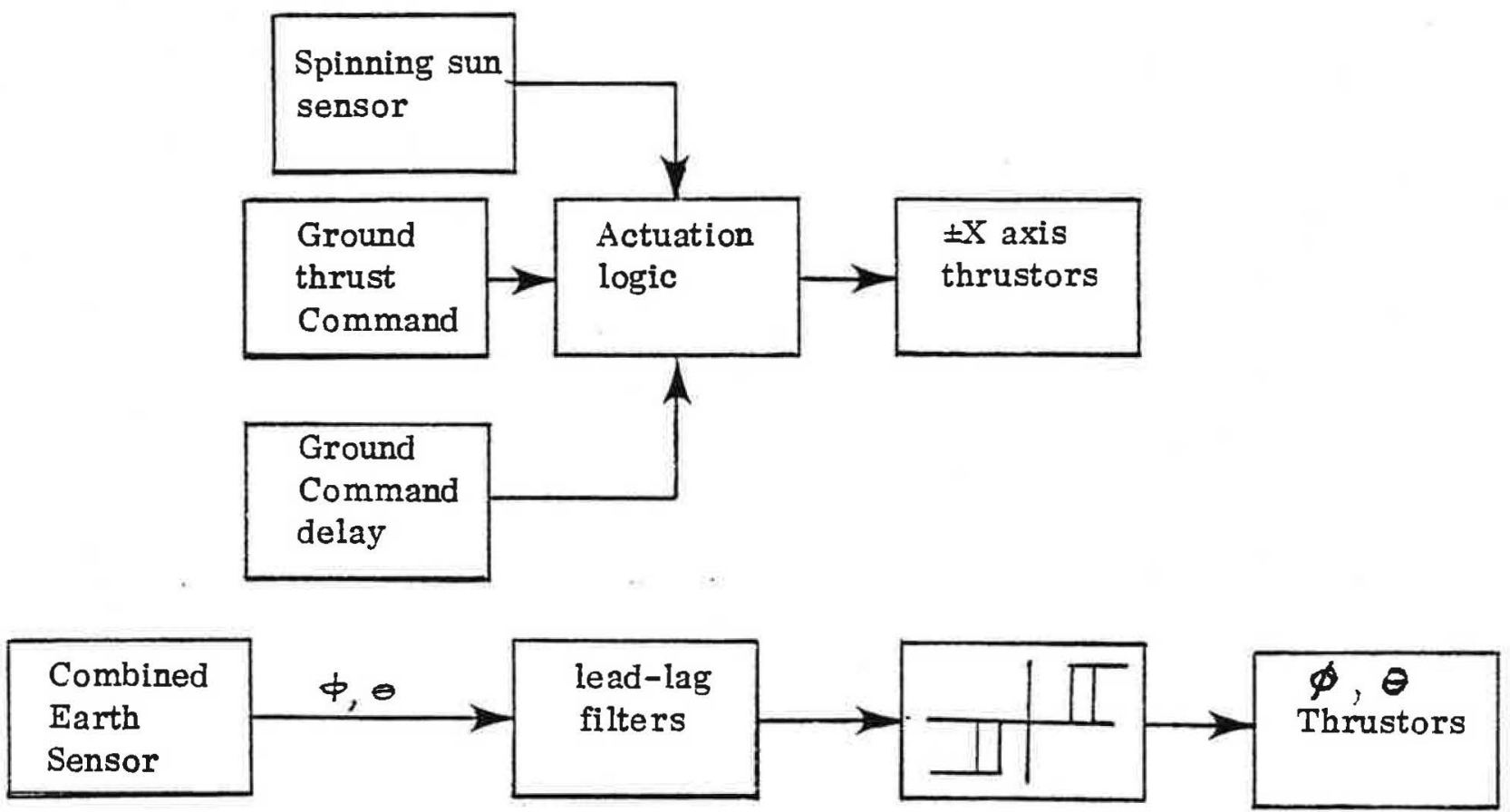

\subsubsection{NORMAL ON-ORBIT OPERATION}

During normal operation, the vehicle spins about the satellite-Earth line, with vehicle attitude controlled about axes normal to that line. Earth sensor information, after processing, is used to drive reaction wheels for lateral axis control.

Vehicle spin rate is uncontrolled; variations in rate occur primarily as the vehicle accumulates angular momentum due to external torques. Periodic, autonomous or ground-controlled, unloading of the accumulated angular momentum to re-establish the desired spin rate is accomplished by jet thrusting. Spin rate information indicating the unload requirement is obtained from tachometers monitoring wheel speed over a spin cycle or from body-mounted sun sensors when the vehicle spin axis is normal to " the vehicle-sun line, i. e., twice each orbit.

Unload occurs no more frequently than every 10 days, 0.5 foot-pound-second being unloaded by jets firing as couples while the vehicle is rotating (Appendix H). Two 0.2 - pound thrusters, 6 feet apart, and firing for 0.42 second will unload 0.5 foot-poundsecond. 
Wheel torquing is based on attitude errors from the CES followed by filtering to achieve loop stabilization. Resolution must be made of desired pitch and roll torque outputs into wheel drives for the two operating wheels (the three wheels have their spin axes in the vehicle $\mathrm{X}-\mathrm{Y}$ plane, the axes are $120^{\circ}$ apart).

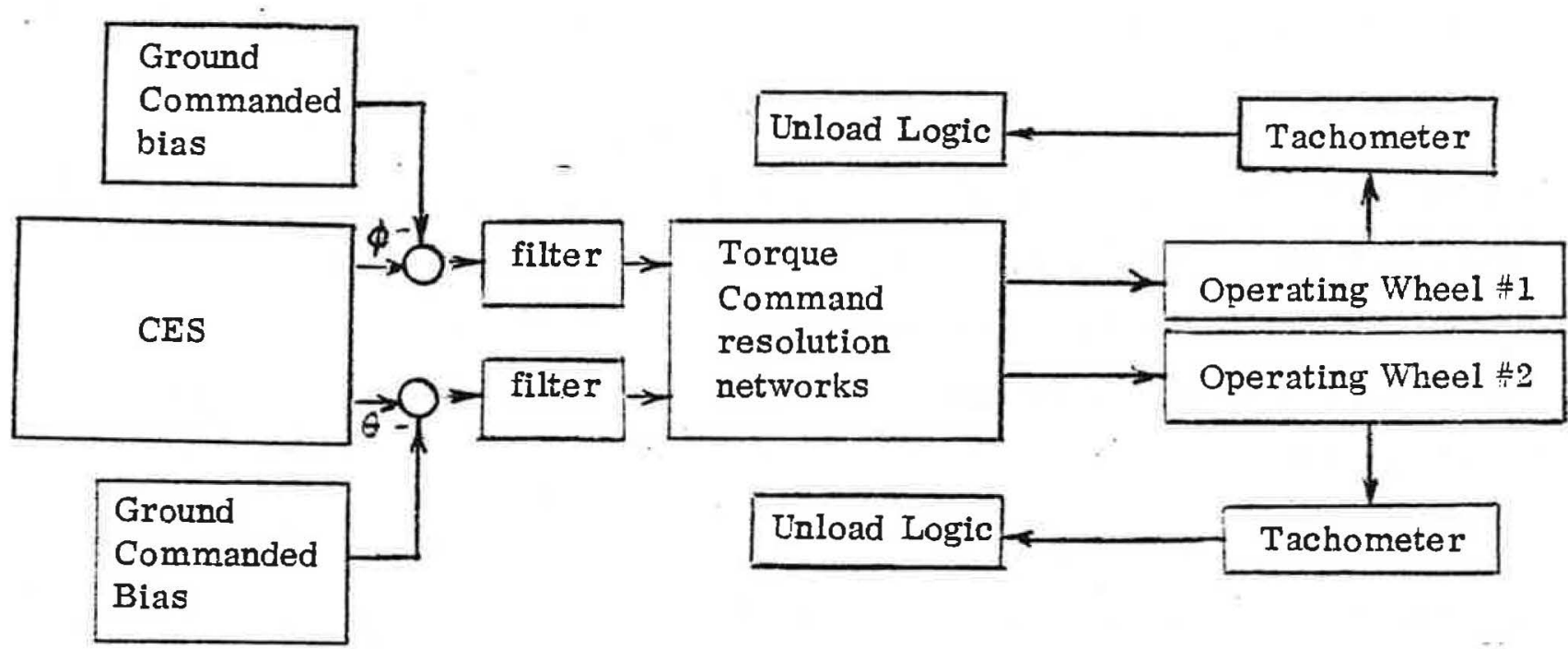

\subsubsection{ORBIT MAINTENANCE}

As a natural result of the forces on the SV, its orbit will become distorted. Ref. 2 indicates an ellipticity increase from zero to 0.01 in about 5 years. Ellipticity corrections are achieved by sequential firing along the orbit at orbit apogee (or perigee) and perigee (or apogee). Ref. 2 suggests reduction of ellipticity to 0.006 (not 0.0 ) requiring $30 \mathrm{ft} / \mathrm{sec}$. (Ideal reduction to $\epsilon=0$ requires $63.4 \mathrm{ft} / \mathrm{sec}$.) Fuel for low orbit corrections is budgeted. The technique for along-velocity-vector firing described in Section 7.3.2 is used to achieve the ellipticity reduction. The on-orbit design options which were explored, the control laws, and the results of control simulations are given in Appendix $\mathrm{H}$.

2. Global Positioning System, Space Vehicle, Critical Design Review, Mission Analysis Working Group. Rockwell International, Space Division, 2-6 June 1975. 


\subsection{0 $\quad$ ACS COMPONENTS}

The components used in the AVCS have been selected based on their suitability for the intended application; advantage is taken of their prior development and application in NDS vehicles.

7.4.1 COMBINED EARTH SENSOR (CES) BARNES 13-169

The static earth sensor portion of the CES is an internally redundant nomoving-part horizon sensor which is designed for operation at $11,000 \mathrm{~nm}$ altitude and operates on energy in the range 14-16 $\mu$. Radiant energy is converted into electrical signals by thermopiles which are sampled every quarter second and logically checked for "validity" . Error signals due to sun, moon, and failed internal components are thereby rejected.

Pitch and roll sensor outputs are analog and errors at null are $0.1^{\circ} 3 \sigma$. A linear output signal range exists for pitch/roll displacements of \pm 4 degrees.

The spinning earth sens or part of the CES looks along the $-\mathrm{X}$ axis and senses intersections of its line-of-sight with the Earth. A redundant unit also looks along the $-\mathrm{X}$ axis.

Unit weight is $13.5 \mathrm{lbs}$. It consumes 4 watts max.

7.4.2 REACTION WHEELS (RW) - BENDIX

The reaction wheels are basically Bendix's OGO pitch and roll wheel design with increased motor torque. Components parameters are:

$\begin{array}{ll}\text { Unit Angular Momentum } & 0.75 \mathrm{ft} . \mathrm{lb} . \text { sec. at } 490 \mathrm{rpm} \\ \text { Stall torque } & 20 \mathrm{in} .-\mathrm{oz} . \\ \text { Weight } & 11 \mathrm{lbs} . \\ \text { Maximum power } & 10 \text { watts }\end{array}$

7.4.3 SPIN SUN SENSOR - ADCOLE 18980

This unit, with digitized output, looks in the vehicle $\mathrm{X}-\mathrm{Z}$ plane with total field-of-view of $180^{\circ}$. Angular resolution of sun location is $1 / 2^{\circ}$ and transition accuracy is $\pm 15^{\circ}$. The unit weight is $1.3 \mathrm{lbs}$. and consumes 2 watts. 
The CEA contains all AVCS electronic functions listed below. Estimated unit weight is $18.5 \mathrm{lbs}$ and estimated maximum power 7.5 watts. The performing characteristics of the individual CEA elements shall be:

a. Mode Select Logic (MSL) - The MSL shall decode a 14-bit binary serial digital word received, via ground command from the TT\&C subsystem and appropriately route the information to the desired CEA element.

b. Jet Control Electronics (JCE) - The JCE shall be utilized to perform initial earth acquisition and, if necessary, earth reacquisition. In addition, the JCE shall be utilized for temporary backup attitude control.

(1) JCE Signal Interface -- The JCE shall have the following signal interfaces:

(a) Ground command signals from the MSL.

(b) An analog signal from each axis of the two-axis horizon sensor.

(c) An enable/disable signal to activate and deactivate the JCE.

(d) Output signals to the JSL commanding specific thruster firing.

(e) Signals from the spin earth sensor.

(2) JCE Functional Characteristics - The JCE shall contain jet control electronics including gains, shaping, and deadbands.

c. Wheel Control Electronics (WCE) - The WCE shall be utilized to provide on-orbit space vehicle pointing control. 
(1) WCE Signal Interface - The WCE shall have the following signal interfaces:

(a) Override attitude bias command signal from the MSL.

(b) An analog signal from each axis of the two-axis horizon sensor.

(c) A ground command signal, from the MSL, indicating deactivation of a wheel causing appropriate compensation to be switched in.

(d) An enable/disable signal to activate and deactivate the WCE.

(e) Output control signals to the reaction wheels.

(2) WCE Functional Characteristics - The WCE shall contain control electronics to activate the reaction wheels thereby providing control torques to the vehicle. Gains, shaping, inverters and signal mixing shall be included in the control electronics. Logic shall also be provided that switches, upon ground command, a failed wheel out and appropriate compensation in.

d. Momentum Dump Logic (MDL) - The MDL shall be utilized to desaturate (dump momentum) the reaction wheels periodically in order to maintain continuous vehicle control.

(1) MDL Signal Interface - The MDL shall have the following signal interfaces:

(a) Signals from the reaction wheel tachometers indicating each reaction wheel spin speed.

(b) An enable/disable signal to activate and deactivate the MDL.

(c) Ground commanded attitude override commands from the MSL. 
(d) Output signals to the JSL commanding specific thruster firings. These signals shall also turn the thruster heaters on automatically $60 \pm 5$ minutes before thruster firing.

(e) Signals from the spin sun sensor.

(2) MDL Functional Characteristics - The MDL shall contain control electronics to monitor the angular velocity of each reaction wheel and command thruster pulsing. Also, it monitors SV spin speed using spin sun sensor outputs. The threshold level at which an axis will be "dumped" shall be selectable by ground command.

e. Attitude Bias Electronics (ABE) and Commandable Thruster Firing Logic (CTFL) - The ABE shall be utilized to bias the pitch and roll attitude vai ground command. The CTFL shall provide for open loop thruster firing via ground command.

(1) ABE Signal Interfaces - The ABE shall have the following signal interfaces:

(a) Commands from TT\&C

(b) Bias commands to WCE and JCE

(c) Enable/Disable discrete inputs from TT\&C

(2) CTFL Signal Interfaces - The CTFL shall have the following signal interfaces:

(a) Commands from TT\&C

(b) Output commands to the JSL

(c) Enable/Disable discrete inputs from TT\&C

f. Jet Select Logic (JSL) - The JSL shall be utilized to receive thruster commands from the other CEA units and route these commands to the required thruster logic and valve driver amplifiers to perform these functions. A Jet Watchdog Logic (JWL) shall be provided to insure 
that a runaway jet due to an electronic failure is automatically shut down in less than one minute.

\subsection{RCS DESCRIPTION}

The Nuclear Powered GPS makes direct use of the propulsion components and modular assemblies from the solar-powered spacecraft with modifications to the extent suggested by reduced propellant and thrust level requirements and aft-end jet location.

Because the $13^{\prime \prime}$ tanks with an accumulator provide more than sufficient impulse margin, the $61 / 4$ " pressurant accumulator was eliminated and the propellant per tank reduced to a nominal load of $27.5 \mathrm{lb}$. The increased ullage volume of the propellant tank retains the blowdown ratio of 3 to 1 . Table 7.5-1 details the propellant budget and indicates a $24 \%$ contingency reserve.

Table 7.5-1. Propellant Budget

\begin{tabular}{|c|c|c|c|}
\hline Maneuver & $\begin{array}{l}\Delta V \text { Req'd } \\
\mathrm{ft} / \mathrm{sec}, 3 \\
\end{array}$ & $\begin{array}{l}\text { Avg. Is } \\
\text { (lbf. sec/lb) }\end{array}$ & $\begin{array}{c}\mathrm{H}_{2} \mathrm{H}_{4} \mathrm{Req}^{\prime} \mathrm{d} \\
\mathrm{lb} \\
\end{array}$ \\
\hline $\begin{array}{l}\text { Null, Roll, Pitch and Yaw } \\
\text { Separation Rates }\end{array}$ & $\begin{array}{l}\left(0.5^{\circ} / \mathrm{sec}\right. \\
\text { each axis) }\end{array}$ & 100 & 0.1 \\
\hline IUS Injection Error & Radial $=75$ & 205 & 12.5 \\
\hline Correction & Normal $=12$ & 200 & 3.1 \\
\hline & Tangential $=16$ & 200 & 4.1 \\
\hline $\begin{array}{l}\text { Circularization of } \\
\text { Drift Orbit }\left(4^{\circ} / \text { Day }\right)\end{array}$ & Tangential $=23.5$ & 200 & 6.1 \\
\hline Momentum Dump & $\begin{array}{l}\left(0.5 \mathrm{ft} . \mathrm{lbf}_{\mathrm{.}} \mathrm{sec}\right. \\
\text { every } 10 \text { days })\end{array}$ & 125 & 0.3 \\
\hline Ellipticity Correction & T angential $=60$ & 200 & 15.5 \\
\hline
\end{tabular}

Total Required

Total Usable (2 Tanks)

Contingency/ Reserves
$41.7 \mathrm{lb}(75.8 \%)$

55.0

$13.3 \mathrm{lb}(24.2 \%)$ 
The dual valve 0.2 - lbf thrusters for attitude control are retained in a modular configuration where eight thrusters in each of two modules provide three-axis attitude control as well as translational $\Delta V$ capability. The $5 \mathrm{lbf}$ thrusters are eliminated since the nuclear-powered spacecraft is non-rotating during injection error correction and high torques for precession are not required. The $0.2 \mathrm{lbf}$ is also sufficient for nodal correction of orbit non-circularity.

The thruster modules have been moved aft from the center of mass to the separation plane of the satellite for four reasons:

1) Electronic equipment occupies the central volume at the plane of the center of mass.

2) The cylindrical power system radiator is designed as an integral unit, preferably with no cutouts that would compromise its performance.

3) Installation of the RCS modules is facilitated by using the annular segments of space between the radiator and the thrust cone $90^{\circ}$ from the two heat sources.

4) The penalty incurred by translational thrusting along the $\mathrm{X}$ axis using thrusters offset somewhat from the CM (requiring rotational stabilization with other jets) is small because of the larger moment arms in the stabilizing jets.

Figure 7-5-2 illustrates jet arrangement and utilization and indicates a necessary and sufficient level of redundancy. Added flexibility is provided by the fact that individual thrusters in either the backup or prime subset can be activated to replace single failed thrusters in the other subset.

The schematic arrangement of components shown in Figure 7.5-3 is essentially that used on the Solar Power GPS. The accumulators and 5 Ibf thrusters with their latch valves have been removed and an interconnect latch valve has been added. The intermodule line is installed last, utilizing mechanical couplings with redundant seals. 


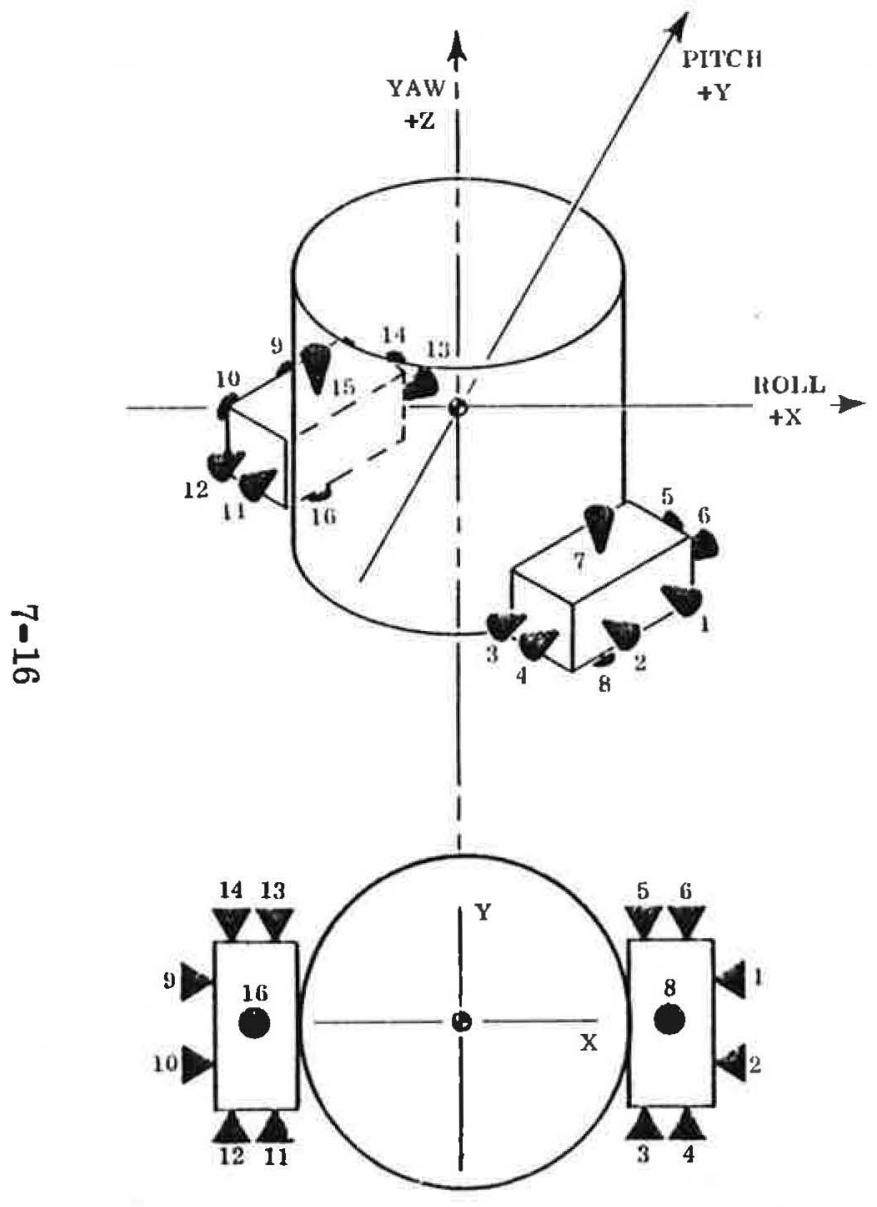

VIEW INTO AF'T END

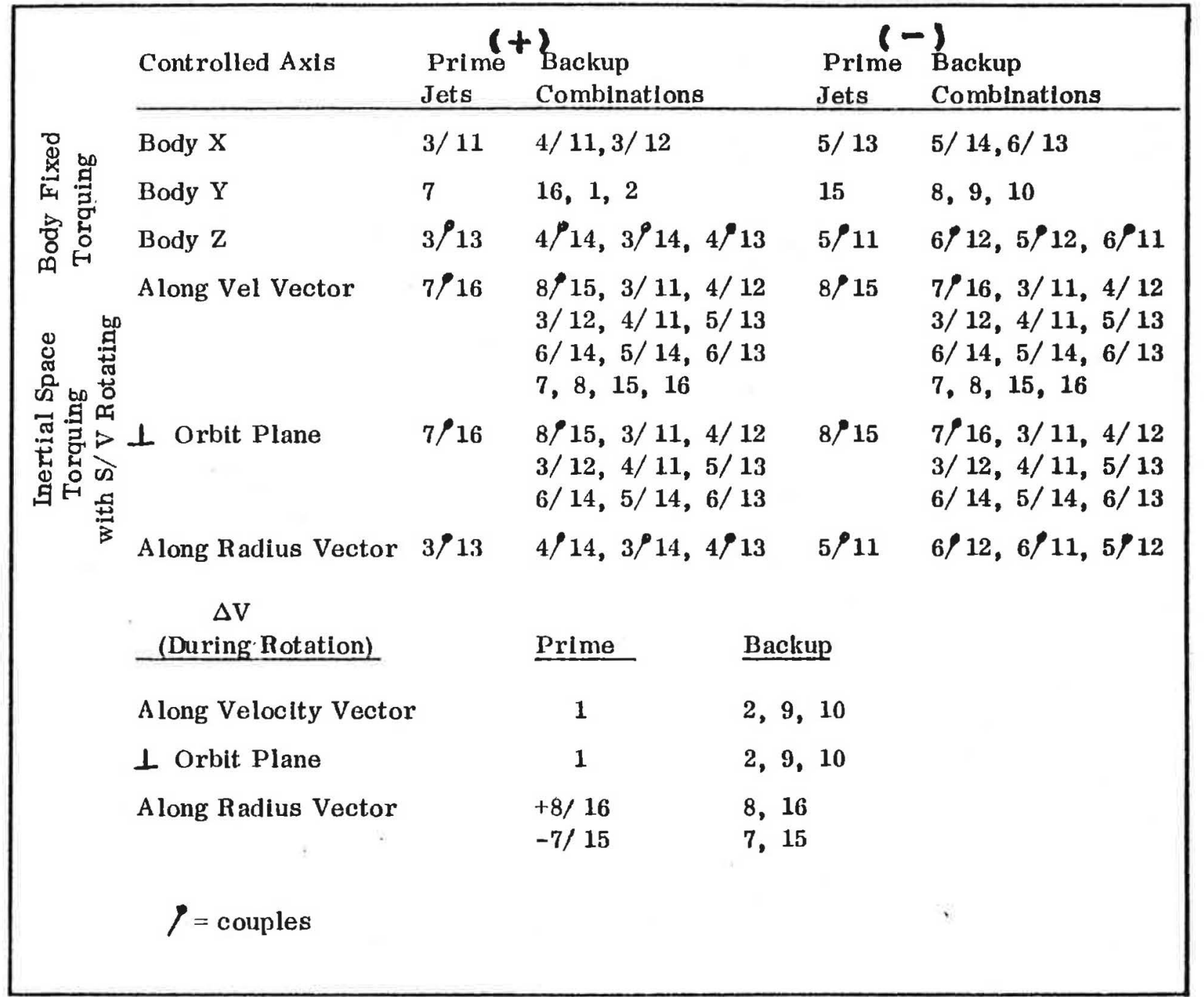

Figure 7.5-2. Jet Orientation/Utilization 


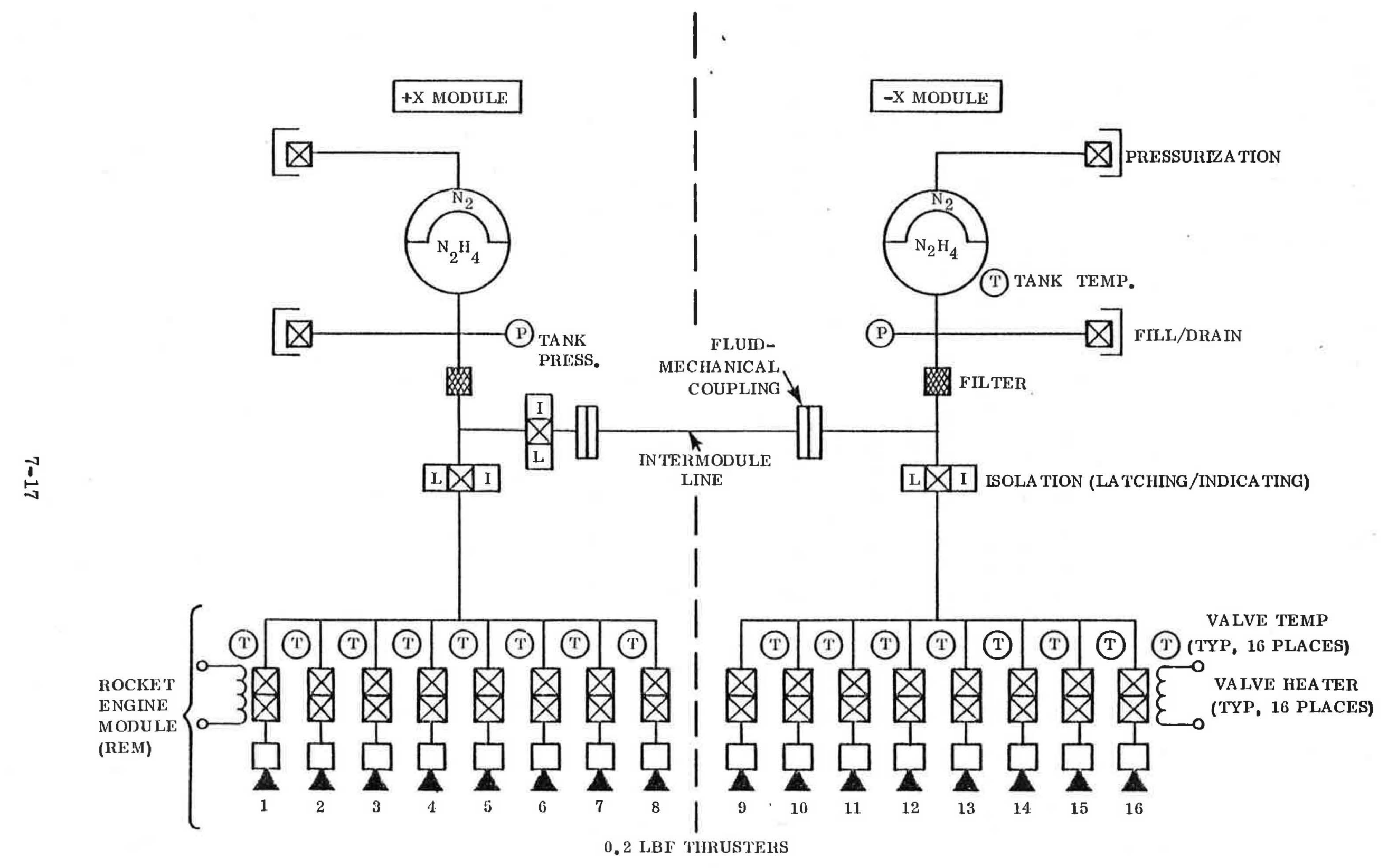

Figure 7.5-3. Reaction Control Fluid Schematic 
The total loaded weight of both modules is $89.1 \mathrm{lb}$ with the nominal propellant load of 55.0 lb of hydrazine. Table 7.5-4 itemizes the component weight and indicates nominal value and heater power levels.

Thrusters are activated through driver and logic circuits located in the AVCS Control Electronics Assembly.

7.6 CHANGES CAUSED BY USE OF THE BRAYTON POWER SYSTEM

The angular momentum of the Brayton power system (0.68 ft. Ib. sec.) is very much less than that of the Rankine power system (17.6 ft. lb. sec.), so that it is impractial to use the former for counterbalancing the yaw rotation of the BSV. Therefore, a large fixed speed reaction wheel with an angular momentum of $10 \mathrm{ft}$. $\mathrm{lb}$. sec. along the yaw axis is included for this purpose. This allows the RSV to rotate at $0.51 \mathrm{RPM}$ with full momentum cancelling. Because the Brayton rotating unit has been mounted horizontally, with its angular momentum in the XZ plane, a slight canting of the reaction wheel, at an angle of 4-degrees to the yaw axis, will cancel its momentum as well.

A suitable unit is a Bendix manufactured wheel of the ATS type which has a unit weight of $19.5 \mathrm{lbs}$, AM of $8.5 \mathrm{ft}$. $\mathrm{lb}$. sec, and consumes 10 watts at $1450 \mathrm{rpm}$. Because the SV is turning at $0.5 \mathrm{rpm}$, i. e., somewhat more slowly than an SV with an Organic Rankine Cycle engine, there is about a 10-watt savings in the reaction wheels used for attitude control, so no AVCS net power difference exists. 
Table 7.5-4. RCS Weight and Power Summary

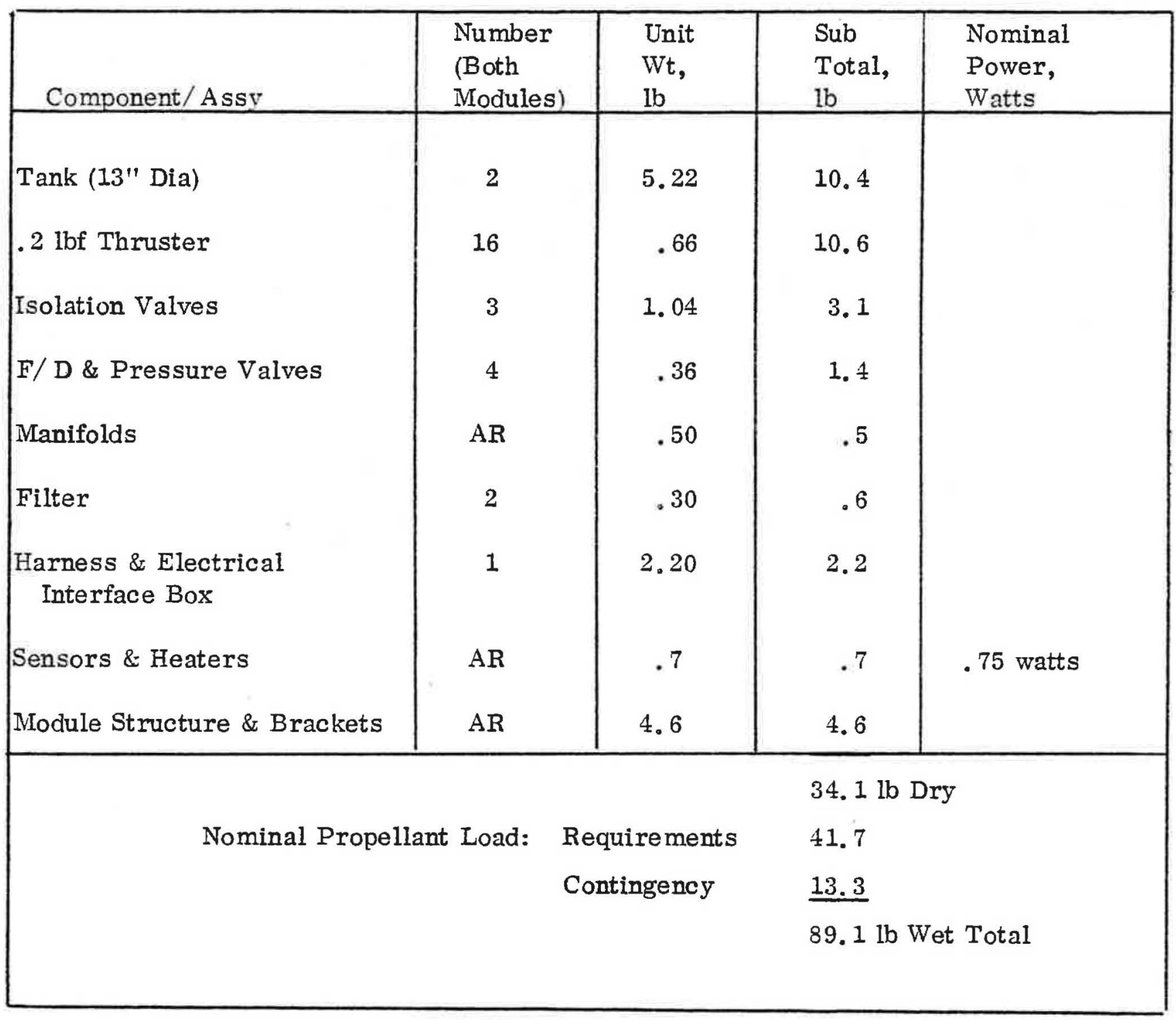





\section{SECTION VIII}

\section{THERMAL DESIGN AND ANALYSIS}

8.1 $\quad$ PROBLEMS ADDRESSED

The major thermal design problems which were addressed in the study are as follows:

1) Heat pipe performance in spinning mode.

2) Equipment shelf thermal performance.

3) Overall spacecraft thermal variations in eclipse orbit.

4) Temperature variation and control of frequency standards.

These are each discussed in the following paragraphs.

\subsection{PERFORMANCE OF RADIAL HEAT PIPE IN SPINNING MODE}

The reduction in pumping capacity of an ATS-type heat pipe spinning about an axis normal to its longitudinal axis was evaluated. For the GPS application it is proposed to orient heat pipes in a radial direction normal to the vehicle spin axis to provide thermal control of the frequency standard clocks mounted on the upper shelf. The pipe evaporators will be located 10 inches from the spin axis; the condensors will be 33 inches from the axis. (See sketch Figure 8.2-1). A grooved ATS heat pipe, which uses ammonia as the working substance, provides a capillary pumping head of about 1.4 psf. With the evaporator inboard, this pumping head will be reduced by the centrifugal forces acting on the fluid in accordance with

$$
\Delta \mathrm{P}=\frac{\rho_{\omega}}{\mathrm{g}}\left(\mathrm{r}_{2}^{2}-\mathrm{r}_{1}^{2}\right)
$$

where

$$
\begin{aligned}
\rho & =\text { density of liquid ammonia, about } 40 \mathrm{lbs} / \mathrm{ft}^{3} \\
\omega & =\text { angular velocity } \\
r & =\text { distance to end points from spin axis }
\end{aligned}
$$


This equation was used to generate the curve shown in Figure 8.2-1, where it may be noted that the percentage loss in pumping capacity is neglibible providing spin rates of about one RPM are not exceeded.

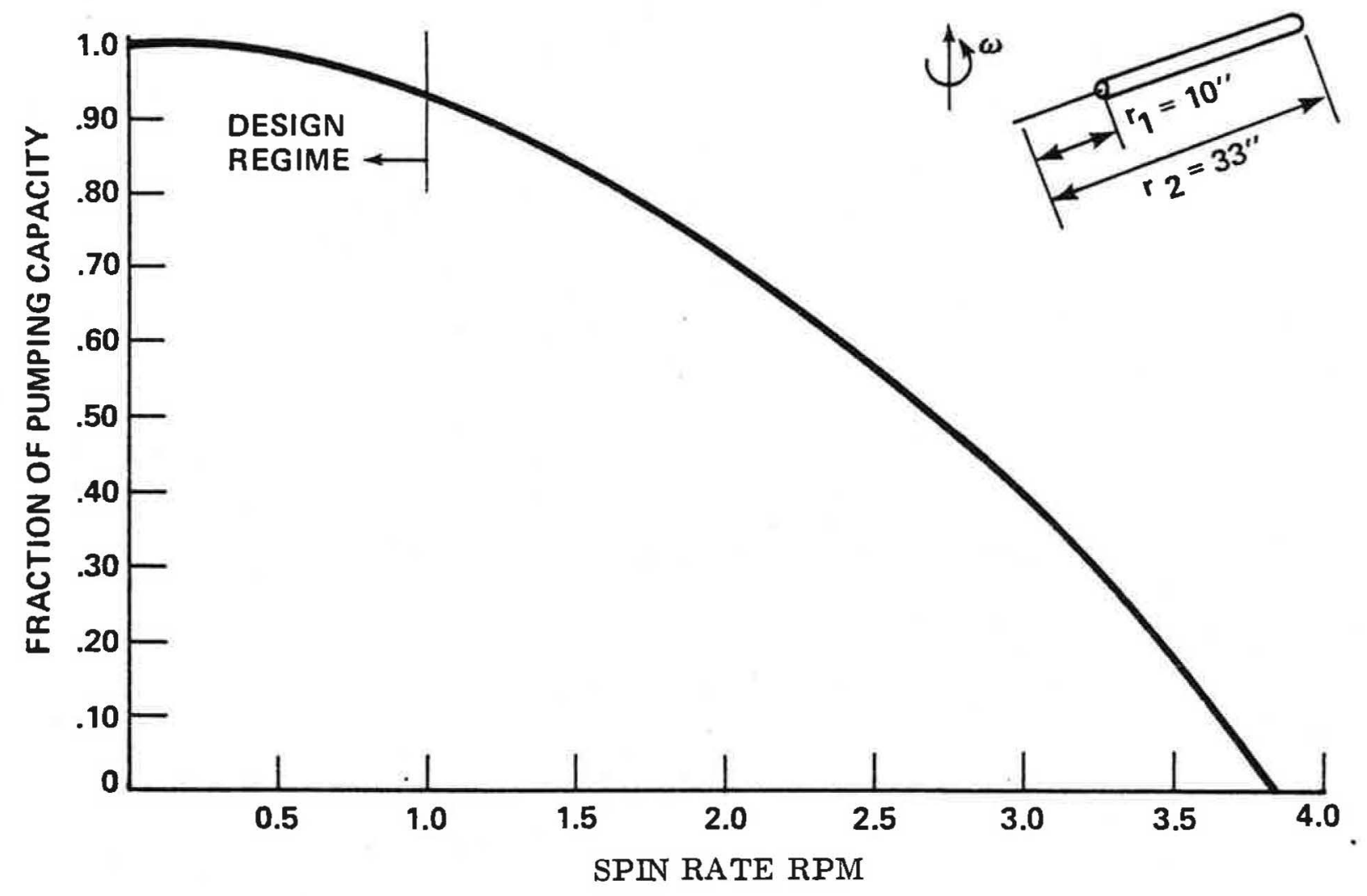

Figure 8.2-1. Pumping Capacity of ATS-6-Type Heat Pipe (Ammonia in Aluminum) Rotating About An Axis Normal To Its Axis

\subsection{EQUIPMENT SHELF TEMPERATURE CONTROL}

Dissipation of electronic equipment thermal power is achieved by transferring heat along heat pipes which are thermally coupled to a coolant header. The header is an extension of the power system fluid system. The heat pipes are imbedded in the honeycomb panel and connected to the header wall at the saddle region. Figures 8.3-1 and 8.3-2 show the heat pipe layout for the lower and upper shelves, respectively. 

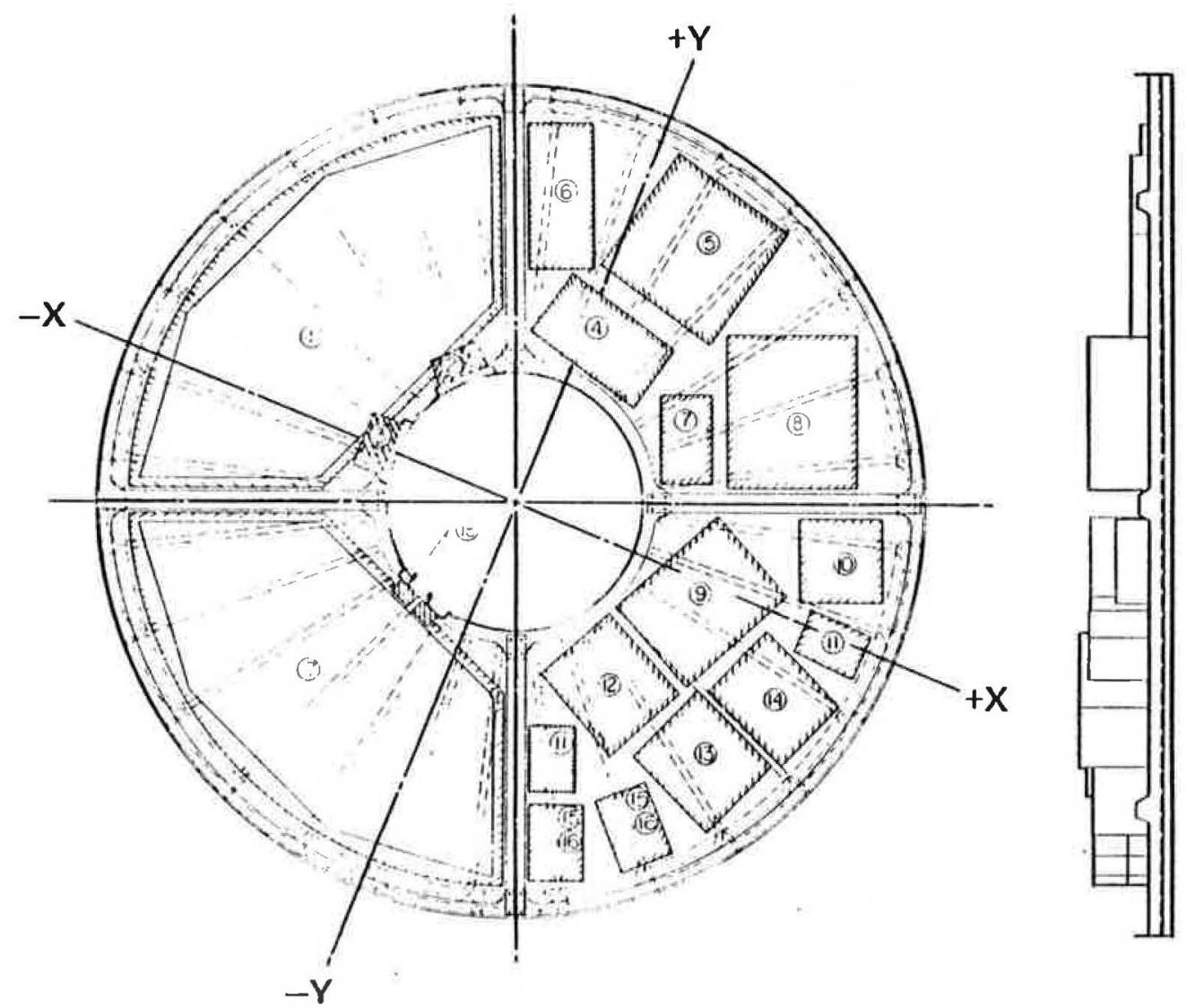

\begin{tabular}{|c|c|c|c|c|c|}
\hline ITEM & NAME & $\begin{array}{c}\text { SIZE } \\
\text { LENGTH } \times \text { WIOTH } \times \text { HEIGHT }\end{array}$ & $\begin{array}{l}\text { WEIGHT } \\
\text { LBS (EA) }\end{array}$ & $\begin{array}{l}\text { DISSIP } \\
\text { WATTS }\end{array}$ & $\begin{array}{c}\text { OTY } \\
\text { USEם } \\
\end{array}$ \\
\hline 1 & NAV SYS $L_{1} H P A$ & $16.0 \times 40.0 \times 2.0$ & 21.5 & 200 & 1 \\
\hline 2 & L, HPA FILTER & $5.3 \times 4.3 \times 2.2$ & 2.5 & - & $i$ \\
\hline 3 & $\mathrm{~L}_{2}$ HPA FILTER & $4.8 \times 4.0 \times 2.0$ & 1.6 & - & $i$ \\
\hline & NÁV SYS $\mathrm{L}_{2} \mathrm{HPA}$ & $9.6 \times 6.0 \times 1.3$ & 4.34 & 14.7 & $i$ \\
\hline 5 & NAV SYS $L_{1}^{2} / L_{2}$ SYN & $10.4 \times 10.3 \times 2.5$ & 7.43 & 10.6 & $i$ \\
\hline 6 & MAV SYS L MOD IPA & $11.25 \times 4.68 \times 1.65$ & 3.08 & 3.4 & 1 \\
\hline 7 & NAV SYS L MOO IPA & $6.7 \times 3.6 \times 1.65$ & 1.7 & 1.5 & $i$ \\
\hline$\theta$ & NAV SYS DIG PROC/BB & $11.7 \times 10.2 \times 5.8$ & 20.8 & 21.1 & $i$ \\
\hline 9 & PCM/TLM UNIT & $10.65 \times 8.28 \times 5.75$ & 12.0 & 8.0 & 1 \\
\hline 10 & NAV DC/DC CONVERTER & $6.0 \times 6.0 \times 3.75$ & 7.6 & 18.1 & i \\
\hline 11 & IT\&C KIA-23 & $5.02 \times 3.6 \times 5.8$ & 4.3 & 2.2 & $i$ \\
\hline 12 & TT\&C CMD DECODEA & $8.8 \times 7.41 \times 5.75$ & 8.0 & 0.8 & $i$ \\
\hline 13 & ITEC SIG COND & $8.28 \times 7.05 \times 5.45$ & 7.0 & 2.0 & i \\
\hline 14 & TT\&C RF ASSY & $8.0 \times 6.5 \times 6.5$ & 5.3 & 2.7 & $i$ \\
\hline 15 & TI\&C TRANSMITIER & $5.82 \times 4.25 \times 2.7$ & 5.0 & 20.0 & 2 \\
\hline 10 & TTRC ACVR DEMOD & $5.82 \times 4.25 \times 2.7$ & 5.0 & 1.8 & 2 \\
\hline 17 & $L_{1}$ HPA FINAL AMPLIFIER & $16.0 \times 40.0 \times 2.0$ & 15.0 & 200 & 1 \\
\hline 18 & CURRENT SENSOR & $2.5 \times 1.7 \times 1.3$ & 0.5 & 1.3 & 2 \\
\hline $\begin{array}{l}19 \\
20\end{array}$ & PWR SYS CONT ELEC & & & & \\
\hline & AVCS CONTROL ELECT & $16.0 \times 10.0 \times 6.0$ & 17.0 & 8.5 & 1 \\
\hline 22 & MOMENTUM WHEEL & 7.50 DIA $\times 4.15$ & 11.0 & 10.0 & $\mathbf{j}$ \\
\hline 23 & CESIUM CLOCK & $16.0 \times 10.0 \times 10.0$ & 34.0 & 24.0 & 2 \\
\hline 24 & EARTH SENSOR & $7.44 \mathrm{DIA} \times 122$ & 13.5 & 3.0 & i \\
\hline
\end{tabular}

Figure 8.3-1. Lower Equipment Shelf Reference Design (Both Spacecraft) 


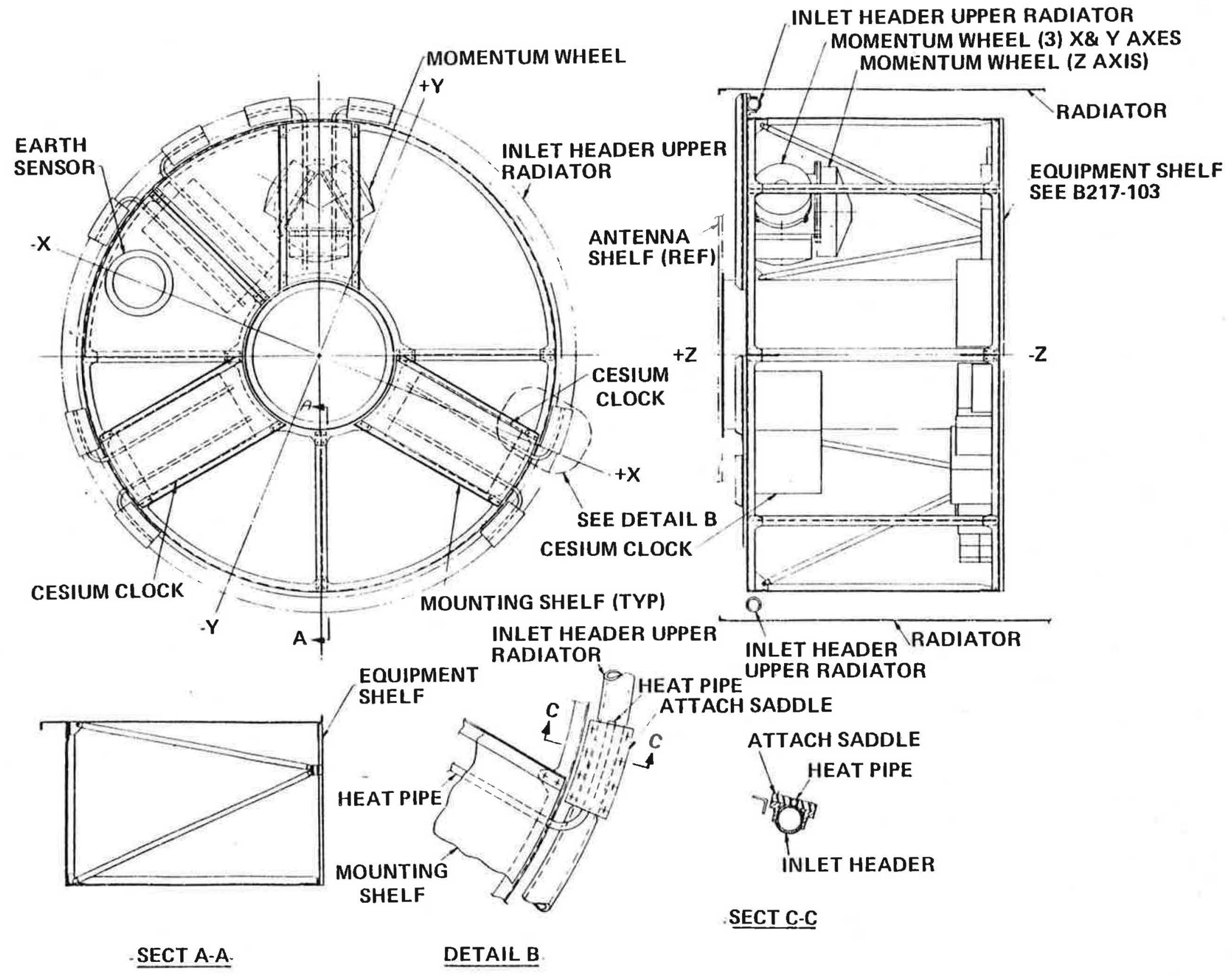

Figure 8.3-2. Equipment Module - Brayton Spacecraft 
Experimental and flight data from ATS-6 heat pipes (grooved aluminum tubing with ammonia for working fluid) reveals that the temperature gradient between the evaporation and condensation regions is small $\left(\sim 1^{\circ} \mathrm{C}\right)$ when the heating level is in the range of interest for this nuclear GPS design: about 30 watts along a 30 " heat pipe. The largest changes in temperature occur across the interfaces which connect the heat pipe to the heat generating components and to the sink. In the current application, an additional gradient is realized across the coolant fluid film adhering to the header wall. ATS-6 experience shows that the contact resistance due to the mounting interfaces is reduced considerably by employing conductive thermal grease and mechanical mounting pressure. The convective heat transfer across the header film will be a function of the flow characteristics and available heat transfer area.

An analysis was conducted to evaluate various header configurations which will provide the required heat transfer from the lower equipment shelf at acceptable gradients. The selected header design for the Organic-Rankine space vehicle provides an annular flow area by a tube-in-tube arrangement. The outer tube has 1.5 in. outer diameter. The flow gap of 0.035 -inch is maintained by conductive wire spacers which run the length of the header. This design, shown in Figure 8.3-3 has the advantage of achieving high values of convection coefficients without sacrificing the heat-transfer surface area in contact with the fluid. This header is fabricated and assembled as part of the power system module, and undergoes the same rigid cleanliness and inspection procedures as the rest of the power system.

The fluid properties for the Dowtherm Aworking fluid of the RSV/PSM are given in Table $8.3-4$. The rate of flow is a specified $322 \mathrm{lb} m / \mathrm{hr}$. Following dissipation in the upper header the temperature at the inlet of the lower header is found to be $31.3^{\circ} \mathrm{C}\left(88.3^{\circ} \mathrm{F}\right)$. The temperature at the outlet is calculated based on a total components dissipation of 532.7 watts as indicated in Figure 8.3-5. It may be assumed that all component heating is transferred to the fluid leading to a temperature rise of $8.2^{\circ} \mathrm{C}\left(14.7^{\circ} \mathrm{F}\right)$ and hence a temperature at the outlet of $39.5^{\circ} \mathrm{C}\left(103^{\circ} \mathrm{F}\right)$. The relative location of the dissipators with respect to the header is selected such that the critical high-power amplifiers are nearest to the inlet region in order to take advantage of the cooler regime. 


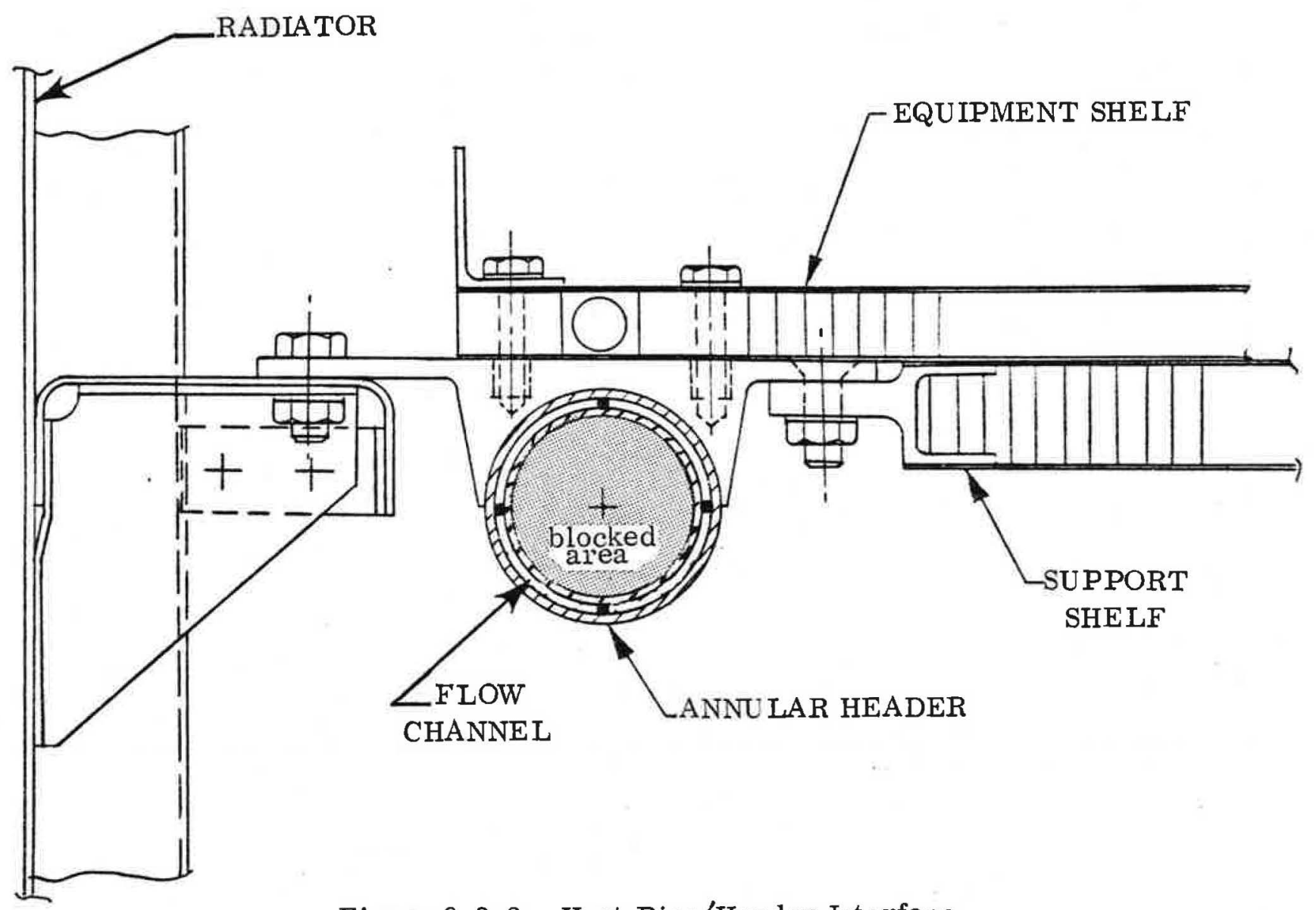

Figure 8.3-3. Heat Pipe/Header Interface

Table 8.3-4. Properties of Dowtherm - A

\begin{tabular}{|c|c|c|c|c|}
\hline $\begin{array}{c}\text { Temp } \\
(\mathrm{OF})\end{array}$ & $\begin{array}{c}\text { Density } \\
\left(1 \mathrm{~b}_{\mathrm{m}} / \mathrm{ft}^{3}\right)\end{array}$ & $\begin{array}{c}\text { Viscosity } \\
\left(1 \mathrm{~b}_{\mathrm{m}} / \mathrm{hrft}\right)\end{array}$ & $\begin{array}{c}\text { Conductivity } \\
\left(\mathrm{Btu} / \mathrm{fthr} \mathrm{O}^{\circ}\right)\end{array}$ & $\begin{array}{c}\text { Specific Heat } \\
(\mathrm{Btu} / \mathrm{lb} \mathrm{O})\end{array}$ \\
\hline 80 & 65.82 & 7.70 & 0.0814 & 0.388 \\
100 & 65.27 & 6.36 & 0.0805 & 0.388 \\
120 & 64.72 & 5.03 & 0.0797 & 0.396 \\
\hline
\end{tabular}




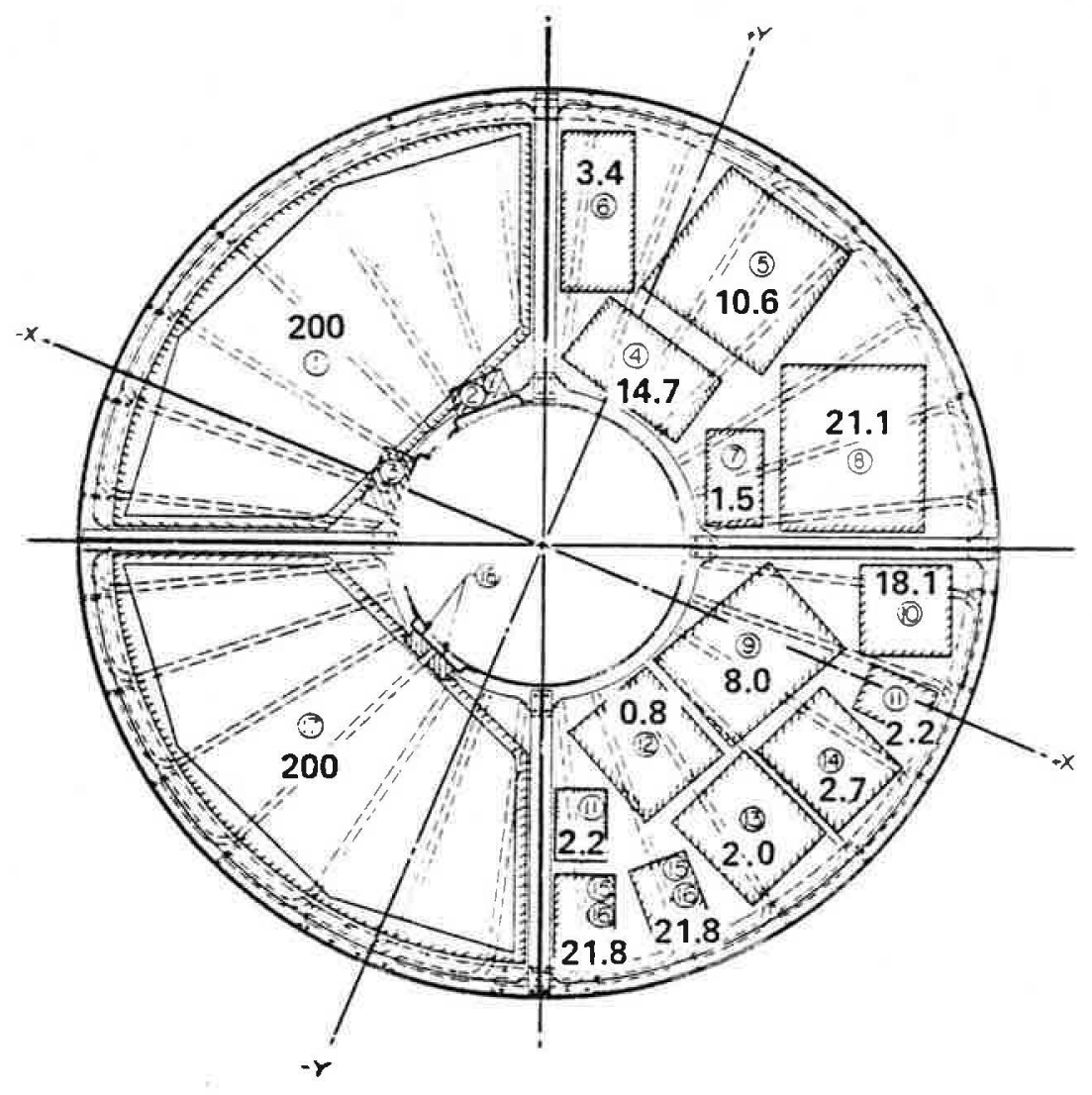

Figure 8. $\dot{3}-5$. Lower Equipment Shelf Component Dissipation (Watts ${ }_{T}$ )

The Reynolds number based on the hydraulic diameter (Do-Di) and average fluid properties is found to be

$$
\operatorname{Re}=\frac{745.5}{\mathrm{D}_{\mathrm{O}}+\overline{\mathrm{D}}_{\mathrm{i}}} ; \mathrm{D}_{\mathrm{O}}, \mathrm{D}_{\mathrm{i}} \text { in inches }
$$

Hence, for the range of diameters under consideration, the flow is laminar and the friction factor may be assumed to be

$$
F=24 / R_{e}=0.032\left(D_{o}+D_{i}\right)
$$

Thus, in terms of the annulus dimensions, the pressure drop $P$ (psf) and the heat transfer coefficient $\mathrm{H}\left(\mathrm{Btu} / \mathrm{ft}^{2} \mathrm{hr} \mathrm{o}^{\mathrm{O}} \mathrm{R}\right)$ are given by

$$
\Delta P=\frac{0.4315}{\left(D_{0}+D_{i}\right)\left(D_{0}-D_{i}\right)}
$$

and

$$
\mathrm{H}=\frac{49.86}{\mathrm{D}_{\mathrm{O}}-\mathrm{D}_{\mathrm{i}}} \quad\left[\begin{array}{ll}
\frac{1}{\mathrm{~L}} & \frac{\mathrm{D}_{\mathrm{o}}-\mathrm{D}_{\mathrm{i}}}{\mathrm{D}_{\mathrm{O}}+\mathrm{D}_{\mathrm{i}}}
\end{array}\right]^{0.33}
$$

where $\mathrm{L}$ is tube length in inches. 
Equations (1) through (4) were used in a parametric evaluation of the annular header. The results are shown in Figures 8.3-6 and 8.3-7. The intent is to minimize the temperature gradient from the annulus walls to the fluid without resorting to excessively large diameters or high pressure drops. Structural and spacecraft envelop limitations indicate a suitable outer diameter of about 1.5 " which yields a gradient of $2^{\circ} \mathrm{C}\left(3.6^{\circ} \mathrm{F}\right)$ in the portion of the header around the shelf quadrants supporting the high power amplifiers.

A thermal model was generated to determine the temperature profile on the lower mounting shelf and the results are shown in Figure 8.3-8. It is noted that the temperatures are in the vicinity of $40^{\circ} \mathrm{F}\left(104^{\circ} \mathrm{F}\right)$ due to the inherently high temperature of the fluid. Acceptance and qualification test levels for the components can be in excess of $40^{\circ} \mathrm{C}$, as specified in MIL SPEC 1504A. This is possible since no batteries and, apparently, no other temperature-critical components are on the shelf.

Similar considerations for the Brayton spacecraft led to a header design which utilizes a tube with internal fins as shown in Figure 8.3-9. Comparison is made with a standard circular tube of the same diameter. The design parameters were selected to correspond to a 1.75" O. D tube but, as can be seen in Figure 8.7-9, only a slight increase in the diameter is needed to achieve allowable pressure drops without significantly affecting the temperature gradient from the tube wall to the gas. Figure 8.3-10 gives the components baseplate temperature.

A substantial reduction of the baseplate temperature of the high power amplifiers can be achieved by using an auxiliary heat pipe immediately below the radial heat pipes saddles and running continuously around the shelf periphery. The purpose of this heat pipe is to more evenly distribute the dissipation along the header and thus reduce the heat flux to the header in the region of the amplifiers. The temperature profile in this case is as shown in Figure 8.3-11. It is noted that the reduction in the amplifier temperature is achieved at the expense of higher baseplate temperatures in the vicinity of the fluid outlet. Clearly, a redistribution of components is required with this approach, so that the least temperature-sensitive boxes are located in the last 
SIGN PARAMETERS

FILM COEFFICIENT $\times$ HEAT TRANSFER AREA

ANNULUS DIMENSIONS
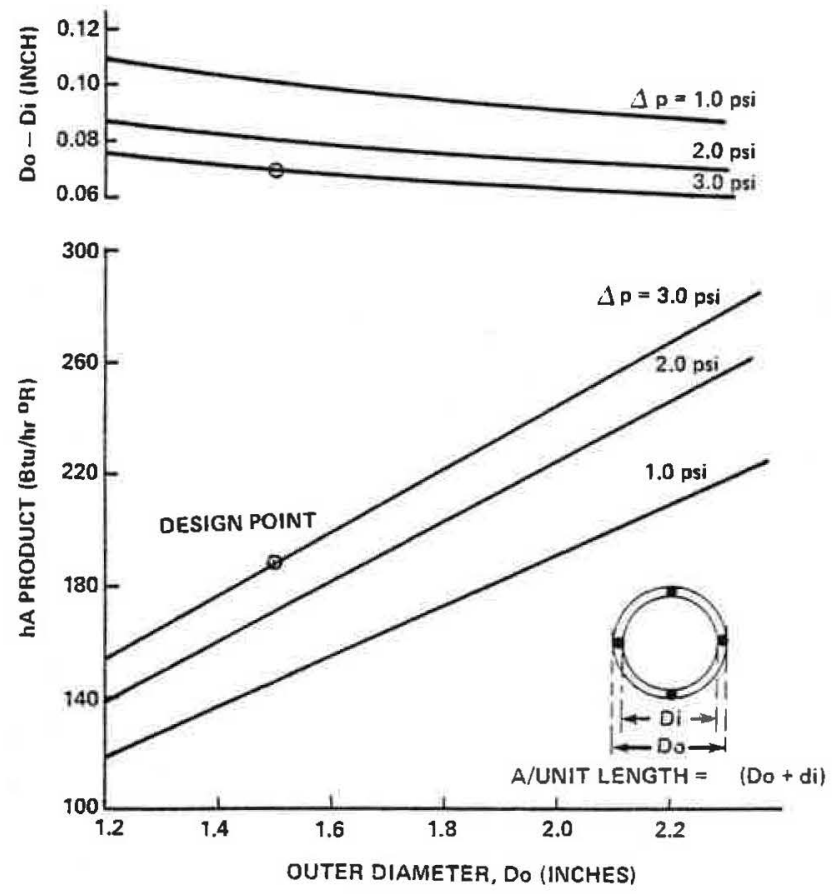

Figure 8.3-6. Annular Header - Equipment Shelf (Organic - Rankine)

FILM TEMPERATURE GRADIENT

(From Tube Walls to Fluid)

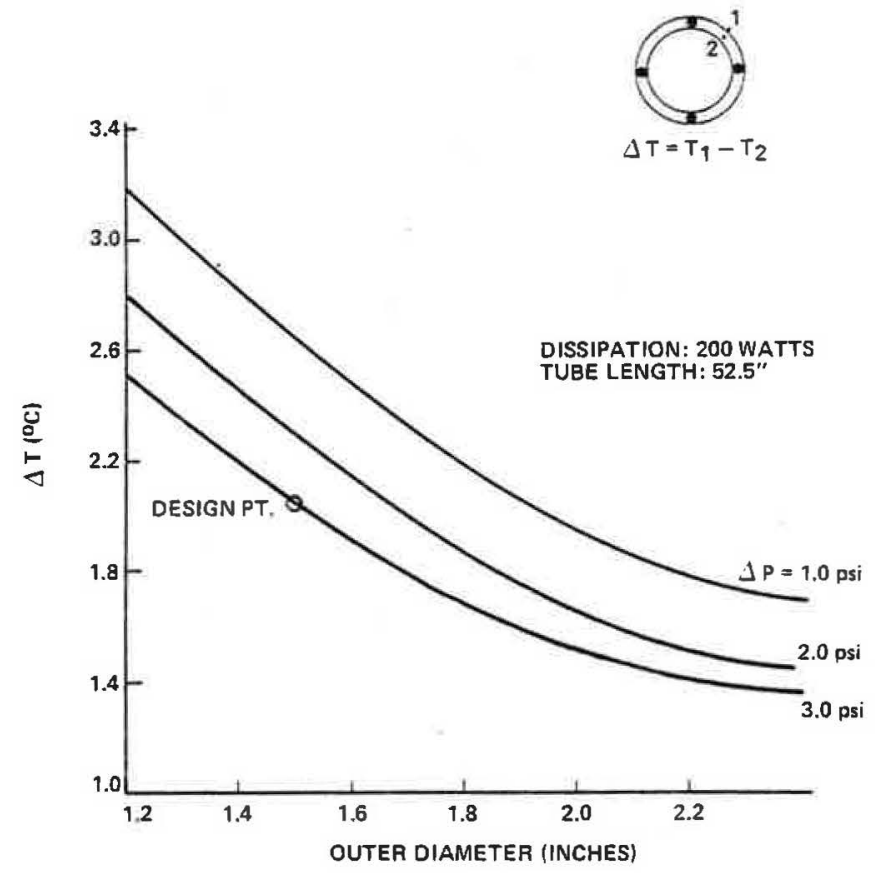

Figure 8.3-7, Annular Header - Equipment Shelf (Organic - Rankine) 


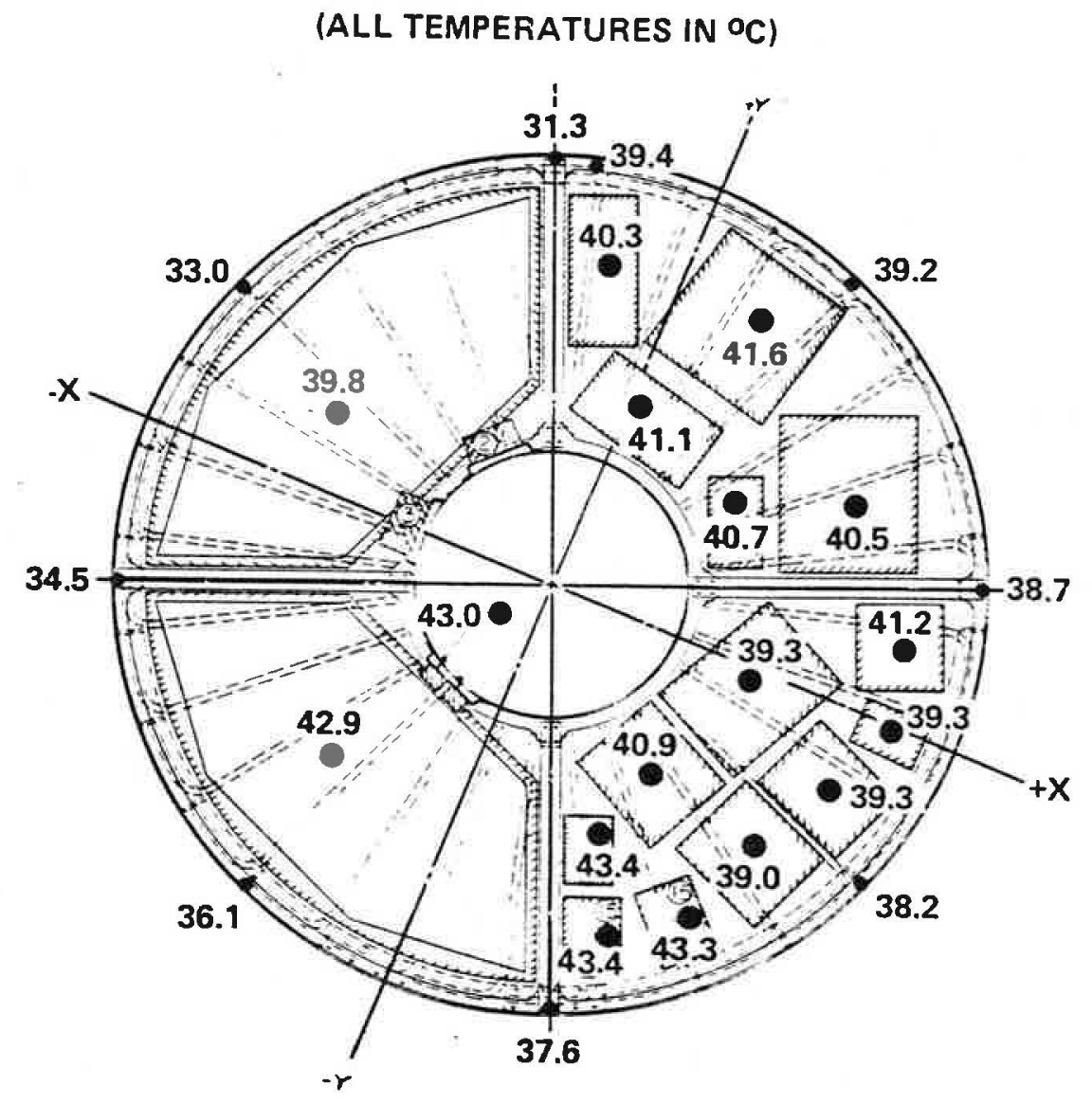

Figure 8.3-8. Organic - Rankine Spacecraft Lower Equipment Shelf Temperature Map

quadrant of the equipment shelf. This also illustrates the desirability of further reduction of the fluid temperature at the outlet of the radiator. A potential additional radiator area of some 25 square feet is available by use of the antenna mounting plate as a radiator extension area, which was not taken advantage of in this analysis.

Temperature control of the upper shelf on which the cesium clocks and momentum wheels are mounted is achieved by the same method as that of the lower shelf. The header, however, is a circular tube of 1-inch, I. D. for both RSV and BSV versions, and the design in both cases includes an auxiliary circular heat pipe for enhancing the thermal interface between the radial heat pipe saddles and the coolant header wall. The function of the auxiliary heat pipe will be to distribute the dissipation over the entire header area and hence eliminate local hot spots. Steady state temperatures based on the power listing shown in Figure 8.3-1 are shown in Figure 8.3-12. 


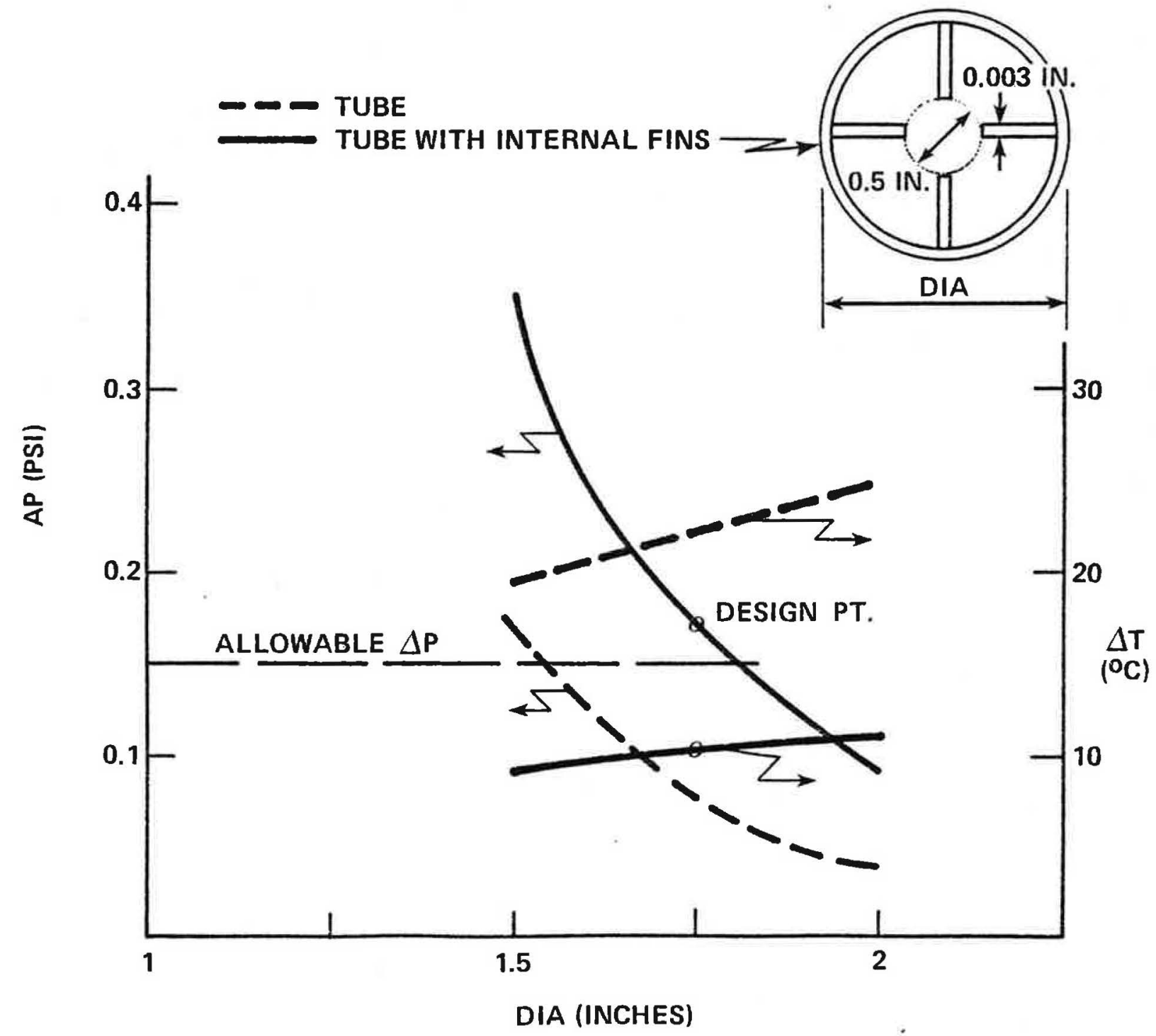

Figure 8.3-9. Equipment Shelf Header Design Parameters (Brayton Spacecraft) 
(FINNED CIRCULAR HEADER $13 / \% "$ O.D.

(ALL TEMPERATURES IN ${ }^{\circ} \mathrm{C}$ )
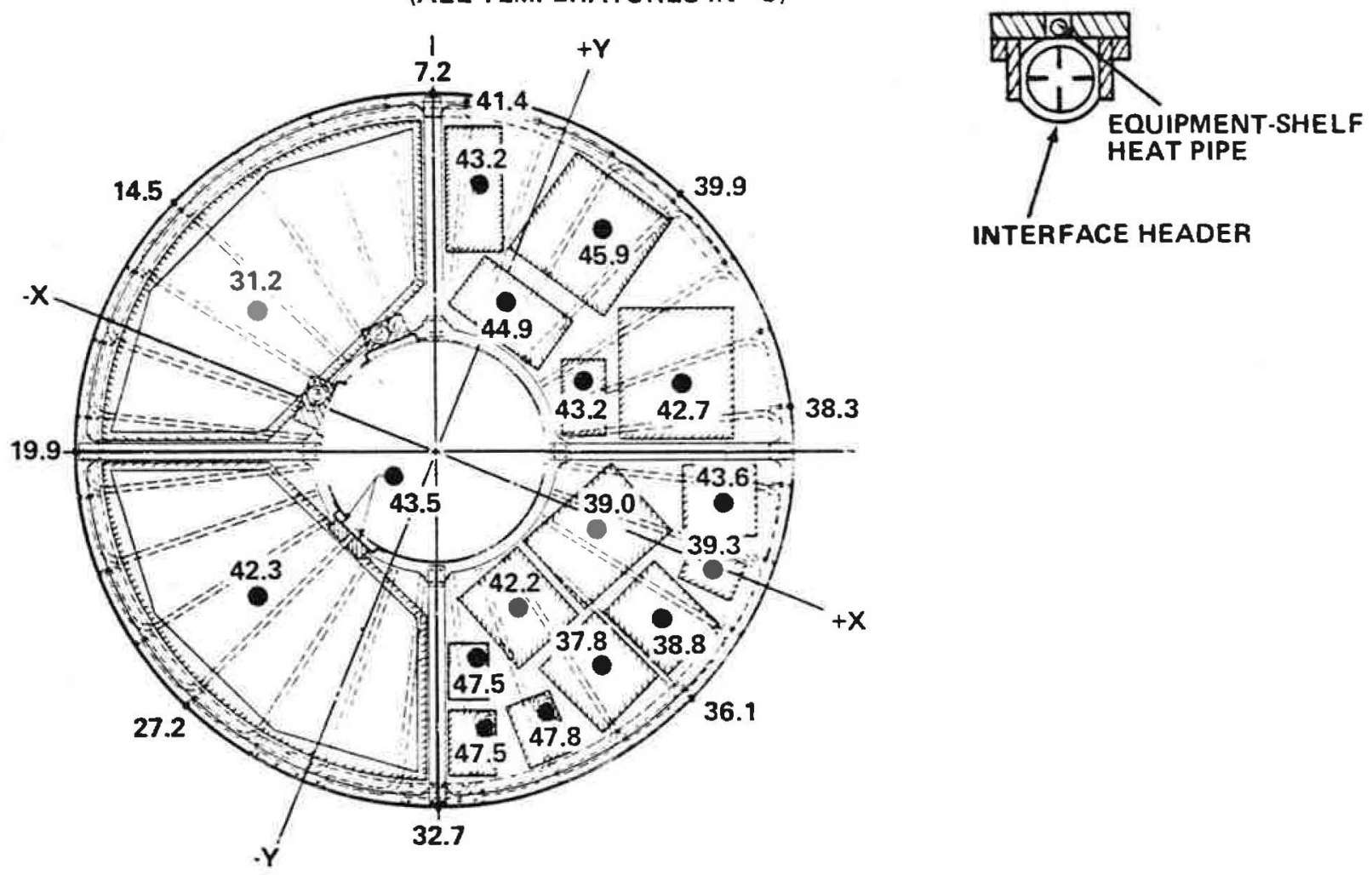

Figure 8.3-10. Brayton Spacecraft Lower Equipment Shelf Temperature Map

(With Auxilliary Circular Heat Pipe)

(ALL TEMPERATURES IN ${ }^{\circ} \mathrm{C}$ )

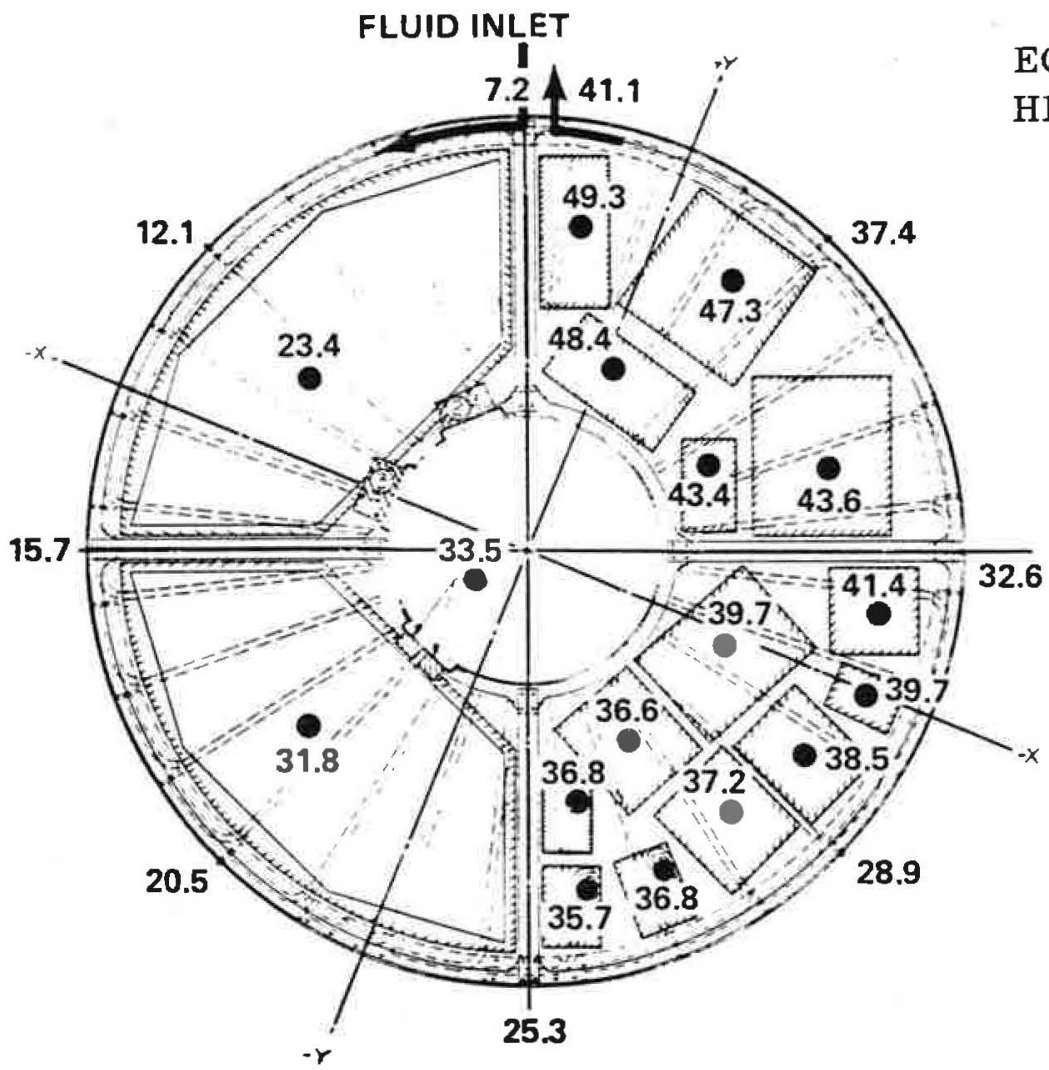

EQUIPMENT-SHELF

HEAT PIPE

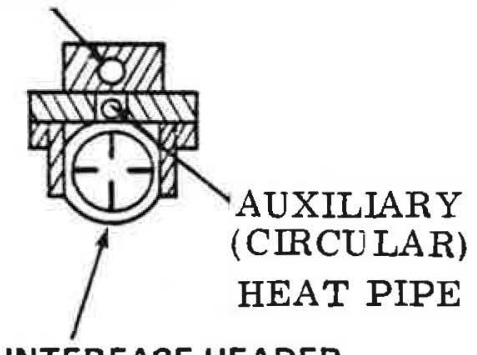

INTERFACE HEADER

Figure 8.3-11. Brayton Spacecraft Lower Equipment Shelf Temperature Map 
UPPER EQUIPMENT SHELF TEMPERATURE MAP

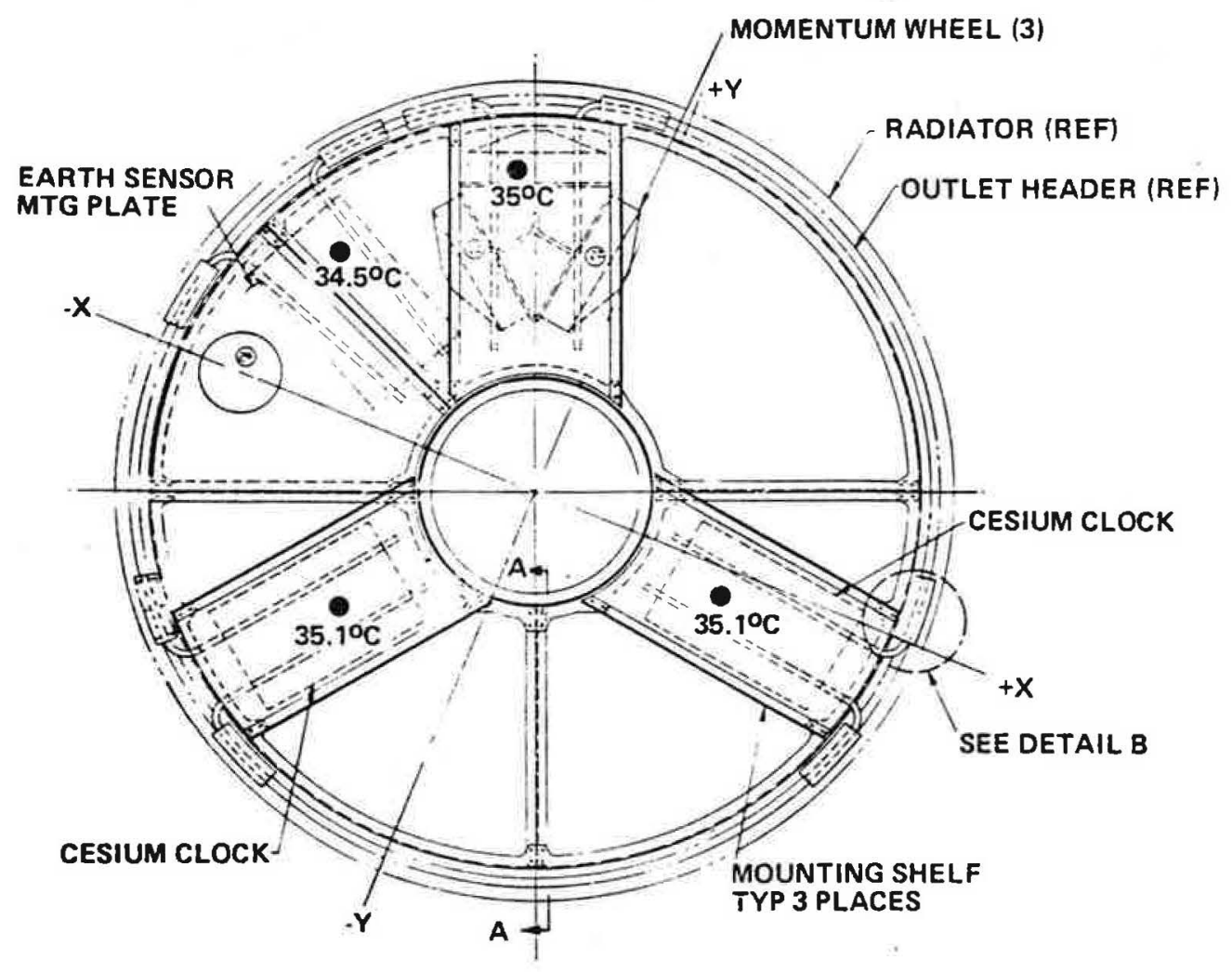

Figure 8.3-12a. Organic Rankine Spacecraft

\section{UPPER EQUIPMENT SHELF TEMPERATURE MAP}

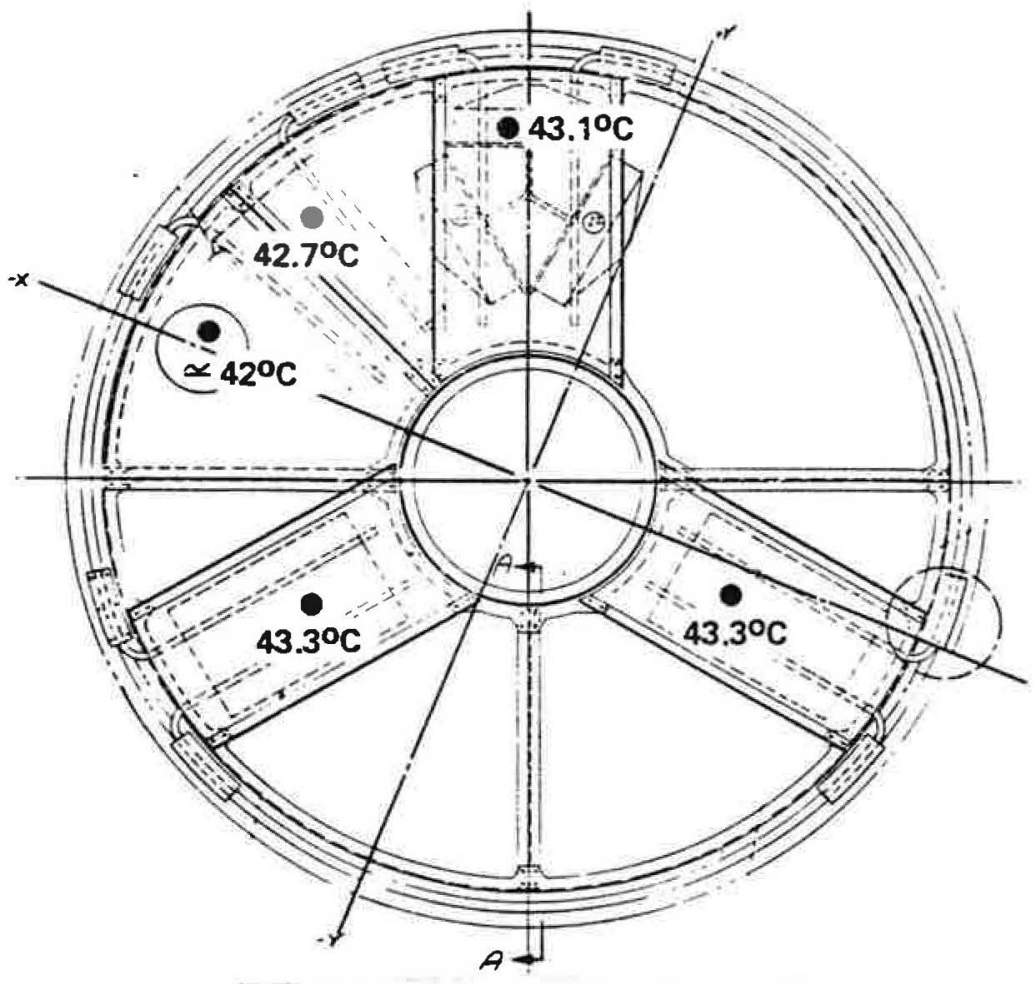

Figure 8.3-12b. Brayton Spacecraft 
Overall spacecraft temperature variations during the worst case eclipse orbits were determined for a variety of spacecraft surface finishes. Because the selected design approach results in essentially the entire spacecraft being cooled by the power system fluid loop, the vehicle was treated as a single-node isothermal body for the purpose of the calculation. Since eclipse transients have time constants of the order of an hour, and the fluid loop transit time through the spacecraft is of the order of seconds, this is believed to be a reasonable assumption for the spacecraft as a whole.

Minimization of the overall spacecraft temperature variation will have the beneficial effect of minimizing the variations of the critical frequency standards, discussed in the next subsection.

The resulting temperature profile for various surface finishes is given in Figure 8.4-1. An absorptance to emittance ratio $(\alpha / \epsilon)$ of $0.4 / 0.76$ refers to a silvered teflon coating which is available in the form of an adhesive tape. $\alpha / \epsilon=0.3 / 0.9$ are standard degraded properties values for a limited class of white paints which include SG-13 and Z-93. The aluminum side of vapor-deposited aluminum of Kapton has an $\alpha / \epsilon$ ratio of about $0.11 / 0.035$. The Kapton side of a 2 -mil sheet has an $\alpha / \epsilon$ of $0.36 / 0.76$.

Figure 8.4-1 indicates that a silvered teflon coating offers the most satisfactory selection. It combines a low overall temperature $\left(\sim 24^{\circ} \mathrm{C}\right)$ and a low worst-case orbit variation as seen in Figure $8.4-2\left(4^{\circ} \mathrm{C}\right.$ from maximum to minimum). In addition, it is superior to white paints in the stability of optical properties and shows only slight dependence of absorptance on the angle of incidence. These characteristics are important for accurate orbit predictability. 


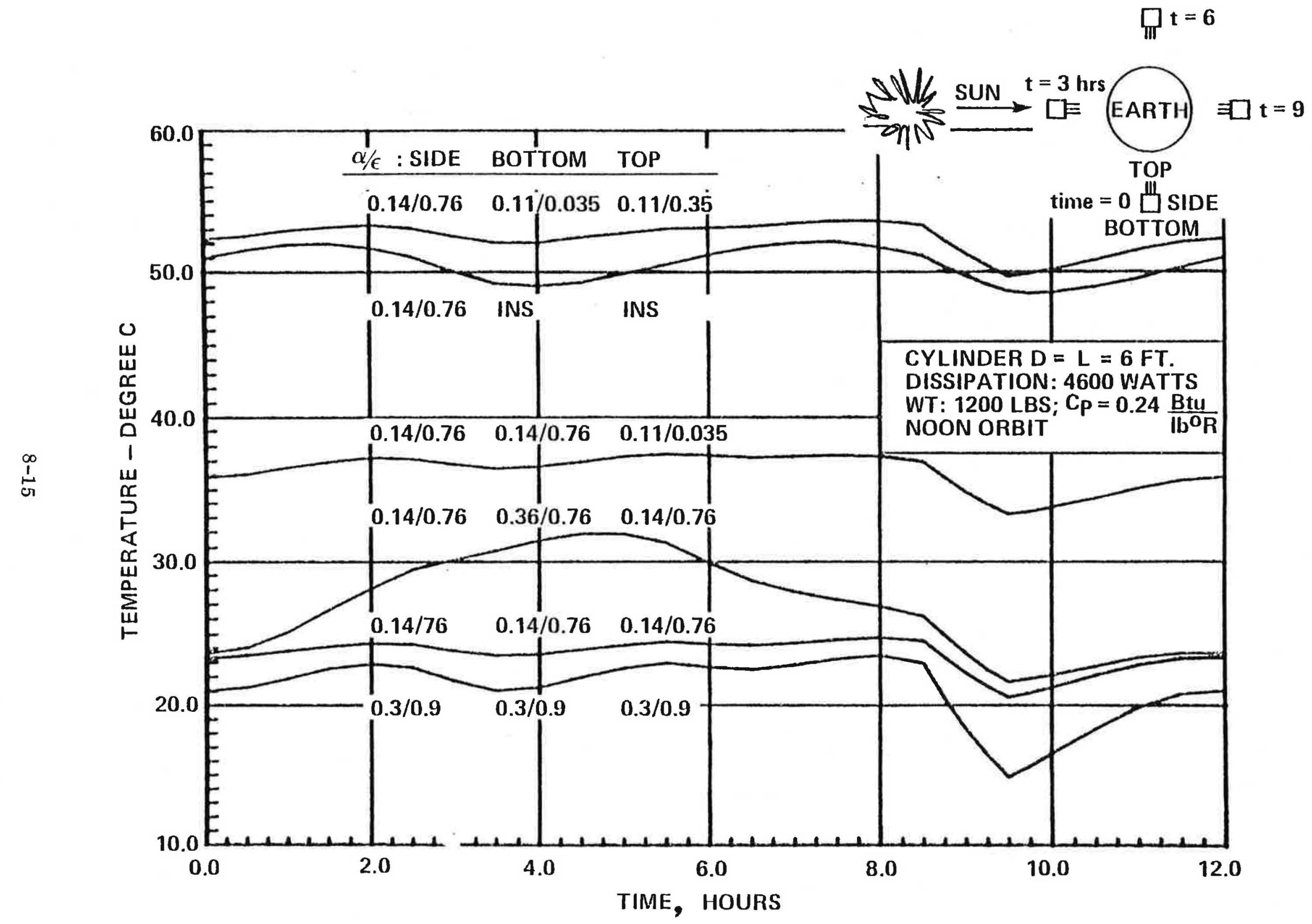

Figure 8.4-1. Overall Spacecraft Temperature Transient in Maximum Eclipse Orbit 
ALL SURFACES $\alpha / \epsilon=.14 / .76$ (Ag-Teflon)

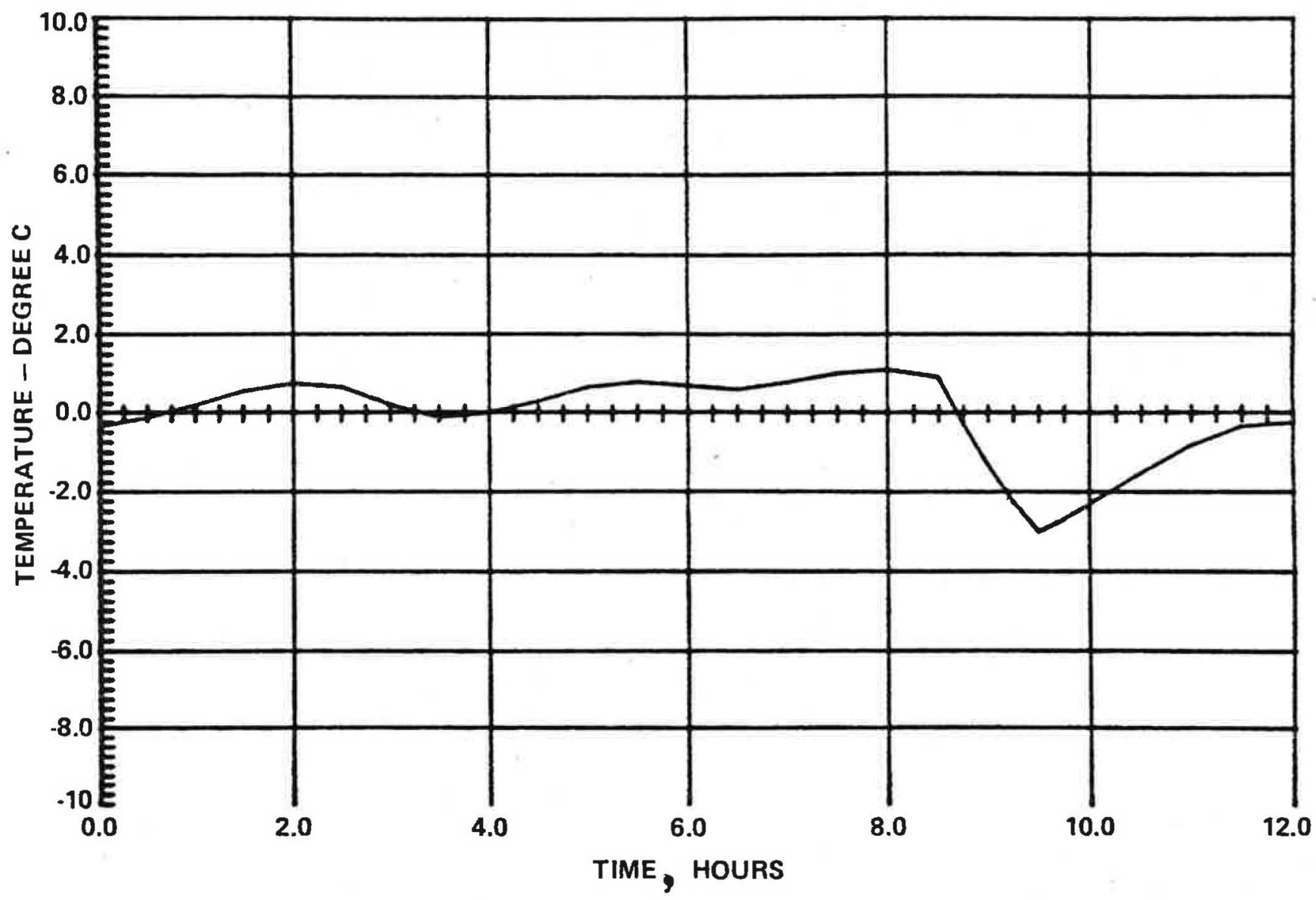

Figure 8.4-2. N/GPS Orbital Temperature Variation

\subsection{ACTIVE THERMAL CONTROL OF FREQUENCY STANDARDS}

The design of the heat path between the clocks and the header is directed towards minimizing the gradient so as to maintain the clock temperatures in the neighborhood of $40^{\circ} \mathrm{C}$. This approach to coupling results in a variation on the clock baseplate temperature which nearly parallels the orbital variation of the sink although reduced to approximately $2^{\circ} \mathrm{C}$ in the absence of active control (See Figure 8.5-1).

A heater/thermostat system can be employed at the clock baseplate for an active control which would minimize the orbital temperature variations still further. Calculations were made based on a best estimate for the conductance from the baseplate to the header fluid, which is found to be about $7.0 \mathrm{Btu} / \mathrm{hr}^{\circ} \mathrm{R}$ for the RSV, and a clock dissipation of 24 watts. A heater element rated at 30 watts and thermostatically controlled at $38^{\circ} \mathrm{C}\left(\mathrm{ON}\right.$ for $\mathrm{T}<38^{\circ} \mathrm{C}$ and $\mathrm{OFF}$ for $\mathrm{T} \geq 38^{\circ} \mathrm{C}$ ) operating at $13 \%$ duty cycle 
can maintain the temperature variation at less than $0.2^{\circ} \mathrm{C}$. This is shown in Figure 8.5-1 for the maximum eclipse orbit. Also shown in Figure 8.5-1 is the effect a change in the clock-to-fluid conductance would have after the thermostat set point is fixed. This could occur during launch, or after a period of time at temperature in space. Clearly, the most effective design would be one for which the set point can be shifted by ground command during orbital operation.

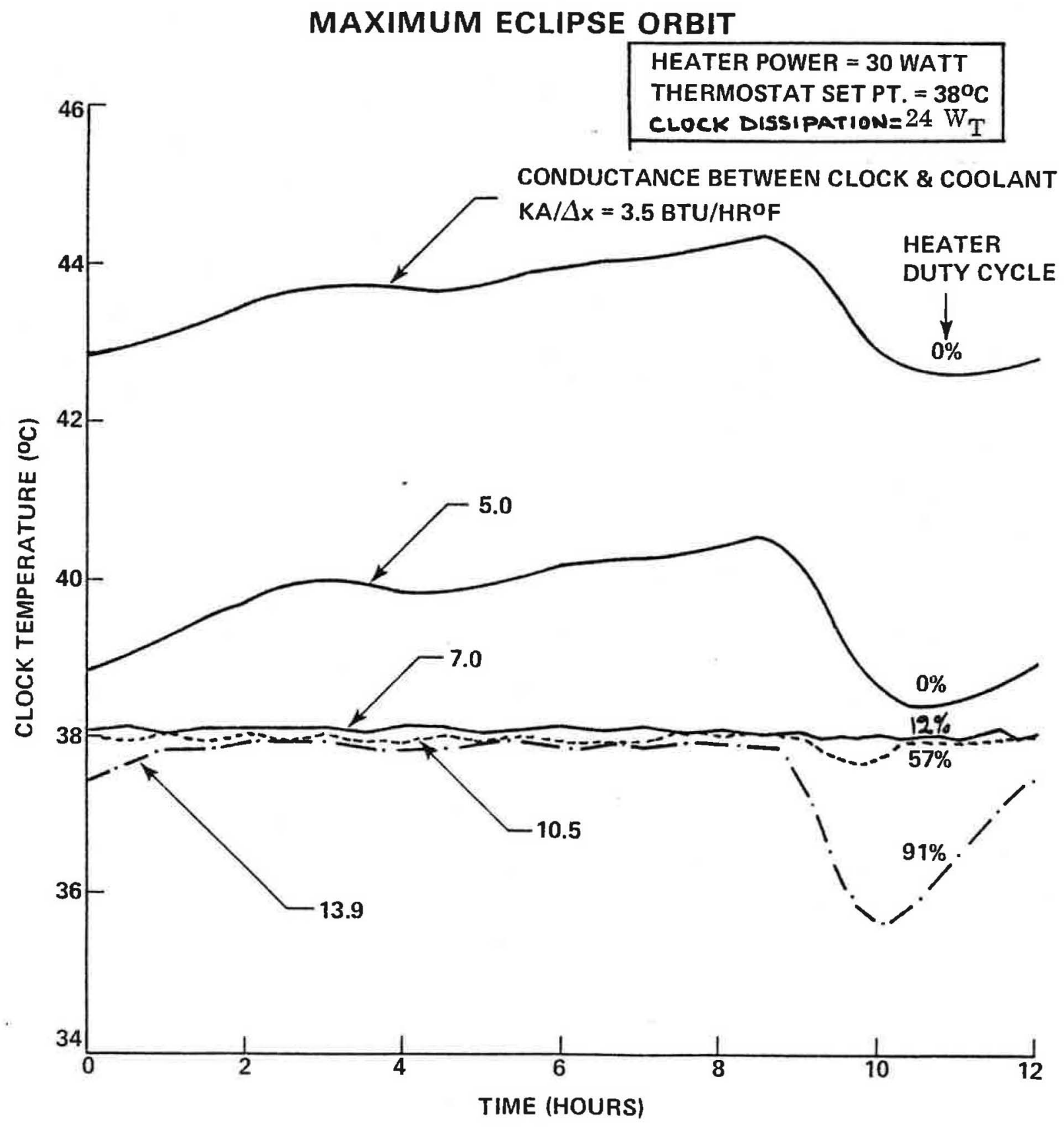

Figure 8.5-1. CS Frequency Standard Temperature Variations with Heater/Thermostat Control 



\section{SECTION IX}

\section{HIGHER ALTITUDE VERSION}

\subsection{HIGHER ALTITUDE VERSION}

The nuclear-powered reference designs are quite insensitive to operating orbit altitude, and could be flown in higher orbit without modification, except for the Earth sensor coverage angle. However, in order to preserve RF illumination levels on the Earth without substantial radiated power increases would require modification of the navigation antenna. Table 9.1-1 lists the variation in the angle subtended by Earth for various altitudes, together with a nominal antenna diameter for the L1 signal based on first order optical considerations. At some altitude between 48 and 120 hour orbital periods, a reflector-type antenna would become more effective for the L1 - $19 \mathrm{~cm}$ wavelength.

Table 9.1-1. Higher Altitude Versions

\begin{tabular}{|c|c|c|c|}
\hline $\begin{array}{c}\text { ORBIT } \\
\text { PERIOD }\end{array}$ & ALTITUDE & $\begin{array}{c}\text { EARTH } \\
\text { ANGLE }\end{array}$ & $\begin{array}{c}\text { NOMINAL } \\
\text { ANTENNA } \\
\text { DIAMETER }\end{array}$ \\
\hline DAYS & N.MI. & DEGREES & METERS \\
\hline $1 / 2$ & 10,900 & 26.9 & 0.49 \\
1 & 19,350 & 17.2 & 0.77 \\
2 & 32,700 & 10.9 & 1.22 \\
5 & 62,950 & 5.92 & 2.24 \\
10 & 102,000 & 3.74 & 3.56 \\
\hline
\end{tabular}

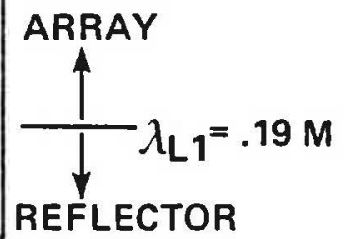

For the 120-hour alternative orbit suggested by the original groundrules, a reflector antenna with diameter only slightly smaller than the spacecraft diameter is adequate to maintain essentially the same illumination levels as are achieved from the 12-hour orbit. This illustrated in Table 9.1-2, conservatively 
assuming an antenna efficiency of $40 \%$. The half-power beamwidth of the 1.7 meter reflector is 8.0 degrees, compared to the Earth angle of 5.9 degrees, so that illumination should be fairly uniform over the surface of the Earth.

Table 9.1-2. Fixed Antenna Design for 120-Hour Orbit

SPACECRAFT DIAMETER $=1.82 \mathrm{M}$

ANTENNA DIAMETER $=1.7 \mathrm{M}$

HALF.POWER BEAMWIDTH $=8.0$ DEG.

ANTENNA GAIN $(\eta=.40)=25.0 \mathrm{db}$

$L_{1}$ SIGNAL COMPARISON

\begin{tabular}{|c|c|c|c|c|c|}
\hline \multirow[b]{3}{*}{ PRNSA OUTPUT } & \multicolumn{2}{|c|}{120 HR. ORBIT } & & \multicolumn{2}{|c|}{12 HR. ORBIT } \\
\hline & $\mathbf{P}$ & C/A & UNIT & $\mathbf{P}$ & $\mathrm{C} / \mathrm{A}$ \\
\hline & \multicolumn{2}{|c|}{20.9} & $d B w$ & \multicolumn{2}{|c|}{20.9} \\
\hline WORST CASE SIGNAL RATIO & -4.8 & -1.8 & $\mathrm{~dB}$ & -4.8 & -1.8 \\
\hline ANTENNA GAIN & \multicolumn{2}{|c|}{25.0} & dB & \multicolumn{2}{|c|}{13.7} \\
\hline NET LINK LOSSES & \multicolumn{2}{|c|}{-197.3} & dB & \multicolumn{2}{|c|}{186.8} \\
\hline USER SIGNAL & -156.2 & -153.2 & $d B w$ & -157.0 & -154.0 \\
\hline
\end{tabular}

Figure 9.1-3 illustrates the spacecraft configuration as modified for the 120 hour-orbit.

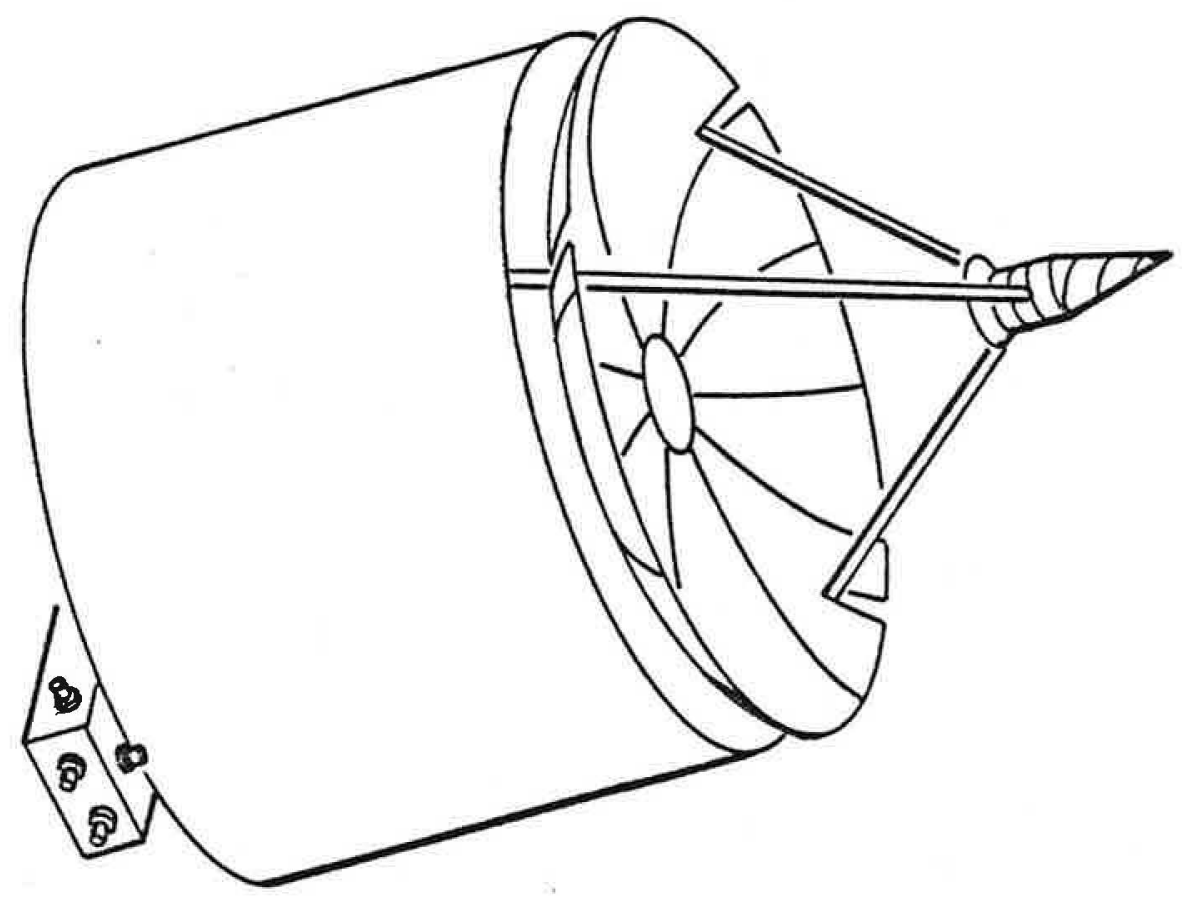

Figure 9.1-3. Spacecraft Design for 120-Hour Orbit 


\section{SECTION X}

\section{WEIGHT AND COST SUMMARY}

\subsection{WEIGHT AND COST SUMMARY}

The weight breakdowns for the two reference spacecraft are given in Tables 10.1-1 and 10.1-2. As can be seen the higher converter weight of the BSV is almost exactly compensated by the somewhat heavier radiator, so that the weight of the two spacecraft are within a few pounds of each other. The weight of the four spacecraft cluster plus adapter would therefore come to approximately $4400 \mathrm{lbs}$, or a nominal 5000 pounds with adequate margin. Including the 32,000 lbs two-stage IUS, a total of 37,000 lbs, would be the shuttle-chargeable launch weight. Also chargeable to the mission would be the heat-source cooling kit required until the power system is started in parking orbit. This would consist of a water tank plus a pump, valves and controls. Based on a four-spacecraft heat load of $12.8 \mathrm{kw} T$ (BSV), the water consumption rate would be approximately $45 \mathrm{lb} / \mathrm{hr}$, assuming that the resultant water vapor would be vented overboard through the shuttle auxiliary 4 -inch vent port. This cooling kit would fit within the IUS support structure; however, it has not as yet been designed in detail.

Cost estimates for the two reference spacecraft were derived from the SAMSO cost model, 1973 update (Tables 10.1-3 and 10.1-4). These costs are relevant when compared to each other; however, their relevance to actual spacecraft costs may be questioned because of the dated nature of the data in the cost model. For example, no allowance for inflation was made in the cost estimating relationship (CER's) because this would have distorted them considerably beyond the level of commonly known costs. 
Table 10.1-1. Weight Breakdown, Organic Rankine Spacecraft

TOTAL SPACECRAFT WEIGHT, LBS.

1008.2

ANTENNA ASSEMBLY

SHELF

ANTENNAS

EQUIPMENT MODULE

STRUCTURE

CYLINDER SYSTEM

RINGS

RIBS

TOP SHELF

BOTTOM SHELF

EQUIPMENT

CS. FREQ. STDS.

REACTION WHEELS (3)

SENSORS

ACS CONTROL BOX

SHELF EQUIPMENT

POWER SYSTEM MODULE

STRUCTURE

CYLINDER

RING

28.0

RIBS

34.0

SUPPORT SHELF

30.8

BOTTOM COVER

12.2

24.5

REACTION CONTROL SYS.

TANKS \& PROPELLANT

THRUSTERS

LINES \& COMPONENTS

RADIATOR SYSTEM

RINGS

LONGERONS

SHELL

HEADERS

SPIRAL TUBE

POWER SYSTEM

PCU

MHW

8.1

30.0

38.1

24.0

5.1

9.2

15.0

25.5

68.0

33.0

16.1

18.5

145.0

$\underline{280.6}$

$78.8 \stackrel{359.4}{=}$

65.4

10.6

13.1

15.6

9.8

33.9

5.0

7.8

$\underline{129.5} \stackrel{\underline{610.7}}{\underline{\underline{n}}}$

89.1

4 S $/ C+$ ADAPTER $=4040+360=4400$ LBS. 
Table 10.1-2. Weight Breakdown, Brayton Powered Spacecraft

TOTAL SPACECRAFT WEIGHT, LBS.

1001.6

ANTENNA SUBASSEMBLY

SHELF

ANTENNAS

EQUIPMENT MODULE

STRUCTURE

CYLINDER SYSTEM

RINGS

RIBS

TOP SHELF

BOTTOM SHELF

EQUIPMENT

CS FREQ. STDS

REACTION WHEELS (4)

SENSORS

ACS CONTROL BOX

SHELF EQUIPMENT

POWER SYSTEM MODULE

STRUCTURE

CYLINDER

DOMES

RINGS

RIBS

8.1

30.0

SUPPORT SHELF

$12 . \quad 65.0 \stackrel{356.6}{=}$

4.0

8.8

15.0

25.5

68.0

291.6

44.0

16.1

18.5

145.0

BOTTOM COVER

INSULATION

$\underline{139.4} \stackrel{606.9}{=}$

45.0

17.4

27.7

22.0

12.8

24.5

2.8

REACTION CONTROL SYS.

TANKS \& PROPELLANT

THRUSTERS

LINES \& COMPONENTS

RADIATOR SYSTEM (AI)

RINGS

65.4

10.6

13.1

LONGERONS

SHELL

HEADERS \& TRANSFER

92.6

89.1

VERT. TUBES

POWER SYSTEM

HSA

10.2

36.9

14.2

12.7

MINI-BRU

RECUPERATOR

DUCTING

INSULATION

CONTROL BOX

101.0

273.0

38.1

PARASITIC LOAD

38.0

84.0

15.0

15.0

15.0

5.0 
Table 10.1-3. Recurring Costs -- Organic Rankine Spacecraft

(COSTS IN K\$)

\begin{tabular}{|l|c|c|c|c|c|c|c|}
\hline & $\begin{array}{c}\text { Normalized } \\
\text { CER } \\
\text { Costs }\end{array}$ & $\begin{array}{c}\text { Complexity } \\
\text { Factor }\end{array}$ & $\begin{array}{c}\text { Complexity } \\
\text { Weighting }\end{array}$ & $\begin{array}{c}\text { Technology } \\
\text { Factor }\end{array}$ & $\begin{array}{c}\text { Technology } \\
\text { Weighting }\end{array}$ & $\begin{array}{c}\text { Composite } \\
\text { Factor }\end{array}$ & $\begin{array}{c}\text { Adjusted } \\
\text { Costs }\end{array}$ \\
\hline Structure, TC & 400 & 1.11 & 0.445 & 0.90 & 0.400 & 1.00 & 400 \\
Commun/Nav & 500 & 1.51 & 0.550 & 0.83 & 0.310 & 1.23 & 614 \\
TT \& C & 275 & 1.17 & 0.445 & 0.65 & 0.435 & 0.92 & 254 \\
EPS & $950^{*}$ & - & - & - & - & 1.00 & $950^{*}$ \\
ACS/RCS & 458 & 1.20 & 0.550 & 0.69 & 0.350 & 1.00 & 459 \\
\hline & \\
& $\begin{array}{l}\text { Total Subsystems Direct Cost } \\
\text { Program Level Cost @ .30 } \\
\text { Burden + G\&A @ 1.06 }\end{array}$ \\
& $\begin{array}{l}\text { Fee @ .13 } \\
\text { Total Spacecraft Recurring Cost } \\
\text { Nuclear Fuel @ \$400/WT } \\
\text { Total Unit Recurring Cost }\end{array}$ \\
&
\end{tabular}

- Power System Contractor Estimate $+\$ 200 K$ For Heat Sources and Other EPS Functions

Table 10.1-4. Recurring Costs -- Brayton Spacecraft

(COSTS IN K\$)

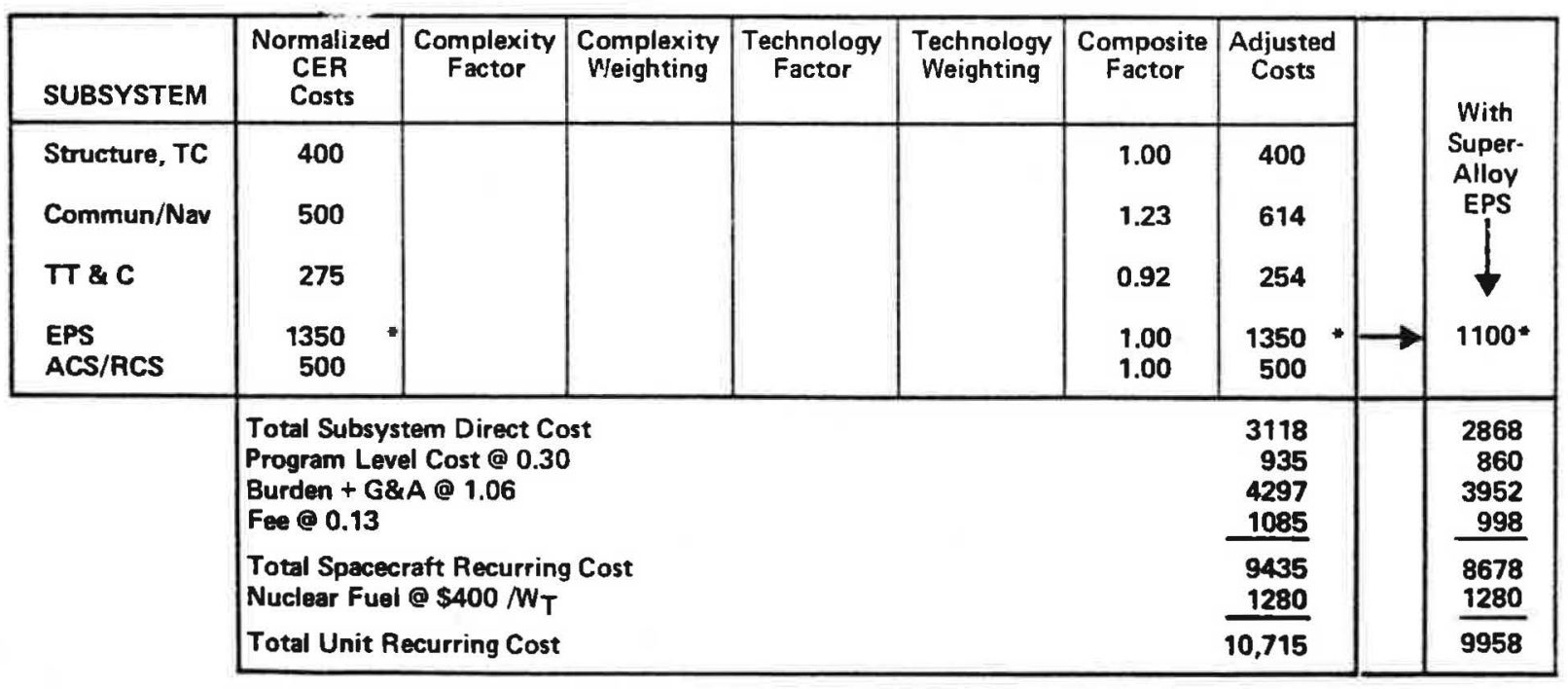

-Power System Contractor Estimate $+150 \mathrm{~K}$ for Heat Source and Other EPS Functions 
Costs of the power systems themselves were based on ERDA-supplied estimates, and adjusted as indicated in the Tables.

Nuclear fuel costs are based on quantity use which would be typical of the GPS program. Fuel costs were not burdened in the analysis, since the nuclear fuel itself would be part of an intergovernmental transfer and would not show up on the contractor's books. However, costs for heat source fabrication were included in the contractor cost estimates.

The nonrecurring development costs of the first nuclear-powered GPS spacecraft were similarly estimated (Table 10.1-5) although three of the five subsystem costs were arbitrarily assigned, and no claim can be made for their accuracy. It is assumed herein that the power system development is funded separately, and is not included herein.

Table 10.1-5. Brayton and Organic Rankine; Non-Recurring Costs *

(COSTS IN K\$)

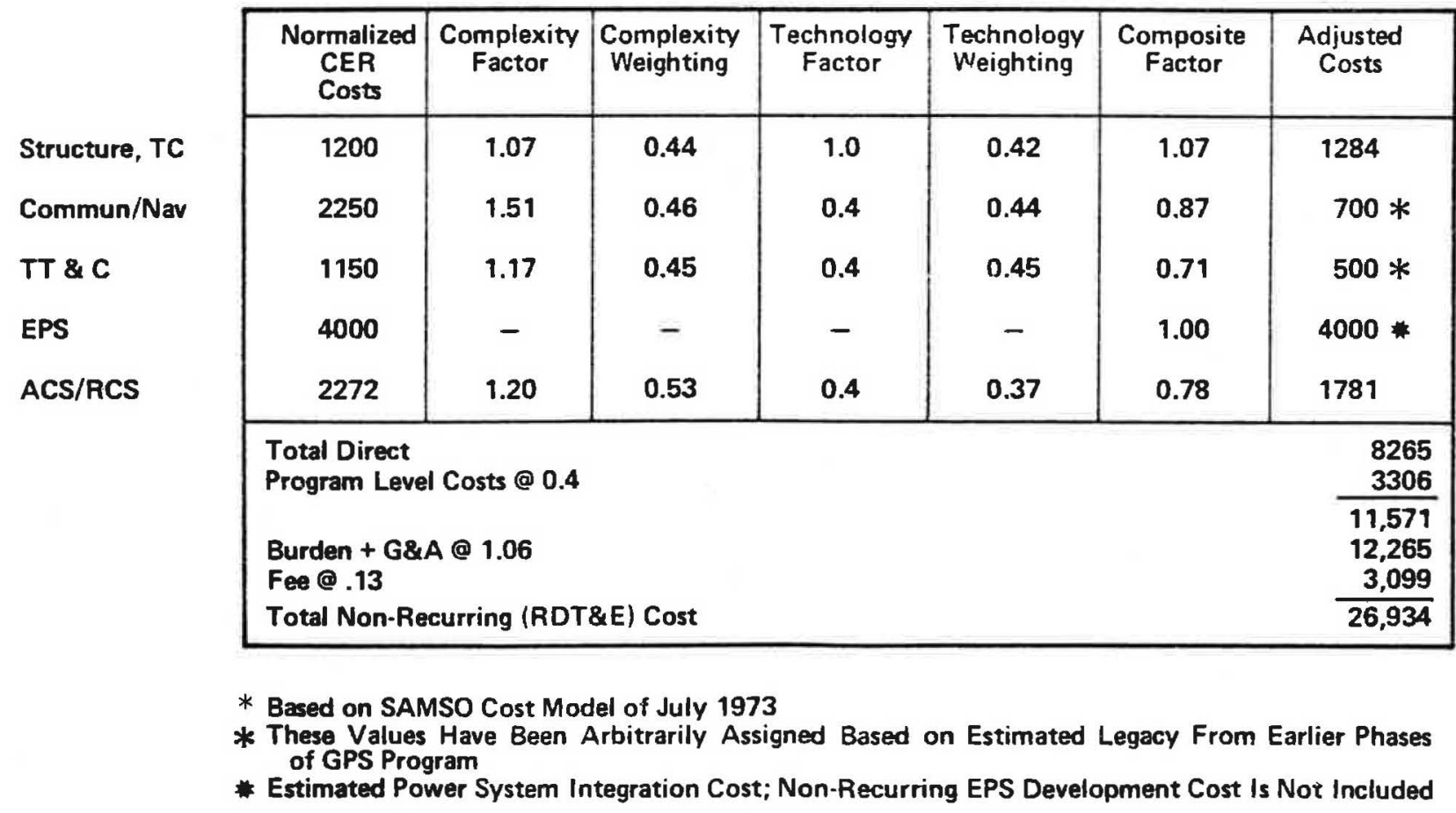





\section{SECTION XI}

\section{CRITICAL PARAMETERS -- RESULTS}

\section{$11.1 \quad$ POWER TO USER}

The determination of user power for the two reference designs, including the two versions of the Brayton design, is dependent on the prime spacecraft power less the fixed power requirements, and taking into account the efficiency of conversion of DC-to-RF of the high power amplifiers.

The fixed power requirements (i. e., not dependent on specific design) are shown in Table 11.1-1. Because the reference-design vehicles are not equipped with batteries or other power storage capability, attention to peak as well as average power requirements is necessary.

The AVCS power consumption varies as a result of build-up in speed of the momentum wheels over the ten-day unload cycle. Following momentum unload, wheel power consumption is close to zero, gradually building to a maximum near 20 watts over 10 days. (In practice, wheel peak power consumption could be reduced to roughly half this value by pre-loading angular momentum in the direction opposite to the normal build-up, so that consumption would vary from 10 watts to zero and back to ten watts before the next unload period).

Telemetry and command power consumption is based on NDS values, with the peaks occurring during telemetry transmission periods.

Losses in the electric power subsystem are based on the prime power source of 3-phase AC (100 or 120 watts) from the generators. The assumed AC-to-DC conversion losses of $5 \%$ are considerably less than the typical losses experienced by DC-toDC converters. The losses accounted for in Table 11.1-1 are based only on the power required by the fixed elements listed in the table. 
Table 11.1-1. Fixed Power Budget

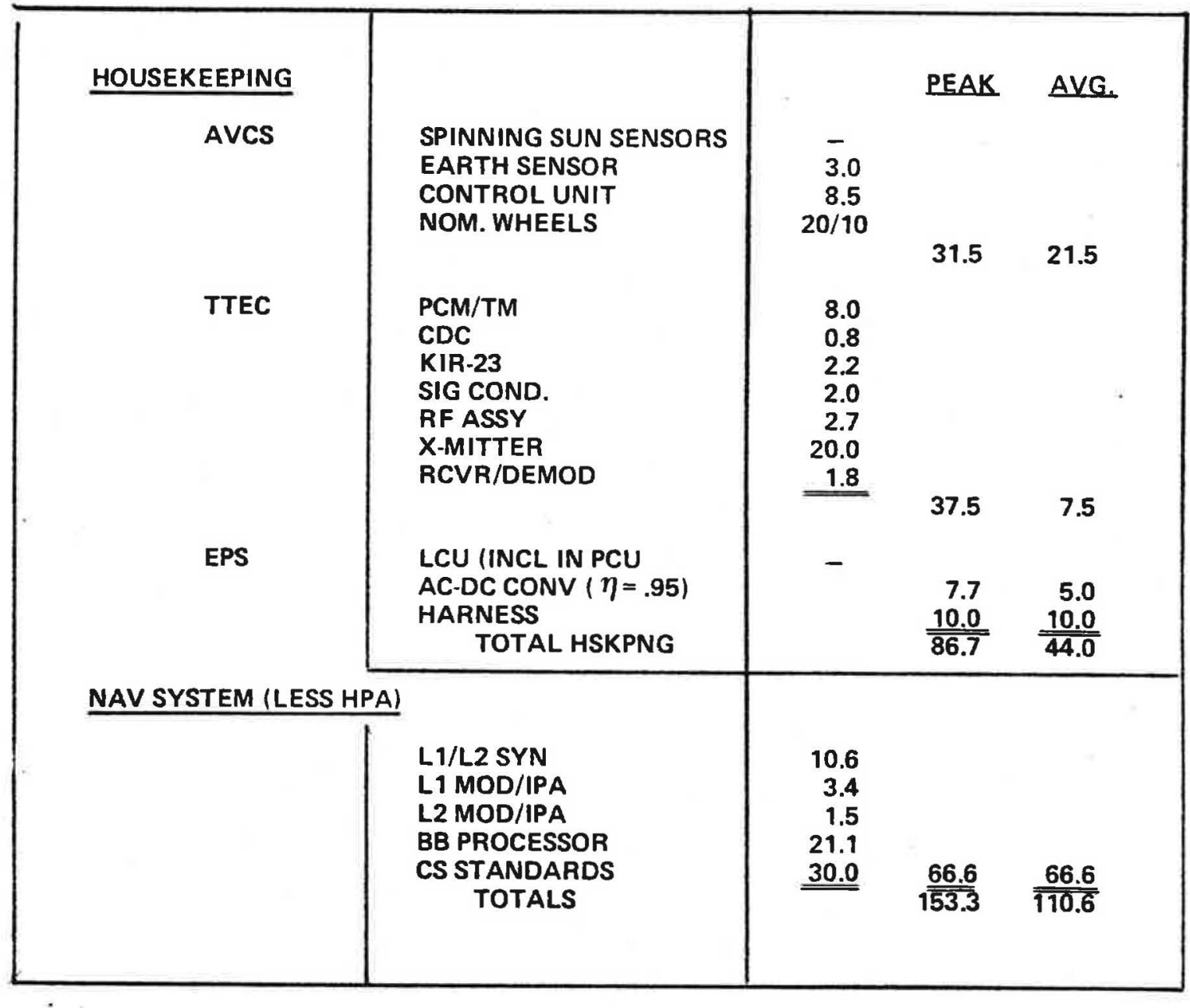

The fixed power requirements of the navigation system are also shown in Table 11.1-1, based on NDS values, and assuming that only one of the two frequency standards is operating at any given time. The remainder of the spacecraft prime power is assumed to be available for powering the final amplifiers of the navigation transmitters.

The operating efficiency of these solid state amplifiers is dependent on their junction temperatures, which depends in turn on their baseplate temperatures. An estimate of this temperature dependence was made * as shown in Figure 11. 1-2. From the equipment shelf temperature calculations as shown in Figures 8.3-8 and 8.3-11 the efficiency values assumed for the two reference designs are shown in Figure 11.1-2. This was rounded off to 0.3 for the RSV and 0.4 for the BSV, in Table 11.1-3.

* Personal communication of Capt. Wayne LeCompte, SAMSO/YE 


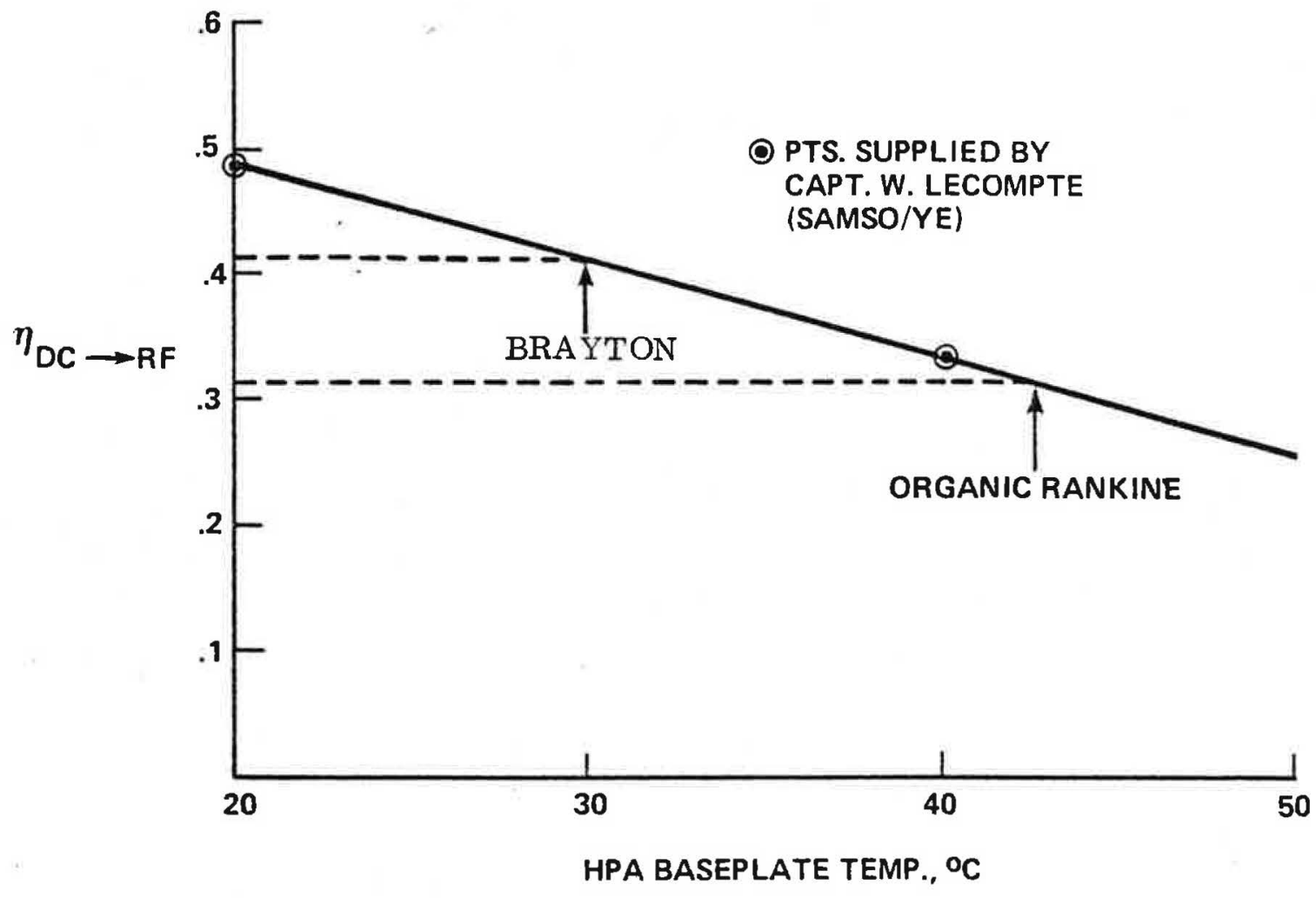

Figure 11.1-2. High Power Amplifier Efficiency Variation With Temperature

Table 11.1-3. Total Power Budget (Watts)

\begin{tabular}{|c|c|c|c|c|}
\hline \multirow[b]{2}{*}{ SYSTEM } & \multirow{2}{*}{$\begin{array}{l}\text { ORGANIC } \\
\text { RANKINE }\end{array}$} & \multicolumn{3}{|c|}{ BRAYTON } \\
\hline & & $\mathrm{Cb}$ & SUPERALLOY & $\mathrm{Cb}$ \\
\hline $\begin{array}{l}\text { BOL THERMAL POWER } \\
\text { BOL AC POWER } \\
\text { 7-YR AC POWER }\end{array}$ & $\begin{array}{r}4800 \\
875 \\
823\end{array}$ & $\begin{array}{r}3200 \\
735 \\
678\end{array}$ & $\begin{array}{r}3200 \\
665 \\
614\end{array}$ & $\begin{array}{r}2400 \\
490 \\
452\end{array}$ \\
\hline $\begin{array}{l}\text { HPA } \eta D C \rightarrow \text { RF } \\
\text { HPA POWER INPUT } \\
\text { OPTION (4) } \\
\text { OPTION (2) + } 1 \mathrm{~dB} \\
\text { OPTION (2) }\end{array}$ & $\begin{array}{c}0.3 \\
644 \\
- \\
-\end{array}$ & $\begin{array}{c}0.4 \\
483 \\
- \\
-\end{array}$ & $\begin{array}{c}0.4 \\
- \\
430 \\
-\end{array}$ & $\begin{array}{c}0.5 \\
- \\
- \\
277\end{array}$ \\
\hline TOTAL FIXED POWER & $153 / 111$ & $153 / 111$ & $153 / 111$ & $153 / 111$ \\
\hline $\begin{array}{l}\text { 7-YR POWER MARGIN } \\
\text { BOL POWER MARGIN }\end{array}$ & $\begin{array}{r}26 / 68 \\
78 / 120\end{array}$ & $\begin{array}{r}42 / 84 \\
99 / 141\end{array}$ & $\begin{array}{r}31 / 73 \\
82 / 124\end{array}$ & $\begin{array}{r}23 / 64 \\
60 / 102\end{array}$ \\
\hline
\end{tabular}


Table 11. 1-3 shows the resulting overall power budget. Both the 4800 watt $_{\mathrm{T}}$ RSV and the $3200 \mathrm{w}_{\mathrm{T}}$ BSV can achieve the so-called option (4) level of powerto-user (Ref. Section 2.1) with adequate power margin for seven years. The superalloy BSV, on the other hand, can only achieve option (2) plus $1 \mathrm{~dB}$ in the $\mathrm{L}_{1}$ (P) link (i. e. - 154 $\mathrm{dBw} \mathrm{L}_{1}(\mathrm{C} / \mathrm{A})$ and $-162 \mathrm{dBw} \mathrm{L}_{1}(\mathrm{P})$, although at a lower cost (Ref. Section 10).

Although not carried through in the design detail phase, a $2400 \mathrm{~W}_{\mathrm{T}}$ Brayton vehicle of the same size would be capable of a baseplate temperature low enough to achieve a high-power-amplifier efficiency approaching 0.5. If feasible, option (2) might be achieved by such a vehicle with a power margin similar to that of the other designs, as shown in Table 11.1-3.

\subsection{MEAN MISSION DURATION (MMD)}

From the discussion of Section 2.2, it is apparent that the major gains to be achieved in MMD will come about from an extension of the design lifetime of the life-limiting subsystems. Because the power degradation of the solar array in the $\phi 1$ NDS is the current life-limiting element, replacement by a nuclear power system with a 7-year design lifetime will result in an evident gain in this critical parameter. The exact value of this gain will depend on the reliability achieved by the nuclear power system, as well as by the other subsystems in the spacecraft.

If it is assumed that the failure rates of the other subsystems are identical to those of the NDS and are constant for 7 years, then the dependence of MMD on the reliability of the nuclear power system can be calculated, as shown in Figure 11.2-1. Reliability design goals for the nuclear power systems have been set at 0.95 , which would lead to an MMD of approximately 5.9 years. Even if this goal is not fully achieved, however, an MMD in excess of 5.5 years appears assured. No more precise claims can be made at this time, obviously, without full-system test data on the nuclear power system and a more detailed study of the other subsystems as applied in the reference designs. 


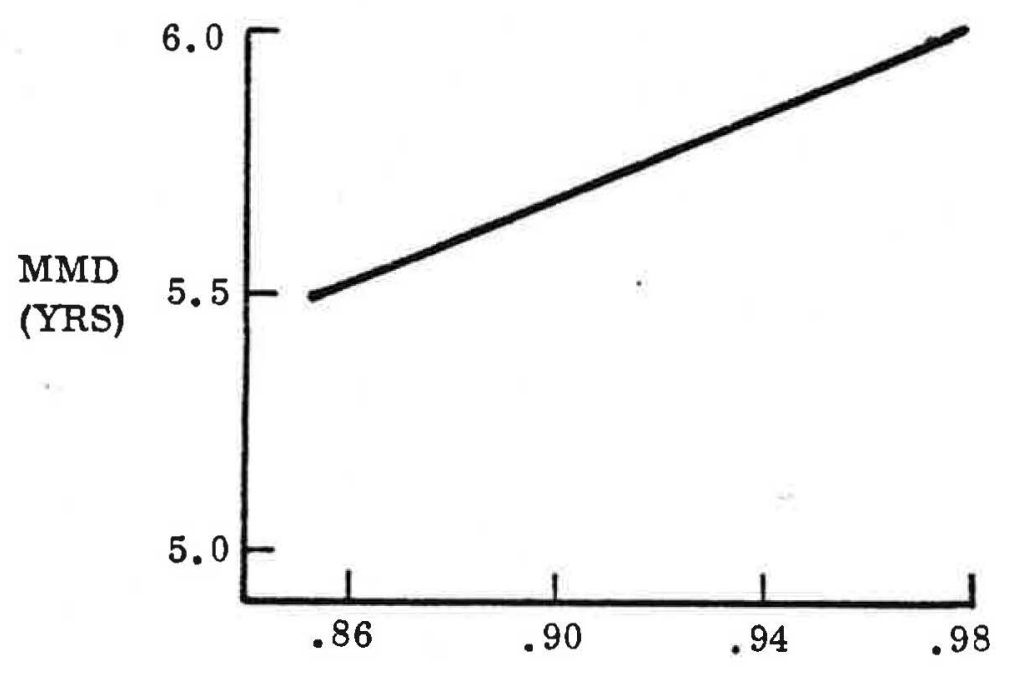

Figure 11.2-1. Variation of Spacecraft MMD with Reliability of Power System

\section{3}

\section{ORBIT PREDICTABILITY}

Three major sources of errors in spacecraft ephemerides predictions which may not be modelable, and hence correctable by extended periods of ground observation, have been evaluated. These are:

1. Errors in predicting surface temperatures.

2. Errors in alignment or pairing of jet firings during momentum unload.

3. Errors in calculating solar forces due to spacecraft misalignment (i. e. , spacecraft axis not precisely aligned with axis of rotation) .

These are the major effects which are believed to apply to the reference nuclear-powered spacecraft; not necessarily to all spacecraft in general. In particular, item 3) will apply to rotating spacecraft only. Stationary spacecraft will have a counterpart effect due to attitude control errors or control deadband.

The magnitude of these effects have been estimated, as reported in Appendix $\mathrm{J}$, and summarized in Table 11.3-1. In all cases errors in the more important (in terms of translating into user position errors) radial direction are very small, and errors in the transverse direction, also small, can probably be reduced still further. 
Table 11.3-1. Maximum Orbit Ephemerides Errors Due to Unpredicted Spacecraft Conditions

\begin{tabular}{|c|c|c|}
\hline \multirow[t]{2}{*}{ ERROR SOURCE } & \multicolumn{2}{|c|}{ ERROR RATES (ft/day) } \\
\hline & $\bar{R}$ RIRECTION & $\frac{\text { TRANSVERSE }}{\text { TO }}$ \\
\hline THERMAL EFFECTS & 0.03 & 0.30 \\
\hline $\begin{array}{l}\text { 1. PREDICTION ERROR IN TEMPERATURE OF } \\
\text { EARTH.VIEWING FACE }=10^{\circ} \mathrm{C}\end{array}$ & 0.025 & 0.25 \\
\hline $\begin{array}{l}\text { 2. PREDICTION ERROR IN TEMPERATURE OF } \\
\text { SPACE-VIEWING FACE }=2^{\circ} \mathrm{C}\end{array}$ & 0.005 & 0.05 \\
\hline $\begin{array}{l}\text { 3. MEAN TEMPERATURE DIFFERENCE ON } \\
\text { OPPOSITE SIDES OF CYLINDER DUE TO } \\
\text { HELICAL COOLING COIL (ORGANIC } \\
\text { RANKINE) }=10^{\circ} \mathrm{C}\end{array}$ & $2.8 \times 10^{-4}$ & $2.8 \times 10^{-3}$ \\
\hline UNLOAD PERTURBATIONS & 0.0132 & 1.3 \\
\hline 4. JET MISALIGNMENT $=0.5^{\circ}$, ATTITUDE ERROR $=\jmath^{\circ}$ & & \\
\hline $\begin{array}{l}\text { a. IMPULSE PERPENDICULAR TO ORBIT PLANE } \\
\text { b. IMPULSE ALONG RADIUS VECTOR }\end{array}$ & $\begin{array}{l}0.0016 \\
0.0016\end{array}$ & $\begin{array}{l}0.155 \\
0.155\end{array}$ \\
\hline 5. JET MISMATCH $=5 \%, A T T I T U D E$ ERROR $=10$ & & \\
\hline $\begin{array}{l}\text { a. IMPULSE PERPENDICULAR TO ORBIT PLANE } \\
\text { b. IMPULSE ALONG RADIUS VECTOR }\end{array}$ & $\begin{array}{l}0.005 \\
0.005\end{array}$ & $\begin{array}{l}0.5 \\
0.5\end{array}$ \\
\hline SPACECRAFT MISALIGNMENT (CONICAL ROTATION MODE) & 0.065 & 0.13 \\
\hline 6. ALIGNMENT ERROR $=0.5^{\circ}$, UNIFORM REFLECTIVITY & 0.025 & 0.05 \\
\hline 7. ALIGNMENT ERROR $=0.5^{\circ}$. NON-UNIFORM REFLECTIVITY & 0.025 & 0.05 \\
\hline 8. ALIGNMENT ERROR $=0.5^{\circ}$, SELF SHADOWING EFFECT & 0.04 & 0.08 \\
\hline SIMPLE TOTAL, ALL EFFECTS (FT./DAY) & 0.11 & 1.73 \\
\hline (M/DAY) & 0.03 & 0.53 \\
\hline
\end{tabular}

For example, the thermal effects are due mainly to the assumption that the temperature of the Earth-viewing face is not predictable to better than $10^{\circ} \mathrm{C}$. This is based largely on the fact that this is the only surface of the spacecraft which is not in intimate thermal contact with the power system coolant loop. However, it is unlikely that the thermal model will be so much in error. In addition, this adds to the desirability of using this face as a radiator extension, thus controlling its temperature much more closely. Also, unload perturbations can be reduced to absolute zero by use of of magnetic torqueing in place of jet firings. The small daily angular momentum buildup of the reference vehicle $(\sim .02 \mathrm{ft}-\mathrm{lb}-\mathrm{sec})$ makes this a possible option (Ref. Appendix H). 
Errors due to jet misalignment or mismatch are liberally estimated, resulting in the rather large attitude error which was postulated. In addition, impluses along the radius vector will be made much less frequently than those normal to the orbit plane, perhaps no more frequently than every 50-to-100 days. Therefore, the simple sum of these two errors results in a clear overestimate of the total error which may be expected from this source.

Errors due to the misestimation of solar pressure forces are likely to be the most significant errors which are encountered by most three-axis controlled spacecraft. The fact that these are so small in the reference case is due to the averaging effects of the spacecraft rotation, as detailed in Appendix J. The most significant residue of this effect is due to the variability of the self-shadowing effect of the thrust cone extension and the antennas because of spacecraft misalignment. Clearly shadowing due to the thrust cone can be minimized at very little cost by minimizing the length of this extension. Shadowing due to the antennas can be effectively eliminated by use of a radome-type cover, as was considered earlier in the study for the other design approaches. However, the magnitude of this effect is so small that the dielectric cover is judged to be unnecessary.

11.4 TEMPERATURE CONTROL OF FREQUENCY STANDARDS

The results of this analysis are discussed in Section 8. It must be noted here that these results are only preliminary. A complete multi-node transient thermal analysis which models the entire spacecraft is required before a definitive conclusion is possible. In particular, power transients as well as orbit-cycle transients will effect the temperature distribution of the spacecraft in ways which are not readily apparent in simplified models.

11.5

VULNERABILITY

The study ground rules specified that the power system only was to be evaluated for vulnerability to $\mathrm{x}$-rays and the control system to nuclear radiation.

The selection of teflon-coated silver tape as the surface thermalcontrol material would appear to provide an adequate level of hardness as shown in 
Table 11.5-1. This table, taken from the SAMSO Survivability Conference of 23 March 1977, summarizes the hardness of various materials on the basis of JCS multiples, with silvered teflon corresponding to "second-surface aluminized plastics" in physical response.

The two power systems differ somewhat in the design of their control systems. The Organic Rankine system uses discreet components in a frequency discriminator which drives a transistor comparator. This in turn generates control signals which switch parasitic load resistors in or out as required to maintain a fixed alternator speed. A check of the components used in the controller as currently designed revealed the switching transistors to be the only component of potential concern, although no hardness data on the specific devices (2N5927) could be located. In general, however, switching transistors which have been qualified to JCS standards are available and could be substituted for the specified devices.

The Brayton system uses a digital control system for greater control flexibility, which to the present time has only been designed to the subsystem block diagram level. Therefore, component checks could not be conducted.

In general, it may be concluded that the power system should be able to meet JCS survivability standards or greater with the proper selection of control system components.

Table 11.5-1. Nuclear Effects on Surfaces*

\begin{tabular}{|c|c|c|c|c|}
\hline & $\begin{array}{l}\text { SOLAR } \\
\text { CELLS }\end{array}$ & $\begin{array}{l}\text { TRANSPARENT } \\
\text { MATERIALS }\end{array}$ & $\begin{array}{c}\text { THERMAL CONTROL } \\
\text { MATERIALS }\end{array}$ & MIRRORS \\
\hline $10 x$ & HARDENED & $\begin{array}{l}\text { FUZED SILICA } \\
\text { QUARTZ }\end{array}$ & FUZED SILICA & \\
\hline \multirow{3}{*}{$\begin{array}{l}3 \times \mathrm{JCS} \\
1 \times \mathrm{JCS}\end{array}$} & HARDENED & LOW Z GLASSES & $\begin{array}{l}\text { LOW Z PAINTS } \\
\text { SECOND SURFACE } \\
\text { ALUMINIZED } \\
\text { PLASTICS }\end{array}$ & $\mathrm{Al}$ on $\mathrm{Be}$ \\
\hline & STIANDARD & GERMANIUM & HI Z PAINTS & Al on GLASS \\
\hline & STANDARD & GERMANIUM & HI Z PAINTS & Au on GLASS \\
\hline
\end{tabular}

* FROM SAMSO SURVIVABILITY CONF. OF 23 MARCH 1977 
A. SUMMARY OF GPS PROGRAM AND PHASE I SPACE VEHICLE 



\section{A.1 Navstar System Description}

The Global Positioning System (GPS) is a space-based radio positioning navigation system that will provide extremely accurate three-dimensional position and velocity information tozether with system time to suitably equipped users anywhere on or near the earth. The GPS consists of three major sezments: Space Segment, Control Sezment, and User Segment. Figures A-1, A-2, and A-3 provide a simplified overview of the GPS concept.

A functional flow diagram of the GPS system and its interfaces is given by figure A-4, (taken from figure 1 of IRN 14726). Note that the satellites are controlled by the Air Force Satellite Control Facility (AFSCF) which maintains

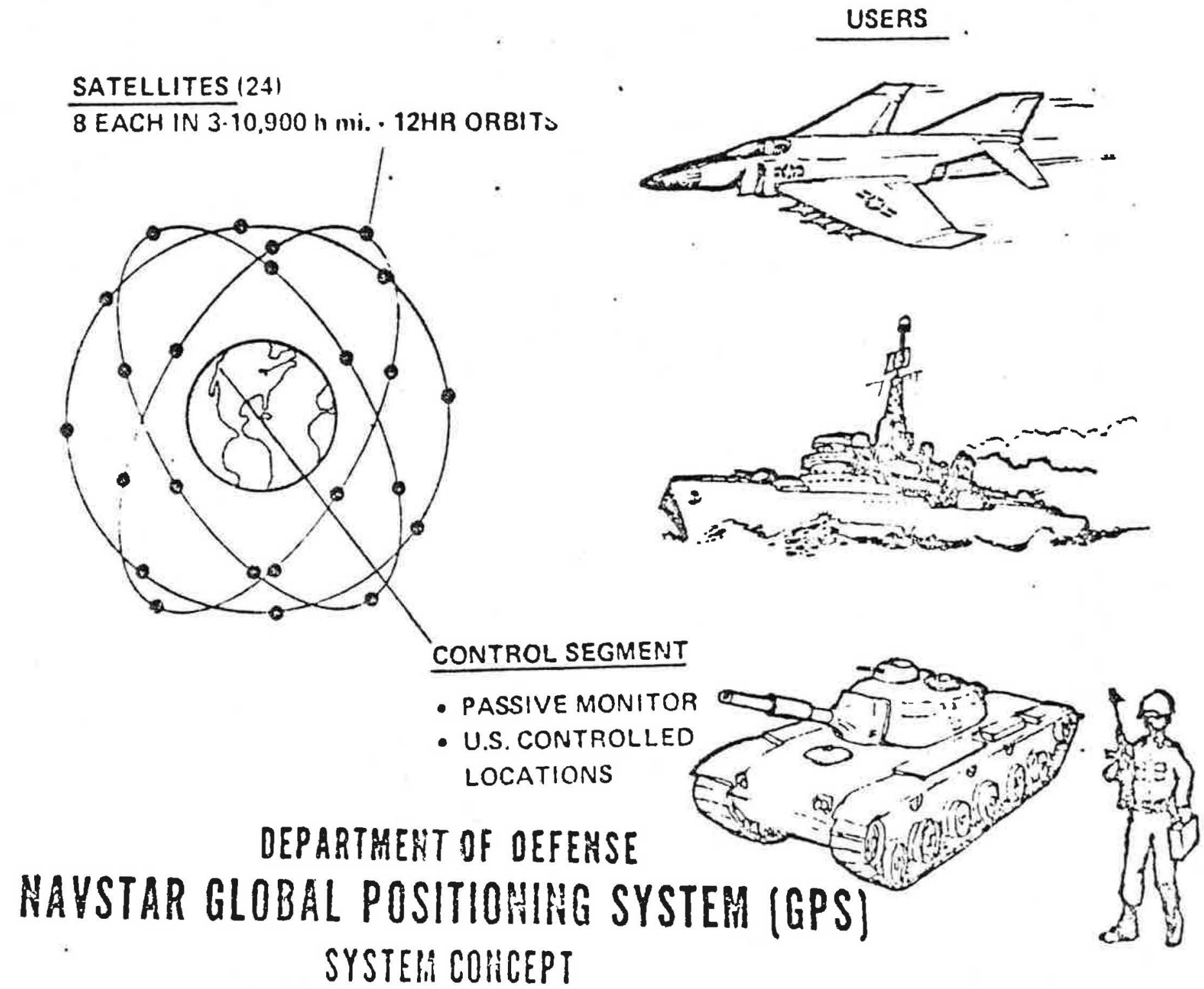

FIGURE A-1. 


\section{GLOBAL POSITIONING SYSTEM (GPS)}

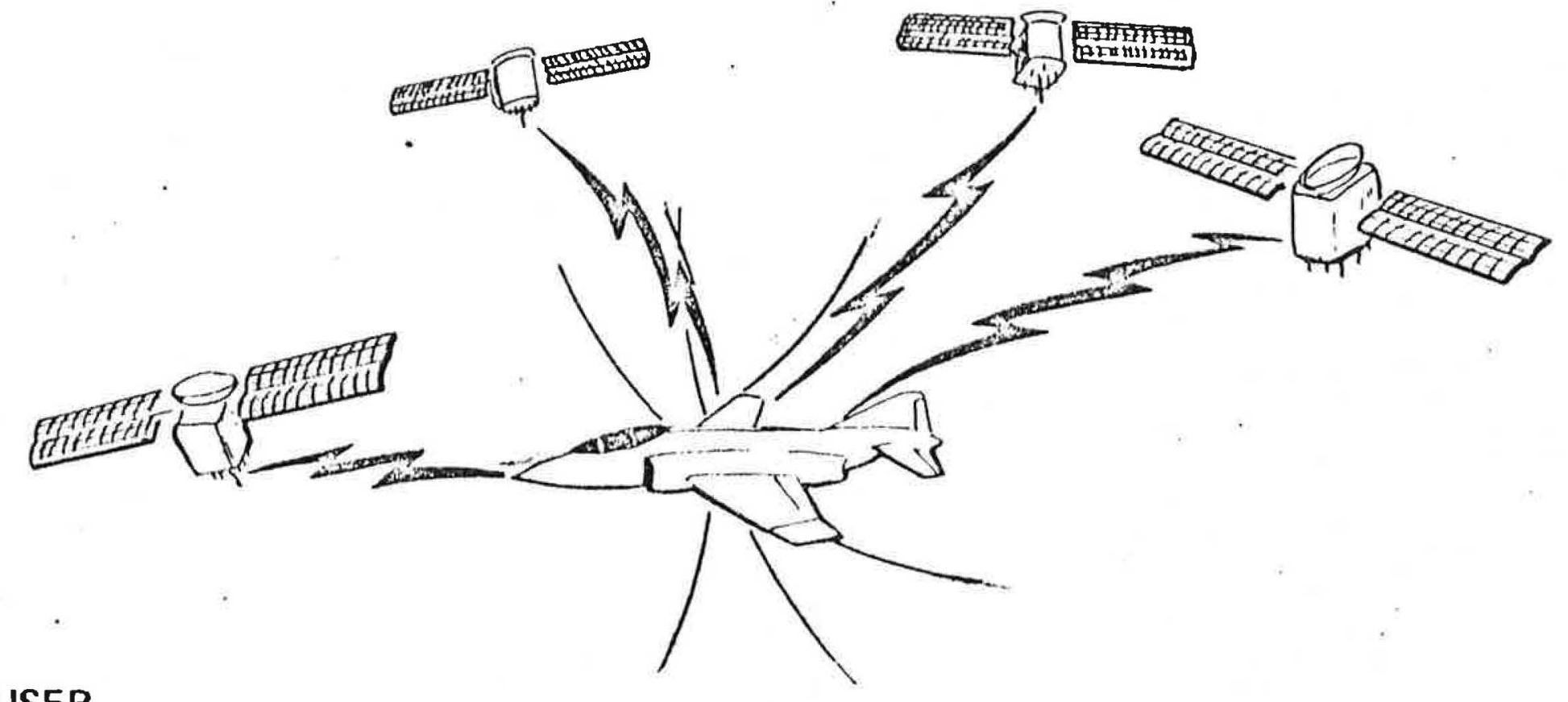

USER

- RECEIVES SYNCHRONIZED POSITIONING SIGNALS FROM SATELLITES ' (continuous, global coverage)

- COMPUTES 3 DIMENSIONAL POSITION AND VELOCITY

- OPERATES IN COMMON GRID WITH OTHER USERS 


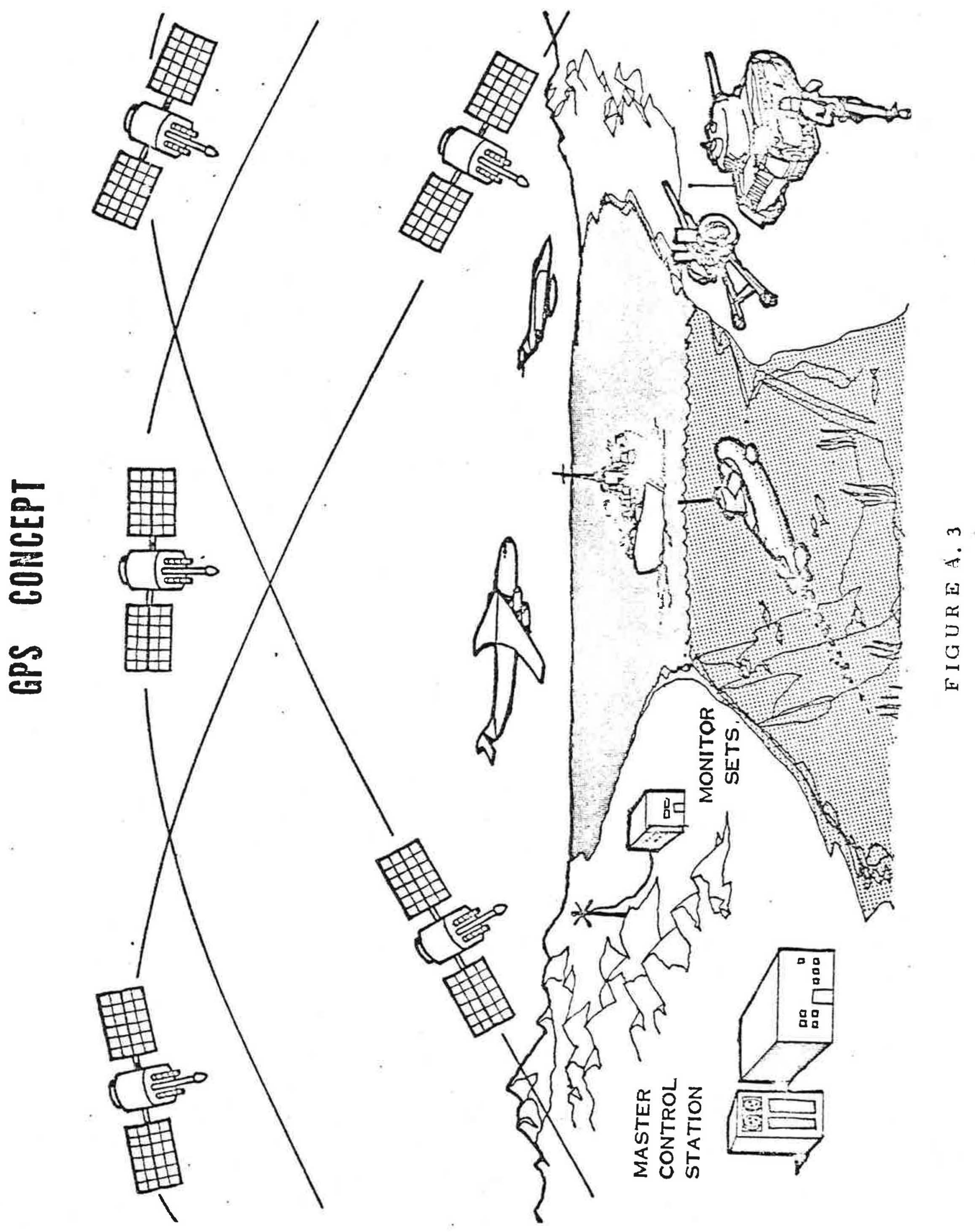




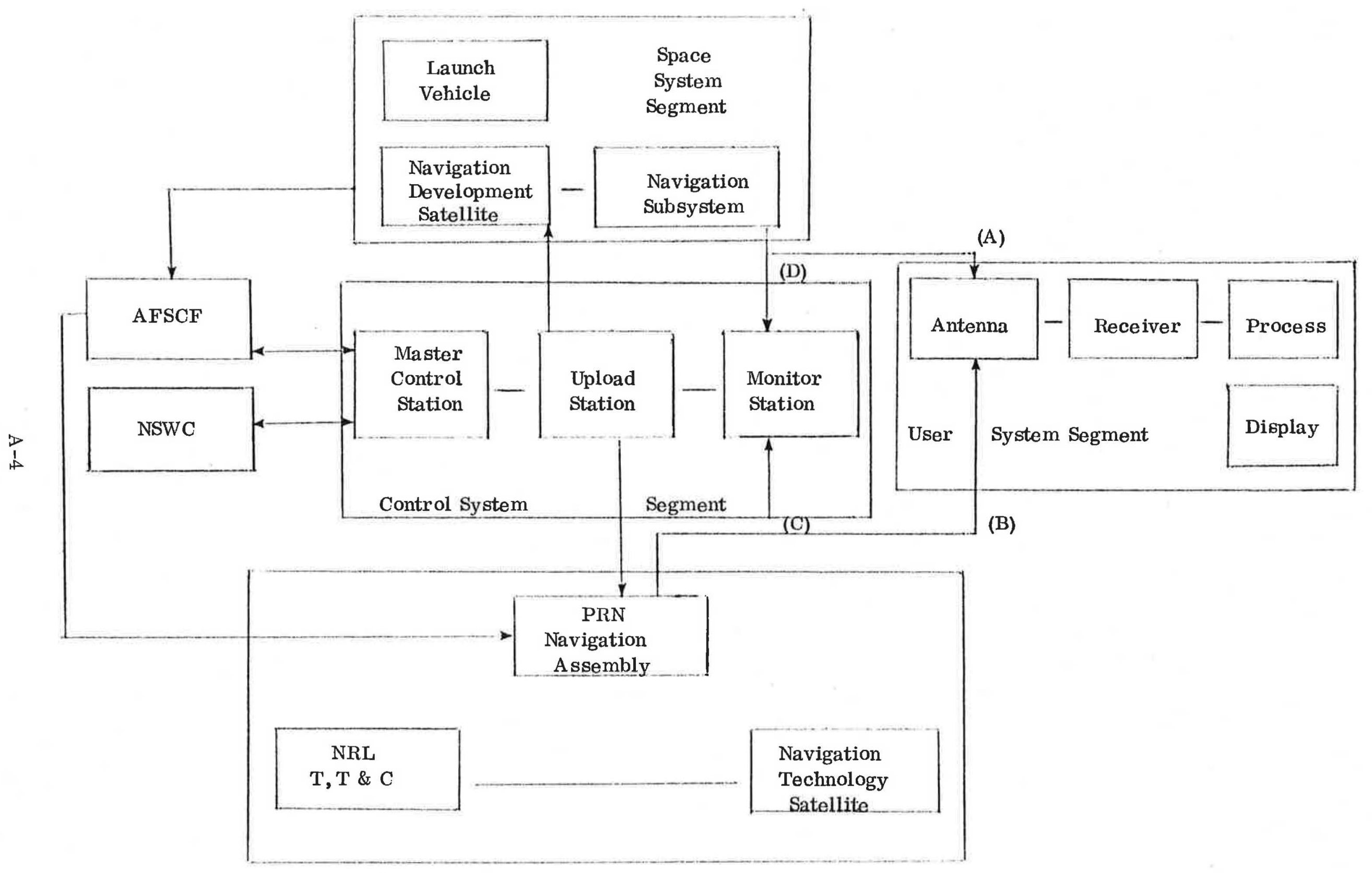

Navigation Technology Segment

FIGURE A-4 TUNCTIONAL FLOW DIAGRAM GPS :YYSTE M 
S-band telemetry and command links with each satellite in the constellation. A separate Upload Station to be located in CONUS will transmit satellite ephemerides, system time, and propagation delay data to the satellites periodically as required to update the system. A description of the NAVSTAR system, taken from sections 1.5 and 2. 2 of the Joint Program Management Plan, is attached for reference as Appendix A.

The system has been approved for Phase I implementation by DSARC I (Figure A-5). ' Phases II and III will be considered for approval by DSARC II and III as shown in Figure A-5.

\section{A.2 Space Segment}

The operational GPS will deploy satellites in circular, 10,900 nautical mile orbits inclined at approximately 63 degrees and having a twelve hour period. This deployment will provide the satellite coverage for continuous, threa-dimensional positioning and velocity determination. Each satellite will transmit a composite signal at two L-band freqruencies consisting of a protected navigation signal and a clear acquisition navigation signal. The signals contain navigation data such as satellite ephemeris, atmospheric propagation correction data, and satellite clock bias information. Using the L-ioand navigation signals permits the user to determine the iozospheric group delay or other electro magnetic disturbances in the atmosphere which may affect the transmitted signals.

The user is required to effectively synchronize his clook with system time through a combination of physical and computation corrections. Users with very precise clocks could range to three different space vehicles. However, to avoid requiring each user to be equipped with a very precise clock, an additional indepdendent range measurement is utilized. Thus, measurement data from four space vehicles will be used to compute the user's three-dimensional position and system time (see Figure A-6). In addition, the user measures doppler shift of the four indepdendent navigation signals to determine his threedimensional velocity and the drift of his clock. When more than four (4) satellites are within the user's line of sight, the user will select the optimum subset o: four satellites to ase in his position determination. Some of the criteria that may be used to select this subset are:

\footnotetext{
a. Relationships of satellites to user to insure optimum geometry.

b. Minimum iozospheric and tropospheric delay uncertainty.

c. Remaining view time of space vehicle.
} 


\section{NAVSTAR GLOBAL POSITIONING SYSTEM SCHEDULE}

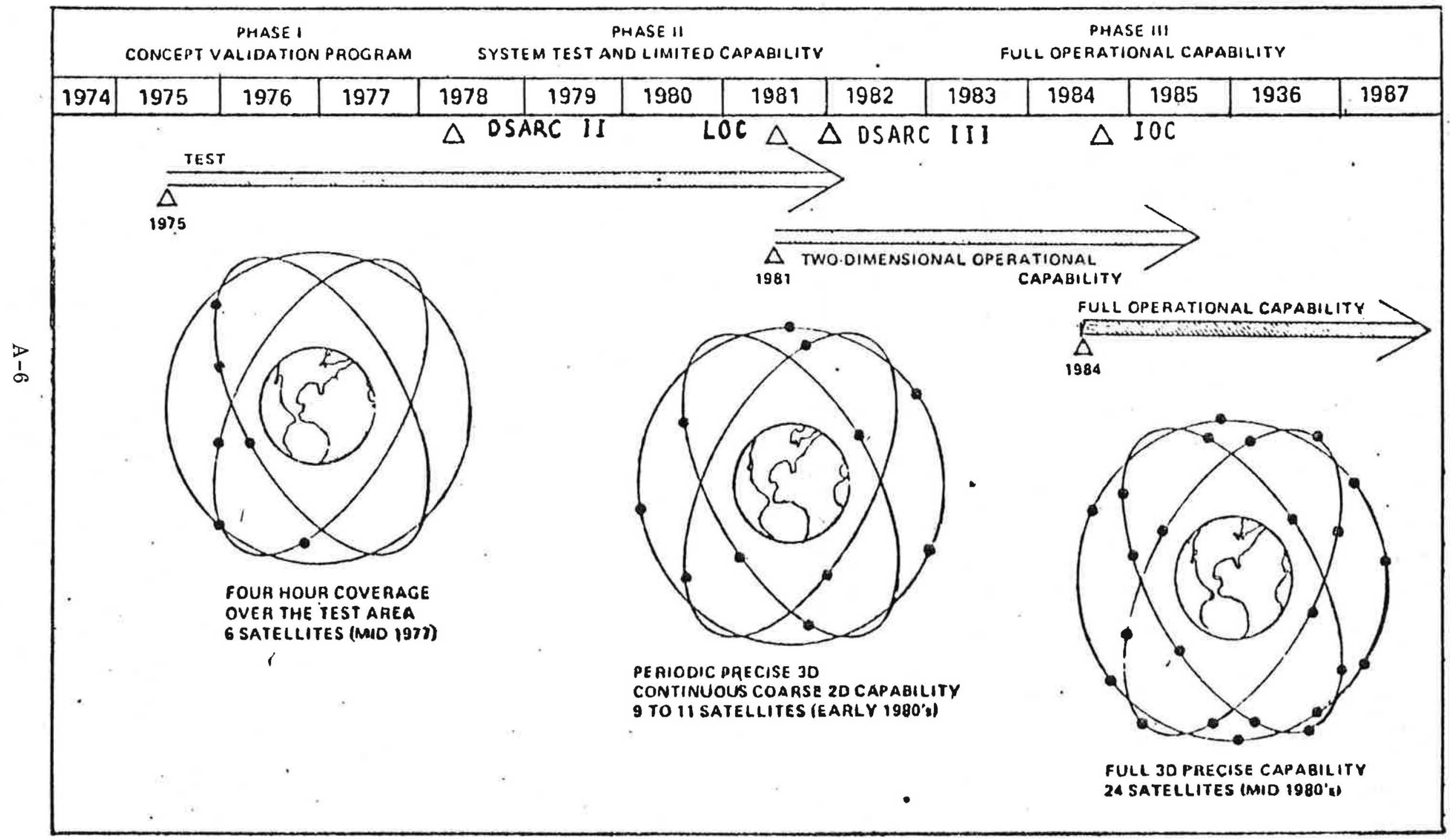

FIGURE A-5 


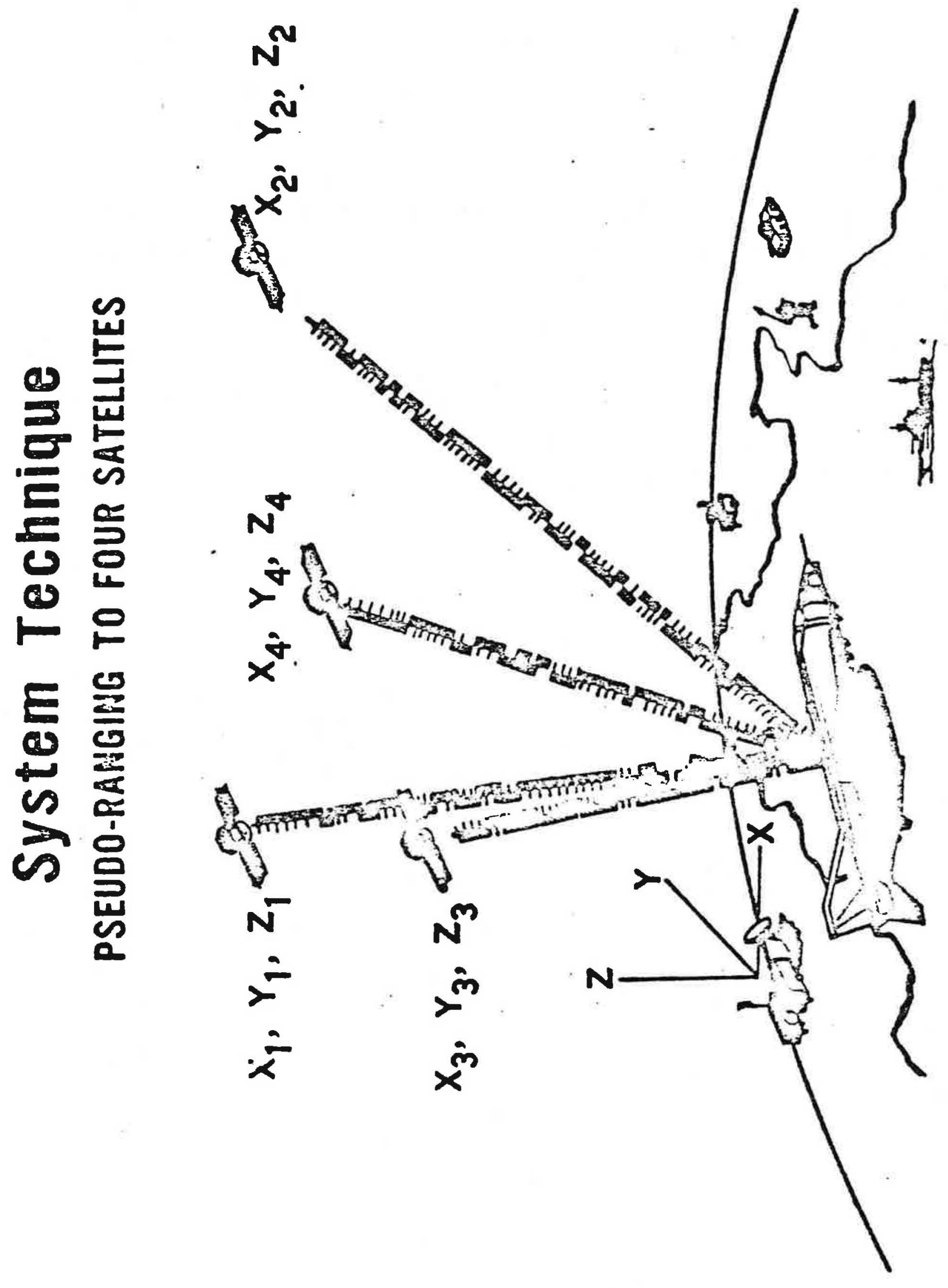




\section{A.3 Control Sezment}

Four widely separated Mozitor Sets (MS), located on U.S. territory, will passively track all satellites in view, and accumulate ranging data from the navigation signals. This information will be processed at a Master Control Station (MCS), to be located in the Central Continental United States for use in satellite orbit determination and systematic error elimination. The oroit determination process derives prozressively refined information defining the gravitational field and solar pressure parameters influencing the satellite, the clock drift of the satellite, the electronic delay characteristics of the signal, the location of the ground station, and other observable system influences.

An Upload Station (ULS), co-located with the Central Continental United States MCS, will transmit the satellite ephemerides, clock drifts, and propagation delay data to the satellites as required.

\section{A.4 User Sezment}

Using the navigation signal from each of four satellites, the user's receiver will measure four independent pseudo-ranges and pseudo-range rates to the satellites. The user's receiver/processor will then convert these pseudo-range and pseudo-range rates to three-dimensional position, velocity, and system time. This position soluticn is in World Geodetic System - 72 coordinates, an earth-centered, earth fixed coordinate system, which can be converted to any coordinate frame in units of measure the user requires.

\section{A.5 Concept}

The GPS is a space-based radio navigation system that will provide GPSequippped users with the capability to precisely determine their three-dimensional position, velocity, and time. Each GPS space vehicle (SV) will generate and transmit two GPS Navigation signals. Also, each GPS user receiver will generate replicas of the desired set of SV generated navigation signals. When the user's signal matches in time with the received SV signal, a measurement of signal trans mit time and user clock asynchronization can be made. All signals eminating from the GPS space vehicles will be synchronized by the GPS Coztrol Segment. 


\section{A.6 GPS Performance}

The user's single position fix accuracy is a function of the user's position relative to the GPS SV constellation. The relationship between the user's navigation accuracy and his relative position to the SV constellation is referred to as Geometric Dilution of Precision (GDOP). User Equivalent Range Error (UERE) is defined as the uncorrelated portion of the o.bserved user range error. Thus, the user's actual navigational accuracy is obtained by multiplying the UERE by the GDOP for the user's position. During Phase I with primarily six SVs available, the user's single position fix navigation accuracy will be approximately three times the UERE (average GDOP of 3). The components of UERE for Phase III and Phase I of the GPS are shown in Table A.1. The Space Vehicle Group Delay components of the Phase I Error Budget will be applicable for two hours after all SVs are updated. All other components of the Error Budget will be applicable for twenty-four hours after all SVs are updated. All values are one sigma $(1 \sigma)$ errors given in meters.

\begin{tabular}{|c|c|c|}
\hline \multicolumn{3}{|c|}{$\begin{array}{l}\text { TABIE A.1 } \\
\text { GPS ERROR BUDGET }\end{array}$} \\
\hline & Phase III & Phase I \\
\hline Space Vehicle Ephemeris & 1.6 & 3.9 \\
\hline Atomospheric Delay & 2.6 to $5.6 *$ & 2.6 to $5.6 *$ \\
\hline Space Vehicle Group Delay & 1 & 2.8 \\
\hline Receiver Noise & 1.6 & 1.6 \\
\hline Multipath & $\underline{1.3 \text { to } 3.0 *}$ & 1.3 to $3.0 *$ \\
\hline Tołal R.S.S. & 3.9 to 5.9 & 5.9 to 8.2 \\
\hline \multicolumn{3}{|c|}{$\begin{array}{l}* \text { These error quantities represent the use of different atmospheric delay correction methods } \\
\text { and different user environmental conditions (values in meters). }\end{array}$} \\
\hline
\end{tabular}

\section{A. $7 \quad$ GPS Error Analysis}

\section{Ephemeris}

Space vehicle ephemeris error is defined as the difference in actual satellite 
ephemeris and the satellite ephemeris computed by the user from the data provided in the Navigation Signal data frame. Since the GPS satellites will be in 12-hour oroits, the effect of satellite in-track and cross-track ephemeris errors will be reduced. For example, a three foot ephemeris in-track error projects to a one foot error in range. Thus, the primary error so'xrce that will effect the user's position determination is satellite radial position error. In addition, ephemeris errors common to the four range measurements cause a user clock calibration error, rather than a user position error because the user clock asynchronization is common to all four measurements.

The Naval Surface Weapons Center's (NSWC) operational experience with orbit determination for Transit satellites (600 nmi altitude) has produced the following conclusions:

a. Observed versus predicted values in the orbit determination process check within $2 \mathrm{~m}$ (rms).

b. Extremely accurate laser tracking by NASA of Transit satellites agrees with NSWC ephemerides within 1-2 m (rms).

c. Long baseline interferometry experiments conducted by MIT agree with Transmit/Geoceiver surveys to within $1 \mathrm{~m}$ (rms).

Based upon NSWC's experience and the more favorable geometry of GPS satellites, there should be low risk in achieving the satellite ephemeris accuracies specified in the error budget.

\section{Propagation Errors}

Atmospheric delay error is defined as the difference between actual signal propagation delay through the ionsphere and troposphere and the delay determined by the user employing suitable ionosphere and troposphere models. Since the ionospheric delay is a function of frequency, two coherent signals can be used to calibrate the delay. In this dual frequency calibration mode, the ionospheric delay uncertainty is a function of the difference in noise in the receiver channels. The variation with frequency is shown in Figure A-7. For GPS, the ranging error is at most three times the interchannel noise. Interchannel noise can be minimized if a single measuring circuit is time-shared between channels. A 
FREQUENCY DEPENDENCE

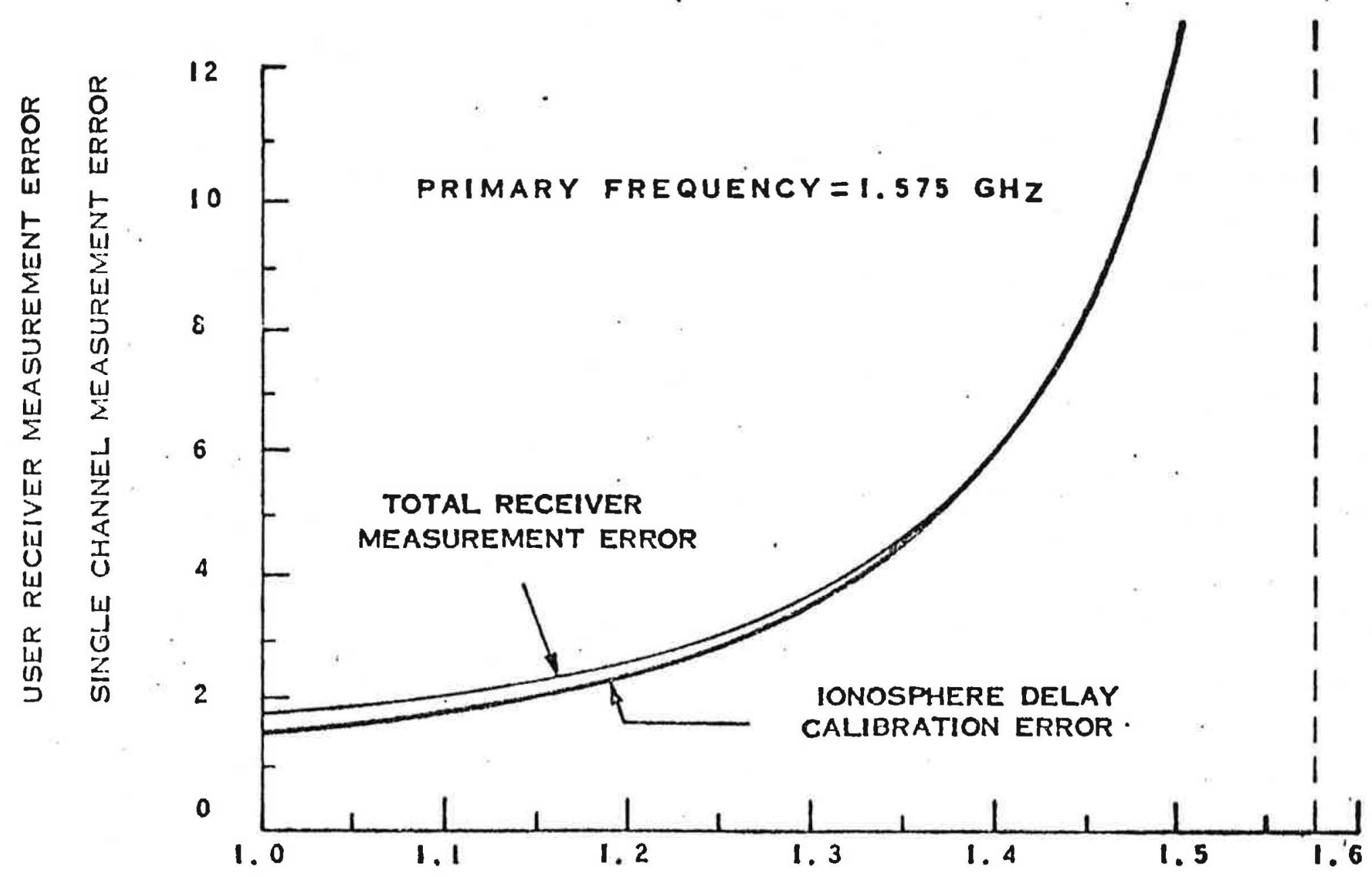

SECONDARY FREQUENCY. GHZ

USER RECEIVER MEASUREMENT ERROR

FIGURE $A-7$ 
sophisticated modeling process can produce an equivalent error. It is currently anticipated that users demanding the best GPS accuracy will use dual frequency calibration. A less sophisticated ionospheric model will be available for medium accuracy users. The tropospheric propagation delay model will be contained in the user software. A conservative estimate fora user's tropospheric model requiring no external data indicates a vertical uncertainty of 1 meter $(1 \sigma)$ with satellites at the zenith increasing to $2 \frac{1}{2}$ meters $(1 \sigma)$ for a satellite at 95-deg. elevation angle.

\section{Space Vehicle Delays}

Space vehicle group delay error is defined as the sum of delay uncertainty in the SV; such as unmodeled clock drift and uncalibrated delay in signal equipment. The satellite groxp delay is undistinguishable from clock errors and will be accounted for in the clock calibration process. Immediately after an orbit determination solution, the satellite slock uncertainties will be the same for each satellite and so highly correlated between satellites there is little or very slight effect on the user. In Phase I, after two hours, the magnitude of the accumulated clock drift will be approximately 7 nanoseconds ( 7 feet using a $1 \times 10^{-12}$ clock in each satellite).

\section{User Errors}

Receiver noise and resolution errors are the contributions of noise and measurement errors in the user navigation receiver hardware and software which perturb the correct navigation solution. The User Equipment Definition and Experiments test program at White Sands Missile Range demonstrated that a receiver noise level of five feet is conservative and obtainable.

Multipath error is the effect of several propagation paths from the SV to the user which corrupt the measurement of line-of-sight distance. Operational environments may cause multipath arrivals that are comparable in amplitude to the line-of-sight signal. However, the GPS receiver searches for the first signal to arrive and not the most powerful signal. Therefore, the likelihood of tracking a multipath signal that is delayed more than $1 \frac{1}{2}$ code chips $(\approx 19 \mathrm{~m}$ for $\mathrm{P}$ code) is drastically reduced. If the time of arrival is sufficiently 
close to that of the line-of-sight signal (less than $11 / 2$ chips), the user receiver will not easily be able to discriminate between the signals. The magnitude of the resulting ranging error will depend strongly on the location and nature of reflecting surfaces in the user environment.

A-8 Signal Structure

The primary navigation signal of the GPS is a composite waveform consisting of a Precision (P) Signal and a Clear Acquisition (C/A) Signal transmitted in phase quadrature. The $\mathrm{P}$ signal is used by the precision military user and will resist jamming, spoofing, and multipath and will be deniable to unauthorized users by employing nonlinear encoding. The C/A signal will serve as an aid to the acquisition of the P Signal, and will also provide a navigation signal in the clear to both the military and civil user. This signal may also employ System techniques to deny the high accuracy of this signal to unauthorized users.

The navigation signals are transmitted on two channels: L 1 and L2. Channel L1, the Primary Navigation Signal, is nominally centered at $1575 \mathrm{MHz}$ and shall carry both the P and the C/A Signals. Channel L 2, the Secondary Navigation Signal, is nominally centered at $1230 \mathrm{MHz}$ and shall carry the P Signal or C/A Signal. On the L 2 channel, either $\mathrm{P}$ or $\mathrm{C} / \mathrm{A}$ can be selected through a telemetry controlled switch.

Both the $\mathrm{P}$ and the $\mathrm{C} / \mathrm{A}$ Signals are pseudorandom noise bi-phase shift keyed (PN/BPSK) continuous sinusoidal carriers. Both signals carry system data. The chipping rates of the PN codes are 10.23 Mbps and $1023 \mathrm{kbps}$ for the P and the C/A Signals respectively. The system data rate is 50 bps. Each Space Vehicle is assigned a unique set of PN codes. The P code repeats each seven (7) days and the C/A code repeats each millisecond. This waveform is specifically designed to allow system time to be conveniently and directly extracted in terms of standard units of days, hours, minutes, and interger multiples and submultiples of the second. 
The system data contains information which allows the user to navigate successfully with GPS. It will provide space vehicle ephemerides, system time-of-week, space vehicle clock behavior data, system status messages, and C/A to P signal handover information. The data stream will be common to both the $\mathrm{P}$ and the C/A Signal on both L 1 and $\mathrm{L} 2$.

The P Signal is a continuous sinusoidal carrier, bi-phase modulated according to the modulo-2 sum of a PN code and a synchronous data bit stream. The P codes will be generated such that no space vehicle shall duplicate the output of any other space vehicle before seven (7) days have elapsed in system time. The period of the code will be sufficiently long (267 days) to accommodate 37 different initial code phases, each displaced by a minimum of seven $(7)$ days $(i=1,2 \ldots . .37)$. The same basic code generator will be used by all GPS P Signal transmitters with each one assigned one of the 37 possible unique code phases.

The C/A Signal is a continuous sinusoidal carrier, bi-phase modulated according to the modulo-2 sum of a 1023 bit Gold code sequence and a synchronous data bit stream. All of the 36 legitimate codes within the 1023 bit Gold code family will be used for each C/A signal transmitter. The codes will be selected to have low envelope cross-correlation with any other code in the Gold family for all possible shifts of the code and doppler conditions.

\section{A-9 $\quad$ GPS Design Goals}

Tables A.2, A. 3, and A. 4 provide design goals for the GPS user equipment for Phase I, Phase II, and Phase III, respectively. These goals are subject to designto-cost trade-offs and other considerations.

$\mathrm{A}-10$

SPACE SEGMENT; NDS DATE.

$\mathrm{A}-10.1$

REQUIREMENTS

The GPS Space System Segment/User Systems Segment interfaces are defined in Rockwell Document IRN 14726 and in the NAVSTAR GPS Joint 
TABLE A. 2 PHASE 1 DESIGN GOALS FOR NAVSTAR/GPS

\begin{tabular}{|c|c|c|c|c|c|c|c|c|c|}
\hline \multirow{2}{*}{\multicolumn{2}{|c|}{ Desizr. Porameter: }} & A & B & C & D & $\mathbf{E}$ & $\mathbf{F}$ & $\begin{array}{c}\text { G/M } \\
\text { (No Dev) }\end{array}$ & $\begin{array}{c}\text { s } \\
\text { (No Dev) }\end{array}$ \\
\hline & & $\begin{array}{c}\text { High } \\
\text { Performance } \\
\text { Aircraft } \\
\text { w'Iligh AJ }\end{array}$ & $\begin{array}{c}\text { High } \\
\text { Performarice } \\
\text { Airctaf: }\end{array}$ & $\begin{array}{l}\text { lyission } \\
\text { Support } \\
\text { Veticites }\end{array}$ & $\begin{array}{l}\text { Land and } \\
\text { Sea Vehicles } \\
\text { W High AJ }\end{array}$ & $\begin{array}{c}\text { Man } \\
\text { Backpack }\end{array}$ & Submarir:e & $\begin{array}{l}\text { Space } \\
\text { Missile }\end{array}$ & $\begin{array}{l}\text { Spartan, } \\
\text { Very-Low } \\
\text { Cost }\end{array}$ \\
\hline \multirow{5}{*}{ 冚 } & $\begin{array}{ll}11 ! \quad x \\
\quad \\
y\end{array}$ & $6.9 \mathrm{~m}$ & $6.9 \mathrm{~m}$ & $15.20 \mathrm{~m}$ & $18.25 \mathrm{~m}$ & $18.25 \mathrm{~m}$ & $6-9 \mathrm{~m}$ & 16 & $\begin{array}{c}30-100 \mathrm{~m} \\
\text { (cep) }\end{array}$ \\
\hline & $\begin{aligned} 121 \quad & i \\
& \vdots \\
& i\end{aligned}$ & $0.1 \mathrm{kt}$ & $0.1 \mathrm{kt}$ & $0.5 \mathrm{kt}$ & So req & No req & $0.1 \mathrm{kt}$ & $.012 \mathrm{~m} ; \mathrm{sec}$ & No req \\
\hline & Time & $18-25 \mathrm{nsec}$ & $18.25 \mathrm{nsec}$ & 18.25 riser & $\begin{array}{l}1 \mathrm{sec} \\
\text { displayed }\end{array}$ & $\begin{array}{l}1 \mathrm{sec} \\
\text { displayed }\end{array}$ & $18-25$ osec & TBD & $\begin{array}{l}1 \mathrm{sec} \\
\text { displayed }\end{array}$ \\
\hline & ${ }^{31}$ Time-to-first- & $80.180 \mathrm{sec}$ & $80.180 \mathrm{sec}$ & $200.300 \mathrm{sec}$ & $100 \mathrm{sec}$ & $100 \mathrm{sec}$ & $80.100 \mathrm{sec}$ & $400 \mathrm{sec}$ & $600 \mathrm{sec}$ \\
\hline & $(4)_{N J}$ margin & $\begin{array}{l}P-54 \\
C: A 34\end{array}$ & $\begin{array}{l}\text { P }-54 \\
\text { C.A } 34\end{array}$ & $\begin{array}{l}\text { P.NA } \\
C+34\end{array}$ & $\begin{array}{l}P-44 \\
C+34\end{array}$ & $\begin{array}{l}P-44 \\
\text { C/A } 34\end{array}$ & $\begin{array}{l}p-44 \\
\text { cis } 34\end{array}$ & $\begin{array}{r}(5)_{29.8} \\
29.8\end{array}$ & $\begin{array}{l}\text { P.NA } \\
\text { C/A TBD }\end{array}$ \\
\hline \multicolumn{2}{|r|}{ Size } & $.2 \mathbf{m}^{\mathbf{3}}$ & $.2 \mathrm{~m}^{3}$ & (i) $11 \mathrm{~m}^{3}$ & $.018^{3}$ & $.018 \mathrm{~m}^{3}$ & $.2 \mathrm{~m}^{3}$ & $.2 \mathrm{~m}^{3}$ & $.04 \mathrm{~m}^{3}$ \\
\hline \multicolumn{2}{|r|}{ Height } & .56 .1 & $56.1 \mathrm{k!}$ & $22.5 \mathrm{k}_{6}$ & $10.5 \mathrm{~kg}$ & $10.5 \mathrm{~kg}$ & 56.1 & . $30 \mathrm{~kg}$ & $18 \mathrm{~kg}$ \\
\hline \multicolumn{2}{|r|}{ Power } & $632 \mathrm{w}$ & $632 \mathrm{~W}$ & $151 w$ & $30 \mathrm{w}$ & $30 w$ & $632 w$ & $80 w$ & $30 w$ \\
\hline \multicolumn{2}{|r|}{ MI'BF } & 600 & 500 & 500 & 500 & 500 & 500 & TBD & TBD \\
\hline \multicolumn{2}{|r|}{ DTC } & 'IBD & THD & 525.010 & TBI) & TBD & TBD & TBD & TBD \\
\hline & $\begin{array}{l}(11) \\
121\end{array}$ & (3) & $\begin{array}{l}\text { taneuus } \\
- \text { Coc }\end{array}$ & $\begin{array}{l}\text { tions of Tabl } \\
\text { racking }\end{array}$ & $s-(15-101-11$ & $\begin{array}{ll}\text { (b) C/I } \\
\text { (6) Des }\end{array}$ & $-11 z$ & $197.1 \mathrm{dol}$ & 14 units) \\
\hline
\end{tabular}


Table A. 3 Phase II 5 s!gn Goals for NAVSTAR /GPS

Pericalc 3-D

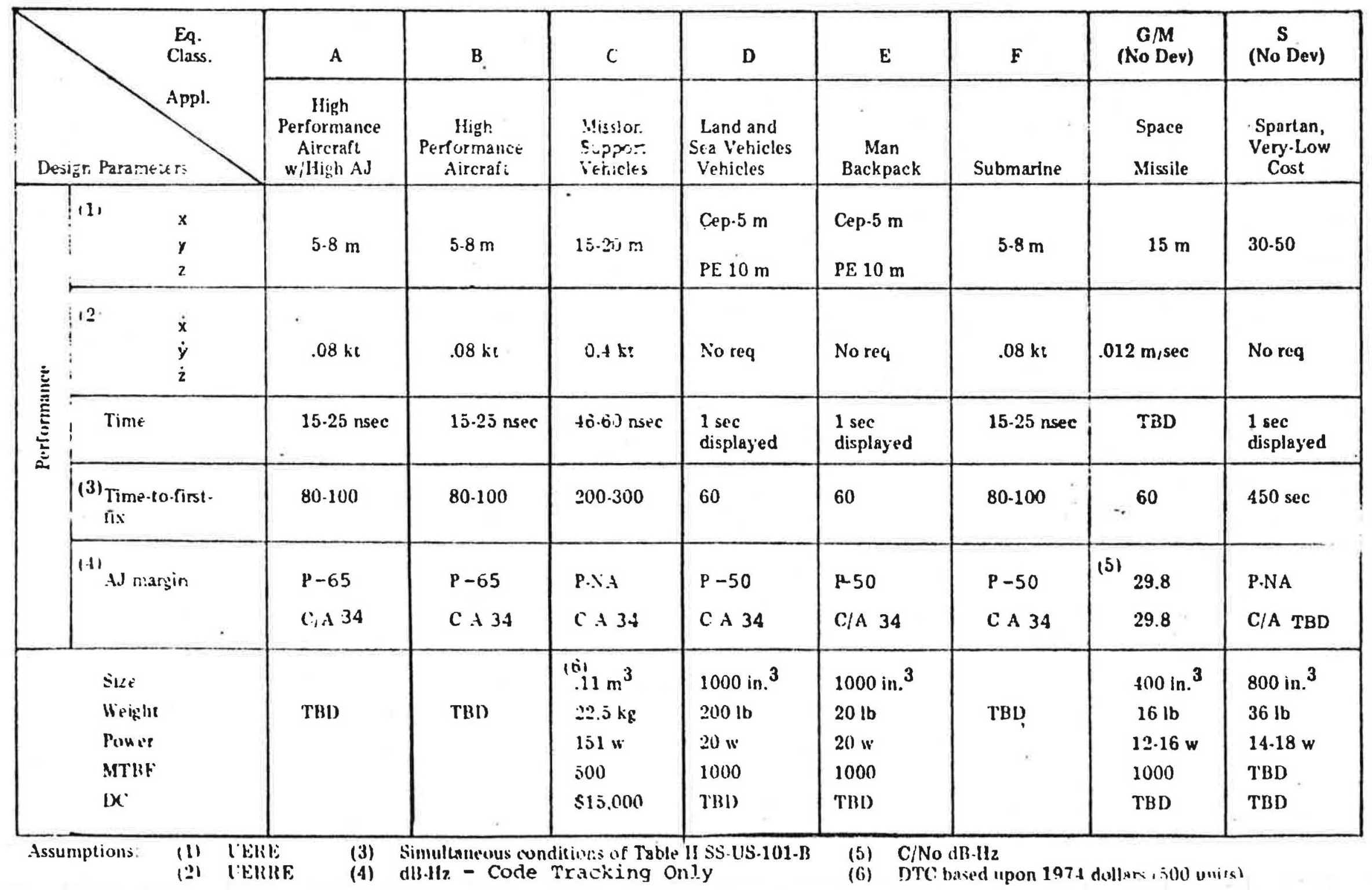


Table A.4 Ptase III Design Goals for NAVSTAR/Gg

World-Wide 3-D

\begin{tabular}{|c|c|c|c|c|c|c|c|c|c|}
\hline \multirow{2}{*}{\multicolumn{2}{|c|}{ Design Paramelers }} & A & B & C & D & $\mathbf{E}$ & $\mathbf{F}$ & $\begin{array}{c}G / M \\
\text { (No Dev) }\end{array}$ & $\begin{array}{c}\text { S } \\
\text { (No Dev) }\end{array}$ \\
\hline & & $\begin{array}{c}\text { High } \\
\text { Performance } \\
\text { Aircraft } \\
\text { w!ligh AJ }\end{array}$ & $\begin{array}{l}\text { High } \\
\text { Performarce } \\
\text { Aircrals }\end{array}$ & $\begin{array}{l}\text { Mission } \\
\text { Support } \\
\text { Vehicles }\end{array}$ & $\begin{array}{l}\text { Land and } \\
\text { Sea Vehicles } \\
\text { w/lligh AJ }\end{array}$ & $\begin{array}{c}\text { Man } \\
\text { Backpack }\end{array}$ & Submarine & $\begin{array}{l}\text { Space } \\
\text { Missile }\end{array}$ & $\begin{array}{l}\text { Spartan, } \\
\text { Very-Low } \\
\text { Cost }\end{array}$ \\
\hline \multirow{5}{*}{ 宸 } & $\begin{array}{l}\text { (1) } \\
y \\
z\end{array}$ & $4.7 \mathrm{~m}$ & 4-7 m & $15.20 \mathrm{~m}$ & $\begin{array}{l}3 \text { m Cep } \\
3 \text { m PE }\end{array}$ & $\begin{array}{l}3 \mathrm{~m} \mathrm{Cep} \\
3 \mathrm{mPE}\end{array}$ & $4.7 \mathrm{~m}$ & $10 \mathrm{~m}$ & $30 \mathrm{~m}$ \\
\hline & $(2)$ & $.05 \mathrm{kt}$ & $0.5 \mathrm{kl}$ & $.2 \mathrm{kt}$ & No req & No req & $.05 \mathrm{kt}$ & $: 012 \mathrm{~m} / \mathrm{sec}$ & No req \\
\hline & Time & 12.21 nsec & $12.21 \mathrm{nsec}$ & $\$ 5.60$ nsec & $\begin{array}{l}1 \text { sec } \\
\text { displajed }\end{array}$ & $\begin{array}{l}1 \mathrm{sec} \\
\text { displayed }\end{array}$ & 12.21 nsec & TBD & $\begin{array}{l}1 \mathrm{sec} \\
\text { displayed }\end{array}$ \\
\hline & $\begin{array}{l}\text { (3) Time-to-first } \\
\text { fix }\end{array}$ & $80-180$ & 80.180 & $200-300$ & 30 & 30 & $80-180$ & 60 & 300 \\
\hline & If'AJ margin & $\begin{array}{l}P-74 \\
\text { C:A } 34\end{array}$ & $\begin{array}{l}p-74 \\
c+34\end{array}$ & $\begin{array}{l}\text { P.A.A } \\
\text { CA } 34\end{array}$ & $\begin{array}{l}p-58 \\
\text { C/A } 34\end{array}$ & $\begin{array}{l}p-58 \\
c_{i}+34\end{array}$ & $\begin{array}{l}\text { P-58 } \\
\text { C.A } 34\end{array}$ & (5) $\begin{array}{r}29.8 \\
29.8\end{array}$ & $\begin{array}{l}\text { P.NA } \\
\text { C:A TBD }\end{array}$ \\
\hline & $\begin{array}{l}\text { Siza } \\
\text { Height } \\
\text { luuer } \\
\text { MTBF } \\
\text { D'TC }\end{array}$ & $\begin{array}{l}\text { THL) } \\
\text { THD } \\
\text { 'IHL } \\
\text { 'IHL) } \\
\text { 'IUD }\end{array}$ & $\begin{array}{l}\text { TBD } \\
\text { TBD } \\
\text { TBD } \\
1200 \\
\text { TKD }\end{array}$ & $\begin{array}{l}\text { (6) } \\
\text { THD } \\
1200 \\
\$ 15,000\end{array}$ & $\begin{array}{l}500 \mathrm{in.}^{3} \\
8.12 \mathrm{lb} \\
10 \mathrm{w} \\
2000 \\
\mathrm{TBD}\end{array}$ & $\begin{array}{l}500 \mathrm{in}^{3} \\
8.12 \mathrm{lb} \\
10 \mathrm{w} \\
2000 \\
\mathrm{IBD}\end{array}$ & $\begin{array}{l}\text { TBD } \\
\text { TBD } \\
\text { TBD } \\
12110 \\
\text { I'BD }\end{array}$ & $\begin{array}{l}250 \mathrm{in.}^{3} \\
10 \mathrm{lb} \\
8.12 \mathrm{w} \\
2000\end{array}$ & $\begin{array}{l}800 \text { in. }^{3} \\
\text { TBCD } \\
\text { TBD } \\
\text { TBD }\end{array}$ \\
\hline & $\begin{array}{l}\text { (1) } \\
\text { (2) }\end{array}$ & $\begin{array}{l}(3) \\
(4)\end{array}$ & $\begin{array}{l}\text { muleancerus st } \\
\text { 3.11. - Cod }\end{array}$ & $\begin{array}{l}\text { ims of Tul } \\
\text { cacking }\end{array}$ & 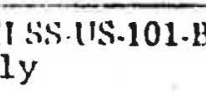 & $\begin{array}{l}\text { (5) } \\
\text { (i) }\end{array}$ & $\begin{array}{l}\text { No dlB-Hz } \\
\text { Tic based up }\end{array}$ & 1974 dollars ( & units: \\
\hline
\end{tabular}


Program Management Plan. The key satellite performance requirements as they relate to the objectives of this study are:

- earth coverage right hand circularly polarized (RCP) beams at two frequencies: L-1 and L-2 (1575. $42 \mathrm{MHz}$ and 1227. $6 \mathrm{MHz}$ ).

- generation of pseudo-random-noise (PRN) phase-modulated protected $(\mathrm{P})$ signals and clear acquisition (C/A) signals which are BPSK (bi-phase shift key) modulated in phase quadrature with separate PRN codes.

- direct sequence spectrum spreading at chipping rates of 10.23 Mbps for the P signals and 1.023 Mbps for the C/A signals.

- $\quad$ code repetition periods of 7 days for the $P$ signals and 1 millisecond for the $\mathrm{C} / \mathrm{A}$ signals

- frame lengths of 1500 bits

- operating modes in accordance with the following table Table A-5. Transmitter Operating Modes

\begin{tabular}{|c|c|c|c|c|}
\hline \multirow[b]{2}{*}{ Mode } & \multicolumn{2}{|c|}{$\mathrm{L}_{1 \text { Link }}$} & \multicolumn{2}{|c|}{$\mathrm{L}_{2}$ Link } \\
\hline & $P$ & $\mathrm{C} / \mathrm{A}$ & $\mathbf{P}$ & $\mathrm{C} / \mathrm{A}$ \\
\hline $\begin{array}{c}1 \\
2 \\
3 \\
4 \\
5 \\
6 \\
7 \\
8 \\
\text { Standby }\end{array}$ & $\begin{array}{l}\text { Normal } \\
\text { Normal } \\
\text { Normal } \\
\text { Normal } \\
\text { Normal } \\
\text { Normal } \\
\text { Note } 3 \\
\text { Note } 3 \\
\text { Note } 3\end{array}$ & $\begin{array}{l}\text { Normal } \\
\text { Normal } \\
\text { High } \\
\text { High } \\
\text { Normal } \\
\text { High } \\
\text { Note } 3 \\
\text { Note } 3 \\
\text { Note 3 }\end{array}$ & $\begin{array}{l}\text { Normal } \\
\text { Note } 4 \\
\text { Normal } \\
\text { Note } 4 \\
\text { Note } 3 \\
\text { Note } 3 \\
\text { Normal } \\
\text { Note } 4 \\
\text { Note } 3\end{array}$ & $\begin{array}{l}\text { Note } 4 \\
\text { Normal } \\
\text { Note } 4 \\
\text { Normal } \\
\text { Note 3 } \\
\text { Note } 3 \\
\text { Note } 4 \\
\text { Normal } \\
\text { Note } 3\end{array}$ \\
\hline \multicolumn{5}{|c|}{$\begin{array}{l}\text { Notes: 1. High power will be on the NDS space vehicle only. } \\
\text { 2. Mode control shall be effected through the TT\&C system. } \\
\text { 3. No RF carrier is transmitted } \\
\text { 4. Modulation not present. } \\
\text { 5. Modes } 7 \text { and } 8 \text { are for ground checkout. }\end{array}$} \\
\hline
\end{tabular}


- Minimum user carrier power levels of $-163 \mathrm{dBW}$ for $\mathrm{L} 1-\mathrm{P}$, $-160 \mathrm{dBW}$ for L-1 C/A (Normal), $-158 \mathrm{dBW}$ for L-1 C/A (high power), and $-166 \mathrm{dBW}$ for $\mathrm{L}-2 \mathrm{P}$ or $\mathrm{C} / \mathrm{A}$.

- Maintenance of phase coherence between all signals within 5 nanoseconds (one sigma)

- Maintenance of equipment group delay variation (uncertainty) within 1.5 nanoseconds (one sigma) for either L-1 or L-2 and maintenance for either L-1 or L-2 and maintenance of differential group delay between L-1 and L-2 within 1.5 nanoseconds (one sigma).

- Provision of a highly accurate frequency standard adjustable in steps no larger than $4 \times 10^{-12} \Delta \mathrm{f} / \mathrm{f}$ over a range of $\pm 2 \times 10^{-9} \Delta \mathrm{f} / \mathrm{f}$ around a nominal output frequency of $10.23 \mathrm{MHz}$ (10.22999999545 $\mathrm{MHz} \pm 10^{11}$ adjusted for relativistic effects) with a frequency drift not to exceed one part in $10^{12}$ per day.

- Provision of navigation signal data at 50 bps containing, as a minimum, space vehicle ephimerides, system time, space vehicle and clock behavior data, transmitter status information and $\mathrm{C} / \mathrm{A}$ to $\mathrm{P}$ signal handover information. The data stream shall be common to both the $\mathrm{P}$ and $\mathrm{C} / \mathrm{A}$ signals on both the L1 and L2 links.

- Maintenance of space vehicle attitude with the $+\mathrm{Z}$ axis pointed toward earth center with an accuracy of $\pm 0.5^{\circ}$ except during momentum dump when the accuracy may be $\pm 1.0^{\circ}$.

The major navigation signal is carried by the L-1 link. The L-2 link is provided at a different frequency so that users requiring greater precision can compare the two signals to correct for atmospheric propagation errors. 
External and internal interfaces for the GPS Space Vehicle (SV) are defined in the Prime Item Development Specification (CID-SV-101A) volumes 1 through 11). The SV subsystems are identified as shown by figure $A-8$ (block diagram) and, for the NDS, by figure A-9 (exploded view). External space vehicle interfaces are defined by figure $\mathbf{A}-10$ and internal interfaces are defined by figure $A-I I$.

The key interfaces are described briefly below.

A-11 Navigation Subsystem

The navigation subsystem RF signal characteristics are given by table A. 6 and a (non-redundant) block diagram of the navigation subsystem is given by figure A-12 The LCU (Load Control Unit) and DC/DC Converter are part of the electrical power subsystem (EPS). Nominal DC current and voltage requirements and nominal DC power requirements are given by table A. 7. The antenna gain for the $\mathrm{I}-1$ band varies from $+12.2 \mathrm{~dB}$ on-axis $\left(\theta=0^{\circ}\right)$ to $+13.7 \mathrm{~dB}$ off-axis $\left(\theta=14.3^{\circ}\right)$. Corresponding gains for the L-2 band are +9.7 and +11.3 respectively. Diplexer losses can be inferred from the power levels shown on the block diagram.

It should be noted that the high-power mode specified in table 2.1-1, and many of the different mode combinations requested, are a result of a desire to use more power at the beginning of mission life, before solar array degradation has limited the available power, and a further desire to apportion the reduced power subsequently available according to priority.

Other mission requirements which have been identified for particular emphasis in this study lie in the areas of mean-mission duration, orbital stability, spacecraft vulnerability and the thermal stability of on-board clocks. Mean-mission duration for the phase I navigation development satellite (NDS) is currently estimated at 4.54 years against a minimum requirement of 5 years. Orbital stability, as reflected by the predictability of the space vehicle ephemerides has one-sigma error 


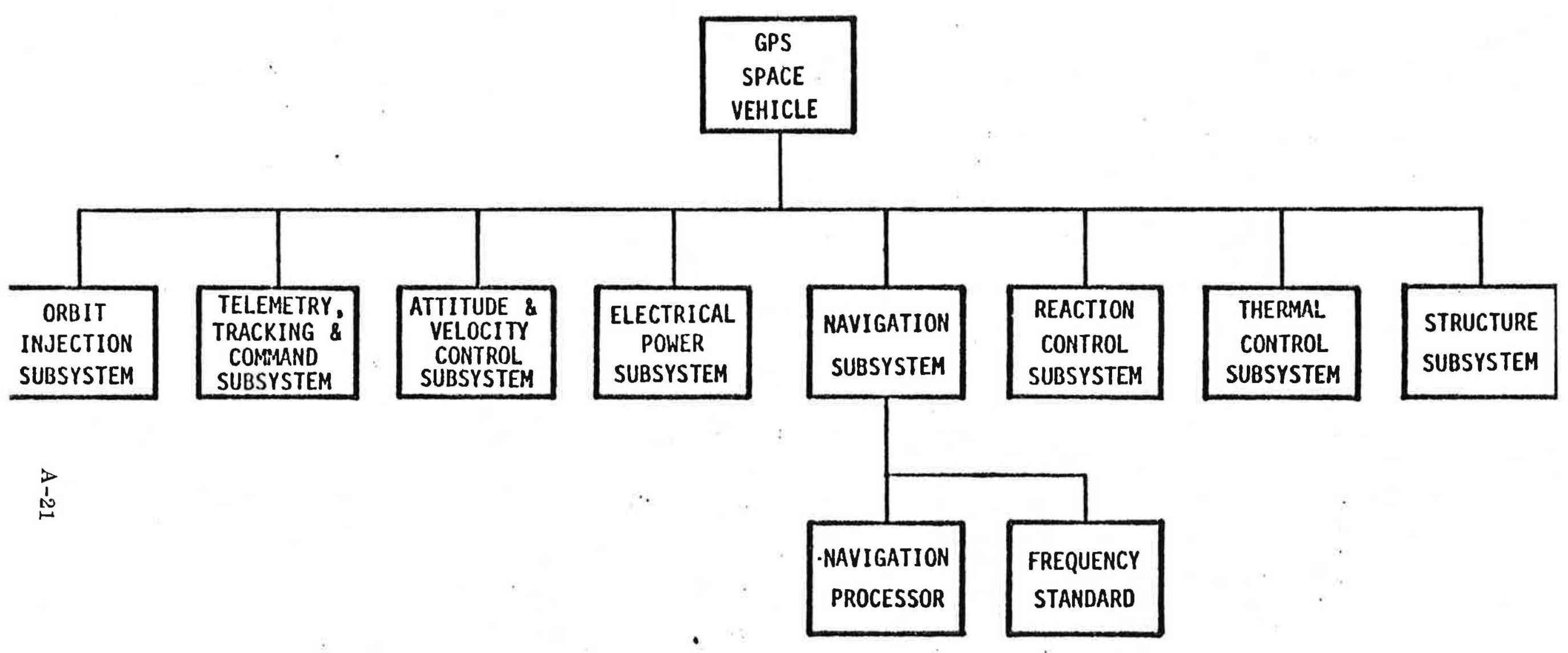

FIGURE A-8. SV SUBSYSTEM DESCRIPTION 


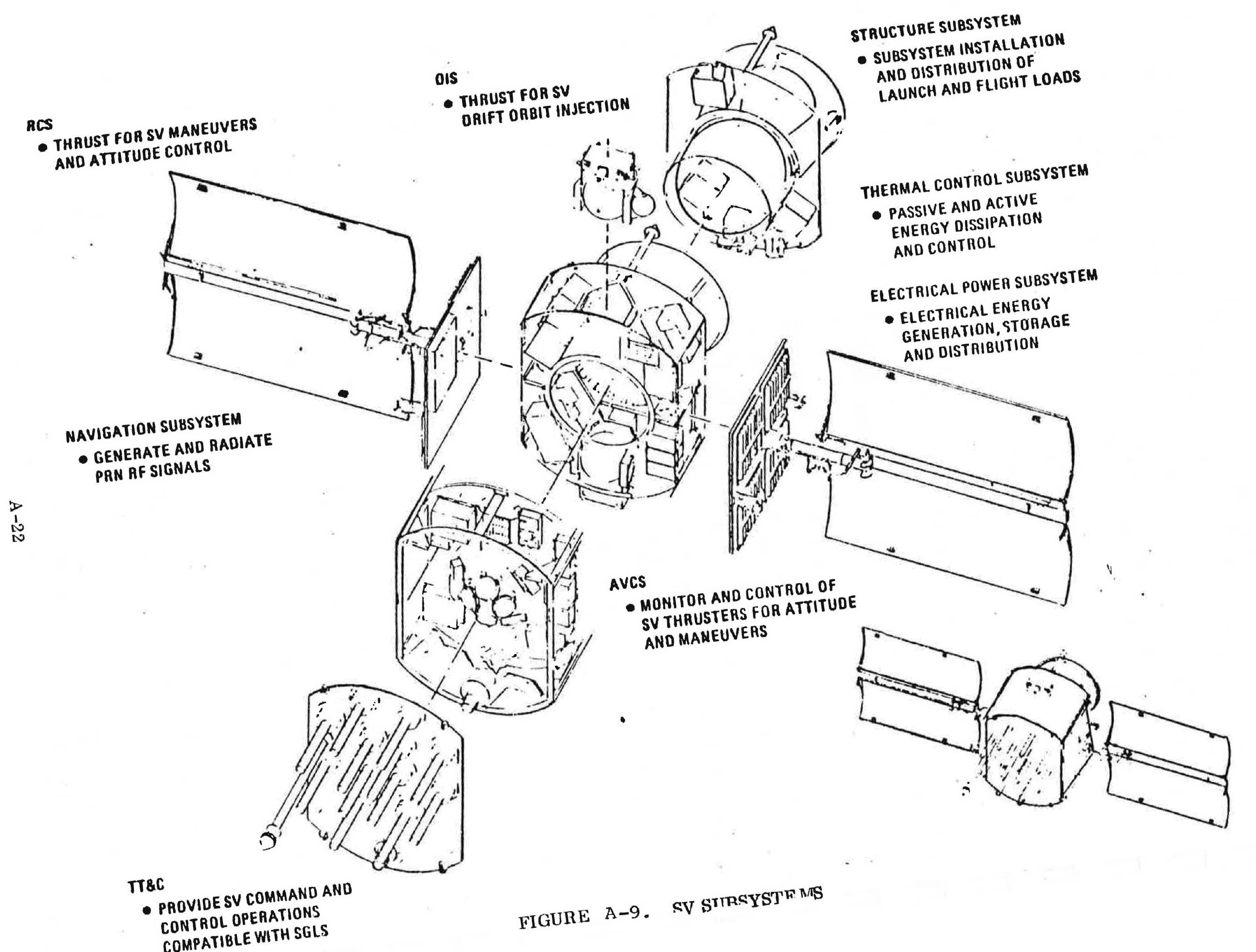




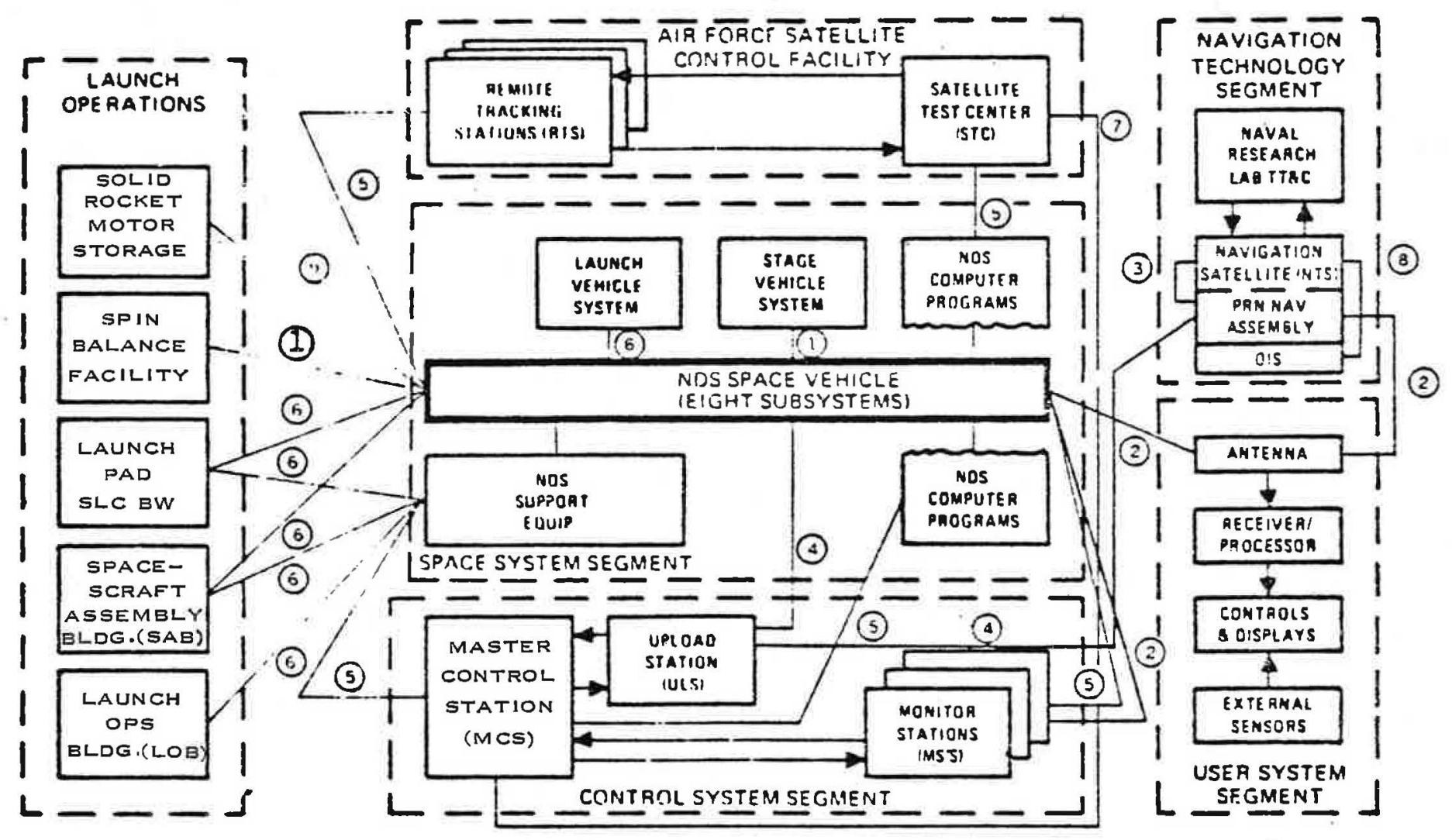

NUMBER

(1) $160309 \cdot 60.2002$.

(2) ICD $M H 08-00002-400$

(3) ICO $\mathrm{HH} 08.00003-400$

(4) ICD MHO8-00004-400

(5) ICD мно8-00006-400

(b) ICD TBO

(7) SD 75-GP-0007

(8) ICD MOH-00009-400

(9) (PRD)
IITLE

MAVSTAR GLOBAL POSITIONING SYSTEM

NDS SPACE VEHICLE/STAGE VEHICLE

SPACE VEHICLE NAV SUBSYSTEM AND NTS PRN NAV ASSY/USER ANO MONITOR SYSTEM SEGMENTS

PRN NAV ASSY/NTS PHYSICALAND FUNCTIONAL REQUIREMENTS

SPACE VEHICLE NAV SUBSYSTEM AND NTS PRN NAV ASSY/UPLOAD STATION

SIMULATORS/CONTROL AND USER SYSTEM SEGMENTS AND AFSCF

BOOSTER-FAIRING/STAGE VEHICLE AND SPACE

VEHICIE/SITE AGE

AFSCF/MCS COMPUTER PROGRAMS

ORBIT INJECTION SYSTEM/NTS PHYSICAL AND FUNCTIONAL REQUIAEMENTS

PROGRAM REQUIRENENTS DOCUMENT 


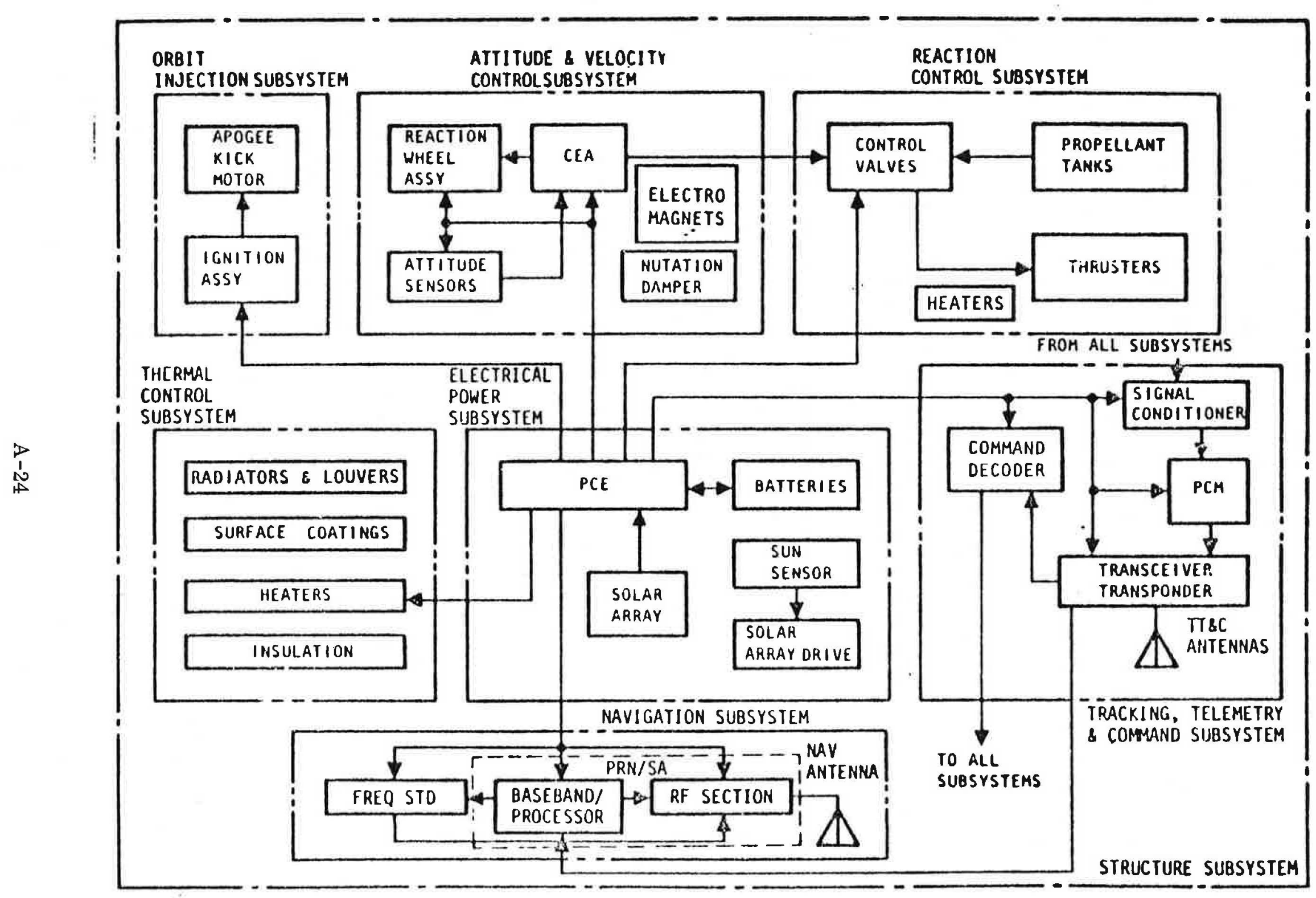

FIGURE A-11. INTERNAL SV INTERFACES 
TABLE A. 6.

NAV SUBSYSTEM RF SIGNAL CHARACTERISTICS

\begin{tabular}{|c|c|c|}
\hline Parameter & $\mathrm{L}_{1} \diamond$ & $\mathrm{L}_{2}$ \\
\hline Channel Allocation & $20.46 \mathrm{MHz}$ & $20.46 \mathrm{MHz}$ \\
\hline Nominal carrier frequency & $1575.42 \mathrm{MHz}\left(f_{1}\right)$ & $1227.6 \mathrm{MHz}\left(f_{2}\right)$ \\
\hline Carrler frequency accuracy & 2 part in $10^{9}$ & 2 part in $10^{9}$ \\
\hline $\begin{array}{l}\text { Minimum EIRP at f }+10.23 \mathrm{MHz} \text { to } \\
\text { end of life (Including eclipge } \\
\text { operations) }\end{array}$ & $\begin{array}{l}P \quad+23.8 \mathrm{dBW} \\
C / A+26.8 \mathrm{dBW}\end{array}$ & $\begin{array}{l}P \quad+19.1 \mathrm{dBW} \\
\mathrm{C} / \mathrm{A}+19.1 \mathrm{dBW}\end{array}$ \\
\hline $\begin{array}{l}\text { Minlmum EIRP at } \mathrm{f}_{0} \pm 10.23 \mathrm{MHz} \text { for } \\
\text { h1gh power mode }\end{array}$ & $\begin{array}{l}P \quad+23.8 \mathrm{dBW} \\
\mathrm{C} / \mathrm{A}+28.8 \mathrm{dBW}\end{array}$ & $\begin{array}{l}P \quad+19.1 \mathrm{dBW} \\
\mathrm{C} / \mathrm{A}+19.1 \mathrm{dBW}\end{array}$ \\
\hline Antenna polarization & $\begin{array}{l}\text { RIght hand circular } \\
\text { polarization }\end{array}$ & $\begin{array}{l}\text { Right hand circular } \\
\text { polarization }\end{array}$ \\
\hline $\begin{array}{l}\text { Maximum axtal ratio over the } \\
\text { band }\end{array}$ & $0.8 \mathrm{~dB}$ & $1.5 \mathrm{~dB}$ \\
\hline $\begin{array}{l}\text { Phase nolse measured by a P.L.L. } \\
\text { receiver with } 10 \mathrm{~Hz} \text { single sided } \\
\text { loop bandwidth. }\end{array}$ & $100 \mathrm{mr}$ & $100 \mathrm{mr}$ \\
\hline
\end{tabular}

objectives of $3.9 \mathrm{~m}$ for Phase I and $1.6 \mathrm{~m}$ for Phase II (Reference section 2.2.2 of the Joint Program Management Plan.) The spacecraft vulnerability objective is to meet JCS standards with the Phase III spacecraft. Thermal stability of the on-board clocl:s impose a temperature-change requirement for the mounting plate of the clocks of $<4$ degrees/day and $<5$ degrees/hour. Current phase I satellites achieve \pm 2.5 degrees/ day; however, even tighter control is thought to be desirable. 


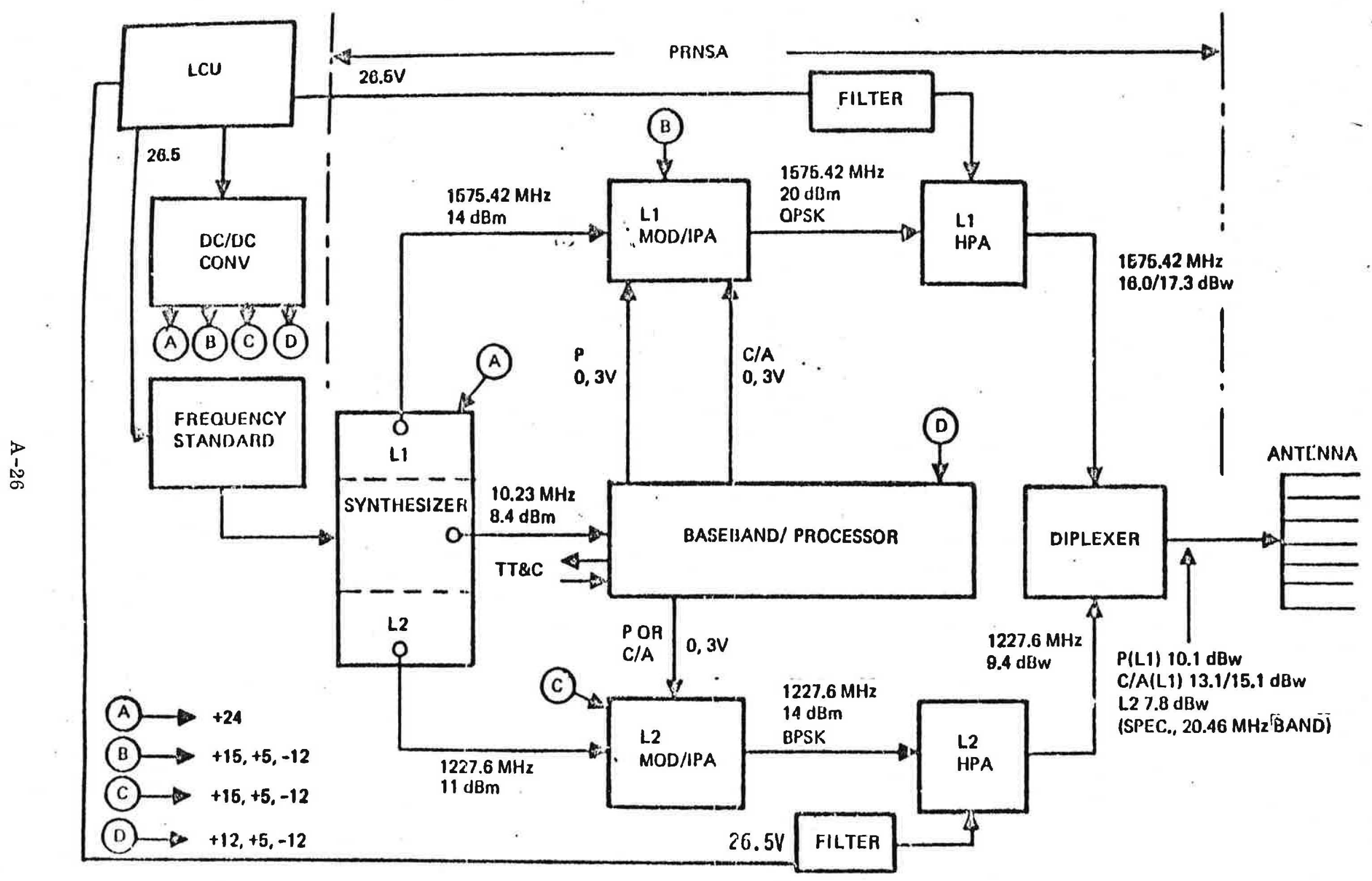

FIGURE A-12. NAGIVA TION SUBSYSTEM PRNSA BLOCK DIAGRAM 
(a) Nominal DC Voltage/Current Requirements

\begin{tabular}{|c|c|c|c|c|c|c|c|c|}
\hline \multirow[b]{2}{*}{ Units (I) } & \multirow[b]{2}{*}{$\begin{array}{l}\text { Bus } \\
+26.5 \mathrm{~V}\end{array}$} & \multicolumn{5}{|c|}{ Regulated Voltages ${ }^{(2)}$} & \multirow[b]{2}{*}{$\begin{array}{l}\text { Power } \\
\text { Leads }\end{array}$} & \multirow[b]{2}{*}{ Remarks } \\
\hline & & $+24 \mathrm{~V}$ & $+15 \mathrm{~V}$ & $+12 \mathrm{~V}$ & $+5 \mathrm{~V}$ & $-12 \mathrm{~V}$ & & \\
\hline $\begin{aligned} I_{1} \text { Band HPA } & (\text { Nam) } \\
& \text { (INCR) }\end{aligned}$ & $\begin{array}{l}4.64 \\
2.44\end{array}$ & & & & & & 2 & $\begin{array}{l}\text { (3) } \\
\text { (3) }\end{array}$ \\
\hline$I_{2}$ Band HPA & $\begin{array}{l}0.85 \\
0.90\end{array}$ & & & & & & 2 & \\
\hline $\mathrm{L}_{I}$ Band MOD/IPA & & & $0.14 \mathrm{~A}$ & 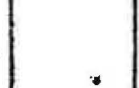 & $0.12 \mathrm{~A}$ & $0.15 \mathrm{~A}$ & 6 & Group B* \\
\hline$I_{2}$ Band MOD/IPA & & & $0.05 \mathrm{~A}$ & & $0.045 \mathrm{~A}$ & $0.09 \mathrm{~A}$ & 6 & Group C* \\
\hline $\mathrm{L}_{1} / \mathrm{L}_{2}$ Synthesizer & & $0.44 \mathrm{~A}$ & & & & & 2 & Group A* \\
\hline PRN Baseband/Processor & & & & $0.21 \mathrm{~A}$ & $2.74 \mathrm{~A}$ & $0.4 \mathrm{~A}$ & 8 & Group D* \\
\hline Frequency Standard & $0.93 \mathrm{~A}$ & & & & & & 3 & (4) \\
\hline
\end{tabular}

NOTES: * Selectable DC/DC Converter Primary and Back-up groups to each NAV Subsystem unit

(1) See Paragraph 3.1.3

(2) DC/DC Converter (Specified in Yolume 4)

(3) Selectable Primary and Back-up Input to each unit

(4) Selectable input to each of 3 units

(b) Nominal DC Power Requirements (Watts)

\begin{tabular}{|c|c|c|c|c|c|c|c|}
\hline \multirow[b]{2}{*}{ Unfts $^{(1)}$} & \multicolumn{6}{|c|}{ Operating Modes (2) } & \multirow[b]{2}{*}{ Stdby ${ }^{(3)}$} \\
\hline & 1 & 2 & 3 & 4 & 5 & 6 & \\
\hline$L_{1}$ Band HPA & 123.0 & 123.0 & 187.6 & 187.6 & 123.0 & 187.6 & - \\
\hline$L_{2}$ Band HPA & 23.8 & 23.8 & 23.8 & 23.8 & -- & - & -- \\
\hline $\mathrm{L}_{1}$ Band MOD/IPA & 3.4 & 3.4 & 3.4 & 3.4 & 3.4 & 3.4 & -- \\
\hline $\mathrm{L}_{2}$ Band MOD/IPA & 1.6 & 1.6 & 1.6 & 1.6 & - & -- & - \\
\hline $\mathrm{L}_{1} / \mathrm{L}_{2}$ Synthes1zer & 10.6 & 10.6 & 10.6 & 10.6 & 10.6 & 10.6 & - \\
\hline PRN Baseband/Processor & 21.0 & 21.0 & 21.0 & 21.0 & 21.0 & 21.0 & 21.0 \\
\hline Frequency Standard & 24.5 & 24.5 & 24.5 & 24.5 & 24.5 & 24.5 & 24.5 \\
\hline
\end{tabular}

NOTES: (1) See Paragraph 3.1.3

(2) See Paragraph 3.2.1.2

(3) See Paragraph 3.2.1.2 
A-11.1 Electrical Power Subsystem

The primary EPS interface definitions are shown in Figure $A-13$ and the nominal electrical load budget is given by table A.7, Electrical power for vehicle loads is supplied from the main bus at $27 \pm 1$ volt $d . c$. The voltage at the load interfaces will be at $26.5(+1.5,-1.0)$ volts $d . c$. Power is provided through a twowire system (power and return) and there is no sharing of the power loads. Main bus dynamic impedance is shown in figure $A-14$ and transient voltage limits are shown by figure $A-15$.

A-11.2 Attitude And Velocity Control Subsystem (AVCS)

Figure A-16 shows the AVCS component block diagram and figure A-17 defines the AVCS top level interfaces. These figures are taken from CID-SV101A, Volume 5. (Prime Item Development Specification).

The figures are quite complete and self-descriptive. Note, however, the relatively small size of the reaction wheels, $\mathrm{H}=0.8 \mathrm{ft}-\mathrm{lb}-\mathrm{sec}$ each.

The two 5-lb thrusters are used for orbit correction during initial insertion, as well as for later orbit maneuvers as required. The smaller thrusters are also available for orbit corrections and maneuvers, but are used mainly for unloading of the momentum wheels.

A-11. $3 \quad$ Thermal Control Subsvstem (TCS)

The thermal control subsystem consists of the following major components:
a. Louver Panel Assemblies (7)
b. Thermal Doublers
c. Space External Insulation Blanket Assemblies
d. Spacecraft Tunnel Insulation Blanket Assembly
e. Battery Radiators (3)
f. Battery Heater/Thermoswitch Assembly (3)
g. OIS Nozzle Cap
h. OIS Plume (Support Skirt Close Out) Shield 


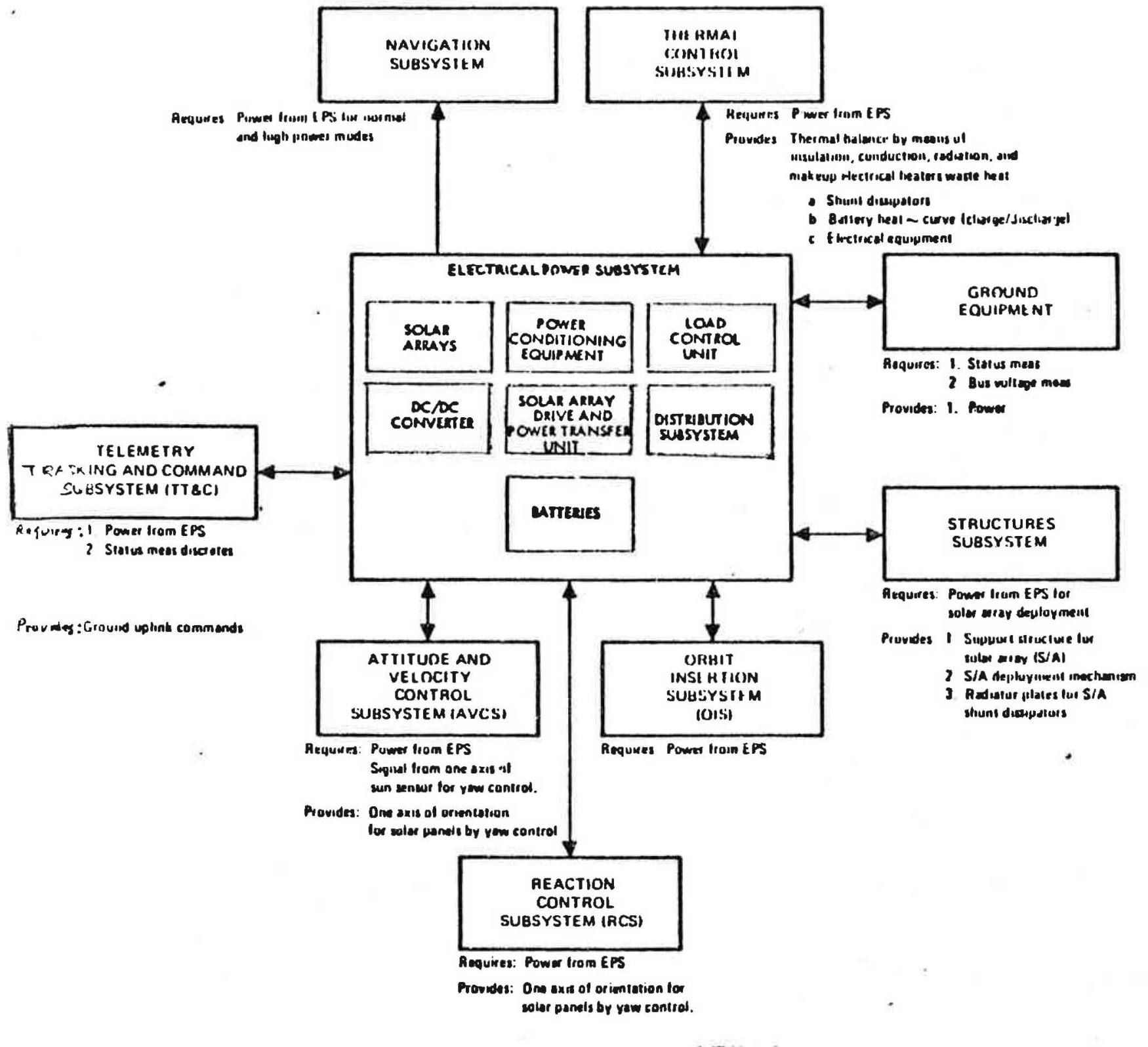

FIGURE A-13 EPS INTERFACES 
TABLE A-8. NOMINAL ELEC'TRICAL LOAD BUDGET

\begin{tabular}{|c|c|c|c|c|c|c|}
\hline \multirow[b]{2}{*}{ Subsystem } & \multicolumn{6}{|c|}{ Mission Phase } \\
\hline & $\begin{array}{c}\text { (1) } \\
\text { Prelaunch \& } \\
\text { Launch (avg) }\end{array}$ & \begin{tabular}{l}
\multicolumn{1}{c}{$(2)$} \\
Transfer \\
Orbit (avg)
\end{tabular} & $\begin{array}{l}\text { (3) } \\
\text { Orbit } \\
\text {-Phasing !avg) }\end{array}$ & $\left\{\begin{array}{l}\text { (4) } \\
\text { Normal NAV } \\
\text { Non Eclipse } \\
\text { Orbit (avg) }\end{array}\right.$ & \begin{tabular}{l} 
On Orbit \\
\multicolumn{1}{|c}{$(5)$} \\
Normal NAV \\
Eclipse \\
Orbit (avg)
\end{tabular} & $\begin{array}{l}\text { (6) } \\
\text { figh-Power } \\
\text { Av Non- } \\
\text { clipse } \\
\text { prbit (avg) }\end{array}$ \\
\hline EPS & 12 & 12 & 21 & 20 & 20 & 20 \\
\hline RCS & 9 & 8 & 8 & 1 & 1 & 1 \\
\hline AVCS & - & 10 & 33 & 33 & 33 & 33 \\
\hline TTSC & 60 & 15 & 15 & 11 & 11 & 11 \\
\hline NAV & - & - & - & 227 & 227 & 292 \\
\hline THEPMAL & - & 41 & 41 & 21 & 13 & 16 \\
\hline TOTAL (Watts) & 81 & $86 \star^{\circ}$ & 118 & 313 & 305 & 373 \\
\hline $\begin{array}{l}\text { * } 133 \mathrm{~W} \text { for the fi } \\
\text { NoTE: Subsystem }\end{array}$ & $\begin{array}{l}\text { rs of transf } \\
\text { are naminal }\end{array}$ & $\begin{array}{l}\text { fer arbit } \\
\text { values an }\end{array}$ & are for refer & rence only. & & \\
\hline
\end{tabular}




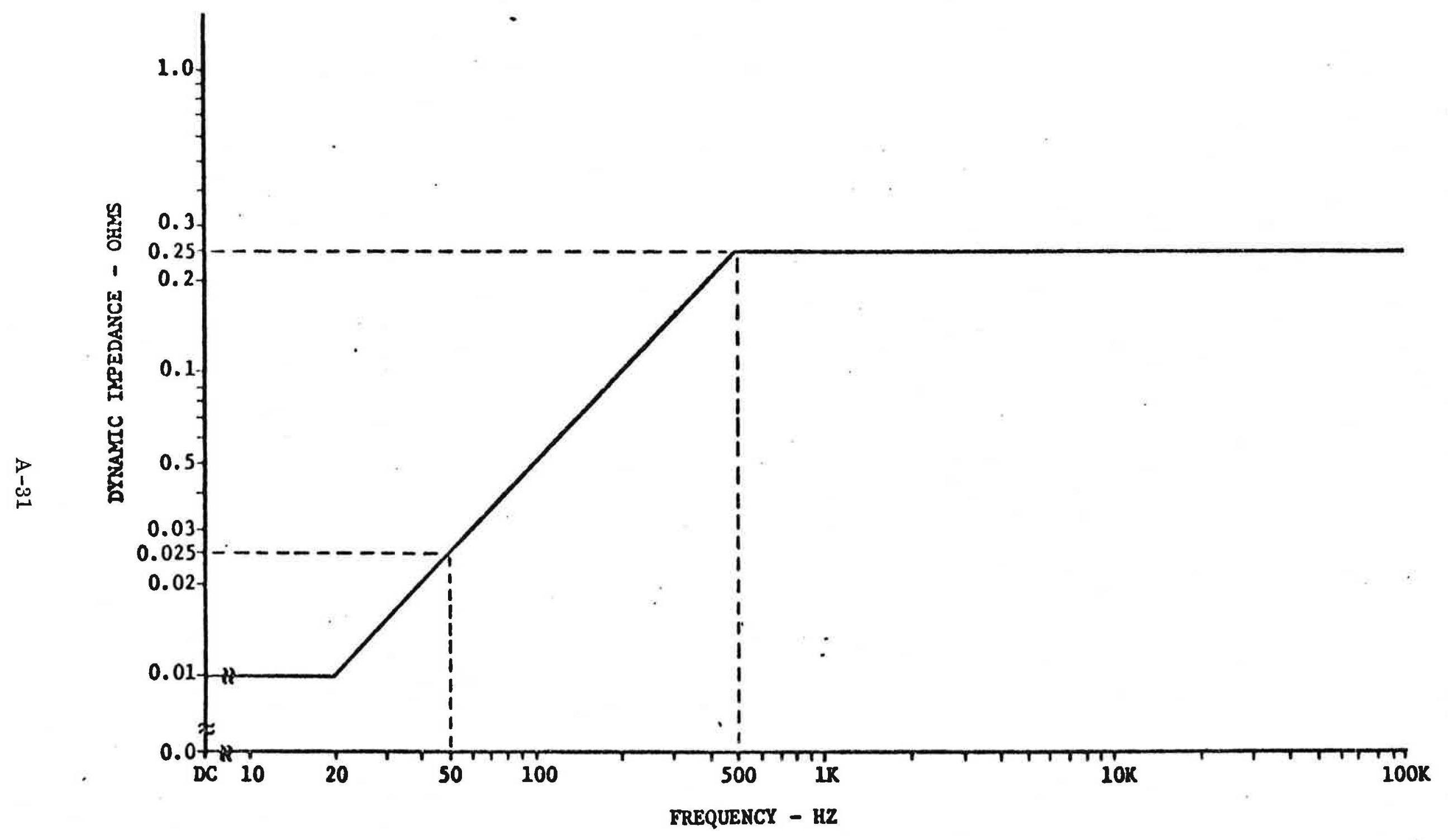

FIGURE A-14. EPS MAIN BUS DYNAMIC SOURCE IMPEDANCE 


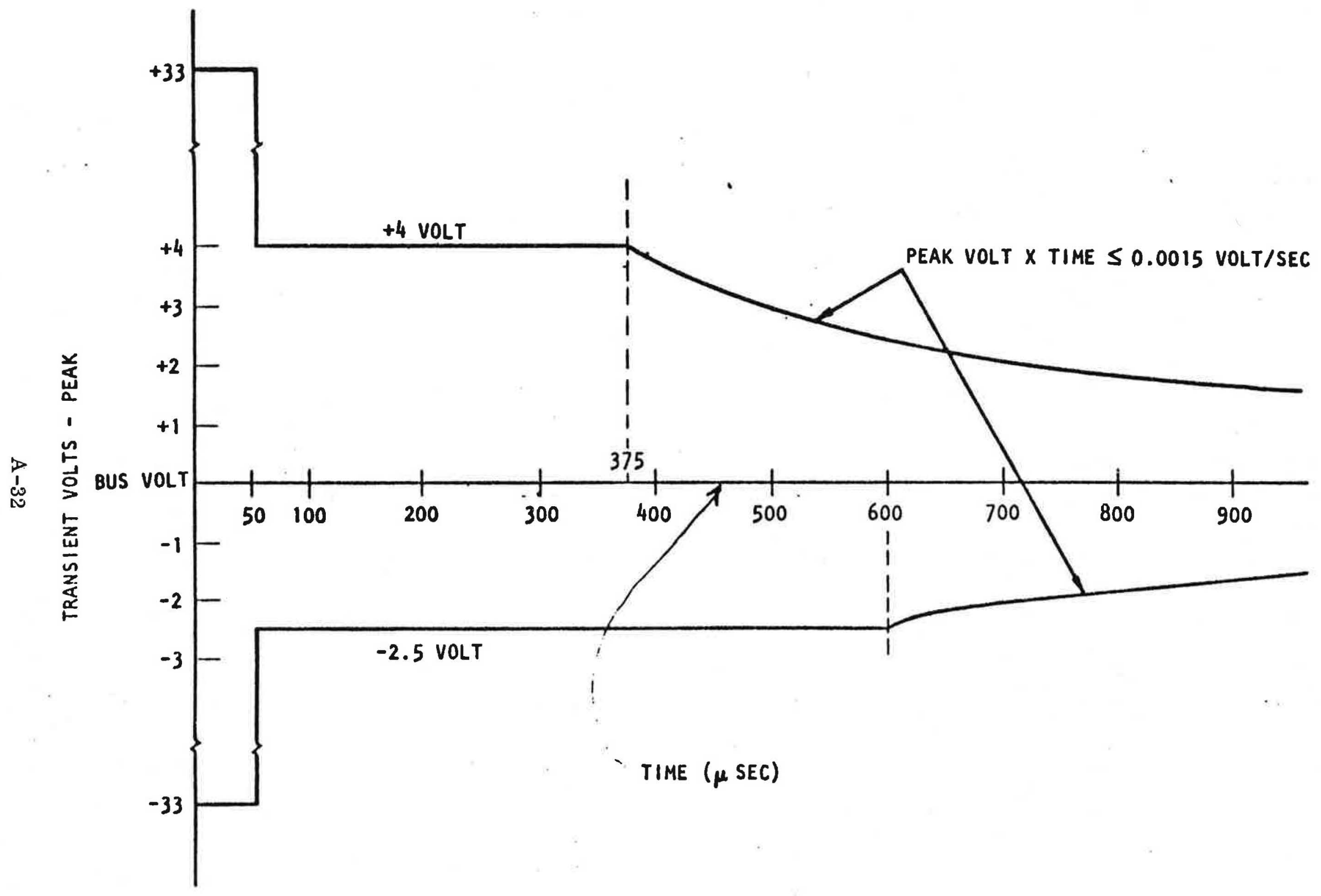

FIGURE A-15. TRANSIENT VOLTAGE LIMITS 


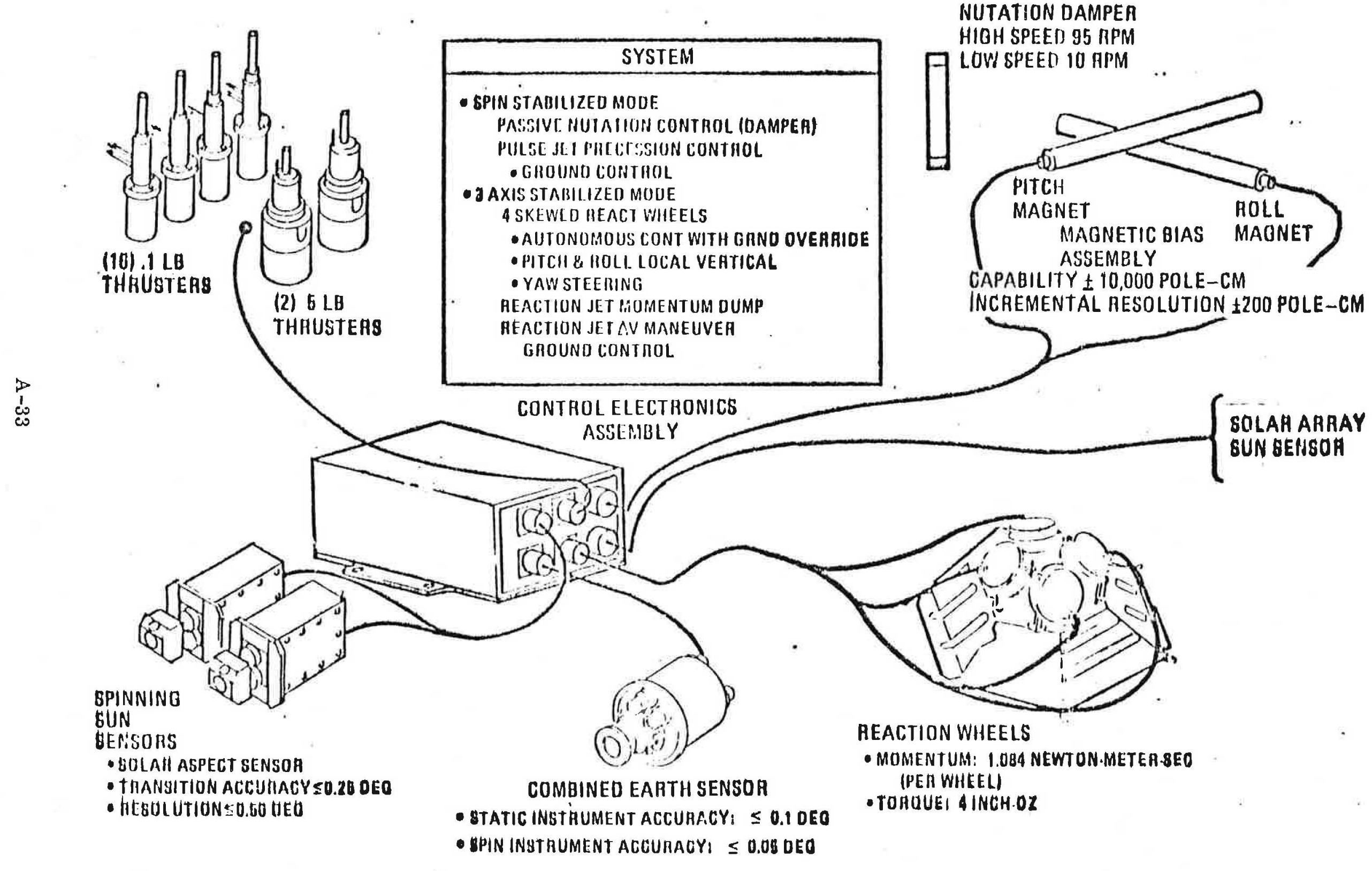

FIGURE A-16. AVCS TOP LEVEL INTERFACES 


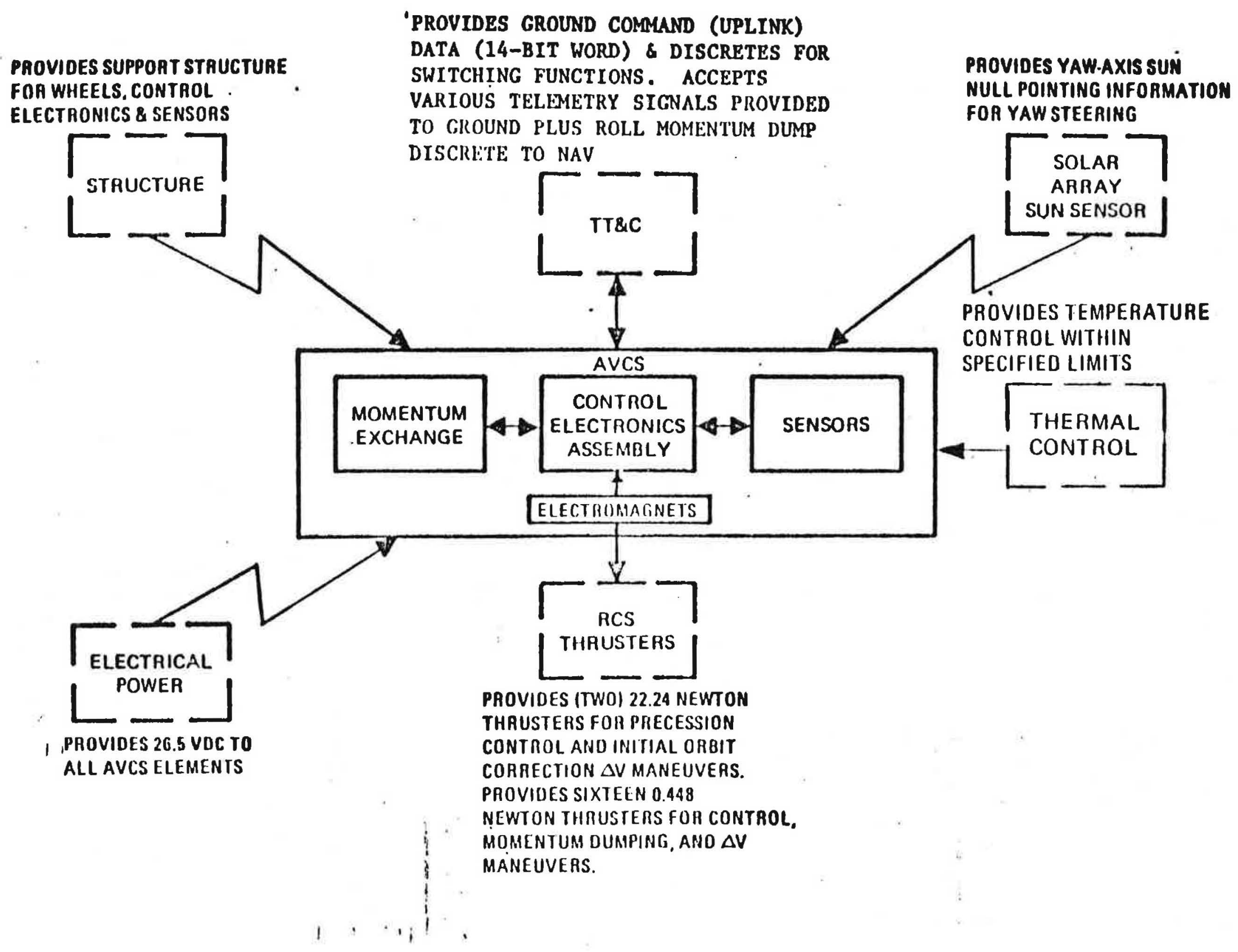

FIGURE 3 17. AVCS TOP LEVEL INTERFACES 
i. OIS Nozzle Throat Heater, Vendor Supplied

j. RCS Component Heater/Thermoswitch Assemblies (10)

k. Surface coatings, finishes, tapes, etc.

Most of the thermal control requirements are those commonly applied to spacecraft components, with the critical exception of the navigation frequency standards. Although the baseplate temperature limits of these "on-board clocks" are 20 to $45^{\circ} \mathrm{C}$, the permitted temperature variation is not to exceed $4^{\circ} \mathrm{C} /$ hour and $5^{\circ} \mathrm{C} /$ day.

The rubidium frequency standard in current use on the Phase I space vehicle ( $\mathrm{SV}$ ) is mounted (within a double oven) to one of the radiator shear panels on the "Y" face of the spacecraft. These panels are subject to control by the thermal louvers, which are located on the shear panels, 3 louvers on the $+Y$ and 4 on the $-Y$ panels. The louvers are mounted outboard, over silvered FEP Teflon radiators. The louvered area is approximately $1.318 \mathrm{~m}^{2}\left(14.2 \mathrm{ft}^{2}\right)$. Maximum orbital heat rejection load capability is 454 watts.

The efficacy of the radiators in limiting temperature excursions of the clocks is substantially improved by the yaw steering of the spacecraft, which keeps the $\mathrm{Y}$ faces out of direct sunlight. Therefore, in the absence of variations of internal dissipation, the major remaining sources of time-varying thermal input are due to:

1.) reflected sunlight and thermal radiation from the solar array edges and panels at an angle,

2.) nominal direct sunlight due to control deadband, and due to temporary re-orientation required during noon orbit and when coming out of eclipse.

A-11.4 Telemetry and Command Subsystem (TT\&C)

Figure A-18 shows a block diagram of the telemetry and command subsystem taken from the Command and Measurement Requirement List (Drawing \# V 505-910006). The command and measurement requirements for the current GPS Phase I Spacecraft are summarized in table A.8. As may be seen from this table, the Electrical Power Subsystem (EPS) command and measurement budget is fairly generous and preliminary study has indicated that a nuclear power subsystem would be able to operate within this budget. 


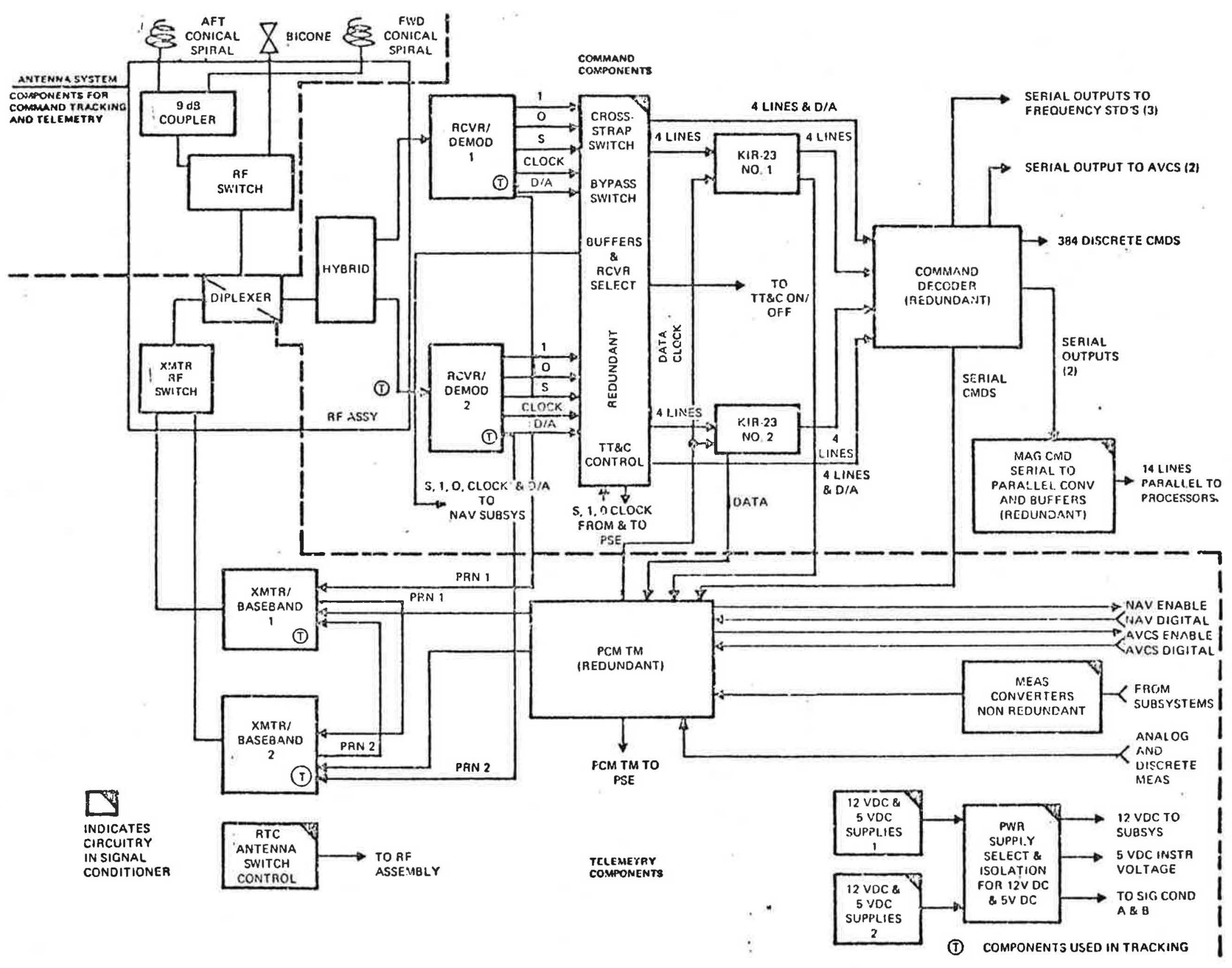


Table A-8.

NAVSTAR Command and Measurement Requirement Summary

\begin{tabular}{|c|c|c|c|}
\hline \multirow[b]{2}{*}{ Subsy stem } & \multicolumn{3}{|c|}{ Number of Commands } \\
\hline & Discrete & Magnitude & Hardwar \\
\hline AVCS & 98 & $33 *$ & 2 \\
\hline EPS & 68 & - & 9 \\
\hline NAV & 88 & - & - \\
\hline OIS & 4 & - & - \\
\hline $\operatorname{RCS}$ & 22 & - & 12 \\
\hline TCS & 18 & - & - \\
\hline $\mathrm{T}, \mathrm{T} \& \mathrm{C}$ & 53 & 127 & 16 \\
\hline Subsy stem & & surements & \\
\hline AVCS & & & 20 \\
\hline EPS & & & 17 \\
\hline NAV & & & - \\
\hline OIS & & & - \\
\hline RCS & & & 8 \\
\hline TCS & & & - \\
\hline $\mathrm{T}, \mathrm{T} \& \mathrm{C}$ & & & - \\
\hline
\end{tabular}

* Plus 10 other AVCS command groups defined by changing the bit patterns within the data field of the uplink command word. 



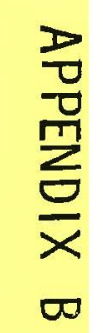

B. CALCULA TION OF MAXIMUM ALLOWABLE USER POWER 

B. $1 \quad$ Case of Uniform Increases in All Links

The GPS load summary and navigation subsystem power requirements, as obtained from the Rockwell July 1976 Design Review, are given by Table B-1.

From the EPS Load Summary Chart, it is seen that average (low power/ high power mode) total spacecraft power requirements are $300 / 372$ watts and that the NAV subsystem uses $233 / 306$ watts of this total. The LP/HP mode is utilized in the L1 links only. The L2 link power output is constant. It is first assumed that any increased spacecraft power capability resulting from use of a nuclear power supply would first be used to maintain the high power mode continuously. The analysis presented therefore concentrates on this mode. Additional increases in power capability would next be directed to increased output of the L1 and L2 High Power Amplifiers (HPA's). RF power output is given by the L1/L2 Link Budgets previously shown in Table 4.1-1. Note that the L1 HPA provides two similtaneous signals (a protected (P) signal and a clear acquisition (C/A) signal) and that L1 HPA power has to be shared between these two signals and self-generated noise.

Using the values for the net L-1 HPA and L-2 HPA RF output and adding the losses shown on the link margin charts results in the following HPA gross RF output values.

\begin{tabular}{|c|c|c|}
\hline & $\underline{\mathrm{L}-1 \mathrm{HPA}}$ & $\underline{\mathrm{L}-2 \mathrm{HPA}}$ \\
\hline Net RF Power & $16.66 \mathrm{dBW}$ & $8.24 \mathrm{dBW}$ \\
\hline Losses & $1.33 \mathrm{~dB}$ & $1.28 \mathrm{~dB}$ \\
\hline Gross RF Power & $\begin{array}{l}17.99 \mathrm{dBW} \\
(63.0 \text { watts })\end{array}$ & $\begin{array}{l}9.52 \mathrm{dBW} \\
\text { (9.0 watts) }\end{array}$ \\
\hline
\end{tabular}

Total RF output is therefore 72 watts which requires 232 watts for the HPA's and power filters and leaves 74 watts for the rest of the NAV subsystem with a power division between the L1 and L2 HPA's of . 875/.125.

Table B-2 shows the effect of increased spacecraft power on the critical parameter (CP) of "power to user" assuming equal ratio increase of power in all four links. Note that a $3.0 \mathrm{~dB}$ (doubling) of spacecraft power capability results in a $4.3 \mathrm{~dB}$ increase in power to user. The $0.1 \mathrm{~dB}$ difference between the L-1 and L-2 links is not 


\section{GPS Load Summary}

Subsystem

EPS

$\mathrm{RCA}$

AVCS

TT\&C

NAV

Thermal

Normal Mode Load

High Power Load
Power Demand

(watts on orbit)

15. 2

0.5

21.3

11. 0

233.0

13,4

299. 4 Watts

372. 0 Watts

Navigation Subsystem Power

Component

L 1 HPA

I 2 HPA

Power Filters

L 1 Mod 1 PA

L $2 \operatorname{Mod} 1$ PA

L 1 /L2 Synthesizer

Baseband Processor

Frequency Standard

Power Converter

Normal Mode Power

Incremental HPA

Power Filter

High Power Mode Power
DC Watts

131.0

27.0

1.0

2.8

1. 0

8.4

14.4

31. 8

15.5

232. 9 Watts

72.0

0.6

305.5 Watts 


\section{TABLE B-2 - USER POWER VERSUS TOTAL DC SPACECRAFT POWER}

\begin{tabular}{|c|c|c|c|c|c|c|c|c|c|}
\hline $\begin{array}{c}\text { Total } \\
\text { DC Power } \\
\text { (Watts) }\end{array}$ & $\begin{array}{l}\text { Total } \\
\text { HPA Power } \\
\text { (Watts) }\end{array}$ & $\begin{array}{r}\mathrm{L}-1 \\
\mathrm{RF} F \\
\text { (Watts) } \\
\end{array}$ & $\begin{array}{l}\text { EPA } \\
\text { ower } \\
\text { (dBW) not }\end{array}$ & $\begin{array}{c}\mathrm{L}-1 \\
\mathbf{P} \text { User } \\
\text { Power } \\
\text { (dBW) }\end{array}$ & $\begin{array}{c}\text { L-1 } \\
\text { C/A User } \\
\text { Power } \\
\text { (dBW) }\end{array}$ & $\begin{array}{r}\mathrm{L}-2 \\
\mathrm{RF} \\
\text { Wattg) }\end{array}$ & $\begin{array}{l}\text { HPA } \\
\text { Power } \\
\text { (dBW) net }\end{array}$ & $\begin{array}{l}\text { L-2 } \\
\text { P User } \\
\text { Power } \\
\text { (dBW) }\end{array}$ & $\begin{array}{c}\text { L-2 } \\
\text { C/A user } \\
\text { Power } \\
\text { (dBW) }\end{array}$ \\
\hline 370 & 230 & 63.0 & 16.7 & -162.4 & -157.8 & 9.0 & 8.2 & -165.6 & -165.1 \\
\hline 400 & 260 & 71.5 & 17.3 & -161.8 & -157.2 & 10.2 & 8.8 & -165.0 & -164.5 \\
\hline 450 & 310 & 85.8 & 18.0 & -161.1 & -156.5 & 12.3 & 9.6 & -164.2 & -163.7 \\
\hline 500 & 360 & 100.1 & 18.7 & -160.4 & -155.8 & 14.4 & 10.3 & -163.5 & -163.0 \\
\hline 550 & 410 & 114.4 & 19.3 & -159.8 & -155.2 & 16.4 & 10.8 & -163.0 & -162.5 \\
\hline 600 & 460 & 128.7 & 19.8 & -159.3 & -154.7 & 18.4 & 11. 3 & -162.5 & -1620 \\
\hline 650 & 510 & 143.0 & 20.2 & -158.9 & -154.3 & 20.5 & $11.8^{\circ}$ & -162.0 & -161.5 \\
\hline 700 & 560 & 157.3 & 20.7 & -158.4 & -153.8 & 22.6 & 12.2 & -161.6 & -161.1 \\
\hline 750 & 610 & 171.6 & 21.0 & -158.1 & -153.5 & 24.6 & 12.6 & -161.2 & -160.7 \\
\hline increa $8 \theta$ & & & & $\begin{array}{l}4.3 \mathrm{~dB} \\
\text { increa } 8 \theta\end{array}$ & $\begin{array}{l}4.3 \mathrm{~dB} \\
\text { Increase }\end{array}$ & & & $\begin{array}{l}4.4 \mathrm{~dB} \\
\text { increase }\end{array}$ & $\begin{array}{l}4.4 \mathrm{~dB} \\
\text { increase }\end{array}$ \\
\hline
\end{tabular}

* Reference state 
significant and is due to round-off in the calculations. Rounded off values have been used for spacecraft power and HPA power.

The rate of increase varies from $0.7 \mathrm{~dB} / 50$ watts at the low power end of the table to $0.3 \mathrm{~dB} / 50$ watts at the high power end of the table and further "diminishing returns" would be expected for additional power increases. Figure B-3 is a graphical presentation of this user power versus spacecraft power relationship.

\section{B.2 Case of Power Increases in One Link Only}

This analysis shows the effect on "power to user" when additional spacecraft power is used to enhance a single link instead of being proportionally divided between the L1 (P), L1 (C/A) and L2 (P or C/A) links as evaluated in the previous section.

The assumptions used in this analyses are the same as those stated previously i.e. Reference state total spacecraft DC power 370 watts, total HPA DC power 230 watts $(203 \mathrm{~W}-\mathrm{L} 1,27 \mathrm{~W}-\mathrm{L} 2)$.

In table $\mathrm{B}-4$, all $\mathrm{DC}$ power increments above the reference state are applied to the L 1 HPA and alternatively used to enhance the (P) link or (C/A) link. Resultant increments in user power as a function of DC spacecraft power are tabulated. As indicated by table 2, a $3.0 \mathrm{~dB}$ increase in spacecraft power can result in either a 9. $6 \mathrm{~dB}$ increase in power to the $\mathrm{L} 1$ (P) link user or a $5.8 \mathrm{~dB}$ increase in power to the L 1 (C/A) link user.

In Table B-5, all DC power increments above the reference state are applied to the L 2 HP and used to enhance the (P) link or (C/A) link. Resultant increments in user power as a function of DC spacecraft power are tabulated. As indicated by table B-5, a $3.0 \mathrm{~dB}$ increase in spacecraft power can result in an $11.8 \mathrm{~dB}$ increase in the $\mathrm{L} 2(\mathrm{P})$ or $(\mathrm{C} / \mathrm{A})$ link. Note that $6.0 \mathrm{~dB}$ of increase is accomplished in the first 80 watts of DC power increment.

Figure B-6, shown below, illustrates the power division relationships. Nominal (reference state) values are shown. In the tabulations L 2 HPA DC input power is held constant while L 1 HPA DC input power is increased (Table B-4) then L-1 H PA DC input power is held constant while L 2 HPA DC input power is increased (Table B-5). 
3
0
0
0
0
0
0
0
0
0
0
0

$755:=1-1 \mathrm{C} / \mathrm{A}$

Flux Density Limit_.r.

$-160$

$=105$

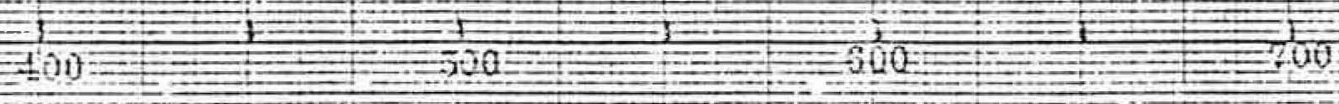

SPACECRAFT POWER NVATTS)

FIGURE B-3. POWER TO USER VS SPACECRAFT POWER 
TABLE B-4. USER POWER VERSUS TOTAL SPACECRAFT POWER (Concentrated in one L 1 Link)

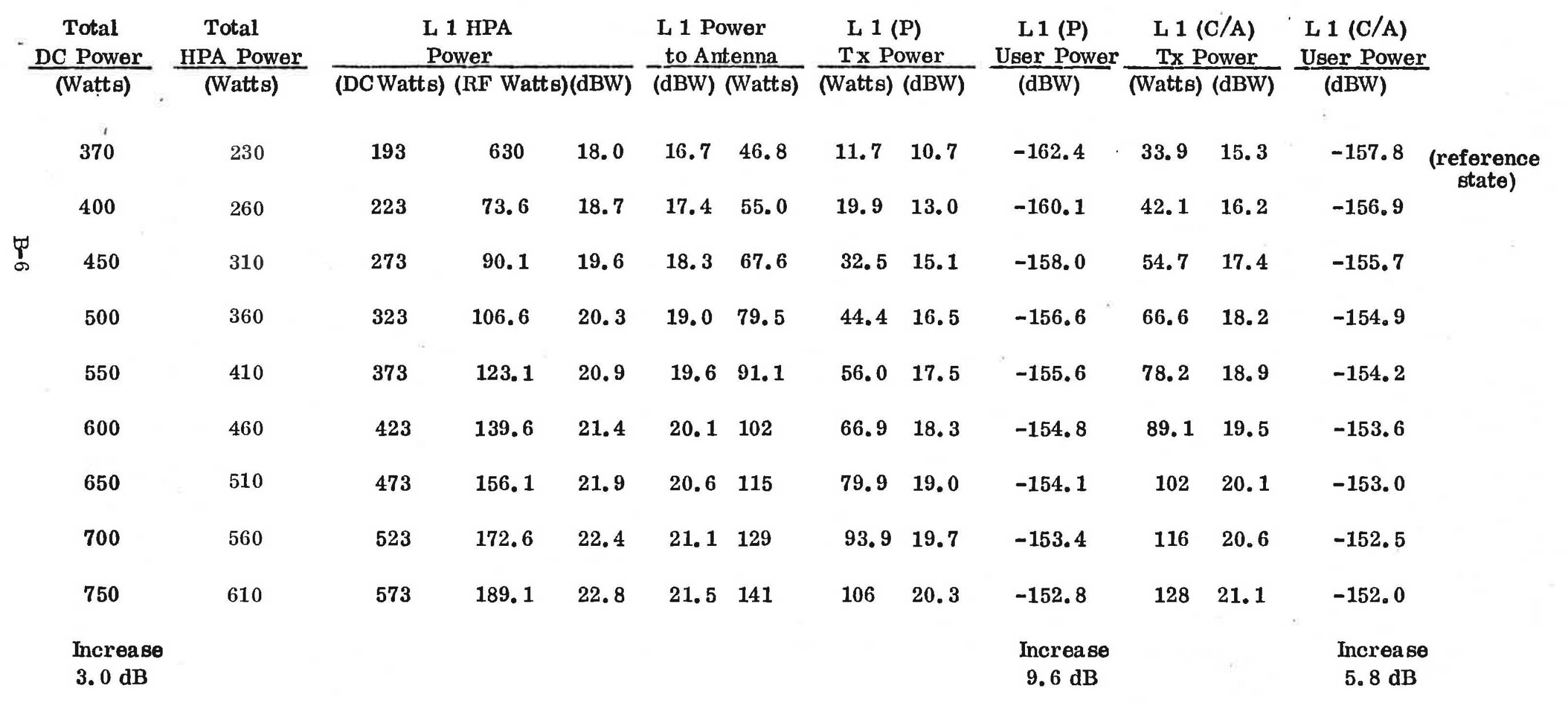


TABLE B-5. USER POWER VERSUS TOTAL SPACECRAFT POWER

\begin{tabular}{|c|c|c|c|c|c|c|c|c|c|}
\hline $\begin{array}{c}\text { Total } \\
\text { DC Power }\end{array}$ & $\begin{array}{l}\text { Total } \\
\text { HPA Power }\end{array}$ & $\begin{array}{r}\mathrm{L} \\
\mathrm{Po}\end{array}$ & $\begin{array}{l}2 \mathrm{HF} \\
\text { ver }\end{array}$ & & & $\begin{array}{l}\text { L } 2 \text { Power } \\
\text { to Antenna }\end{array}$ & $\begin{array}{c}\text { L } 2 \text { (P) } \\
\text { (User Power) }\end{array}$ & $\begin{array}{r}\text { L } 2(\mathrm{C} / \mathrm{A}) \\
\text { (User Power) }\end{array}$ & \\
\hline (Watts) & (Watts) & (DC Watts) & (RF & $\overline{\text { Watts }}$ & $(\mathrm{dBW})$ & $(\mathrm{dBW})$ & (dBW) & (dBW) & \\
\hline 370 & 230 & 27 & & 9.0 & 9.5 & 8.2 & -165.6 & -165.1 & $\begin{array}{c}\text { (reference } \\
\text { state) }\end{array}$ \\
\hline 400 & 260 & 57 & & 18.8 & 12.7 & 11.4 & -162.4 & -161.9 & \\
\hline 450 & 310 & 107 & & 35.3 & 15.5 & 14.2 & -159.6 & -159.1 & \\
\hline 500 & 360 & 157 & . & 51.8 & 17.1 & 15.8 & -158.0 & -157.5 & \\
\hline 550 & 410 & 207 & & 68.3 & 18.3 & 17.0 & -156.8 & -156.3 & \\
\hline 600 & 460 & 257 & & 84.8 & 19.3 & 18.0 & -155.8 & -155.3 & \\
\hline 650 & 510 & 307 & . & 101 & 20.0 & 18.7 & -155.1 & -154.6 & \\
\hline 700 & 560 & 357 & & 118 & 20.7 & 19.4 & -154.4 & -153.9 & \\
\hline 750 & 610 & 407 & & 134 & 21.3 & 20.0 & -153.8 & -153.3 & \\
\hline $\begin{array}{l}\text { Increase } \\
3.0 \mathrm{~dB}\end{array}$ & & & & & & & $\begin{array}{r}\text { Increase } \\
11.8 \mathrm{~dB}\end{array}$ & $\begin{array}{l}\text { Increase } \\
11.8 \mathrm{~dB}\end{array}$ & \\
\hline
\end{tabular}




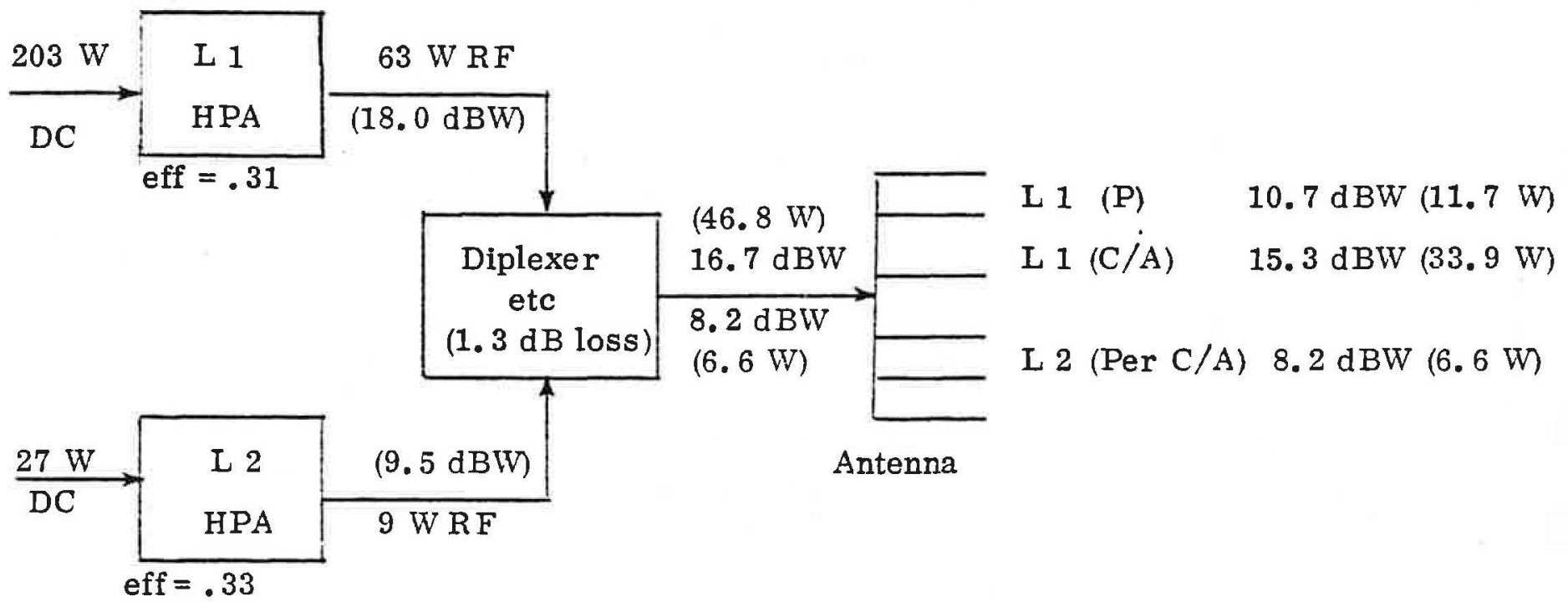

FIGURE B-6

Figure B-7 shows a graphical representation of "power to user" versus spacecraft power for the condition where all incremental power increases are concentrated in one link.

The impact of increased "power to user" can be reflected in either increased jamming protection or decreased user terminal sensitivity. In a spread spectrum communication system, jamming protection, expressed as a jammer to signal ratio $(\mathrm{J} / \mathrm{S})$, is a function of the processing gain in the receiver correlator and the allowable error rate threshold expressed as an energy per bit to noise density ratio $\left(E_{b} / N_{o}\right)$. Assuming a bit error rate (BER) threshold of $10^{-5}$, coherent PSK modulation and allowing $2.0 \mathrm{~dB}$ for non-theoretical equipment performance, the $E_{b^{\prime}} / N_{0}$ threshold is $11.6 \mathrm{~dB}$. Standard curves showing the BER to $E_{b} / N_{o}$ relationship given by Figure B-8. Correlator processing gain is equal to the chip rate (10.23 Mbps for $\mathrm{P}$ links, $1.023 \mathrm{Mbps}$ for $\mathrm{C} / \mathrm{A}$ links) divided by the bit rate $(50 \mathrm{bps})$. Processing gains and $\mathrm{J} / \mathrm{S}$ ratios for the nominal (reference state) and maximum improvement (750 watts of available spacecraft power) are tabulated below. 


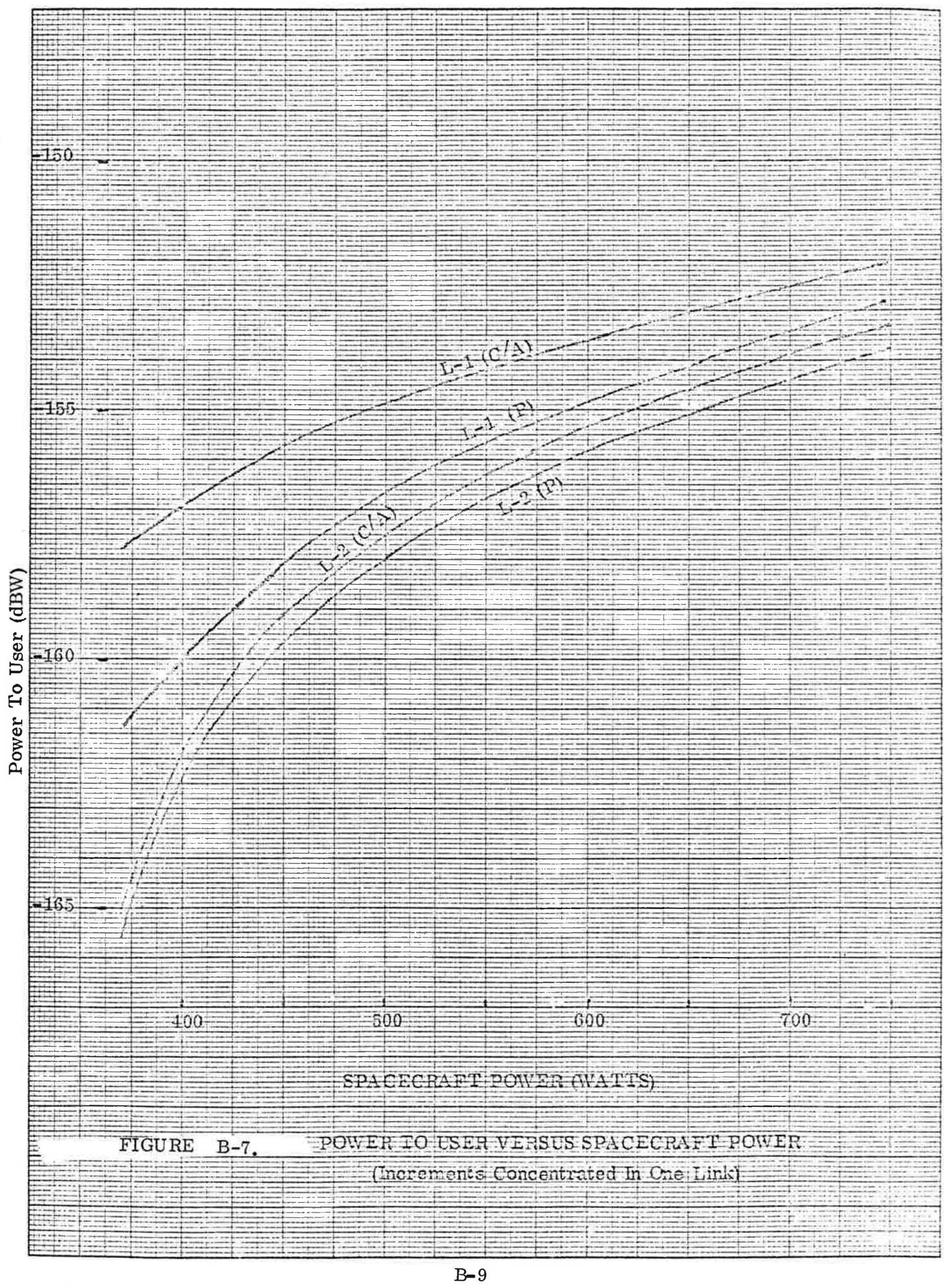




\section{$\underline{\text { L } 1 \text { (P) Link }} \underline{\text { L } 1 \text { (C/A) Link }}$ L 2 (P) Link $\underline{\text { L } 2 \text { (C/A) Link }}$}

PG (dB)

$E_{b} / N_{o}$ threshold (dB)

$\mathrm{J} / \mathrm{S}$ (reference) (dB)

Link Enhancement $(\mathrm{d} B)$

$J / S$ (enhanced) (dB)
53.1

$\underline{11.6}$

41.5

$\underline{9.6}$

51.1

(dB)

$$
J / S(d B)=P G(d B)-\frac{E_{b}}{N_{o}} \mid T
$$

43.1

53.1

$\underline{11.6}$

41.5

$\underline{11.8}$

53.3

37.3

Note that with incremental power concentrated in one link, only one of the $\mathrm{L} 1$ or both of the $\mathrm{L} 2 \mathrm{~J} / \mathrm{S}$ enhancements is possible.

B-3 Power Flux Density Limitations

The GPS system has legal power flux density limitations and increases in spacecraft power will be effective only insofar as these limitations are not exceeded. Attachments 1, 2 and 3 taken from the White House Office of Telecommunications Policy (OTP) Radio Frequency Management Regulations and Procedures show the spectrum sharing in the L-1 and L-2 bands and define a power flux density limitation for either band of $-152 \mathrm{dBW} / \mathrm{m}^{2} / 4 \mathrm{kHz}$. Current GPS signals at the best performance evaluation angle of $40^{\circ}$ are estimated to have power flux densities of $-154.3 \mathrm{dBW} / \mathrm{m}^{2} / 4 \mathrm{kHz}$ for the L-1 (C/A) signal and $-156.6 \mathrm{dBW} / \mathrm{m}^{2} / 4 \mathrm{kHz}$ for the $\mathrm{L}-2$ (C/A) signal. These values are taken from figures 2 and 3 of Interface Release Notice (IRN) \#14726.

As may be seen, these values do not allow for much in the way of user power growth. User's are counting on an unjammed $\mathrm{C} / \mathrm{N}_{\mathrm{O}}$ of $34 \mathrm{~dB}-\mathrm{Hz}$ the raw bit error rate is $10^{-3}$ and this raw rate is corrected by a Hamming Code (3226) with a concatenated algorithm to give $10^{-7}$ BER at $50 \mathrm{bps}$ which is the correlated data rate. The projected system with 10 satellites 
will not produce self-interference significantly above thermal noise $\left(\mathrm{N}_{0}\right.$ estimated at $-200 \mathrm{dBW} / \mathrm{Hz}$ ), but extensive receiver filtering is required to eliminate adjacent interference. It was commented that the users work primarily with the L-1 link using a 4-channel CDMA (Code Division Multiple Access) receiver with approximately 4\% of the time spent on each visible satellite and $20 \%$ of the time at L-2 for ionosphere delay corrections. While other waveforms and other frequencies are being considered for later phases of its program, no attention is being given to frequency hopping as opposed to the current direct sequence method of spectrum spreading.

As a cross-check on the power flux density status, the powe $\mathrm{r}$ distribution of a direct sequence spread spectrum is of the form $\left(\frac{\operatorname{Sin} x}{X}\right)^{2}$ as indicated by figure 2.8 of attachment 4 taken from "Spread Spectrum Systems" by Dixon (Wiley 1976). Figure 2.9 shows the effect on correlation function of the usual bandwidth restriction as used in GPS (Bandwidth equal to two times chip rate). The power distribution has a peak to average factor of 1.53 within the main lobe and $10 \%$ of the power lies autside the restricted bandwidth and is contained in the side lobes. Peak power density is therefore $1.53 \times 0.9$ or 1.38 times the density that is obtained assuming uniform spreading which equals a worst case increase of $1.4 \mathrm{~dB}$. Calculations on the L-1 C/A link (worst case) are as follows:

$\begin{array}{ll}\text { specified user power } & -158.0 \mathrm{dBW} \text { (HP mode) } \\ \text { uniform spreading factor } & -63.1 \mathrm{~dB} \text { (over 2.046 MHz) } \\ \text { gam of } 1 \mathrm{M}^{2} \text { aperture at } \mathrm{L}-1 & +25.5 \mathrm{~dB} \\ \text { peaking factor } & +1.4 \mathrm{~dB} \\ 4 \mathrm{kHz} \text { reference bandwidth } & +36.0 \mathrm{~dB}-\mathrm{Hz} \\ \text { correction for difference } & \\ \text { between standard space loss } & \\ \text { and worst case space loss in } & \\ \text { Rockwell link budgets } & +3.9 \mathrm{~dB}\end{array}$

which correlates with the value given previous $1 y$. 
The significance of the $34 \mathrm{~dB}-\mathrm{Hz}$ carrier to noise density $\left(\mathrm{C} / \mathrm{N}_{0}\right)$ ratio and the $10^{-3}$ raw bit error rate (code corrected to $10^{-7}$ ) can be seen from the following calculations:

Specified L-1 (P) link user power

$-163.0 \mathrm{dBW}$

Receive System Noise density (4 dB NF)

$-200.0 \mathrm{dBW} / \mathrm{Hz}$

$\mathrm{C} / \mathrm{N}_{\mathrm{o}}$

37. $0 \mathrm{~dB}-\mathrm{Hz}$ ( $3 \mathrm{~dB}$ above stated requirement)

Processing gain chip rate bit rate

$53.1 \mathrm{~dB}$

Chip rate $(10.23 \mathrm{Mbps})$

$73.1 \mathrm{~dB}$

$\mathrm{E}_{\mathrm{b}} / \mathrm{N}_{\mathrm{o}}$

$10^{-3}$ BER Threshold

$17.0 \mathrm{~dB}$

$6.8 \mathrm{~dB}$ reference Figure $\mathrm{B}-8$

(PSK coherent modulation)

correlation loss

1. $0 \mathrm{~dB}$ reference IRN 17426

sec. $3.3 .7 .27(d)$

Operating Margin

$9.2 \mathrm{~dB}$

As seen from the bit error rate vs $E_{b} / N_{0}$ curves of Figure $B-8$, improvements in bit error rate by 3 orders of magnitude require only a $2 \mathrm{~dB}$ increase in $E_{b} / N_{o}$ and the system could probably operate without coding corrections under its present link power budgets.

A $4 \mathrm{~dB}$ noise figure receiver at $\mathrm{L}$-band is not excessively expensive; more cost involved in the receiver's filtering than in its low noise preamplifier. 


\section{Probability of Bit Error vs Signal to Noise Ratio}

,

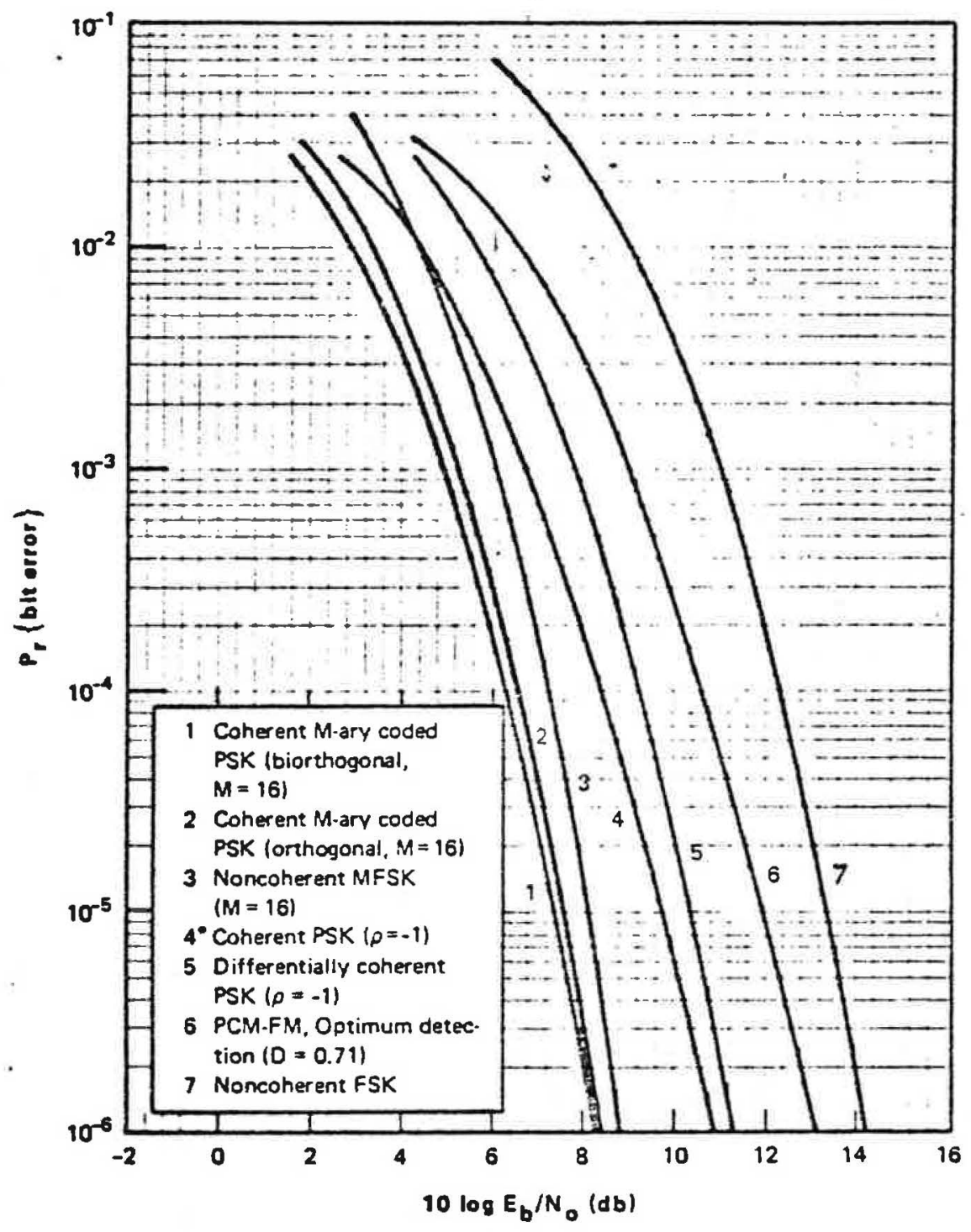

- A comparison of $M$-ary and binary signaling techniques relating signal to noise ratio to the probability of bit error; ex. $10^{-3}$ means one bit lost or in error per thousand bits.

\begin{tabular}{llr}
\hline $\begin{array}{c}\text { Oigital Modulation } \\
\text { Methods }\end{array}$ & $\begin{array}{c}\text { Required Power } \\
\text { SER }=10^{-5}\end{array}$ & $\begin{array}{c}\text { Required } \\
\text { Bandwidth }\end{array}$ \\
\hline 16-ary coded PSK & $0 \mathrm{~dB}$ (reference) & $\begin{array}{c}4 \times \text { bit rate } \\
\text { 16-ary MFSK }\end{array}$ \\
Quadriphase & $2.8 \mathrm{~dB}$ more & $4.5 \times$ bit rate \\
Coherent PSK & $2.5 \mathrm{~dB}$ more & $1 \times$ bit rate \\
PCM/FM & $4.6 \mathrm{~dB}$ more & $2 \times$ bit rate \\
FSK & $6.0 \mathrm{~dB}$ more & $3 \times$ bit rate \\
\hline
\end{tabular}

$E_{b} / N_{o}$ and the terminal sensitivity factor $C / K T_{s}$ are related by the bit rate $R_{b}$ as expressed by:

$$
\frac{C}{K_{s}}=\frac{C_{b}}{N_{0}} \cdot \frac{1}{T_{b}}=\frac{E_{b}}{N_{0}} \cdot R_{b}
$$

whare $T_{b}=R_{b}{ }^{-1}$, the bit duration time; $N_{0}=K T_{5}$. the noise power density: and $C_{T_{b}}=E_{b}$.

Courtesy of C.L.Cuccia, W.J.Gill, L.H.Wilson, Philco - Ford. 


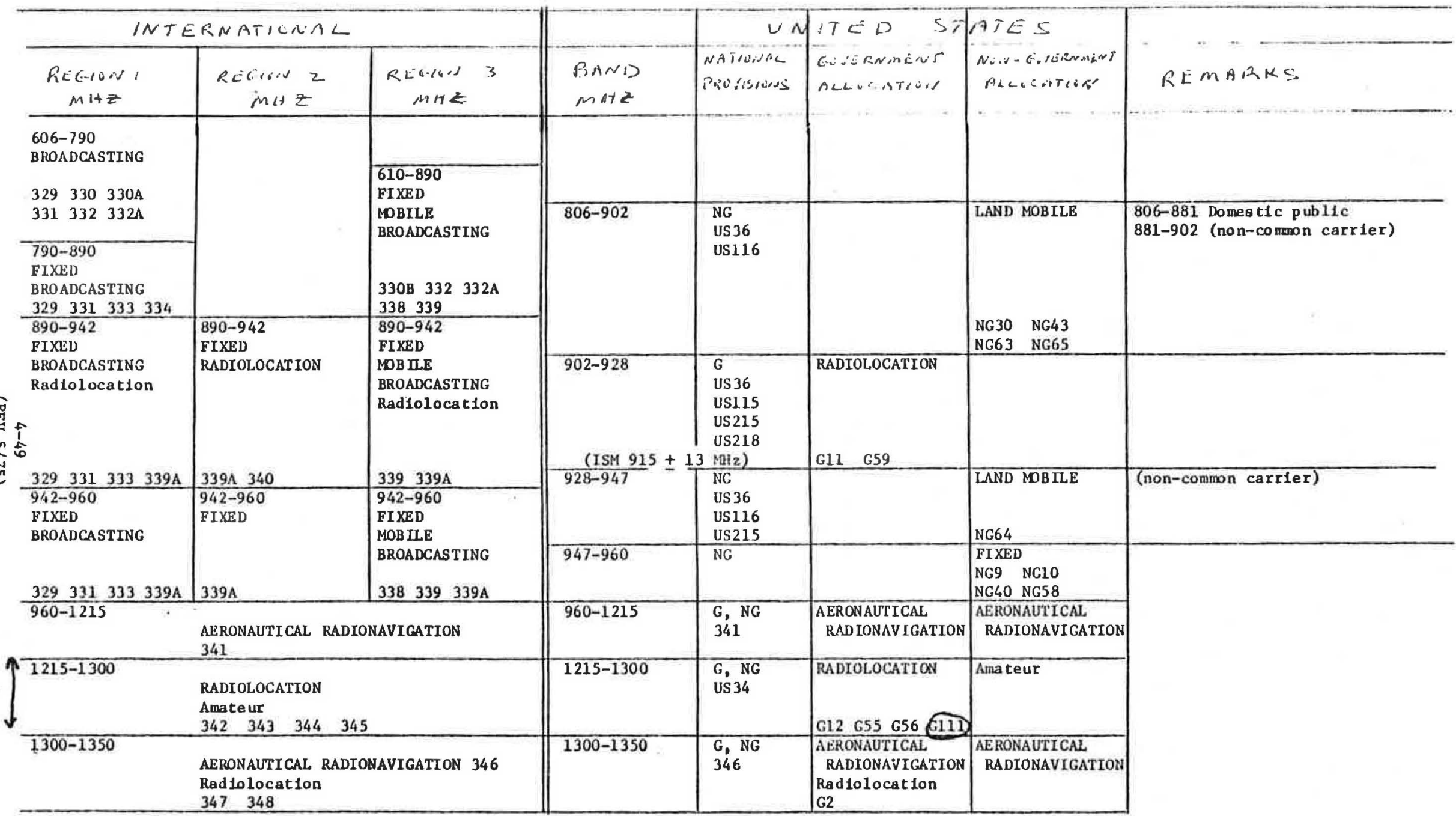

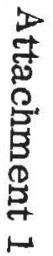




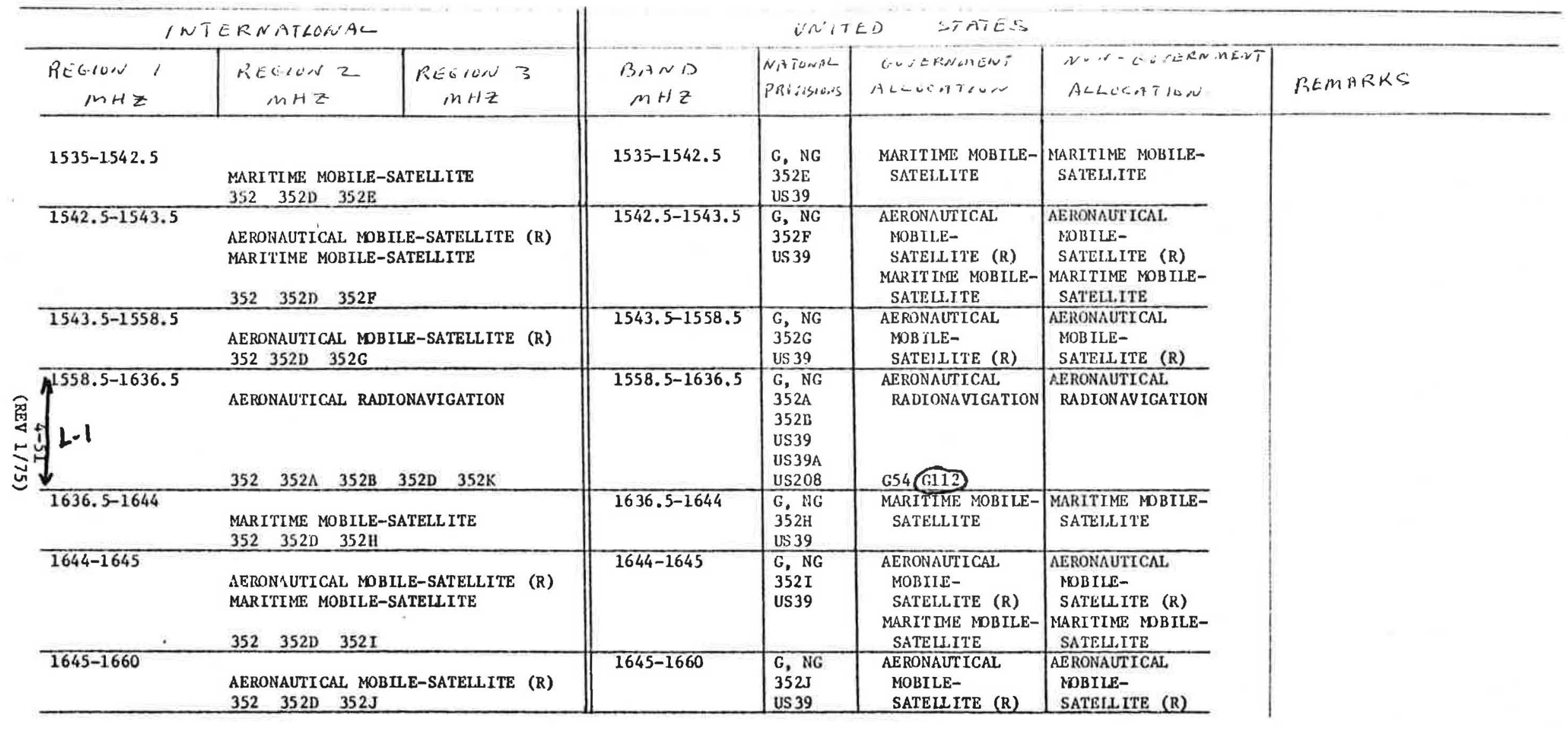


G109 AlI assignments in the band 157.0375-157.1875 MHz are subject to adjustment to other frequencles in this band as long term U.S. maritime VHF planning develops, particularly that planning incident to support of the National VEF-FM Radlotelephone Safety and Distress System (See Doc. 15624/1-1.9.111/ $1.9 .125)$.

G110 Goverment ground-based stations in the aeronatical radionavigation service may be authorfzed between 3500 and $3700 \mathrm{MHz}$ where accommodation in the 2700-2900 MHz band is not tecinically and/or economically feasible.

G111) In the band $1215-1250 \mathrm{MHz}$, the frequency $1227.6 \mathrm{MHz}$ with emissions 1 imited to $+12 \mathrm{MHz}$ bandwidth, is also 3llocated to the Radionavigation Satellite Service, for satellite down link transmlssions only. The power flux density at the earth's surface from such transmissions shall not exceed $-152 \mathrm{dBW} / \mathrm{m}^{2} / 4 \mathrm{kHz}$. The Radionavigation Satellite Service shall not cause harmful interference to the Amateur Service and shall accept any harmful interference that may be caused by the Amateur Service.

(6112) In the band 1558.5-1636.5 Miz, the frequency $1575.42 \mathrm{MHz}$ with emission $11 \mathrm{mited}$ to $\pm 15 \mathrm{MHz}$ bandwith is also allocated to the Radionavigation Satellite Service for satellite down link transmisslons only. The power flux denslty at the earth's surface from such transuisstons shall not exceed $-152 \mathrm{dBW} / \mathrm{m}^{2} / 4 \mathrm{kHz}$. 


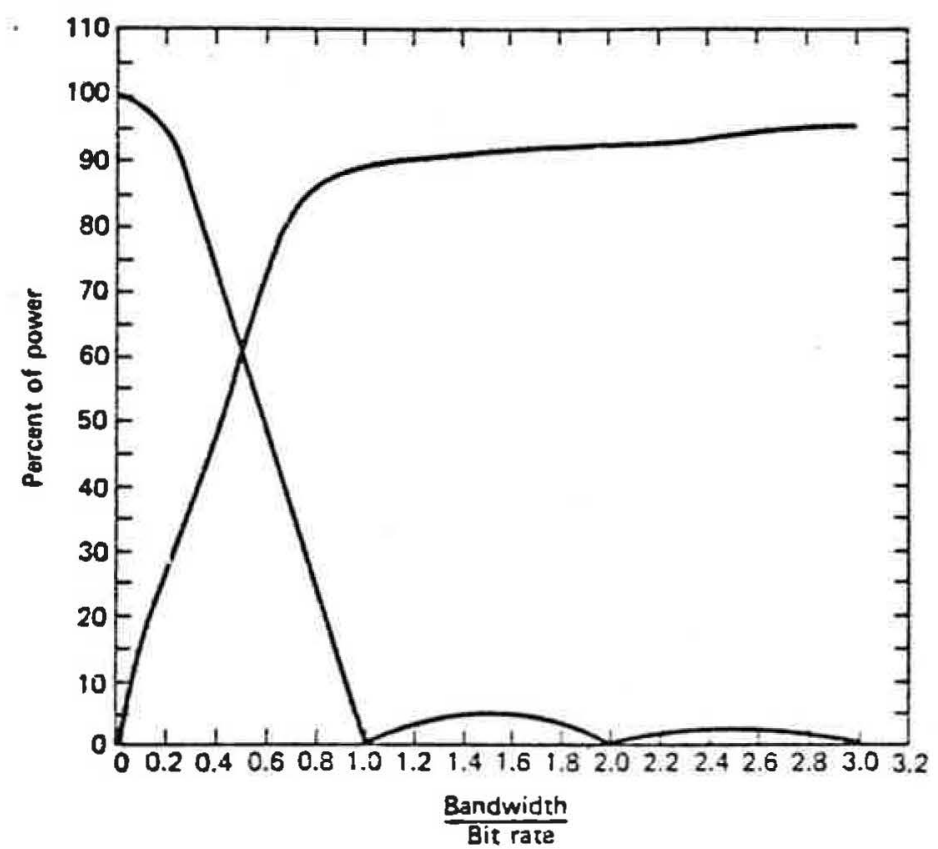

Figure 2.8 Power distribution in $[(\sin x) / x]^{2}$ spectrum.

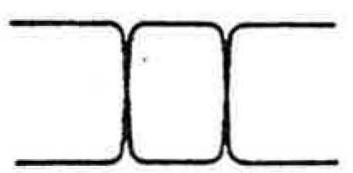

Bw $\gg \frac{2}{T}$

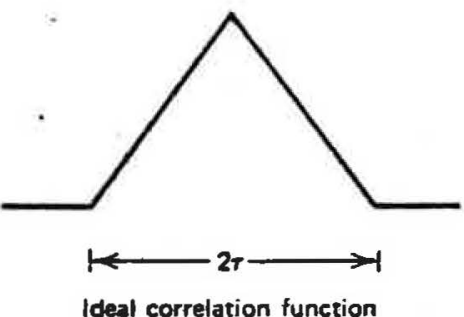

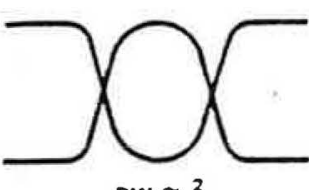

$\mathrm{BW} \cong \frac{2}{7}$

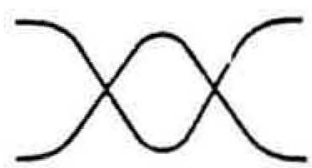

$\mathrm{BW} \cong \frac{1}{r}$

(a)

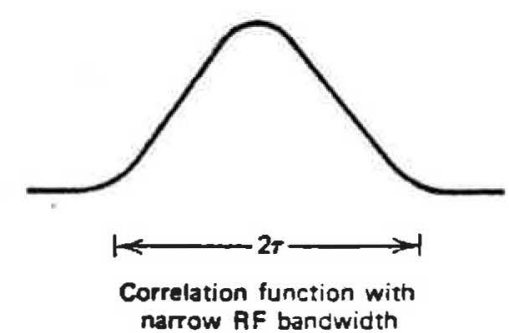

(b)

Figure 2.9 RF bandwidth restriction and its effect on direct sequence signals: (a) RF envelope of direct sequence signal for various RF bandwidths. (b) effect of bandwidth restriction on cortelation function. 

C. EFFECT OF SOLAR PRESSURE ON GPS ORBIT PREDICTABILITY 



\section{C-1 Introduction}

The GPS space vehicles are in nominal 12-hour orbits with $63^{\circ}$ inclination. Perturbations to the orbits are caused by earth non-spherǿcity, lunar and solar gravitational effects, solar and vehicle emitted radiation pressures, impingement of particles, and vehicle mass expulsions. Reference 1 asserts that the total effect of these perturbing forces are sufficiently well-modelled that, after adequate ground-tracking, the actual. vehicle position can be predicted within 1 to 2 meters (RNS). Unfortunately, there is no statement in Ref. 1 as to the length of time for which such accurate ephemerides may be predicted.

\section{C-2 Analysis}

Our interest here is the incremental effect of solar pressure on the vehicle. The analytical approach taken is to write the equations which describe the incremental accelerations of the satellite resulting from the incremental forces. These differential er uations are non-linear and are therefore integrated numerically to determine actual incremental motions due to the incremental forces. The results of the computerized integrations are given in the attached figures.

C-3 Relative Motion of the Satellite with Respect to the Earth

Let: 0 be an inertial fixed point

$F_{1}=$ the radius vector from 0 to the earth's center

$\bar{r}_{2}=$ the radius vector from 0 to the satellite

$\bar{r}_{s}=$ the radius vector from 0 to the sun

$\bar{r}_{m}=$ the radius vector from 0 to the moon

$m_{i}=$ the mass of the body at $\bar{r}_{i}$

$G=$ gravitational constant

$\bar{F}_{s}=$ force due to solar pressure 
Then, if the earth's oblateness is neglected and there are no forces due to solar pressure, equation (1) gives the acceleration of the earth due to gravitational forces from the satellite, the moon, and the sun.

(1)

$$
\frac{d^{2}}{d t^{2}} \bar{r}_{1}=G m_{2} \frac{\left(\bar{r}_{2}-\bar{r}_{1}\right)}{\left|\bar{r}_{2}-\bar{r}_{1}\right|^{3}}+G m=\frac{\left(\bar{r}_{1}-\bar{r}_{1}\right)}{\left|\bar{r}_{s}-\bar{r}_{1}\right|^{3}}+G m_{m} \frac{\left(\bar{r}_{m}-\bar{r}_{1}\right)}{\left|\bar{r} m-\bar{r}_{1}\right|^{2}}
$$

Similarly, the acceleration of the satellite is given by (2).

(2)

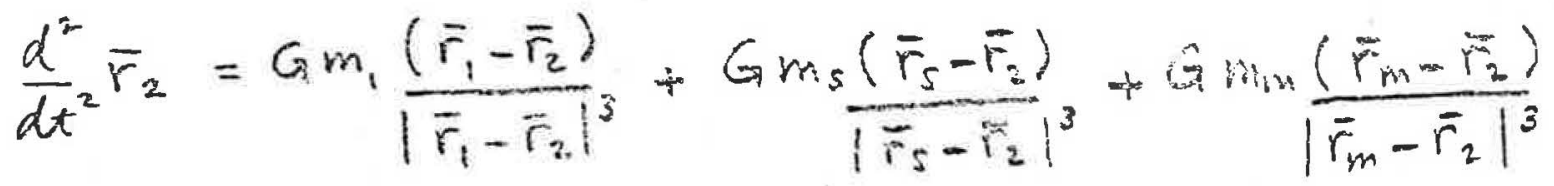

The relative acceleration of the satellite with respect to the earth is obtained

by subtracting equation (1) from equation (2). Subtracting, and letting $\bar{r}=\bar{r}_{2}-\bar{r}_{1}$ we have:

(3)

$$
\begin{aligned}
\frac{d^{2}}{d t^{2}} \bar{r}+\frac{G\left(m_{1}+m_{2}\right)}{r^{3}} \bar{r} & =G m_{s}\left[\frac{\left(\bar{r}_{s}-\bar{r}_{2}\right)}{\left|\bar{r}_{s}-\bar{r}_{2}\right|^{3}}-\frac{\left(\bar{r}_{s}-\bar{r}_{1}\right)}{\left|\bar{r}_{s}-\bar{r}_{1}\right|^{3}}\right] \\
& +G m_{m}\left[\frac{\left(\bar{r}_{m}-\bar{r}_{2}\right)}{\left|\bar{r}_{m}-\bar{r}_{2}\right|^{3}}-\frac{\left(\bar{r}_{m}-\bar{r}_{1}\right)}{\left|\bar{r}_{m}-\bar{r}_{1}\right|^{3}}\right]
\end{aligned}
$$

Let the terms on the right be called $\overline{\mathrm{R}}$ so that:

(4)

$$
\frac{d^{2}}{d t^{2}} \bar{r}+\frac{G\left(m_{1}+m_{2}\right)}{r^{3}} \bar{r}=\bar{R}
$$


When solar pressure is seen by the satellite the equation of motion correspond is; to (4) is:

(5)

$$
\frac{d^{2}}{d^{2}} \bar{r}^{\prime}+\frac{G\left(m_{1}+m_{2}\right)}{r^{\prime 3}} \bar{r}^{\prime}=\bar{R}^{\prime}+\frac{F_{s}}{m_{2}}
$$

where primed values denote the values obtained with solar pressure. The acceleration of the earth due to solar pressure is negligible.

C-4 Relative Motion of the Solar-Forced-Satellite to the Unforced-Satellite (the incremental motion)

Subtracting equation (4) from (5) yields:

(6)

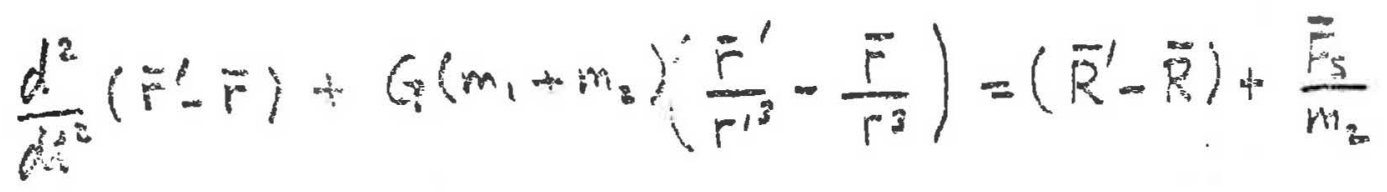

Let $\quad \overline{\Delta r}=\bar{r}^{\prime}-\bar{r}$

and $\quad r^{\prime}=r+\dot{d} r$

Then $\quad \frac{r^{\prime}}{r^{3}}-\frac{\vec{r}}{r^{3}}=\frac{\bar{r}+\dot{A r}}{(r+d r)^{3}}-\frac{\bar{r}}{r^{3}}$

(7)

$\approx \frac{A F^{2}-3 \frac{2 . r}{r} \bar{r}}{r^{3}}$

But $\oint r$ is approximately equal to the projection of $\overline{\Delta r}$ on $\vec{T}$ when $|\overline{\Delta r}|$ is small compared to $|r|$.

Therefore $\delta P \cong \Delta P \cdot|\overrightarrow{\mid}| \overrightarrow{\mid}$ and the equation of incremental motion (6)
approximated by:

(8)

$$
\frac{d^{2}}{d t^{2}} \overline{\Delta r}+\frac{G\left(m_{1}+m_{2}\right)}{r^{3}}\left[\Delta r-3 \frac{\delta r}{r}\right]=\left(\tilde{R}^{\prime}-\tilde{R}\right)+\frac{r_{s}}{r_{2}}
$$

$C-3$ 


\section{C-5 Calculation of the Incremental Motion}

In solving equation (8) we simplify by first assuming that the term $\bar{R}^{\prime}-\bar{R}$ which is the sum of increments in solar and lunar gravitational forces, is negligible. This assumption is shown to be valid in Attachment $\mathrm{C}-1$ for the condition in which the incremental motions are small.

For simplicity, the satellite orbit is assumed basically circular and the satellite mass is assumed small with respect to the earth. Then, the function $\frac{G\left(m_{1}+m_{2}\right)}{r^{3}}$ equals the square of the sateliite, orbital rate $\omega$.

Equation (8) then reduces to:

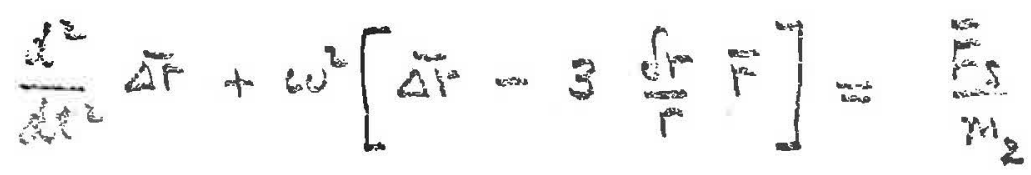

The coordinate system used for the numerical evaluations is shown in the sketch below. The sun lies on the $+X$ axis, the orbit is tilted through an angle $i$ about the $Z$ axis. $\theta$ is the vehicle orbit angle measured from the $+Z$ axis. Unit vectors $\bar{I}, \bar{J}, \bar{K}$ lie along the $\mathrm{X}, \mathrm{Y}$, and $\mathrm{Z}$ axes, respectively.

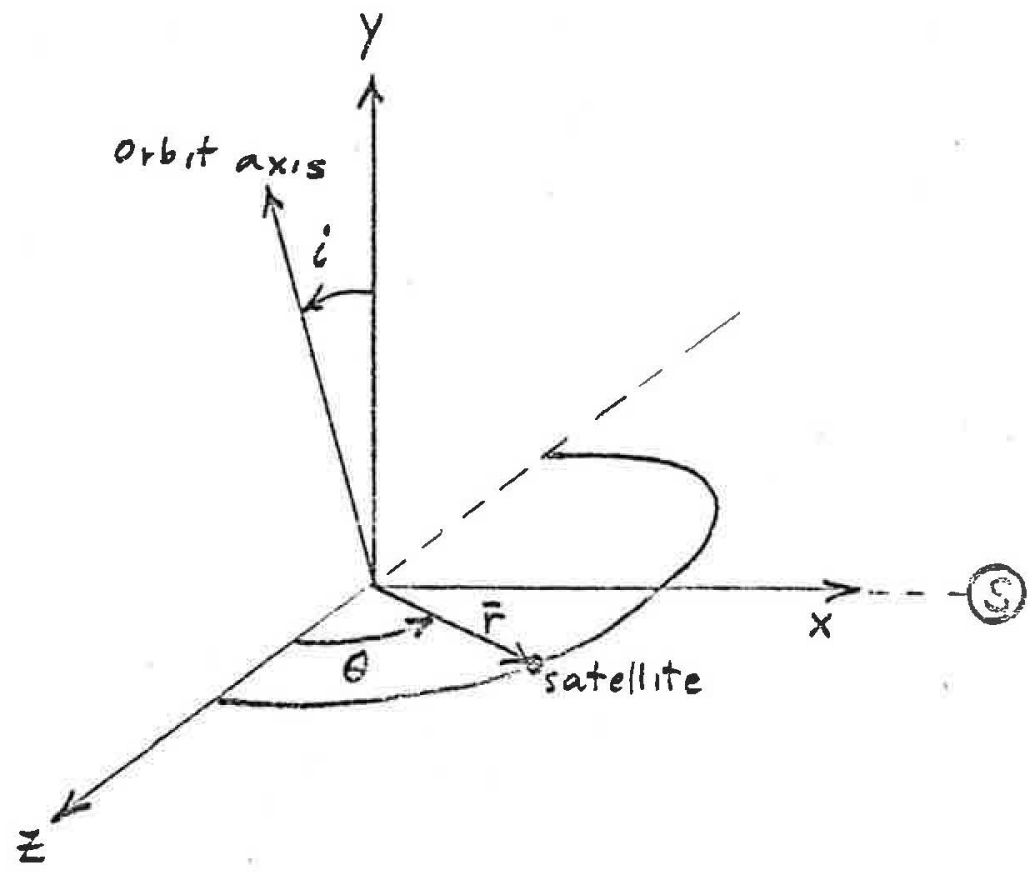


Then, the radius vector $\bar{r}$ is given by:

$$
\bar{r}=(\cos i)(\sin \theta) \bar{b}+(\sin i k \sin \theta) \bar{j}+(\cos \theta) \bar{k}
$$

Tha vector $\overline{\Delta r}$ is given by:

$$
\Delta T=\Delta Y \bar{b}+\Delta Y \bar{J}+\Delta F \vec{F}
$$

Incorporating these relations in equation (9) gives the equations of incremental motion:

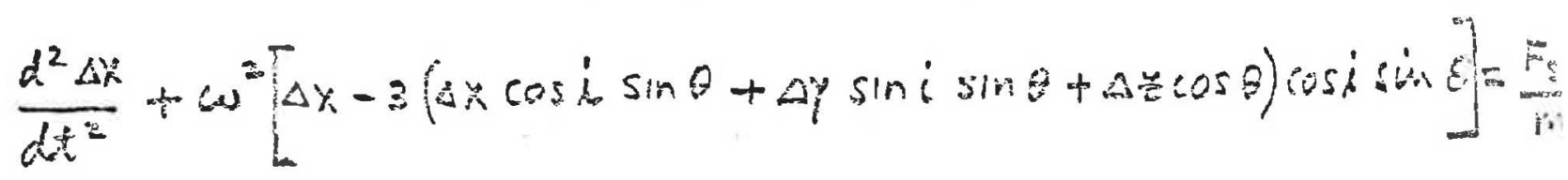

12)

$$
\begin{aligned}
& \frac{d^{2} \Delta y}{d t^{2}}+\omega^{2}\left[\Delta y-3\left(\Delta x \cos i \sin \theta+\Delta y \sin i \sin \theta^{2}+\Delta z \cos \theta\right) \sin i \sin \theta=\frac{F}{d x^{2}}\right. \\
& \frac{d^{2} \Delta z}{d t^{2}}+\omega^{2}[\Delta-3(\Delta x \cos i \sin \theta+\Delta y \sin i \sin \theta+\Delta z \cos \theta) \cos \theta]=\frac{F}{m}
\end{aligned}
$$

It should be noted that equations (12) may also be regarded as differential equations which describe the motion of a satellite with a given solar pressure force relative to the satellite with a different solar pressure force, i.e., in equation (6) the term $\frac{F_{2}}{m_{2}}$ can represent the increment in acceleration due to incremental solar pressure forces. With this interpretation, equation (12) can be used to determine the errors in predicted satellite location due to mis-estimates of the solar pressure forces.

C-6 Simulation Results

Numerical solutions to equation (12) for a 12-hour orbit were determined using the digital computer program, given in Attachment $\mathrm{C}-2$, for the values for $\mathrm{i}=0^{\circ}, 30^{\circ}, 60^{\circ}$ and $90^{\circ}$. 
Solar force per unit mass $\left(F / \mathrm{sx}^{\prime}\right)$ was set at $1.4 \times 10^{-7} \mathrm{ft} / \mathrm{sec}^{2}$, based on a nominal total solar force of $70 \times 10^{-7} \mathrm{lb}$ and satellite mass of 50 slugs. The incremental effect of an incremental solar force of $\frac{1}{100}$ of the basic solar force was also simulated. The output plots give the components of the incremental displacement of the satellite a!.ung. and normal to the radius vector.

Fig. $\mathrm{C}-1$ thru $\mathrm{C} 4$ show the increase in amplitude of the displacement, resulting from full steady solar pressure force, as the satellite orbit plane approaches the sunline. An orbit-to-orbit inc rease is shown when the inclination $i ; 90^{\circ}$. This follows from equation (9) wherein $\dot{C}$, the increment in radius vector length, is zero if the displacement $\Delta r$ is normal to the radius vector. In this event $\left(i=90^{\circ}\right.$ ) the equations are linear in $\overline{\Delta r}$ and represent simple undamped harmonic oscillations with

as forcing function. As $i$ approaches zero so that $\overline{\Delta r}$ and $\bar{r}$ are not normal there is an increase in $E r$ which allows "instability", e.g., if $\bar{\Delta} r$ is aligned with $\bar{r}$ then and equation (9) represents an unstable second-order system. The term

represents the increment in the gravitational force of the earth on the satelite due to the increment in satellite orbit radius vector. In effect there is an inherent "gain" in the solar pressure forces due to the change in gravitational effect.

Eig. C-5 depicts the time variation of the error in estimation of satellite position when an error of $1 \%$ is made in estimating constant solar pressure forces. Again, increasing errors occur as the inclination, i, approaches zero. After three orbits ( $11 / 2$ days), errors up to 4 feet are obtained when $i=0^{\circ}$. The errors oscillate during each orbit. A comparison of the results for full and partial solar pressure forces shows that the displacements are fairly linear to the solar pressure forces.

Fig. C -6 shows the effect of mis-estimating the solar pressure force on the satellite, when $i=90^{\circ}$, due to an attitude error of the vehicle. The vehicle nominally points toward the center of the earth (along $\overline{\mathrm{r}}$ ) but actually points 0.5 degree out of the $\mathrm{YZ}$ plane. A component of the solar pressure force is thereby exerted along the radius vector and is fixed relative to the vehicle. The assumed unknown disturbance acceleration is directed along $\overline{\mathrm{r}}$ with magnitude $1.4 \times 10^{-7} \mathrm{x}$ sin $0.5^{\circ}$. A gradual increase in satellite displacement, accumulating at the rate of about $1 / 2$ foot per orbit, is noticed.

C-7 Conclusions

1. The effect of solar pressure forces, on a vehicle in nominal circular orbit, is to destabilize the basic vehicle motion so that increasing displacement increments occur with time.

2. Large variations in displacement occur during each orbit. (Just how long the destabilization remains is unknown.)

3. The destabilized motion increases with approach of the orbit plane to the sun-line. 

force.

4. The displacements increase relatively linearly with total solar pressure

5. Cyclic variation in solar pressure force at orbit frequency can cause increasing displacement amplitudes.

6. Small changes in solar pressure force can cause increasingly large displacement error oscillations.

Because facilitating ephemeris predictability is an important goal in GPS design, the GPS design should be as accurately modellable as possible. If a fixed percentage error is really necessary, one should minimize the basic solar pressure force by minimizing the surface area. Random changes in vehicle attitude, in external configurtion, and in radiation from the vehicle should be minimized in order both to promote predictability before launch and to aid on-orbit evaluation of the forces on the vehicle. GPS design and/or operation should be such as to minimize force changes on the vehicle resulting from attitude perturbations or variations in radiation to or from the vehicle. 


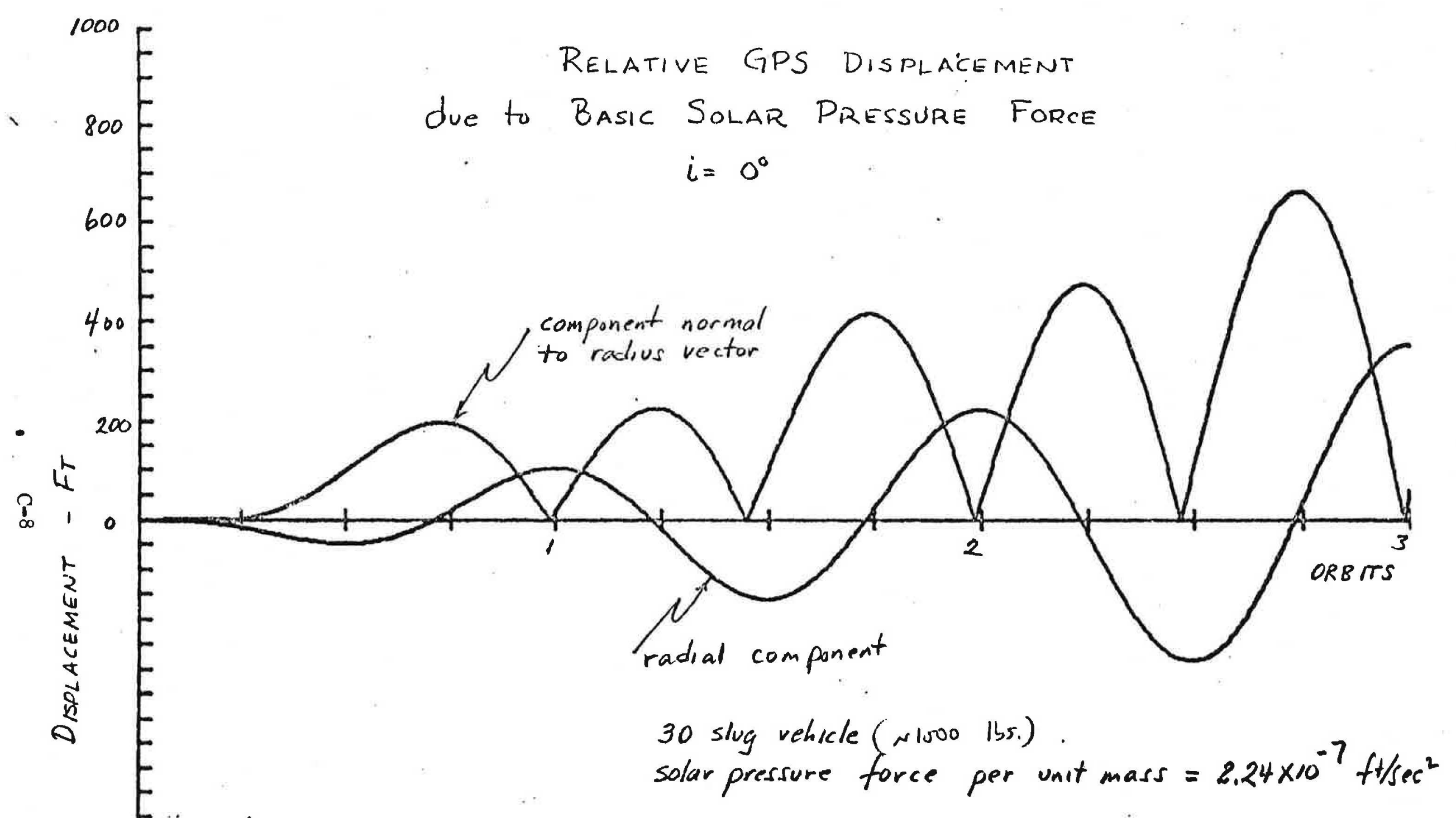

F IGURE C-1 


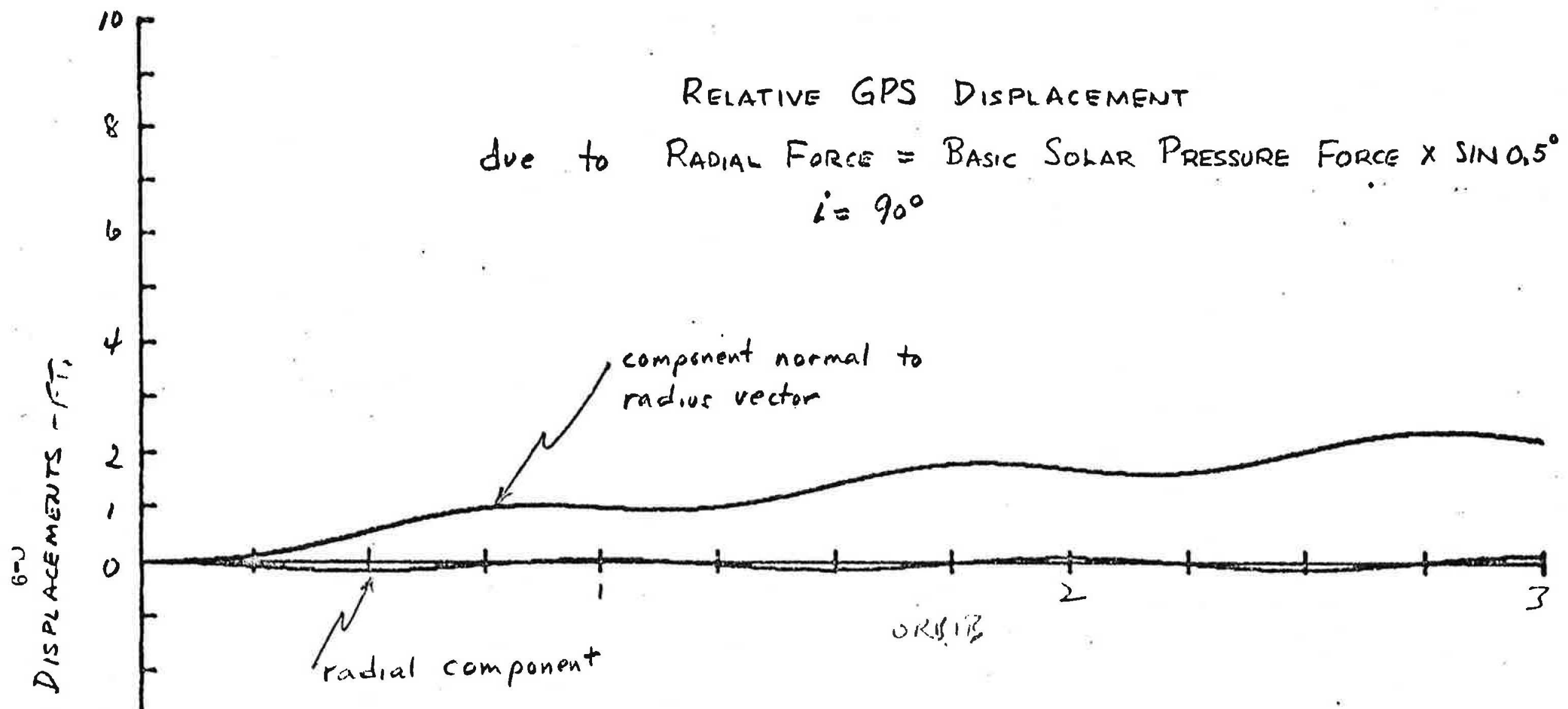

30 slug vehicle, N1500 13.)

$\frac{\text { solarpressurc force }}{\text { vetincle mass }}=2.24 \times 10^{-7} \mathrm{ft} / \mathrm{sec}^{2}$

FIGURE C-2 


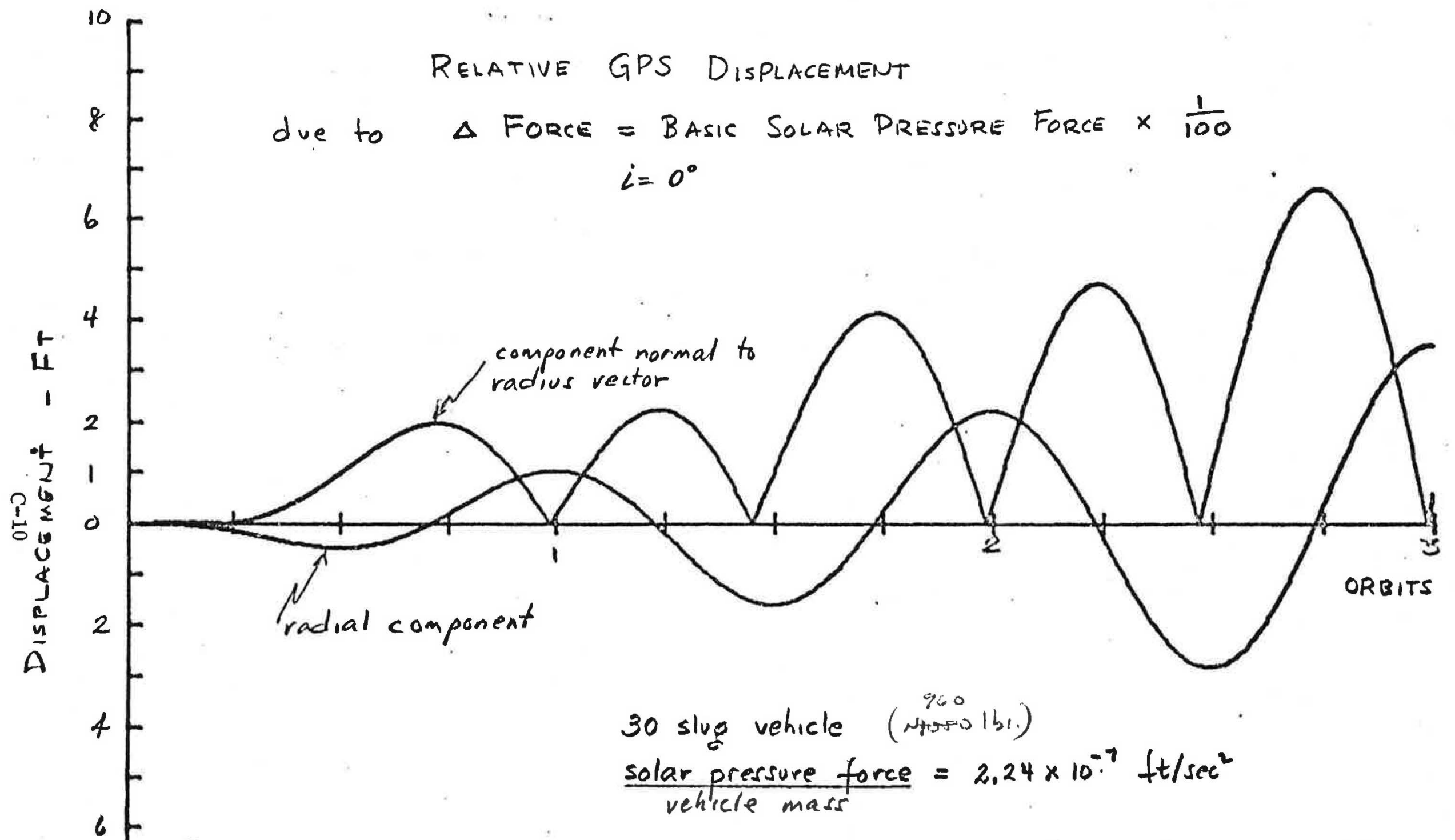

FIGURE C-3 

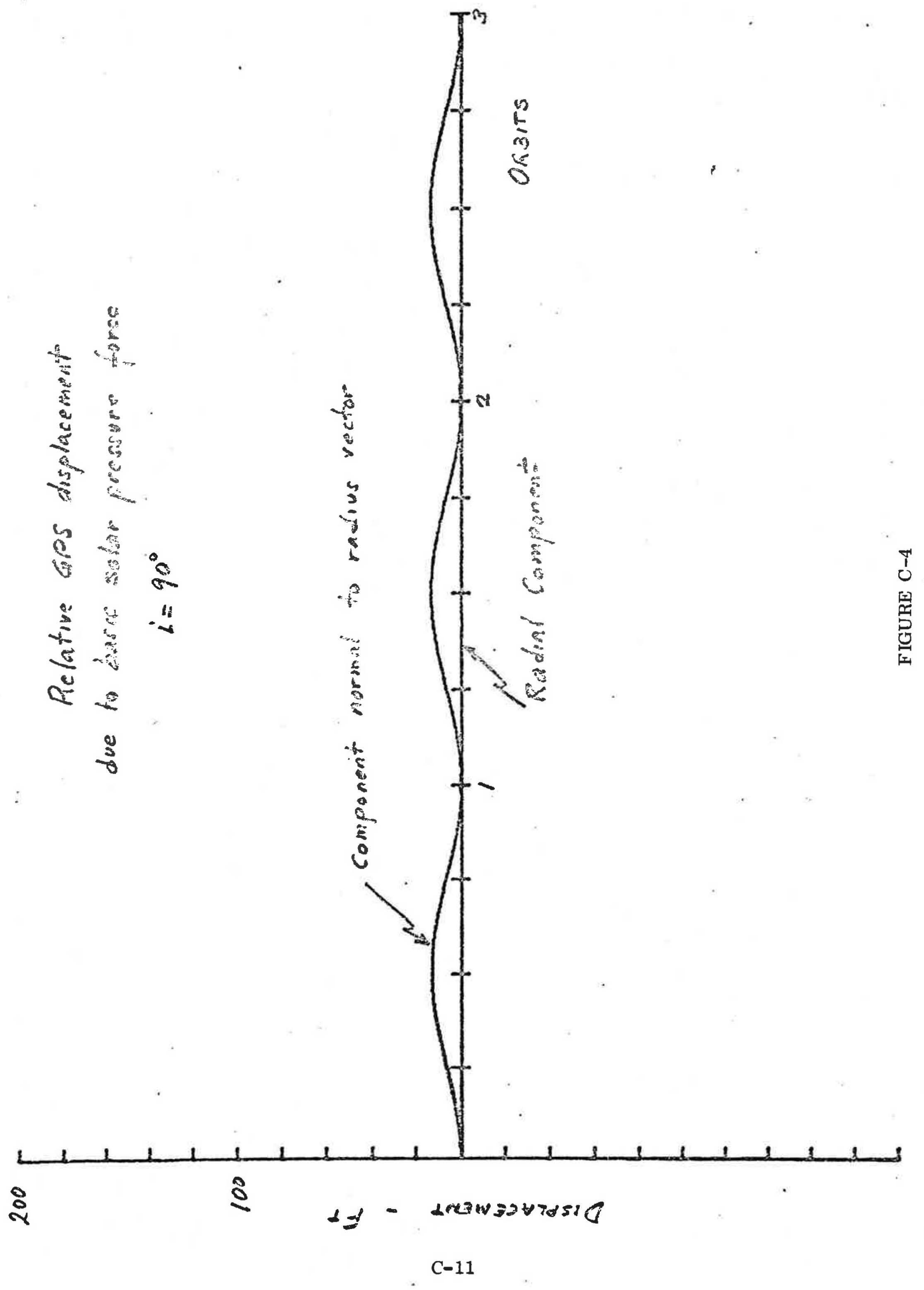

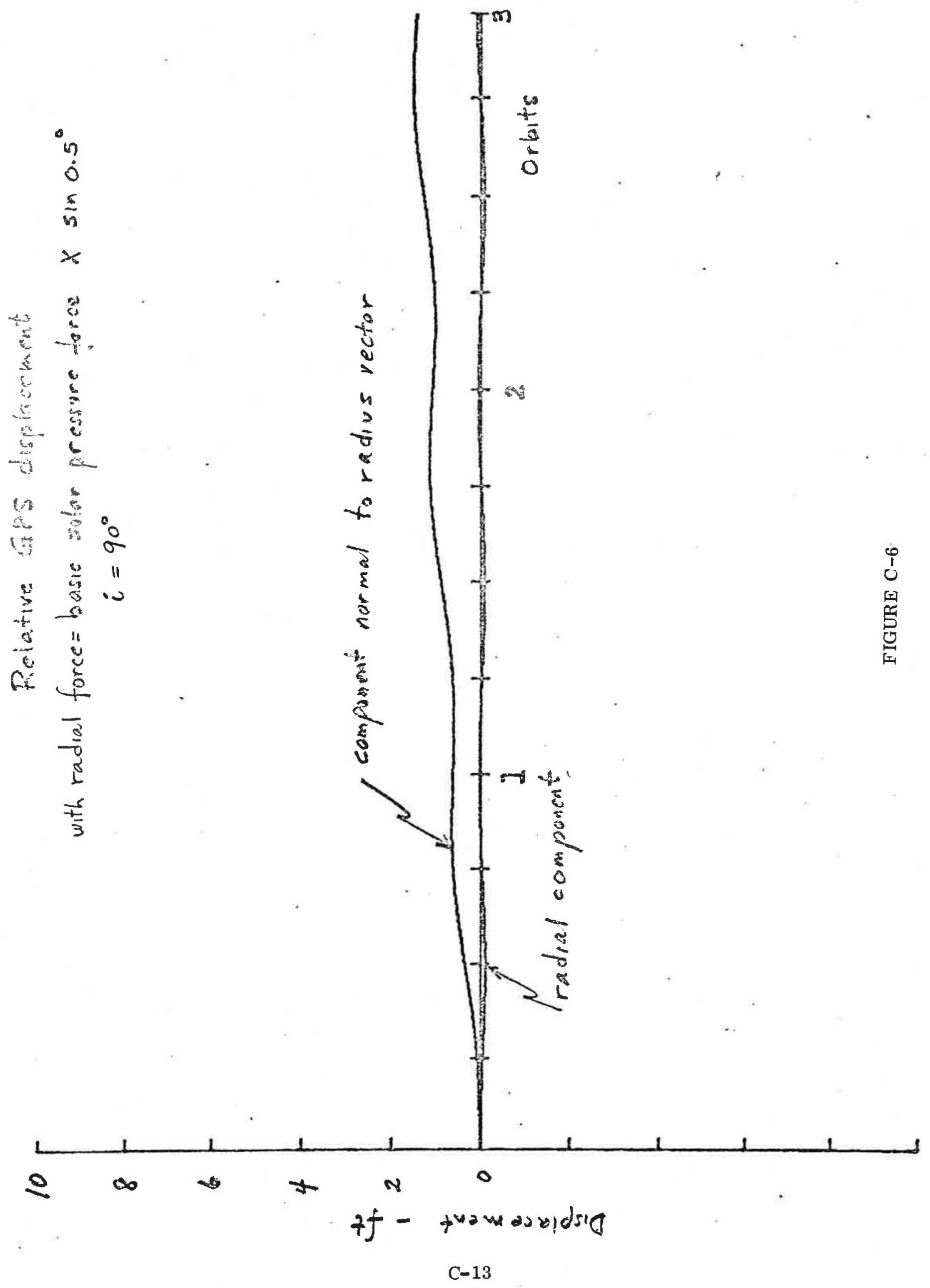
ATTACHMENT C-1

Evaluation of Approximation in Equation (S)

The incremental accelerations due to sun and moon gravity are:

$$
\begin{aligned}
& \bar{R}^{\prime}-\bar{R}=G m_{s}\left[\frac{\bar{r}_{s}-\bar{r}_{2}^{\prime}}{\left|\bar{r}_{s}-\bar{r}_{2}\right|^{3}}-\frac{\bar{r}_{s}-\bar{r}_{1}}{\left|\bar{r}_{s}-\bar{r}_{1}\right|^{3}}\right] \therefore G_{m_{m}}\left[\frac{\left(\bar{r}_{m}-\bar{r}_{2}^{\prime}\right)}{\left|\bar{F}_{m}-\bar{r}_{2}^{\prime}\right|^{3}}-\frac{\left(\bar{r}_{m}-\bar{r}_{1}\right)}{\left|\bar{r}_{m}-\bar{r}_{1}\right|^{3}}\right]
\end{aligned}
$$

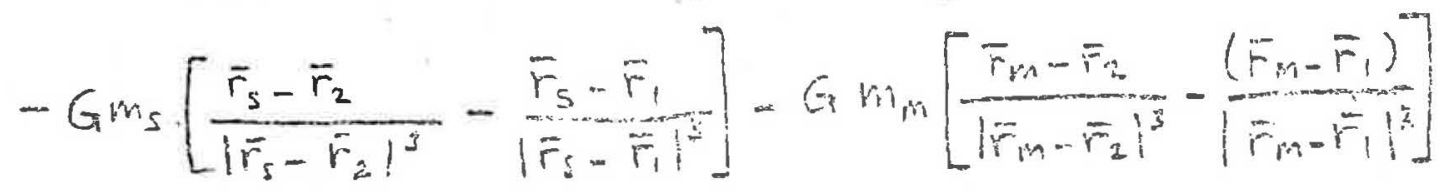

$$
\begin{aligned}
& =G_{m}\left[\frac{\left(\bar{r}_{s}-\bar{r}_{2}^{\prime}\right)}{\left|\bar{r}_{s}-\bar{r}_{2}^{\prime}\right|^{3}}-\frac{\left(\bar{r}_{s}-\bar{r}_{2}\right)}{\left|\bar{r}_{s}-\bar{r}_{2}\right|^{3}}\right]+G_{m}\left[\frac{\left(\bar{r}_{m}-\bar{r}_{2}^{\prime}\right)}{\left|\bar{r}_{m}-\bar{r}_{2}^{\prime}\right|^{3}}-\frac{\left(\bar{r}_{m}-\bar{r}_{2}\right)}{\left|\bar{r}_{m}-\bar{r}_{2}\right|^{2}}\right]
\end{aligned}
$$

Consider first the term associated with the acceleration due to solar forces.

Let $\quad \bar{R}_{s}=\bar{F}_{s}-\bar{F}_{2}, \quad \vec{R}_{s}^{\prime}=\bar{r}_{s}-\bar{F}_{i}^{\prime}, \quad\left|\bar{R}_{s}^{\prime}\right|=F_{s}+s$ so that $\bar{R}_{s}^{\prime}-\bar{R}_{s}=F_{2}^{\prime}-\bar{F}_{2}=\overline{\Delta i}_{i}^{\prime}$.

$$
\begin{aligned}
& \left(\bar{R}^{\prime}-\bar{R}\right)_{\text {SUN }}=G m=\left[\frac{\left(\bar{r}_{s}-\bar{r}_{2}^{\prime}\right)}{\left|\overline{r_{s}}-\bar{r}_{2}^{\prime}\right|^{3}}-\frac{\left(\bar{r}_{s}-\bar{F}_{2}\right)}{\left|\overline{r_{s}}-\bar{F}_{2}\right|^{3}}\right] \\
& \frac{\left(\bar{R}^{\prime}-\bar{R}\right)_{S \text { SN }}}{G M_{s}}=\frac{\bar{R}_{s}^{\prime} R_{s}^{3}-\bar{R}_{s} R_{s}^{\prime 3}}{R_{s}^{\prime 3} R_{s}^{3}} \\
& =\frac{\left(\bar{R}_{s}+\overline{\Delta r}\right) R_{s}^{3}-\bar{R}_{s}\left(R_{s}+\delta\right)^{3}}{R_{s}^{3}\left(R_{s}+\delta\right)^{3}} \\
& =\frac{\overline{\Delta r}-\bar{R}_{5}\left(3 \frac{\delta}{R_{5}}+3\left[\frac{\delta}{R_{5}}\right]^{2}+\left[\frac{\delta}{R_{5}}\right]^{3}\right.}{R_{5}^{3}\left(1+\frac{\delta}{R_{5}}\right)^{3}}
\end{aligned}
$$

C-14 
Attachment C-1

Evaluation of Approximation in Equation (8)

But $f=\Delta r$ and $\Delta r$ is assumed small. So that:

$$
\begin{aligned}
& \frac{\left(\bar{R}-\overline{R_{i}}\right)_{s}}{G m_{s}} \cong \frac{\overline{\Delta r}-\bar{R}_{s}=\frac{\delta}{R_{s}}}{R_{s}^{3}}
\end{aligned}
$$

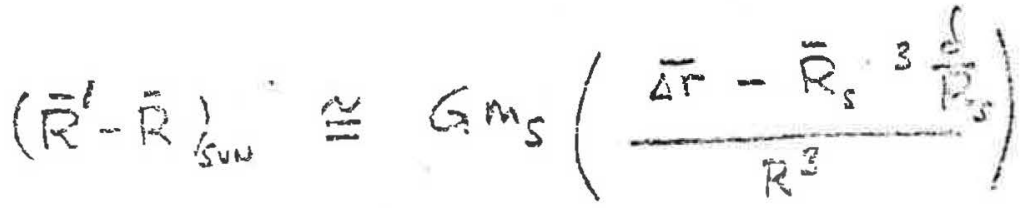

and

so that the amplitude of $\left(\overline{\beta^{\prime}}-\bar{R}\right)_{5,3}$ is limited by:

$$
\mid\left(R^{2}+\left.\right|_{\text {sur: }}\left|\leq \frac{G m_{s}}{R_{s}^{3}} \cdot\right| \psi r a r \mid\right.
$$

But $\frac{G m_{S}}{R_{S}^{3}}=\omega_{e}^{2 \cdot}$ the square of the earth's orbital rate about the sun.

$$
\begin{aligned}
\therefore\left|\left(\bar{R}^{\prime}-\bar{R}\right)_{s w+1}\right| & \leq \omega_{e}^{2} 4|\Delta r| \\
& \leq 1.58 \times 10^{-13} \Delta r \quad \mathrm{ft} / \mathrm{sec}^{2}
\end{aligned}
$$

The equivalent relation for the incremental acceleration due to the moon is:

$$
\left|\left(\bar{R}^{\prime}-\bar{R}\right)_{\text {Ra O }}\right|=\frac{G m_{M}}{R_{M}^{3}} \cdot 4 \Delta r \mid
$$

where $\bar{R}_{M}=\bar{r}_{M}-\bar{r}_{2}$. Thus the ratio of incremental accelerationgdue to the moon and sun is:

$$
\frac{\frac{m_{M}}{R_{p H}^{3}}}{\frac{m_{s}}{R_{s}^{3}}}=\frac{m_{M}}{M_{s}} \cdot \frac{R_{s}^{3}}{R_{M}^{3}}=\frac{\frac{m_{m}}{m_{e}}}{\frac{m_{s}}{m_{e}}} \cdot\left(\frac{R_{s}}{R_{m}}\right)^{3}
$$

$C-15$ 


\section{Attachment C-1}

Evaluation of Approximation in Equation (8)

where $m_{e}=$ earth mass.

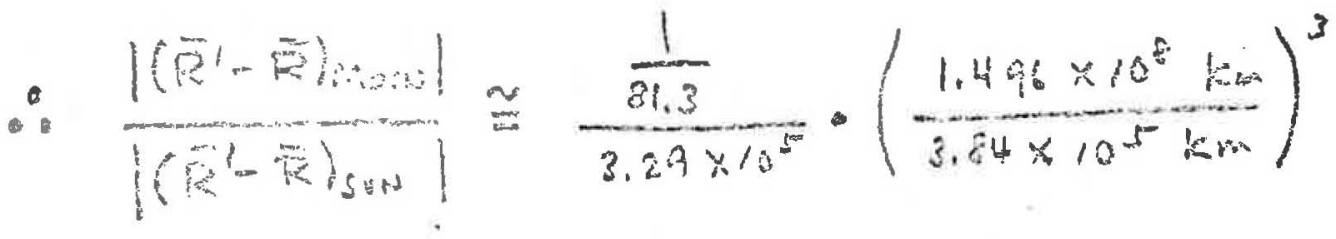

$$
\begin{aligned}
& \cong 2.2
\end{aligned}
$$

But the basic acceleration due to solar pressure force on the GPS is about $1.4 \times 10^{-7} \mathrm{ft} / \mathrm{sec}^{2}$ which is large compared to $10^{-13} \mathrm{ft} / \mathrm{sec}^{2}$. Thus, for small values of $\Delta \mathrm{r}$ (say $\leq 10^{4} \mathrm{ft}$ ) with the basic solar pressure force, or for accelerations at least $10^{3}$ greater than the incremental solar and lunar accelerations, the incremental accelerations due to sun and moon gravity may be neglected. 
160 SET DEGREES

119 HINDOH $0,1086,-490,480$

120 fixis 90,20

130 MOUE 0,0

$140 \quad I=0$

$150 \quad N=0$

$1600=1$

$170 \quad \mathrm{Y} \theta=0$

$183 \quad Y 8=0$

$190 \quad \times 1=0$

$200 \quad 11=0$

$21021=0$

$22020=6$

$230 \mathrm{E}=0$

$240 \quad T 2=0$

$250 \quad C=-1.45-7$

$26001=2 * P I /(12 * 3600)$

$27002=01+2$

$280 T I=2 \times P I / 01 * 0 ; 368$

$280 \quad i=0$

$309 n=0$

310 PRINT

320 REM

$33 B j=\operatorname{CoS}(I) \times S I N(A)$

$340 K=5 I N(I) * S I N(A)$

$350 L=005(A)$

$360 \quad P 2=3$

37 B $=20 \mathrm{~N}+Y 0 * k+20 \mathrm{k}$

380 H $=c-02$ (

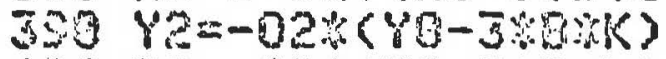

$40322=02 \times(20-3.021)$

480 RI=YOKL-208k

$420 R=20 \% J-20 \%$

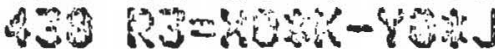




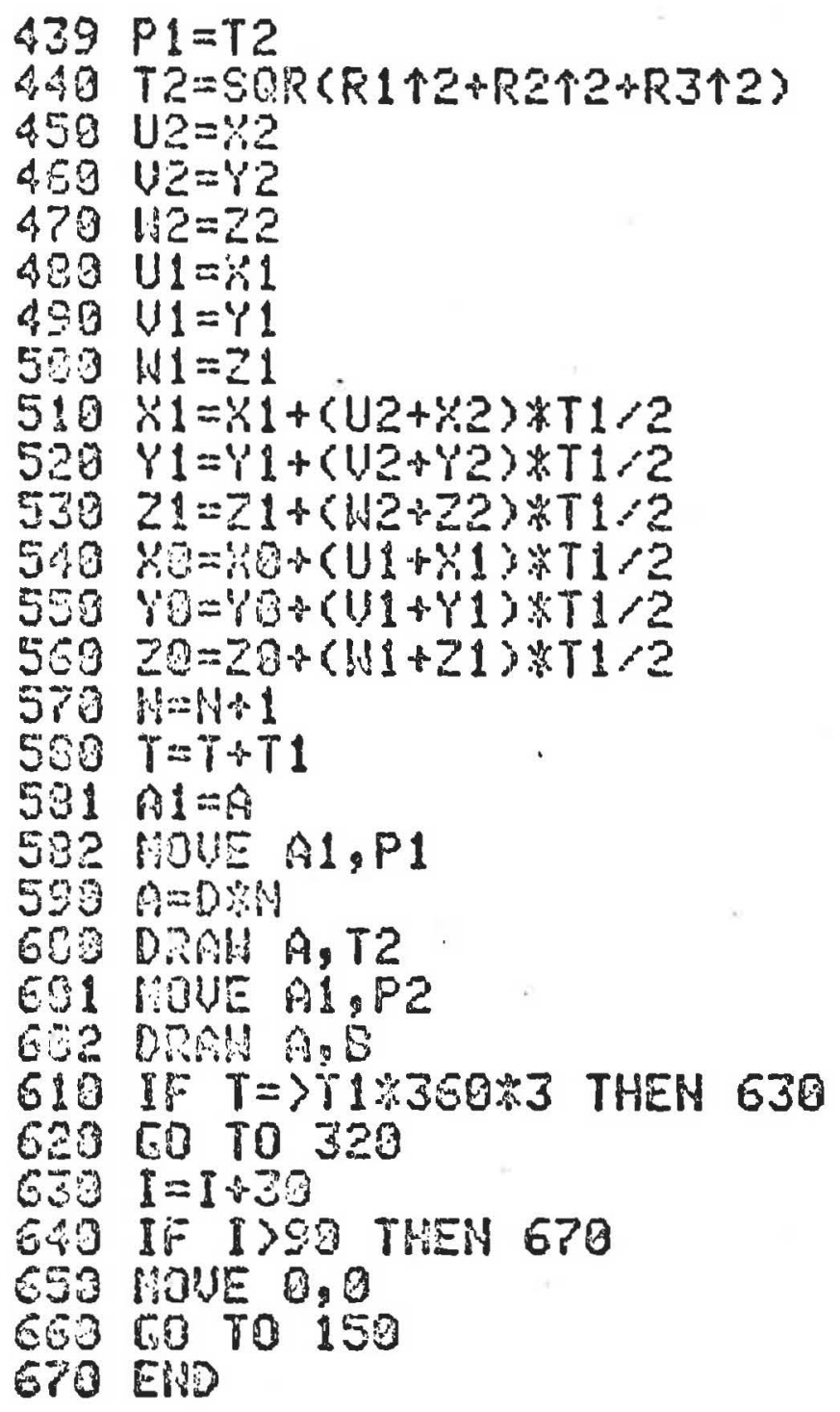


D. AN EXAMPLE OF THE EFFECT OF EPHEMERIS ERRORS ON USER POSITION DE TERMINA TION 

D- $1.0 \quad$ INTRODUCTION

Several previous studies have determined GPS orbit pertubations as a function of external forces. Components of these pertubations along and normal to the satellite radius vector were determined. This study considers the relative effects of these components in causing user errors in position determination.

The specific situation examined assumes three satellites in 12 hour orbit available to the user. The satellites are assumed equally spaced in azimuth and at equal angles above the user's horizon. Ranges to the satellites are measured perfectly and two of the satellites' exhemerides are known perfectly. The ephemeris of the third satellite is in error in its radial distance or in distance normal to its radius vector.

D-2.0 ANALISIS

Figure D1 illustrates the geometry of the situation:

the user is located at $0,0,0$

$\theta$ is the angle above the horizon for the satellites

$R_{e}$ is the earth's radius

$\mathrm{K}_{\mathrm{S}}$ is the satellites' radial distance to the center of the earth

a is the satellites' range to the user (measured perfectly)

$\psi \quad$ is the angle from the local horizontal plane $X Y$ to the intersection of spheres of radius $a^{\prime}$ centered on the two perfectly located satellites A and B. A and B are symmetrically located with respect to the $\mathrm{XZ}$ plane.

c is the user position determination error.

$\Phi$ is the angle between the plane containing satellites $A, B$ and the user and the $X$ axis.

$\beta \quad$ is the angle between the satellite $C^{\prime}$ s radius vector and the vector from the user to the satellite

$\delta_{\mathcal{L}}$ is satellite $C$ 's ephemeris error normal to the radius vector

$\delta_{R}$ is satellite $C$ 's ephemeris error along the radius vector. 
The user calculates bis position based on the intersection of spheres centered on the individual satellites, presumed at locations specified by the individual ephemerides. The radius of the spheres are the range measurements made by the user.

of

In the situation here, the user calculates his location as the intersection

1) the line which passes through $0,0,0$, lies in the $\mathrm{XZ}$ plane, and is inclined by angle $\psi$ with respect to the $X$ axis, and

2) a sphere of radius a centered on the ephemeris location for sateilite $\mathrm{C}$.

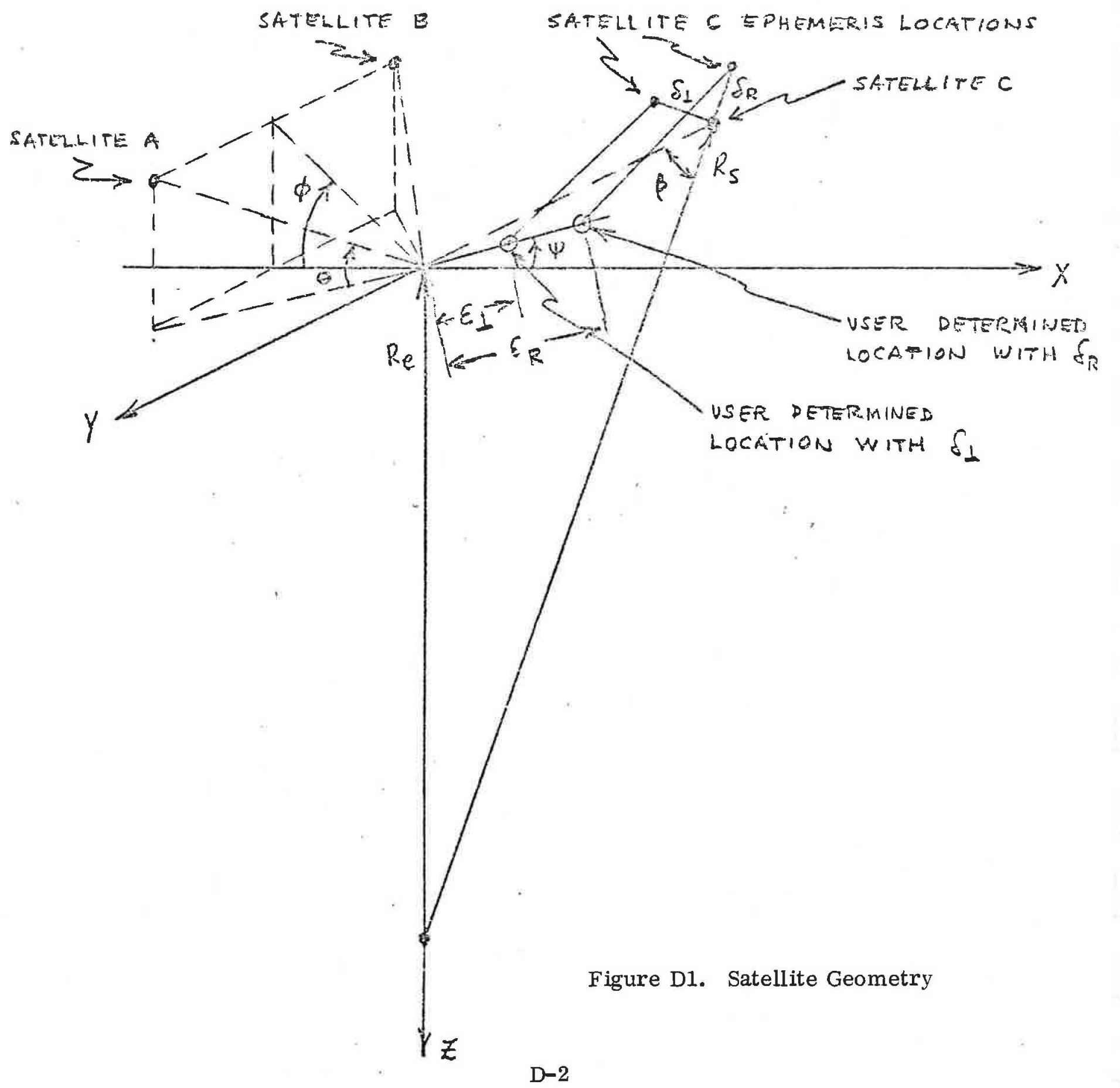


The following equations therefore apply:

$$
\begin{aligned}
& \phi=\tan ^{-1}\left(\frac{1}{2 \tan \theta}\right) \\
& \frac{a}{R_{e}}=-\operatorname{sen} \theta+\sqrt{\left(\frac{R_{s}}{R_{e}}\right)^{2}-\cos ^{2} \theta} \\
& \cos \beta=-\frac{1-\left(\frac{R_{s}}{R_{e}}\right)^{2}+\left(\frac{a}{R_{e}}\right) \sin ^{2} \theta}{\frac{a}{R_{e}} \cdot \frac{R_{s}}{R_{e}}} \\
& \frac{\varepsilon_{1}}{\varepsilon_{\perp}}=\frac{\sin \beta}{\cos (\theta-\psi)} \\
& \frac{\varepsilon_{R}}{\delta_{R}}=\frac{\cos \beta}{\cos (\theta-\psi)}
\end{aligned}
$$

Values of $\frac{\varepsilon}{\delta_{-}}$and $\frac{\varepsilon}{\delta R}$ were computed as function of angle $\theta$ using the program listed in Figure 2. The results in that figure indicates that ephemeris errors in radial displacement are about 5 times as important as errors in normal-to-radius displacement -- for the conditions a: sumed.

It may be noted that the calculated errors due to $\delta_{I}$ occur when $\delta_{\perp}$ lies in the $\mathrm{XZ}$ plane. When $\delta_{\mathcal{L}}$ is normal to that plane, no user position error exists. To emphasize this fact, the second column in Figure D2 is labelled "Maximum Position Error ...".

$D-3.0$

CONCLUSIONS

Errors in GPS satellite radial position are several times as significant as equal errors in normal to radial vector position in user determination of position. When choice is possible between incurring radial or normal errors, normal errors should be accepted. 


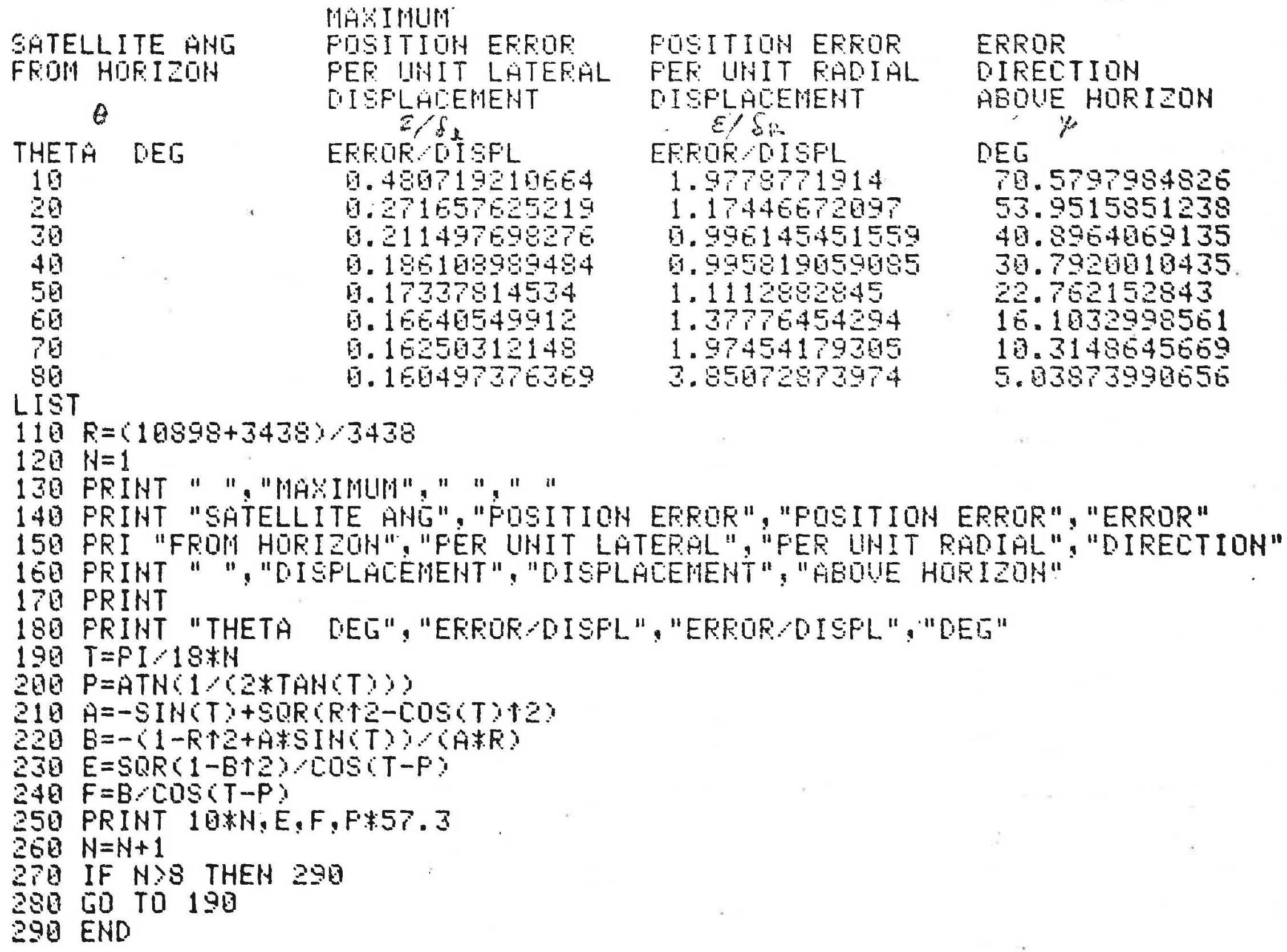

FUSITIOA EREOR

FEF: UHIT FAOIAL

CISFLACENENT $\varepsilon / S$

\section{EFFUF ISFL}

1.9775771914

1. 17446e.72097

0.996145451559

6. 995819059605

1.111208045

1.37F76454294

1.97454179365

3.95072873974

ERROR

DIRECTION ABOUE HORI ION \%

DEE

7B. 5797984826

53.9515851238

40.8964069135

30.7926018435

22.782152843

18. 1832998561

10.3148045669

5.03873998656

$120 \mathrm{H}=1$

130 PRINT " " "MASIMLIN", " " " "

140 PRINT "SATELLITE AHG" "FUSITIOH EFRUR" "FUSITIOH ERROR" "ERROR"

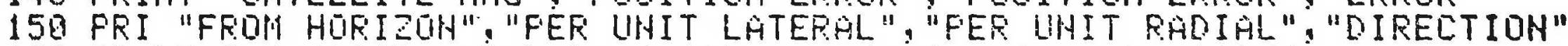

16 PRIHT " " "OISFLACEMENT" "DISFLACEMEHT" "ABOUE HUR IEOH"

170 PRINT

180 FRINT "THETA [EG": "EFFUR OISFL", "ERFURYOISFL": "OEG"

$190 T=P I 18 * 4$

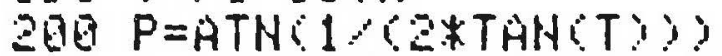

$218 A=-S I H C T Y+S O R C R T-C O S(T) T 2 Y$

$220 B=-(1-R+2+A$ SIH $B$ (T) (AFR)

$230 E=\operatorname{SQR}(1-B+2) \operatorname{COS}(T-F)$

$240 F=B \cos (T-P)$

250 PRINT 10WN,E,F,F\$57.3

$260 \mathrm{~N}=\mathrm{N}+1$

270 IF $N$ S THEH 290

280 5O TO 190

290 END 
E. ORBIT PREDICTION UNCERTAINTY OF DUAL-SPIN AND BIAS-MOMENTUM SPACECRAFT

$m$ 



\section{E1.0 Introduction}

A dual-spin configuration has been proposed for a candidate GPS. This arrangement has the advantages of all dual-spin systems as well as the potential advantage of highly predictable forces due to solar pressure, especially when the despin section is enclosed in a spinning radome. The purpose of this study is to make an estimate of potential predictability of the GPS orbit as influenced by solar pressure on, and attitude control requirements of, such a vehicle.

Total vehicle angular momentum is a parameter in this study. When that angular momentum is sufficiently small, it can be stored in a reaction wheel (rather than the spinning motion of the vehicle itself) so that a bias-momentum system is equivalent, on a dynamic basis, to a low-momentum dual-spin system. It is considered here in that sense.

\section{E-2.0 Analyses \\ E-2.1 Vehicle Angular Error Rate due to Solar Pressure Torques}

The precession rate $\dot{\epsilon}$ equals the external torque (the solar pressure torque $\mathrm{T}$ ) divided by the vehicle angular momentum $\mathrm{H}$.

$$
\begin{aligned}
\dot{\epsilon} & =\frac{T}{H} \\
& =1.2 \times 10^{-3} \mathrm{deg} / \text { day/inch offset }
\end{aligned}
$$

when: vehicle sunlit area $=35 \mathrm{ft}^{2}$

vehicle spin inertia $=200$ slug $\mathrm{ft}^{2}$

vehicle spin rate $=6 \mathrm{rad} / \mathrm{sec}$

axial offset between vehicle c.p. and c.m. = 1 inch

Reasonable offsets of 6 inches may be expected, depending upon vehicle attitude relative to the sun. So that $\dot{\epsilon} \max \cong .007^{\circ} /$ day. 


\section{E-2.2 $\quad$ Precession Requirements Due to Orbit Nodal Regression}

The orbit line-of-nodes rate due to earth oblateness (Reference 1) is:

$$
\dot{\Omega}=\frac{-J n \cos i}{a^{2}\left(1-e^{2}\right)^{2}}
$$

where: $\quad J=1.624 \times 10^{-3}$

$$
\begin{aligned}
\mathrm{a}=\text { semi-major axis/earth radius } & =\frac{10898+3438}{3438} \\
\mathrm{n}=\text { mean angular velocity } & =2 \pi \mathrm{rad} / 12 \text { hours } \\
\mathrm{i}=\text { inclination } & =63^{\circ} \\
\mathrm{e}=\text { eccentricity } & =0
\end{aligned}
$$

so that $\dot{\Omega}=-0.0305^{\circ} /$ day

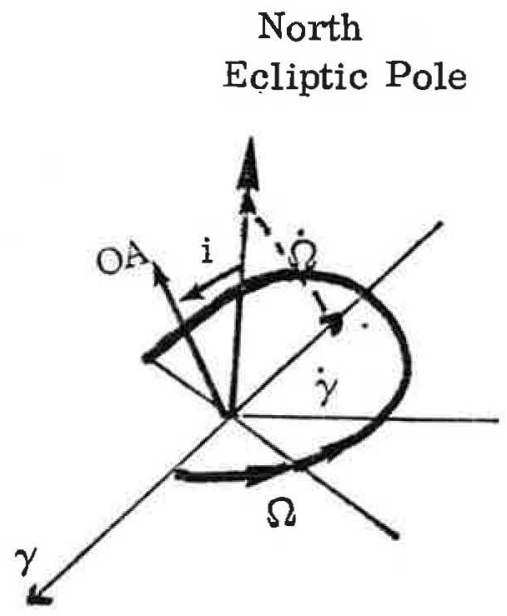

Therefore the orbit precession rate is:

$$
\begin{aligned}
\dot{\gamma} & =\dot{\Omega} \sin i \\
& =0.0272^{\circ} / \text { day }
\end{aligned}
$$

If a $0.25^{\circ}$ error is allowed for despin section earth pointing out of a total error budget of $0.5^{\circ}$, then attitude corrections must be made every $\frac{0.25}{0.0272}=9.2$ days 
a. Angular momentum corrected every 9.2 days is

$$
\begin{aligned}
\Delta H & =\epsilon \mathrm{H} \\
& =5.23 \mathrm{ft}-\mathrm{lb}-\mathrm{sec} . \text { for a } 1200 \mathrm{ft}-\mathrm{lb} \text { sec. vehicle with } \\
\epsilon & =0.25 \mathrm{deg} .
\end{aligned}
$$

b. Fuel use/ $\Delta H$ correction $=\frac{\Delta H}{I_{s p} d}$

$$
=0.0087 \mathrm{lb} \text { for } I_{\mathrm{sp}}=200 \text { sec. with } 3 \mathrm{ft} \text {. moment } \operatorname{arm}\left(\mathrm{d}=\mathrm{A}_{\mathrm{.}}\right)
$$

c. Fuel use/year $=0.0087 \times \frac{365}{9.2}$

$=0.34 \mathrm{lb}$. for $I_{\mathrm{sp}}=200 \mathrm{sec}$. with $3 \mathrm{ft}$. moment arm

d. Jet pulses/day $=\frac{\Delta H}{\mathrm{fdt}} \approx \frac{1}{9.2}$

$=20 /$ day for $0.1 \#$ thrusters, with $3 \mathrm{ft}$. moment arm with 0.1 sec. on-time

E- 2.3 Estimate of Uncertainty in Predicting Solar Pressure Forces

The relative fixity of a spinning vehicle's attitude in inertial space permits an accurate evaluation of the solar pressure forces by ground observers. Observation noise and changes in vehicle attitude relative to the sun-line prevent perfect evaluation. Appendix E-Acontains an evaluation of the rates of change of the attitude relative to the sun-line due to motion of ihe Earth about the sun. The maximum rate of change is low, only $1^{\circ} /$ day, as shown in Appendix E-A, and therefore permits accurate estimation of future solar forces since the solar forces change at correspondingly low rates.

Estimation errors due to the changing sun aspect of the vehicle are primarily due to uncertainty as to the characteristics of the new areas which become sunlit, and the old areas which lose the sun, as time proceeds. It is assumed here that the daily increment in solar force is known within $5 \%$, the persisting force is assumed known exactly. The maximum percentage change in sunlit area, over one day, for a cylindrical vehicle with height $1.5 \mathrm{x}$ as great as diameter is:

$$
\% \text { change }=100 \times \frac{(1.5)(D) \sin 1^{\circ}}{\pi D^{2} / 4}=5 \%
$$


when the circular face looks directly at the sun (orbit plane normal to sun-line). Also,

$$
\% \text { change }=100 \times \frac{\pi \mathrm{D}^{2} / 4 \sin 1^{\circ}}{(1.5 \mathrm{D})(\mathrm{D})}=0.91 \%
$$

when the cylinder axis is normal to the sun line (orbit plane contains the sun-line).

Thus the total percentage force uncertainty varies between $5 \% \times 5 \%=$ $0.25 \%$ and $5 \% \times 0.9 \%=0.045 \%$, depending upon the orbit axis inclination to the sunline. It may be recalled (Reference 2) that the largest error in estimating a satellite's ephemeris is caused by mis-estimating solar forces which occur when the orbit plane contains the sun-line, and the smallest when the sun-line is normal to the orbit plane. Thus the largest uncertainty in estimating solar force occurs when the effect of those uncertainties is least and vice versa.

E-2.4 Displacement Uncertainty Due to Solar Force Uncertainty

The solar force uncertainty after 9.2 days (the required time between firings of the jets to force the vehicle spin axis to remain within $0.25^{\circ}$ of the precessing orbit axis) causes a displacement error uncertainty which depends upon a) the mean force uncertainty and b) the sensitivity of displacement to incremental force. The latter is a function of the angle (i) between the orbit plane and the sun-line.

In Section 2.3, the force uncertainties of $0.25 \%$ per day and $0.045 \%$ per day were determined for angles $i=90^{\circ}$ and $0^{\circ}$, respectively. After 9.2 days these uncertainties could accumulate to $2.3 \%$ and $0.415 \%$, with mean values over that interval of $1.15 \%$ and $0.21 \%$. From Reference 2 (which evaluates errors for a 50 slug SV ), the maximum displacement errors for 9.2 days for a 30 slug SV and for $1 \%$ force error are:

$$
\begin{aligned}
& \text { Max errors after } 9.2 \text { days per } 1 \% \text { force error; } \\
& \begin{aligned}
\mathrm{i}=90^{\circ} \quad \text { normal to radius vector } \quad \delta_{1} & =0.2 \mathrm{ft} \times \frac{5}{3} \\
& =0.33 \mathrm{ft} \\
\text { along radius vector } \quad \delta_{\mathrm{R}} & =0
\end{aligned}
\end{aligned}
$$




$$
i=0^{\circ}
$$

$$
\begin{aligned}
& =46 \mathrm{ft} \\
\text { along radius vector } \quad \varepsilon_{\mathrm{R}} & =13 / 4 \mathrm{ft} / \mathrm{day} \times \frac{5}{3} \times 9.2 \mathrm{day} \\
& =27 \mathrm{ft}
\end{aligned}
$$

normal to radius vector $\quad \delta_{1}=3 \mathrm{ft} /$ day $\times \frac{5}{3} \times 9.2$ day

Using the estimated actual daily errors of $0.25 \%$ and $0.045 \%$ : Maximum error after 9.2 days:

$$
\begin{aligned}
& i=90^{\circ} \quad \text { normal to radius vector } \quad \delta_{1}=0.33 \times 1.15 \\
& =0.38 \mathrm{ft} \\
& \text { along radius vector } \quad \delta_{\mathrm{R}}=0 \\
& i=0^{0} \quad \text { normal to radius vector } \quad \delta_{1}=46 \times 0.21 \\
& =9.6 \mathrm{ft} \\
& \text { along radius vector } \quad \delta_{R}=27 \times 0.21 \\
& =5.7 \mathrm{ft}
\end{aligned}
$$

Values for intermediate angle $i$ are estimated as:

Maximum error after 9.2 days:

$$
\begin{array}{lll}
\mathrm{i}=60^{\circ} & \text { normal to radius vector } & \delta_{1}=4 \mathrm{ft} \\
& \text { along radius vector } & { }_{1} \delta_{\mathrm{R}}=3 \mathrm{ft} \\
\mathrm{i}=30^{\circ} & \text { normal to radius vector } & { }^{\delta_{1}}=7 \mathrm{ft} \\
& \text { along radius vector } & { }_{\delta_{\mathrm{R}}}=5 \mathrm{ft}
\end{array}
$$

All these values are maximum or $3 \sigma$ values.

E-2.5 Estimation of Orbit Disturbances Due to Unmatched or Misaligned Thrusters

The GPS thrusters fired every 9.2 days move the vehicle spin axis $0.25^{\circ}$ to account for vehicle orbit plane nodal regression. In order to minimize $\Delta \mathrm{V} \quad$ effects due to jet firing, the jets are fired in pairs so as nominally to apply couples to the vehicle. Due to mismatch between jets there will be a 
$\Delta V \quad$ along an axis normal to the nominal line-of-thrust. Evaluation of these orbital disturbances was accomplished using the computer program of Reference 1. That program, altered as required for the impulse disturbances, is documented in Appendix E-B. Appendix E-B also contains computer results for jet mismatches when line-of-thrust is normal to the orbit plane, along the radius vector, and along the vehicle's velocity vector. Jet misalignment effects are determined from jet mismatch effects by considering that the misalignment causes an error impulse along a normal axis. The effects of that error impulse are the same regardless of their source --mismatch or misalignment.

Computer runs were made for twe sets of conditions. In the first, the required 0.25 degree reorientation was achieved by a single thrusting. In the second, two thrustings were made, one-half orbit apart. It was assumed here that the jet mismatches/misalignments remained unchanged during this time interval. A resultant compensating effect occurs yielding a decided improvement in orbit perturbation. * A comparison of the resultant orbit perturbations indicates that minimum perturbation, due to residual delta V, occurs when the line-ofthrust of the jets is normal to the orbit plane; indeed there is a negligible effect due to thrust mismatch. A misorientation of the jets by 0.5 degrees can add an increment to the along-orbit velocity. The incremental impulse I equals sin $0.5^{\circ}$ time the sum of the individual jet impulses $2 \mathrm{~F} \Delta \mathrm{t}$. Since the total precession angular momentum $\Delta \mathrm{H}$ equals the impulse $2 \mathrm{~F} \Delta \mathrm{t}$ times the moment arm d (taken as 3 feet) we have:

and

$$
\Delta \mathrm{H}=2 \mathrm{Fd} \Delta \mathrm{t}
$$

$$
\begin{aligned}
I & =\frac{\Delta H}{d}\left(\sin 0.5^{\circ}\right) \\
& =\frac{5.23 \mathrm{ft} \mathrm{lb} \mathrm{sec}}{3 \mathrm{ft}}\left(\sin 0.5^{\circ}\right) \\
& =.0152 \mathrm{lb} \mathrm{sec}
\end{aligned}
$$

* This is due to the approximate equality of orbit period before and after the two thrustings. 
In Appendix E-B the orbit perturbation obtained with impulses of 0.029 lb sec, displacements, along and normal to the radius vector are $20 \mathrm{ft}$ and 55 $\mathrm{ft}$, respectively, for a vehicle with a $1200 \mathrm{ft} \mathrm{lb}$ sec angular momentum with delta $\mathrm{V}$ along the orbit. Displacements are much smaller for an equal delta V along the radius vector. Since orbit effects are roughly proportional to the perturbations, and the perturbations (jet impulses) are proportional to vehicle angular momentum $\mathrm{H}$ we can approximate * orbit effects by:

Max. displacement along radius vector $=\frac{\mathrm{H}}{1200} \times 20 \times \frac{0.0152}{0.029} \mathrm{ft}$

Max. displacement normal to radius vector $=\frac{\mathrm{H}}{1200} \times 55 \times \frac{0.0152}{0.029} \mathrm{ft} * *$

E-2.6 Estimate of Uncertainty in Predicting Orbit Perturbations of a Bias Momentum System

Bias momentum stabilized vehicles, like dual spin vehicles, tend to respond slowly to external torques. Since the precession rate is proportional to vehicle angular momentum, and stored angular momentum is limited by allowable wheel weight to about $50 \mathrm{ft}-1 \mathrm{~b}-\mathrm{sec}$, they are far less fixed in space than reasonable dual-spinners.

Using the numerics in Section E-2.1, a 50-ft-1b-sec vehicle will have a precession rate, due to solar pressure torques, of $\dot{\epsilon}_{\max }=\frac{1200}{50} \times 0.007 \%$ day $=$ $0.168^{\circ}$ day. for a 6 -inch offset between c.p. and c.m. If vehicle reorientation is required when the precession angle is 0.25 , then jet thrusting will be required every $\frac{.25}{.168}=1.49$ days.

* When spin angular momentum goes below about $300 \mathrm{ft} \mathrm{lb} \mathrm{sec}$, the period at which jets must be fired to reorient the vehicle due to precession occasioned by soiar pressure torques approaches 9 days, so that the total orbit disturbances are not closely approximated by jet firings to follow the precessing orbit.

** The along radius vector component increases slowly with time due to the change in orbit period from the nominal period. It may conservatively be estimated to double in 9.2 days. Doubled values are used in the Table 1 compilation. 
Total jet impulses would be $\frac{1200}{50}=\frac{1}{24}$ as large as required for the $1200 \mathrm{ft} \mathrm{lb} \mathrm{sec} \mathrm{dual-spinner,} \mathrm{and} \mathrm{all} \mathrm{vehicle} \mathrm{effects} \mathrm{will} \mathrm{be} \mathrm{in} \mathrm{the} \mathrm{same} \mathrm{proportion.}$ Over a 9.2 day interval $\frac{9.2}{1.49}=6.16$ firings would be required so that the total orbital effects due to jet ${ }^{1}$ hrustings may be expected to be approximately $6.16 \times \frac{1}{24} \times \frac{1}{2}=\frac{1}{78}$ as great as those obtained with a $1200 \mathrm{ft} \mathrm{lb}$ sec vehicle. (The $\frac{1}{2}$ factor takes into account the spread-out over time effects of the thrustings.)

Uncertainities in solar pressure forces are more difficult to estimate : the attitude is not as constant, the tracking time between thrustings is less, differerent vehicle surfaces see the sun over the orbit, and the vehicle is not quite as thermally uniform as the dual-spinner. Assumptions of uncertainties are:

a) Daily increment in solar force known within $10 \%$ (5\% for spinner)

b) Uncertainty in basic solar force due to attitude uncertainty of $0.1^{\circ}$ is $0.17 \%$.

Using the technique of Section E-2.3, these values lead to mean percentar? errors of $0.17 \%+\frac{10 \% \times 5 \% \times 9.2}{2}=2.47 \%$ when the vehicle orbit plane is normal to the sun-line and $0.17 \%+\frac{2}{10 \% \times 0.9 \% \times 9.2}=0.585 \%$ when the vehicle axis is normal to the sun-line. Just as is true for the delal-spinner, the greatest uncertainty is due to the inability to know precisely the change in solar pressure force due to the changing solar aspect of the vehicle. days is:

Thus, using the numerics of Section E-2.4, the maximum error after 9.2

$$
\begin{aligned}
& \mathrm{i}=90^{\circ} \quad \text { normal to radius vector } \quad d_{1}=0.33 \times 2.47 \\
& =0.82 \mathrm{ft} \\
& \text { along radius vector } \quad \delta_{R}=0 \\
& \mathrm{i}=0^{\circ} \quad \text { normal to radius vector } \quad \delta_{1}=46 \times 0.585 \\
& =26.9 \mathrm{ft} \\
& \text { along radius vector } \quad \delta_{\mathrm{R}}=27 \times 0.585 \\
& =15.8 \mathrm{ft}
\end{aligned}
$$


Estimates of maximum errors for intermediate angles $i$ are:

$\begin{array}{lll}\mathrm{i}=60^{\circ} & \text { normal to radius vector } & \delta_{1}=12 \mathrm{ft} \\ & \text { along radius vector } & \delta_{R}=8 \mathrm{ft} \\ \mathrm{i}=30^{\circ} & \text { normal to radius vector } & { }_{\mathrm{R}}^{\delta_{1}}=20 \mathrm{ft} \\ & \text { along radius vector } & { }_{\delta_{\mathrm{R}}}=13 \mathrm{ft}\end{array}$

E-2.7 Estimated Orbit Displacements Over 9.2 Days

This section determines the 9.2 day total estimates in orbit displacements based on mis-estimations of solar pressure forces and mismatched and misaligned thrusters. 9.2 days is chosen because it represents a complete thrusting and non-thrusting cycle. Errors determined by combining results from Sections $2.3,2.4,2.5$, and 2.6 may be considered as those achievable in an operation in which after tracking to establish the true initial conditions a) a twopart jet thrusting cycle is instituted to re-orient the vehicle and b) a 9.2 day orbital drift occurs -- the ephemeris for the complete cycle being estimated before the jet thrusting. Tracking and curve-fitting is employed during the 9.2 has orbital drift to establish the "true" initial conditions of the orbit at the start of the next cycle and the "true" vehicle solar pressure force parameters.

Maximum errors in vehicle ephemeris over 9.2 days are taken as the sum of jet thrusting and solar pressure force mis-estimates. Values of error components are compiled in Table E-1. Since all component error values used in Table 1 are maximum, they may reasonably be considered as $3 \sigma$ values so that $1 / 3$ the maximums are taken as the expected or $1 \sigma$ values. These results are plotted in Figure $E-1$ as a function of orbit plane inclination $i$ to the sunline. 


\section{ESTIMATED ORBIT UNCERTAINTIES FOR STORED MOMENTUM VEHICLES}

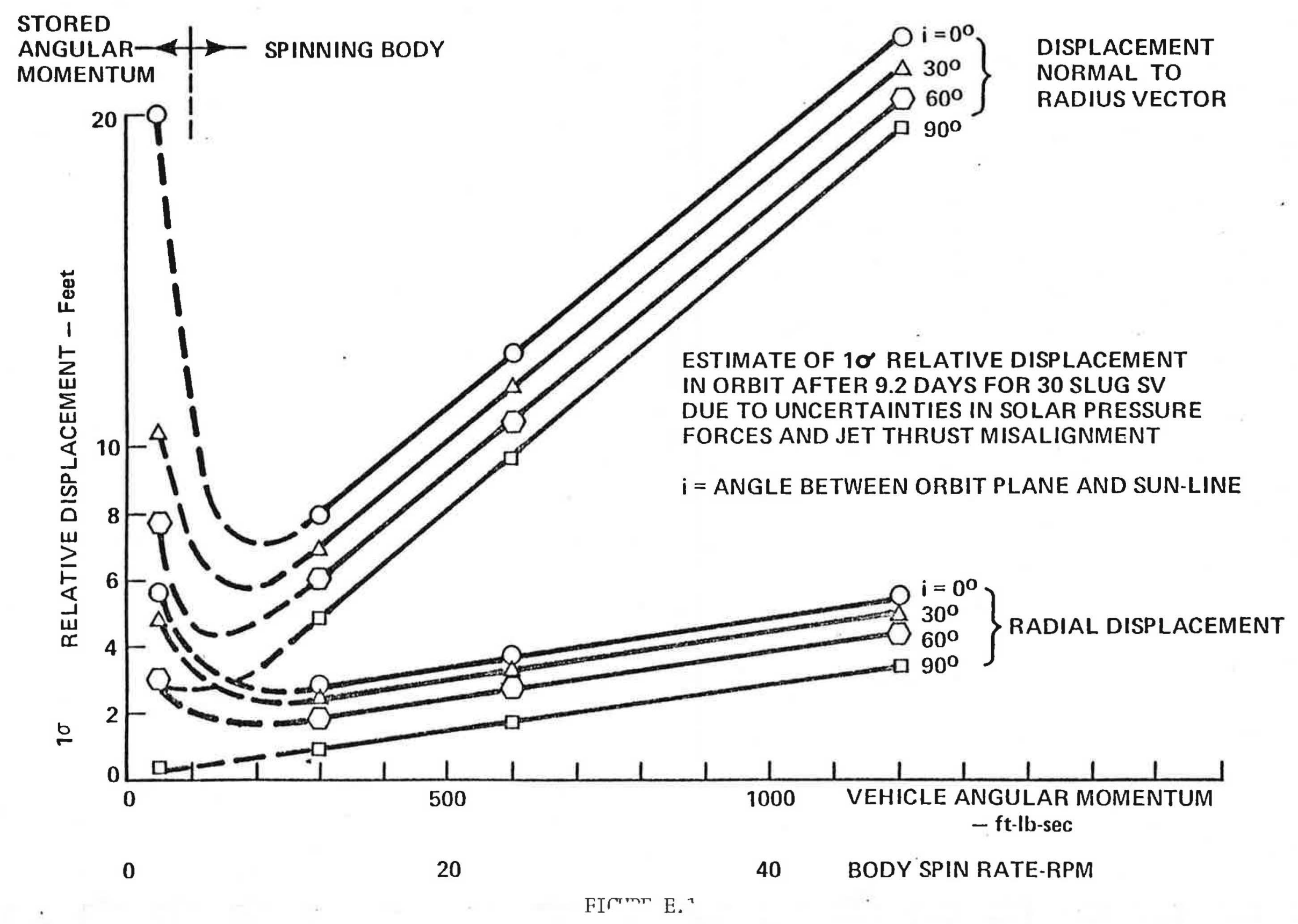




\section{E-3.0 Conclusions}

A major contributor to GPS ephemeris uncertainty for a dual-spin satellite is due to the imperfection in the required jet thrustings. These thrustings force the satellite spin axis to remain normal to the orbit plane. The best direction for the thrustings is normal to the orbit plane. Because jet misorientation is the prime imperfection it is worthwhile to investigate means of increasing the precision of control and determination of actual jet thrust directions.

Estimated $1 \sigma$ ephemeris errors as low as 3 feet in radial distance and 8 feet in distance normal to the radius vector are attainable with dual-spin vehicles. Bias momentum system estimates are about twice these values but suffer from greater uncertainty in the estimates themselves.

Persons with actual experience in estimating on-orbit vehicle characteristics should be consulted to (hopefully) confirm or (hopefully not) reject the computational assumptions of this study.

\section{$\underline{\text { References }}$}

1. Basic Physics of the Solar System by V. M. Blanco and S.W. McCuskey. (Addison-Wesley Publishing Company - 1961).

2. Effect of Solar Pressure on GPS Orbit Predictability, Appendix C. 


\section{APPENDIX E-A}

Determination of rate of change of angle between the sun-line orbit axis, as a function of time of year.

The geometry of the situation is shown in figure A-1. Axis systems are denoted by subscripts:

$I \quad$ is the inertially fixed sy stem with $Z_{I}$ pointing to the north ecliptic pole and $\mathrm{Y}_{\mathrm{I}}$ to the first point of Aries.

e is an earth centered inertial system with $Z_{e}$ pointing to the north pole and $\mathrm{Y}_{\mathrm{e}}$ along $\mathrm{Y}_{\mathrm{I}} \cdot \mathrm{Z}_{\mathrm{e}}$ is displaced $\mathrm{i}_{\mathrm{e}}$ $\left(=23.5^{\circ}\right)$ from $Z_{I}$.

$\mathrm{OA}$ is the orbit axis system with $\mathrm{Z}_{\mathrm{OA}}$ pointing along the orbital rate vector. $\mathrm{Z}_{\mathrm{OA}}$ is displaced by angle $\mathrm{i}\left(=63^{\circ}\right)$ from $\mathrm{Z}$
lies in the from $\mathrm{Y}_{\mathrm{e}}$.

$\Phi \quad$ is the earth centered system with $Z_{\Phi}$ along $Z_{I}$ and $Y_{\Phi}$ pointing from the earth to the sun so that $\mathrm{Y}_{\Phi}$ is displaced by angle $\Phi$ from $\mathrm{Y}_{\mathrm{r}}$.

With these definitions the angle $\alpha$ between the orbit axis and the sunline is given by:

$$
\alpha=\cos ^{-1} V_{\Phi Y}
$$

where:

$$
\left[\begin{array}{c}
\mathrm{V}_{\Phi \mathrm{X}} \\
\mathrm{V}_{\Phi \mathrm{Y}} \\
\mathrm{V}_{\Phi \mathrm{Z}}
\end{array}\right]=\left[\begin{array}{ccc}
\mathrm{c} \Phi & \mathrm{s} \Phi & 0 \\
-\mathrm{s} \Phi & \mathrm{c} \Phi & 0 \\
0 & 0 & 1
\end{array}\right]\left[\begin{array}{ccc}
\mathrm{ci}_{\mathrm{e}} & 0 & -\mathrm{si}_{\mathrm{e}} \\
0 & 1 & 0 \\
+\mathrm{Si}_{\mathrm{e}} & 0 & \mathrm{ci}_{\mathrm{e}}
\end{array}\right]\left[\begin{array}{ccc}
\mathrm{c} \Omega & -\mathrm{s} \Omega & 0 \\
\mathrm{~s} \Omega & \mathrm{c} \Omega & 0 \\
0 & 0 & 1
\end{array}\right]\left[\begin{array}{ccc}
\mathrm{ci} & 0 & \mathrm{si} \\
0 & 1 & 0 \\
-\mathrm{si} & 0 & \mathrm{ci}
\end{array}\right]\left[\begin{array}{l}
0 \\
0 \\
1
\end{array}\right]
$$

and $\mathrm{c}=\cos , \mathrm{s}=\sin$. 
Thus:

$$
\alpha=\cos ^{-1}(-s \Phi c i e s s i+c \Phi s \Omega \text { si }+ \text { cis } \Phi s i e)
$$

And:

$-s \alpha \frac{\partial \alpha}{\partial \Phi}=-c_{e}$ c $\Omega$ si $c \Phi-s \Omega$ si s $\Phi+$ ci si $_{e}$ c $\left.\Phi\right)$

These functions were calculated using the program of Figure.E-A-2 with the results plotted in Figure E-A-1. The maximum rate of change

$\frac{\partial \alpha}{\partial \phi}$ is seen to be unity. 


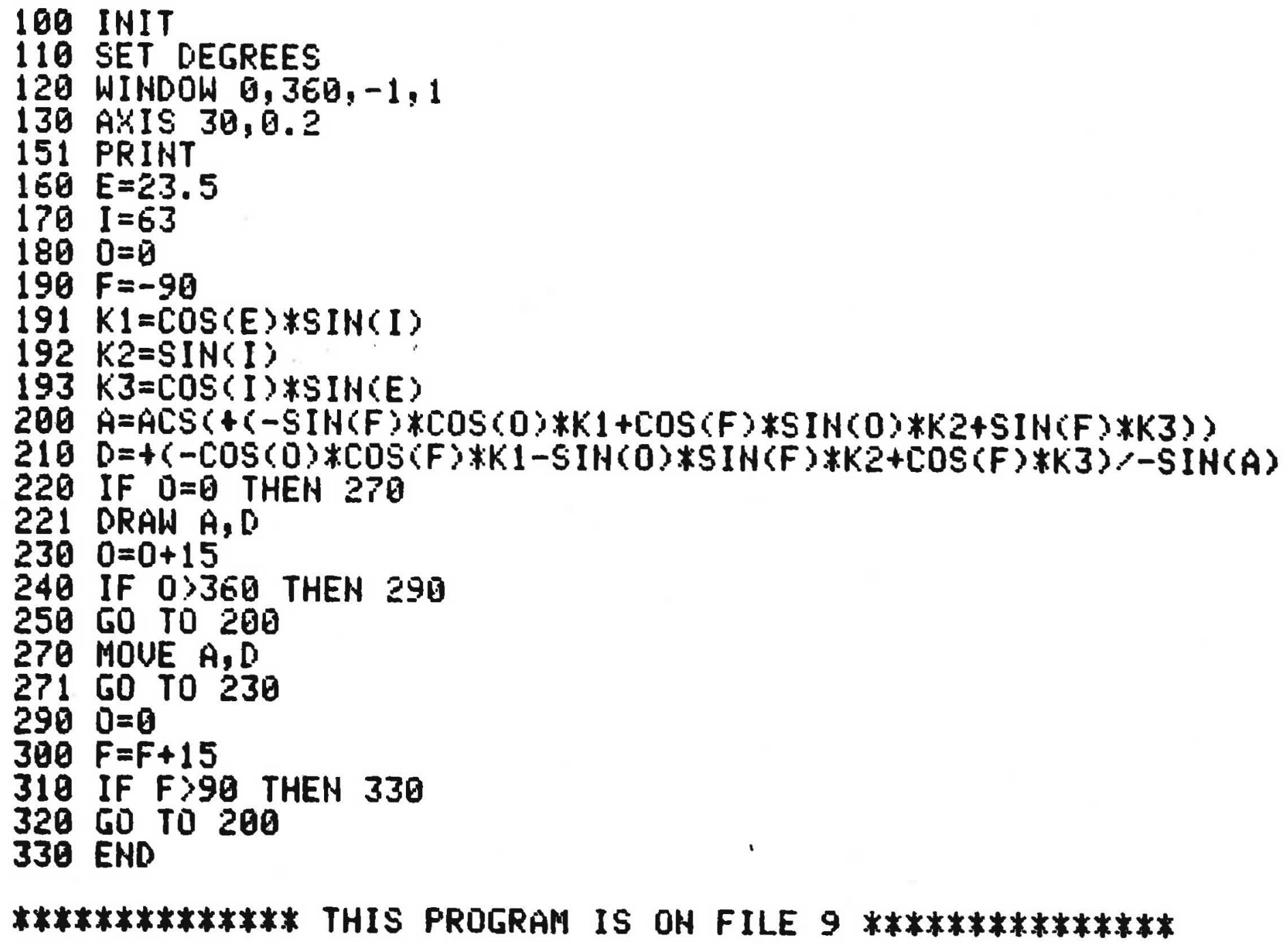




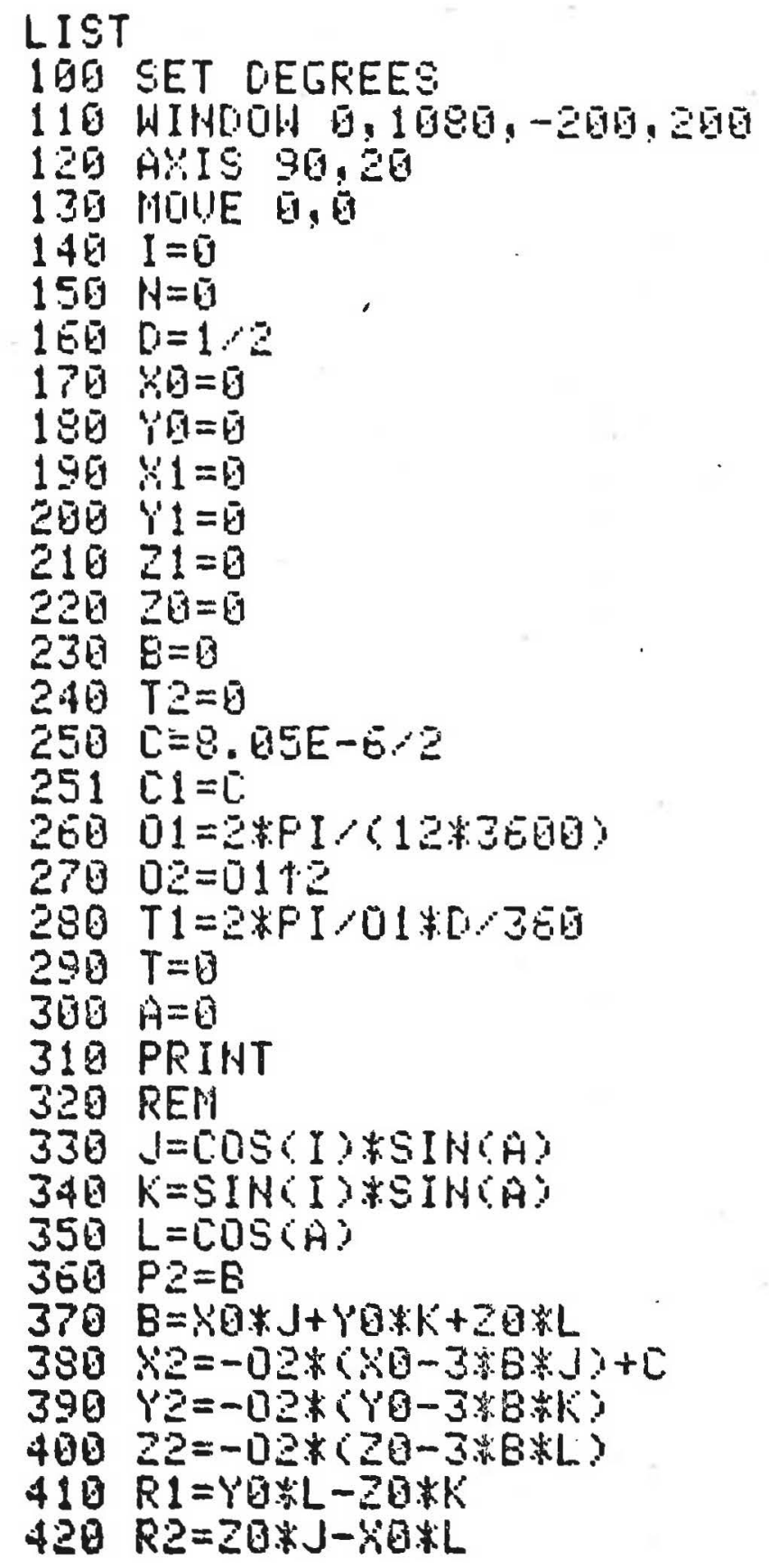


$439 R 3=.40 * K-Y \overline{0} * 1$

$439 \quad \mathrm{~F} 1=T 2$

$440 T 2=S T R(R 1+2+R 2+2+R 3+2)$

450 Uट $=32$

$46, \quad 12=Y 3$

$470 \quad 12=22$

$485 \quad U 1=R$

$490 \quad v 1=Y_{1}$

$560 \quad N 1=21$

$510 \quad K 1=31+40+32+1 / 2$

$520 \quad Y 1=Y 1+(U 2+Y 2) * T 1 / 2$

$530 \quad 21=21+(\omega 2+22)+T 12$

$540 \quad 80=80+C U 1+M 1 ?+112$

$550 \quad Y \bar{B}=Y 0+C y 1+41 \% T 12$

$56020=20+61+21)+112$

$570 \mathrm{H}=\mathrm{H}+1$

$5807=T+T 1$

$581 \mathrm{Al}=\mathrm{A}$

582 HOUE $A 1, P 1$

$590 \quad A=[1 *+14$

600 DRAN A,T2

601 NOUE A1:P2

602 DRAN A, B

610 IF $A=21080$ THEH 630

$611 C=0$

612 IF $A=180$ THEN ES0

613 IF $A=180.5$ THEH 680

614 IF $A=0.5$ THEN 650

620 G0 ro 320

$630 I=I+30$

640 IF I $\geqslant 90$ THEN BPO

650 MOUE B. 0

660 GO TO 150

670 END

$680 \mathrm{C}=\mathrm{C} 1$

681. Go TO 620 


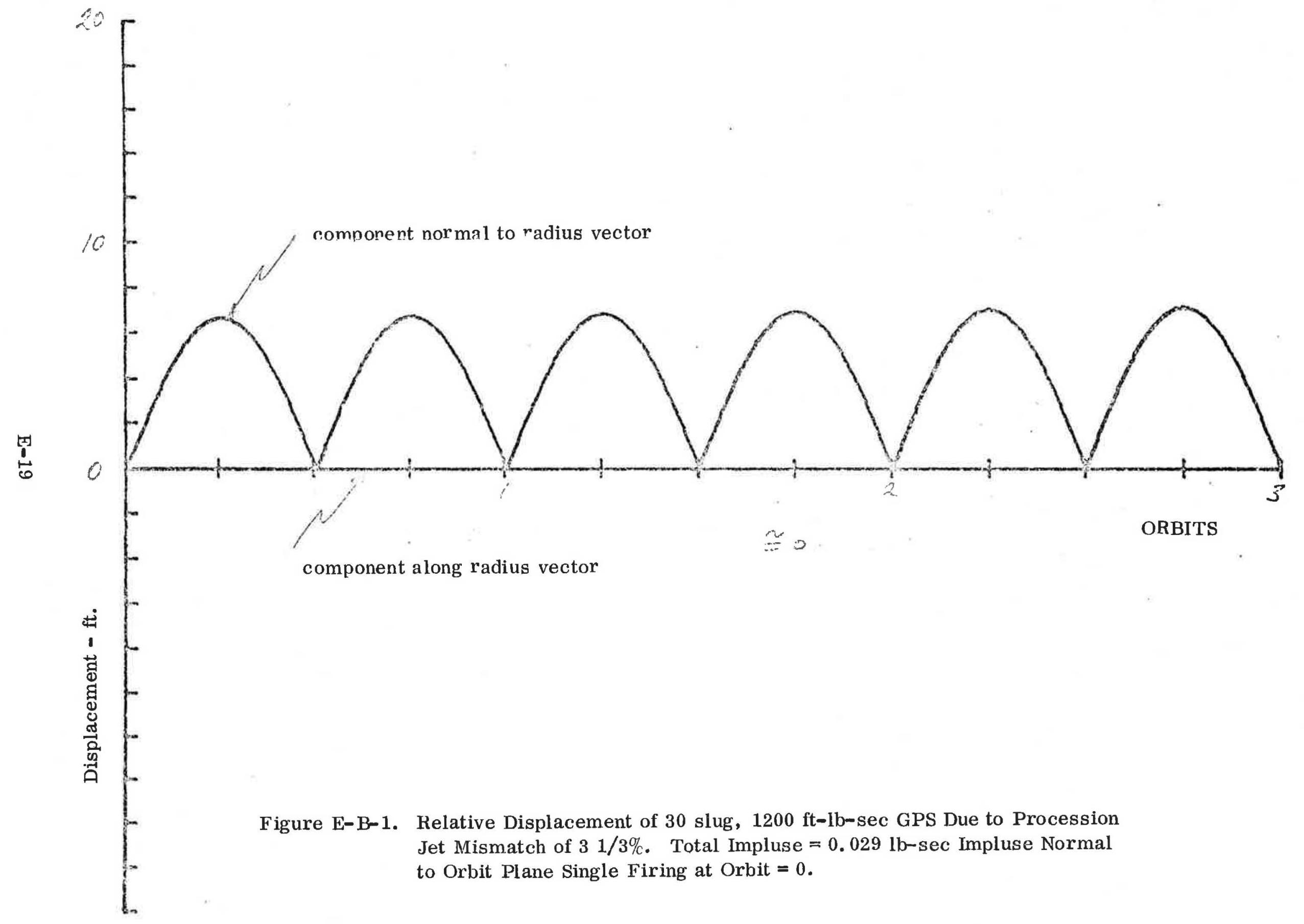




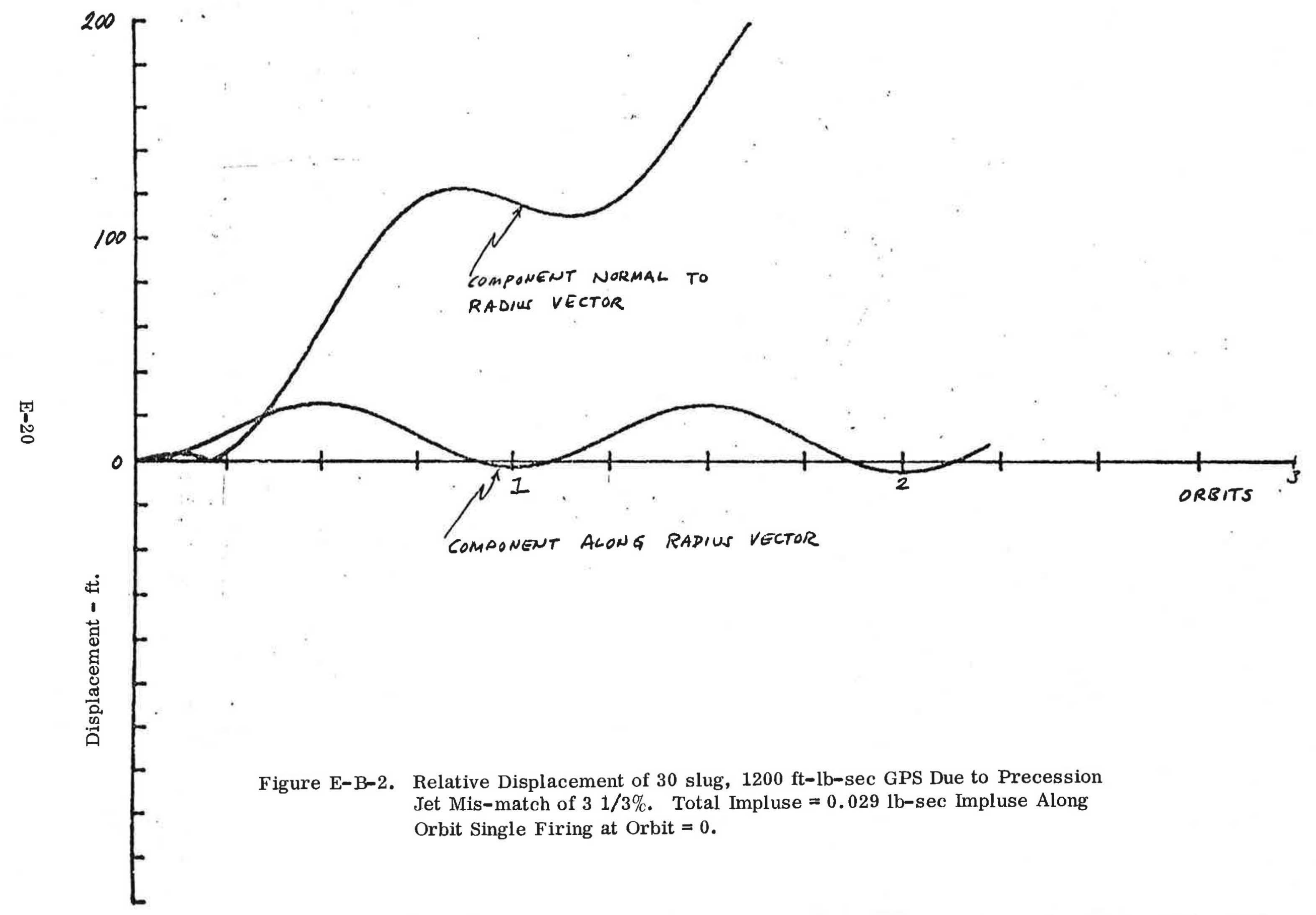




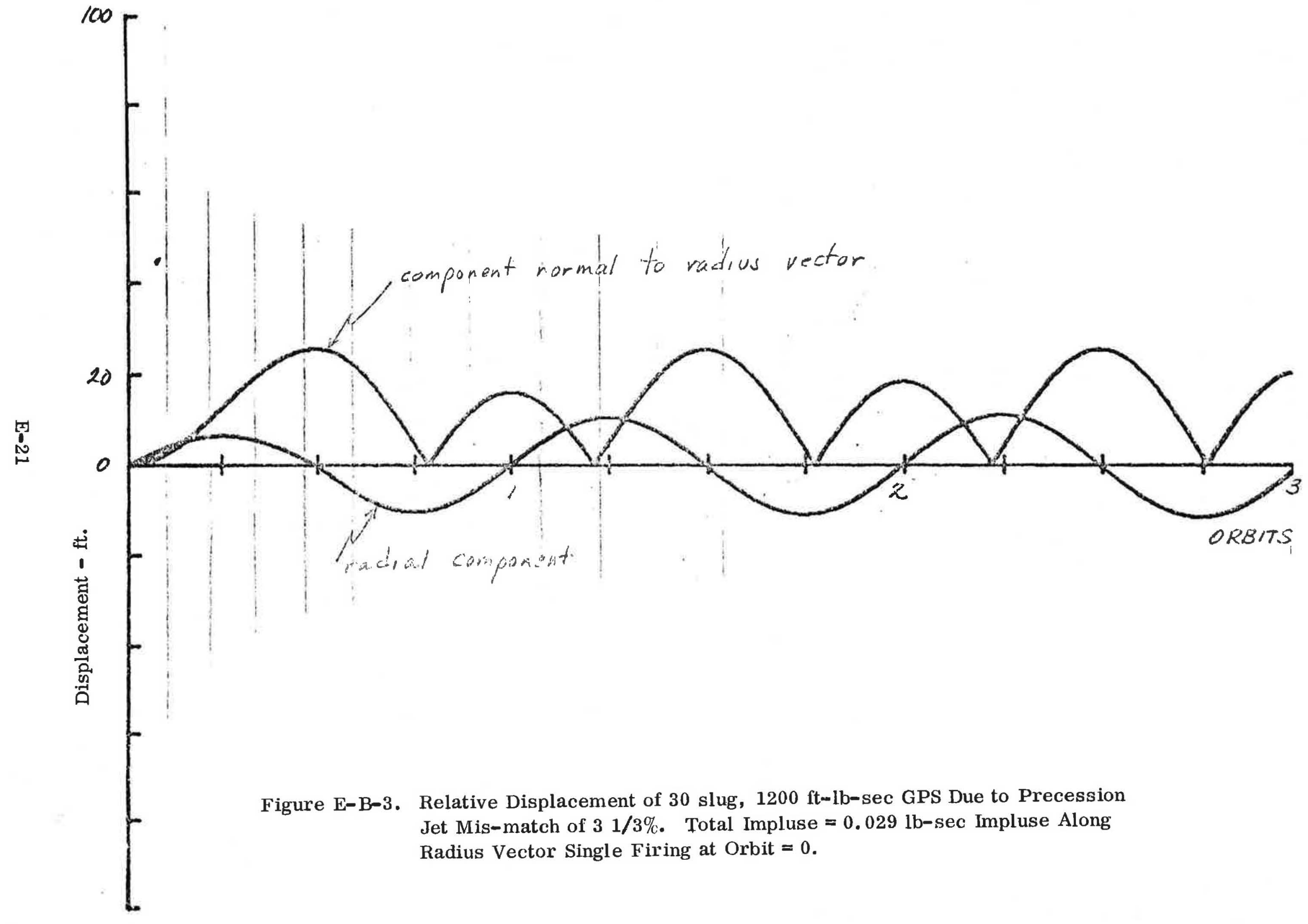




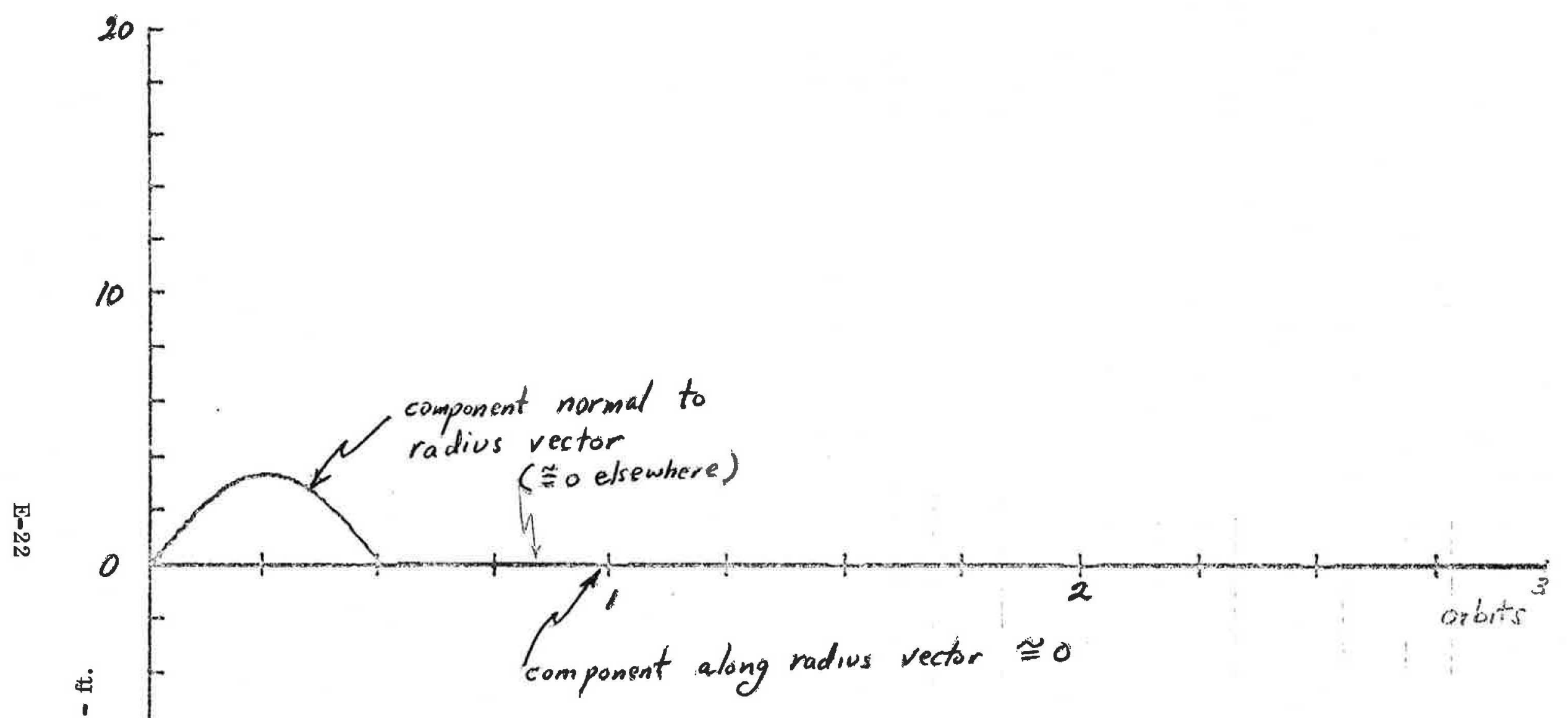

Figure E-B-4. Relative Displacement of $30 \mathrm{slug}, 1200 \mathrm{ft}-\mathrm{lb}-\mathrm{sec}$ GPS Due to Precession Jet Mis-match of $31 / 3 \%$. Total Impluse $=0.029 \mathrm{lb}$-sec Impluses Normal to Orbit, Two Equal Firings: One at Orbit $=0$, One at Orbit $=1 / 2$. 


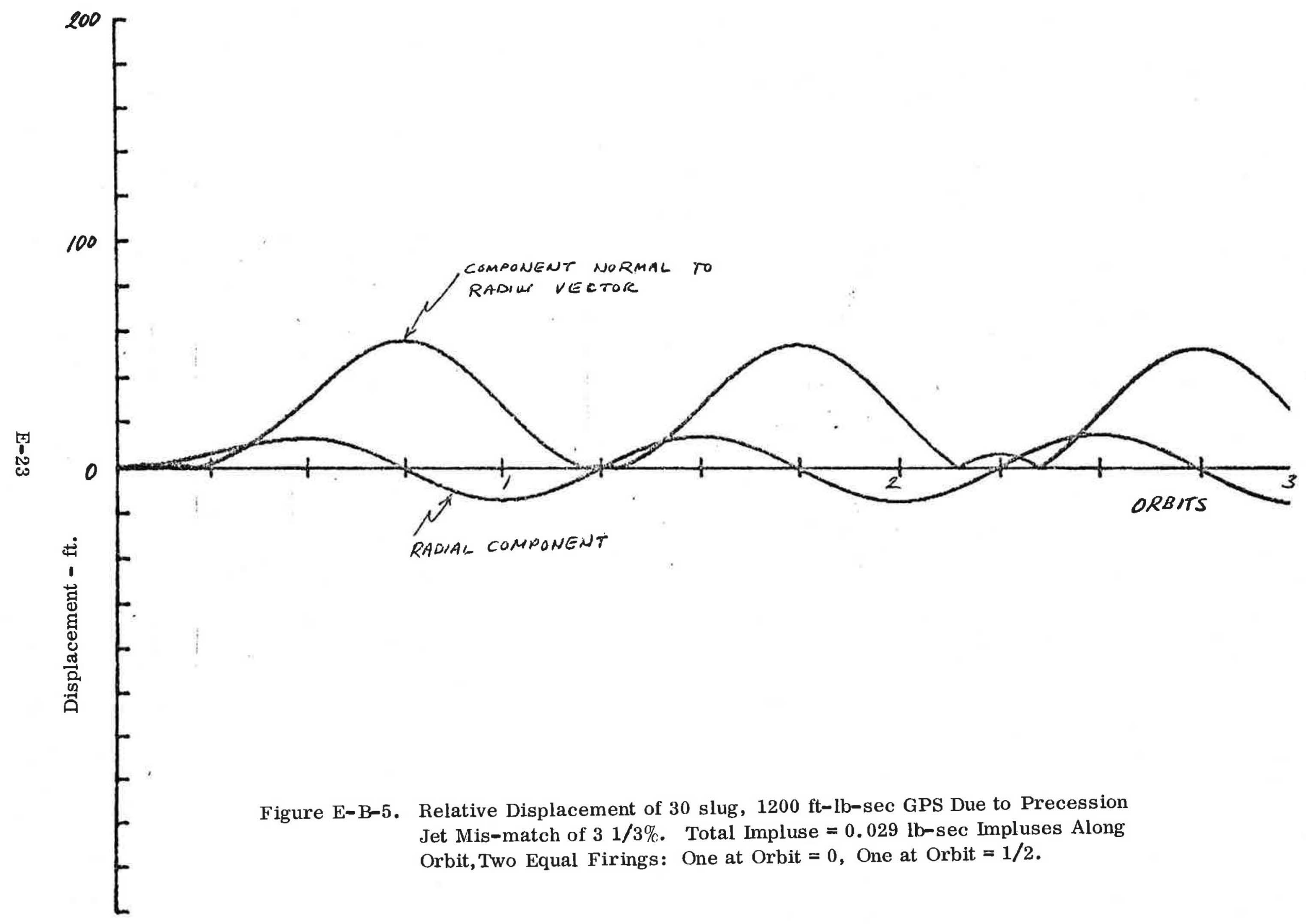




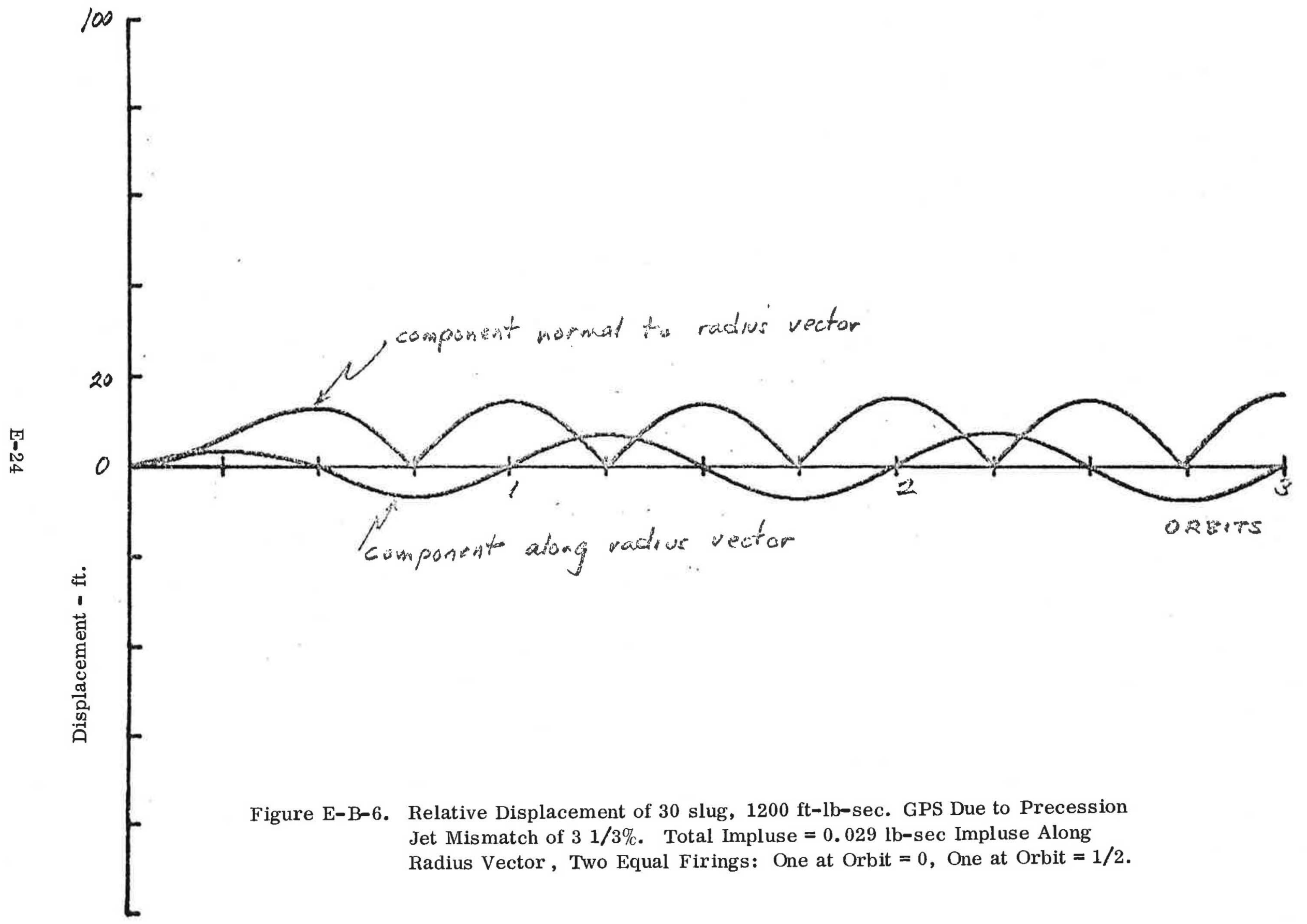


F. ACS ELEMENTS FOR BIAS-MOMENTUMCONTROLLED GPS SPACECRAFT 

F1 Introduction

This appendix documents the ACS requirements for a GPS/SV with biased angular momentum control. Two vehicle orientations are considered: 1) the vehicle axis points to the Earth and 2) the vehicle axis is normal to the orbit plane. In both, the bias momentum is normal to the orbit plane. The orientations impose similar sensor requirements, but due to different solar aspects require different bias momentum requirements. These have been evaluated for the two configurations assuming the vehicle as cylindrical, $8 \mathrm{ft}$. long and $6 \mathrm{ft}$. in diameter about the yaw axis, and covered with white paint. Center-of-mass displacement from the vehicle geometric center is assumed as 3 inches maximum. As shown below, the actuator sizing depends upon the allowable time between jet thrustings for vehicle unload of stored momentum.

\section{F2 Sensor Complement}

Since the bias momentum GPS control requirements differ from the NDS require ments only in needing an earth pointing signal about the single continuously controlled axis rather than the two axis requirement of NDS, the sensor complement of NDS will be suitable for the bias momentum system. While probably not optimum, it will be proposed here for simplicity.

$$
\text { Table E-1 AVCS Physical Characteristics }{ }^{\dagger}
$$

\begin{tabular}{|c|c|c|c|c|c|}
\hline \multirow{2}{*}{ Component } & \multirow{2}{*}{$\begin{array}{l}\text { Qty Per } \\
\text { Ship }\end{array}$} & \multicolumn{3}{|c|}{ Estimated Unit Size (c m) } & \multirow{2}{*}{$\begin{array}{l}\text { Weight Per } \\
\text { Ship } \neq \\
\text { Lbs }\end{array}$} \\
\hline & & Length & Width & Height & \\
\hline Control Electronics Assembly & $1 *$ & 38.1 & 22.9 & 15.3 & 18.5 \\
\hline Reaction Wheel & 1 & $\mathrm{~N} / \mathrm{A}$ & $\mathrm{N} / \mathrm{A}$ & $\mathrm{N} / \mathrm{A}$ & $\mathrm{N} / \mathrm{A}$ \\
\hline Combined Earth Sensor & $1 *$ & 31.0 & $18.9 \mathrm{dia}$ & $\mathrm{N} / \mathrm{A}$ & 13 \\
\hline Spin Sun Sensor & 2 & 5.1 & 3.9 & 3.1 & 2.5 \\
\hline Nutation Damper & 1 & 61.0 & 6.0 dia & $\mathrm{N} / \mathrm{A}$ & $2.5 \neq$ \\
\hline Electromagnets & $2 *$ & 43.0 & 2.5 dia & $\mathrm{N} / \mathrm{A}$ & 2 \\
\hline
\end{tabular}

* Internally redundant

+ from Rockwell GPS design CID-SV-101A Vol 5, Part 11975 June 06

₹ from Rockweli GPS design 16 GP 6296

\$. estimated

F3 $\underline{\mathrm{Z} \text { Axis to Earth Orientation }}$

An evaluation was made of the vehicle's angular momentum accumulation in the orbit plane (i.e., normal to the bias momentum). The program of Fig. F1 was utilized and graphical results are given in Fig. F2. Over the set of conditions shown, the maximum accumulation $\Delta \mathrm{H}$ of $0.035 \mathrm{ft} \mathrm{lb} \mathrm{sec}$ per orbit is noted for the condition $\mathrm{i}=45^{\circ}$ with zero center-of-mass offset. 


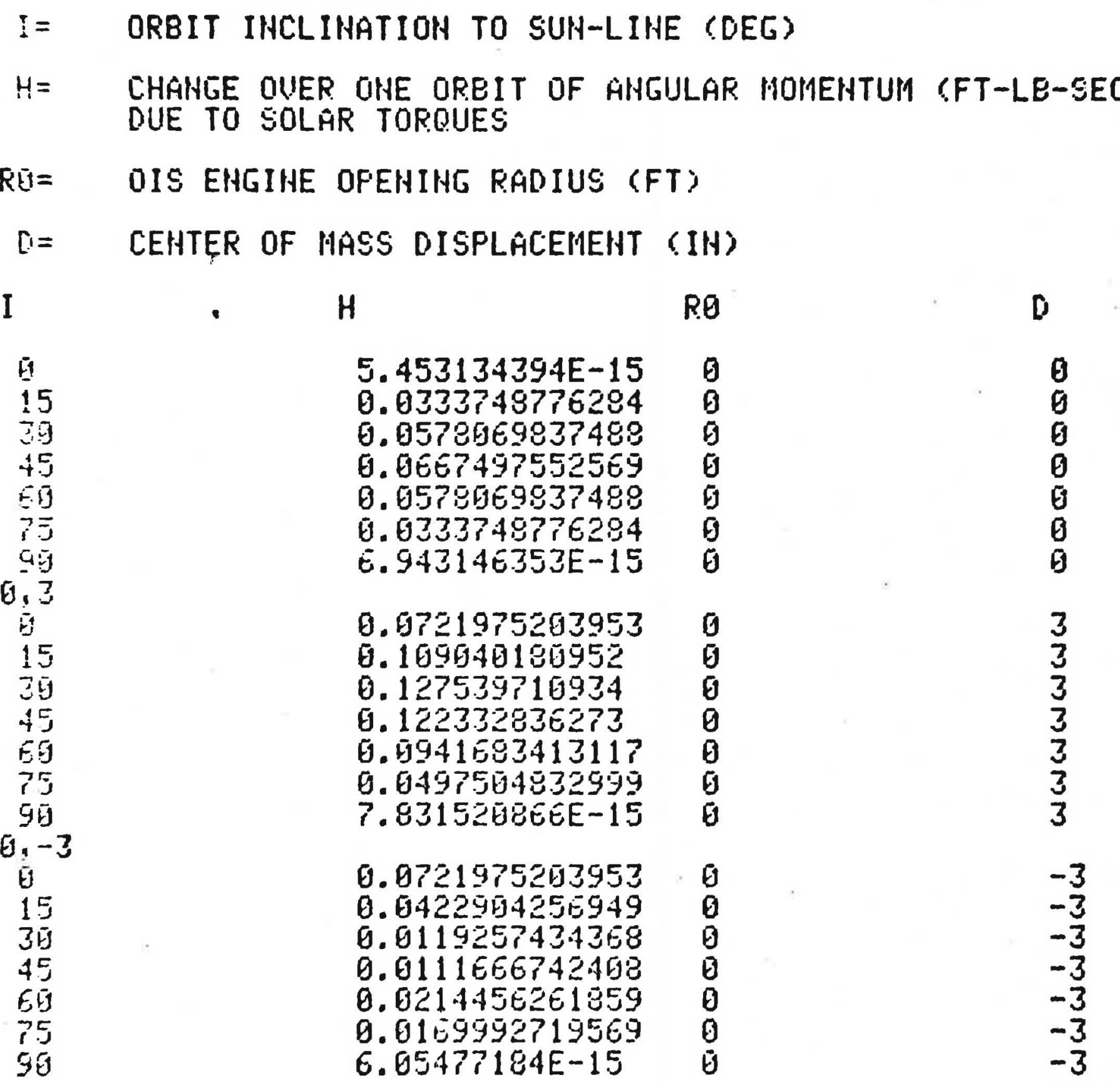




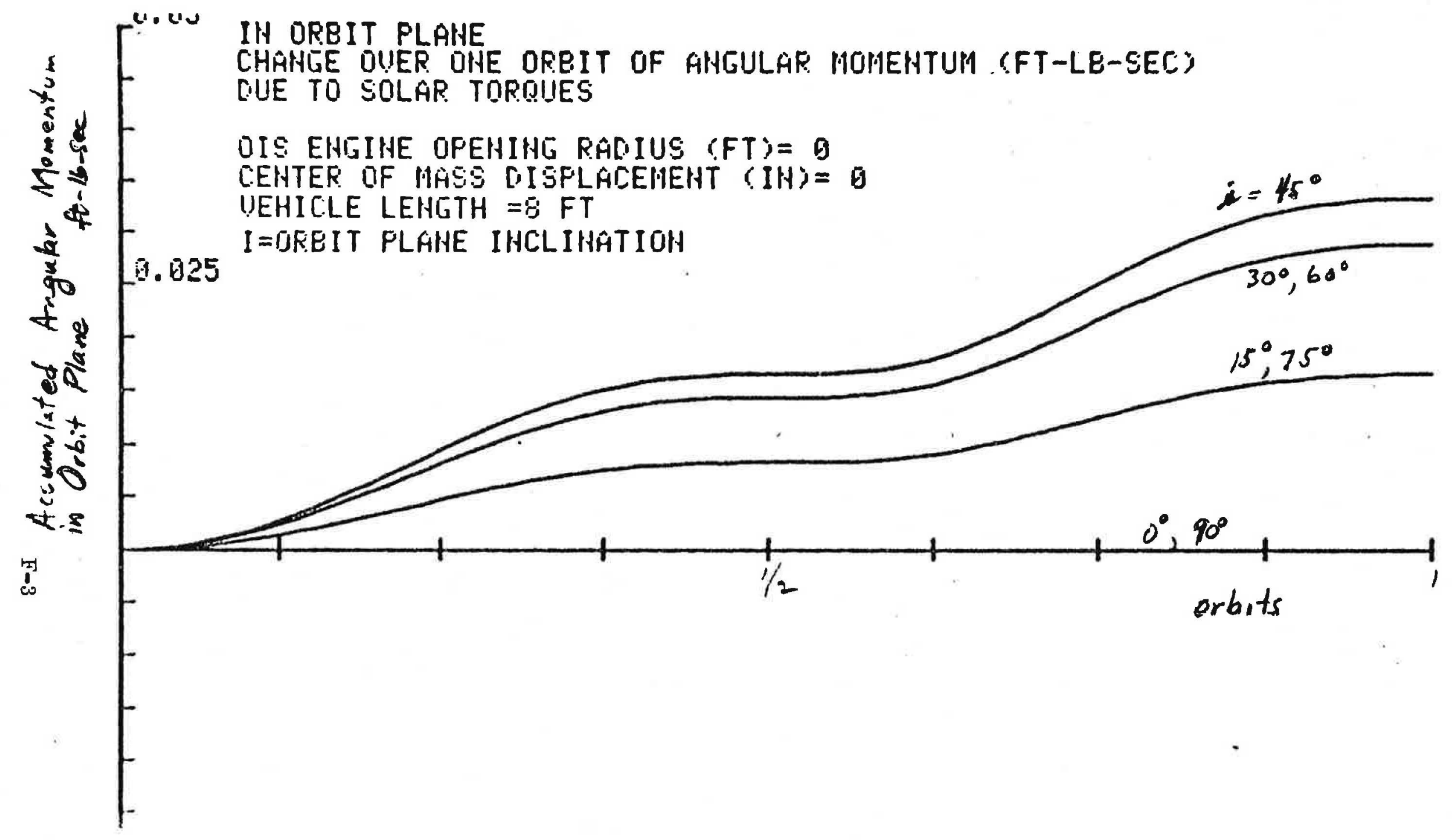

Figure 2. (Sheet 1 of 3 ) 


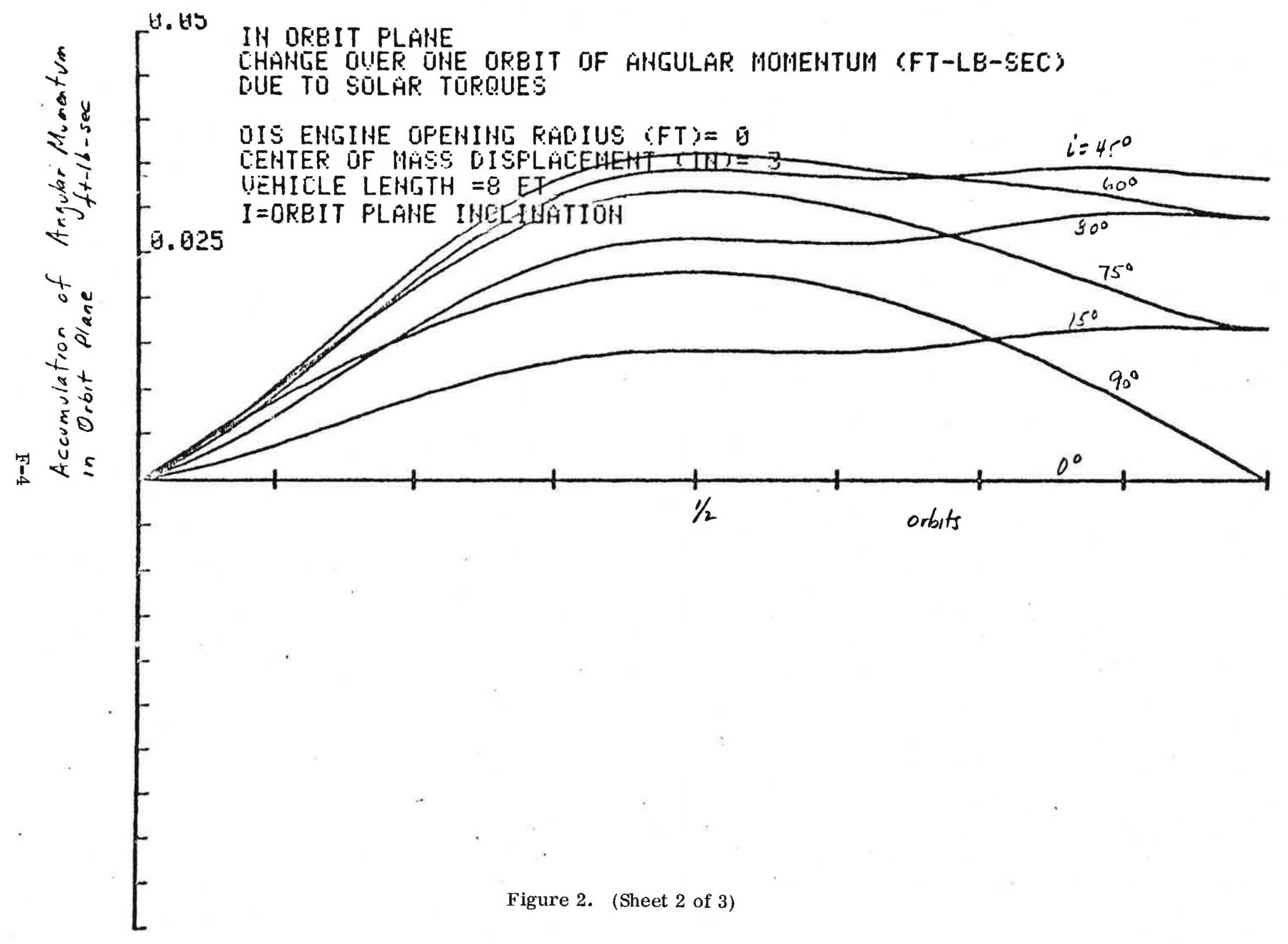




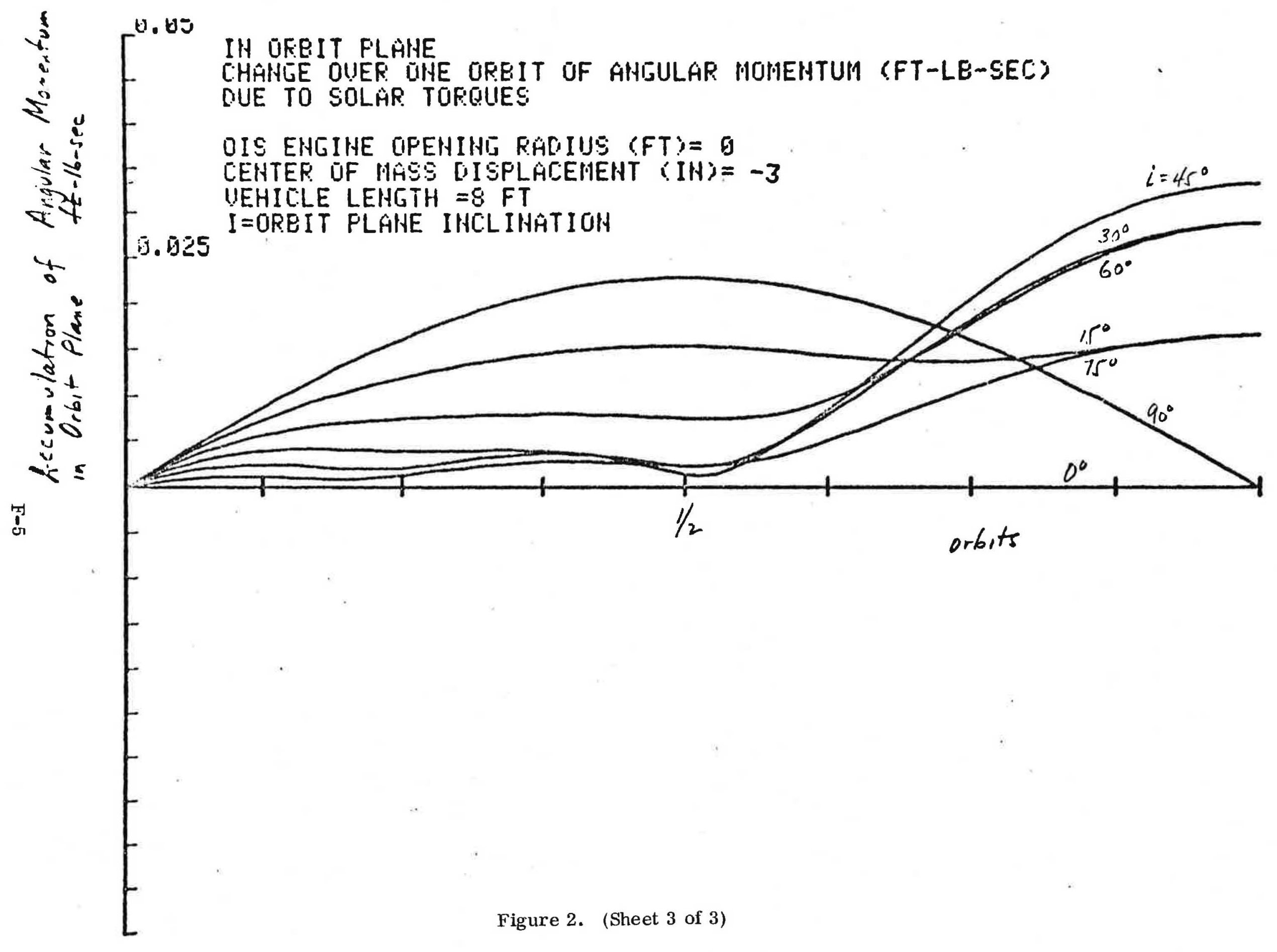


If $\Delta \theta$ is the permitted angular error in nointing to the nadir, then the relation between bias momentum $\mathrm{H}$ and the time interval $\Delta t$ between reorientations (achieved by jet firing) is:

$$
\Delta t=\frac{H \Delta \theta}{(\Delta H / \text { orbit })}
$$

If $\Delta \theta=0.25 \mathrm{deg}$

$$
\Delta \mathrm{H} / \text { orbit }=0.035 \mathrm{ft} \mathrm{lb} \mathrm{sec}
$$

then $\Delta t=0.125 \mathrm{H}$ orbits

or $\Delta t_{d}=0.062 \mathrm{H}$ days

Table F-2 below puts numerics in this equation and also shows the incremental $\mathrm{H}$ required by a GPS which contains an Organic Rankine cycle engine with angular momentum of $17.6 \mathrm{ft} \mathrm{lb}$ sec. The weight of wheel with that incremental $\mathrm{H}$ is included.

Table $\mathrm{F}-2$

\begin{tabular}{cccc}
\hline $\begin{array}{c}\Delta t \\
\text { days } \\
\text { between reorientations }\end{array}$ & $\begin{array}{c}\mathrm{H} \\
\mathrm{ft} \mathrm{lb} \mathrm{sec}\end{array}$ & $\begin{array}{l}\text { Incremental } \mathrm{H} \\
\text { with O.R. engine } \\
\mathrm{ft} \mathrm{lb} \mathrm{sec}\end{array}$ & $\begin{array}{c}\text { Wheel Weight } \\
\text { lbs } \\
\text { (Estimated) }\end{array}$ \\
\hline & & & \\
1 & 16 & 1.6 & 10 \\
2 & 32 & 14.4 & 21 \\
3 & 48 & 30.4 & 24 \\
5 & 80 & 62.4 & 35 \\
7 & 112 & 94.6 & 45 \\
\hline
\end{tabular}

It should be noted that, in configuration layouts for this on-orbit orientation, the wheels and/or power supply rotating machine are oriented with their spin axes normal to the booster thrust axis.

The above calculations are based on the assumption that the nuclear engine's coolant flow contributes no net angular momentum to the syste $m$ other than about the bias momentum axis. Angular momentum due to coolant flow either causes vehic le angular errors or is removed by jet action, nulled by auxiliary wheels or nulled by suitable slight inclination of the reaction wheel or rotating machine. Preset inclination of the rotating machine appears to be the most satisfactory alternative if the angular errors are unacceptable.

\section{F4 Z Axis Normal to Orbit Plane Orientation}

An evaluation was made of the vehicle's angular momentum accumulation in the orbit plane. A modification of the program of Ref. 1 was utilized and tabular results are given in Fig. 2. Over the set of conditions shown, the maximum accumulation $\Delta H$ of $0.127 \mathrm{ft}$ lb sec per orbit is noted for the condition $\mathrm{i}=30^{\circ}$ with center-of-mass offset of 3 in. 
Table F-3 below gives the data for this orientation equivalent to that given in Table F-2 for its orientation.

\begin{tabular}{|c|c|c|c|}
\hline \multicolumn{4}{|c|}{$\begin{aligned} \Delta \mathrm{t}_{\mathrm{d}}= & 0.0172 \mathrm{H} \mathrm{d} \\
& \text { Table } \mathrm{F}-3\end{aligned}$} \\
\hline $\begin{array}{c}\Delta \mathrm{t} \\
\text { days } \\
\text { Between reorientations } \\
\end{array}$ & $\begin{array}{c}\mathrm{H} \\
\mathrm{ft} \mathrm{lb} \text { sec } \\
\end{array}$ & $\begin{array}{l}\text { Incremental } \mathrm{H} \\
\text { with } \mathrm{O} . \mathrm{R} \text {. engine } \\
\mathrm{ft} \mathrm{lb} \mathrm{sec}\end{array}$ & $\begin{array}{c}\text { Wheel Weight } \\
\text { lbs } \\
\text { (Estimated) }\end{array}$ \\
\hline 1 & 58.4 & 40.8 & 25 \\
\hline 2 & 116.8 & 99.2 & 45 \\
\hline 3 & 175.2 & 167.6 & 65 \\
\hline
\end{tabular}

Basic spinner ACS component complement, used on Hughes HS-333 Anik-A.

Wt.\#

(2) Earth Sensors 1.20

(2) Sun Sensors 0.24

On-board dynamic balance mechanism 19.99

Motor bearing ass'y. $\quad 9.82$

Nutation damper SN6 $\underline{0.98}$

Total weight $32.23 \mathrm{lbs}$.

As shown, practically all the required weight is due to mechanical components not ordinarily considered as ACS elements. More important, these weights are not reviewable for validity or improvement without detailed explicit design; they therefore, must be accepted at face value.

"Standard" spinners and dual-spinners use only two jets, one radial for velocity control, the other axial for attitude control. It has already been shown that axially oriented jets, firing in pairs as couples, are most effective in minimizing orbit perturbations due to required jet thrusting for attitude control. Thus, at least two jets axially oriented and opositely firing are required as part of the jet complement. 



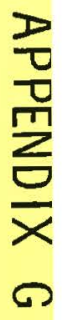

G. ORGANIC RANKINE CYCLE INPUT DATA *

* From Sunstrand Energy Systems, Rockford, Ohio 



\section{G-1 GPS System Performance}

System performance was analysed using the computer programs developed for the KIPS program. These model the interactions of the components and allow individual components to be sized to meet given mission requirements. Alternatively, the system performance in an off-design mode can be determined with no change in component configuration. The design program is controlled by an optimization procedure which allows component designs to be varied to meet preselected constraints on the system or to maximize efficiency, or minimize weight or some compromise between the two.

The optimization procedure consists of a computerized mathematical model of the system incorporating both cycle analysis and component design. The logic is arranged in the form of a mainline program which reads input data, performs a cycle analysis, and prints output. The mainline is the vehicle used to call subroutine elements which contain mathematical relationships describing working fluid properties, component design logic, and a multivariable optimization routine.

The operation of the program is illustrated by the flow chart shown in Figure G-1. For the salient inputs such as condenser temperature, regenerator effectiveness, heat transfer component pressure losses, prime mover design variables, and radiator component probabilities, a cycle analysis is undertaken and cycle efficiency and system weight calculated. A criterion function (PAOFFl defined as follows is then computed: 


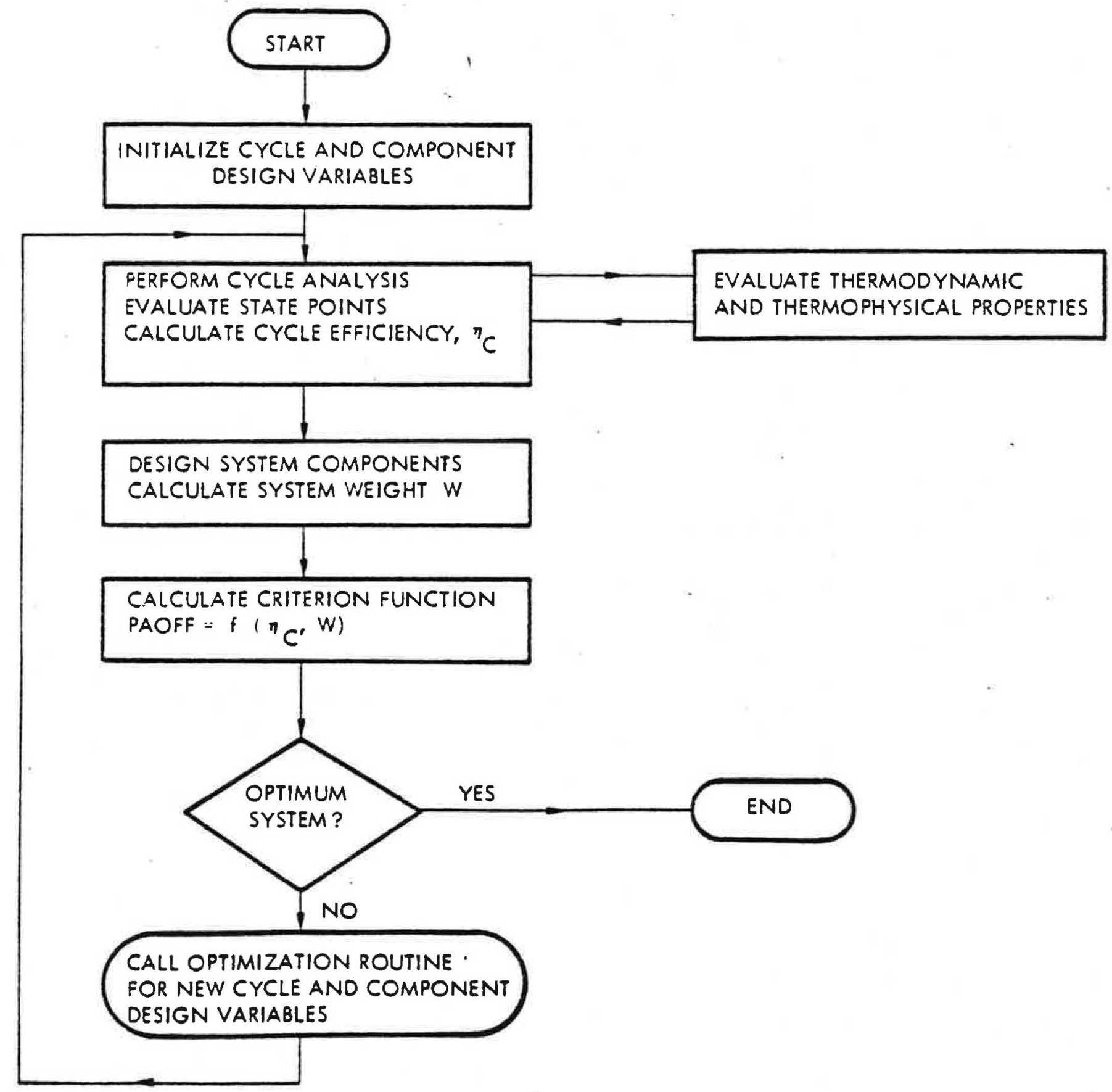

Figure G-1 Basic Flow Chart of Rankine Cycle System Design Optimization Program 


$$
\text { PAOFF }=\frac{(I-C)}{D_{C}}+(C) W
$$

Where: $\quad C=$ weighting factor

$$
\begin{aligned}
& a_{c}=\text { system efficiency } \\
& W=\text { system weight }
\end{aligned}
$$

The inputs to the program are then varied by the optimization subroutine and the cycle calculation and component design procedure repeated until PAOFF is minimized. By conducting the optimization with values of the weighting factors between zero and unity a locus of optimum systems can be generated as a function of system weight and efficiency as shown by the general curve in FigureG-2. Each point on the curve corresponds to a different optimum system design, the relative importance of efficiency and weight being different. The modular construction of the program coupled with the generalized approach to optimization enables Sundstrand to optimize the design of Rankine cycle engines required for various applications in a very rapid manner. The basic mainline program is tailored to call the subroutines containing the logic for each component included in the particular system. Sunstrand has developed a comprehensive inventory of computer subroutines to support the overall system design optimization program. The optimizations have been performed about a representative system comprising a turbo-alternator-feed pump combined rotating unit (CRUl, a vapor-liquid regenerator, a jet condenser, and Iiquid radiator. 


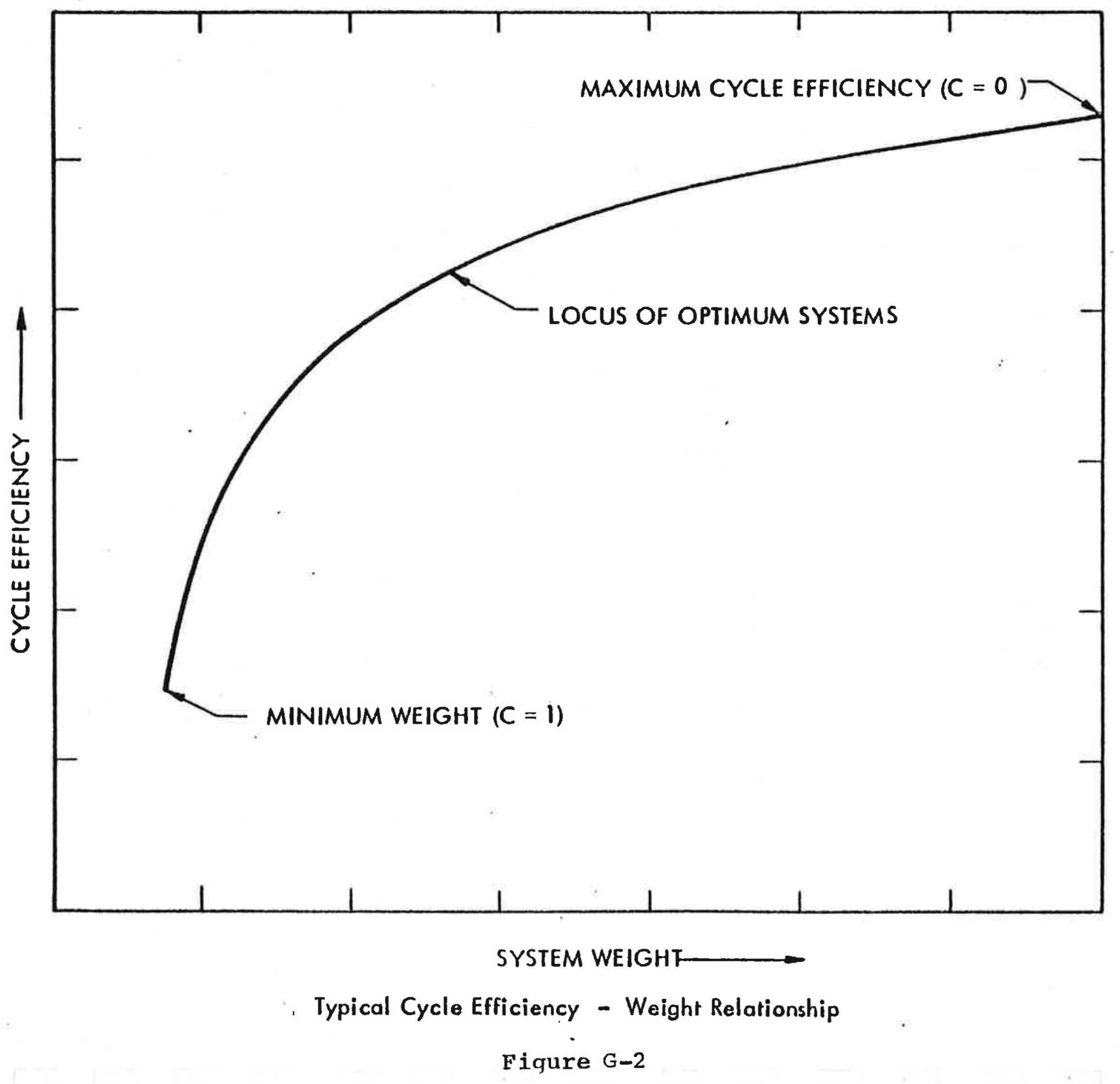


By interconnecting detail design and evaluation subroutines for the various system components with the correct logical criteria, the mathematical model can duplicate the operating characteristics of a real system. This technique offers a utility much more powerful than single parameter trade-offs. The information gained correctly reflects not just the first order variations, but becuase the complete system is being studied, it also shows effects not available in single component parameter trade-offs. For the GPS study the only components whose design was allowed to change from the KIPS system were the jet condenser, turbine nozzles, pump impeller, radiator and radiator bypass valve. The radiator outlet temperature was fixed by the mission requirements at $86^{\circ} \mathrm{F}$, and the liquid/vapour flow ratio of the jet condenser was preserved at the same value $(9: 1)$ as for the KIPS system. The system schematic,figureg-3was modified to include a heat addition between the radiator and bypass valve equal to the total electrical output less 200 watts. Sample printouts from the design and off-design programs are presented in figures 4 and 5 respectively showing relavent operating conditions. Based on the current KIPS Heat Source Assembly heat loss of $200 \mathrm{Btu} / \mathrm{hr}$ per module, the end-of-mission heat input, heat rejected and power output is slightly higher than shown in figuresG-4and $G-5$. 


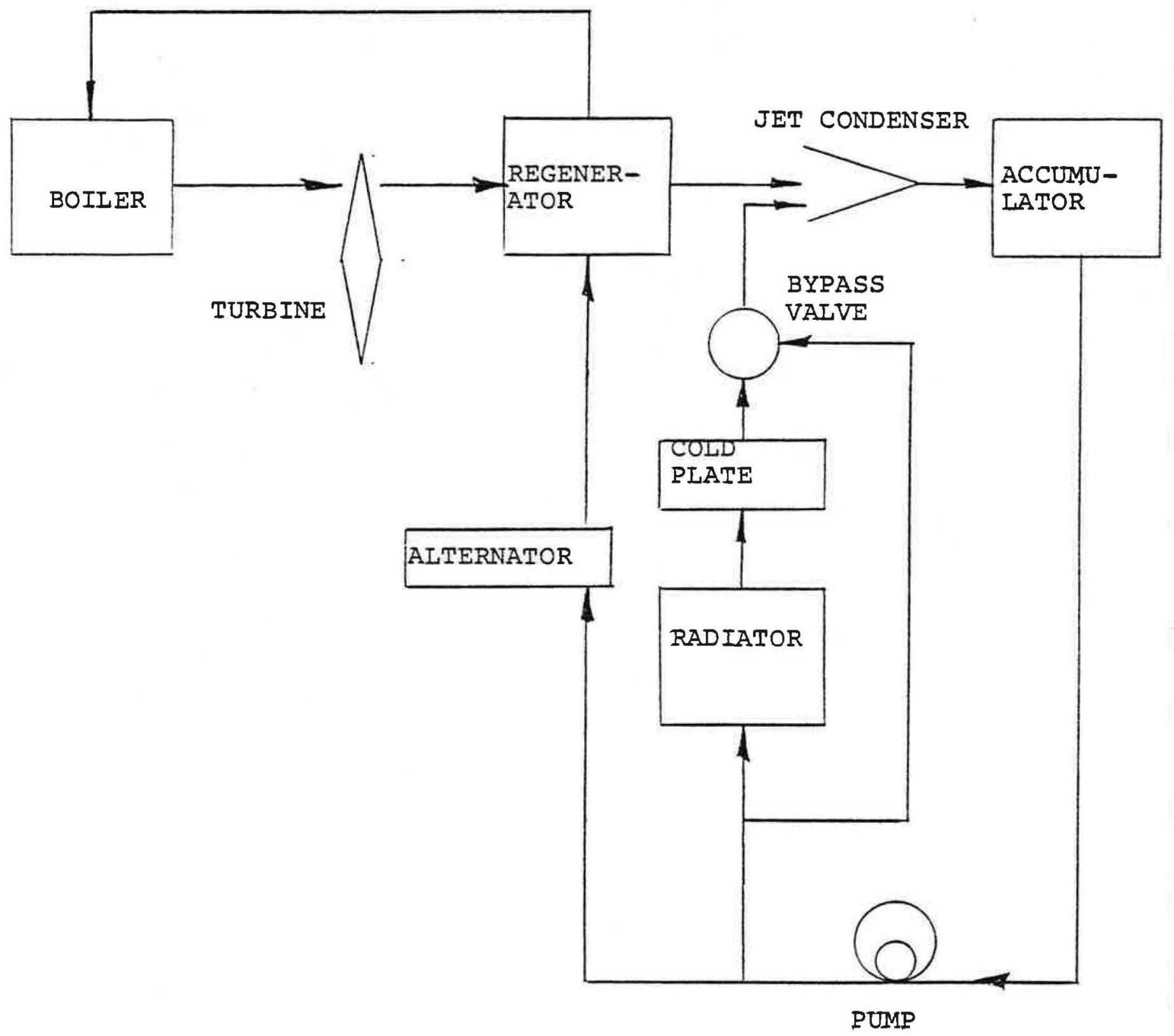

FIGURE G-3 GPS SYSTEM SEHEMATIC

$\mathrm{G}-6$ 
FAIRCHILU GLOBAL POSIJIONING SYSIEM OKC .. KIPS/MOUIFIEU .... OUTPUT

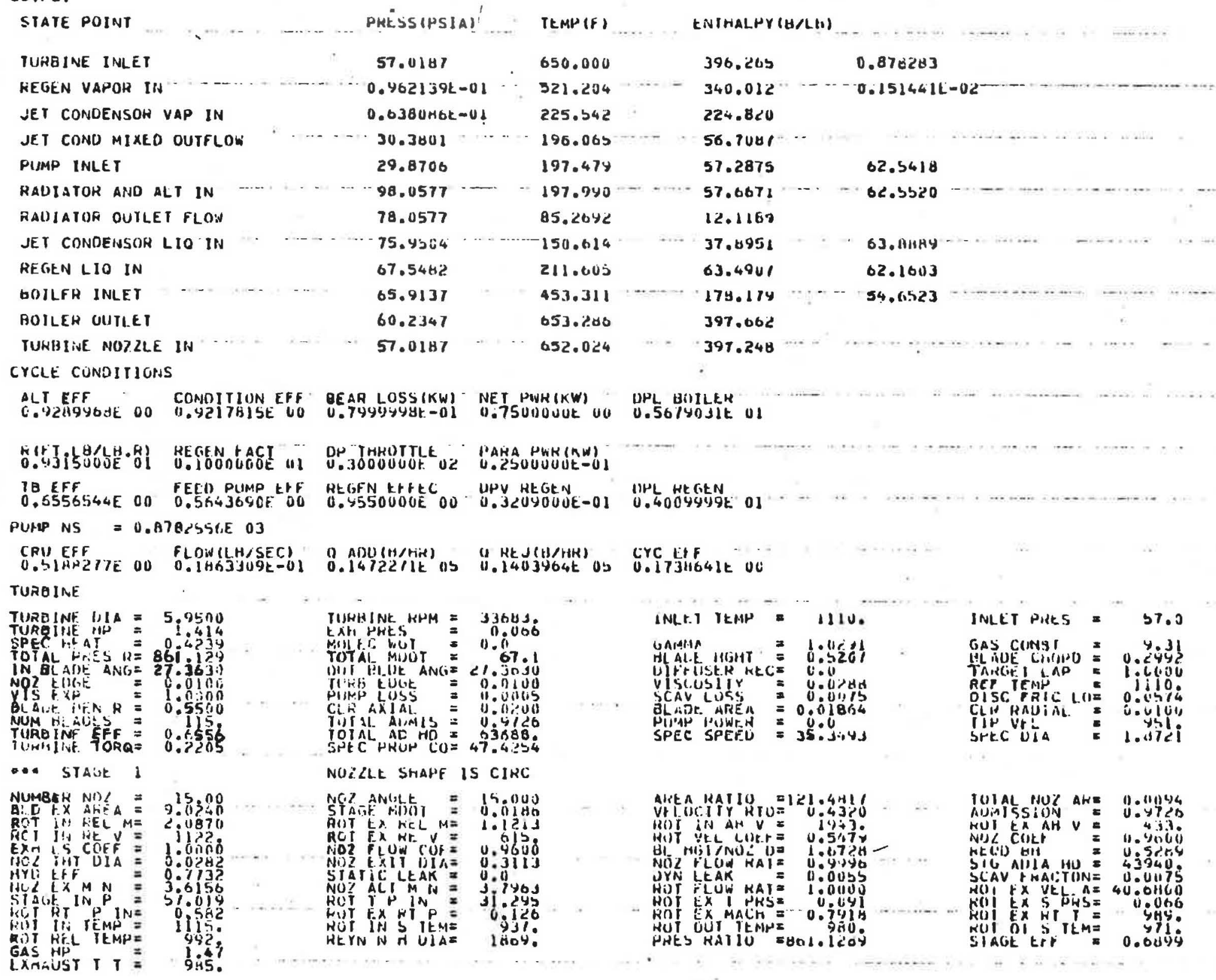




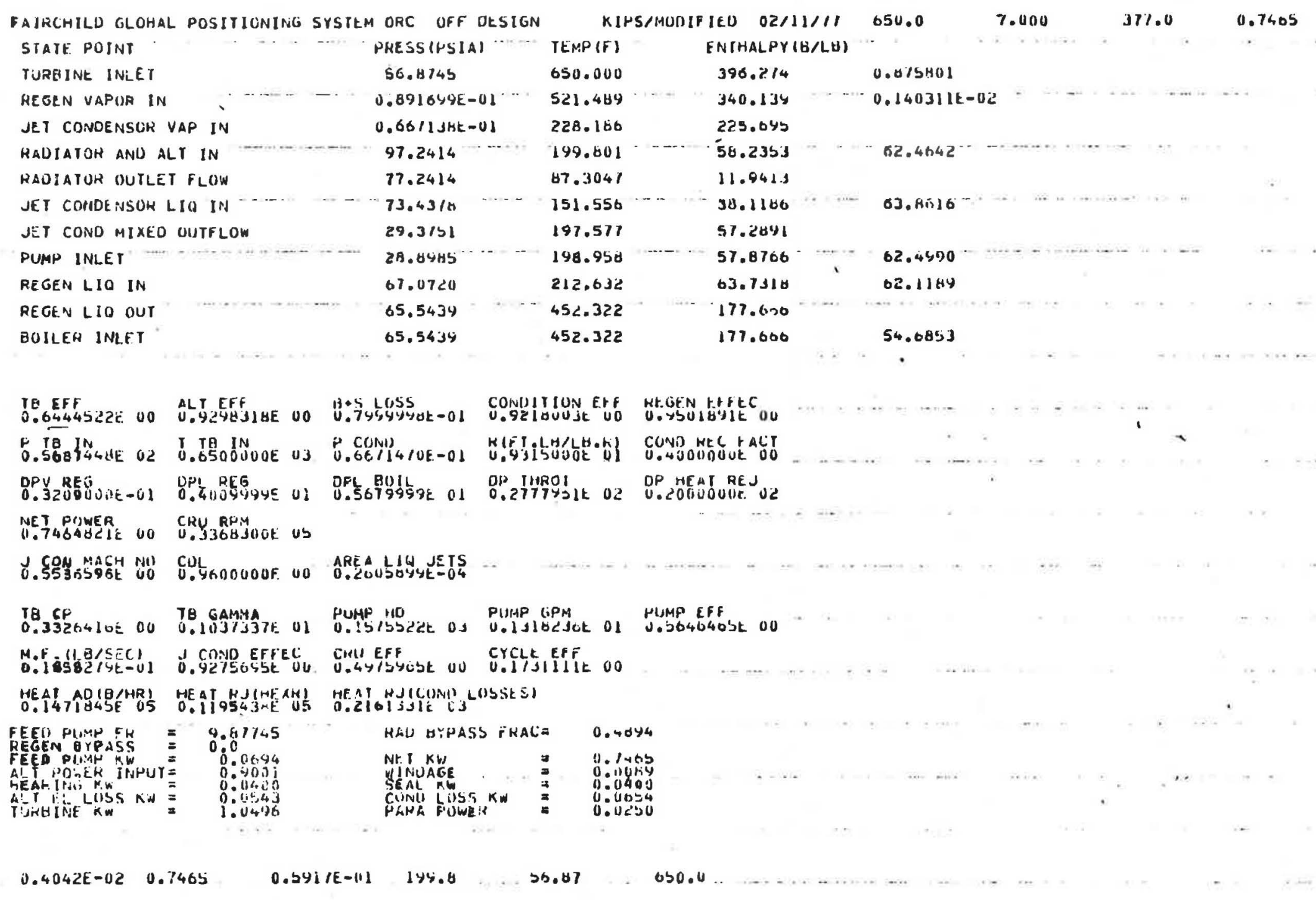

FIGUREG-5 IFF-DESIGN PROGRAM,-END-OF-MISSION 
G-2 Radiator Design Procedure

The radiator design procedure is illustrated by the basic flow chart shown in figure -6 . The logic is computerized and integrated with the Rankine cycle system design optimization program. Radiator variables under control of the multi variable optimization routine include the following:

1) header probability of non puncture

2) inside tube probability of non puncture

3) outside (spacesidel tube probability of non puncture

4) liquid pressure drop

5) fin efficiency

Other quantities such as overall probability of nan puncture, radiator diameter, material and fluid properties and armor correlation coefficients are input to, or calculated by, the systems program.

The design procedure consists of satisfying the heat transfer, armor thickness and friction pressure drop equations for a specified heat load, fluid condition, heat sink temperature and probability of no puncture in a prescribed time period. Heat transfer coefficients for the inside of the radiator tubes are determined using heat transfer data from reference 1 shown in figureG-7for tubes with dimpled surfaces. The purpose of the dimpled surface is to extend the turbulent heat transfer into lower Reynolds numbers then is possible with a plain tube. An equivalent heat transfer coefficient on the outside surface is defined in terms of the Stefan radiant. heat transfer equation. The overall heat transfer coefficient 




Figure $G-6$ Radiator Design Procedure 
Fig. 10-3. Flow inside dimpled flartened pubes, surface FTO-1.

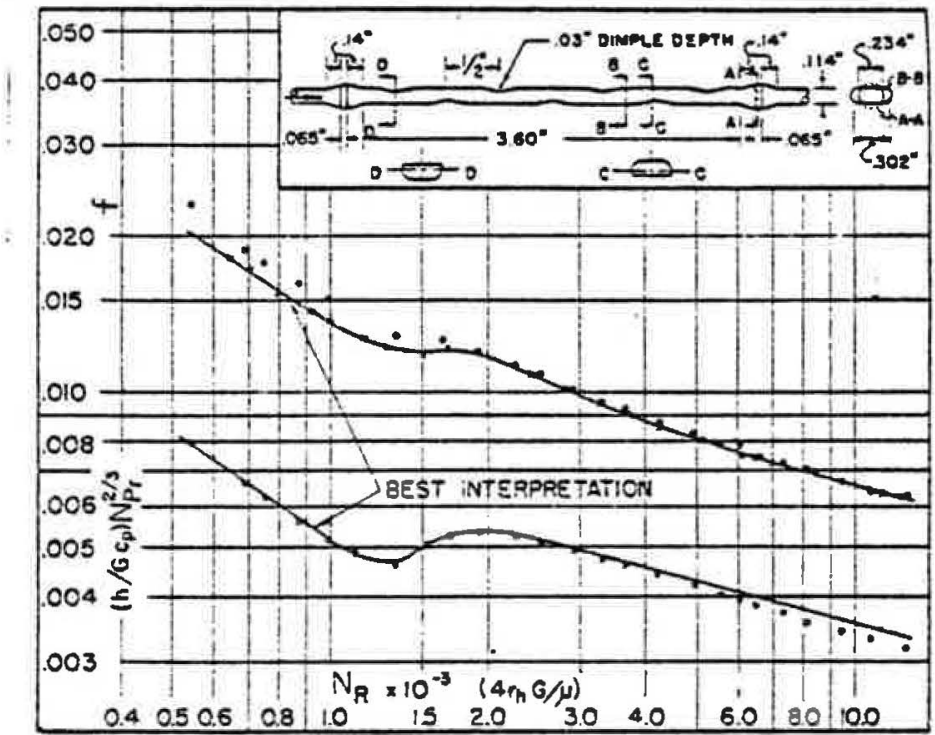

Tube ID before flattening $=0.234 \mathrm{in}$.

Tube inside dimension peroendicular to flats $=0.114 \mathrm{in}$.

Tube inside dimension parallel to flats $=0.302 \mathrm{in}$.

Length of flat along tube $=3.60 \mathrm{in}$.

Length of section from flat-to-cireular-to-flat eross section $=0.345 \mathrm{in}$.

Dimple depth $=0.03 \mathrm{in}$.

Minimum distance between dimpies $=0.5$ in.

Flow passage hydraulic diameter, $4 r_{h}=0.01116$ it

Minimum free-flow area per tube $=0.000169 \mathrm{ft}^{2}$

\section{FIGUREG-7GEAT TRANSFER AND PRESSURE DROP DATA}


is computed using the inside and outside film coefficients together with the conduction term and an overall efficiency term for the extended surface. This latter quantity is determined from the input fin efficiency and a fin length calculation per reference 2. A fin thickness of 0.025 inches is used to match the KIPS design.

Tube pressure drop is determined from the friction factor data given in figure G-7. Armour thickness on the header and tube backside surface is derived from data in reference 3 , while the armour requirements of the tube space side came from reference 4 .

In order to determine the optimum configuration, varions radiator diameters and number of tubes were selected. Sample computer printouts are presented in figures G-8 through G-10 for one, two and three tubes. 


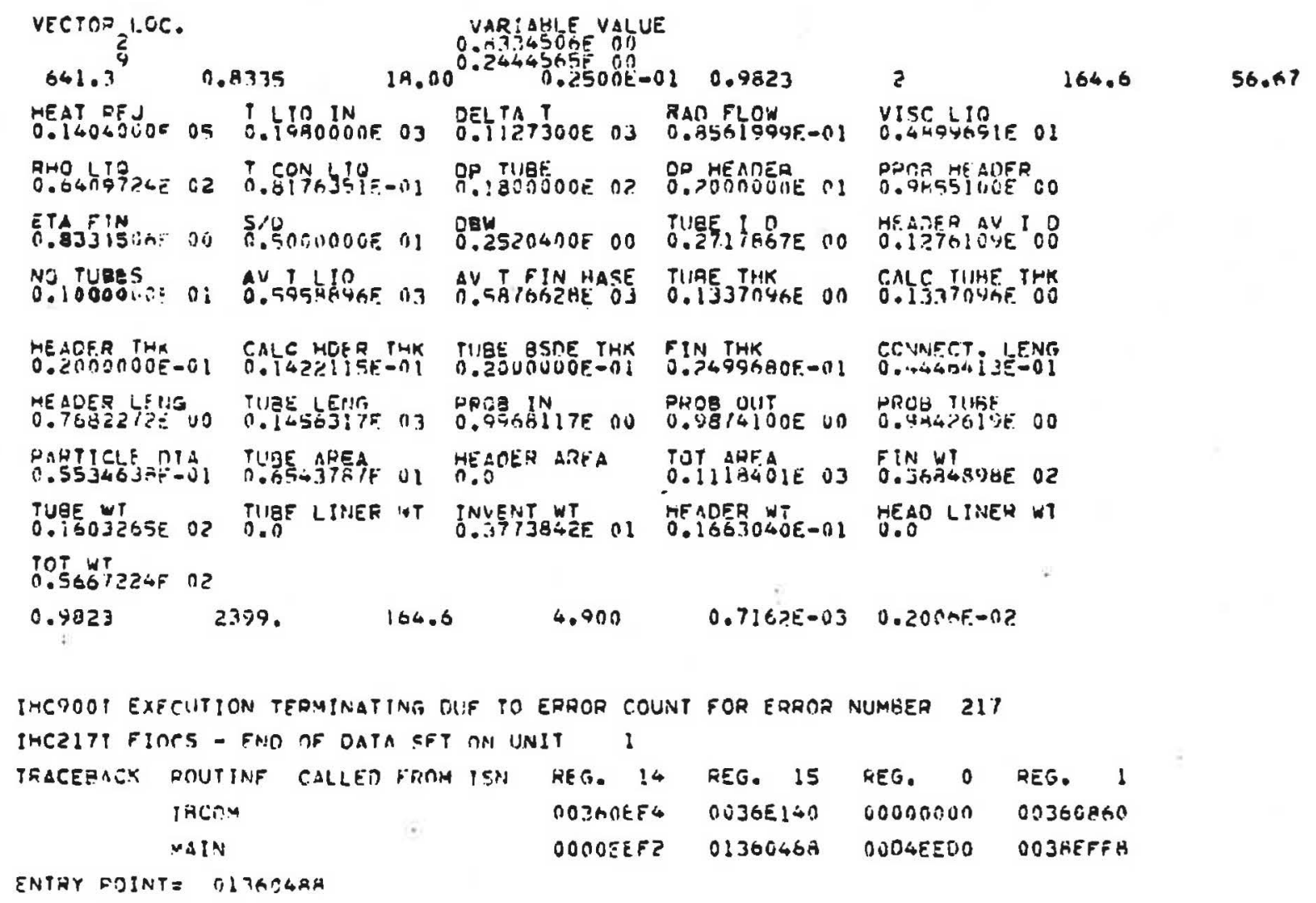

FIGUREG-8RADIATOR DESIGN, ONE TUBE 


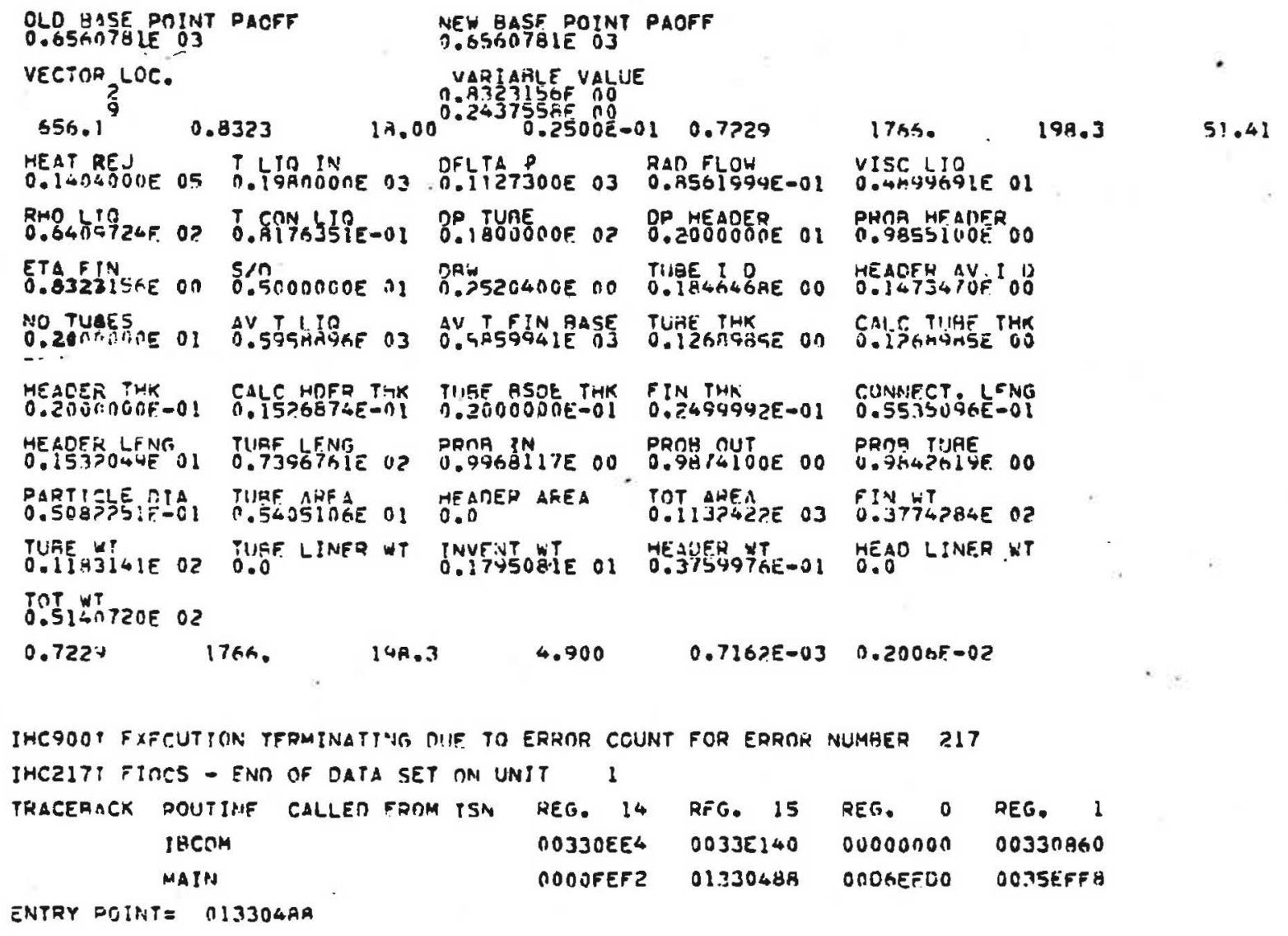

FIGUREG-9RADIATOR DESIGN, TWO TUBES 


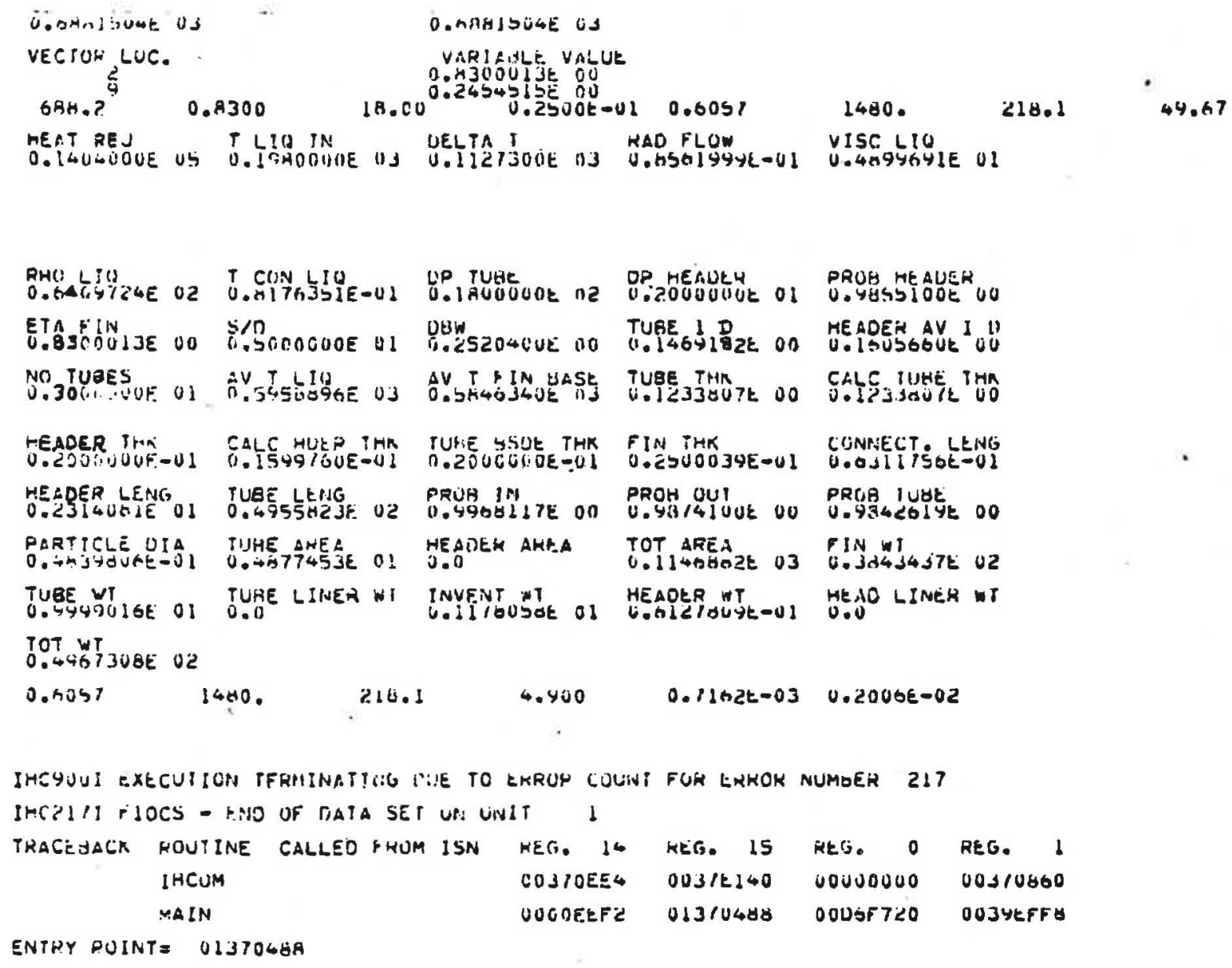

FIGUREG-IORADIATOR DESIGN, THREE TUBES 
G-3. MULTIVARIABLE ORTIMIZATION TECHNIQUE

The general form for all optinization techniques is:

$$
\underline{X}(n+1)=\underline{x}(n)-A(\underline{x})^{-1} f(\underline{x})
$$

Where $x \quad$ is a vector of model parameters

$f(x)$ is the payoff function

$A(X)$ is a matrix determined by the system under study which carries the information the optimization technique requires to find a minima of the payoff.

There are many optimization methods available with the basic difference being the logic used to form the $A(\underline{x})$ matrix. The multivariable optimization technique used for most problems studied by Sundstrand uses a slope-curvature technique to search out a minimum by two modes. The first is a numerical evaluation of the first and second partial derivatives of the payoff function with respect to each of the parameters under study. The second search mode then uses the gradient vector defined by the first mode to vary the system parameters toward an optimum point. This process continues until no worthwhile improvement is possible. 


\section{References}

1) Kays and London, Compact Heat Exchangers AliGraw Hill

2) "Analysis of Temperature Distribution and Radiant Heat Transfer Along a Rectangular fin of Constant Thickenss" S. Iieblein, NASA TN-D-196

3) "A Penetration Criterion for Double Walled Structures Subject to Metearoid Impact" J.P.D. Wilkinson Paper 68-1058, AIAA 5\# Annual Meeting

4) "Results of Hypervelocity Impacts Into space Radiator Materials " N. Clough \& J. Diedrich AIAA Specialists Conference on Ranking Space Power Systems. NASA Lewis 1965 

H. CONTROL SYSTEM DESIGN FOR A YAW ROTA TOR GPS SPACECRAFT 

H-1.0 INTRODUCTION

A candidate nuclear-powered GPS Space Vehicle spins about the earth pointing axis. This arrangement aids in making the effective solar pressure forces quite predictable because the vehicle responds to the average, over time, of the instantaneous solar pressure forces. These instantaneous solar pressure forces vary with vehicle solar aspect and vehicle surface characteristics. The effect of these variations is made more predictable with spin because the effective solar force on the vehicle due to the changing solar aspect becomes a function only of the angle between the sun-line and spin axis even for a vehicle whose surface is not a surface of revolution. In addition, those surface characteristics which are a function of temperature, are maintained nearly constant because the surface is approximately isothermal over many spins. Thus, the surface characteristics vary slowly over each orbit.

The nuclear power systems of interest contain (speed controlled) rotating . machines which are assumed aligned with the vehicle spin axis. When the vehicle's and rotating machine's spin angular momenta are equal and oppositely directed, the vehicle responds to extermal torque as if it were non-rotating. Without the compensating effect of the rotating machine's angular mo mentum, there would be either a need for continual momentum unload as the vehicle moves in orbit, or an alternate compensation through on-board control rotating devices. Thus, the inherent angular momentum of the nuclear power system may be employed advantageously by eliminating or reducing the need for counter-rotating devices in the proposed operating mode.

The purpose of this study is to verify that relatively simple, lightweight control systems can be designed suitable for the required use-- $0.5^{\circ}$ earth pointing accuracy with spin rate nominally fixed with a basic value in the range of 0.2 to $2 \mathrm{rom}$.

\section{H-2.0 REQUTRED MOMENTUM STORAGE}

Solar pressure torques cause angular momentum accumulation in the vehicle. The torques are functions of vehicle solar aspect and surface properties. Appendix $H-B$ contains an analysis of the effect of these torques in causing the angular momentum buildup which requires storage in the vehicle control torques.

The satellite is assumed to be $6 \mathrm{ft}$ in diameter and $5 \mathrm{ft}$ long. A 3 -ft black "hole" in the skin is made available for the orbit insertion engine. The vehicle $\mathrm{cg}$ is taken as displaced 3 inches (an estimated maximum value) along the axis of symmetry from the geometric center. The skin is painted white. Under these conditions Fig. $\mathrm{H}-\mathrm{B}-4$ indicates that the required storage capability is approximately $0.025 \mathrm{ft} \mathrm{lb} \mathrm{sec}$ times the number of orbits desired between momentum unloads. $F i_{g}$. H-B-4also indicates that the hole in the skin causes only a negligible portion of the total angular momentum storage requirement. The maximum storage requirement occurs when the orbit plane is inclined about 45 degrees from the sun-line. 
In this study a 10-day interval between unloads will be posited so that the required storage is $0.5 \mathrm{ft} \mathrm{lb} \mathrm{sec}$. The actual implementation would include at least a $50 \%$ contingency resulting in a $0.75 \mathrm{ft} \mathrm{lb}$ sec on-board capability.

(In the event that the nuclear engine combined rotating unit (CRU) is not along the vehicle axis, as postulated in 2.0 , an increment must be made to the required storage of magnitude $2 \mathrm{rlQ}$. )

\section{H-3' ON-ORBIT CONTROL SYSTEM DESCRIPTION}

The approach taken in the normal operation control of the satellite, which spins about the satellite-earth line, is to control the vehicle attitude about axes normal to that line and to allow the vehicle spin rate to be uncontrolled. Variations in spin rate will occur primarily as the vehicle accumulates angular momentum due to external torques and secondarily during vehicle attitude transients. Periodic unloading of the accumulated angular momentum to reestablish the desired spin rate is accomplished by jet thrusting; spin rate information indicating the unload requirement is available from body-mounted sun sensors when the vehicle spin axis is normal to the vehicle-sunline, i.e., twice each orbit.

Thus, continuous control is only required about the two axes normal to the spin axis. Body-fixed horizon sensors are used to measure the angle "components" about the vehicle $\mathrm{X}$ and $\mathrm{Y}$ axes, $\mathrm{Z}$, and the local vertical. Derivatives of the horizon sensor outputs may be used to stabilize the control loons. Four different non-redundant torquer configurations may be considered (see Fig. H-l).

a. Two reaction wheels may be used. One wheel torques the vehicle about its $\mathrm{X}$ axis; the other about its $\mathrm{Y}$ axis.

b. Two single-gimballed control moment gyros may be used. With one gimbal axis along the vehicle $X$ axis, the other along the vehicle $Y$ axis and spin of both wheels nominally along the vehicle $Z$ axis, the units act very much like high torque reaction wheels.

c. One double-gimballed reaction wheel may be used. One gimbal axis is along the vehicle $X$ axis, the other along the vehicle $Y$ axis.

d. One single-gimballed reaction wheel may be used. The wheel spin axis is normal to the vehicle spin axis, and the gimbal axis parallel to the vehicle spin axis. Along the wheel axis the unit acts as a reaction wheel; normal to the wheel axis the unit acts as a control moment gyro.

Variable wheel speeds in 2 and 3 above could also be considered.

In all configurations the addition of a single like unit, reaction wheel or CMG, will provide redundancy without periormance degradation.

H-3.1 Control Loop

The vehicle-fixed earth sensors scan in the vehicle $X Z$ and vehicle $Y Z$ planes to provide vehicle angular positions $(\theta, \phi)$ about $Y$ (pitch) and $X$ (roll) axes. Because 


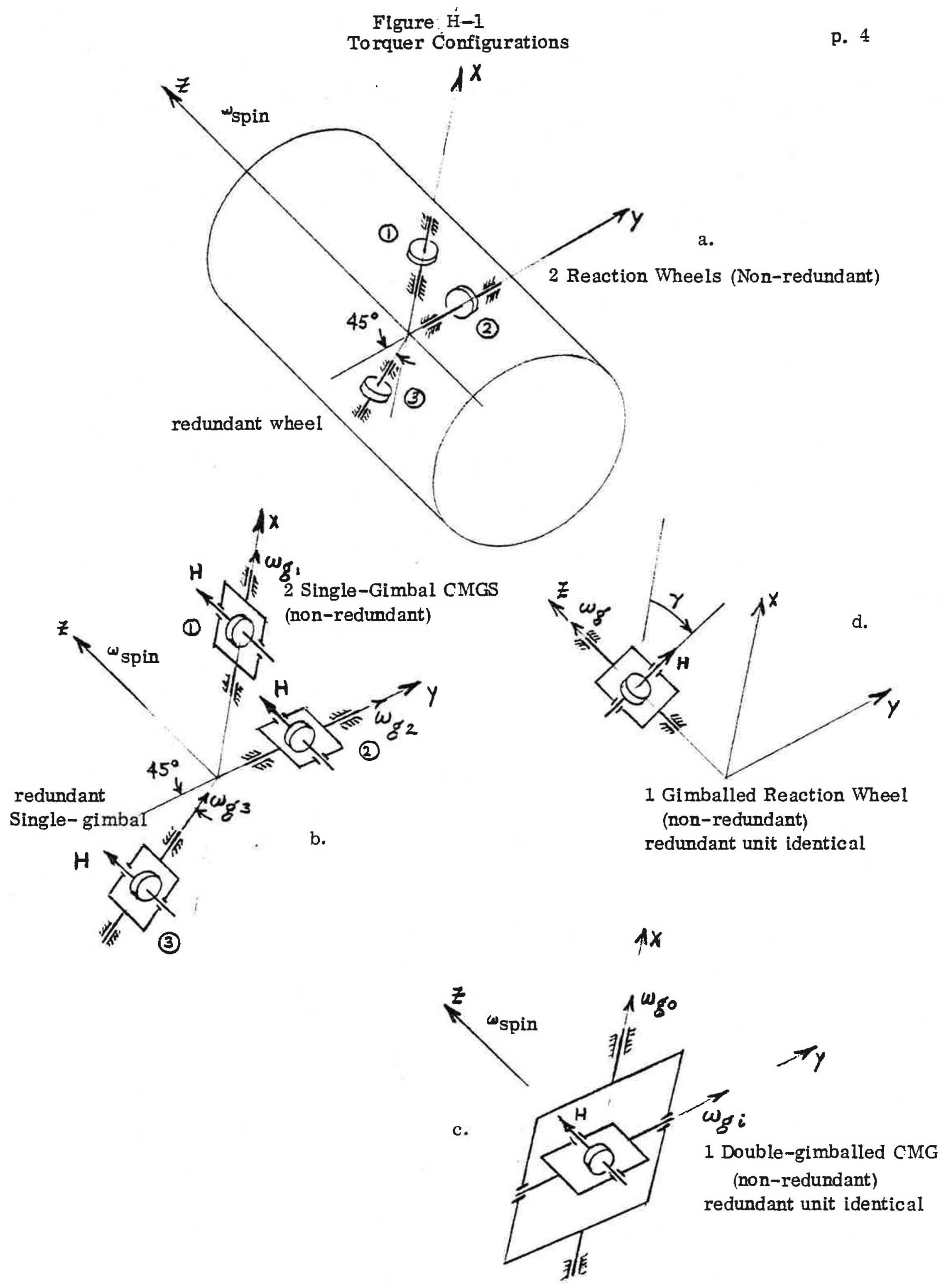


the vehicle is rotating about its $Z$ axis sequential $\theta$ or $\phi$ measurements are not about the same inertial axes. Thus, derivatives of $\phi$ and $\theta$ are not really inertial rates but are only rates of change of earth sensor outputs.

Over small fractions of a vehicle spin cycle the outputs $\theta, \dot{\theta}_{\rightarrow} \phi \quad \phi$ are approximately the same as would be obtained if the vehicle were non-rotating. This suggests the use of the "standard" and simple control law:

$$
\begin{aligned}
& \mathrm{T}_{\mathrm{cx}}=\mathrm{X} \text { axis control torque }=-\mathrm{K}_{1} \dot{\phi}-\mathrm{K}_{2} \phi \\
& \mathrm{T}_{\mathrm{cy}}=\mathrm{Y} \text { axis control torque }=-\mathrm{K}_{1} \dot{\theta}-\mathrm{K}_{2} \theta
\end{aligned}
$$

where $K_{1}, K_{2}$ are constant.

Because the vehicle is rotating in yaw, there is an $\bar{\omega} \times \overline{\mathrm{H}}$ torque generated due to the stored angular momentum in the torquer configuration, $S_{i}$. e., stored $X$ (or $Y$ ) angular momentum $\mathrm{H}_{\mathrm{x}}$ (or $\mathrm{H}_{\mathrm{y}}$ ) results in $\mathrm{Y}$ (or $\mathrm{X}$ ) axis torques. These torques are predictable within the uncertainty in the yaw spin rate $\omega_{s}$, and compensating terms $c$ an be added into the basic control laws to reduce the torque effects:

$$
\begin{aligned}
& \mathrm{T}_{\mathrm{cx}}=\mathrm{X} \text { axis control torque }=-\mathrm{K}_{1} \dot{\phi}-\mathrm{K}_{2} \phi-\omega_{\mathrm{s}} \mathrm{H}_{\mathrm{y}} \\
& \mathrm{T}_{\mathrm{cy}}=\mathrm{Y} \text { axis control torque }=-\mathrm{K}_{1} \dot{\theta}-\mathrm{K}_{2} \theta+\omega_{\mathrm{s}} \mathrm{H}_{\mathrm{x}}
\end{aligned}
$$

Whether the compensating terms are included or not in the control laws, they define a lower bound on the required to rquer output capability as a function of spin speed and desired momentum storage capability.

To retain the similarity of the control loop for this rotating vehicle to the control loop for a non-rotating vehicle, the bandwidth of the loop should be made high compared to the spin rate, i.e.

$$
\sqrt{\frac{K_{2}}{I}} \gg w_{5}
$$

\section{H-3.2 Commands for Singly Redundant Torquer Configurations}

\section{E-3.2.] $\quad$ Reaction Wheels}

For wheels mounted as shown in Figure $\mathrm{H}-1 \mathrm{a}$, the wheel motor to rque commanded on the individual wheels are:

$$
\begin{aligned}
& \text { Motor torque on Wheel } 1=-T_{\text {ex }} \\
& \text { Motor torque on Wheel } 2=-T_{\text {cy }} \\
& \text { Motor torque on Wheel } 3=0 \text { (wheel power OFF }
\end{aligned}
$$


mands are:

In the event of failure of Wheel 1 , it is turned off and the wheel torque com-

Motor Torque on Wheel $2=-\mathrm{T}_{c y}+\frac{\mathrm{T}_{\mathrm{cx}}}{\frac{.707}{}}$

Motor torque on Wheel $3=\frac{-T_{c x}}{0.707}$

0.707

mands are:

In the event of failure of Wheel 2 , it is turned off and the wheel torque com-

$$
\text { Motor torque on Wheel } 1=-T_{c x}+\frac{T_{c y}}{.707}
$$

Motor torque on Wheel $3=-\mathrm{T}$

$$
\frac{c y}{.707}
$$

When two wheels fail, the system has failed.

\section{H-3.2.2 Single Gimballed Control Moment Gyros}

For gyros mounted as shown in Fig. $\mathrm{H}-1 \mathrm{~b}$, the commanded gimbal rates are:

$$
\begin{aligned}
& \text { Gimbal rate of gyro } 1=\frac{T_{c y} / \cos \gamma_{1}}{H} \\
& \text { Gimbal rate of gyro } 2=\frac{-T}{\mathrm{H}} / \cos \gamma_{2} \\
& \text { Gimbal rate of gyro } 3=0 \text { (gyro power OFF) }
\end{aligned}
$$

where is gimbal displacement of unit i.

rates are:

In the event of failure of gyro 1 , it is turned off and the commanded gimbal

$$
\begin{aligned}
\text { Gimbal rate of gyro } 2= & \left.\frac{\left(T_{c x}\right.}{H}-\frac{T_{c y}}{.707 \mathrm{H}} / \cos \gamma_{3}\right) / \cos \gamma_{2} \\
\text { Gimbal rate of gyro } 3= & \frac{T_{c y}}{.707 \mathrm{H}}
\end{aligned}
$$


In the event of failure of gyro 2 , it is turned off and the commanded gimbal rates are:

$$
\begin{aligned}
& \text { Gimbal rate of gyro } 1=\left(\frac{T_{c y}}{H}+\frac{T_{c x}}{.707 \mathrm{H}} / \cos \gamma_{3}\right) / \cos \gamma_{1} \\
& \text { Gimbal rate of gyro } 3=\frac{-T_{c x}}{.707 \mathrm{H}} / \cos \gamma_{3}
\end{aligned}
$$

When two gyros have failed, the system has failed.

\section{$\mathrm{H}-3.2 .3 \quad$ Gimballed Reaction Wheel}

For the unit orientation shown in Fig. $\mathrm{H}-1 \mathrm{c}$ the commanded gimbal rates and motor torques are:

$$
\begin{aligned}
& \text { Motor torque } \left.=-T_{c x} \cos \gamma+T_{c y} \sin \gamma\right] \\
& \text { Gimbal rate }=\frac{-1}{H}\left[-T_{c x} \sin \gamma+T_{c y} \cos \gamma\right]
\end{aligned}
$$

In the event of failure of the operating unit its non-operating redundant equal is activated. It uses the same commands.

H-3.2.4 Double-Gimballed CMG

For the unit orientation shown in Fig. $\mathrm{H}-1 \mathrm{~d}$, the commanded gimbal rates are:

$$
\begin{aligned}
& \text { Outer gimbal rate command }=\frac{{ }^{T} c y-T_{c x} \sin Y_{0} \tan \gamma_{1}}{\mathrm{H} \cos Y_{i} \cos Y_{0}} \\
& \text { Inner gimbal rate command }=\frac{-T_{c x}}{H \cos Y_{i}}
\end{aligned}
$$

In the event of failure of the operating unit, its non-operating redundant equal is activated. It uses the same commands.

\section{H. -3 System Performance}

With the intent of verifying the basic contro' concept, a series of tests of loop stability and performance were made, by digital simulation, for two of the torquer configurations--the reaction wheel set and the gimballed reaction wheel. (The two CIVG sets act dynamically very much like reaction wheels, of either high or low torque capability, and their performance can be estimated from the performance of the reaction wheels.) 
The simulation is documented in Appendix $\mathrm{H}-\mathrm{C}$.

Parameters used in the simulation were:

$\begin{array}{ll}\text { Vehicle spin inertia } I_{z} & 100 \mathrm{slug}-\mathrm{ft}^{2} \\ \text { Vehicle lateral inertia } I_{x}, I_{y} & 90 \mathrm{slug}-\mathrm{ft}^{2} \\ \text { Vehicle spin rate } \mathrm{W}_{\mathrm{z}} & 0.1 \mathrm{rad} / \mathrm{sec} \\ \text { Vehicle orbital period } & 12 \mathrm{hours} \\ \text { Control System natural freq. } \omega_{n} & \begin{array}{c}1 \mathrm{rad} / \mathrm{sec} \\ \quad K_{z}=\omega_{n}^{2} I\end{array} \\ & 90 \mathrm{ft} \mathrm{lb} / \mathrm{rad} \\ & \\ \text { Control System damping ratio } \zeta & 1 \\ \quad K_{1}=2 \zeta \omega_{n} I & 180 \mathrm{ft}-\mathrm{lb} / \mathrm{rad} / \mathrm{sec} \\ \text { Reaction Wheel torque capability } & 20 \mathrm{in}-0 \mathrm{z} \\ \text { Reaction Wheel Maximum } \mathrm{H} & 0.75 \mathrm{ft}-\mathrm{lb}-\mathrm{sec} \\ \text { Gimballed wheel torque capability } & 20 \mathrm{in}-\mathrm{oz} \\ \text { Gimballed wheel maximum } \mathrm{H} & 0.75 \mathrm{ft}-\mathrm{lb}-\mathrm{sec} \\ \text { Gimballed wheel gimbal rate (max) } & 1 \mathrm{rad} / \mathrm{sec}\end{array}$

Loop stability and error closure toward zero was verified with both torquer configurations using initial attitude errors. However, no attempt was made at optimizing nor has a wide variety of possible situations been treated. Typical results are given in Fig. $\mathrm{H}-2$.

H-4.0 TORQUER DISCUSSION

Provided that the associated size, weight, power costs are acceptable, reaction wheels would undoubtedly be the preferred storage devices/torquers. This results from the success experienced in their on-orbit operations. In most of the on-orbit experience the wheels have been operated in quasi-steady speed, low torque (hence low power) modes. Application of this experience to the present use implies only low power operation of the wheels. This may be the determining factor in selection or non-selection of wheels as control system torquers.

As noted in $\mathrm{H}-3.1$ the wheel torque must be at least $U \mathrm{H}$. If a maximum $2 \mathrm{rpm}$ vehicle spin rate is assumed, and $\mathrm{H}$ is taken as $0.75 \mathrm{ft} \mathrm{lb} \mathrm{sec}$, the wheel torque requirement $\tau_{\mathrm{W}}$ is given by:

$$
\begin{aligned}
T_{w} & =\omega_{s} H=2\left(\frac{2 \pi}{60}\right) \quad 0,75(10,2) \quad \mathrm{in} .02 \\
& =30 \mathrm{in} \mathrm{oz}
\end{aligned}
$$


H-2

(1 of 3 )
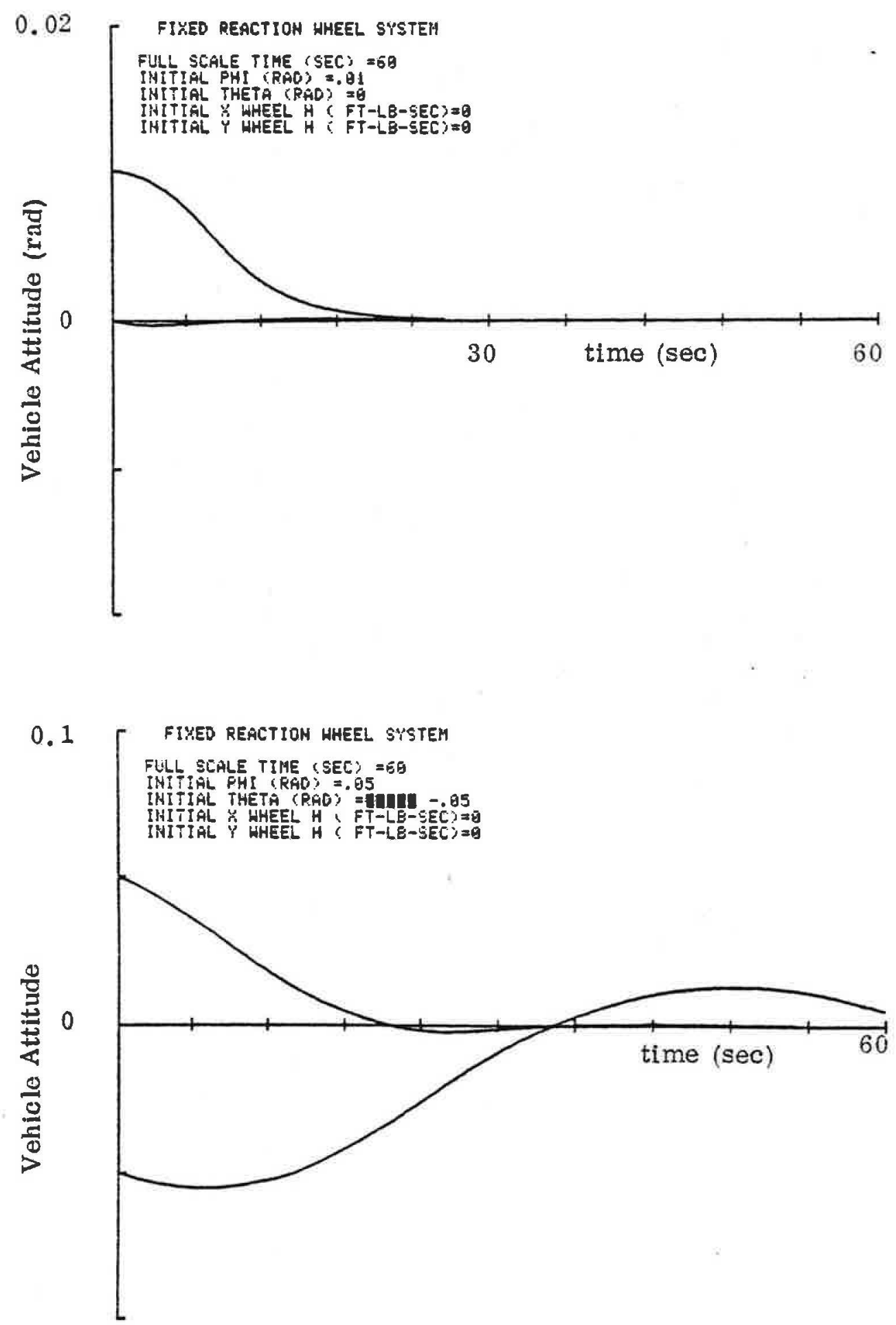
Fig. $\mathrm{H}-2$

(2 of 3)
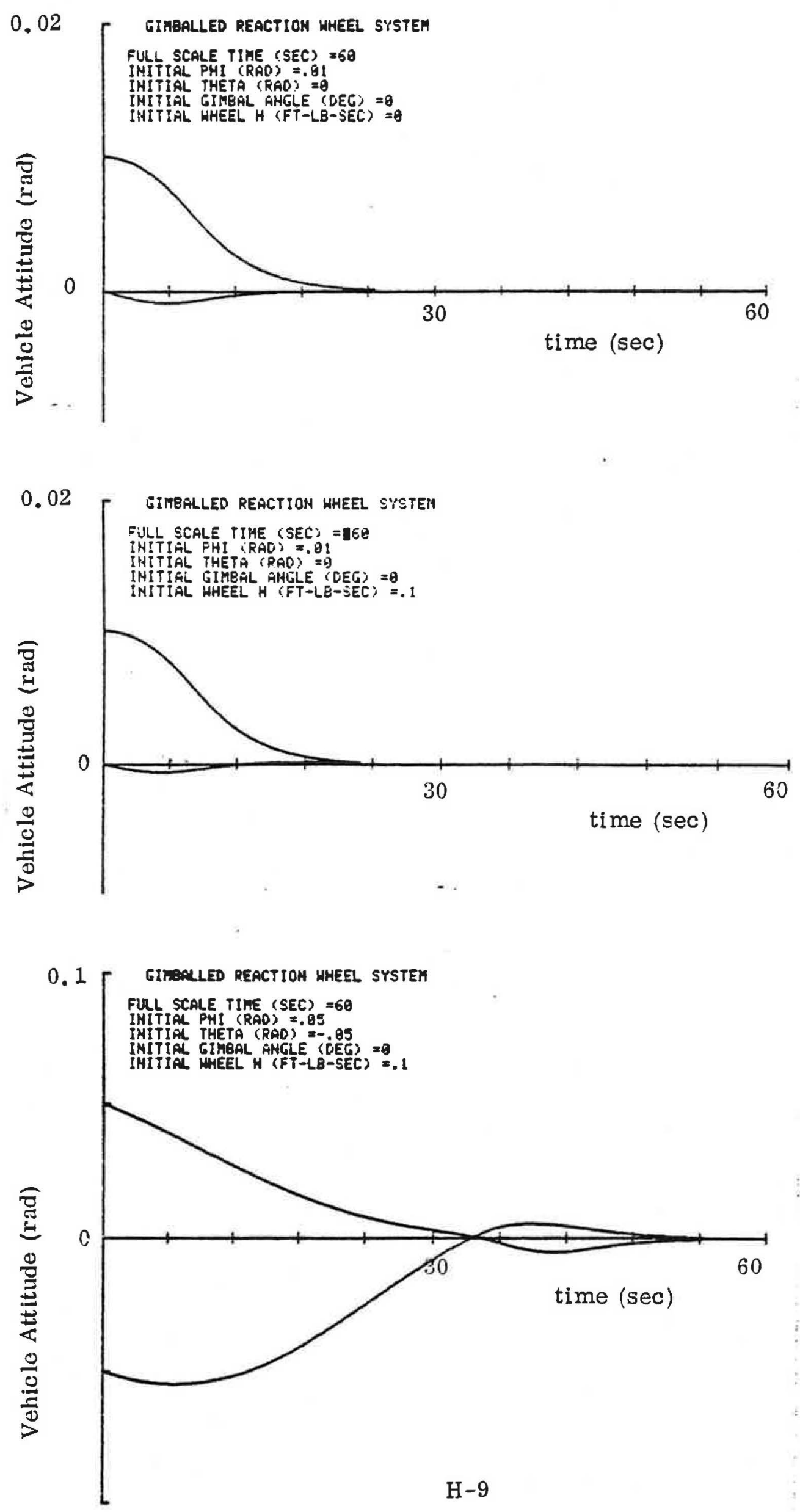

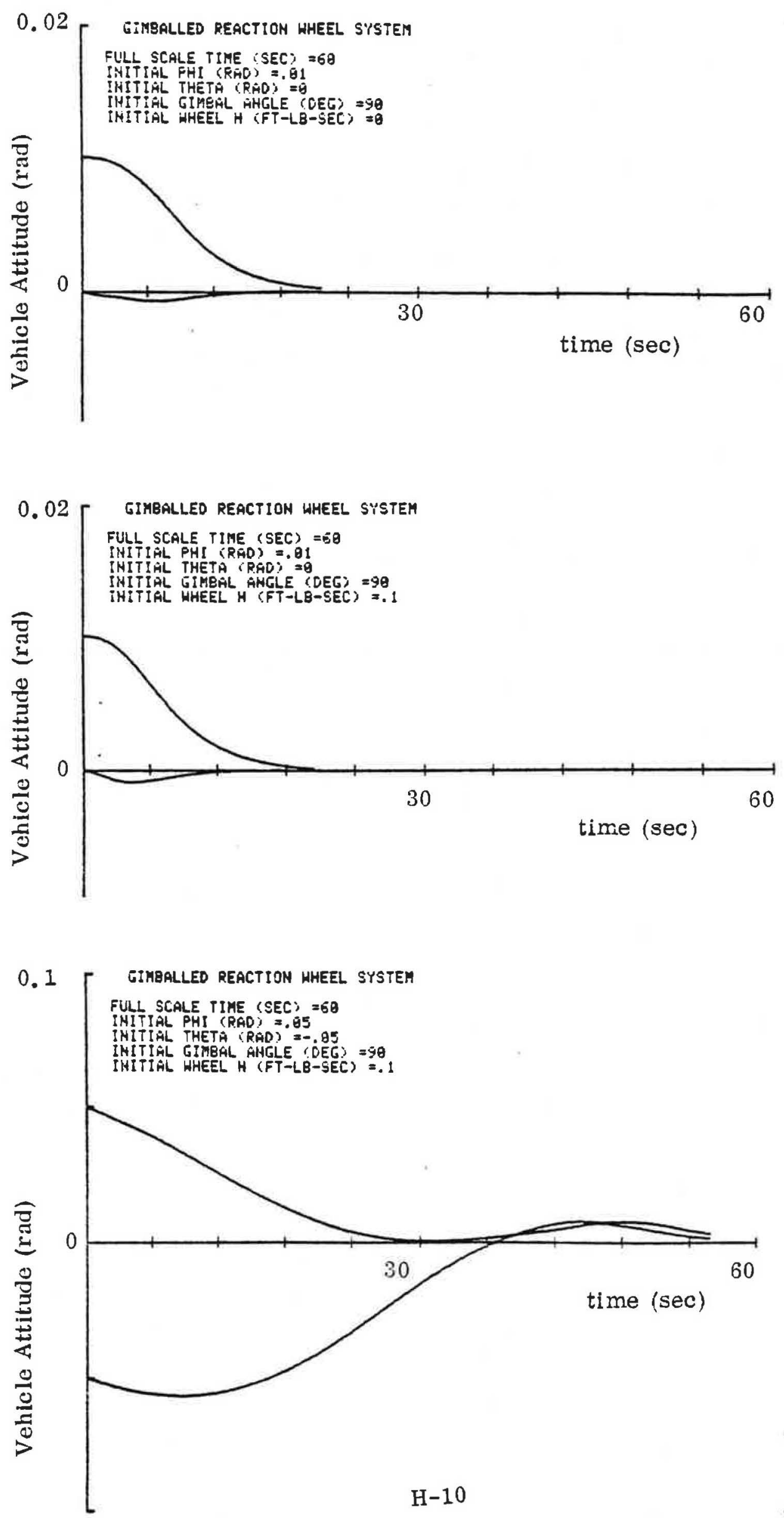
The resulting wheel motor input power $\mathrm{P}_{\mathrm{W}}$ requirements are given by the product of to rque $T_{W}$, speed $W_{W}$, and reciprocal of efficiency $\eta_{W^{*}}$. An efficiency of $50 \%$ may be assumed. Also, the wheel $\mathrm{H}$ is the product of wheel $\mathrm{W}^{\mathrm{W}}$ speed and spin

moment of inertia $I_{W}$.

$$
\mathrm{P}_{\mathrm{w}}=\mathrm{T}_{\mathrm{w}} \quad \mathrm{w}_{\mathrm{w}}^{/ \eta}{ }_{\mathrm{w}}
$$

Maximum power is the refore given by:

$$
\begin{aligned}
P_{w} \max & =2\left(w_{s} H\right) w_{w} \\
& =2\left(w_{s} H^{2} / I_{w}\right)
\end{aligned}
$$

Spin rate $\omega$ is primarily a function of the angular momentum of the nuclear engine's rotating machine, the wheel angular momentum $\mathrm{H}$ depends upon the storage requirement, and the wheel speed $\omega_{\mathrm{W}}$ (or equivalently its moment of inertia $I_{\mathrm{W}}$ ) depend upon its physical size. The latter is the only element at the designer's option unless the spin rate is adjusted by the use of auxiliary $\mathrm{Z}$ axis wheels).

If an Organic Rankine engine with $\mathrm{H}_{\mathrm{or}}=17.6 \mathrm{ft} \mathrm{lb} \mathrm{sec}$ is assumed for a 100
slug $\mathrm{ft}^{2}$ vehicle, then:

$$
\omega_{\mathrm{W}}=49 \text { (P watts) } \mathrm{rpm}
$$

With 10 watts estimated as allowable power, the maximum allowable wheel speed is $490 \mathrm{rpm}$ and the approximate wheel weight is 11 pounds.

The gimballed reaction wheel does not have the to rquing requirements of the reaction wheel system because momentum transfer from axis to axis during spin is accomplished by gimbal rotation relative to the vehicle. Thus, higher wheel speeds may be used. The wheel assembly weight would be about 6 pounds and an additional $4 \mathrm{lbs}$ might be expected for the gimbal, its torquer, position sensor, its cover, etc. A singly redundant reaction wheel configuration would the refore weigh about ten pounds more and require about 10 watts more than a singly redundant gimballed reaction wheel system.

There appears to be small advantage in using CMGs as actuators when the Organic Rankine engine, with its large angular momentum, is used. So me improvement in transient response would be achieved due to the large torque output of these units. If a Brayton engine, with its rotating machine's angular momentum of $0.68 \mathrm{ft}$ lb sec is used, the angular momentum of the CMGs can be profitably used to set the vehicle spin rate. Thus, with $\mathrm{CMG} \mathrm{H}=1 \mathrm{ft} \mathrm{lb} \mathrm{sec}$, one can achieve a spin rate of $0.25 \mathrm{rpm}$ when 2 single gimballed CMGs are used and a spin rate of $0.16 \mathrm{rpm}$ when one double gimballed CMG is used.

H-5.0 CONCLUSION

A simple, lightweight control system for an earth-pointing rotating GPS satellite has been defined and its stability and error closure toward zero verified. Of all the control loop elements, only the torquer differs from that in the existing GPS satellites. Fixed or gimballed reaction wheel configurations are suitable, the former being preferred if the costs due to its higher weight and power are acceptable. 


\section{APPENDIX H-B}

\section{CALCULATION OF ANGULAR MOME NTUM ACCUMULATION}

DUE TO SOLAR PRESSURE

\section{H-B.1.0 SOLAR PRESSURE TORQUES - NOMINAL}

A simplified model of the vehicle configuration was developed for use in assessing the level of cyclic and bias solar torques and resulting angular momentum accumulation during earth orbit. The model consists of flat and cylindrical surfaces equivalent in area to those of the proposed configuration, detailed in Fig. H-B-1, where surface properties are desc ribed.

The equations used have been derived based on the usual photon momentum transfer theory and the appropriate geometric treatment of surfaces exposed to solar flux. Normal $\left(F_{n}\right)$ and tangential $\left(F_{t}\right)$ components of force are defined for flat and cylindrical surfaces as follows:

\section{Flat Plate}

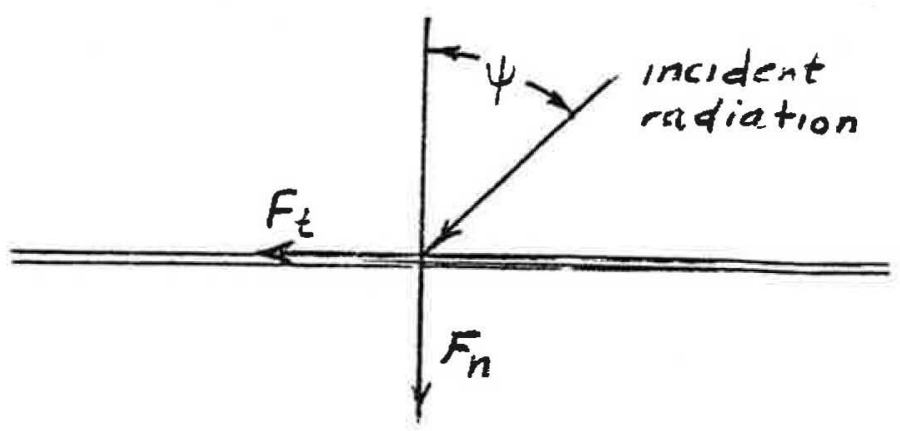

where:

$$
\begin{aligned}
& F_{n}=\left[(1+s p) \cos ^{2} \psi+\frac{2}{3} p(1-s) \cos \psi\right] P_{f} A_{p} \\
& F_{\frac{1}{n}}=[(1-s p) \cos \psi \sin \psi] P_{f} A_{p} \\
& F_{p}=\text { solar pressure, } \mid \times 10^{-7} 16 / f^{2} \\
& A_{p}=p: a-p \text { ar }=a, f^{2}
\end{aligned}
$$




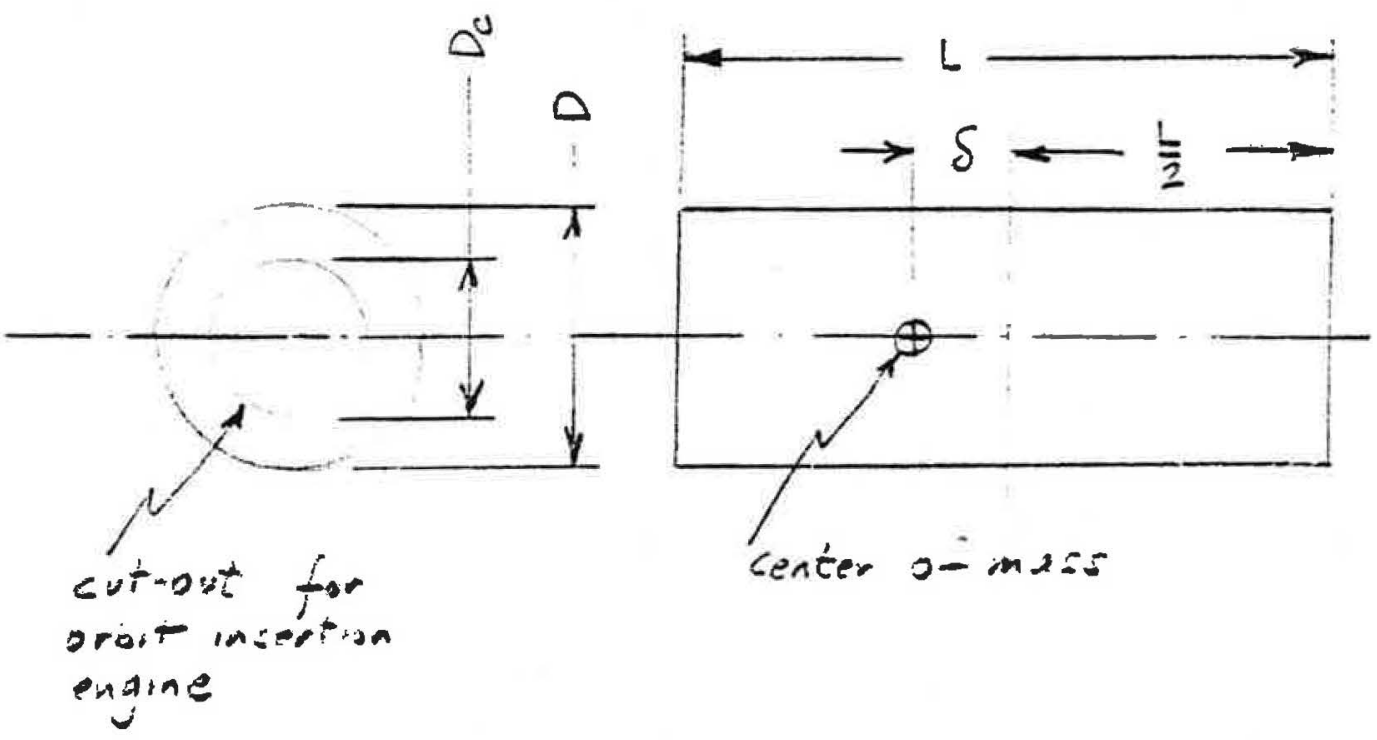
$\mathrm{L}=5 \mathrm{ft}$
$D=6 \mathrm{ft}$
$D_{c}=0$ or $4 \mathrm{ft}$
$\delta=3$ inches

vehicle painted white all over $\quad s=0, \rho=0.75$

cutout for OIS $\quad s=0, \rho=0.1$

( $s=$ specularity, $\rho=$ reflectivity)

Fig. $H-B-1$ Simplified Vehicle Model 


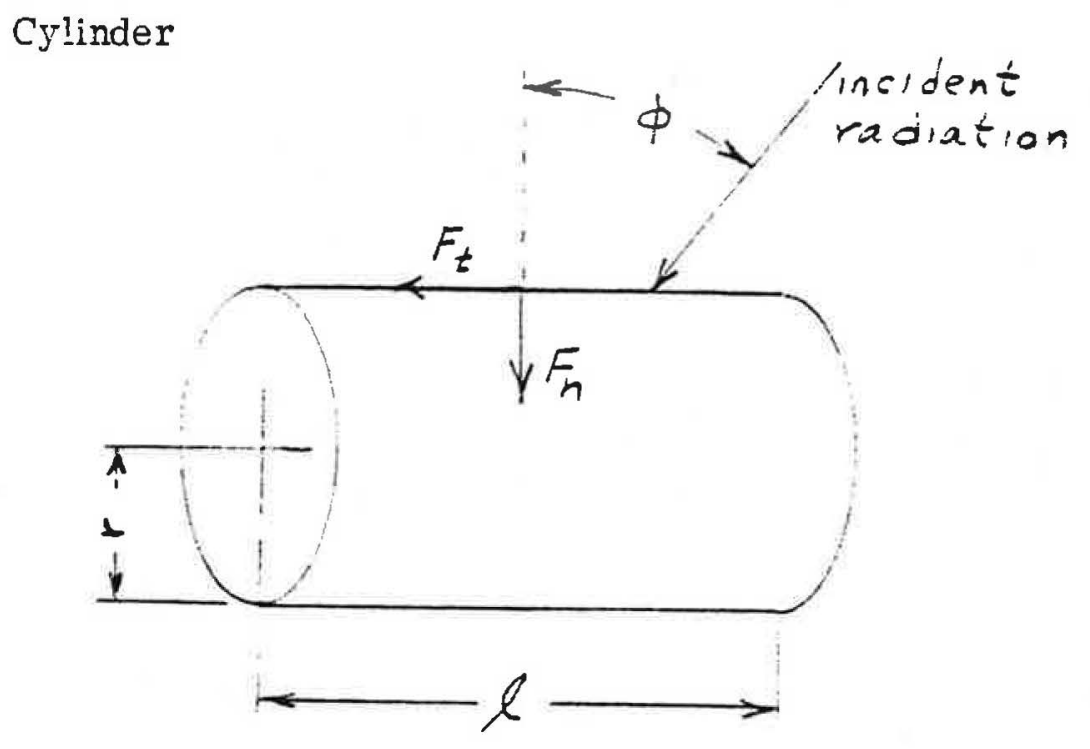

$$
\text { where } \begin{aligned}
F_{n} & \left.=\left[(1+s \rho / 3) \cos ^{2} \phi+\rho ! 1-s\right) \frac{\pi}{6} \cos \phi\right] P_{\leqslant} A_{c} \\
F_{t} & =[(1-s \rho) \cos \phi \sin \phi] P_{\dot{f}} A_{c} \\
A_{c} & =2 r l
\end{aligned}
$$

and where

$$
\begin{aligned}
& p=\text { fraction of incident radiation reflected } \\
& s=\text { fraction of reflect radiation which is specular }
\end{aligned}
$$

H-B.2 CALCULATION OF TORQUES DUE TO SOLAR PRESSURE

The vehicle moves in an orbit inclined angle $i$ to the sun-line. It is located angle $\Omega$ from the ascending node with respect to an inertial reference system $X_{s}, Y_{s}$, $Z$, where $Z_{s}$ looks at the sun, and $Y_{s}$ points along the ascending line-of-nodes.' $T$ he vehicle, with coordinate system $X_{v}, Y_{v}, Z_{v}$, has its $-Y_{v}$ axis pointing to the earth and $X$ pointing along the orbit axis $X_{O A}$. The orbit axis system has its $y$ axis $Y_{o A}$ along the inertial $Y_{S}$ axis. The geometry is illustrated in Fig. B-2.

Transformation relating vectors $\mathrm{V}_{\mathrm{V}}, \mathrm{V}_{\mathrm{S}}, \mathrm{V}_{\mathrm{OA}}$ in the vehicle, sun inertial, and orbit axis system are given below: 
(s)

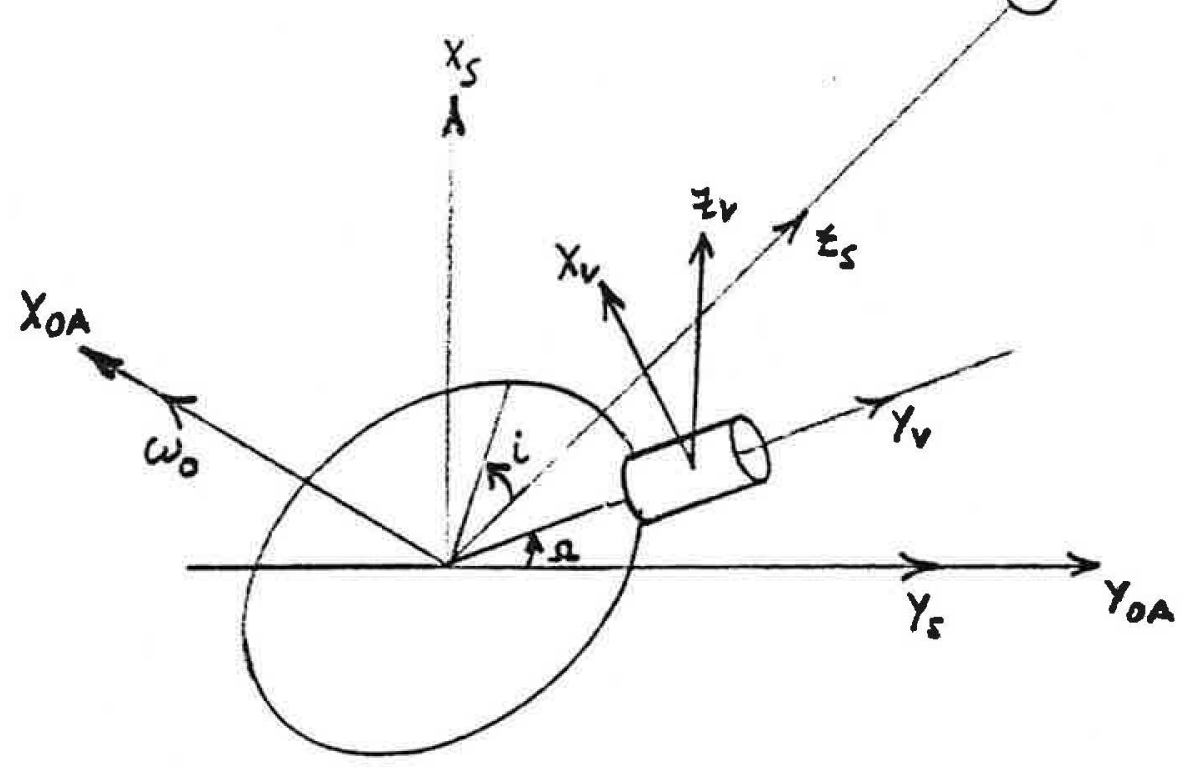

$U_{\text {nit }}$ vectors along coordinate axes in space $i: \bar{\tau}_{i}, \bar{f}_{i}, \bar{k}_{i}$

Vehicle in-orbit geometry

fig $\mathrm{H}-\mathrm{B}-2$ 


$$
\begin{aligned}
& \left(V_{s}\right)=\left[\begin{array}{ccc}
c i & 0 & 5 i \\
0 & 1 & 0 \\
-s i & 0 & c i
\end{array}\right]\left[\begin{array}{ccc}
1 & 0 & 0 \\
0 & c \Omega & -s \Omega \\
0 & s \Omega & c \Omega
\end{array}\right]\left(V_{v}\right) \\
& \left(V_{0 A}\right)=\left[\begin{array}{ccc}
1 & 0 & 0 \\
0 & c \Omega & -s \Omega \\
0 & s \Omega & c \Omega
\end{array}\right]\left(V_{V}\right)
\end{aligned}
$$

where $c=\cos \quad s=\sin$

Thus:

$$
\begin{aligned}
& \left(V_{s}\right)=\left[\begin{array}{ccc}
c i & \text { sis } \Omega & \text { sic } \Omega \\
0 & c \Omega & -s \Omega \\
-5 i & c i s \Omega & c i c \Omega
\end{array}\right]\left(V_{v}\right) \\
& \left(V_{v}\right)=\left[\begin{array}{ccc}
c i & 0 & -s i \\
s i s \Omega & c \Omega & c i s \Omega \\
s i c \Omega & -5 \Omega & c i c \Omega
\end{array}\right]\left(V_{s}\right)
\end{aligned}
$$

H-16 
The normal component of solar force on the cylindrical portion of the vehicle $\left(\bar{F}\right.$ ) lies normal to the vehic le $Y_{V}$ axis and in the $Y_{V} Z$ plane. It has a component away from the sun. Thus, the unit vector $\bar{e}_{n c}$ along ${ }_{\mathrm{F}} \mathrm{s}$ is given by:

$$
\begin{aligned}
\bar{e}_{n c} & =\frac{\bar{y}_{v} \times\left(\bar{y}_{v} \times \bar{s}_{s}\right)}{\left|\bar{y}_{v} \times\left(\bar{y}_{v} \times \overline{\bar{E}}_{s}\right)\right|} \\
& =\frac{s i \bar{c}_{v}-c i c \Omega \bar{k}_{v}}{\left(s i^{2}+(\operatorname{cic} \Omega)^{2}\right)^{1 / 2}}
\end{aligned}
$$

The tangential component of solar force on the cylindrical portion of the vehicle $\left(F_{t c}\right)$ is directed along the $-Y_{v}$ axis when $0 \leq \Omega<\pi$ and along the $+\bar{Y}_{v}$ axis when $\pi \leq \mathrm{tc} \Omega \leq 2 \pi$

$$
\bar{e}_{n, t}=\left\{\begin{array}{lll}
-\bar{j}_{v} & \text { for } & 0 \leq \Omega<\pi \\
+\bar{j}_{v} & \text { for } & \pi \leq \Omega<2 \pi
\end{array}\right.
$$

The normal component of solar force on the flat faces of the vehicle $\left(\overline{\mathrm{F}}_{\mathrm{nf}}\right)$ lies along the vehicle $\mathrm{Y}_{\mathrm{V}}$ axis. (It causes no vehicle torques because the vehicle $\mathrm{nf}^{\mathrm{C}} \mathrm{g}$. is assumed on the $Y_{v}$ axis.) The tangential component of solar force on the flat faces of the vehicle is directed parallel to $\bar{e}_{n c}$.

The moment arm vector for $\overline{\mathrm{F}}_{\text {tc }}$ is directed opposite the $\bar{\Xi}_{\text {pc }}$ vector and has half the diameter of the vehicle as magnitude. Due to the vehic le center-of-mass displacement $(\delta)$ along the $+Y$ axis, from the vehicle geometric center, the moment arm vector for $\bar{F}$ is $-\delta P_{v}$. The moment arm vector for $\bar{F}_{\text {tf }}$ on the $+Y_{v}$ face is $(L / 2-\delta) J_{V}$. For $\overline{\mathrm{F}}_{\mathrm{tf}}$ on the $-\mathrm{Y}_{\mathrm{v}}$ face, the moment arm vector is $-(\mathrm{L} / \mathrm{tf}+\delta) \overline{\mathrm{J}}_{\mathrm{v}}$.

Total torque on the vebicle is given as the sum of individual moment arm vectors crossed with their force vectors:

$$
\begin{aligned}
\bar{F}_{t o t a l}= & \frac{D}{2}\left(-\bar{e}_{n c}\right) \times\left|\bar{F}_{t_{c}}\right| \bar{j}_{v} M \\
& -\delta \bar{f}_{v} \times\left|\bar{F}_{n c}\right| \bar{e}_{n c} \\
& +\left(\frac{L}{2}+\delta M\right) \bar{f}_{r} \times\left|\bar{F}_{t+1}\right| \bar{e}_{n c}(-M)
\end{aligned}
$$

where: $M=-1$ for $0 \leqslant \Omega<\pi$

$M=1$ for $\pi \leq \Omega<2 \pi$ 
Evaluation of the force magnitudes requires evaluation of the acute angles between the sun-line and the flat surface normal: $\quad \phi^{\prime}=\cos ^{-1}\left|\bar{z}_{\sigma} \cdot \bar{k}_{s}\right|$ and the acute angle between the sun line and the cylinder normal: $\quad \phi=\frac{\pi}{2}-\phi^{\prime}$

Accumulation of angular momentum in inertial space is calculated by taking the components of torques about vehicle axes, resolving them into the orbital axis system and integrating. The total accumulation is the sum of squares of the individual accumulations.

The above equations have been programmed for digital computer simulation, with the program documented in Figure $\mathrm{H}-\mathrm{B}-3$, and results for various orbit inclinations $i$ are given in Figure H-B-4. 
190 REM THIS PROGRAM IS ON FILE 13

120 OIM $F(3,3)$

$130 \quad 08=0$
$158 \quad H 6=0$

$168 \begin{array}{ll}H 7=0 \\ H 7\end{array}$

170 HB=6 : CHGHCE OUER ONE ORBIT OF ANCULAF MOMEHTUH (FT-LE-SEC)

IGQ PRINT"

200 PRINT

III INPUT Ro

OIS EHGINE GPEHINC RADIUS $(F T)=*$

II FRINT :

CENTER OF MÁSS DISPLACEHEHT $\langle I M\rangle="$

$214 \lim _{0=0} 12$

$225 I=9$

$226 C=8.05$

240

245 HOUE $\theta, C$

247 HOUE $\theta, C$,

248 PRINT $\mathrm{C} / 2$

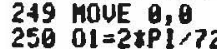

$268 \mathrm{HJ}=0$
$270 \quad \mathrm{HZ}=0$

$270 \quad \mathrm{H}=0$

$306 \quad \mathrm{Q}=1.0 \mathrm{E}-3$

$340 \quad T=12 \$ 3600 * 01 /(2 \$ P I)$

$350 \quad L=5$

$3 \in \theta$
370

出

\section{R1 $=0.75$
390 Al $=2 \$ R+1$}

$\begin{array}{ll}390 & A 1=24 R \\ 400 & S 2=8\end{array}$

$\begin{array}{ll}400 & \mathrm{~S} 2=0 \\ 410 & \mathrm{R} 2=0.75\end{array}$

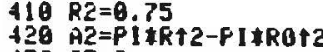

$430 \quad 53=0$

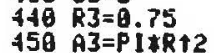

$406 \quad S 4=0$

470 R $=0.1$

4. $A 4=P$ I IROT2

491 IF

$49200=1$. $0 E-1 \theta$

$569 \mathrm{PI}=\mathrm{PI} / 2-A C S(G B S(\operatorname{CoS}(1)+5 I N(0))$ )

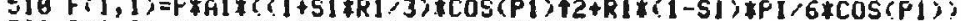

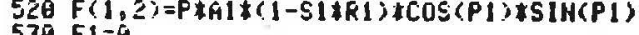

$536 \quad F 1=9$
$5 \neq 0 \quad F 2=8$

$560 \mathrm{H}=1$

570 IF 0 0PI THEH 600

580 F2 =ACS (ABSC COS (I) $\operatorname{SIN}(0) \%$

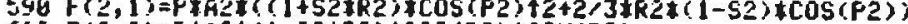

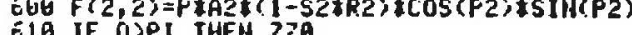

Eld IF OPI THEN 7 ro

E2a $F(2, T)=F 1+F(2,1)$

$646 \quad F l=F(2,1)$

$668 \mathrm{H}=\mathrm{H}+1$

670 If $\mathrm{H}=22$ IHEH 770

67560 T0 738

$\begin{array}{ll}660 & A 2=A 3 \\ 690 & S 2=S 3\end{array}$

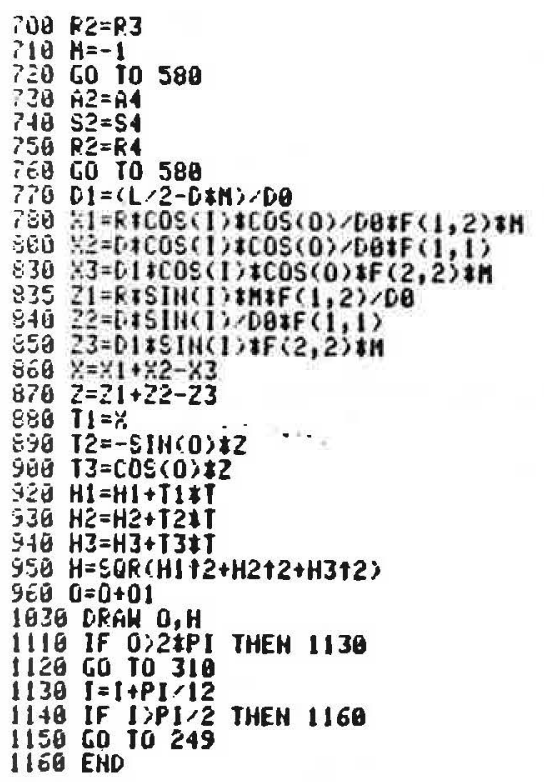



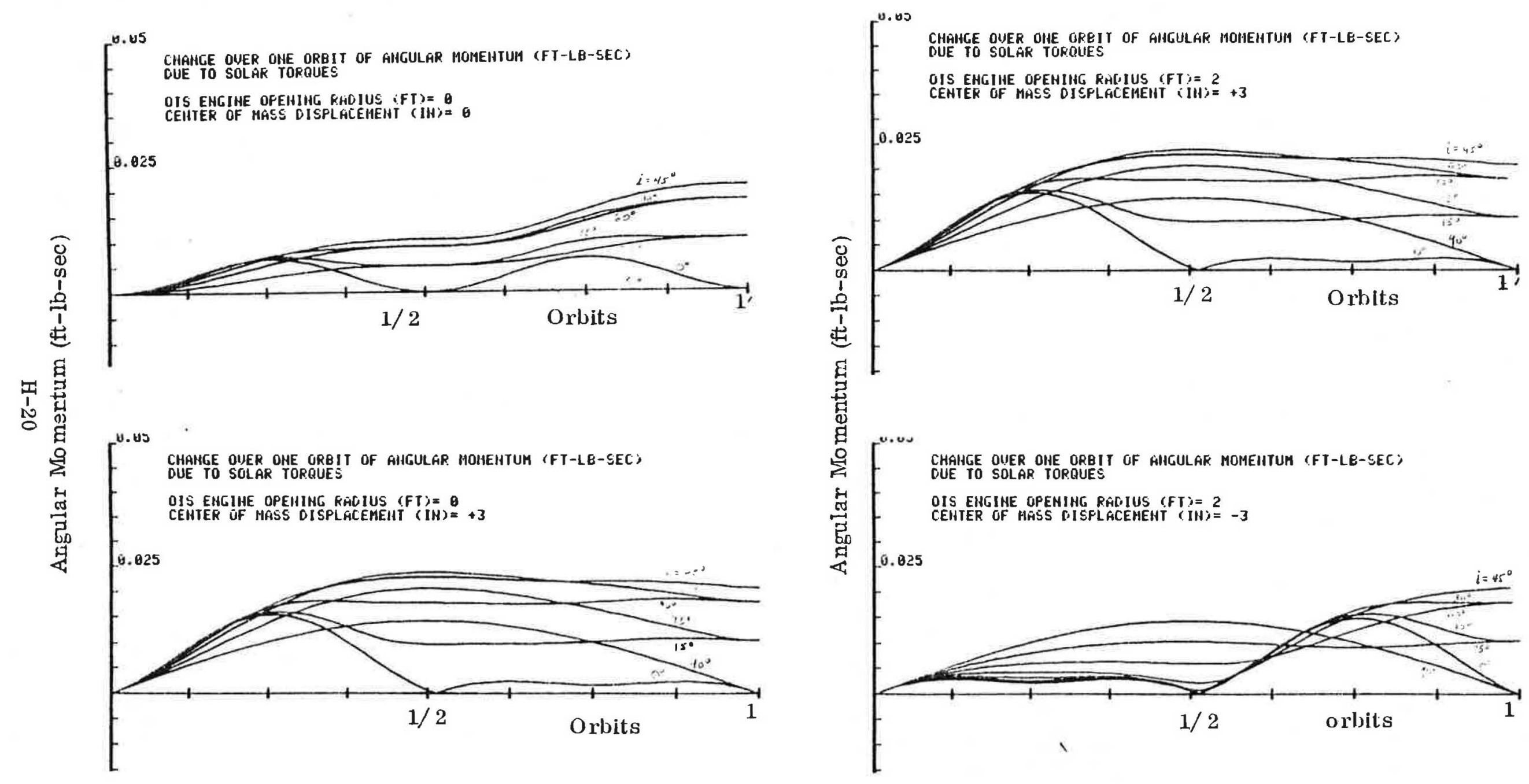

Figure H-B-4 


\section{APPENDIX H-C \\ CONTROLLED VEHICLE DYNAMIC SIMULATION}

The computer program is documented in Figure $\mathrm{H}-1$.

H-C.1.0 COORDINA TE FRAME DEFINITIONS AND TRANSFORMA TIONS

The earth centered inertial system (I) has its $X_{I}$ axis pointing toward the first point of Aries, $\gamma$, and its $Z_{I}$ axis along the vehicle orbit axis.

The reference system $(R)$ with origin at the spacecraft center of mass has its $Z_{R}$ axis point to the earth center below, and its $X_{R}$ axis in the direction of motion. The vehicle is located angle $Y$ from $\gamma$ measured about the orbit axis.

The rotated reference system (RR) has its $Z_{R R}$ axis along the $Z_{R}$ axis and is rotated $\gamma$ about the $\mathrm{Z}_{\mathrm{RR}}$ axis.

The vehicle body axes (B) are nominally along the rotated reference axes but are displaced by rotation $\phi$ about $\mathrm{X}_{\mathbf{R R}}$, then angle $\theta$ about the rotated $\mathrm{Y}_{\mathrm{RR}}$ axis.

Based on these definitions, the following transformations apply:

$$
\left(V_{R}\right)=\left[\begin{array}{ccc}
-s \gamma & c \gamma & 0 \\
0 & 0 & -1 \\
-c \gamma & -s \gamma & 0
\end{array}\right]\left(V_{I}\right)=E\left(V_{I}\right)
$$

where: $\left(V_{I}\right)$ = vector $V$ components in coordinate system $I$

$\mathrm{s}=\sin$

c $\quad=\cos$

$$
\left(V_{R R}\right)=\left[\begin{array}{ccc}
c \gamma & s \gamma & 0 \\
-s \gamma & c \gamma & 0 \\
0 & 0 & 1
\end{array}\right]\left(V_{R}\right)=F\left(V_{R}\right)
$$

Let: $\quad\left(\mathrm{V}_{\mathrm{B}}\right)=\mathrm{A}\left(\mathrm{V}_{\mathrm{I}}\right)$

Then: $\quad\left(\mathrm{V}_{\mathrm{B}}\right)=\mathrm{AE}^{\top} \mathrm{F}^{\top}\left(\mathrm{V}_{\mathrm{RR}}\right)$

also: $\quad\left(V_{R R}\right)=\left[\begin{array}{ccc}c \theta & 0 & s \theta \\ s \phi s \theta & c \theta & -s \phi c \theta \\ -c \phi s \theta & s \phi & c \phi c \theta\end{array}\right]\left(V_{B}\right)=F E A^{\top}\left(V_{B}\right)$ 


\section{$\mathrm{H}-\mathrm{C} .2 .0$ DYNAMICS}

The total spacecraft momentum vector, $\overline{\mathrm{H}}$, is comprised of on-board wheel momentum and momentum due to spacecraft rates:

$$
\bar{H}=[I] \bar{\omega}+\bar{h}
$$

where $[I]$ is spacecraft inertia tensor expressed in body coordinates

$\bar{\omega}$ is spacecraft angular rate expressed in body coordinates

$\bar{h}$ is total on-board wheel momentum expressed in body coordinates

$$
\begin{aligned}
& {[I]=\left[\begin{array}{ccc}
I_{x x} & -I_{x y} & -I_{x z} \\
-I_{x y} & I_{y y} & -I_{y z} \\
-I_{x z} & -I_{y z} & I_{z z}
\end{array}\right]} \\
& \bar{\omega}=\left[\begin{array}{lll}
w_{x} & w_{y} & \omega_{z}
\end{array}\right]^{T} \\
& \bar{h}=\left[\begin{array}{lll}
h_{x} & h_{y} & h_{z}
\end{array}\right]^{T}
\end{aligned}
$$

From Euler's equation of motion, the external torque on the spacecraft causes a rate of change of total momentum:

$$
\begin{aligned}
\bar{T} & =\frac{d}{d t}(\bar{H}) \\
& =[I] \dot{\bar{\omega}}+\dot{\bar{h}}+\bar{\omega} \times([I] \bar{\omega})+\bar{\omega} \times \bar{h}
\end{aligned}
$$

$S$, Iving for the derivative of inertial rate:

$$
\dot{\bar{\omega}}=[I]^{-1}[\bar{T}-\dot{\bar{h}}-\bar{\omega} \times([I] \bar{\omega})-\vec{\omega} \times \bar{h}]
$$

$\mathrm{H}-\mathrm{C}-3.0 \quad$ KINEMLTICS

Euler symmetric parameters are used as the Kinematic variables.

Thus:

$$
\left[\begin{array}{l}
\dot{\xi} \\
\dot{\eta} \\
\dot{\xi} \\
\dot{x}
\end{array}\right]=\frac{1}{2}\left[\begin{array}{cccc}
0 & w_{3} & -w_{2} & w_{1} \\
-w_{3} & 0 & w_{1} & w_{2} \\
w_{2} & -w_{1} & 0 & w_{3} \\
-w_{1} & -w_{2} & -w_{3} & 0
\end{array}\right]\left[\begin{array}{l}
\bar{\eta} \\
\eta \\
\xi
\end{array}\right]
$$


The direction cosine values $\lambda_{i j}$ from equations $C-1, C-4$ are used for initialization with $\boldsymbol{\gamma}=0$.

$$
\begin{aligned}
& \Phi=\cos ^{-1} \frac{1}{2}\left(a_{11}+a_{22}+a_{33}-1\right) \\
& \xi=\left(a_{23}-a_{32}\right) \frac{\sin (\Phi / 2)}{2 \sin \Phi} \\
& \eta=\left(a_{31}-a_{13}\right) \frac{\sin (\Phi / 2)}{2 \sin \Phi} \\
& \xi=\left(a_{12}-a_{31}\right) \frac{\sin (\Phi / 2)}{2 \sin \Phi} \\
& x=\cos (\Phi / 2)
\end{aligned}
$$

During the run the direction cosines are obtained from the symmetric parameters by:

$$
\begin{aligned}
& a_{11}=\xi^{2}-\eta^{2}-\xi^{2}+x^{2} \\
& a_{21}=2(\xi x-\xi x) \\
& a_{31}=2(\xi \xi+\eta x) \\
& a_{12}=2(\xi \eta+\xi x) \\
& a_{32}=-\xi^{2}+\eta^{2}-\xi^{2}+x^{2} \\
& a_{32}=2(\eta \zeta-\xi x) \\
& a_{13}=2(\xi \xi-\eta x) \\
& a_{23}=2(\eta \xi+5 x) \\
& a_{33}=-\xi^{2}-\eta^{2}+\xi^{2}+x^{2}
\end{aligned}
$$

H-C. 4.0 CONTROL LOOP

H-C.4.1 Sensor

The earth sensor is assumed perfect. Assuming small attitude errors its outputs are angles $\phi, \theta$ relative to the $\mathbf{R R}$ system. Thus, from C-5, C-2:

$$
\begin{aligned}
& \phi=\sin ^{-1} F E A^{\top}(3,2)=\sin ^{-1} E A^{\top}(3,2) \\
& \theta=-\sin ^{-1} F E A^{\top}(3,1)=-\sin ^{-1} E A^{\top}(3,1)
\end{aligned}
$$


Attitude output rates $\dot{\phi}, \dot{\theta}$ are due to motion of the body relative to the RR system. They are given by:

$$
\begin{aligned}
& \dot{\phi}=\omega_{1}+E A^{\top}(2,1) \omega_{0}-E A^{\top}(3,1) \dot{\gamma} \\
& \dot{\theta}=\omega_{2}+E A^{\top}(2,2) \omega_{0}-E A^{\top}(3,2) \dot{\gamma}
\end{aligned}
$$

where $W_{0}$ is orbital rate.

This follows from the equation:

$$
\bar{\omega}_{B / R R}+\bar{\omega}_{R R / R}+\bar{\omega}_{R / I}=\bar{\omega}_{R / I}
$$

where $\bar{\omega}_{i / j}$ is angular rate of $i$ with respect to $j$.

$$
\therefore \quad \bar{\omega}_{B / R R}=\bar{\omega}_{B / I}-\bar{\omega}_{R / I}-\bar{\omega}_{R R / R}
$$

The components of these vectors in the base space are given by $(\mathrm{C}-12)$.

H- C. 4.2 Actuators

Only non-redundant configurations are modelled. The wheels are assumed to achieve their commanded to rque perfectly (see H-3.3) with limits of torque and total angular momentum.

The gimballed reaction wheel achieves its commanded motor torque and gimbal rate (see $\mathrm{H}-3.2 .3$ ) perfectly within the wheel torque, wheel angular momentum and gimbal rate limits.

${ }_{\mathrm{H}}^{\mathrm{H}} \mathrm{C} .4 .3$ Control Law

The control law simulated is:

$$
\begin{aligned}
& T_{c x}=X_{\text {axis }} \text { control torgue }=-k_{1} \phi-k_{2} \dot{\phi} \\
& T_{C y}=Y_{\text {axis }} \text { contral torgue }=-K_{1} \theta-k_{2} \dot{\theta}
\end{aligned}
$$

where: $K_{1}, K_{2}$ are constant

and $\sqrt{\frac{K_{1}}{I}}=10 \times$ spin rate. 
160 REA THIS FILE 11 CALLLS OH FILE ?

196

1), IS RERIUATIUE OF U9CI'

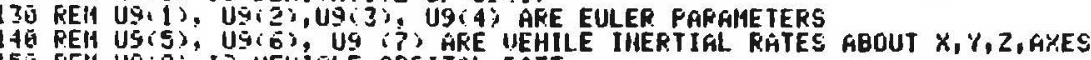

SË REH U9is; IS UEHICIE OPEITAL UEHTL

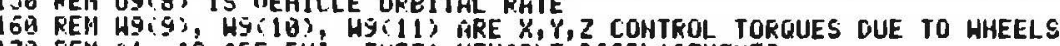

17. REH $\mathrm{Hl}$, T.Z GRE FHI, THETA UEHICLE OISPLACEMEHTS

¿10 REY H = INIEGRATIOH STEF SIZZ

230 REH HE = HAYIIAUH IHTECRATIOH STEP SIZE FOF CURREHT INTEGRATION STEP

230 R.EH HZ = THTEGRATICH STEP CONTKOL OARIAELE. WHEH HZ=O STEF

240 REM SIZE FIYEC, HHEN HZ $=$ EQ STEP SIZE RUTOHATICALLY ADJUSTED

ZEG FEH MATRIY A: THO CUSUE IHERTIAL) = (U SUB BASE:

(UTB REFEREHCE AŇES)

(E)

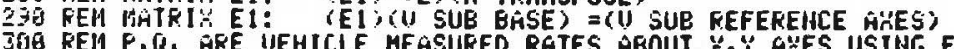

310 RELA THE HEGATIUE OF THE INTEGFAL OF CONTROL TOROUES IS ERUGL TO

320 REH WHEEL AHGULAR MOHEHTUH IHCREMENT

330 REH TS IS EHD TIME

35 REH .....

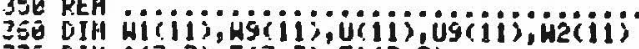

370 OIH A $A(3,3), E(3,3)$, Ei ( 3,3$)$

390 CATH $0,0,1,0.04,98,90,100,0.75$

$400 \mathrm{HG}=\mathrm{H}$

$\begin{array}{ll}461 & R 1=1 \\ 418 & H E=H\end{array}$

tie $E=\theta$

$430 \quad \begin{aligned} & 4 \\ & +40\end{aligned} 49=0$
$49=0$

心

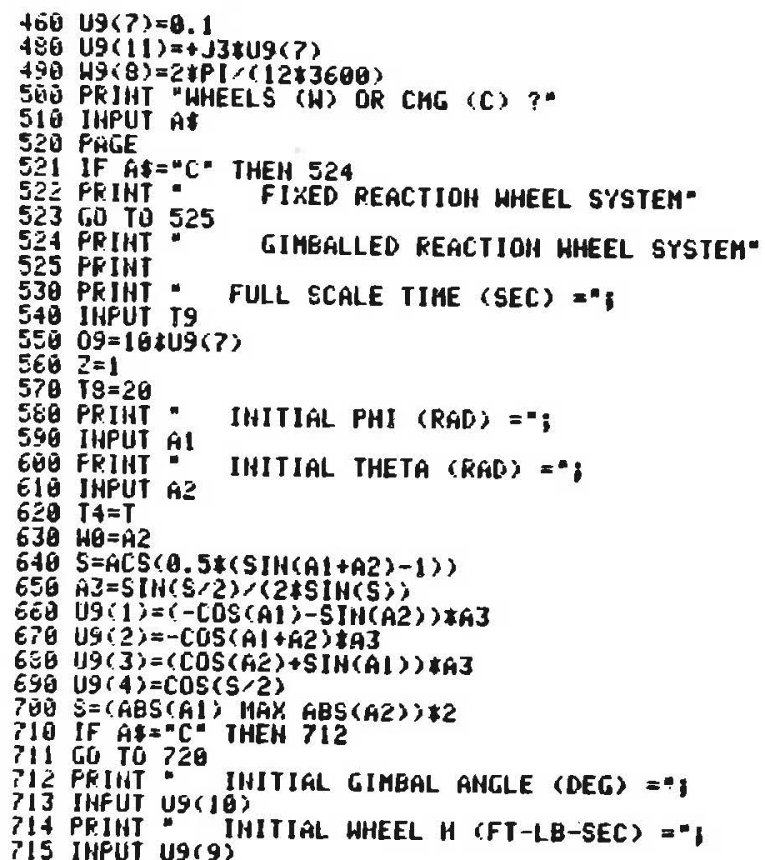

$716 \quad 49(9)=-49(9)$

0) $/ 57.3$

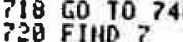

721 PRIHT " IHITIGL $X$ HHEEL $H(F T-L B-S E C)=*$

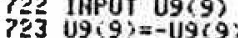

?ะ4 PRIIHT I IHITIAL Y HHEEL H (FT-LB-SEC) $=\cdots$ 725 IHPUT U9(10) 73600 (1) $750(10$

7406 FIMD 12

756 HIHOOW T, T9,-S, S

760 A\%IS T9/16, $S / 2$

370 LOUE T, AI

760 DELETE 100,780

T9O APFEMD 880

SÜ REH 
100 REM THIS IS ON FILE 12 AHD IS USED WITH FILE 11 $110 \quad T 8=\mathrm{T}$

$120 \quad H=H 8$
$130 \quad H 1=N 9$

$146 \quad U=U 9$

150 RE

$180 \mathrm{~T}=\mathrm{TB}+0.5 \mathrm{fH}$

268 U $(1)=U(1)+0.5 * H * H 1(1)$

218 MEKT I

220 COSUB 700

$240 \quad W 2(1)=H 9(1)$

250 US $(1)=U(1)+8.5 * H \neq H 2(1)$

GE. MET 1

$286 \mathrm{HS}=\mathrm{B}$

$281 \quad H=0$

256 FOR $J=1$ TO 11

310 If $A 1=0$ THEH 332

336 If $H S)(S / A 1)+2$ THEN 350

331 CO TO 348

$335 \mathrm{~N}=1 \mathrm{H}+1$
3350
$348 \mathrm{HO}=(\mathrm{S}, \mathrm{AL})+2$

50 HIEXT

IF $N=11$ THEN 460

379 If $H 2=0$ THEN 468

$396 \mathrm{H}=0.5 \mathrm{HH}$
$460 \mathrm{HB}=\mathrm{H}$

II

no

\section{CO 10150}

(6.4HZ) 2 THEH 460

$440 \mathrm{HQ}=2+\mathrm{H}$

$406 T=T 0+H$

480 FOR II TO 11

垈

5 IG HEXY I

530 FOR I 701 TO

$546 \quad S=$ (HI (I $)+H 9$ (I) ) $4 H / 6$

550 US $(I)=U(I)+S$

570 MEXT 1

5 SO GO 10620

610 END 626 IF T=TS THEN 610

E3G CRAH T, AI

ONG MOLE T4, HO

ESG DRAH T,A2

$E 76$ HQ $=A 2$

690 TH 60110

70 GEM

$71001=09+2 \pm J]$

$7 \leq 0202 * 2 \div 09 \% \mathrm{JI}$

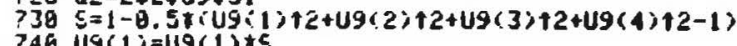

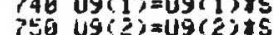

论 U9 $43 ;=49(3) * 5$

780 H9 (1) $=0.54(U 9(7) * U 9(2)-U 9(6) * U 9(3)+U 9(5) * U 9(4))$

790 Hg (2) $=0.5 t(-U 9(7)$ *US $(1)+U 9(5) * U 9(3)+U 9(6) * U 9(4)$
203 H9. 3$)=0.57(49(6) * U 9(1)-U 9(5) \times 49(2)+49(7)$ tU9( 4$)$

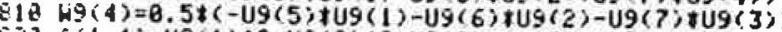

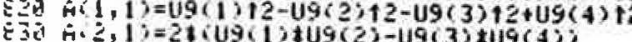

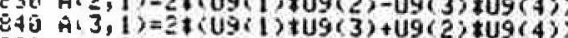

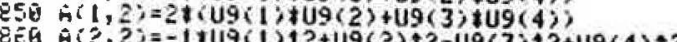

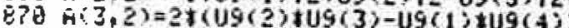

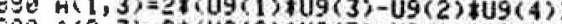

Go $A(3,3)=-1$ tug (1) $+2-U 9(2)+2+U 9(3) 12+U 9(4) 12$

(a)

$930 \mathrm{E}(2,3)=-1$

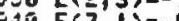

$956 E(3,2)=+E(1,2)$

TÉ FOR I=1 TO

$9 ? 6$ FOR $J=1$ TO 3

990 FOR $\mathrm{K}=1$ TO 3

$1010 \mathrm{E}(\mathrm{I}, J)=E(\langle I, j)+S$

1020 HEXT K

1048 HEXT I

$1050 \mathrm{Al}=\mathrm{HSH}(\mathrm{EL}(3,2)$

(0)

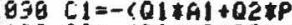

O $C Z=-C 01+A 2+02+0 ;$

(

II0E If U9( 9 ) $=0$ THEH 1108

$110849(16)=8$

1110 H9: 10$)=1$ U9 $(9) *(-\operatorname{C1} * \operatorname{SIH}(U 9(1 \theta))+\cot \cos (U 9(1 \theta))$

$11: 0$ HS $(1 \theta)=R 1$ HIH HQ $(10)$

$1+40 H g(9)=t(C 1+\operatorname{Cos}(U 9(10))+C 2 t \operatorname{SIH}(U 9(1 \theta)))$

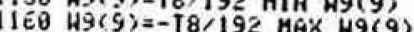

1162 IF $U 9(9)=T 7$ THEH 1166
1160

116460 To 1167

$1 E_{0} \quad H 9(9)=0$ MIN $H 9(9)$

116 IF U $(9)=-T 7$ THEM 1169

$169 \mathrm{KS}(9 ;=0$ MAX $\mathrm{H9}(9)$

ISG C $2=-U 9(9)$ ISIN (US $(10)$ )

(1) $01=J 1+09(5)+C$

$21003=\sqrt{3} * 49(?)-49(11)$

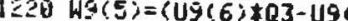

1230 Wg $(E)=(U 9(?)+101-U 9(5)+03+49(9) \neq \operatorname{Cos}(U 9(10))+H 9(10) \neq C 2) / J$

I250 RETURH 
Figure, $\mathrm{H}-\mathrm{C}-1$

99 REM IHIS IS ON FILE 7 AND IS USED HITH FILE 11

100
$181 \quad H=H 8$
$H=H S$

$116 \quad W=49$

I50 REA

$170 \mathrm{~T}=\mathrm{T} \theta+0.5 * \mathrm{H}$

(1)

193 U $U(1)=U(1)+B .5+H \neq H(1)$

200 MENT I

220 GOSUB 3000
230 FOR $I=1$ TO 11

(1)

260 HEYT 1

270 GOSUB 3808

$280 \quad H 9=0$

$281 H=8$

296 FOR J $=1$ TO 11

330 IF

$340 S=H Y(J)-H 2(J)$

350 IF $\mathrm{H} 9$ )(S.AI) $\uparrow 2$ THEN 390

351 CD TO 368

$35 ? \mathrm{~N}=\mathrm{N}+1$

353 60 To 390

390 NEXT $J$

391 IF $N=1$ THEH 510

9 ใH 2 १ THEN 470

430 IF $H \angle=0.2$ THEN 518

$440 H=6.5 \pm H B$
$456=H$

署

\section{CO TO 150}

40 IF H9)(0.4*H2) $\$ 2$ THEN 510

49. HC 3 H THEN 510

$510 \mathrm{~T}=\mathrm{TB}+\mathrm{H}$

530 FOR $I=1$ TO 11

55e

560 MEKT 1

570 GOSUA 3860

580 FOR IxI TO II $)$ (I)

600 U9 $(I)=U(I)+5$

$52 \theta$ MEYTI I

¿40 GOSUE 3000

670 EHD

2000 If $T=3 T$ T THEN 670

20 G GRAH T, ÂI

3005 ORG T4, HQ

2006 MOUE T, AI

2007 H8=A2

2111 Go to

300 B REM 100

3158 QI $=09126 J 1$

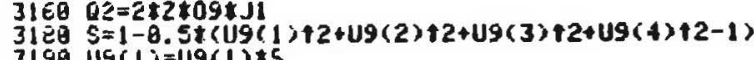

3190 USC $1>=49(1) \$ 5$

$3210 \quad 49(3)=49(3) \neq 5$

$32 \leq 0$ UG $(4)=U 9(4) \pm S$

$3 \equiv 40 \mathrm{Wg}(1)=0.5+(U 9(7)+U 9(2)-49(6)+U 9(3)+U 9(5) \neq U 9(4))$

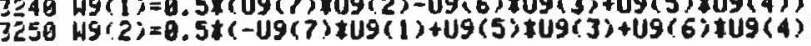

$32 E 0$ HS (3) $=0.5 t(U 9(6) t U 9(1)-U 9(5)$ tU9 (2) $+U 9(7)$ tU9(4))

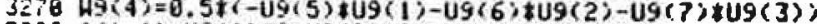

zo

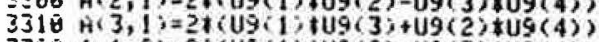

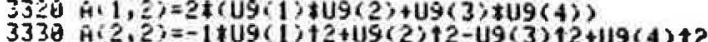

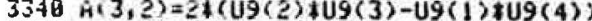

$3358 \mathrm{H}(1,3)=2 \pi(U 9(1)+U 9(3)-U 9(2) \neq U 9(4))$

$\left.336{ }^{H(2,}, 3\right)=2 \pi(U 9(2), 09(3)+U 9(1), 409(4))$

(3). 4 .

$3+10 \mathrm{E}(1,2)=\operatorname{Cos}(U 9(8))$

$3420 \mathrm{E}(2,3)=-1$

$3+40 E(3,2)=+E(1,2)$

3459 FOR $T=1$ TO 3

$34 E 6$ FOR $J=1$ TO 3

340 FOR $K=1$ TO

$3 \rightarrow 96 \mathrm{~S}=\mathrm{E}(\mathrm{I}, \mathrm{K}) \mathrm{X}, \mathrm{A}(\mathrm{J}, \mathrm{K})$

$3500 \mathrm{EI}(\mathrm{I}, J)=E I(I, J)+S$

3519 MENT K

3530 HEXI I

3550 AI $=$ FSH $(E)(3,2))$

U

3

$3596 H 9(1 B)=-(01892+0250)$

3600 HS(11) $=8$

368 ! HS $(9)=\mathrm{T} / 192$ MIH H9(9)

$3 \in Q 2$
$3 \in E 3$
$H S(10)=18,192$
HIH H

$3604 \mathrm{H9}(10)=-18 / 192 \operatorname{MAK} H 9(10)$

3ếS U9(9) $=17$ HIN U $9(9$ )

$30^{\circ}$ U9 $(9)=-17$ HAK U9(9),

उés $U 9(1 \theta)=-1$ ? MAX U9(10)

3610 If $U 9(9)=1$ ? THEN 3614

301260 T0 3616

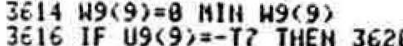

3618 G0 T0 3622

3620 H9 $(9)=\theta$ HAX HQ(9)
$3 \mathrm{E}^{2} 2$ If U9 $(10)=\mathrm{T}$ ? THEH 3626

$3626 \mathrm{H}(10)=\theta$ HIH H9(10)

3629 IF $U 9(10)=-T 7$ THEH 3632

2e $39(10)=0$ HAK H9(18)

$3 \in 40 \quad 01=J 1$ tug ( 5 ; $-49(9)$

3608 Q3 = J3+U9 (?) -09 (ii)

368 G $H 9(5)=(49(6)+03-U 9(7) \div 02+49(9)) / \mathrm{J} 1$

3209 ( $)=(U 5(5)$,

$3 \& 0 ̈$ RETURH 


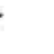


J. ESTIMATE OF POSITION RA TE UNCERTAINTY FOR THE REFEPENCE NUCLEAR GPS SPACECRAFT 



\section{J-1.0 INTRODUCTION}

The candidate nuclear-powered GPS Space Vehicle (SV) as shown in Fig. J-1, is a large squat cylinder, $6 \mathrm{ft}$ in diameter by $6 \mathrm{ft}$ long, with a set of antennae protruding from one cylinder base and a small vehicle launch support structure protruding from the other. Nominally, the antenna farm aligned with the cylinder axis looks down at the Earth and the vehicle spins at relatively fixed rate at about 0.5 to $1 \mathrm{rpm}$.

Differences between predicted and actual vehicle orbits result from imperfect estimation of the forces on the SV. Bias-type differences between expected and actual parameters, such as size and reflectivity, are expected to be determined by the filtering procedures employed, using measured orbital data over extended periods of time. But changes in vehicle forces which are random or with time variations more rapid than the filter time constants, will impede accurate prediction. The purpose of this memo is to consider such forces due to SV error attitude, temperature variation, and error unload impulse, and to evaluate the resultant orbit perturbations.

\section{J-2.0 TEMPERATURE EFFECTS ON BODY FORCE}

\section{J-2.1 Evaluation of Normal Body Force due to Heat Transfer by Diffuse Radiation}

A small area element of a black body $\mathrm{dS}_{1}$ at temperature $\mathrm{T}_{1}$ radiates at the rate of $(\sigma / \pi) \mathrm{T}_{1}{ }^{4} \cos \theta \mathrm{dS} \mathrm{S}_{1} \mathrm{dw}$ into an element of solid angle $\mathrm{dW}$. The solid angle of an element with area $\mathrm{dS}_{2}$ normal to the direction $\theta$ is $\mathrm{dW}=\mathrm{dS}_{2}$. Therefore, the

rate at which radiation passes from $\mathrm{dS}_{1}$ to $\mathrm{dS}_{2}$ is

$$
\frac{2}{r^{2}}
$$

$$
d Q=\frac{\sigma}{\pi} T_{1}^{4} \frac{\cos \theta_{1}}{r^{2}} d s_{1} d s_{2}
$$

Integrating over area 1 and area 2 gives total radiation rate

$$
Q=\int_{s_{1}} \int_{s_{2}} d Q
$$

The $\Delta Q$ into a conical shell zone with thickness $d Q$ is given by

$$
\Delta Q=\frac{\sigma}{\pi} T_{1}^{4} \frac{\cos \theta}{r^{2}}(r \sin \theta) 2 \pi(r \cos \theta) d \theta
$$




\section{N/GPS ORGANIC RANIKINE CVCLE SPACECRAFT PROFILE}

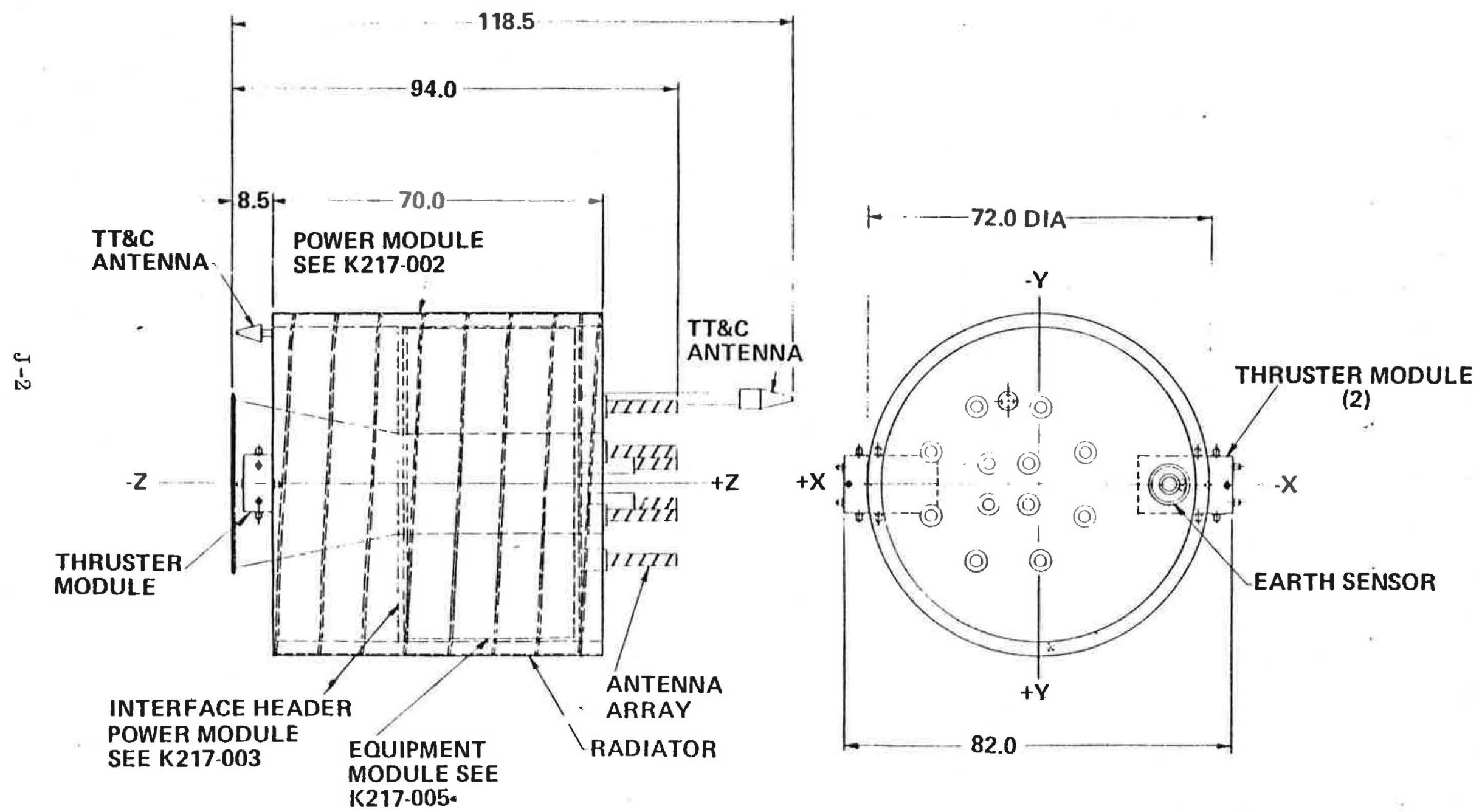


Since the reaction force on the body is proportional to the emitted radiation, the axial force component due to $\Delta \mathrm{Q}$ is proportional to the "axial component" of $\Delta \mathrm{Q}$

$$
\text { i.e. } \Delta Q \text { axial }=\Delta Q \cos \theta
$$

Then, the total axial force due to radiation from $\mathrm{dS}_{1}$ is proportional to

$$
\begin{aligned}
Q_{\text {axial }} & =\int_{0}^{\pi / 2} \frac{\sigma}{\pi} T_{1}^{4} \frac{\cos \theta}{r^{2}}(r \sin \theta) 2 \pi(r \cos \theta) d \theta \\
& =\frac{1}{3} \sigma T_{1}^{4}
\end{aligned}
$$

i. e., the effective axial force is $1 / 3$ that which would be expected in a simple calculation, based on total heat radiated.

Because emitted radiant heat transfer is given by $\sigma T_{1}^{4} \Delta S$

we have:

$$
Q=c \frac{1}{3} \sigma T_{1}^{4} \Delta s
$$

and

$$
\begin{aligned}
& \frac{d Q}{d t}=\frac{1}{3} \sigma\left(4 T^{3}\right) \Delta S \\
& \frac{\Delta Q}{Q}=4 \frac{\Delta T}{T}
\end{aligned}
$$

The tabulation below indicates numerics when the nominal emitting area temperature is $100^{\circ} \mathrm{C}$ (i.e., $373^{\circ} \mathrm{K}$ ). A one-degree centigrade temperature change is shown to cause about a one percent change in radiated heat.

TABLE J-2

\begin{tabular}{|l|l|c|}
\hline$\frac{\Delta \mathrm{T}}{\mathrm{T}}$ & $\frac{\Delta \mathrm{Q}}{\mathrm{Q}}$ & $\Delta \mathrm{T}\left({ }^{\circ} \mathrm{C}\right)$ \\
\hline 0.03 & 0.12 & 11.28 \\
.02 & 0.08 & 7.45 \\
.01 & 0.04 & 3.73 \\
.005 & 0.02 & 1.86 \\
.001 & 0.004 & 0.37 \\
\hline
\end{tabular}




\section{J-2.2 Sensitivity of SV Displacements to Surface Te mperature Change}

\section{J-2.2.1 Flat Surfaces}

If $Q_{I}$ is the rate of total internally generated heat, $Q_{S}$ is the rate of heat input from the sun, ${ }_{\mathrm{E}}$ is the radiated power from the antenna, then the steady-state radiation rate $\mathrm{Q}_{\mathbf{r}}$ from the $\mathrm{SV}$ is:

$$
Q_{r}=Q_{I}+Q_{S}-\dot{E}_{T}
$$

To approximate the heat radiated from the SV's earth-viewing face, the emitted radiation is assumed uniformly distributed over the external surface. Then the rate at which heat is radiated from that earth-viewing face is $\mathrm{Q}_{E V F}$ :

$$
Q_{E V F}=\left[\frac{\pi R^{2}}{2\left(\pi R^{2}\right)+2 \pi R L}\right] Q_{R}
$$

where: $\quad R$ is vehicle radius

and $\quad \mathrm{L}$ is vehicle length

The heat rate $Q_{E V F}$ may be normalized by comparing it to the rate of solar energy radiation to the surface when the sun line is normal to the surface.

$$
\text { i.e. } \quad F_{\text {evn }}=\frac{Q_{E V F}}{E\left(\pi R^{2}\right)}
$$

where: $\quad E$ is the solar constant (1353 watts/ $\mathrm{m}^{2}$ ).

Therefore, the radiation force due to diffuse emission of heat at rate $\mathrm{Q}_{E V F}$ is given by:

$$
\begin{aligned}
F_{\text {ev }} & =K Q_{E V F} / 3 \\
& =K F_{\text {evn }}(E)\left(\pi R^{2}\right) / 3 \\
& =K^{1} F_{\text {evn }}\left(\pi R^{2}\right) / 3
\end{aligned}
$$

where $\mathrm{K}$ is the constant which relates power emitted to radiation force $=7.42 \times 10^{-10} \mathrm{lb} /$ watt and $\mathrm{K}^{1}$ is solar pressure $=1 \times 10^{-7} \mathrm{lb} / \mathrm{ft}^{2}$

The GPS SV has the parameters: mass $=1105 \mathrm{lbs}, \mathrm{Q}_{\mathrm{I}}=4800$ watts, $\mathrm{Q}_{\mathrm{S}}=500$ watts, and $\dot{\mathrm{E}}_{\mathrm{T}}=200$ watts, so that $\mathrm{Q}_{\mathrm{R}}=510$ watts. 
With $\mathrm{R}=3 \mathrm{ft}$ and $\mathrm{L}=6 \mathrm{ft}, \mathrm{Q}_{\mathrm{EVF}}=850$ watts and $\mathrm{F}_{\text {evn }}=0.25$. But a $4 \%$ change in $Q_{E V F}$, which corresponds to about a 4-degree centigrade change in surface te mperature, causes a change in $F_{\text {evn }}$ of 0.01 .

By use of the orbit perturbation equations given in App. C, we have calculated the effect of constant radial forces due to diffuse radiant heat transfer from the earthviewing face of the SV. The computer simulation, given in Appendix J-A, indicates a buildup in perturbation displace ments, linear with radial force. With radiated heat inc rement given in normalized form, the perturbation displacement rates are:

$$
\begin{aligned}
& \text { Radial displacement }\left(\mathrm{ft} / \text { day when } F_{\text {evn }}=0.01\right) \cong 0.01 \\
& \text { Normal-to-radius-vector displacement }\left(\mathrm{ft} / \text { day when } \mathrm{F}_{\text {evn }}=0.01\right) \cong 0.1
\end{aligned}
$$

Another way of stating these results is: as a result of a $4^{\circ} \mathrm{C}$ mis-estimation of the temperature of the SV'S earth-viewing side, there will be an error in predicting the radial displacement of $0.01 \mathrm{ft} /$ day and in normal-to-radius-vector displacement of $0.1 \mathrm{ft} /$ day.

Assuming a $10^{\circ} \mathrm{C}$ error in temperature prediction, the resultant error radial displacement rate is $0.025 \mathrm{ft} /$ day and the resultant error normal-to-radius vector displacement is $0.25 \mathrm{ft} /$ day.

Similarly, the expected $2^{\circ} \mathrm{C}$ prediction error for the SV's space-viewing flat face will yield predictions with errors of $0.005 \mathrm{ft} /$ day and $0.05 \mathrm{ft} / \mathrm{day}$, respectively.

\section{J-2.2.2 Cylindrical Surfaces}

The temperatures of the surface elements on the cylindrical portion of the SV vary slowly with time so that during the course of single vehicle revolution all area elements on the cylinder may be considered isothermal. Therefore, as a result of the vehicle spin all radiation forces, due to cylindrical surface radiation, tend to integrate to zero over a full spin when the spin is about the cylinder axis.

When the spin does not rotate about its cylinder axis there will be non-zero orbit radial forces if there are differences between temperatures of symmetric cylinder areas.

To determine the orbit radial forces on the vehicle we first evaluate the total force in a direction normal to a plane containing the cylinder axis. In accordance with eq 6 , each s mall area has an "axial" $Q$ equal to $1 / 3$ the total $Q$ emitted from that area. Integrating the components of these $Q^{\prime} s$, over half the cylinder, to find their total resultant in a direction normal to the plane one obtains:

$$
\begin{aligned}
Q=Q_{x} & =\int_{0}^{L} \int_{0}^{\pi / 2} 2\left(\frac{1}{3} \sigma T_{1}^{4}\right)(R d \theta d L) \cos \theta \\
& =\frac{2}{3} \sigma T_{1} R L
\end{aligned}
$$


If the temperature of the opposite side of the cylinder is $\mathrm{T}_{2}$ then its total normal $Q$ is:

$$
Q_{-x}=\frac{2}{3} \sigma T_{2} R L
$$

The difference $\Delta Q_{X}=Q_{X}-Q_{-X}$ is proportional to the net radiation force normal to the plane which divide the two halves of the cylinder.

Thus $\Delta \mathrm{Q}_{\mathrm{X}}=\frac{2}{3}$ GR L $\left(\mathrm{T}_{1}^{4}-\mathrm{T}_{2}^{4}\right)$

if $\mathrm{T}_{2}=\mathrm{T}_{1}+\Delta \mathrm{T}$

$\Delta Q_{X} \bumpeq \frac{2}{3} \sigma R L\left(4 T^{3} \Delta T\right)$

so that $\frac{\Delta Q_{X}}{Q_{X}}={ }^{4} \frac{\Delta T}{T}$

With total radiation $Q_{R}$ from the satellite, the radiation from the cylindrical section $Q_{C F}$ is:

$$
Q_{C F}=\left[\frac{2 \pi R L}{2 \pi R L+2 \pi R^{2}}\right] Q_{R}
$$

Also, for uniform heat radiation over the cylinder:

$$
Q_{C F} \cong 2 \pi R L \sigma T_{1}^{4}
$$

Thus:

$$
\frac{Q_{x}}{Q_{C F}}=\frac{\frac{2}{3} \sigma R L T_{1}^{4}}{2 \pi R L \sigma T_{1}^{4}}=\frac{1}{3 \pi}
$$

and

$$
\Delta Q_{x}=\frac{4 \Delta T}{T}\left(\frac{Q_{C F}}{3 \pi}\right)
$$

Define the normalization as before:

$$
F_{\text {cf n }}=\frac{Q_{c f}}{E\left(\pi R^{2}\right)}
$$

Then

$$
\Delta Q_{x}=4 \frac{\Delta T}{T}\left(\frac{E\left(\pi R^{2}\right)}{3 \pi}\right) F_{c f_{n}}
$$

$\mathrm{J}-6$ 
If the SV does not rotate about its cylinder axis but about an axis displaced by an a mount $\alpha$ then there will be a net radiation in force over a spin, along the actual spin axis, due to the net axial radiation $\Delta Q_{X^{*}}$. This force $F_{S A}$ will be given by:

$$
\mathrm{F}_{\mathrm{SA}}=\mathrm{K} \Delta \mathrm{Q}_{\mathrm{X}} \sin \alpha
$$

and

$$
F_{S A}=k^{\prime}\left(\frac{\pi R^{2}}{3 \pi}\right) F_{c f_{n}}\left(\frac{4 \Delta T}{T}\right) \sin \alpha
$$

With the SV parameters in Sec. J-2.1, $\mathrm{F}_{\mathrm{CFM}}=0.955$.

With $\alpha=0.5^{\circ}$ and $\Delta \mathrm{T}=10^{\circ} \mathrm{C}, \mathrm{T}=373^{\circ} \mathrm{K}$ one has

$$
F_{S A}=k^{\prime} \frac{\pi R^{2}}{3}\left(2.82 \times 10^{-4}\right)
$$

The computer results mentioned in Sec. 2.1 show that radial forces of this magnitude have negligible effect on the SV'S orbit since the radial errors accummulate at $2.8 \times 10^{-4}$ $\mathrm{ft} /$ day and normal-to-radial errors are 10 times greater.

\section{J- $3.0 \quad$ UNLOAD PERT URBATIONS}

Periodically, unload is required for the momentum which has accummulated on the SV. Approximately $0.5 \mathrm{ft} \mathrm{lb} \mathrm{sec} \mathrm{will} \mathrm{be} \mathrm{unloaded} \mathrm{during} \mathrm{a} \mathrm{single} \mathrm{unload} \mathrm{by} \mathrm{firing,}$ for $0.42 \mathrm{sec}$, as a couple, a pair of $0.2-\mathrm{lb}$ jets spaced $6 \mathrm{ft}$ apart (Section 7). When these jets are misoriented (or mismatched), their firing causes a net impulse on the vehicle perpendicular (or aligned with) their nominal thrust direction. The orbital effects of such jet errors have been considered inApp. E ' for a somewhat larger vehicle and for larger error impulses than obtained by the vehicle here considered. But the errors do change linearly with mass and impulse so that the results of that reference can be used to obtain the perturbations of interest here.

For the present vehicle, a jet mismatch of $5 \%$ can be assumed and a jet misorientation of 0.5 degrees can be assumed. Then the error impulses are:

$$
\begin{array}{ll}
\text { Jet mismatch impulse } & =(0.2 \#)(0.42 \mathrm{sec})(.05) \mathrm{lb} \mathrm{sec} \\
& =0.0042 \mathrm{lb} \mathrm{sec} \\
& \\
\text { Jet misalignment impulse } & =(2 \text { jets })(.2 \# / \text { jet })(.42 \mathrm{sec})(\sin 0.5 \mathrm{deg}) \mathrm{lb} \mathrm{sec} \\
& =.00146 \mathrm{lb} \mathrm{sec}
\end{array}
$$


As indicated in Table J-3, and in App.E, error impulses along the velocity vector cause the greatest orbit perturbations. The AVCS system design, therefore, achieves in-orbit-plane momentum unload by firing jets normal to the orbit plane, and achieves normal-to-orbit-plane unload by firing orbit-radius pointing jets. A vehicle attitude error, $\alpha$, during firing can cause a component of the error impulse, $\Delta \mathrm{I}$, to be seen along the velocity vector. The along-velocity-vector impulse is $\Delta \mathrm{I} \sin \alpha$. Resultant orbit error rates are shown in the table.

J-4.0 ORBIT PERTURBATIONS DUE TO SV MISA LIGNMENTS

The purpose of this section is to estimate the incremental (error) solar forces on the SV due to misorientation of the vehicle. As a result of the control loop and the spin about the nominal earth pointing axis, the actual motion will be one in which the vehicle axis of symmetry will describe a cone in space, the cone half-angle is the attitude error.

Two error sources are considered below. With respect to the first, intuitively, it may be predicted the average error forces on the cylindrical body of the SV over a spin cycle, will be near zero, since the forces do not change greatly for small attitude changes with respect to the sun and there is an inherent cancelling of errors between attitudes obtained one-half spin cycle apart. With regard to the second - changes in vehicle self-shadowing - it may be observed that the errors are small.

J-4.1 SOLAR FORCES

A simplified model of the vehicle configuration was developed for use in assessing the level of solar forces during earth orbit. The model consists of flat and cylindrical surfaces equivalent in area to those of the proposed configuration, detailed in Fig. J-4 where surface properties are described.

The equations used have been derived based on the usual photon momentum transfer theory and the appropriate geometric treatment of surfaces exposed to solar flux. Normal $\left(\mathrm{F}_{n}\right)$ and tangential $\left(\mathrm{F}_{t}\right)$ components of force are defined for flat and cylindrical surfaces as follows:

Flat Plate

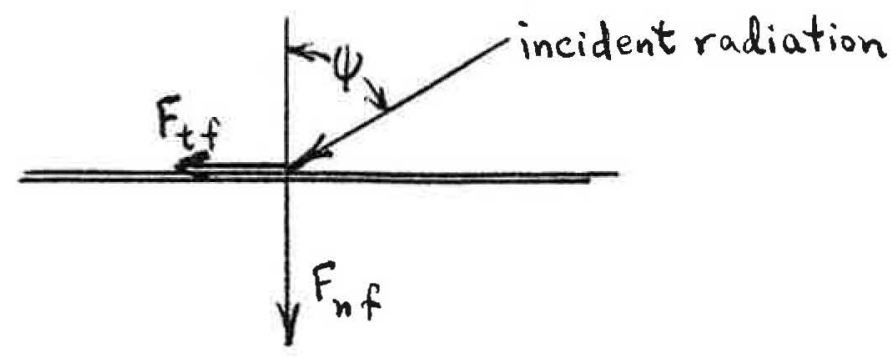


TABLE J-3

\begin{tabular}{|c|c|c|c|c|c|}
\hline & \multirow{3}{*}{$\begin{array}{l}\text { App. E } \\
\text { Vehicle } \\
\text { Spinner) }\end{array}$} & \multicolumn{4}{|c|}{ Reference-Design N/GPS } \\
\hline & & $\begin{array}{l}0.5^{\circ} j \\
\operatorname{misali} \\
\end{array}$ & $\begin{array}{l}\text { et } \\
\text { gnment }\end{array}$ & $\begin{array}{l}5 \% \text { jet } \\
\text { mismatc }\end{array}$ & \\
\hline & & $\begin{array}{l}\text { no att. } \\
\text { error }\end{array}$ & $\begin{array}{l}1 \text { degree } \\
\text { att error }\end{array}$ & $\begin{array}{l}\text { no att. } \\
\text { error }\end{array}$ & $\begin{array}{l}1 \text { degree } \\
\text { att error }\end{array}$ \\
\hline $\begin{array}{ll}\text { vehicle mass } & \text { slugs } \\
\text { error impulse } & \text { lb } \mathrm{sec} \\
\end{array}$ & $\begin{array}{r}30 \\
0.029 \\
\end{array}$ & $\begin{array}{c}34.4 \\
0.0015 \\
\end{array}$ & $\begin{array}{l}34.4 \\
0.0015 \\
\end{array}$ & $\begin{array}{l}34.4 \\
0.0042 \\
\end{array}$ & $\begin{array}{l}34.4 \\
0.0042 \\
\end{array}$ \\
\hline $\begin{array}{l}\text { impulse along velocity vector } \\
\text { radial component ft/day } \\
\perp \text { to radial component } \mathrm{ft} / \mathrm{day}\end{array}$ & $\begin{array}{c}2 \\
200 \\
\end{array}$ & $\begin{array}{c}0.09 \\
9 \\
\end{array}$ & $\begin{array}{c}0.09 \\
9 \\
\end{array}$ & $\begin{array}{l}0.29 \\
29 \\
\end{array}$ & $\begin{array}{c}0.29 \\
29 \\
\end{array}$ \\
\hline $\begin{array}{l}\text { impulse normal to orbit plane } \\
\text { radial component ft/day } \\
\text { t to radial component } \mathrm{ft} / \mathrm{day}\end{array}$ & $\begin{array}{l}0 \\
0\end{array}$ & $\begin{array}{l}0 \\
0\end{array}$ & $\begin{array}{r}0.0016 \\
0.155 \\
\end{array}$ & $\begin{array}{l}0 \\
0 \\
\end{array}$ & $\begin{array}{r}0.005 \\
0.5 \\
\end{array}$ \\
\hline $\begin{array}{l}\text { impulse along radius vector } \\
\text { radial component ft/day } \\
\perp \text { to radial component } \mathrm{ft} / \mathrm{day}\end{array}$ & $\begin{array}{r}0 \\
\sim 0 \\
\end{array}$ & $\begin{array}{l}0 \\
0 \\
\end{array}$ & $\begin{array}{l}0.0016 \\
0.155\end{array}$ & $\begin{array}{r}0 \\
\sim 0 \\
\end{array}$ & $\begin{array}{r}0.005 \\
0.5 \\
\end{array}$ \\
\hline
\end{tabular}


where:

$$
\begin{aligned}
& F_{n f}=\left[(1+s \rho) \cos ^{2} \psi+\frac{2}{3} \rho(1+s) \cos \psi\right] P_{f} A_{p} \\
& F_{\frac{1}{2} f}=[(1-s \rho) \cos \psi \sin \psi] P_{f} A_{p} \\
& P_{f}=\text { solar pressure, }\left(\times 10^{-7} \mathrm{Bb} / \mathrm{f}^{2}\right. \\
& A_{p}=\text { plate area, } \mathrm{f}^{2}
\end{aligned}
$$

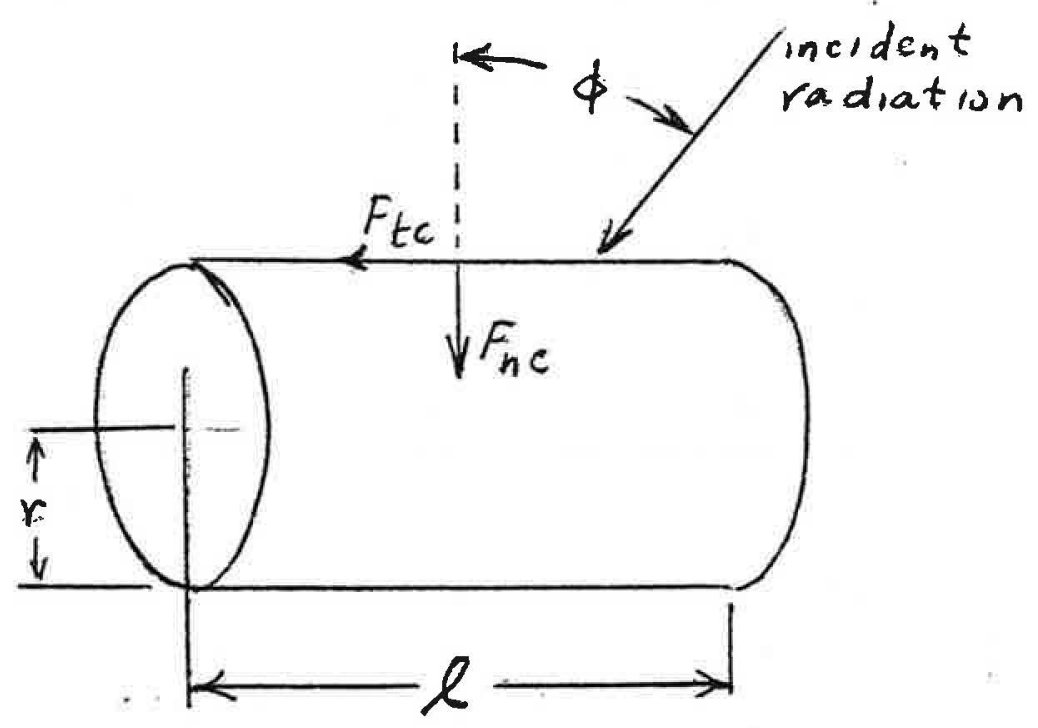

where

$$
\begin{aligned}
& F_{n c}=\left[(1+s \rho / 3) \cos ^{2} \phi+\rho(1-s) \frac{\pi}{6} \cos \phi\right] P_{f} A_{c} \\
& F_{t c}=[(1-s \rho) \cos \phi \sin \phi] P_{f} A_{c} \\
& A_{c}=2 r l
\end{aligned}
$$

and where

$$
\begin{aligned}
& \rho=\text { fraction of incident radiation reflected } \\
& s=\text { fraction of reflected radiation which is specular }
\end{aligned}
$$

$\mathrm{J}-10$ 

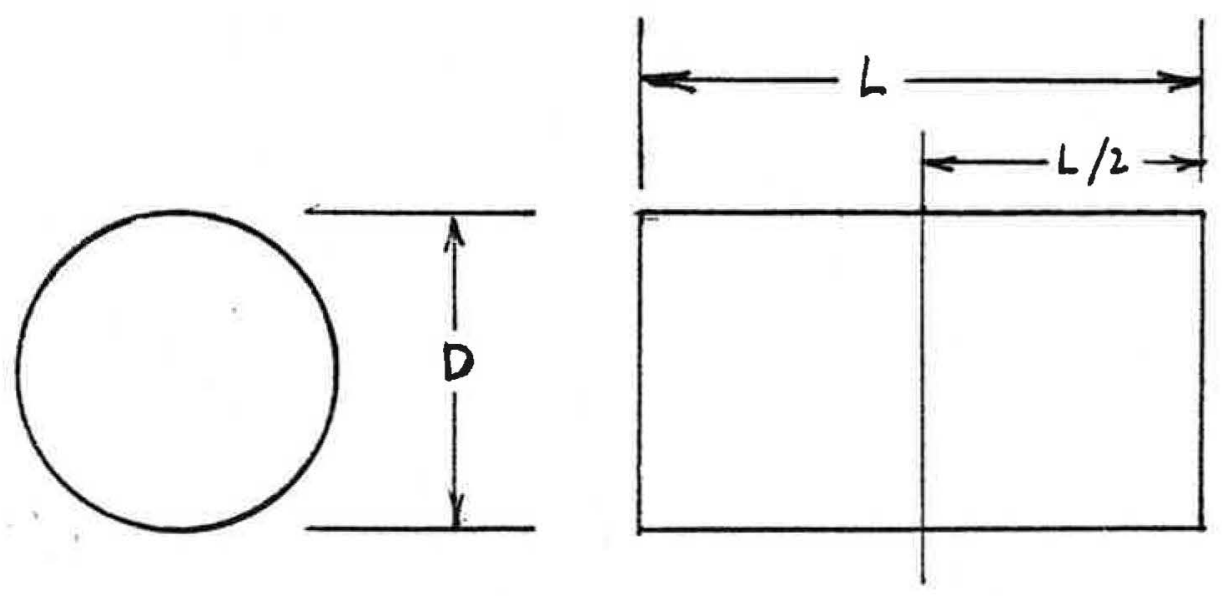

$\mathrm{L}=6 \mathrm{ft}$

$\mathrm{D}=6 \mathrm{ft}$

vehicle covered with Silver-Teflon tape all over

$$
s=1, e=0.88
$$

Fig. $5-4$. Simplified Vehicle Model

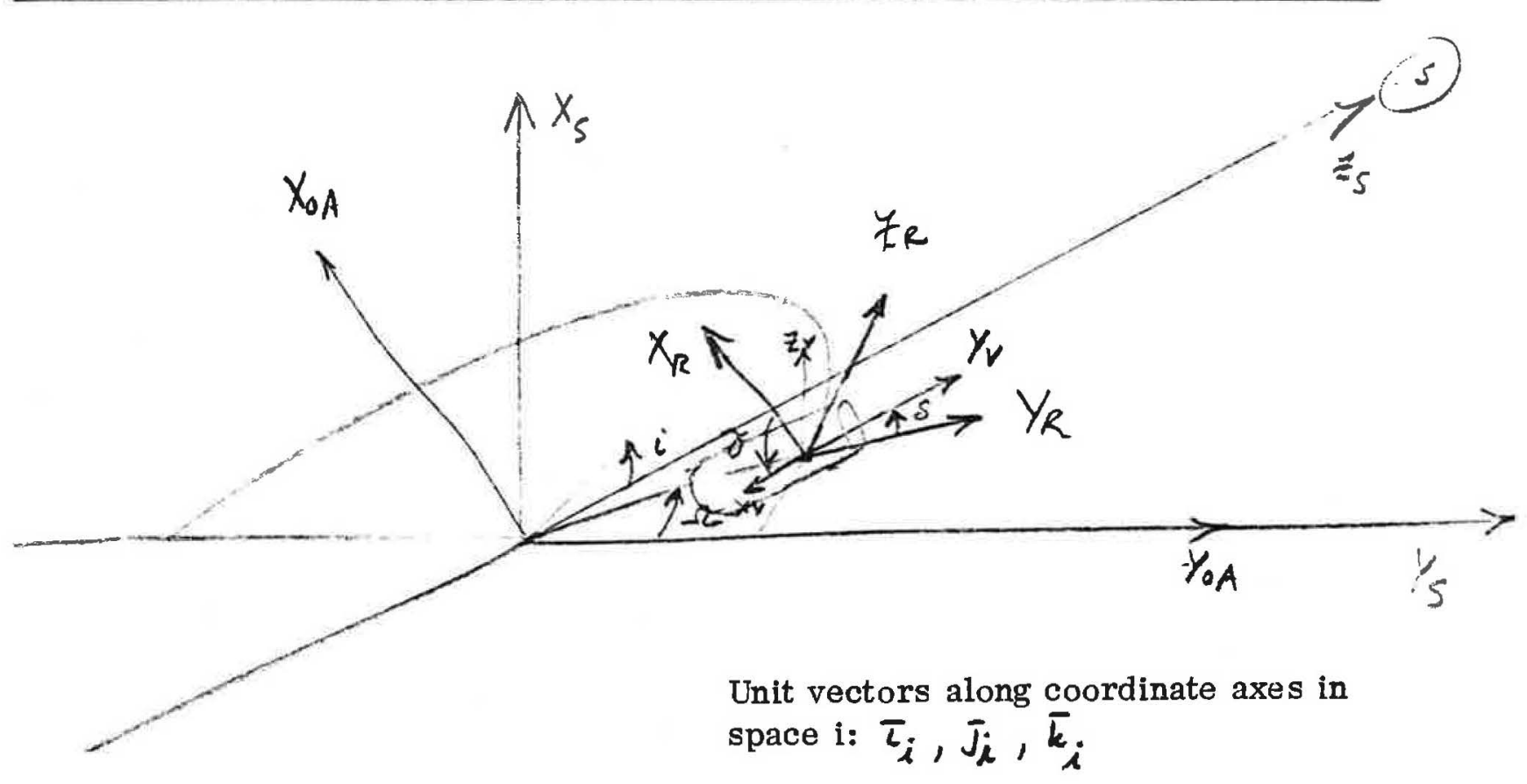

Fig. J-5. Vehicle in-orbit Geometry 
J-4.2 ERROR FORCES ON MAIN BODY CYLINDER DUE TO MISORIENTATION

The vehicle in-orbit geometry is illustrated in Figure $\mathrm{J}-5$. In that figure, $\mathbf{i}$ is the orbit plane inclination to the earth-sun line, the axis $\mathrm{Y}_{O A}$, also denoted $\mathrm{Y}_{\mathrm{S}}$, is normal to the orbit axis $\mathrm{X}_{\mathrm{OA}}$ and the earth-to-sun vector $\mathrm{Z}_{\mathrm{S}}$. The coordinate axis $\mathrm{X}_{\mathrm{S}}$ completes the right-handed triad $X_{S}, Y_{S}, Z_{S}$. The SV is located at angle $\Omega$ from the $Y_{Q A}$ axis taken positively with right-hand sense about the $X_{\Omega A}$ axis. The reference axiS $Y_{R}$ is radially outward from the earth thru the SV's radius vector, the reference axis $X_{R}^{R}$ is parallel to the orbit axis $X_{O A}$, and the reference axis $Z_{R}$ completes the right-handed triad.

The vehicle axes $\mathrm{X}_{\mathrm{V}}, \mathrm{Y}_{\mathrm{V}}, \mathrm{Z}_{\mathrm{V}}$ are located with respect to the reference axis by rotating thru angle $\gamma$ about the $Y_{R}$ axis and then rotating through angle $\delta$ about the rotated $X_{R}$ axis, also called $X_{V} \cdot$ Angle $\delta$ is the $S V$ attitude error angle.

Our goal now is to evaluate the net force over one spin cycle in three directions: radial, along the velocity vector, and normal to the orbit plane.

The normal component of solar force on the cylindrical portion of the SV $\left(\bar{F}_{n c}\right)$ lies normal to the vehicle $\mathrm{Y}_{\mathrm{V}}$ axis and in the $\mathrm{Y}_{\mathrm{V}} \mathrm{X}_{\mathrm{S}}$ plane. It has a component away from the sun. Thus, the unit vector $\mathrm{e}_{\mathrm{nc}}$ along $\mathrm{F}_{\mathrm{nc}}$ is given by

$$
\bar{e}_{n c}=\frac{\bar{y}_{v} \times\left(\bar{y}_{v} \times \bar{z}_{s}\right)}{\left|\bar{y}_{v} \times\left(\bar{y}_{v} \times \bar{z}_{s}\right)\right|}
$$

The tangential component of solar force on the cylindrical portion of the vehicle $\left(\mathrm{F}_{\mathrm{tc}}\right)$ is directed along the $-\mathrm{Y}_{\mathrm{V}}$ axis when $\mathrm{Y}_{\mathrm{V}} \cdot \mathrm{Z}_{\mathrm{S}}$ is positive and along the $-\mathrm{Y}_{\mathrm{V}}$ axis when $\mathrm{Y}_{\mathrm{V}} \cdot \mathrm{Z}_{\mathrm{S}}$ is negative.

$$
\bar{e}_{t c}= \begin{cases}-\bar{j}_{v} & \text { when } \bar{y}_{v} \cdot \bar{z}_{s} \geq 0 \\ +\bar{I}_{v} & \text { when } \bar{y}_{v} \cdot \bar{z}_{s}<0\end{cases}
$$

The normal component of solar force on the flat faces of the $\mathrm{SV}\left(\mathrm{F}_{\mathrm{nf}}\right)$ lies along the vehicle $\mathrm{Y}_{V}$ axis and is positive when $\mathrm{Y}_{V}=\mathrm{Z}_{\mathrm{S}}<0$ i.e., along $\bar{e}_{\text {tc }}$. The tangential component of solar force on the flat faces is directed along $\overrightarrow{\mathrm{e}}_{\mathrm{nc}}$.

Then, the force on the SV's surface is

$$
\bar{F}=\left(\left|\bar{F}_{n c}\right|+\left|\bar{F}_{t f}\right|\right) \bar{e}_{n c}+\left(\left|F_{t c}\right|+\left|\bar{F}_{n f}\right|\right) \bar{e}_{t c}
$$


With the aid of the computer programs listed in Appendix J-C, the net error forces have been calculated as the difference between the average of the force $F$ over a spin revolution and the value of force $\mathrm{F}$ when the misalignment angle $\delta$ is zero. Figures in the App. J-C are graphs of these errors over a full turn. Then the net error forces over the turns are employed in a set of orbit perturbation equations, from App. $C$ to evaluate the orbital effects. Figures in Appendix J-D show how SV orbital positions are perturbed for several values of orbit inclination with respect to the sun line. Maximum displacements occur when the sun lies in the orbit plane. When attitude errors are 0.5 degrees, maximum radial error rates are $0.025 \mathrm{ft} /$ day and maximum normal-to-radiusvector error rates are $0.05 \mathrm{ft} /$ day.

\section{$.7-4.3 \quad$ SELF-SHADOWING}

When on-orbit, there will be predictable shadowing of the antennae and the launch support cone by the SV's main cylindrical body. In addition, there will be unpredictable shadowing due to vehicle attitude uncertainties. We consider bere the effect of such shadowing on the SV's ephemeris. For the present purpose, the vehicle is assumed to have the external configuration shown below.

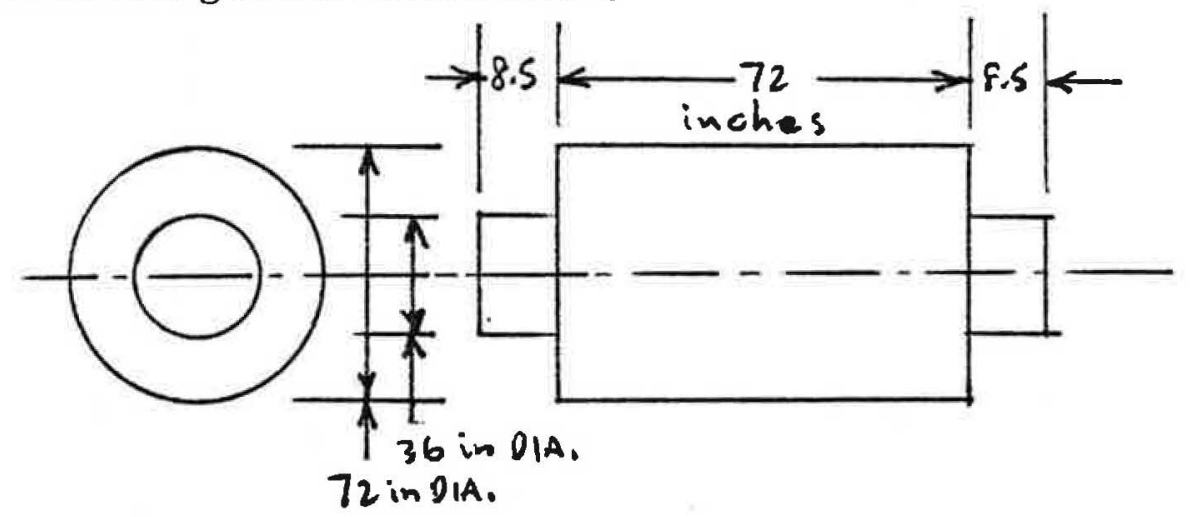

When the sun-line is normal to the cylinder axis within angle $\theta$ part of one or the other of the small cylindrical projections will be shadowed. Small changes $\Delta \theta$ in the sun angle $\theta$ cause small changes in shadowed area by amount $\Delta A=(18 \sin \Delta \theta) 36$ in projected area normal to sun-line.

Angular changes of the SV with respect to the sun-line are caused by spinning of the spacecraft about an axis skewed with respect to the SV's cylinder axis. When the angle of skew is $\Delta \theta$, there is an average deviation, over one vehicle spin, of about $\Delta \theta / 2$ between the nominal sun angle and actual sun angle. Thus, the effective change in vehicle projected area is

$$
\Delta A_{\lambda V G}=(18 \sin \theta / 2) 36
$$


Since the total area normal to sun-line is approximately $\mathrm{A}=72 \times 72 \mathrm{sq}$ in, the fractional area change is

$$
\frac{\Delta A_{\text {AVG }}}{A}=\frac{(18 \sin (\theta / 2)) 36}{72 \times 72}
$$

When $\Delta \Theta=0.5^{\circ}$, the fractional area change is

$$
\frac{\Delta A_{\text {AVG }}}{A} \simeq 0.001
$$

The effect on the vehicle ephemeris, of the force variation due to this fractional area change during times when shadowing occurs (i.e., when $\left.|\tan 0| \leqslant \frac{8.5}{15}\right)$ has been computed with the program documented in. Appendix J-E. The results indicate that with a $0.5^{\circ}$ skew angle, the self-shadowing causes an orbit perturbation rate of 0.04 $\mathrm{ft} /$ day change in radial distance and a $0.08 \mathrm{ft} /$ day change in normal-to-radius distance.

\section{$J=5.0 \quad$ COMPILATION OF RESULTS}

The tabulation of errors on table $\mathrm{J}-5-1$ shows a maximum rate in radial position unpredict ability of about $0.1 \mathrm{ft} / \mathrm{day}$ and in normal-to-radius position of $1.75 \mathrm{ft} /$ day when all bias-type force perturbations are correctly estimated by ground filtering of orbit data.

\section{$\underline{\text { References }}$}

1. Appendix C

2. Appendix E

3. Appendix H 


\begin{tabular}{|c|c|c|c|}
\hline & \multirow[b]{2}{*}{ Error Sources } & \multicolumn{2}{|c|}{ Error Rates } \\
\hline & & \begin{tabular}{|l|} 
Radial \\
Displace- \\
ments \\
ft/day
\end{tabular} & $\begin{array}{l}\text { Normal to } \\
\text { Radial Dis- } \\
\text { placements } \\
\text { ft/day }\end{array}$ \\
\hline Thermal & $\begin{array}{l}\text { 1. } 10^{\circ} \mathrm{C} \text { error prediction in earth- } \\
\text { viewing face temperature. } \\
2.2^{\circ} \mathrm{C} \text { error prediction in space- } \\
\text { viewing face temperature. } \\
3 . \quad 10^{\circ} \mathrm{C} \text { temperature difference on } \\
\text { opposite sides of cylinder plus } 0.5^{\circ} \\
\text { attitude error. }\end{array}$ & $\begin{array}{l}0.025 \\
0.005 \\
.0003\end{array}$ & $\begin{array}{r}0.25 \\
0.05 \\
0.003\end{array}$ \\
\hline $\begin{array}{l}\text { Unload } \\
\text { Perturbations }\end{array}$ & $\begin{array}{l}\text { 1. } 0.5^{\circ} \text { jet misalignment with } 1^{\circ} \text { at- } \\
\text { titude error during firing } \\
\text { a. impulse nominally } \perp \text { to orbit } \\
\text { plane } \\
\text { b. impulse nominally along radius } \\
\text { vector } \\
\text { 2. } 5 \% \text { jet mismatch with } 1^{\circ} \text { attitude } \\
\text { error during firing } \\
\text { a. impulse nominally } \perp \text { to orbit } \\
\text { plane } \\
\text { b. impulse nominally along radius } \\
\text { vector }\end{array}$ & $\begin{array}{l}0.0016 \\
0.0016 \\
0.005 \\
0.005\end{array}$ & 0.155 \\
\hline $\begin{array}{l}\text { Spacecraft } \\
\text { Attitude } \\
\text { Error } \\
\text { (Conical } \\
\text { Motion } \\
\text { of }(\text { ) }\end{array}$ & $\begin{array}{l}\text { 1. } 0.5^{\circ} \text { error-uniform reflectivity } \\
\text { 2. } 0.5^{\circ} \text { error-self-shadowing }\end{array}$ & $\begin{array}{r}0.025 \\
0.04\end{array}$ & $\begin{array}{l}0.05 \\
0.08\end{array}$ \\
\hline
\end{tabular}




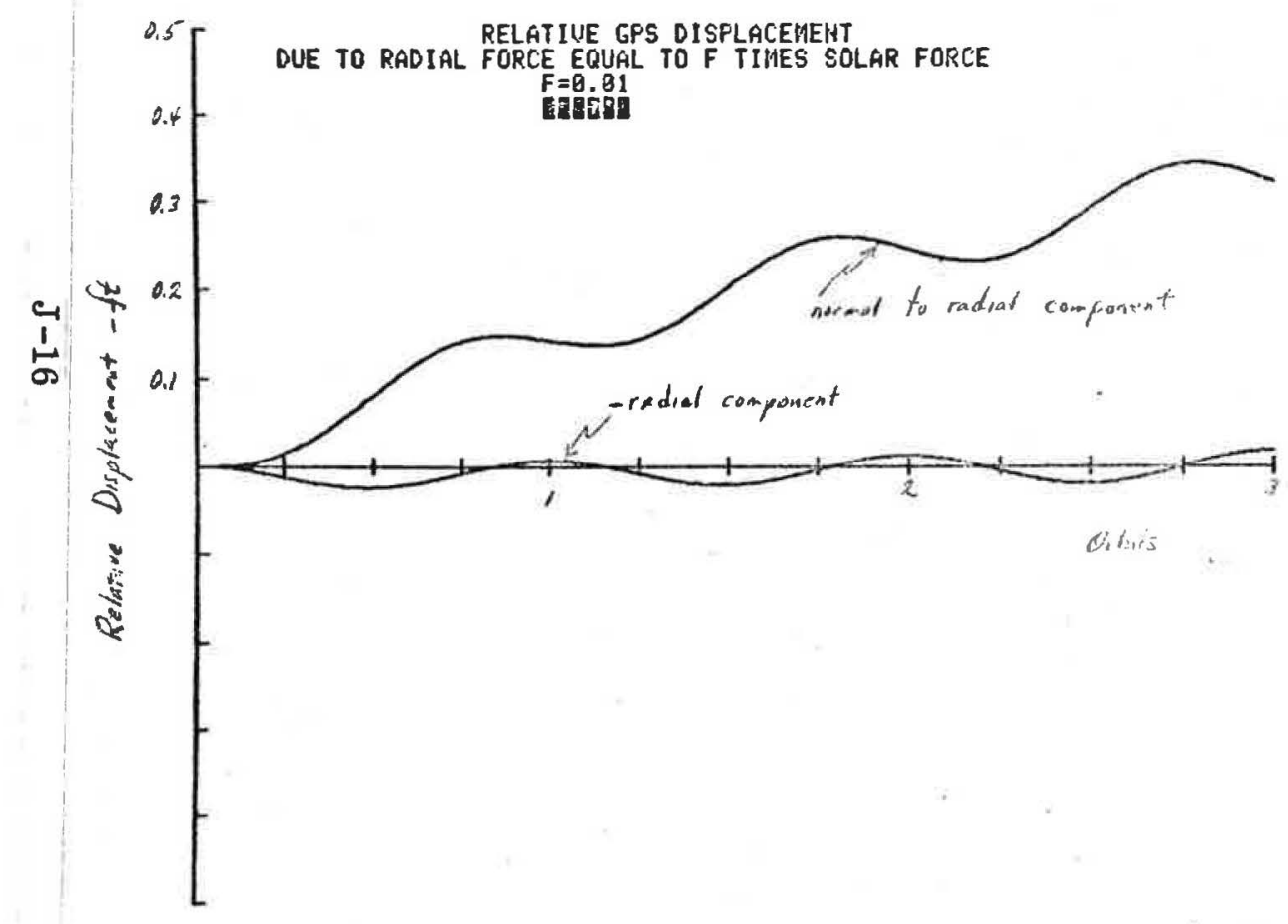

1 REM THIS PROGRAM IS OH FILE 16

10 WIHCOW $\theta, 1980,-0.5,0.5$

$140 \quad I=0$,

$15 \theta \mathrm{N}=\mathrm{B}$

138 Y $Y=9$

190 , $\mathrm{Al}=\mathrm{\theta}$

$210 \mathrm{ZI}=0$

$22829=0$

$249 \quad B=0$

$260 \quad C=P I \leqslant 3+2 /(1185 / 32.17) * 1.0 E-7 / 3$

$265 \mathrm{~F}=9.01$

$27001=2 \$ P I /(12 \$ 360 \theta)$

$28802=0112,01 \pm 0 / 368$

$290 \mathrm{Tl}=2 \mathrm{t}$

$318 \quad \mathrm{~A}=0$

328 PRINT

$340 j=\cos (1) t \sin (A)$

$350 K=S I H(I) \pm S I N(A)$

$3 E \theta L=C \tilde{S}(A)$

$389 \quad P 2=B$
$38=K \theta * J+Y O A K+Z E * 1$

390 ( $2=-027(., \theta-3 * E . J)+C * F * J$

(1)

410

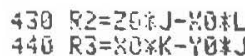

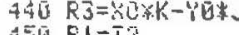

RI $=E R(R 1+2+R Z 12+R 3 \uparrow 2)$

480 U2 $=$

$498 W_{2}=22$

$508 \mathrm{U} U=\mathrm{Y} 1$

$5 ! 9$
5

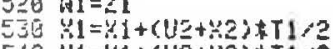

s. $1=1+(4+2) * 11 / 2$

6

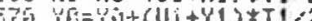

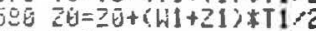

CE: $\mathrm{i}=T+1$

$616 \hat{H} 1=\hat{A}$

E28

ETE DPUA $\hat{H}_{3} T_{2}$

E.

E.T IF $T=31 \% 3 E \theta \div 3$ THEH $3 E \theta$

893 CO TID 320 


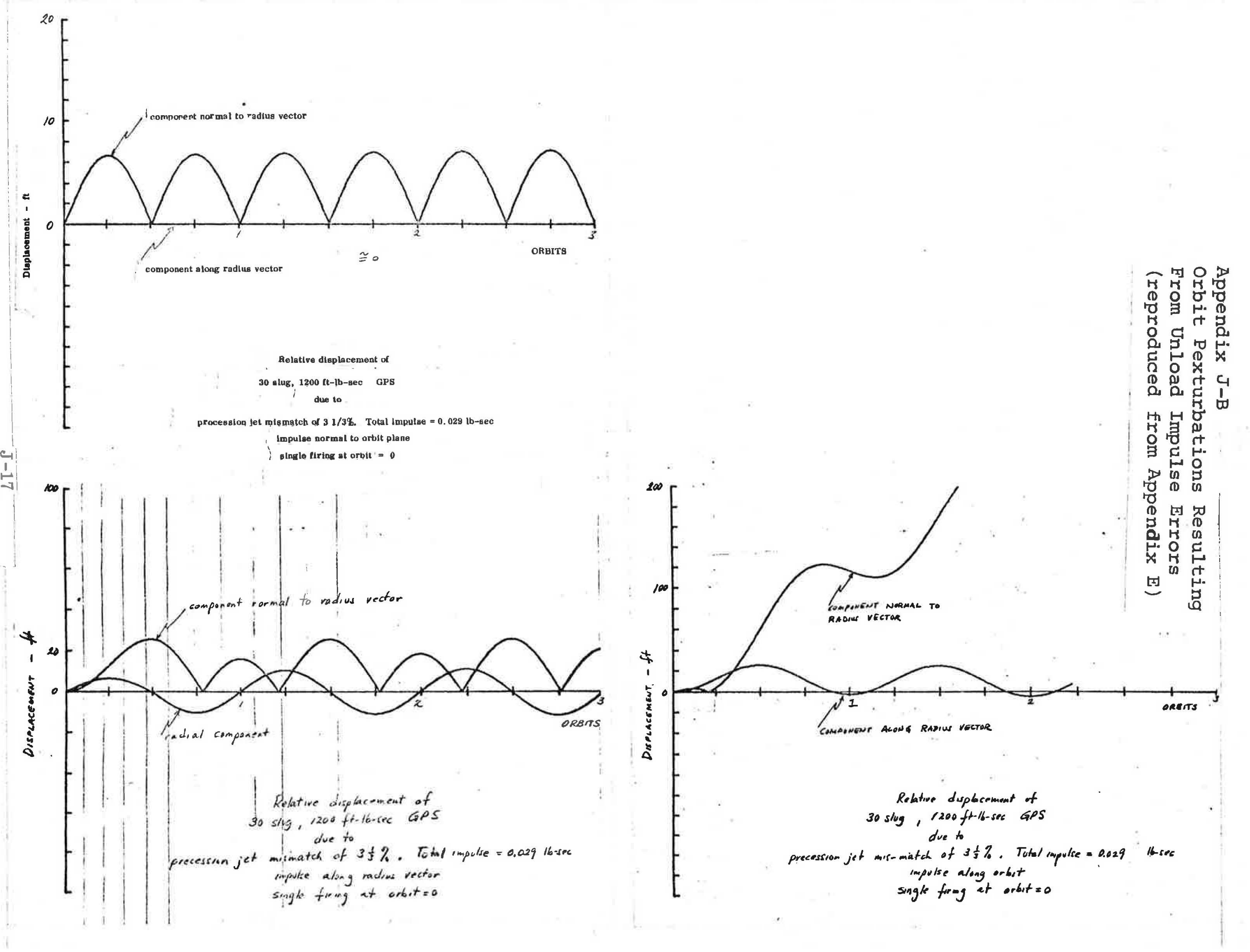


128 REH DUE TO HISAL IGHAEH HHICH RESULTS IH A COHICAL MOTIOH BY THE I

$150 \mathrm{~J}=1$ DERR

151 DIN M(100)

$218 \mathrm{~L}=6$

$\begin{aligned} & 220 \\ & 230 \\ & S 1=1\end{aligned}=0.8$

$240 \mathrm{SL}=1$

$250 \quad R 2=0.88$

$270 \mathrm{G}=0$

$280 \quad 0=0.5$

310 PRIHT 7 CHAHGE OUER ONE SPIN TURM OF 80DY FORCES DUE TO" 328 PRINT

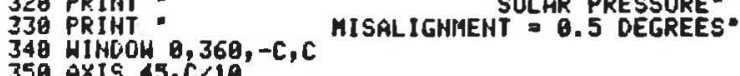

MOUE $\theta_{1} \mathrm{C}$

B日 MOUE $0, \mathrm{C}, 2$

PRIHT $C: 2$

391 MOUE ${ }^{8}-\mathrm{C} / 10$

393 MOUE $8, \theta$

418 Al $=$ PIIR+2

$420 A^{2}=2 \times R+L$

180

SPIN ANGLE(DEG)

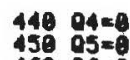

468

530 DI $=$ SIN $(0)$

$556 \mathrm{G1}=51 \mathrm{C}(\mathrm{C})$

$560 \mathrm{G}=\cos (\mathrm{C})$

$58012=\cos (1)$

$59001=51$ (N)

$516 \times 1=02 * G 1 \neq D 1 \pm 12 * 01$

$638 \times 3=C 2 * 014 G 1 \neq 01 \pm 12 * 02$

$65 \theta y=x 1+x 2+x 3+x 4$

$6 \in Q Y_{1}=-G 1 * 01 \neq G 1 \neq 01 \neq 12 * 01$

$68 \mathrm{Y}=-61702 \pm 11$

$690 \quad Y 4=-C 2 \$ 01 \neq C 2 \pm 01 \neq 12 \pm 01$

$700 Y=Y 1+Y 2+Y 3+Y 4$

$71021=-G 1 \neq 01 \div 61 \neq 01 \leqslant 12 \div 02$

$72022 *-G 13012 G 2 * D 1 * 11$

$746 \quad 24=02462501+12201$

$360 \quad A=\operatorname{Sag}(X+2+Y 42+Z+3)$

$765 A=A$ MAK $1.0 E-69$

780 Y=Y/A

Q日e $P \geq=-G 1 * D 1 * 11+D 2 * 12 * 01+G 2 * 01 * 12 * 02$

803 IF P2>O THEN 805
$894 \mathrm{H}=-1$

805 P2=ABS(P2)

820 PI $=S I N(Q)$

$846 \quad F I=S I H(F)$

$850 F 2=\cos (F)$

870 REM

酯

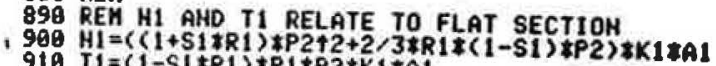

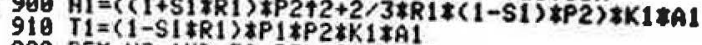

920 REM N2 AHD I2 RELATE TO CYL INDRICAL SECTION

when reflectivity haner wht
angle to sun:

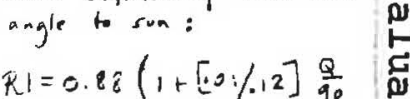

$R_{2}=C_{82}\left(1+\left[\frac{.01}{.12}\right] \frac{E}{90}\right)$ 芩

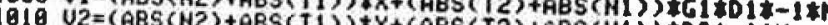

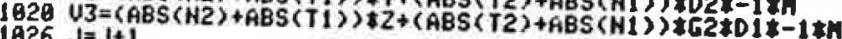

$1026 \mathrm{JJ}=\mathrm{J}+1$ THEN 1180

$1060 \mathrm{UL}=\mathrm{U1}$

$1861 \mathrm{U2}=U 2$
$1863 \mathrm{U3}=\mathrm{U3}$

1080 D=0

$1998 \mathrm{N=1}$

$1180 \substack{\begin{subarray}{c}{0 \\ 0=0} }} \\{0=8,5} \end{subarray}$

1190 o1 =U1-U1

$119102=U 2-U$

$120004 \times 04+01 / 24$
$1210 \quad 05=05+02 / 24$
$1220 \quad 06=06+03 / 24$

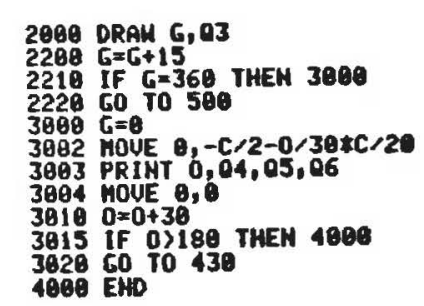



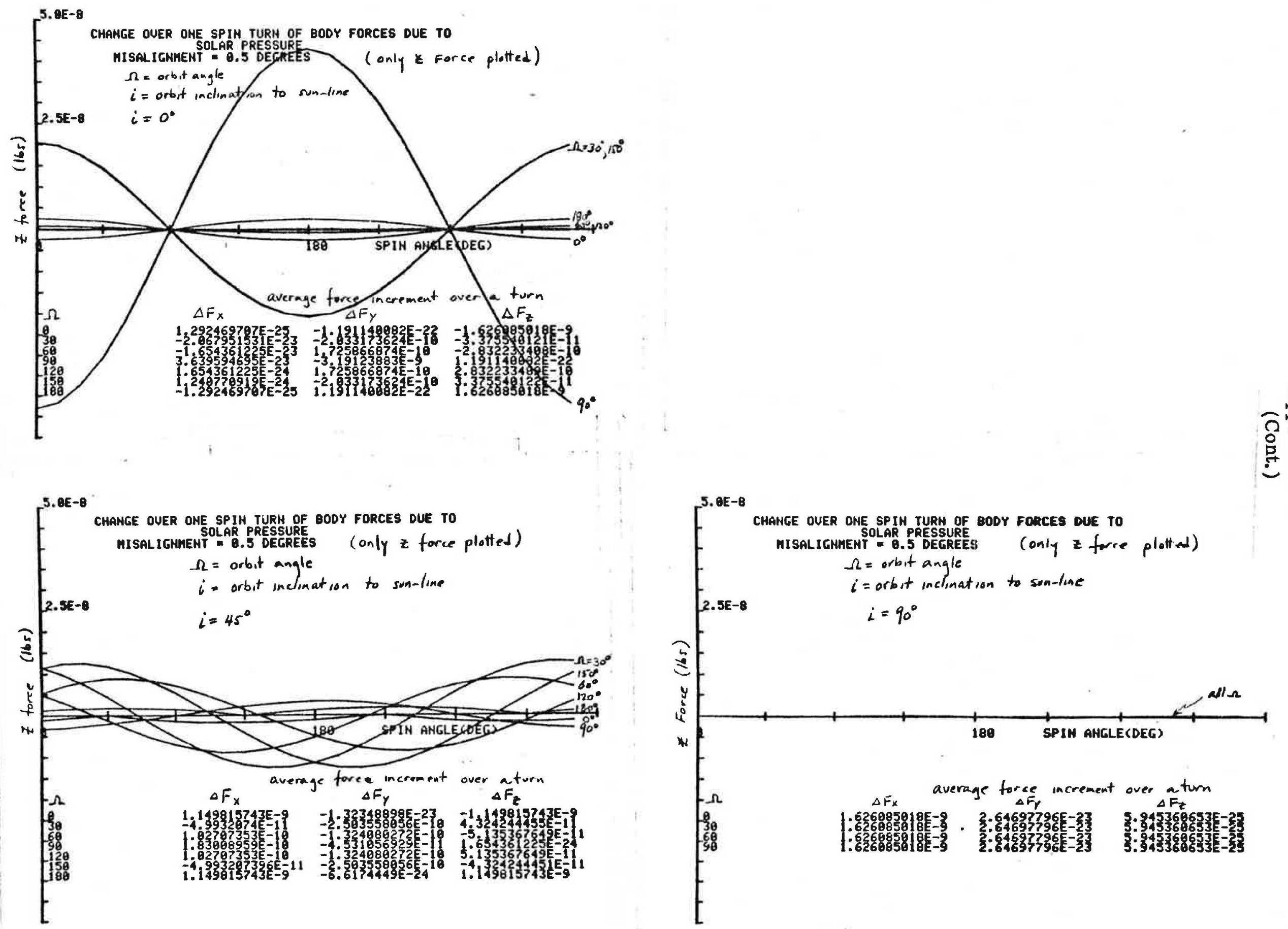


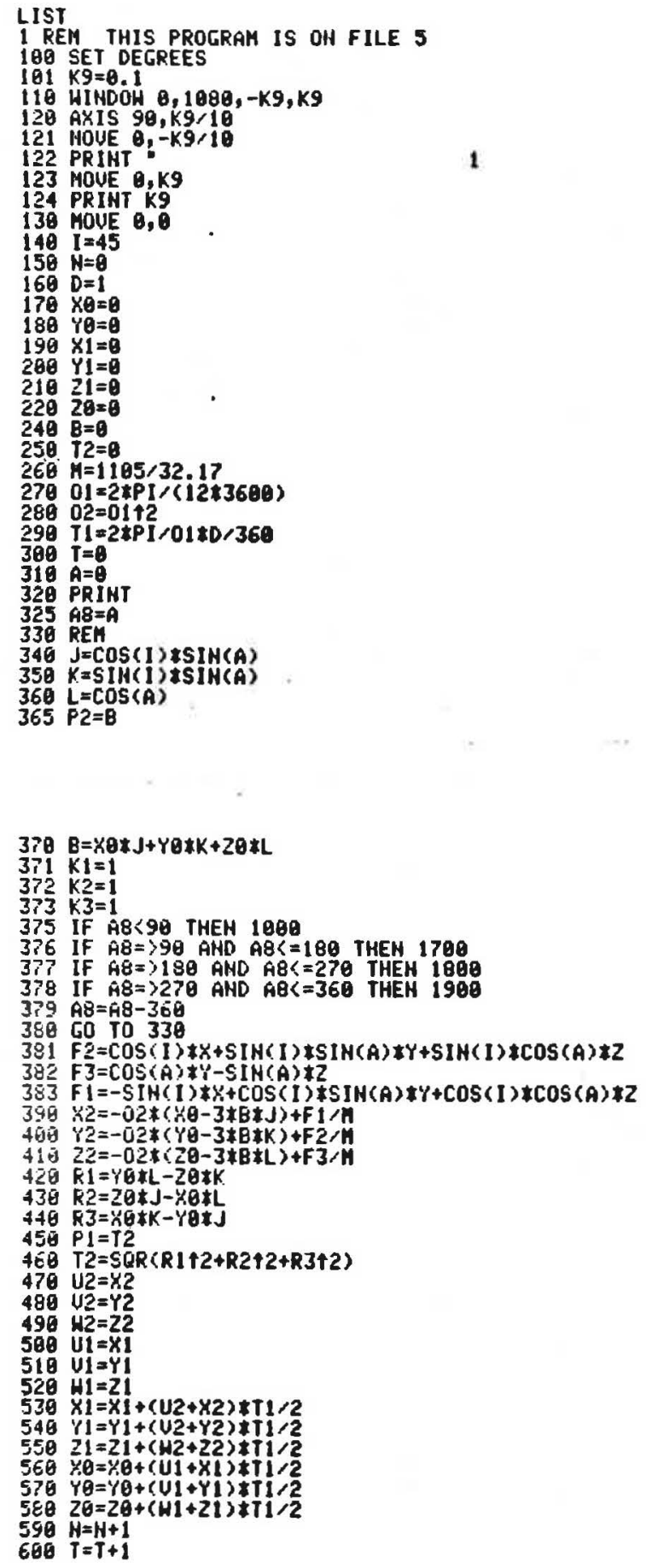


J-D (cont.)
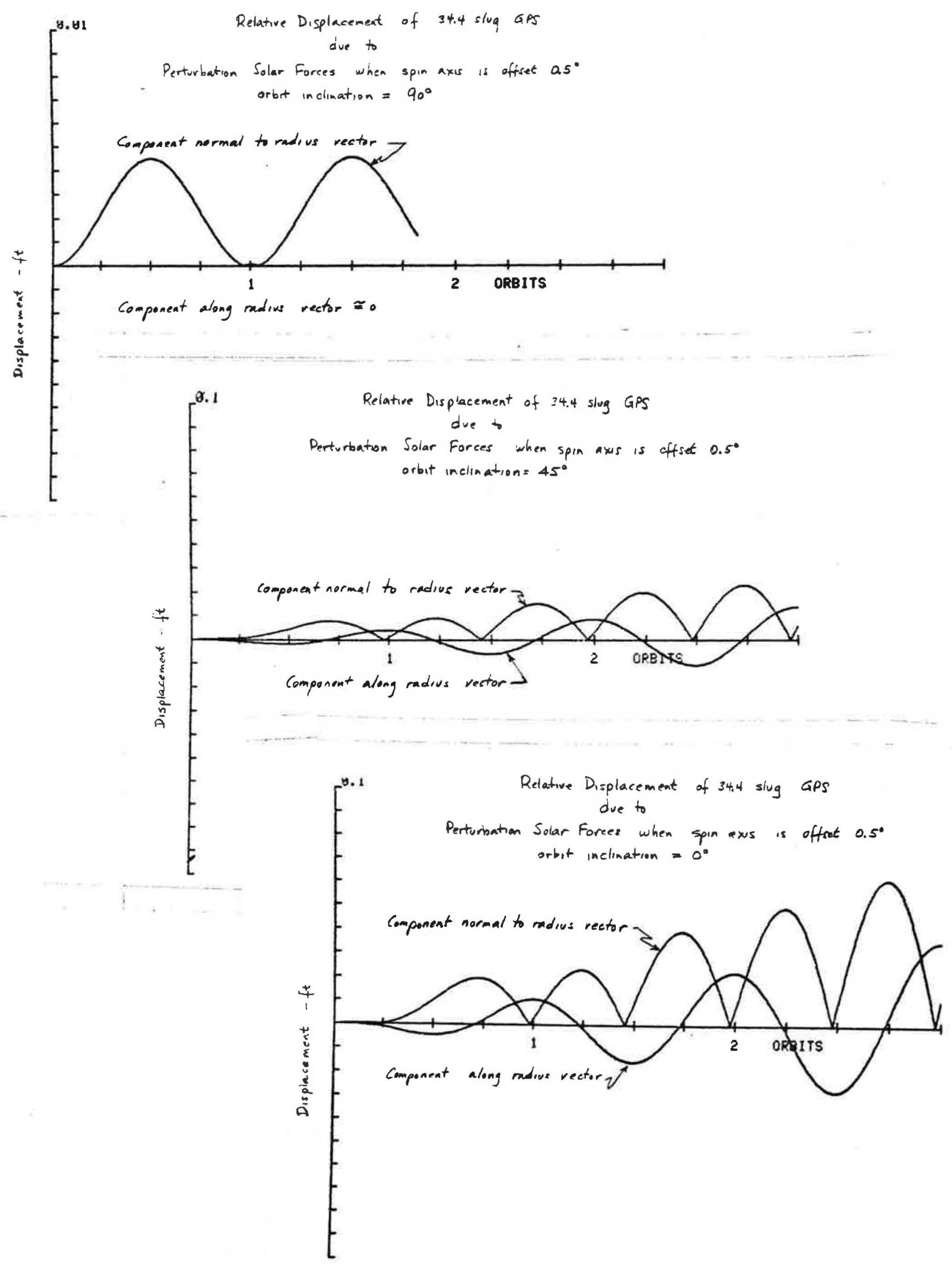


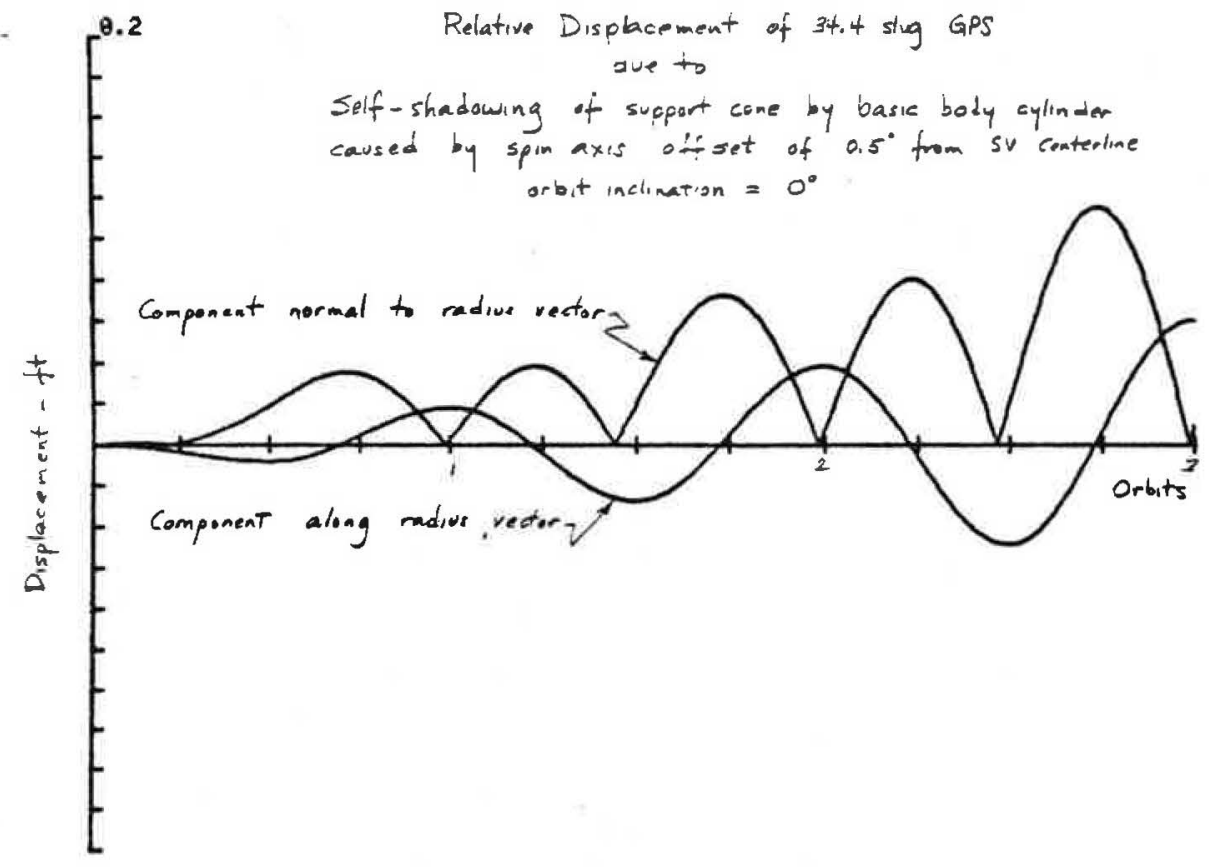

1 REM THIS PROGRAM IS OH FILE 16

100 SET DEGREES

$191 \mathrm{I}=9.2$

110 HINDOH $0,1080,-1, I$

129 AXIS $98,1>10$

138 MOUE 9,1

$140 \quad I=0$

$150 \mathrm{H}=0$

$168 \quad 0=1$

$170 \mathrm{xQ}=0$

$180 \quad 48=0$

$199 \times 1=0$

280 Y $11=0$

218 il

218 in

228 र

$240 \quad B=0$

$25 B \quad T 2=0$

$26 \theta \quad C=-1.8 E-7 * 36 \times(1185,32.17) \div 1 . \theta E-3$

$265 F=A T H C 8.5,18$ )

$2701=2 \pm P I /(12 \$ 3600)$

$288 \quad 02=0142$

$298 \mathrm{TI}=2 \times P I / 01+0 / 368$

$300 \mathrm{~T}=0$

318 p=日

328 PRINT

338 R.EM

$340 . \mathrm{J}=\operatorname{COS}(1) \pm \operatorname{SIN}(\mathrm{A})$

$350 K=S I N(I) \cup S I M(A)$

$360 L=\operatorname{COS}(A)$

$370 \quad P 2=8$

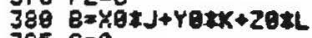

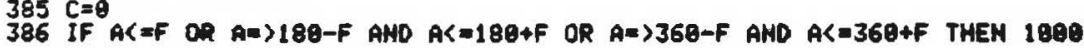

387 If $A=354 \theta-F$ AND $A<=549+F$ OR $A=>73 \theta-F$ AND $A<=729+F$ THEN 1090 388 If $A=>960-F$ AND $A K=990+F$ OR $A=>1680-F$ AND $A<=1690+F$ THEN 1000 $398 \times 2=-02 *(X \theta-3 * B * J)+C$

$460 Y 2=-02 *(Y B-3 * \theta \neq K)$

$418 \quad 22=-02 *(2 \theta-3 * 8 * L)$

420 R1 $=Y \theta * L-Z \theta \neq K$

$438 R 2=2 \theta * J-X \theta * L$

$44 \theta R 3=X 8 \pm K-Y B+1$

$458 \mathrm{Pl}=\mathrm{T} 2$

$468 T 2=S Q R(R 1+2+R 242+R 3+2)$

470 U2 $=\times 2$

489 U2=Y2

$\begin{aligned} & 498 \\ & 580\end{aligned} 41=Z 2$

518 UI $=4$

$528 \quad H 1=21$

$530 x_{1}=x_{1} 1+\left(U 2+x_{2}\right) \pm T 1 / 2$

$546 Y 1=Y 1+(U 2+Y 2)+T 1 / 2$

5 . $Z 1=Z 1+(H 2+Z 2)+T 1 / 2$

568 (a)

$570 \quad Y \theta=Y \theta+(U 1+Y 1)+T 1 / 2$
$588 Z=Z B+(H 1+Z 1)+T 1 / 2$

$590 \quad N=N+1$

$680 T=T+1$

610 Al $x$ A

620 MOUE $A 1, P 1$

$630 A=0+N$

648 DRAH A,T2

650 MOUE A1,P2

668 DRA Ap

$368+3$ THEN 700

680 GO TO 328

$1890 \mathrm{C}=-1.0 \mathrm{E}-7 * 36 /(1165 / 32.17) \$ 1.0 E-3$

1610 Co To 390 
K. RETROFIT DESIGNS FOR A NUCLEAR GPS 

During the first quarter of the study, retrofits to the current Phase I GPS satellite design were considered. Replacements of the solar-array/battery system by radioisotope thermoelectric generators (RTG), organic Rankine cycle (ORC), and Brayton isotope power systems (BIPS) were evaluated. In addition, an ORC with a thermoelectric (TE)" topping" cycle was considered. Each of the systems could be feasibly retrofitted using the space-qualified MHW heat source. Within the weight constraints of the study groundrules, (to $1200 \mathrm{lbs}$ exclusive of apogee motor (AKM) weight), any of the candidate nuclear power systems could be employed. Within the retrofit limitations, operational benefits could be achieved in the form of:

- availability of the high power mode throughout the design life of the satellite

- elimination of the risk of a cold spacecraft during transfer orbit

- reduction in the clock baseplate temperature variation

- possibility of extended satellite design life.

- improvement in predictability of satellite ephemrides

By using an enlarged version of the MHW heat source, which would

provide $25 \%$ additional thermal power, the following added benefits would result on a retrofit basis:

- simultaneous transmission of the L-2 link $\mathrm{P}$ and C/A signals with the C/A link power $3 \mathrm{~dB}$ above its current level

- increased user power to the following levels:

$\begin{array}{rlll}\mathrm{L} 1(\mathrm{P}) & \mathrm{L} 1(\mathrm{C} / \mathrm{A}) & \mathrm{L} 2(\mathrm{P}) & \mathrm{L} 2(\mathrm{C} / \mathrm{A}) \\ -160 \mathrm{dBW} & -157 \mathrm{dBW} & -165 \mathrm{dBW} & -162 \mathrm{dBW}\end{array}$

K-2 CANDIDATE RADIOISOTOPE POWER SYSTEMS

K-2.1 GENERATOR OPTIONS

Three advanced radioisotope power systems now in development under ERDA sponsorship form the bases for the power systems considered in this study. These are the: 1.) selenide RTG 2.) mini-Brayton cycle (BIPS) and 3.) organicRankine cycle (ORC) power systems. These are shown schematically in Figures $\mathrm{K}-1, \mathrm{~K}-2$ and $\mathrm{K}-3$. 


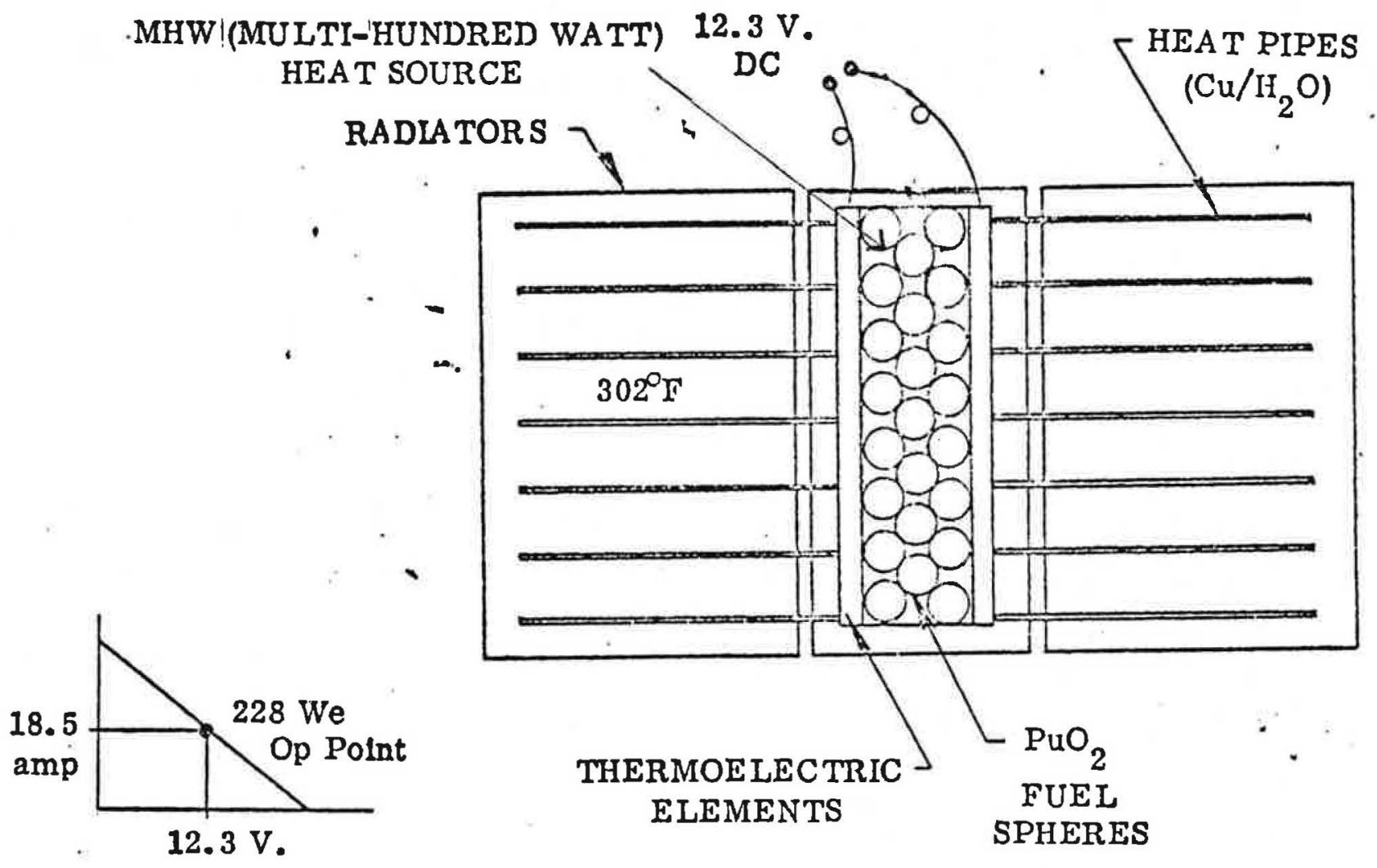

FIGURE $\mathrm{K}-1$. RTG SCHEMA TIC

Current program plans call for carrying both dynamic generator systems through the ground demonstration phase in 1978 (Figure K-4) • Following a period of ground testing, one of the two systems will be selected for flight development and testing. The selenide RTG will be developed independently, to the schedule shown in Figure K-5. Both static and dynamic systems are now scheduled for flight test readiness in FY 81 .

\section{K-2.2 HEAT SOURCE OPTIONS}

An ERDA management decision of December 1975 has specified that the Multi-Hundred-Watt heat source (MHW/HS) shall be used for the 1981 test flight program, and for applications through 1983. A new-design heat source will be phased into programs thereafter. The MHW/HS has been qualified for launch and is currently in use aboard the LES 8 and 9 satellites, powering the MHW/RTG's. It is also scheduled for use on the Mariner-Jupiter/Saturn mission (MJS-77) due to be launched in mid 1977.

Because this heat source is already space-qualified, it imposes minimai schedule risk for missions in the 1981-83 time frame. Consequently, it was used as the baseline heat source in the retrofit phase of the study herein reported. 


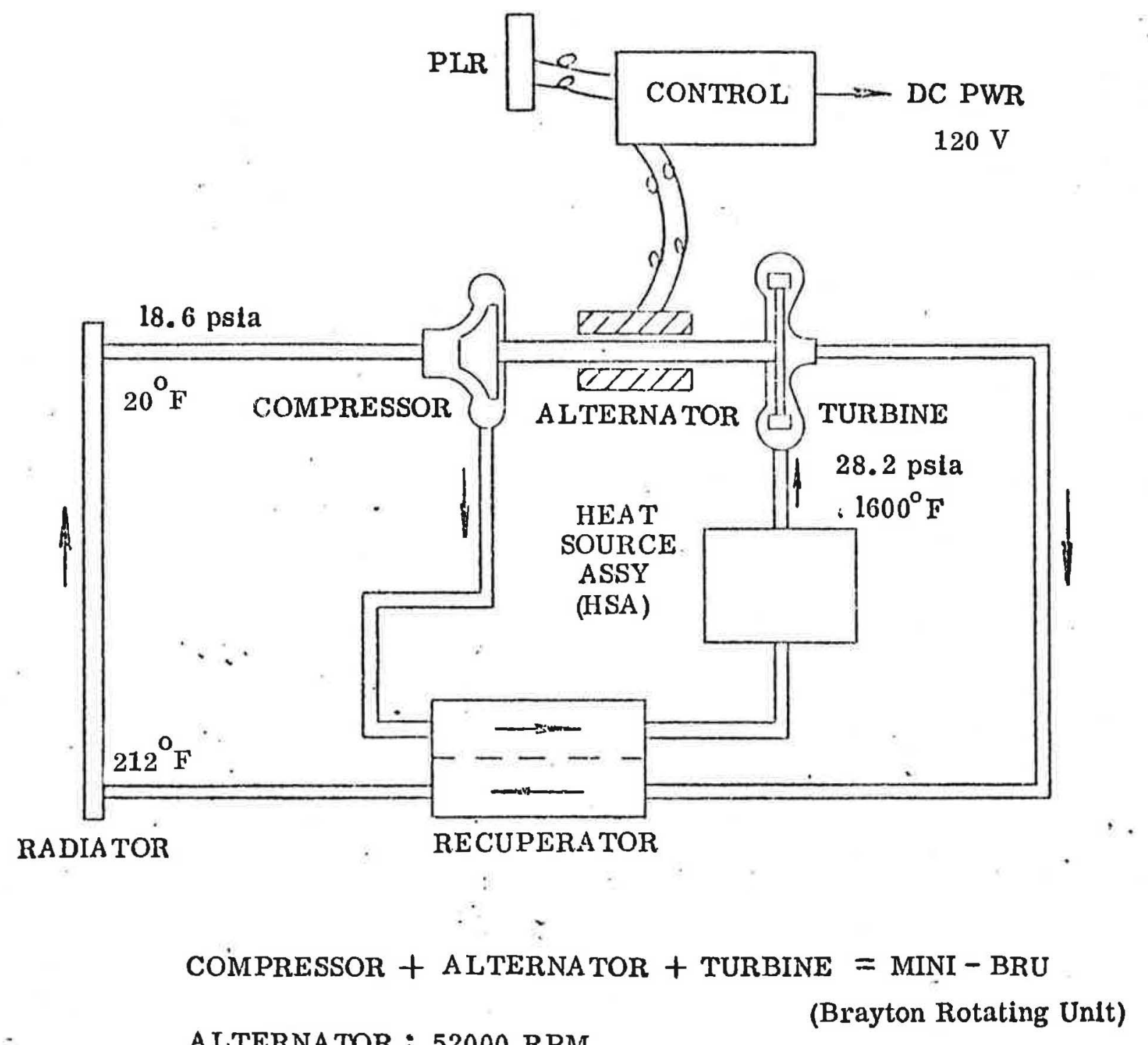

ALTERNATOR : 52000 RPM

$1.16 \mathrm{lb}$ of $\mathrm{Xe} / \mathrm{He}$ gas mixture 


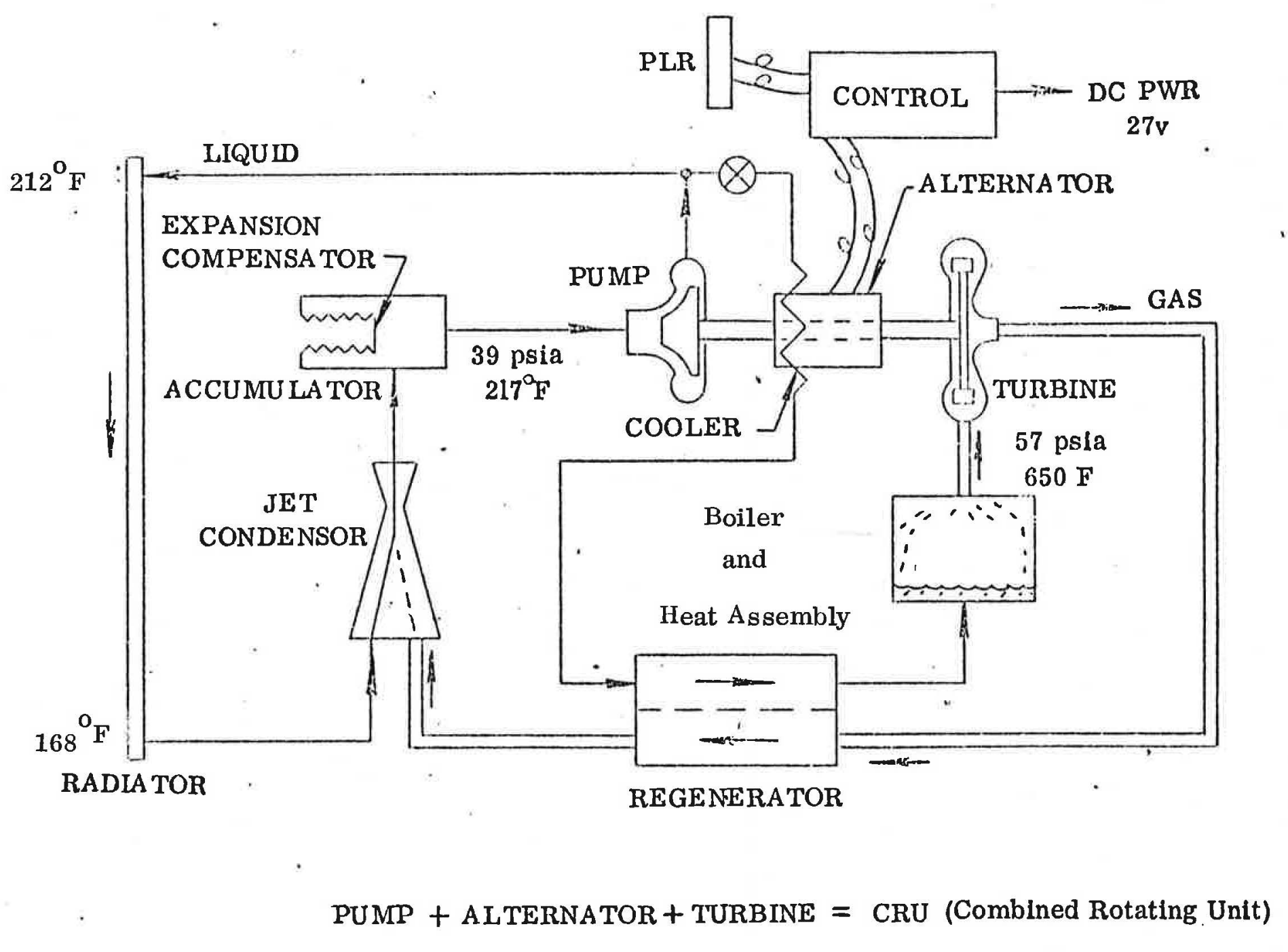

Working Fluid: Dowtherm A 


\begin{tabular}{|l|c|l|l|l|l|l|l|}
\hline \multicolumn{1}{|c|}{ MILESTONES } & FY75 & FY76 & FY77 & FY78 & FY79 & FY80 & FY81 \\
\hline DEV. FLT. CONCEPT & & & & & & & \\
\hline SYS. GROUND DEMO. & & & & & & & \\
\hline SYS. SELECTION & & & & A & & & \\
\hline FLT. SYS. DESIGN AND QUAL & & & & & & & \\
\hline FLT. SYS. PRODUCTION & & & & & & & \\
\hline DELIVERY FOR FLT. TEST & & & & & & & $\Delta$ \\
\hline
\end{tabular}

Figure K-4 Dynamic Generators Development Schedule

\begin{tabular}{|l|l|l|l|l|l|l|}
\hline \multicolumn{1}{|c|}{ MILESTONES } & FY77 & FY78 & FY79 & FY80 & FY81 \\
\hline TECHNOLOGY READY & & & & & & \\
\hline FLT. SYSTEM DESIGN & & & & & & \\
\hline RTG PRODUCTION AND DELIVERY & & & & & & \\
\hline FLT. SYSTEM PRODUCTION & & & & & & \\
\hline
\end{tabular}

Figure K-5 Selenide RTG Development Schedule 
The MHW/HS as qualified produces 2400 thermal watt $\left(\mathrm{w}_{t}\right)$ at BOL, built up of an assembly of 24 fuel sphere assemblies (FSA's) (Figure K-6). Each FSA contains a plutonium-dioxide $\left(\mathrm{PuO}_{2}\right)$ sphere which produces $100 \mathrm{w}_{\mathrm{t}}$ of power. These are arranged in four trays of eight spheres each, bolted together and enclosed by an aeroshell reentry structure.

Initial consideration of the retrofit designs was based on the $2400 \mathrm{w}_{\mathrm{t}}$ unit. Subsequently, the addition of another tray of eight FSA's was postulated to bring the thermal power of each heat source to $3200 \mathrm{w}_{t}$, thus enabling the determination of the effects of increased electrical power, where warranted by weight margin.

Based on the current GPS spacecraft power budget of $373 \mathrm{w}_{\mathrm{e}}$ on orbit (reference App. A), it was anticipated that both of the dynamic power conversion systems would meet the basic requirement with a single heat source, while two heat sources, and two RTG's, would be required for use with the thermoelectric converters.

The parametric and physical characteristics of each of the power systems configured for use with both 2400 and $3200 \mathrm{w}_{t}$ heat sources is described in section $\mathrm{K}-2.4$.

K-2.3 BASIC INTERFACE LIMITATIONS

In the retrofit design approach, the spacecraft design is largely determined, without regard for the desires or limitations of nuclear power systems. Therefore, a certain amount of fitting and tailoring is called for, which may be relieved in the new spacecraft designs to be considered in the next phase of the study. Other interface limitations, however, are less likely to be relieved, and are basic to spacecraft operation as presently conceived. The fundamental interface characteristics which were applied to the power systems in the retrofit phase are:

- Volume -- Potential power system mounting locations are limited to two regions, of which only one ultimately proved to be preferable for all systems. This region is the annular volume surrounding the AKM nozzle and thrust cone (Figure $\mathrm{K}-7$ ). 


\section{MHW HEAT SOURCE}

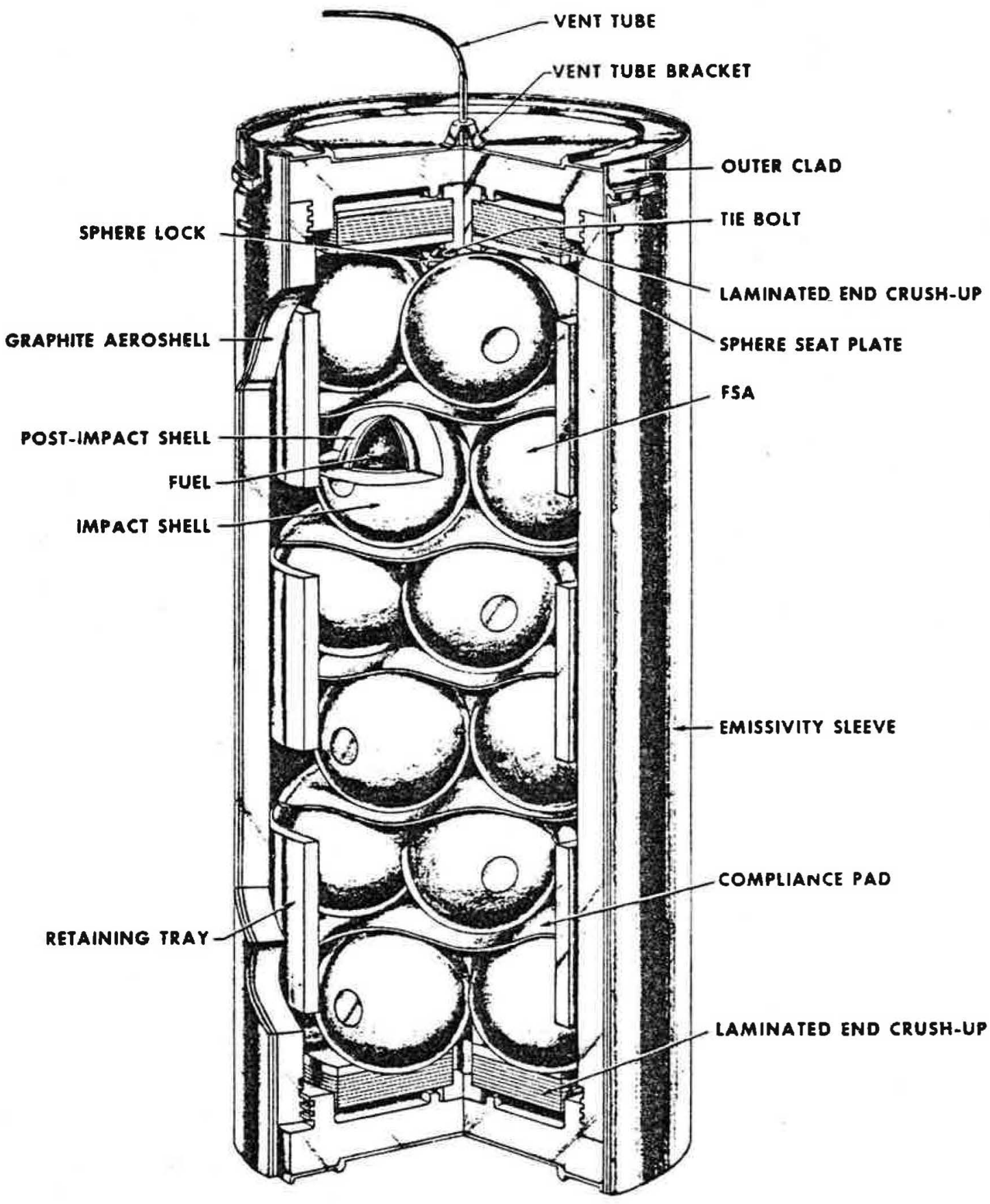

Figure K-6 


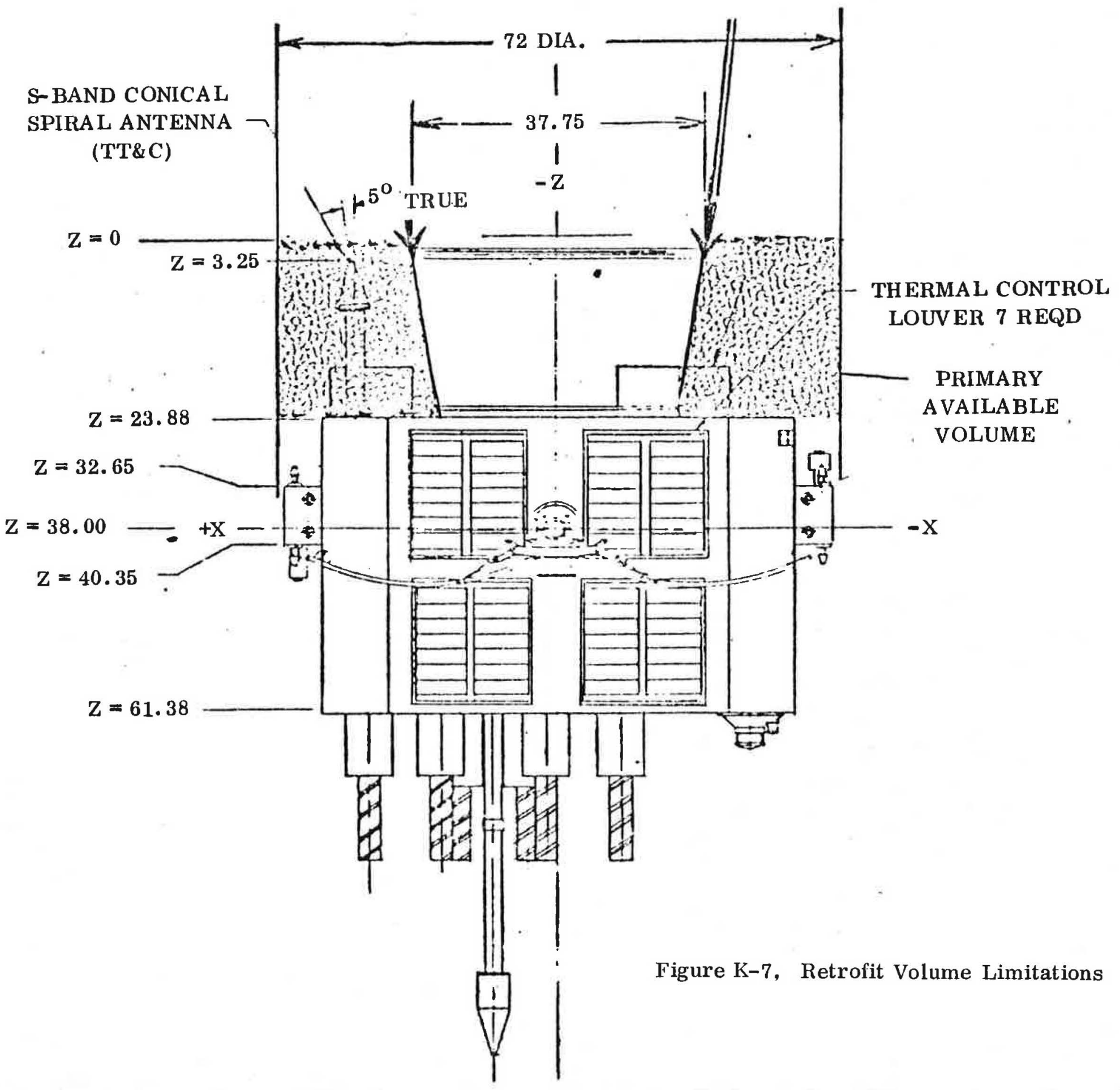


- Launch Power -- Approximately $80 \mathrm{w}_{\mathrm{e}}$ of power is required from $\mathrm{T}-5$ minutes through injection, a maximum of $\mathrm{T}+45$ hours

- Spinning Orbit Transfer -- A spin-stabilized orbit transfer of 45 hours maximum at $95 \mathrm{RPM}$ is used. This requires both lateral g-loading tolerances and dynamic balance of the power system about the spin axis.

K-2.4 POWER SYSTEM DESIGN PARAMETERS

The generator design characteristics to be described were derived by the respective power system development prime contractors with Fairchild guidance and modifications as necessary to meet the various interface characteristics. These contractors are:

Selenide RTG - - Teledyne Energy Systems, Timonium, Maryland

Organic Rankine - Sundstrand Energy Systems, Rockford, Illinois

Mini-Brayton - AiResearch Mfg. Co. of Arizona, Phoenix, Arizona

$\mathrm{K}-2.4 .1 \quad$ Selenide RTG

Because of the particular annular volume limitation of the retrofit design approach, the RTG's must be designed with only two fins which can wrap around the annulus. This suggests a design featuring two columns of selenide thermoelectric converters at opposite sides of the heat source, with heat-pipe-finned radiators extending from these columns, as shown in Figure K-8. The remainder of the heat source surface is thermally insulated, and the entire generator is enclosed by an aluminum housing.

Within the given design limitations two options were considered: fixed generators and deployable generators. Because the latter can radiate from both sides of the radiator, it was believed that the se could offer an additional weight and/ or efficiency advantage.

The fixed generators were finally preferred because:

1.) The weight advantage of the deployed system vanished when the deployment mechanism was included, and 


\section{TELEDYNE RTG DESIGN}

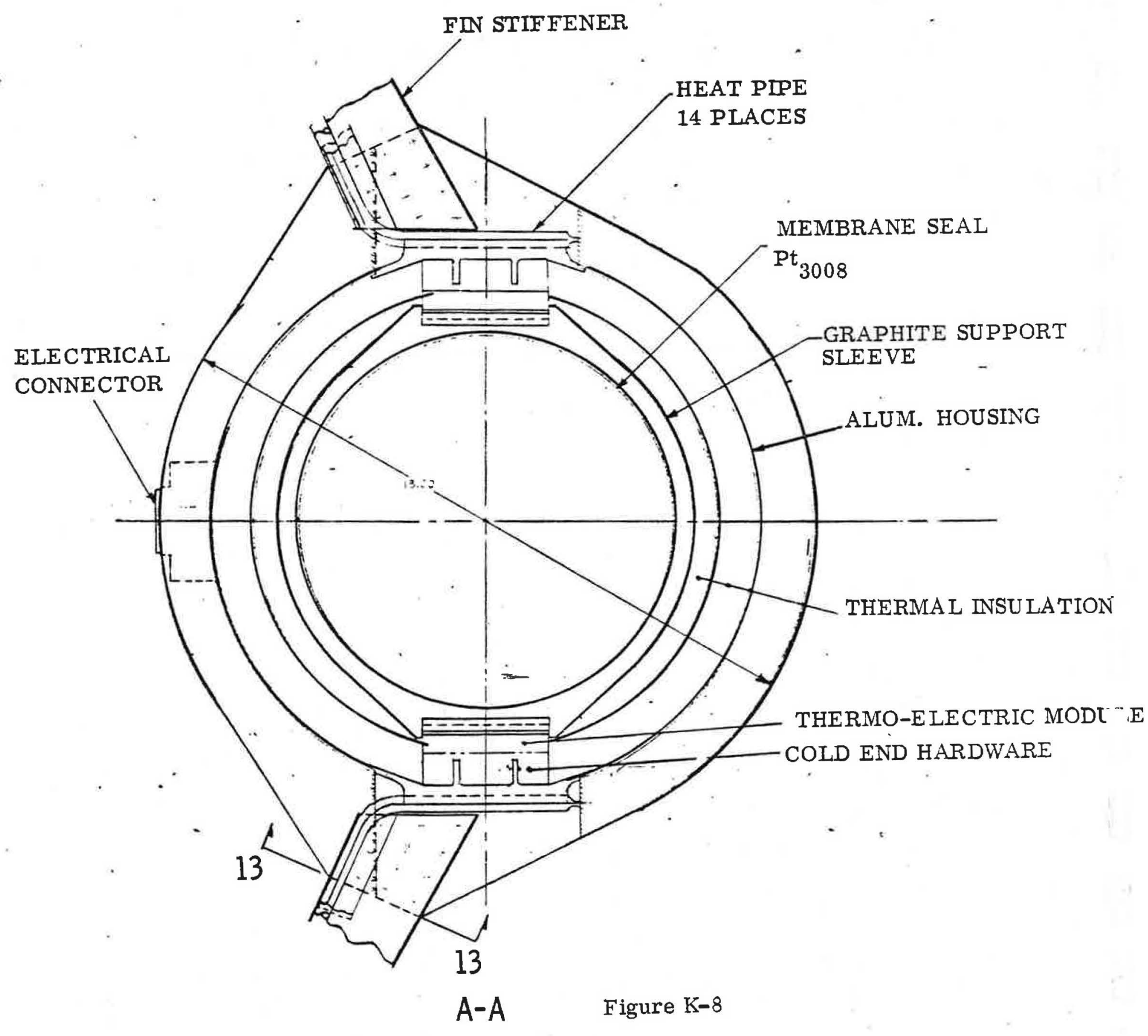


2.) The long spinning transfer orbit with RTG's in the stowed position would result in overheating of the thermoelectric modules, since only one side of the radiators would be effective during this period.

Table,$K-9$ is a summary of the major performance parameters of the RTG unit. The radiator temperature was selected so that the radiator would fit within a semi-space of the available annulus without protruding beneath the $\mathrm{Z}=\mathrm{O}$ station. (The retrofit spacecraft design is shown in section 6.)

Table $\mathrm{K}-10$ shows the detailed weight breakdown for the $2400 \mathrm{w}_{t}$ case, and table $\mathrm{K}-11$ lists the materials and configurations of the individual components in the $2400 \mathrm{w}_{\mathrm{t}}$ version.

K-2.4.2 Organic Rankine Cycle System (ORC)

The ORC system currently under development for the ground demonstration phase of the program is optimized for a $1300 \mathrm{w}_{\mathrm{e}}$ output. This is the KIPS (Kilowatt Isotope Power System) unit, which requires three MHW heat sources. The minimum power requirements of the GPS can be met with a slightly modified KIPS powered by a single MHW heat source.

Added power can be achieved by using the $3200 \mathrm{w}_{\mathrm{t}}$ heat source. The only major component parts of the $1300 \mathrm{w}_{e}$ KIPS system which are reconfigured for these cases are the regenerator and the turbine admission ports, which are specially adjusted for each system. In addition, the components have been repackaged in order to fit within the specified volume, and to provide the necessary dynamic balancing. Of course, connecting plumbing, accumulator, valves, wiring harness, and parasitic load radiator are all re-sized from the KIPS baseline case.

The basic system layout is shown in figures $\mathrm{K}-12$ and $\mathrm{K}-13$ The summary of performance parameters is given in Table $\mathrm{K}-14$ for both $2400 \mathrm{w}_{\mathrm{t}}$ and $3200 \mathrm{w}_{t}$ cases. 


\section{TABLE $\mathrm{K}-9$ \\ PERFORMA NCE SUMMARY \\ SELENIDE RTG* FOR GPS MISSION}

Output power (watts)

BOL

7 years

Load voltage, volts

Specific power (watts/pound), BOL

Steady state temperatures, BOL $\left({ }^{\circ} \mathrm{C}\right)$

Sink

Radiator

Cold junction

Hot junction

Isolation hot frame

Efficiencies, BOL (\%)

Overall

Thermoelectric

Thermal

Shoes/Straps

Fuel inventory (watts $(t)$ )
228

12.3

2. $3^{* *}$ (Non-deployed RTG)

2. $4^{* *}$ (Deployed RTG)

$-69$

185

900

930

9.5

12.0

80.5

98.5

2400

* Two RTG's required

** Support structure not included 
TABLE K-10

SIZE/WIGHT SUMMARY

SELENIDE RTG FOR GPS

\section{Component}

Isolation Hot Frame \& Wedges

Isolation Hot Frame Liner \& Seal

Thermoelectric E lements \& Hot Shoes

Straps \& Cold End Hardware

Insulation

Heat Pipes

Heat Pipe Saddles

$\mathrm{F}$ in (Web)

Fin Ribs

Housing

End Covers

Housing to Fin and Cold End Hardware Transitions

Housing Ribs

Emissive Coating

Connectors and Wiring

Heat Source
$2400 \mathrm{w}_{\mathrm{t}}$

Weight (lbs)

6.3

5.5

4. 0

4. 0

$10 . \dot{4}$

7. 1

1.9

3.8

2.1

5. 0

2. 8

4.3

1.5

2.3

0.3

100.7

22.0

102.0

11.0

$\mathrm{K}-13$. 
TABLE K-11

CONFIGURATION/MATERIAL SUMMARY

LCHPG/GPS II SELENIDE GENERATOR

Item

Heat Source

Isolation Hot Frame

Support Structure

Isolation Hot Frame

Liner

Bellows

Transition Bulkhead

Bi-metallic Seal Ring

Hot Side Dielectric

Hot Straps

Thermoelectric

Thermoelectric

$\mathrm{N}$ element
Material

MJS Helipak

POCO AXF-5Q

Pt -3008

Pt -3008

Inconel 600

Aluminum/Stainless

$\mathrm{A}_{2} \mathrm{O}_{3}$

Molydenum

Copper-Silver Selenide

Current Standard

Gasolinium Selenide
Configuration

Right circular cylinder 7.4 " dia. $\times 16.5^{\prime \prime}$ high with 24 balls $(2400 \mathrm{~W}(\mathrm{t})$

Modified right circular cylinder 7.45" I. D. $x$ 8.25 " O. D. x 16.5 " high

Right circular cylinder with closed bottom, 7.42" I. D. $\times .015$ " wall x 17.0 " high

7. 42 "I. D. $x .010^{\prime \prime}$ thick x 3.9 " developed length

$.010 "$ thick x 7.62" I. D. $x$ 10.37" O. D. ; connects bellows to seal ring

10.37 " I. D. $x 11.10^{\prime \prime}$ O. D., $\mathrm{Al} / \mathrm{ss}$ halves are explosively bonded. Al part welded to housing, ss part welded to transition bulkhead

Either sprayed on straps or separate sheet

$0.060 "$ thick

Partitioned, length $=0.3$ dia. $=.392 "$, number $=$ 128 per generator

Length $=0.3 "$

Diameter $=.472 "$

Number $=128$ per generator 


\section{TABLE $\quad \mathrm{K}-11$ (Cont'd)}

A. Non-Deployable System (Continued)

Item

Cold Straps

Cold End Hardware

Converter Side and End

$\mathrm{T} / \mathrm{E}$ Module Insulation

Housing

Housing Ribs

Radiator Fin

Heat Pipes

Electrical Connectors

Emissive Coating

Converter Gas Fill
Material

Copper

Aluminum rail copper follower $\mathrm{BeO}$ electrical insulation

Min-K TE 1400 , Cotronics Ceramic Board

Fiberfrax Paper

Aluminum

Aluminum

Aluminum

Copper/Water

TBD

$\mathrm{ZnO}+\mathrm{K}_{2} \mathrm{SiO}_{4}$

Binder (IITRI Z-93)

TBD (Xenon or

Vacuum)
Configuration

$0.010 "$ thick, 2 string parallel circuit

Rail with spring loaded follower

Cotronics on hot side Min-K on cold side Cotronics/Min-K interface held at $1600^{\circ} \mathrm{F}$

$0.030^{\prime \prime}$ thick sheets

Right circular cylinder $0.060^{\prime \prime}$ wall x 11.0" O. D. $\times 20^{\prime \prime}$ high

$.060 "$ thick $\times 1 "$ high circumferential ribs number $=7$

Taper from $2 "$ high at RT G to $5 / 32^{\prime \prime}$ high at fin tip. Thickness is 0.020 " with a $1 / 4$ " wide flange on free edge. Number $=7$ per fin

Internal axial grooves $5 / 16^{\prime \prime}$ O. D. , 14 pipes, per RTG (7 on each side)

Hermetically sealed to housing

$0.005^{\prime \prime}$ thick

Sealed between housing, ends, and isolation hot frame liner 
ORC POWER SYSTEM FROM BELOW
(PLUMBING INCOMPLETE FOR CLARITY)

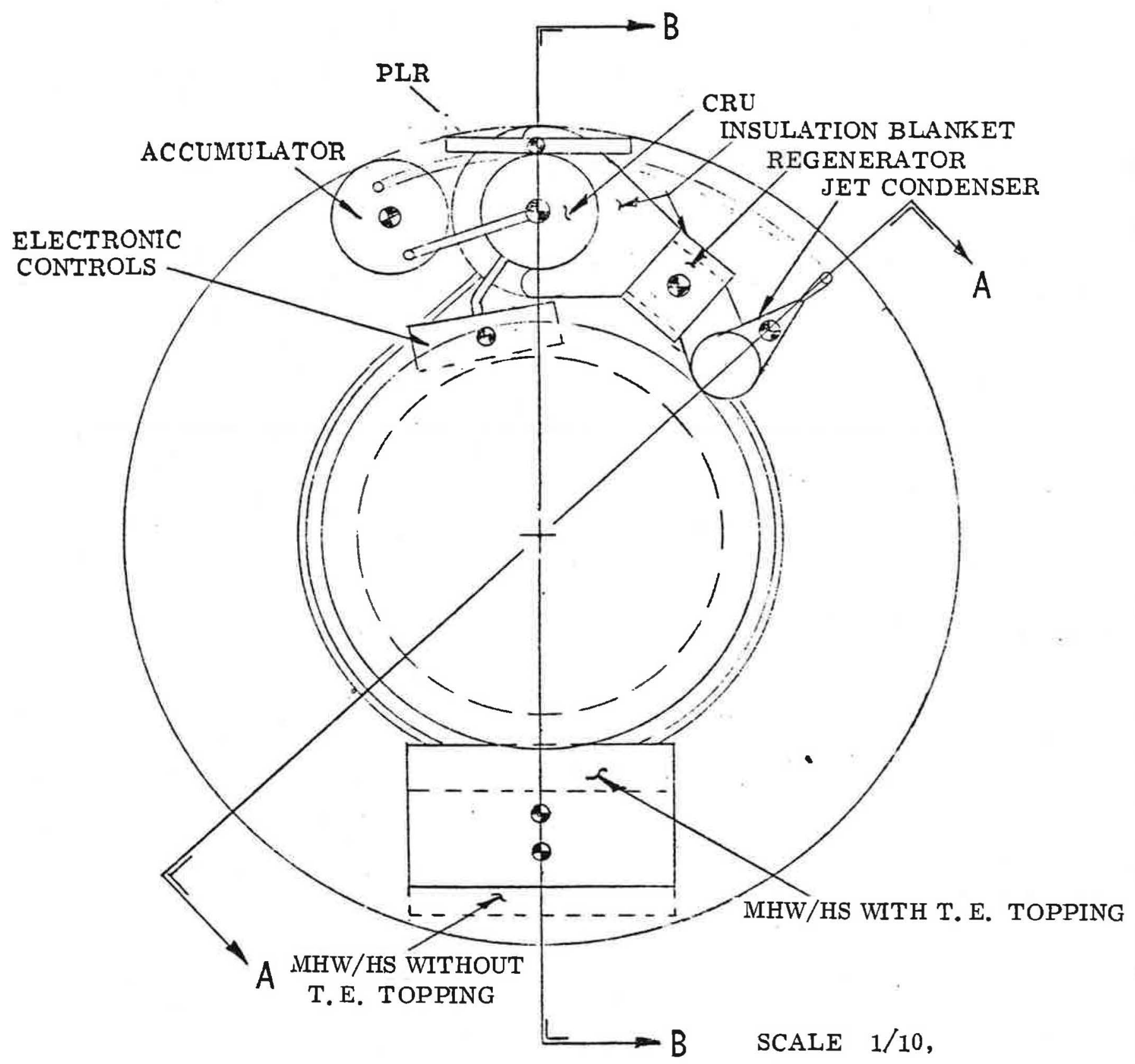

FIGURE K-12 


\section{ROLL OUT OF ORC POWER SYSTEM '(PLUMBING NOT SHOWN FOR CLARITY)}

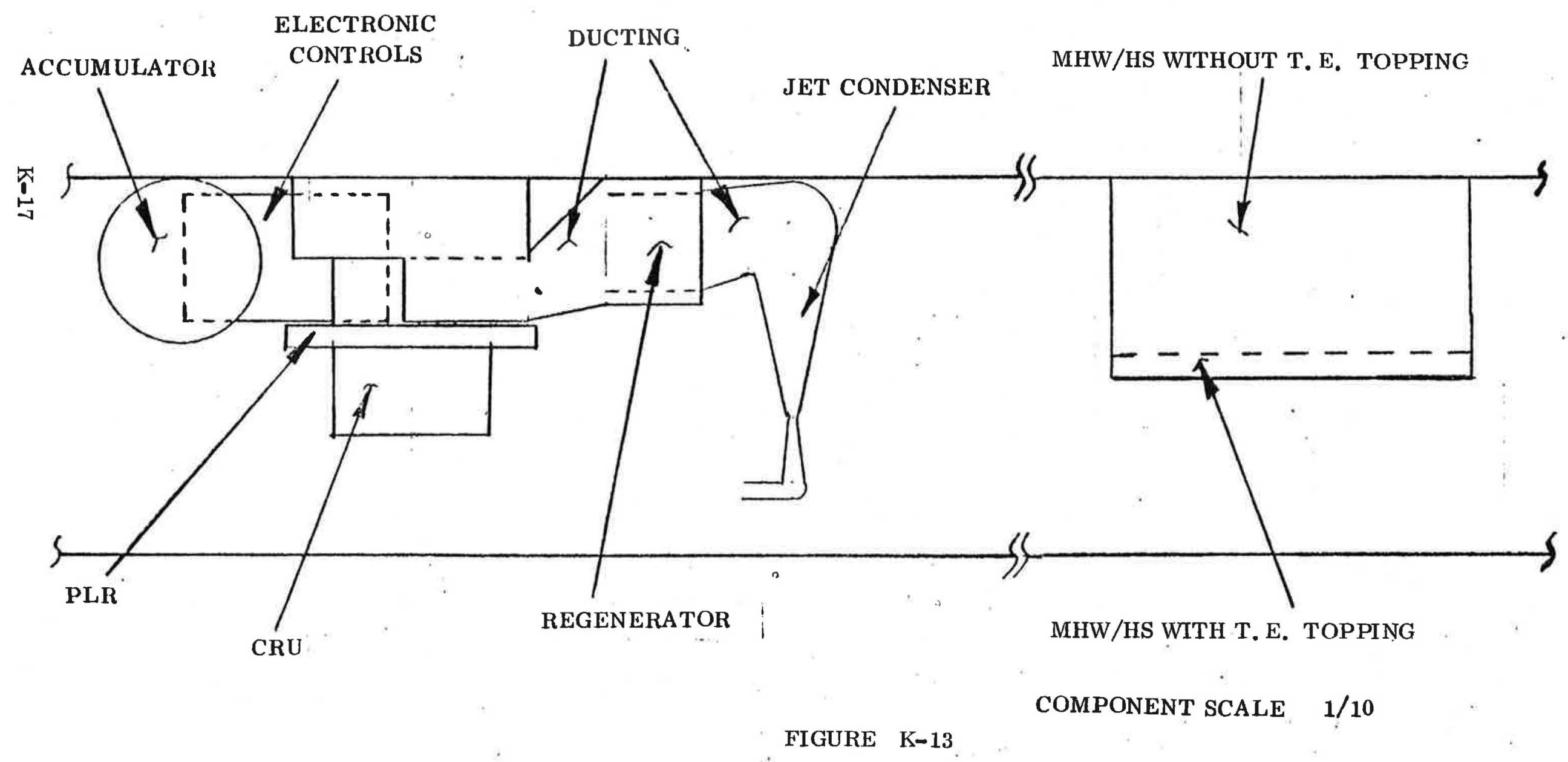




\section{PERFORMANCE AND MASS SUMMARY OF ORC SYSTEM}

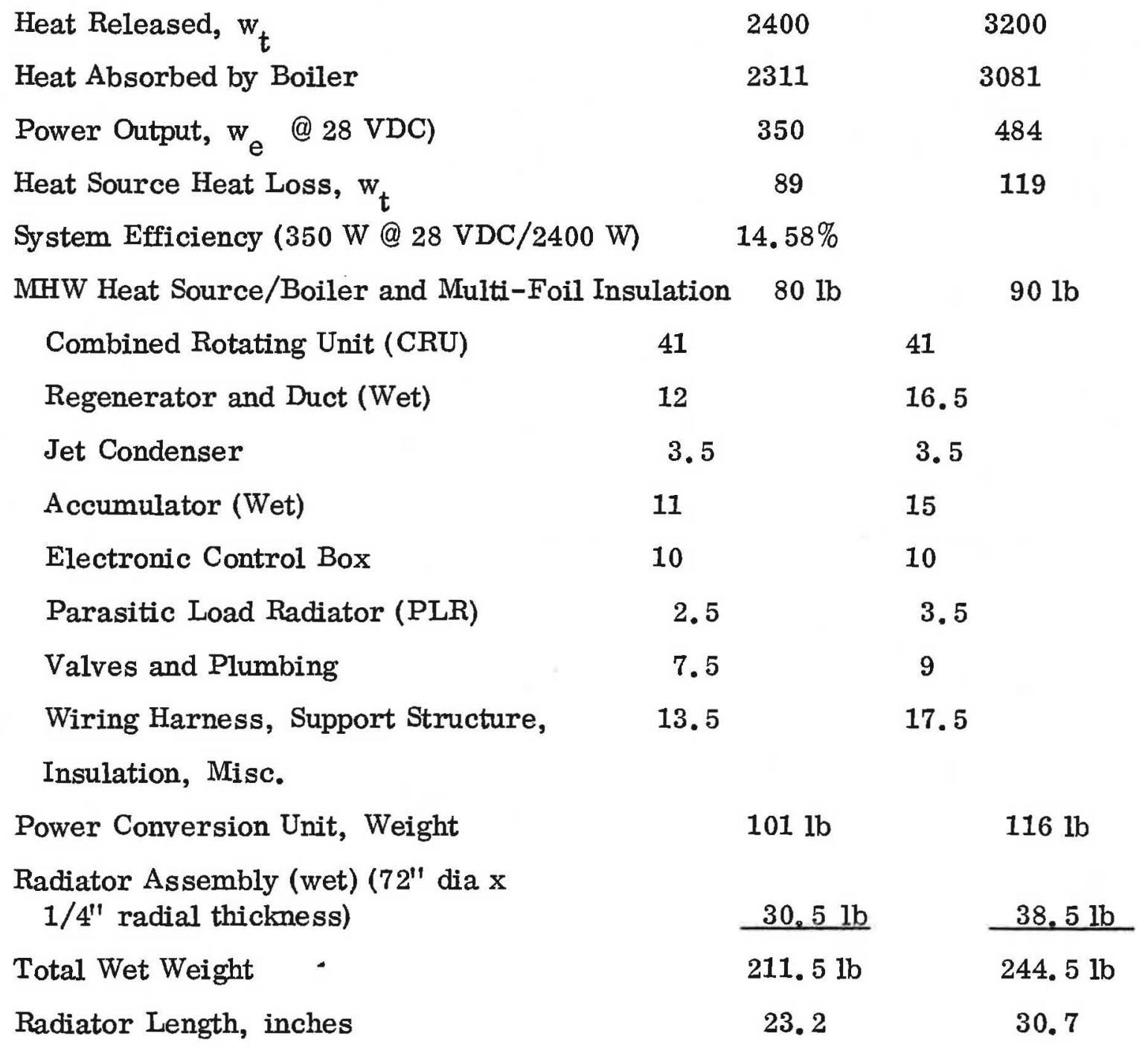


The combined rotating unit (CRU) contains the single-stage turbine wheel, the homopolar inductor rotor, and the rotor of the boiler feed pump. The angular momentum of the CRU at full design speed of 33,700 RPM is $17.6 \mathrm{ft}-\mathrm{lb}-\mathrm{sec}$. In many satellite systems this rather substantial angular momentum might be used for attitude stabilization, by aligning the momentum vector normal to the orbit plane. In the GPS retrofit case as in others, this would involve orienting the CRU laterally, i. e., normal to the Z-axis, in the spacecraft. This can be accommodated in the ORC system by stiffening the lateral bearings at a slight cost in system efficiency.

If the vehicle is spin-stabilized for orbit transfer and orbit injection, as is the GPS spacecraft and many others, a lateral orientation of the CRU would produce a slight coning motion of the vehicle and, therefore, a potential misalignment of the thrust vector and increased propellant consumption. These factors were investigated for the GPS case and found to be negligible (Appendix K-A). Although the coning angle is relatively large during PKM-2 burn ( $\sim 5$ degrees), the maximum increment in $3 \sigma$ orbit trajectory error is less than $5 \%$ (Appendix K-A) which can be easily accomodated. The velocity loss appears during AKM burn for orbit injection and is less than $1 \%$ (Appendix $\mathrm{K}-\mathrm{A}$ ). This can be easily corrected by a corresponding increase in fuel loading of the AKM, now some $10 \%$ off-loaded. Therefore, no impediment appears to the use of the CRU as a bias-momentum wheel from a launch dynamics viewpoint.

Unfortunately, this use of the CRU for attitude stabilization in the retrofit case had to be rejected for reasons relating to the need for continuous spacecraft yaw rotation. In the solar-powered design this yaw steering is needed for solararray orientation. However, it also produces a favorable thermal control condition for the frequency standards. Therefore, even though solar array orientation is no longer required in the nuclear powered case, the thermal control requirement still dictates that yaw steering be retained, at least in the retrofit cases. Consequently, the use of the $\mathrm{ORC}$ in this application will necessitate the inclusion of a counter-rotating momentum wheel to cancel out the angular momentum of the CRU. This will involve a weight penalty of approximately 15 pounds, but negligible power consumption by the wheel, which is to be used in a basically fixed-speed mode. 
K-2.4.2.1. Thermoelectric - ORC Cascaded System

A conceptually simple yet effective modification of the ORC system is to use a thermoelectric topping cycle to increase system efficiency and specific power. This consists of the same MHW heat source with a selenide (or silicongermanium) thermoelectric converter layer between the heat source and the ORC boiler tubes. The physical details of this package have not been worked out, but the performance numbers have been derived, as shown in Table $\mathrm{K}-15$.

The efficiency of the thermoelectric segment is only $5.5 \%$ because the overall system optimizes at a relatively high cold-strap temperature for the selenides. Nevertheless, the turbine inlet temperature requirement of the ORC system is low enough $\left(\sim 340^{\circ} \mathrm{C}\right)$ so that significant performance may still be obtained from the thermoelectrics with a $900^{\circ} \mathrm{C}$ hot junction temperature.

Table K-15

Performance and Mass Summary of ORC Topped by $5.5 \%$ T. E. Generator

Heat Released, $w_{t}$ Heat Absorbed by ORC, $w_{t}$ Power Output, w $w_{\mathrm{e}} @ 28$ VDC

Heat Source Heat Loss, $w_{t}$ System Efficiency (462 W @ 28 VDC/2400 W), \%

MHW Heat Source/Boiler/T. E. G. and Insulations $\mathrm{ORC} / \mathrm{PCU}$

Radiator Assembly (72" dia. x 1/4" radial thickness)

Total

Radiator Length, inches
2400

2179

$\left.\begin{array}{l}\text { ORC } 330 \\ \text { TE } 132\end{array}\right\} 462$

89

19. 25

87

101

28.5

$216.51 \mathrm{~b}$

21. 7
3200

$\left.\begin{array}{l}456 \\ 176\end{array}\right\} 632$

119

19. 7

102

116

36.5

254. $5 \mathrm{lb}$.

29 
K-2.4.3 Brayton Isotope Power System (BIPS)

As with the organic Rankine cycle system, the BIPS is being developed for ground demonstration at $1300 \mathrm{w}_{e}$, although with its higher projected efficiency this is expected to be achieved with two MHW heat sources. The Brayton system differs from the ORC system mainly in two respects: it is a single phase (all gas) cycle, thereby eliminating the boiling/condensing aspects of the Rankine cycle; and it operates at a higher turbine inlet temperature, thereby requiring refractory metals in the hot side of the loop, including the heat source, heat source heat exchanger, turbine housing and connecting ducting.

The system design for the retrofit application was based on the following assumptions:

- $\quad$ single heat source of either 2400 or $3200 \mathrm{w}_{t}$

- Brayton rotating unit (BRU) is fixed, same as for $1300 \mathrm{w}_{+}$BIPS

- recuperator is tailored in size for each case, but similar in geometry and design throughout

- ducting similar to BIPS

- Columbium (C-103) hot loop alloy with $1600^{\circ} \mathrm{F}$ turbine inlet temperature, as for BIPS

- radiator design for 0.99 reliability in 7 years

- $\quad$ sink temperature of $390^{\circ} \mathrm{R}$ with radiator emissivity of 0.8

With these assumptions, a computer analysis was performed by AiResearch/ Phoenix in which several design cases were investigated. The compressor inlet temperature (CIT), recuperator effectiveness $\left(E_{R}\right)$, and the pressure drop $(\Delta P / P)$ of the radiator and recuperator were all varied.

Because the radiator area is known to be a critical parameter, this was first used to generate a typical radiator area vs. output power curve (Figure $\mathrm{K}-16)$. Within the available area for a non-deployable radiator, approximately $360 \mathrm{w}_{\mathrm{e}} \mathrm{would}$ be produced. If a deployable radiator is postulated, the effective radiator area is doubled because radiation can now emit from both faces of the radiator surface, and the output power is increased to approximately $460 \mathrm{w}_{\mathrm{e}}$. Therefore, a deployable radiator, requiring a flexible joint for the working fluid (at $\operatorname{Tma} x=212^{\circ} \mathrm{F}$ ), was considered as an option. 
It must be noted in this connection in particular that the launch power requirement dictates a ground-start up condition for the system, and hence a sy stem in which the hot columbium components are not exposed to oxygen. This requires that the entire system be sealed and either evacuated or flooded in a inert noble gas atmosphere. The radiator is usually assumed to form a part of the enclosing envelope, a much more difficult proposition if it is to be deployable as well. This design problem will be examined in detail in the forthcoming stage of the study within the context of the special nuclear-powered spacecraft. For the retrofit scoping phase no further consideration was given to this aspect of the Brayton system.

From the computer analysis, three candidate sy stems were chosen at each thermal input power, a total of six candidates. The first two in each group were for non-deployed radiators, in which weight and efficiency are traded off. The third case in each group is for the deployed radiator, approximating maximum specific power within the assumptions.

Tables $\mathrm{K}-17$ and $\mathrm{K}-18$ show the weight and power breakdowns for the $2400 \mathrm{w}_{t}$ and $3200 \mathrm{w}_{t}$ cases respectively.

Table K-19 shows the computer summary of the critical cycle parameters for the $413 \mathrm{w}_{\mathrm{e}}$ case.

Figures $\mathrm{K}-20$ and $\mathrm{K}-21$ show the component layouts within the prescribed envelope for the $413 \mathrm{w}_{\mathrm{e}}$ and $453 \mathrm{w}_{\mathrm{e}}$ cases respectively. The smaller recuperator heat exchanger in the latter case necessitates a rearrangement of the components in order to maintain system balance. Fortunately, the electronic control box can be located at any position to assist in the balancing.

The angular momentum of the BRU is $0.68 \mathrm{ft}-\mathrm{sec}$ at design speed of 52,000 RPM. At this value, a factor of 25 less than that of the ORC/CRU, any effects on spacecraft attitude are well within the capability of the normal attitude control system to compensate for, and launch effects would be negligible. 


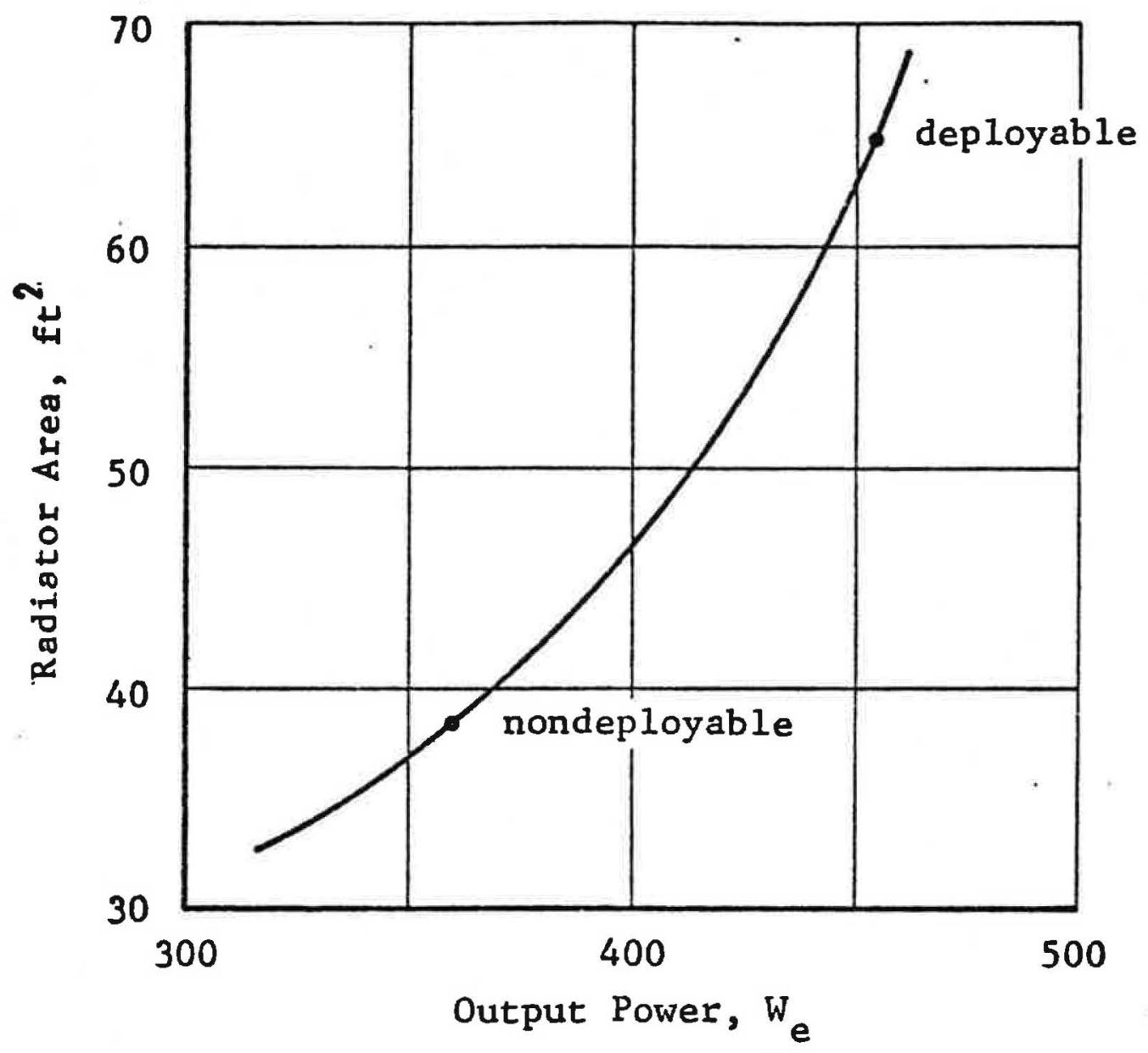

1. Typica1 Characteristics

2. $\mathrm{E}_{\mathrm{R}}=.96$

3. $\Delta \mathrm{P} / \mathrm{P}$ Radiator \& Recuperator $=.024$

4. $10 \%$ Weight Variation

5. $\mathrm{T}_{\text {sink }}=390^{\circ} \mathrm{R}$

6. $\epsilon=.8$ 
TABLE $\quad \mathrm{K}-17$

POWER AND WEIGHT SUMMARY OF BIPS WITH $2400 \mathrm{w}_{t}$ HEAT SOURCE

\begin{tabular}{|l|c|c|c|}
\hline Output Power, we & 359 & 413 & 453 \\
\hline Radiator Area, ft & 38 & 38 & $* 65$ \\
\hline Weight, Ib & 75 & 140 & 60 \\
Recuperator & 35 & 38 & 65 \\
Radiator & 36 & 36 & 36 \\
Mini-BRU & 81 & 81 & 81 \\
HSA & 20 & 20 & 20 \\
Ducting & 28 & 30 & 28 \\
Insulation & 20 & 20 & 20 \\
Structure & 10 & 10 & 10 \\
Controls & 305 & 375 & 320 \\
\hline Total, lb. & & & \\
\hline
\end{tabular}

TABLE K-18

POWER AND WEIGHT SUMMARY OF BIPS WITH $3200 \mathrm{w}_{t}$ HEAT SOURCE

\begin{tabular}{|l|r|r|r|}
\hline Output Power, W Electric & 522 & 563 & 700 \\
\hline Radiator Area, Ft Squared & 38 & 38 & $* 65$ \\
\hline Weight, lb & & & \\
Recuperator & 95 & 140 & 110 \\
Radiator & 38 & 38 & 65 \\
Mini Bru & 36 & 36 & 36 \\
HSA & 101 & 101 & 101 \\
Ducting & 20 & 20 & 20 \\
Insulation & 28 & 30 & 28 \\
Structure & 20 & 20 & 20 \\
Controls & 10 & 10 & 10 \\
\hline Total, Lb. & 348 & 395 & 390 \\
\hline
\end{tabular}

* Deployed Radiator 
TABLE K-19

CYCLE OUTPUT DATA, $413 \mathrm{w}_{\mathrm{e}}$ CASE

\begin{tabular}{|c|c|c|c|}
\hline $\begin{array}{l}\text { COMP INLET TEMP } \\
\text { (DEG R) } \\
.570633 E+03\end{array}$ & $\begin{array}{c}\text { TUKB JNLET TEMP } \\
\text { (UEG R) } \\
\text {.ZUGOOOE॰OA }\end{array}$ & $\begin{array}{l}\text { GROSS PWR OUTPUT } \\
\text { (KW) } \\
.428005 E+00\end{array}$ & $\begin{array}{l}\text { CHU ROTAT.SPEED } \\
\text { (RPM) } \\
.520000 E \text {.05 }\end{array}$ \\
\hline $\begin{array}{c}\text { COMP INLET PRESS } \\
\text { (DSIA) } \\
.248017 E+02\end{array}$ & $\begin{array}{c}\text { TUAB INLET PRESS } \\
\text { (NSIA) } \\
.328622 E+02\end{array}$ & $\begin{array}{c}\text { NET POWER OUTPUT } \\
\text { (KW) } \\
.413377 E \$ 00\end{array}$ & $\begin{array}{c}\text { RECUPERA IOR EFF } \\
(=-\infty) \\
.980000 E+00\end{array}$ \\
\hline $\begin{array}{c}\text { COMP PRESS RATIO } \\
(1--0-1 \\
.146790 E+01\end{array}$ & $\begin{array}{c}\text { TURE PRESS RATIO } \\
(--\infty-1) \\
.142524 E+01\end{array}$ & $\begin{array}{c}\text { ALT .PER IF . WINUAGE } \\
\text { (KW) } \\
.35814 \$ E-01\end{array}$ & $\begin{array}{c}\text { BETA } \\
(-970-0-1 \\
.970938+00\end{array}$ \\
\hline $\begin{array}{c}\text { CUMP EFFICIENCY } \\
(1-0-5) \\
.764010 E+00\end{array}$ & 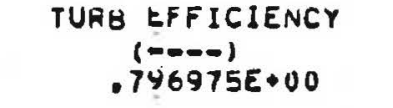 & $\begin{array}{l}\text { ALT.RAOIAL WINOG. } \\
\text { O. }(K=)\end{array}$ & $\begin{array}{l}\text { ALTERNATUR EFF } \\
\left(\begin{array}{l}(-0-1) \\
.877779 E+00\end{array}\right.\end{array}$ \\
\hline $\begin{array}{c}\text { COMP WORK FACTOR } \\
\left(\begin{array}{c}(--1) \\
.787356 E+00\end{array}\right.\end{array}$ & $\begin{array}{c}\text { GQ TURB VEL FACTOK } \\
(-101440 E+01\end{array}$ & $\begin{array}{l}\text { BEAKING POWEK } \\
(K W) \\
.126705 E \nrightarrow 00\end{array}$ & $\begin{array}{l}\text { HLEEU FLOW } \\
\text { (LES/SEC) } \\
.234938 E-02\end{array}$ \\
\hline $\begin{array}{l}\text { COMP WEIGHT FLCW } \\
\text { (LAS/SEC) } \\
\text {.117469E } 1100\end{array}$ & $\begin{array}{l}\text { TURB WEIGHT FLOW } \\
\text { (LUS/SEC) } \\
\text {.11S119E }+00\end{array}$ & $\begin{array}{l}\text { PUMP POWER } \\
(\mathrm{KW}) \\
0 .\end{array}$ & $\begin{array}{c}\text { ACCESSORY POWER } \\
\text { (KW) } \\
.146201 E-01\end{array}$ \\
\hline $\begin{array}{l}\text { COMP POWER } \\
\text { (XW) } \\
.908716 E+00\end{array}$ & $\begin{array}{l}\text { TURB INE PUWER } \\
\text { (KW) } \\
.125883 E+01\end{array}$ & $\begin{array}{c}\text { GROSS CYCLE EFF } \\
(--17-1) \\
.178336 E+00\end{array}$ & $\begin{array}{l}\text { NET CYCLE EFF } \\
(1-0-1) \\
.172240 E \bullet 00\end{array}$ \\
\hline
\end{tabular}




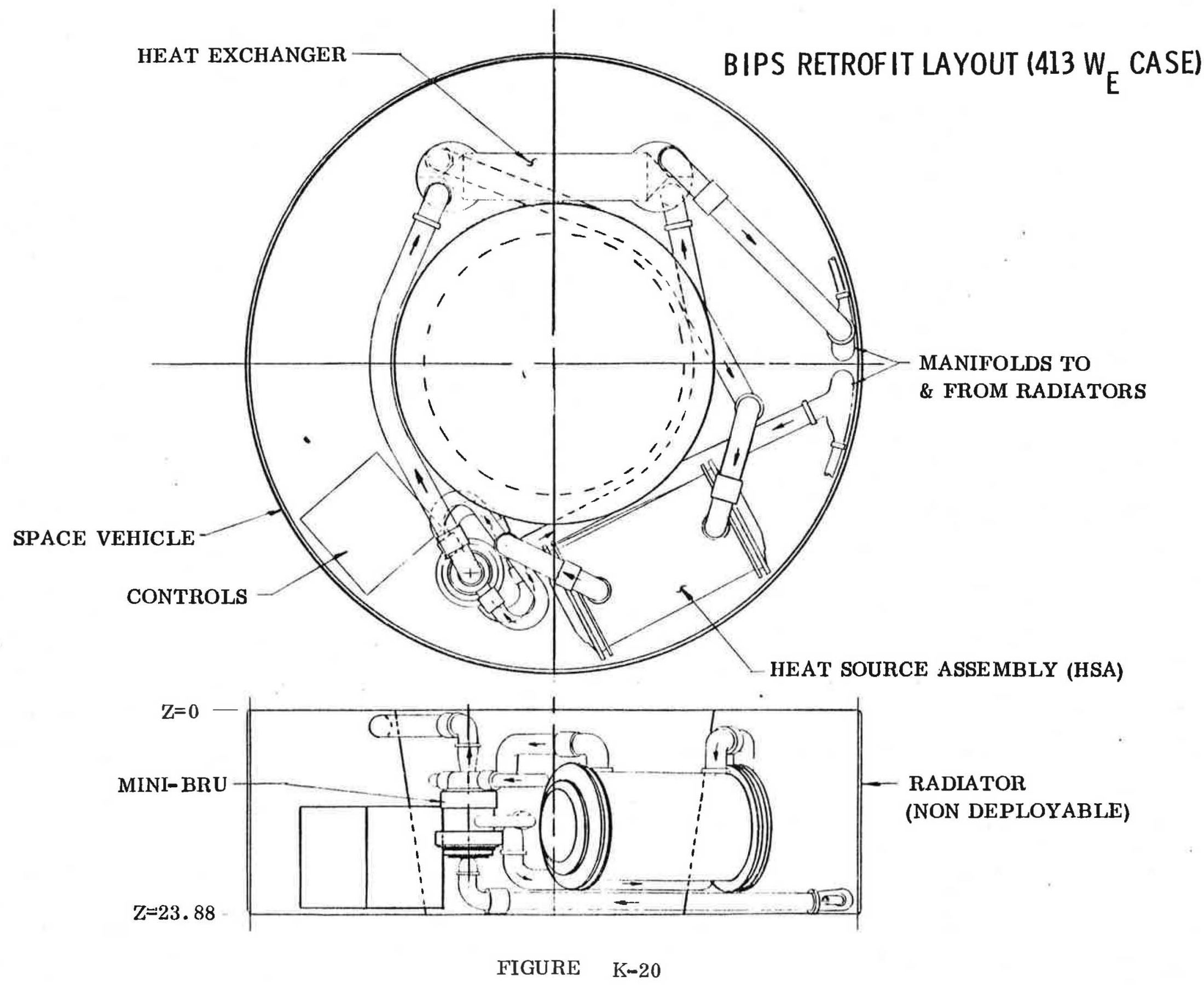




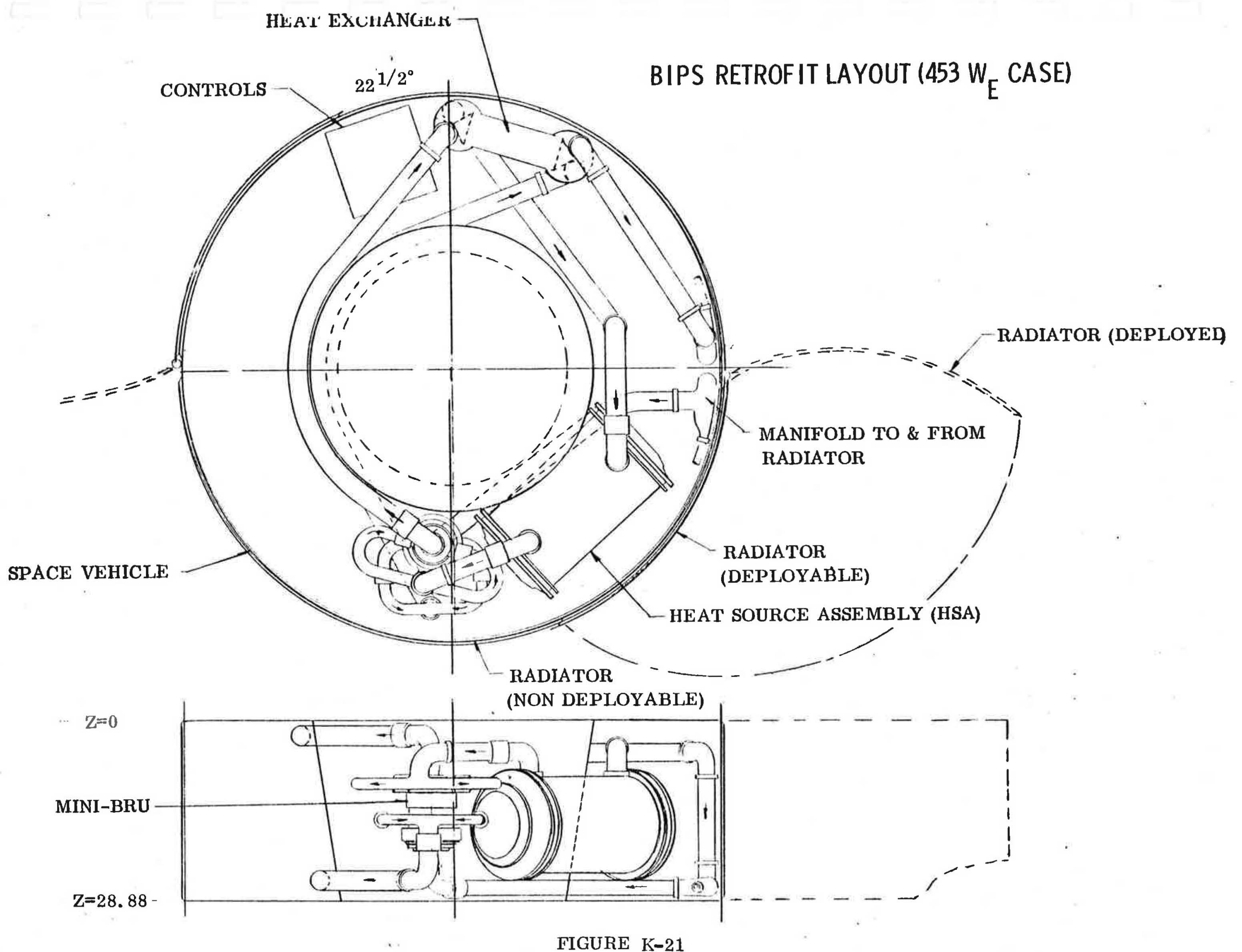


Figures K-22 through K-24 show the major elements of the solar (electrical) power system (EPS) used on the Phase I space vehicle ( $₫ 1 \mathrm{SV}$ ). It was assumed that all of the EPS system elements shown in the figures would be removed except the Load Control Unit, DC/DC converter, sun sensors and current sensor. While being retained, these latter boxes would require some modification for use with any of the isotope systems. The following weight breakdown of the EPS total was derived from the 21 July 1976 weight status report of the $₫ 1$ SV.

$\underline{\text { EPS Item }}$

Solar Array (with deployment mechanism)

Shunt Dissipator

Orientation Drives

Solar Array Control Electronics

Power Conditioning Electronics

Batteries (3@31.5)

Total Removed

Sun Sensor

Load Control Unit

Current Sensor

DC/DC Converter
Weight (1b)

$93.0 \mathrm{lb}$.

9.0

18. 4

6.5

25. 0

96.9

248. 8 .8

15. 0

1. 0

10.0

21 July 76 Status $\quad 275.6$

The weight of the fore and aft stow latches, panel edge rgstraint hooks and other array related hardware which is also removed was assumed to be counterbalanced by cover panels, doubler plates and other secondary structure added to accommodate the retrofitted subsystems. 


\section{GPS SOLAR POWER ELEMENTS}

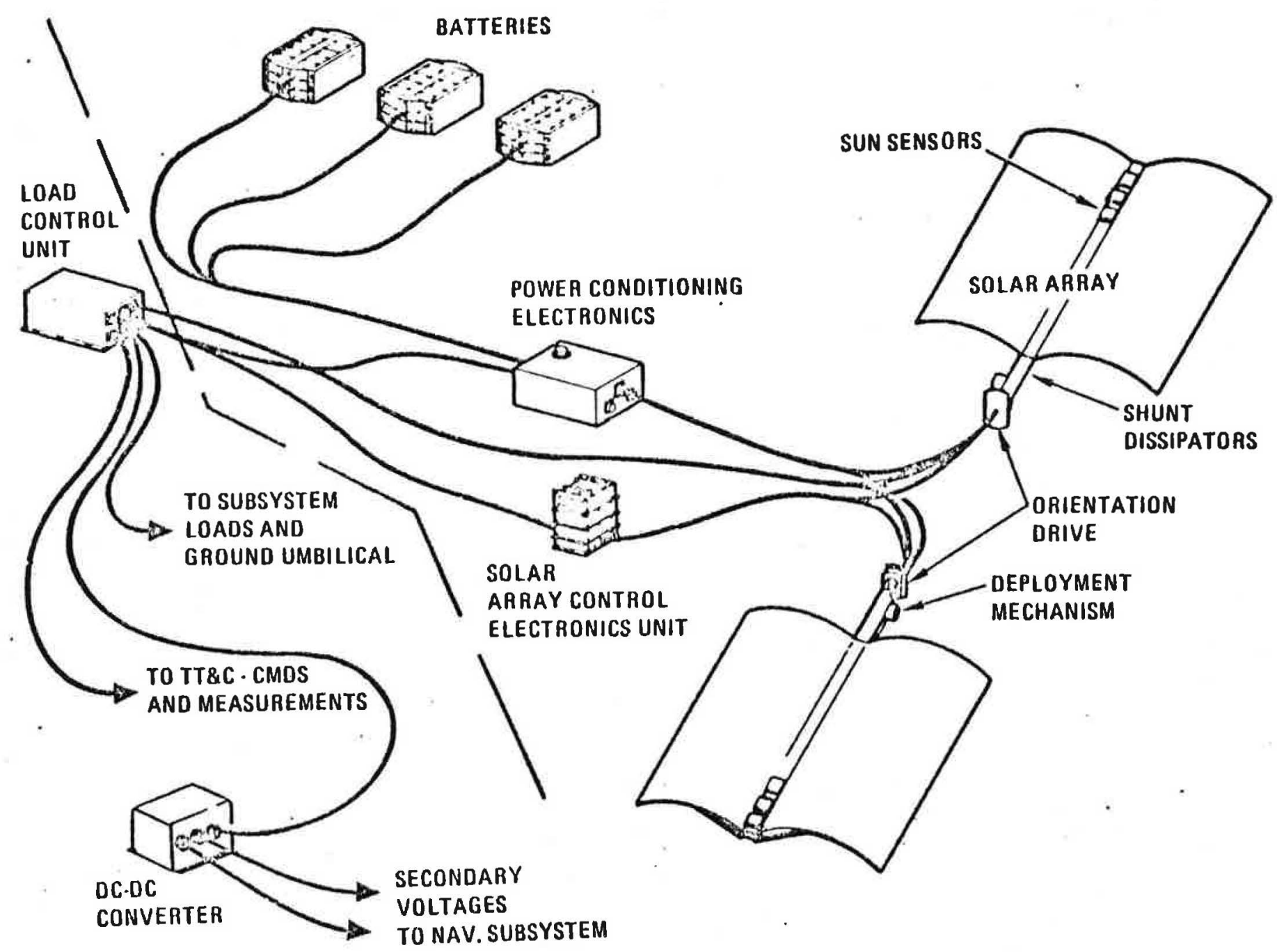

FIGURE K-22 


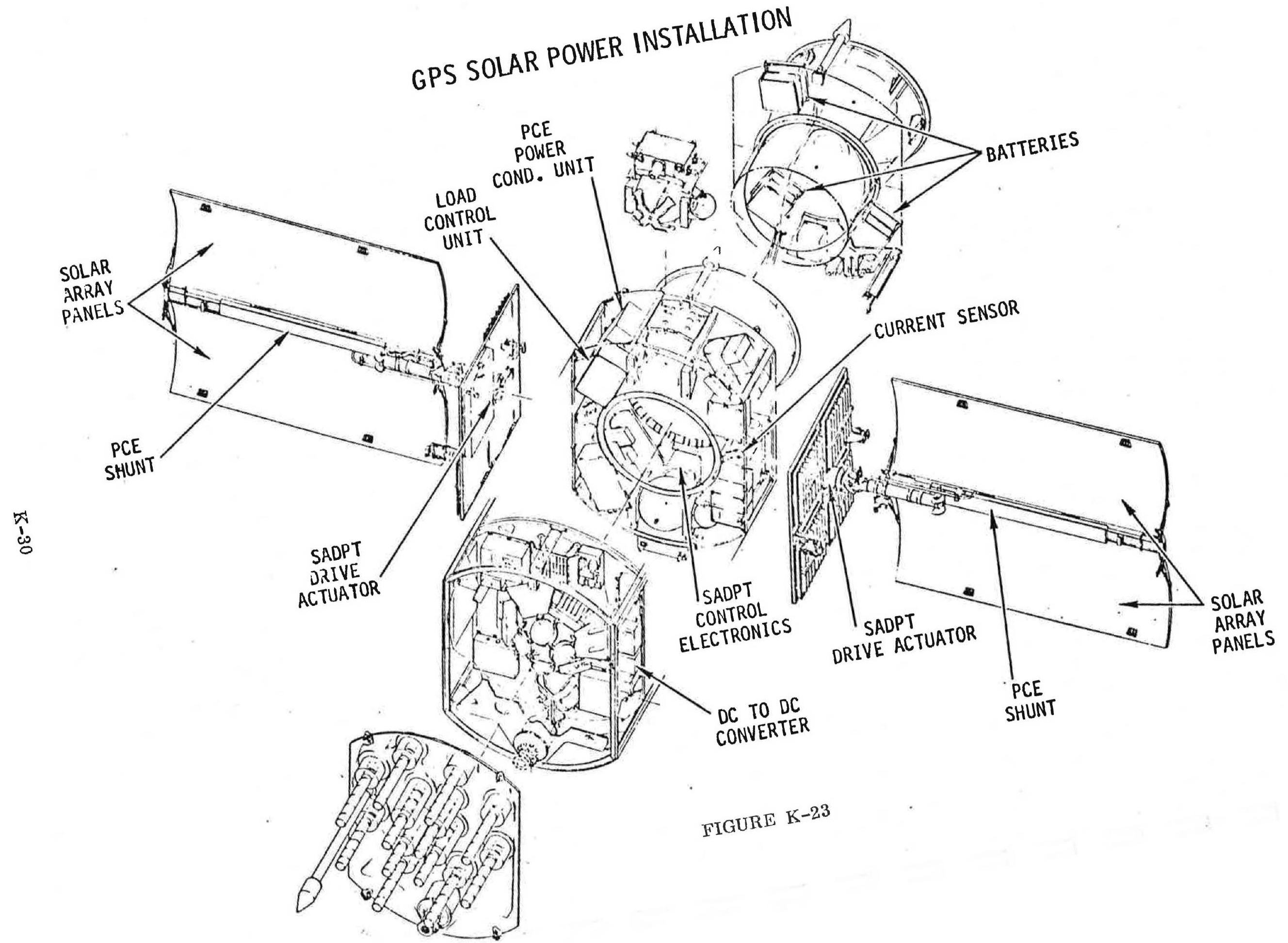




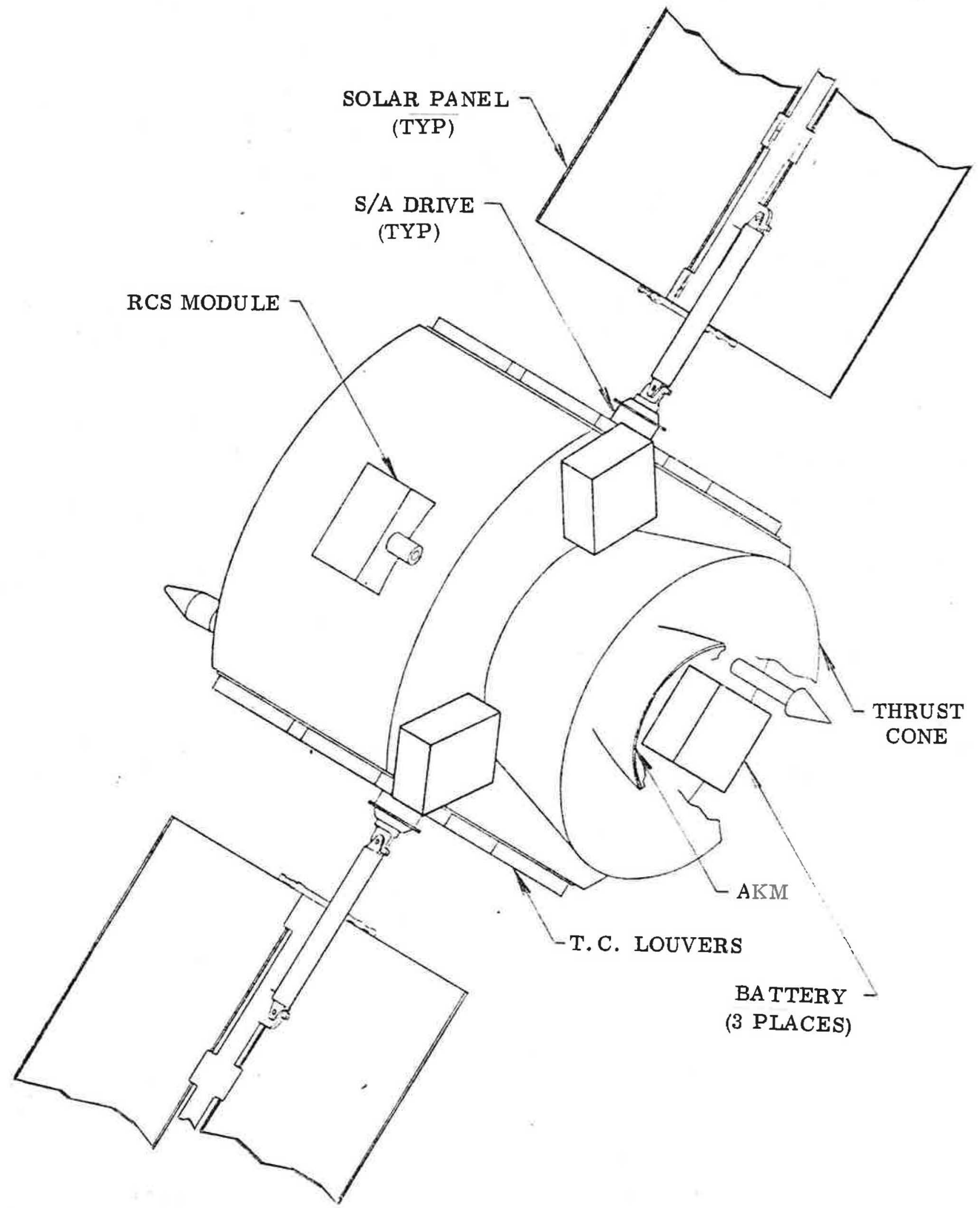

FIGURE K-24 SOLAR/BATTERY POWERED GPS 
It was evident ( reference current GPS Spacecraft layout shown by Figure $\mathrm{K}-25$ ) that the required volume for any of the proposed isotope sy stems precluded their integration within the main body of the satellite. Of the solar power elements being removed only two, the power conditioning unit and the solar array drive electronics, are located in the main body. In addition because the same. structural configuration and louver panels were being retained and the fact that the RCS thruster modules would offer mechanical and jet plume interference, it was clear that the radioisotope system radiators could not be integrated around the main body of the vehicle. A primary desire for the retrofit exercise was to keep structural changes to a minimum, to minimize interference and/or changes to other subsystems, to provide attachment points that tie in with the established structural design and to maximize accessibility to the power subsystem, particularly the heat source.

Accessibility is considered important, particularly if a viable method can be developed for installing the heat source in the radioisotope power subsystem that has already been integrated in the spacecraft, thereby reducing the prelaunch time during which cooling must be provided and access to the spacecraft is limited.

These considerations led to radioisotope power system designs that would occupy the volume between the thrust cone and the dynamic envelope at the aft end of the satellite, with mounting points forward on the aft bulkhead and laterally on the thrust cone. 


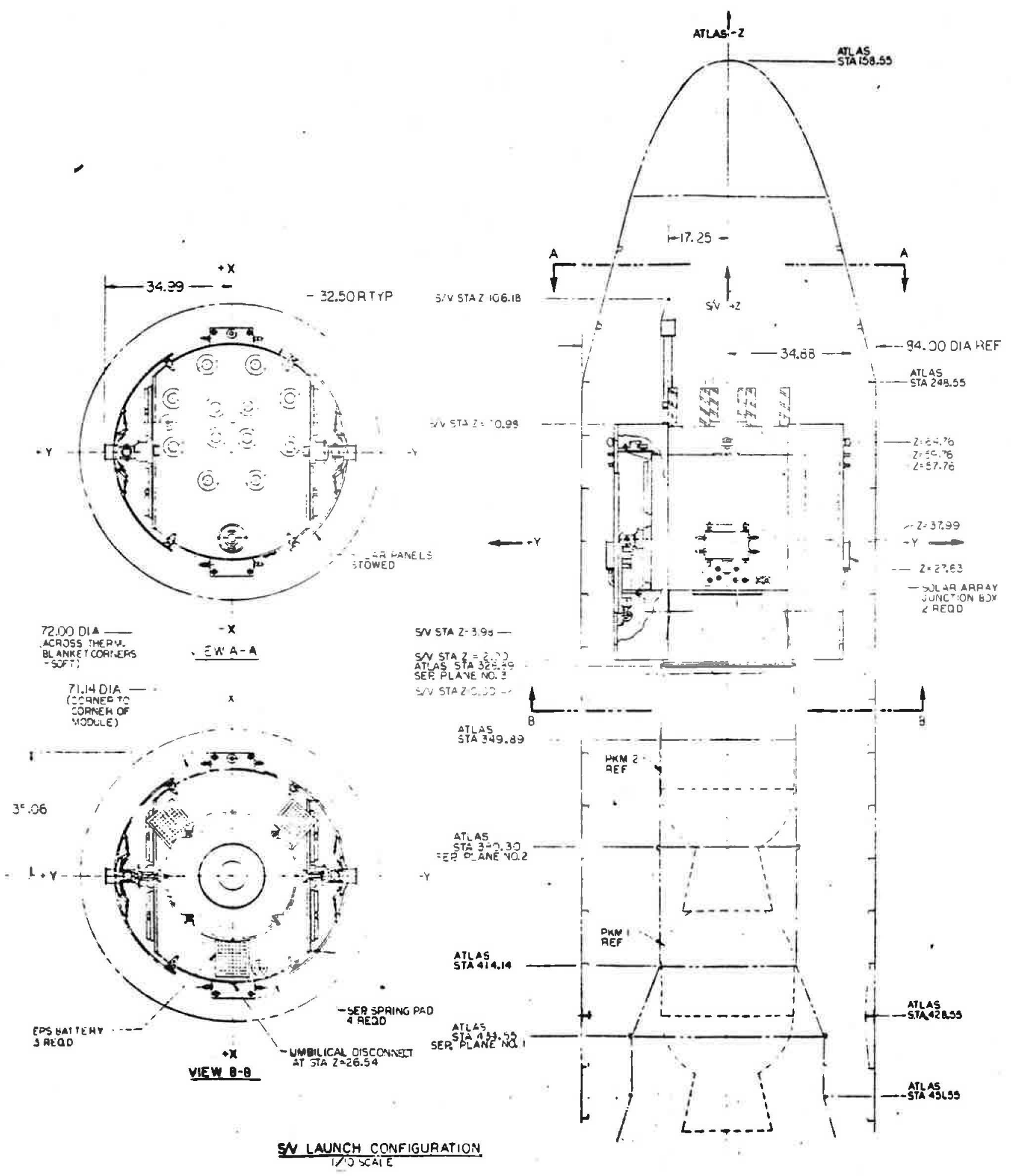

Figure K-25 GPS Space Vehicle Configuration (1 of 2) 


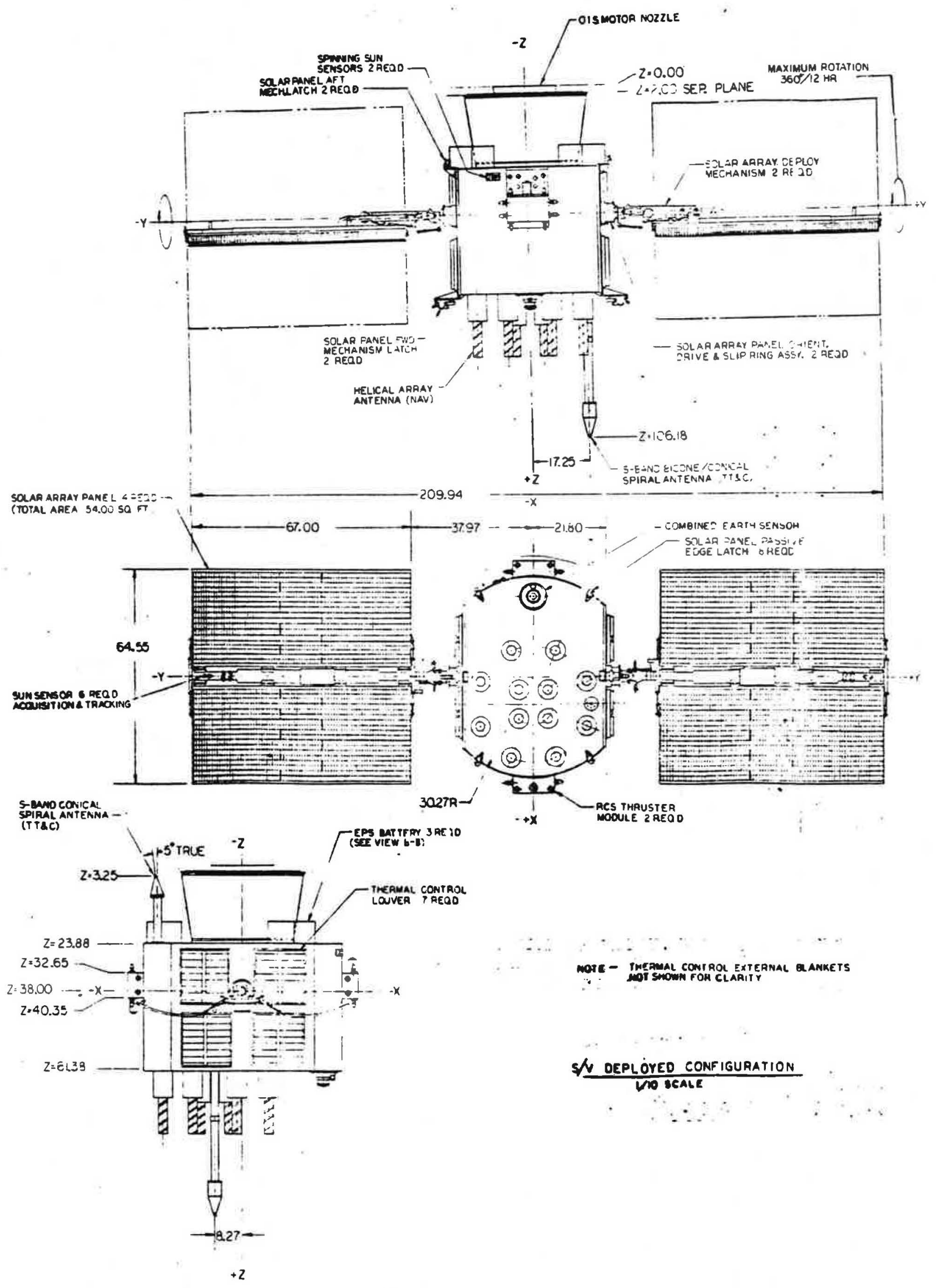

Figure K-25 GPS Space Vehicle Configuration (2 of 2) 
As indicated in section $K-2$ four nuclear powered subsystems were considered for the GPS Phase I design retrofit. Layouts were made to verify that the components could be fitted into the annular volume surrounding the Orbit Insertion Motor nozzle and that required radiator areas could be accommodated without interfering with either the ATLAS-F shroud or the Stage Vehicle System (SVS) attachments. Thermal analyses were also conducted to verify that the radiator area predictions presented by the various nuclear power subsystem contractors were valid and based on consistent assumptions. The radiators for all of the retrofitted nuclear power subsystems form a new, cylindrical surface at the aft end of the spacecraft.

A single electrical interface cable incorporating power, control and telemetry leads runs from the control/monitor unit in each of the subsystems to the Load Control Unit inside the main body of the spacecraft. The power subsystem components are, in general, thermally isolated from the primary structure through the use of insulating washers, pads, etc. that are part of the attachment structure.

Both 2400 and 3200 watt (thermal) sources were considered. System layouts for both approaches are essentially the same.

Descriptions of mechanical interfaces and quantitative data on subsystem power, weight and performance are provided in the following sections.

K-3.2.1 Radioisotype Thermoelectric Gene rator (RT G) Approach

The RTG's were designed with integral radiator wings that are fastened together at their far ends by a splice joint. Figure K-26 shows the tapered stiffeners on the outer surface that parallel the heat pipes around the radiator surface. The generators are supported at their aft ends by tubular struts attached to the thrust cone. A single clevis fitting and a shear panel bracket on the forward end of the generator are fastened to the corner of the aft bulkhead and the GPS shear panel. Side and end views of the RTG 


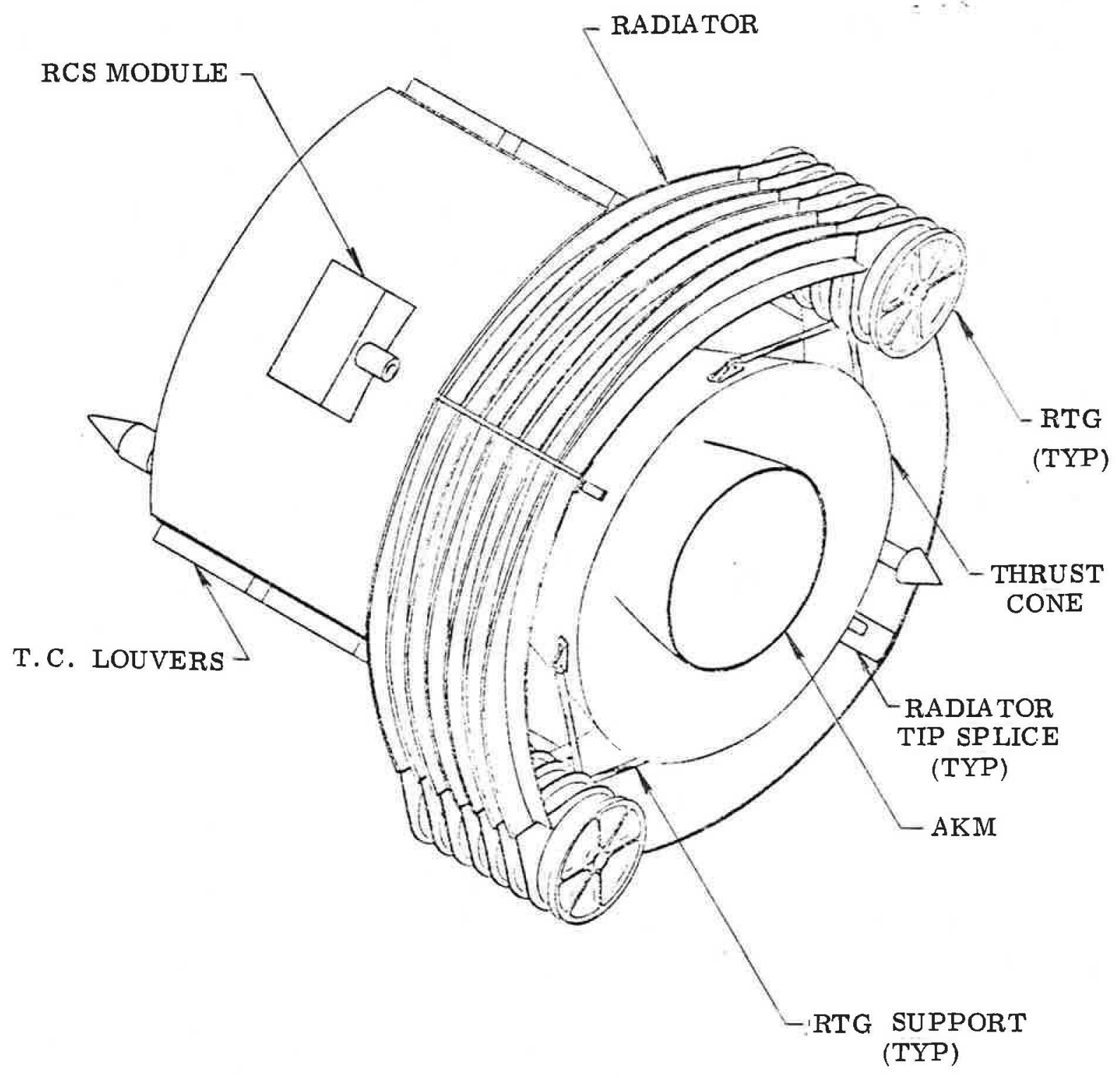

FIGURE K-26, RTG POWERED GPS 
installation are shown by figure $\mathrm{K}-27$. An analysis was made to determine attachment hardware required to mate the RTG components to the spacecraft and, as incicated by Table $K-22$, it was found that attachment hardware weights totalled less than 6 lbs. Provision is made for heat source accessiblity through the end of the spacecraft and thermal insulation is provided to isolate both the GPS spacecraft body and the transfer orbit Stage Vehicle from the Radioisotope heat source thermal load.

6.2.2 Bravton Isotope Power Subsystem (BIPS) Approach

In both the BIPS and ORC units the MHW heat source and associated heat exchanger are attached to the aft bulkhead using a cylindrical bracket incorporating thermal isolators attached to the thrust cone at their aft ends with tubular struts. The loads of other dynamic system components are supported by brackets fastened to the aft bulkhead, the thrust cone or both. Figure K-29 shows a pictorial view of the BIPS retrofit installation with the Brayton Rotating Unit (BRU) oriented with its momentum vector parallel to the spacecraft $Z$ axis (spin axis during transfer orbit and earth pointing axis during operation). Figure $\mathrm{K}-30$ shows an alternate installation with the $\mathrm{BRU}$ momentum vector oriented at right angles to the spacecraft $Z$ axis. Since the momentum vector generated by the BRU is small, either orientation is feasible. Table K-3I. lists the mounting hardware required to mate the BIPS components with the GPS Phase I Spacecraft. As indicated by this table, the mounting hardware weight totals less than 13 lbs.

In the deployable version, the BIPS radiators would utilize bellows joints and spring/damper deployment mechanisms. The cylindrical radiators shown would require some local envelope modification to avoid jet impingement from the $5 \mathrm{lb}$ orbit-correction hydrazine thrusters. Jet plume shielding may also be required but would add negligible weight since this can be accomplished with thin nickel foil. Figure K-32 shows a side and end view of the BIPS installation. 


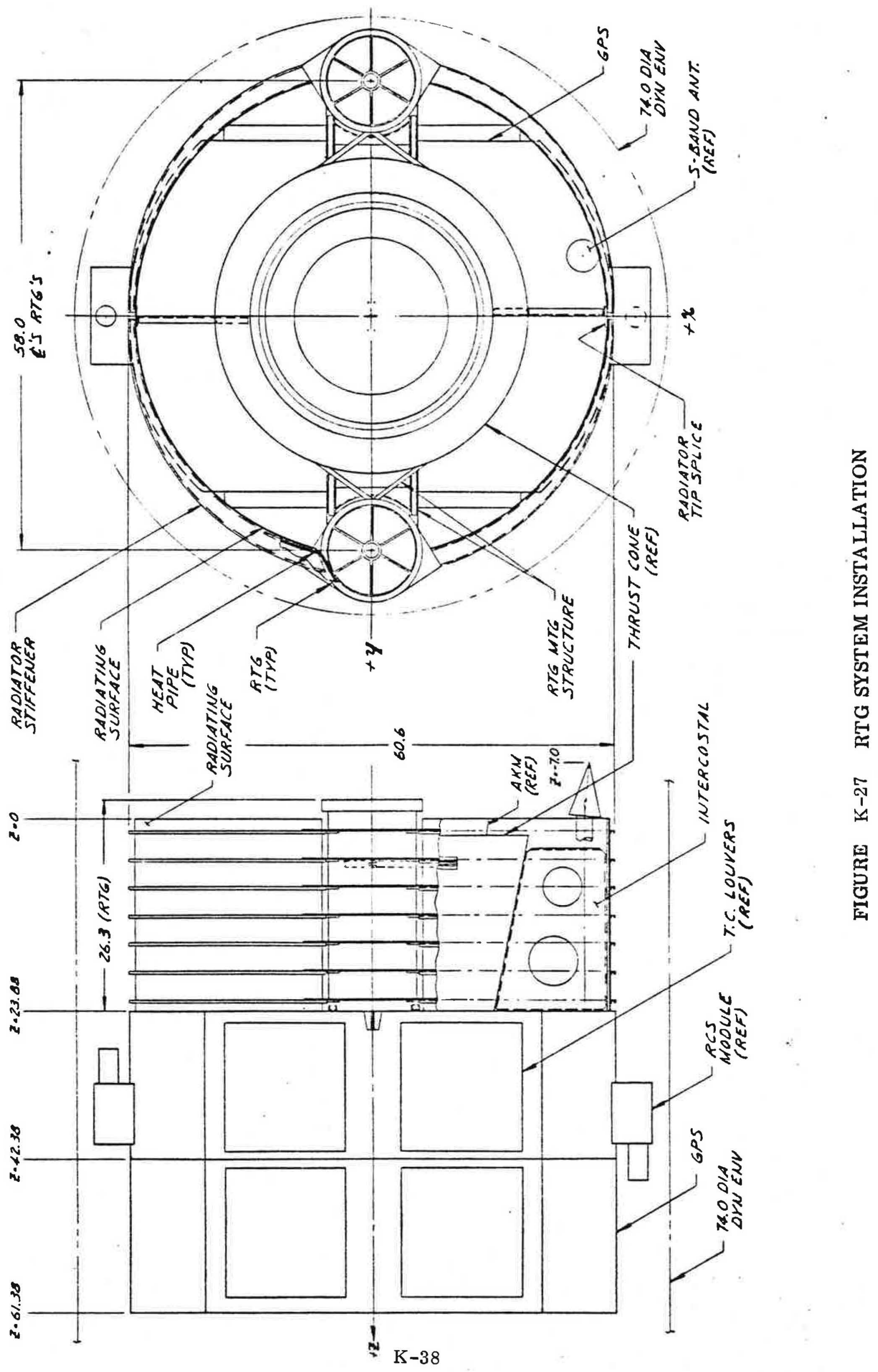


TABLE K-28

WEIGHT BREAKDOWN - RTG ATTACHMENT HARDWARE

\begin{tabular}{|c|c|c|c|c|c|}
\hline ITEM & MTL & $\begin{array}{c}\text { UNIT } \\
\text { WEIGHT }\end{array}$ & $\begin{array}{l}\text { QTY/ } \\
\text { VEH }\end{array}$ & $\begin{array}{l}\text { TOTAL } \\
\text { WEIGHT }\end{array}$ & LOC. \\
\hline $\begin{array}{l}\text { Ftg, Upr Spt, } \\
\text { X-Y Plane }\end{array}$ & $\begin{array}{l}\text { Machined } \\
\quad \mathrm{A} 1\end{array}$ & .14 & 4 & .56 & $\begin{array}{r}\text { Rft } \\
\text { Blkd }\end{array}$ \\
\hline $\begin{array}{l}\text { Shear Web, } \\
X-Y \text { Plane }\end{array}$ & $\begin{array}{l}\text { A1 } \\
\text { Sht. }\end{array}$ & .13 & 2 & .26 & $\begin{array}{c}\text { Aft } \\
\text { Blkd }\end{array}$ \\
\hline $\begin{array}{l}\text { Thrust Ftg. } \\
\text { Z Loading }\end{array}$ & $\begin{array}{l}\text { Machined } \\
\quad \mathrm{Al}\end{array}$ & .29 & 2 & .50 & \begin{tabular}{|l} 
Shear \\
Panel
\end{tabular} \\
\hline $\begin{array}{l}\text { Struts, Lwr Spt, } \\
\text { X-Y Plane }\end{array}$ & $\begin{array}{c}\text { A1 } \\
\text { Tubing }\end{array}$ & .06 & 4 & .24 & $\begin{array}{l}\text { Thrust } \\
\text { Cone }\end{array}$ \\
\hline $\begin{array}{l}\text { Strut End } \\
\text { Fittings }\end{array}$ & $\begin{array}{l}\text { Machined } \\
\text { AI }\end{array}$ & .15 & 8 & 1.20 & $\begin{array}{l}\text { Thrust } \\
\text { Cone }\end{array}$ \\
\hline $\begin{array}{l}\text { Ftg, Strut To } \\
\text { Thrust Cone }\end{array}$ & $\begin{array}{l}\text { Machined } \\
\text { Al }\end{array}$ & .1玉 & 4 & .60 & $\begin{array}{c}\text { Thrust } \\
\text { Cone }\end{array}$ \\
\hline $\begin{array}{l}\text { Doubler, } \\
\text { Thrust Cone }\end{array}$ & $\begin{array}{l}\text { A1 } \\
\text { Sht }\end{array}$ & .09 & 4 & .20 & $\begin{array}{c}\text { Thrust } \\
\text { Cone }\end{array}$ \\
\hline $\begin{array}{l}\text { Radiator Supt } \\
\text { On X-Axis }\end{array}$ & $\begin{array}{c}\text { Sht } \mathrm{Al} \\
\text { Intercostal }\end{array}$ & .60 & 2 & 1.20 & $\begin{array}{l}\text { Aft Blkd } \\
\text { and } \\
\text { ThrustCone }\end{array}$ \\
\hline \begin{tabular}{|c|} 
Bushings, \\
Thermal Isolation
\end{tabular} & $\begin{array}{c}\text { Glass } \\
\text { Reinf. Epoxy }\end{array}$ & - & $A / R$ & .10 & $\begin{array}{l}\text { In Ftg } \\
\text { Lugs }\end{array}$ \\
\hline Attach Hdwre & $\begin{array}{c}\text { Cres } \\
\text { Blts, Nuts }\end{array}$ & .02 & 24 & .48 & $\begin{array}{c}\text { In Ftg } \\
\text { Lugs }\end{array}$ \\
\hline $\begin{array}{l}\text { Wt. of Structure } \\
\text { Provisions }\end{array}$ & & & & $5.34 \mathrm{lb}$ & \\
\hline Contingency & & & $10 \%$ & $.54 \mathrm{lb}$ & \\
\hline $\begin{array}{l}\text { Total Structure } \\
w_{t}\end{array}$ & & & & $5.88 \mathrm{lb}$ & \\
\hline
\end{tabular}




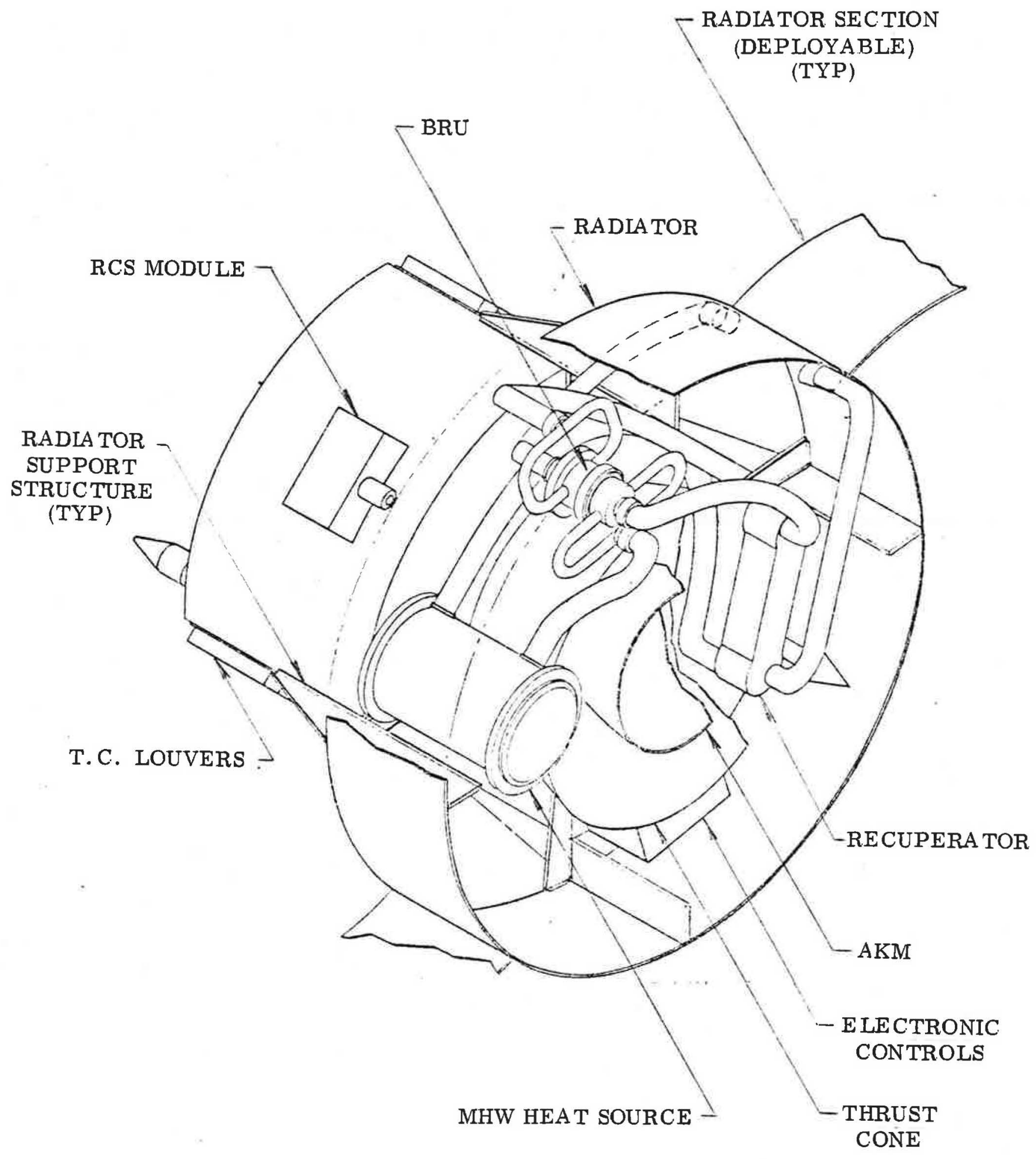

FIGURE K-29, BIPS RETROFIT INSTALLATION 


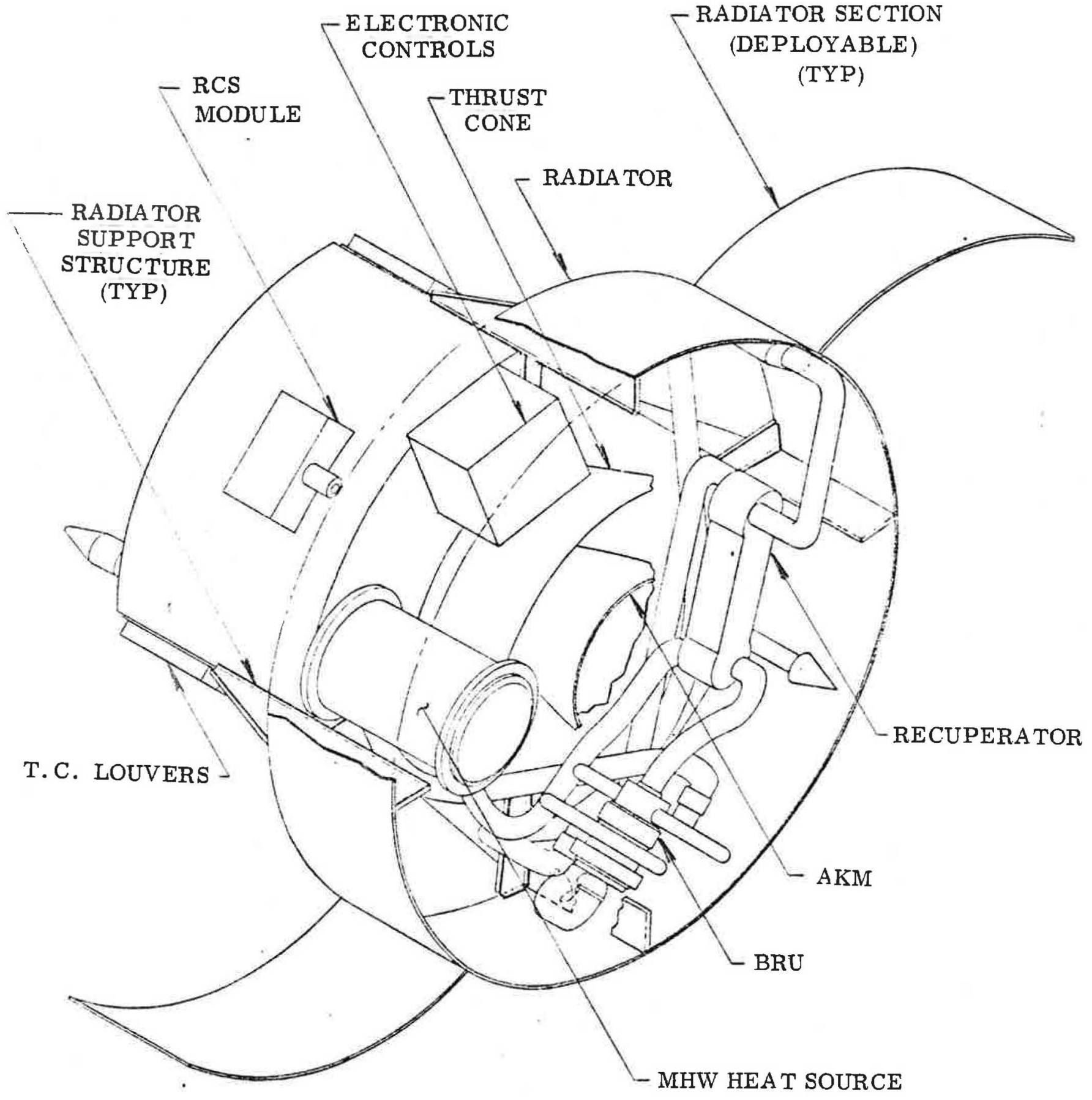

FIGURE \K-30, BIPS RETROFIT INSTALLATION

(ALTERNATE APPROACH) 
TABLE $\mathrm{K}-31$

WEIGHT BREAKDOWN - BIPS MOUNTING HARDWARE

\begin{tabular}{|c|c|c|c|c|c|}
\hline ITEM & MTL & $\begin{array}{c}\text { UNIT } \\
\text { WEIGHT }\end{array}$ & $\begin{array}{l}\text { QTY/ } \\
\text { VEH }\end{array}$ & $\begin{array}{l}\text { TOTAL } \\
\text { WEIGHT }\end{array}$ & LOC. \\
\hline $\begin{array}{l}\text { HSA Support } \\
\text { Cradle }\end{array}$ & $\begin{array}{l}\text { Cres, Saddles } \\
\text { Thermal } \\
\text { Isolators, } \\
\text { A1 Bkts } \\
\end{array}$ & 2.0 & 1 & 2.0 & $\begin{array}{l}\text { Thrust } \\
\text { Cone }\end{array}$ \\
\hline $\begin{array}{l}\text { Stiffeners, } \\
\text { Doublers to Beef } \\
\text { Up Thrust Cone }\end{array}$ & & .5 & 1 & .5 & $\begin{array}{l}\text { Thrust } \\
\text { Cone }\end{array}$ \\
\hline Mini-BRU & $\begin{array}{r}\text { Cres, Ring, } \\
\text { Thermal } \\
\text { Isolation, } \\
\text { Al Pedestal } \\
\end{array}$ & 1.0 & 1 & 1.0 & $\begin{array}{l}\text { Aft } \\
\text { Blkd }\end{array}$ \\
\hline $\begin{array}{l}\text { Doubler For Aft } \\
\text { Blhd H/C Face Sht }\end{array}$ & $\begin{array}{l}\text { Al } \\
\text { Sht }\end{array}$ & .1 & 1 & .1 & $\begin{array}{l}\text { Aft } \\
\text { Blkd }\end{array}$ \\
\hline $\begin{array}{l}\text { Recuperator } \\
\text { Supt Bkts }\end{array}$ & $\begin{array}{l}\text { Cres, } \\
\text { Isolation A1 }\end{array}$ & .8 & 1 & .8 & $\begin{array}{l}\text { Thrust } \\
\text { Cone }\end{array}$ \\
\hline $\begin{array}{l}\text { Thrust Cone } \\
\text { Reinforcement }\end{array}$ & $\mathrm{Al}$ & .5 & 1 & .5 & $\begin{array}{l}\text { Thrust } \\
\text { Cone }\end{array}$ \\
\hline $\begin{array}{l}\text { Control Box } \\
\text { Mounting }\end{array}$ & $\begin{array}{l}\text { Bolts, } \\
\text { Inserts }\end{array}$ & - & - & .1 & $\begin{array}{r}\text { Aft } \\
\text { Blkd }\end{array}$ \\
\hline $\begin{array}{l}\text { Radiator } \\
\text { Support }\end{array}$ & $\begin{array}{l}\text { Alum } \\
\text { Intercostals } \\
\text { and Bkts }\end{array}$ & 1.40 & 4 & 5.6 & $\begin{array}{c}\text { Aft } \\
\text { Blkd, } \\
\text { Longerons }\end{array}$ \\
\hline $\begin{array}{l}\text { Mounting Hdw, } \\
\text { Tube Clamps }\end{array}$ & & & $\mathrm{A} / \mathrm{R}$ & 1.0 & \\
\hline $\begin{array}{l}\text { Wt. of Structure } \\
\text { Provisions }\end{array}$ & & & & $11.6 \mathrm{lb}$ & \\
\hline Contigency & & & $10 \%$ & $1.2 \mathrm{lb}$ & \\
\hline $\begin{array}{l}\text { Total Structure } \\
\text { Wt. }\end{array}$ & & & & $12.8 \mathrm{lb}$ & \\
\hline
\end{tabular}




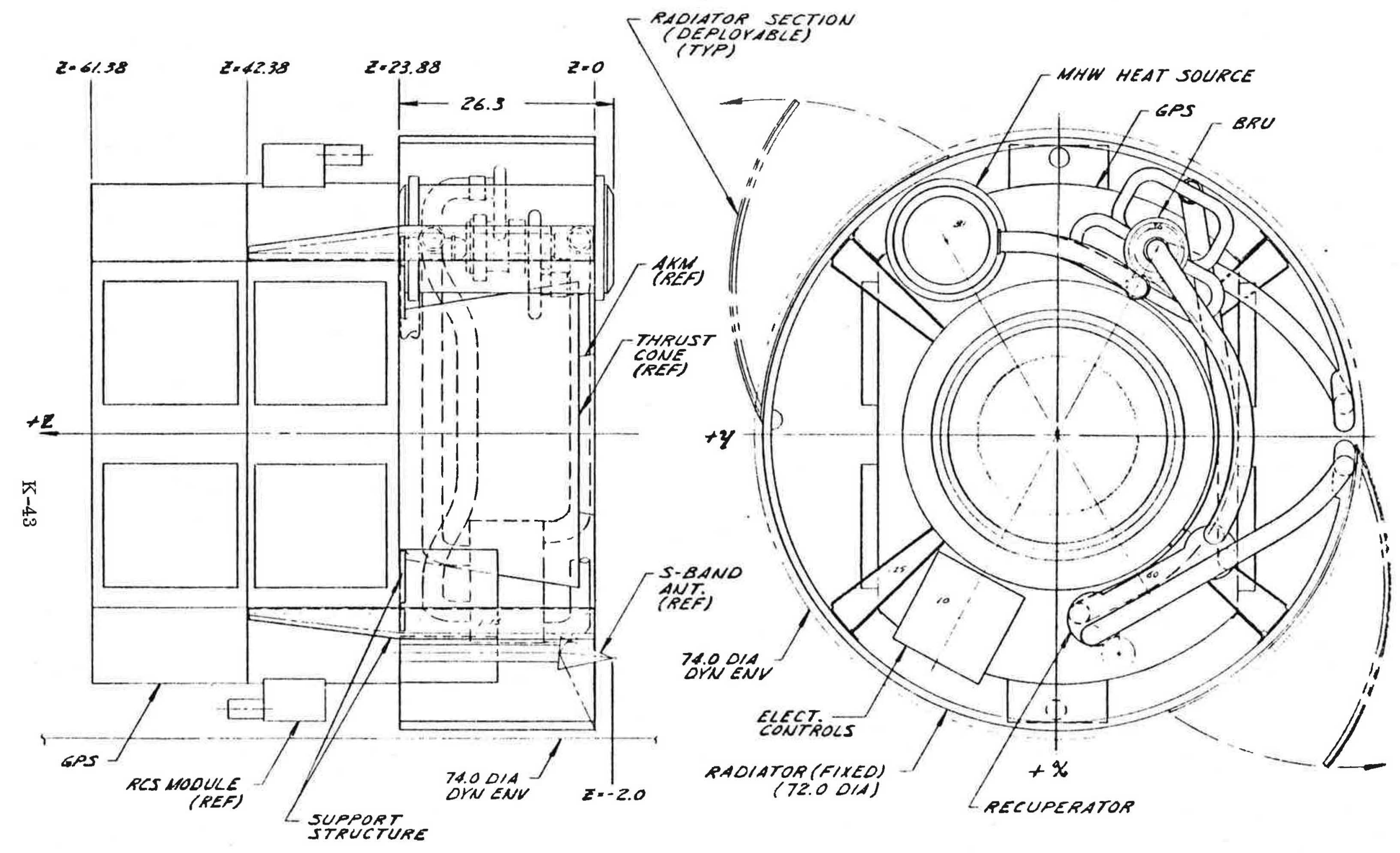

FIGURE K-32

BIPS INSTALIATION (Z AXIS BRU, DEPLOYED RADIATOR) 
K-3.2.3 Organic Rankine Cycle (ORC) Approach

Installation interfaces for the ORC approach are similar to those previously described for BIPS. Figure K-33 provides a pictorial view of the ORC retrofit assembly and figure 6.2-7 shows side and end views. In Figure $\mathrm{K}-34$ the axis of the ORC rotating turbine/generator/pump combined relating unit (CRU) is shown parallel to the $\mathrm{Z}$ axis. As shown in Appendix $\mathrm{K}-\mathrm{A}$, a lateral orientation of the CRU is feasible. However, because of thermal control considerations, all of these retrofit designs maintain the yaw steering requirement. Therefore, the spacecraft must rotate in both yaw and pitch, making it necessary to cancel out the angular momentum of the CRU $(17.6 \mathrm{ft}-\mathrm{lb}-\mathrm{sec})$ in either orientation. A momentum wheel with very low torquing requirement can be provided for this function at a weight penalty of approximately 15 pounds. This is included in the spacecraft weight estimates for both ORC and TE-topped ORC systems.

\section{K-3.2.4 Structure/Mechanical Interfaces}

Layouts of the radioisotope subsystems options based on the 2400 watt heat source show that they fit well within the aft end annular volume bounded by the aft bulkhead, the separation plane, the thrust cone and their respective cylindrical radiators. When the power level is increased to $3200 \mathrm{w}_{t}$, however, the heat source assembly with its associated thermoelectric generator, boiler, or gas heat exchanges protrudes below the separation plane by several inches. The Rankine cycle radiators were equally long. Additional studies, based on detailed drawings of the separation plane area would be required to determine whether or not the thrust cone would need to be modified for the 3200 $w_{t}$ systems. The AKM plume problem in this area is treated below.

Mounting of the radioisotope systems would require new attachment inserts in the aft bulkhead and a number of stiffeners on the thrust cone. Layouts of the support structure for the heat source assemblies, which are located somewhat outboard of the shear panels, suggested attachment at the edge formed by the aft bulkhead and the shear panels. This corner is a strong point and lends itself to the use of brackets with clevis joints at the location of the (removed) aft solar stow latch (Figure K-34). A combination of brackets (with and without shear webs) and struts is then used to support the 


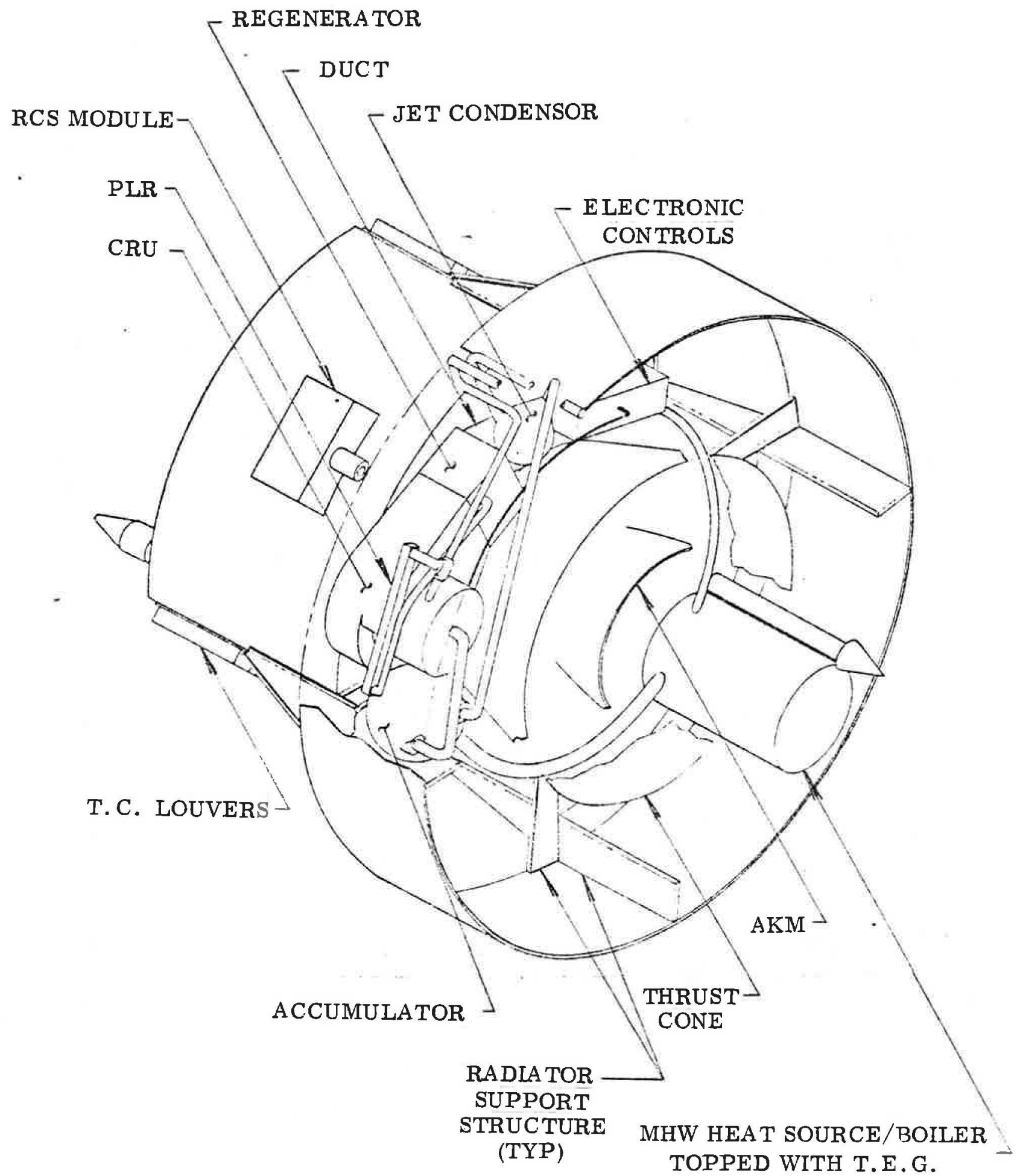

FIGURE K-33 ORGANIC RANKINE ( ORC) POWERED GPS 


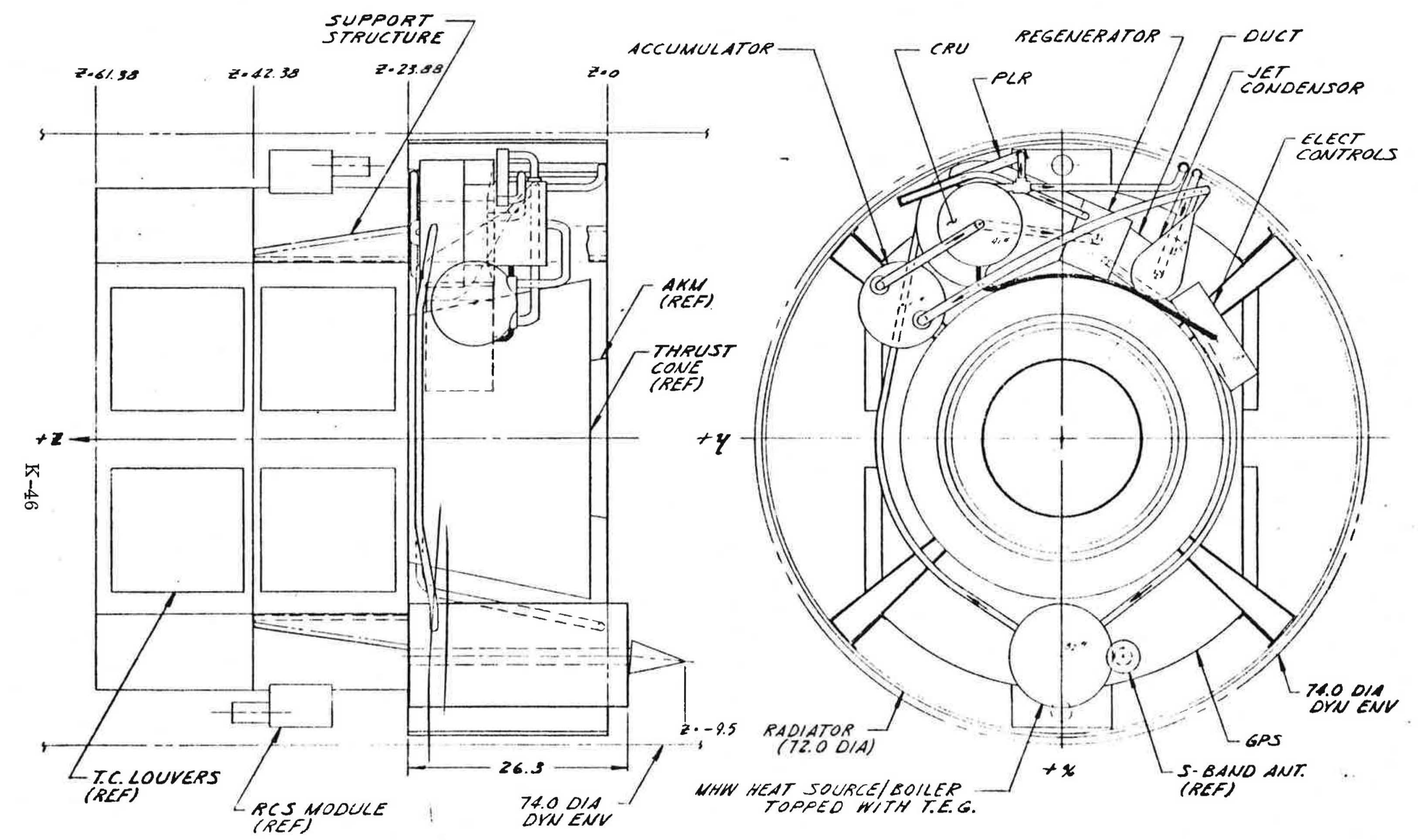

FIGURE K-34 ORC INSTALLATION 
lateral, thrust axis and rotational loads of other components through the bulkhead and/or thrust cone. The support structure weights are based on preliminary stress analysis of selected members.

The arrangement of power system components in the annular volume was constrained by the requirement of a balanced configuration during spin. As the weight data for components becomes more accurate, the locations shown previously will need to be adjusted, but in any case, provision for balance weights will be included. Estimates have been made of the new $C G$ position along the $Z$ axis, but the effect of such a change on the moments of inertia ratio which affects the vehicle during PKM burn or on the AVCS throughout the mission have not been made. The July 21 mass properties indicated CG shifts for the solar powered $\Phi$ I SV between the 36 and 38 inch Z-axis station during the mission. The estimated change resulting from retrofitted isotope power systems were as follows assuming the solar powered GPS at $Z=38.0$ :

\begin{tabular}{|c|c|c|}
\hline & Z Station & $\Delta$ \\
\hline RTG & $33.1 "$ & $4.8 "$ \\
\hline PS & 31.5 & 6.5 \\
\hline $\mathrm{RC}$ & 34.2 & 3.8 \\
\hline
\end{tabular}

These are sizable shifts and their effect will need to be analyzed.

Access to subsystems and components inside the main body of the satellite utilizes four curved panels on the $+X$ and $-X$ sides of the spacecraft. The thrust cone is removed for installation of the AKM in the center of the main body. Access to the radioisotope power systems will be from below between the thrust cone and cylindrical radiators. Orientation of the various heat sources with their cylindrical axis parallel to the SC centerline was generally considered to be desirable. Orientation of other dynamic system components was made to minimize plumbing complexity. Line diameters are as spectfied by the power system subcontractors but, in some cases, line lengths and routing have been modified, leading to small changes in system pressure drops and flow rates. 
The BIPS deployable radiators pose a difficult design problem because of the large size of their gas flow passages. A preliminary layout indicates that the deployable cylindrical sections utilizing flexible bellows joints is a possible solution, although the use of such a radiator with its added mechanical complexity is a clear disadvantage for the BIPS.

$\mathrm{K}-3.2 .5 \quad$ Power Subsystem Interfaces

Each of the radioisotope power subsystems has a power conditioning unit that interfaces with the LCU retained from the solar power system. This unit would also act as the command and telemetry interface and would contain any necessary active monitor/control circuits in the case of the dynamic isotope systems. In the retrofit case it has been assumed that all power matching would be accomplished on the power system side of the interface leaving the LCU with minimum design changes. An example of these latter changes would be removal of the solar array power leads and the added provision for ordinance power.

Internal to the isotope systems, the start up sequence, provisions for prelaunch cooling, launch and ascent temperature profiles and launch phase available power profiles will be studied as part of the redesign effort beyond the retrofit phase.

The effects of internal $G$ forces on the operation of the RTG heat pipes during the spin mode was minimized by a redesign of the heat pipe attachment and radiator curvature as indicated below. In the BIPS gas system, these effects are negligible. In the case of the organic Rankine cycle that has both vapor and liquid loops, the effect require that the jet condenser be mounted at an angle to the axis of rotation in order to correct for the coriolus acceleration of the focussing jet stream.

K-3.2.6 Thermal Control Subsystem Interfaces

An independent assessment of the tradeoff between isotope system radiator size and operating temperature was made (see Appendix K-B). The size of these radiators is limited by aft end dynamic envelope, while the operating temperature has a direct affect on RTG and dynamic cycle efficiency. Parametric data on radiators sized to handle both the 2400 and 3200 watt heat sources are given and confirm contractor designs. 
In the case of the RTG's the (vater/copper) heat pipes were redesigned to account for $\mathrm{G}$ forces and the effective radiating area was increased by moving structural ribs from the inside to the outside of the cylindrical radiator surface. These radiators would operate at a pipe wall temperature of about $304^{\circ} \mathrm{F}$ at the evaporator end with a fairly constant vapor to wall $\Delta \mathrm{T}$ along the pipe length of about 1 to $2^{\circ} \mathrm{F}$. The following parametric data were calculated for these radiators:

\begin{tabular}{l} 
Heat Pipe \\
Vapor Temperature, ${ }^{\circ} \mathrm{F}$ \\
\hline Full Sun \\
Full Shadow \\
${ }^{*} \mathrm{~L}=19^{\prime \prime} @ 2400 \mathrm{w}_{\mathrm{t}},=25 @ 3200 \mathrm{w}_{\mathrm{t}}$
\end{tabular}

Radiator Diameter *

\begin{aligned} $62^{\prime \prime} & 72^{\prime \prime} \\$\hline $271^{\circ} & 226^{\circ} \\ 255^{\circ} & 209^{\circ}\end{aligned}$

The shape and orientation of the pipes were also modified to overcome any "puddles" of water between the evaporator and condensor due to the approximate $10 \mathrm{~g}$ centrifugal force at the $100 \mathrm{rpm}$ spin rate. The $\Delta \mathrm{P}$ required to overcome $10 \mathrm{~g}$ is $131 \mathrm{lbf} / \mathrm{ft}^{2}$ whereas the available capillary pressure within the heat pipes is $2.8 \mathrm{lbf} / \mathrm{ft}^{2}$. The bends where puddles could collect and block heat pipe action were eliminated.

Similar parametric radiator temperature data were calculated for the Brayton and Rankine cycle systems that included additional parameters such as stowed vs. deployed configurations and variable deployed areas (see Appendix K-B).

The heat soakback from the retrofit isotope heat source assemblies into the GPS structure will be a small fraction of the total GPS heat rejection load. A detailed analysis of the conductive paths from the RTG source assemblies through their support structure is given in the Appendix K-B. The total conducted heat load from two RTG's is 26 watts at a GPS structural temperature of $40^{\circ} \mathrm{F}$, decreasing linearly to 16 watts at $100^{\circ} \mathrm{F}$. The conducted heat from the BIPS and ORC systems will be less than half of the RTG value because of their higher source efficiency and lower radiator temperature. In addition, with the solar arrays removed the louver heat rejection capability is increased by 60 watts (at $70^{\circ} \mathrm{F}$ ). The total vehicle heat rejection load is about 300 watts at normal power and 385 watts at high power with $100 \%$ TT\&C. The worst case RTG load would then be $6 \%$ of the total with the structure at $70^{\circ} \mathrm{F}$. 
In retrofitting the radioisotope systems, the thermal subsystem interface would change to reflect the removal of battery heaters, the modification or addition of thermal doublers on the aft bulkhead and changes and/or removal of the external multilayer insulation blankets on the thrust cone. Thermal analysis of the aft end of the spacecraft would include the heat exchange interface between the isotope systems and the AKM during coast and burning phases and AKM plume heating.

This latter interface is an important liability of the retrofit designs. In the $\Phi I$ SV the AKM plume is free to expand. Except for the thrust cone and aft-facing TT\&C antenna no equipment is encountered. In the retrofit designs, particularly the $3200 \mathrm{w}_{t}$ cases where the radiator as well as the heat source assemblies protrude below the separation plane, the plume expansion could result in circulation of hot gases within the annular volume occupied by the power system. The addition of an annular plume deflector causes complications, in that it would upset the radiative heat balance within the power system cavity since about 10 to $20 \%$ of heat rejected is from the back face of the radiators. Analysis however, might show that the relatively short burn time of the AKM ( 35 seconds) does not produce unacceptable temperature or heating levels.

A thermal interface also exists between the radiators and the RCS hydrazine thruster plumes. A 5-lbf precession and $\Delta V$ thruster fires aftward along the- $\mathrm{X}$ face, while two 0.1-lbf thrusters fire aft along both the + and $-\mathrm{X}$ faces*. The primary problem with the larger plume would be radiator heating during steady state firings (maximum 13 minutes), while the added area of the isotope radiators also offers a surface for the development of side forces during on-orbit firing of the 0.1-1bf thrusters. These impingement forces and heating are acceptably small in the $\Phi$ I SV design because of the outboard modular mounting of the jets that provides separation from adjacent surfaces. The outside envelope of the RTG radiators has been made flush with the + and- $X$ panel surfaces to avoid direct plume impingement (see Figure K-27). An iteration on the dynamic systems radiator design would be required to modify their shape in the area of the 5-lbf thruster, or to provide deflection/shielding of this plume.

\footnotetext{
* The other 5-1bf thruster fires forward.
} 
K-3.2.7 Tracking Telemetrv and Command (TT\&C) Interfaces

It is assumed that conditioning circuitry for power system telemetry would be part of the omnibus power conditioning unit included in each of the power subsystems and that sufficient channels would be available in the TT\&C after removal of the solar power yaw and pitch sun sensor and drive position telemetry.

It is possible that the aft-facing TT\&C antenna would either have to be relocated or have its length modified because of possible interference with isotope power components, particulariy those in the dynamic systems. The integration layouts shown in previous figures maintain the antenna in its present location and do not violate its field of view, but power system components are in close proximity to the antenna and any changes could lead to mechanical or field of view interference.

$\mathrm{K}-3.2 .8 \quad$ Attitude/Velocity Control Svstem (AVCS) Interfaces

As already discussed, the thermal control requirements dictate that yaw steering and pitch control be maintained, which permitted the axis of the rotating unit to be retained along the $\mathrm{Z}$-direction.

\section{$\mathrm{K}-4$ Conclusions}

K-4.1 Weight and Power Comparisons

The weight and power capabilities of the candidate nuclear power subsystems were previously defined in section $\mathrm{K}-2$. In summary, these characteristics are:

$$
\text { For } 2400 \mathrm{w}_{t} \text { Sources }
$$

Gross EOL power (watts)

Net EOL power (watts)

Subsystem weight (lbs)

\section{Current}

\begin{tabular}{|c|c|c|c|c|}
\hline Solar & RTG & BIPS & ORC & $\mathrm{ORC} / \mathrm{RTG}$ \\
\hline 410 & 420 & 428 & 331 & 465 \\
\hline 326 & 412 & 420 & 323 & 457 \\
\hline 276 & 249 & 340 & 253 & 258 \\
\hline
\end{tabular}


For $3200 \mathrm{w}_{\mathrm{t}}$ Sources

Gross EOL power (watts)

$559 \quad 662 \quad 457 \quad 635$

Net EOL power (watts)

$548 \quad 651 \quad 446 \quad 624$

Subsystem weight

$285 \quad 412 \quad 286$

296

As may be seen from the above summary, the $2400 \mathrm{w}_{t}$ sources are capable of maintaining the current GPS spacecraft high power mode (373 watts net power required) through end of life and three of the four systems have sufficient power margin to provide some small improvements to the "user power" critical parameter.

The $3200 \mathrm{wt}$ sources are capable of providing significant improvements to the "user power" critical parameter. Power-to-user options vs DC loads are listed in Appendix B, which encompasses spacecraft power levels up to 654 watts. Estimated incremental Navigation subsystem weights corresponding to these options are:

\begin{tabular}{ccc} 
Option & \multicolumn{1}{c}{$\Delta$ Power } & NAV Subsvstem Weight Increment \\
\cline { 3 - 3 }$(1)$ & Baseline & Baseline \\
$(2)$ & $+18 \mathrm{~W}$ & $+2.1 \mathrm{lbs}$ \\
$(3)$ & +54 & 9.9 \\
$(4)$ & +86 & 19.7 \\
$(5)$ & +43 & 24.6 \\
$(6)$ & +50 & 31.8 \\
$(7)$ & +30 & 36.1
\end{tabular}

As indicated in previous sections, the spacecraft housekeeping subsystems required 67 watts D. C. and the basic spacecraft initial on-orbit weight (after AKM firing) is 889 lbs. 
Table $K-35$ and $K-36$ show the impact on overall spacecraft weight of the 2400 watt and 3200 watt sources respectively.

Compared to an initial on-orbit weight budget of 897 lbs. (GPS spacecraft launch weight estimate as of July 21, 1976, was 1665 lbs. which included 8 lbs. of ballast) only marginal improvements are possible and the BIPS approval is not feasible. However, if the initial on orbit weight budget is increased to $1200 \mathrm{lbs}$. (as stated in the study guidelines), any of the nuclear power source approaches is feasible and most of the user power options can be accommodated.

K-4.2 Weight/Reliability Tradeoffs

GPS reliability and failure rates at the subsystem level are as follows:

(reference: Rockwell 7/76 Design Review).

\begin{tabular}{|c|c|c|}
\hline & $R_{5 \text { year }}$ & $\lambda \times 10^{-9} / \mathrm{hr}$ \\
\hline TT\&C & .8927 & 2591.4 \\
\hline AVCS & .9847 & 352.0 \\
\hline EPS & .9628 & 865.5 \\
\hline $\mathrm{RCS}$ & .9945 & 125.9 \\
\hline TCS & 9999 & 2.2 \\
\hline STR & .9992 & 18.3 \\
\hline NAV & .9407 & 1395.7 \\
\hline OIS & .9963 & 73.2 \\
\hline sV & .7885 & 5424.2 \\
\hline
\end{tabular}

The TT\&C subsystem has the lowest reliability and of its components and subassemblies, the following two are the major contributors:

\begin{tabular}{|c|c|c|c|}
\hline & $R_{5 \text { year }}$ & $\lambda \times 10^{-9} / \mathrm{hr}$ & Weight \\
\hline Transponder & .96105 & 907.1 & 16.0 \\
\hline $\mathrm{K}$ R -23 & . 95841 & 909.9 & 8.6 \\
\hline
\end{tabular}

The KIR-23 function is handled in a switched parallel redundant arrangement. As an example 
TABLE K-35.SPACE VEHICLE: POWER/WEIGHT SUMMARY (2400 $\mathrm{w}_{\mathrm{t}}$ SOURCE)

\begin{tabular}{|c|c|c|c|c|c|}
\hline GPS POWER SYSTEM & SOLAR & $\begin{array}{c}\text { RT G } \\
\text { (FIXED) }\end{array}$ & $\begin{array}{c}\text { BIPS } \\
\text { DEPLOYABLE } \\
\text { RADIATOR }\end{array}$ & ORC & $\begin{array}{c}\text { ORC } \\
\text { (TE TOPPING) }\end{array}$ \\
\hline \multicolumn{6}{|l|}{ POWER (WATTS) } \\
\hline NAV Subsystem (HiPower) & 305.5 & 305.5 & 305.5 & 305.5 & 305.5 \\
\hline EPS (Losses + Battery Chrg) & 84.0 & 8.1 & 8.1 & 8.1 & 8.1 \\
\hline Housekeeping & 61.4 & 61.4 & 61.4 & 61.4 & 61.4 \\
\hline Power Conversion Loss & NA & 41.7 & 0 & 0 & 0 \\
\hline TOTAL REQUIRED & 450.9 & 416.7 & 375,0 & 375.0 & 375.0 \\
\hline EOL AVAILABLE POWER, WATTS & 410 & 420 & 428 & 331 & 465 \\
\hline MARGIN & -40.9 & +3.3 & +53 & $(-44)$ & +90 \\
\hline \multicolumn{6}{|l|}{ SV WEIGHTS: } \\
\hline EPS Retained/Added & 275.6 & 249.1 & 339.8 & 253.3 & 257.8 \\
\hline Other Subsystems & 613.2 & 596.5 & 601.5 & $\underline{601.5}$ & 601.5 \\
\hline TOTAL (Less AKM) & 888.8 & 845.6 & 941.3 & 854.8 & 859.3 \\
\hline MARGIN & - & +43.2 & $(-52.5)$ & +34.0 & +29.5 \\
\hline EPS EOL POWER/WEIGHT & 1.49 & 1.69 & 1.26 & 1.31 & 1.80 \\
\hline
\end{tabular}


TABLE K-36. SPACE VEHICLE: POWER/WEIGHT SUMMARY (3200 $\mathrm{w}_{t}$ SOURCE)

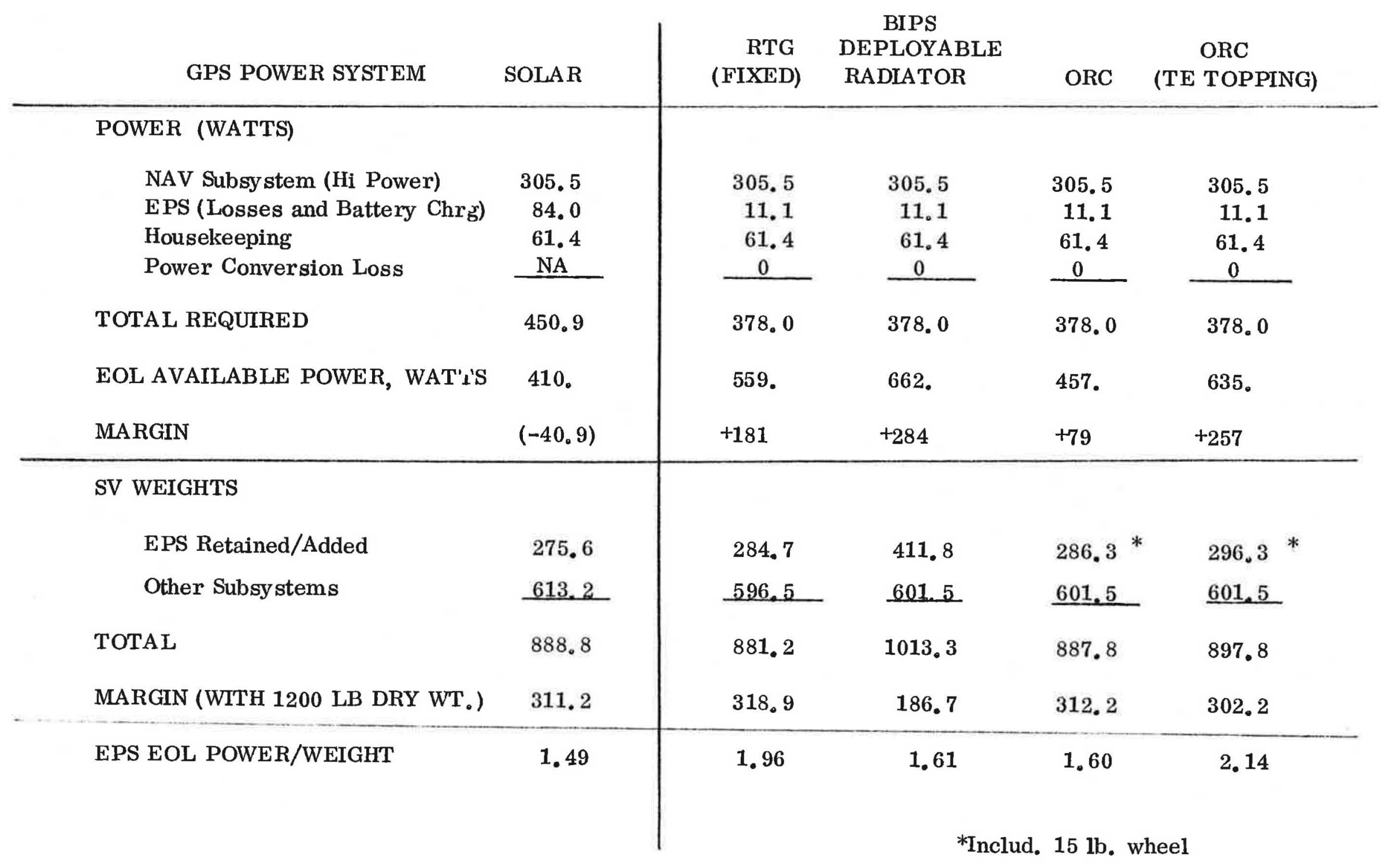


of weight/reliability tradeoff, the effect on MMD of adding a third parallel KIR-23 was calculated. The submodel at that point was:

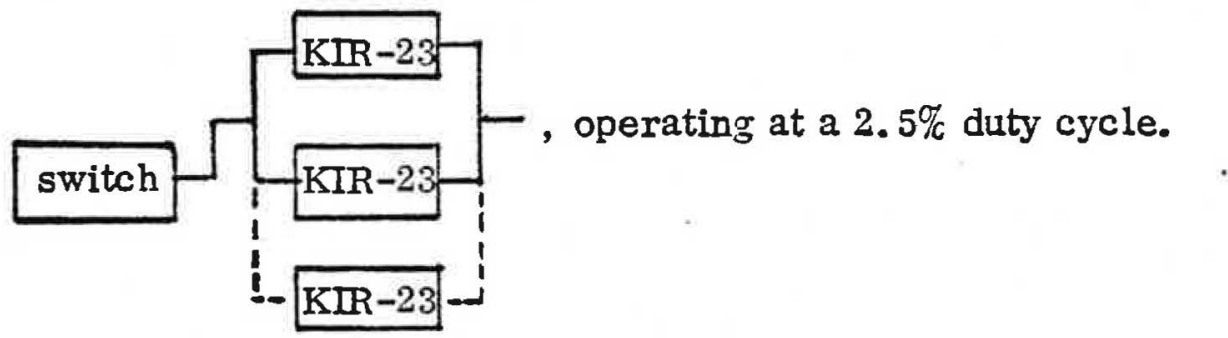

The reliability increased about $3 \%$ from .9584 to about . 9900 which in turn increased the TT\&C subsystem from .8927 to .9222 . This improved the overall SV reliability from .7885 to. $8146\left(\lambda=4682.6 \times 10^{-9} / \mathrm{hr}\right)$, resulting in an increase in MMD from 4.45 to 4.74 years or 3-1/2 months, at a cost of $8.6 \mathrm{lb}$. weight increase. A similar improvement in MMD brought about by an additional TT\&C transponder would cost $16 \mathrm{lb}$. weight increase.

\section{K-4.3 POTENTIAL ADVANTAGES OF RETROFIT DESIGNS}

In addition to the user power improvements documented in section $\mathrm{K}-4.1$, two significant advantages accrue from the use of retrofitted isotope power on the GPS. Both occur in the navigation area, as a result of the removal of the solar panels:

1) Improved Frequency Standard temperature control and,

2) Reduced navigation error over a given time or, conversely, increased time between required updates.

The solar panels on the current GPS spacecraft radiate both reflected solar and infrared energy into the louvered radiators. : It is the orbital fluctuation of this heat load on the louver that thermally controls the frequency standard that is the major cause of variation in its interface temperature. Removal of the solar panels reduces the temperature variation and in turn improves stability of the standard.

Details are given in Appendix K-B of simplified math models for the thermal input/output to the louver and of the frequency standard/louver panel composite structure. The model assumes a constant frequency standard dissipation of 18.6 watts and utilizes experimental performance data for the GPS Fairchild-supplied louver panels. The model calculates a maximum daily variation of $2.6^{\circ} \mathrm{C}$ for the worse case noon orbit compared to a measured 
variation of about $2.2^{\circ} \mathrm{C}$ reported at the 21 July 1976 GPS review. The specified stability tolerance is $4^{\circ} \mathrm{C}$ per day.

The residual thermal load after removal of the solar panels consists of direct solar radiation at small angles in pitch and roll. Following an eclipse the worse case sun angle could approach $8^{\circ}$ over a period of about 8 minutes. The net input under these conditions is a complex function of both sun and louver blade angle due to secondary reflections within the louver mechanism. The resldual load however would be a small fraction of that from the solar array. If, for example, the residual were as high as $13 \%$ the interface temperature variation could be reduced to about $0.3^{\circ} \mathrm{C}$.

The second effect of removing the GPS solar arrays is that the area seeing the sun and therefore the effect of solar radiation pressure is reduced by a factor of $2 / 3$. The advantage in this arises from the fact that navigation accuracy depends on the degree of uncertainty in orbit predictability. Where a fixed percentage error exists in the prediction of solar forces, a reduction in the absolute level of the solar force reduces proportionally this contribution to the total error. Pertrubations to the orbit are caused by earth nonsphericity, lunar and solar gravitational effects, solar and vehicle emitted radiation pressures, particle impingement and vehicle mass expulsions. The total effect of these perturbing forces are sufficiently well-modelled that, after adequate ground-tracking, the actual space vehicle position is currently predicted to within 1 to 2 meters.

The analysis of the predictable effect of solar pressure in Appendix. $K-A$ leads to the following conclusions:

1) In a nominal circular orbit, solar pressure destabilizes the basic vehicle motion so that increasing displacement increments occur with time,

2) Large variatious in displacement occur during each orbit,

3) The destabilized motion increases as the orbit plane approaches the sunline,

4) The displacements increase relatively linearly with total solar pressure force,

5) Cyclic variation in solar pressure force at orbit frequency can cause increasing displacement amplitudes, 
6) Small changes in solar pressure force can cause increasingly large displacement errors.

The following table indicates the maximum magnitude of the relative displacements of the GPS solar configuration from a circular orbit path due to basic solar pressure force only. The displacements are in terms of an increase in the length of the radias vector (i.e., altitude) and a sideward displacement perpendicular to the vector.

\begin{tabular}{|c|c|c|c|c|}
\hline \multirow{3}{*}{$\begin{array}{l}\text { Orbit Inclination } \\
\text { from Sun Line }\end{array}$} & \multicolumn{4}{|c|}{$\begin{array}{l}\text { Maximum Relative Orbit Displacements } \\
\text { Due to Solar Pressure, ft. }\end{array}$} \\
\hline & \multicolumn{2}{|c|}{ Single Orbit } & \multicolumn{2}{|c|}{$\Sigma^{3}$ Orbits } \\
\hline & Altitude" & "Normal* & "Altitude" & Normal ${ }^{*}$ \\
\hline \multirow[b]{2}{*}{$0^{\circ}$ (Ecliptic) } & Error & $\begin{array}{c}\text { Error } \\
\text { (@3/4 orbit) }\end{array}$ & Error & $\begin{array}{c}\text { Error } \\
\text { (a } 3 / 4 \text { orbit) }\end{array}$ \\
\hline & 104 & 192 & 352 & 656 \\
\hline $30^{\circ}$ & 88 & 168 & 304 & 568 \\
\hline $60^{\circ}$ & 53 & 99 & 176 & 320 \\
\hline $90^{\circ}$ & 0 & 24 & 0 & 24 \\
\hline
\end{tabular}

An uncertainty of 1,6 in the solar pressure force would produce an approximately proportional uncertainty in displacement.

The nuclear powered spacecraft however would experience $1 / 3$ the-predictable displacements shown as well as a $1 / 3$ reduction in uncertainty of position prediction. 


\section{APPENDIX $\mathrm{K}-\mathrm{A}$}

DYNAMIC ANALYSES

$\mathrm{K}-59$ 


\section{K-A.1 $\quad$ Rotating Machinery Orientation Tradeoffs}

\section{$\mathrm{K}-\mathrm{A} .1 .1 \quad$ Introduction}

The candidate nuclear power supplies for the GPS space vehicle contain rotating machines. These machines, after start on the ground, remain operating throughout launch and on-orbit life. They can be mounted with their spin axes either along the launch direction or normal to it.

Insertion into the nominal final orbit follows the sequence:

1. Launch by Atlas burn.

2. Separation, spin-up, and launch into drift orbit by two successive PKM burns.

3. Insertion of the spinning payload into nominal final orbit using the orbital insertion system (OIS)

Up to 48 hours of spin may pass between the end of event 2 and the start of event 3. By the start of event 3 , the vehicle normally is rotating stably about its axis $(X)$ of maximum moment of inertia, nutation having been damped out by a passive damper responding to lateral axis accelerations. Stable rotation about the $\mathrm{X}$ axis, or close to it, is necessary for efficient OIS operation.

In this memo, we consider the vehicle motion at start of event 3 as determined by the orientation of the spin axis of the nuclear supply's rotating machine.

\section{$\mathrm{K}-\mathrm{A} .1 .2 \quad$ Analysis}

While in drift orbit, the vehicle is in essentially external torquefree motion which is described by the Euler equations:

$$
\begin{aligned}
& \dot{\omega}_{\mathrm{x}}=-\omega_{\mathrm{y}} \frac{\mathrm{H}_{\ell}}{\mathrm{I}_{\mathrm{x}}} \\
& \dot{\omega}_{\mathrm{y}}=\omega_{\mathrm{x}} \frac{\mathrm{H}_{1}}{\mathrm{I}_{l}}-\omega_{\mathrm{z}} \frac{\mathrm{H}}{\mathrm{I}_{\ell}}+\omega_{\mathrm{x}} \omega_{\mathrm{z}}\left(1-\frac{\mathrm{I}_{\mathrm{x}}}{\mathrm{I}_{l}}\right)-\frac{\mathrm{T}}{\mathrm{I}_{l}}
\end{aligned}
$$


(3)

$$
\omega_{z}=\omega_{y} \frac{H_{x}}{I_{l}}-\omega_{x} \omega_{y}\left(1-\frac{I_{x}}{I_{\ell}}\right)-\frac{T_{d z}}{I_{l}}
$$

Where: $\omega_{i}=$ Angular rate about vehicle axis $i$

$\dot{\omega}=$ Angular acceleration about vehicle axis $\mathrm{i}$

$\mathrm{H}_{1}=$ Angular momentum of laterally oriented rotating machine spin axis, assumed along $\mathrm{Z}$

$\mathrm{H}_{\mathrm{x}}=$ Angular momentum of rotating machine spin axis along nominal spin axis $\mathrm{X}$

$I_{i}=$ Vehicle moment of inertia about axis $\mathrm{i}$

$\mathrm{T}_{\mathrm{di}}=$ Damper torque about axis i

By assumption:

$$
I_{x}>I_{y}=I_{z}=I_{\ell}
$$

$\mathrm{K}-\mathrm{A} \cdot 1.2 .1$

$\underline{\text { Rotating Machine Spin Axis Along Vehicle X Axis }}$

By definition for this configuration: $\mathrm{I}-\mathrm{I}_{\mathrm{x}}>0 ; \mathrm{H}_{\ell}=0$

Because the vehicle is symmetrical about the $\mathrm{X}$ axis, the axes can be redefined so that the $y$ axis will be assumed to feel the damping torques and $\mathrm{T}_{\mathrm{dz}}=0$.

In the steady state condition $\dot{\omega}_{\mathrm{y}}=0$ because the damper torque $\mathrm{T}_{\mathrm{dy}}$ removes rotational kinetic energy from the system whenever accelerated axis motion exists. Thus, $\omega_{y}$ will be constant in steady state.

Since $\dot{\omega}_{\mathrm{y}}$ is zero then from equation (2) $\omega_{\mathrm{z}}=0$ and/or $\omega_{\mathrm{x}}=\frac{\mathrm{H}_{\mathrm{x}}}{\mathrm{I}_{\ell^{-I}}}$ And if $\omega_{z}=0$, from equation (3) $\omega_{y}=0$ and/or $\omega_{x}=\frac{H_{x}}{I_{l}-I_{x}}$.

Two possibilities for the steady state condition therefore exist:

either $\omega_{\mathrm{x}}=\frac{\mathrm{H}_{\mathrm{x}}}{\mathrm{I}_{\ell}-\mathrm{I}_{\mathrm{x}}}$ and $\omega_{\mathrm{z}}, \omega_{\mathrm{y}}$ are constant (because $\dot{\omega}_{\mathrm{y}}=\dot{\omega}_{\mathrm{z}}=0$ ) or $\omega_{\mathrm{y}}=\mathrm{w}_{\mathrm{z}}=0$. 
Now, it is shown that the steady state solution $\omega_{x}=\frac{H_{x}}{I_{R}-I_{x}}$

is impossible for arbitrary initial conditions if any damping has occurred.

Total angular momentum of the system is conserved so that with initial $X$ ixis rate $\omega_{x O}$ and initial lateral axis rate $\omega_{l_{0}}$ (where $\left.\omega_{x}^{2}=\omega_{x}^{2}+\omega_{y}^{2}\right)$ :

$$
\left(\mathrm{I}_{\mathrm{x}} \omega_{\mathrm{XO}}+\mathrm{H}_{\mathrm{x}}\right)^{2}+\left(\mathrm{I}_{\ell} \omega_{0}\right)^{2}=\left(\mathrm{I}_{\mathrm{x}} \omega_{\mathrm{x}}+\mathrm{H}_{\mathrm{x}}\right)^{2}+\left(\mathrm{I}_{\ell} \omega_{l}\right)^{2}
$$

Also, total vel,icle rotational kinetic energy cannot increase:

$$
\frac{I_{x} \omega_{x o}^{2}}{2}+\frac{I_{1} \omega_{x_{0}}^{2}}{2} \geq \frac{I_{x} \omega_{x}^{2}}{2}+\frac{I_{X} \omega_{X}^{2}}{2}
$$

$\mathrm{H}_{\mathrm{x}} \quad$ Combining equation (4) and (5) with the assumed steady state value $\omega_{x}=\frac{x}{I_{x}-I_{x}}$ leads to the condition: $\left(\omega_{x O}-\omega_{X}\right)^{2} \leq 0$.

This is impossible unless $\omega_{x}=\omega_{x O}$ in which instance no damping has taken place bi...ause the equality sign applies. Thus, when damping has occured $\omega_{x} \neq \frac{H_{x}}{I_{z}-I_{x}}$ and the true steady state condition has $\omega_{y}=\omega_{z}=0$ with the angular momentûm of the system concentrated along the $\mathrm{X}$ axis.

K-A.1.2.2 Rotating Machine Spin Axis Aligned Near Vehicle X Axis

In this configuration, $\mathrm{H}_{\mathrm{x}} \neq 0$ and $\mathrm{H}_{2} \neq 0$ but $\mathrm{H}_{\mathrm{x}} \gg \mathrm{H}_{-}$. As in 2.1 , because "he vehicle is symmetrical about the $\mathrm{X}$ axis the axes can be redefined, so that the $\mathrm{Y}$ axi will be assumed to feel the damping torques, and $\mathrm{T}_{\mathrm{dz}}=0$.

In the steady state condition $\dot{\omega}_{\mathrm{y}}=0$ due to the damping action, so that $\omega_{y}$ is a constant. But $\omega_{y}$ must be zero since a non-zero $\omega_{y}$ would change $\omega_{x}$ at constant rate in accorw, nce with equation (1). Therefore, from equation (3) $\omega_{\mathrm{Z}}=0, \omega_{\mathrm{Z}}=$ constant and:

$$
0=\omega_{x} H_{l}-\omega_{z} H_{x}+\omega_{x} \omega_{z}\left(I_{l}-I_{x}\right)
$$

Solutions to equation (6) can be understood if $\mathrm{H}_{C}$ and $\mathrm{H}_{x}$ are assumed components of a single vector $\mathrm{H}$ in the $\mathrm{X} \mathrm{Z}$ plane making an angle $\theta$ with respect to the $\mathrm{X}$ axis. 
$\omega_{\mathrm{x}}$ and $\omega_{\mathrm{f}}$ are components of a total rate vector $\omega$ in the $\mathrm{ZX}$ plane making an angle $\alpha$ with respect to the $\mathrm{X}$ axis.

$$
\text { Then: } \begin{aligned}
\mathrm{H}_{\mathrm{x}} & =\mathrm{H} \cos \theta ; \mathrm{H}_{l}=\mathrm{H}_{\sin } \theta \\
\omega_{\mathrm{x}} & =\omega \cos \alpha ; \omega_{\mathrm{z}}=\omega \sin \alpha
\end{aligned}
$$

Combining these equations with equation (6) leads to:

$$
\sin 2 \alpha=\frac{-2 H}{\omega\left(I-I_{x}\right)} \sin (\theta=\alpha)
$$

for $\theta-\alpha$ and $\alpha$ small:

$$
\alpha=\frac{K}{2+K} \theta
$$

$$
\text { where } K=\frac{2 H}{\omega\left(I_{x}-\frac{I}{l}\right)}
$$

Thus, when damping occurs about the $\mathrm{Y}$ axis, $\alpha$ is at most equal to $\theta$ so that the vehicle's steady state axis of rotation is near the $\mathrm{X}$ axis when there is a small deviation of the $H$ vector from the $X$ axis.

Note that because all vehicle rates are constant the same steady condition also satisfies the dynamic equation when damping occurs along the $\mathrm{Z}$ axis.

\section{K-A.1.2:3 Rotating Machine Spin Axis Along Vehicle Z Axis}

For this configuration, the analyses of section 1.2 .2 applies with $\theta=$ $90^{\circ}$. The resultant steady state motion then has $\omega_{y}=0, \omega_{X}$ and $\omega_{z}$ are constant, and from equation (7) $\alpha=\sin ^{-1} \frac{H}{\left.I_{x}-I_{z}\right) \omega}$. It should be noted that the steady state axis of rotation can be greatly offset ${ }^{X}$ from the $X$ principal axis - the intuitively "obvious " axis. 
The table below shows $\mathrm{x}, \omega_{\mathrm{x}}, \frac{\Delta \mathrm{V}}{\mathrm{V}}$ as a function of $\mathrm{H}$ for the Rockwell GPS wherein $I_{x}-I_{k}=14$ slug ft ${ }^{2}, \omega \cong 9.19 \mathrm{r} / \mathrm{s}$. The function $\frac{\Delta V}{V}$ $(=\cos \alpha)$ shows the fractional loss in thrusting, at the end of drift orbit, incurred as a result of the rotation of the vehicle $\mathrm{X}$ axis about the vehicle axis of rotation. Thrusting is of such long duration that it covers several spin cycles.

\begin{tabular}{|c|c|c|c|}
\hline $\begin{array}{c}\mathrm{H} \\
\text { ft. lb. sec. }\end{array}$ & $\begin{array}{c}\alpha \\
\mathrm{deg} .\end{array}$ & $\begin{array}{c}\omega_{\mathrm{rad} / \mathrm{sec}} \\
\mathrm{V}\end{array}$ \\
\hline 0.5 & 0.22 & 9.19 & 0.0000 \\
1.0 & 0.45 & 9.19 & 0.0000 \\
5.0 & 2.23 & 9.18 & 0.0008 \\
10.0 & 4.46 & 9.16 & 0.0030 \\
15.0 & 6.70 & 9.13 & 0.0068 \\
20.0 & 8.94 & 9.08 & 0.0120 \\
25.0 & 11.2 & 9.01 & 0.0190 \\
\hline
\end{tabular}

\section{K-A.,1.3 Conclusions}

The steady state effects on drift orbit vehicle attitude due to rotating machine orientation have been shown to be small with possible exceptions due to large laterally oriented machines. These are discussed below.

K-A.2 Errors Introduced by Laterally-Oriented Machines

The launch sequence for the GPS includes separation from the Atlas launch stage, rapid spin-up, ignition of the first PKM, separation from the spent PKM, short coast, ignition of the second PKM, and separation from the spent second PKM. Attitude errors and vehicle nutations which develop during these phases propagate into transfer orbit inclination, and apogee and perigee attitude errors.

This study evaluates the effects on the drift orbit trajectory at first apogee, due to an operating, laterally oriented rotating machine in the GPS. 
K-A.2.1 Angular Disturbances Causing Trajectory Errors

The analysis technique in Reference (1) determines the 3 angular deviation, from the desired direction, which occurs during each launch phase. RSS'ing of these increments yields the total 3 misdirection of the vehicle's velocity vector from the desired direction. In addition, the nutation cone angle increment, during each phase, is found. Table D.2-1 contains a summary of the angular disturbances documented in Reference (1).

Here, we are concerned with the effects of operating, laterally oriented, rotating machines in the payload. The approach taken is based on using the techniques and results of Ref. (1). The approach assumes two reasonable propositions:

1. Equal lateral axis angular momenta, due to lateral axis torques, are added to the basic GPS vehicle (i. e., with no lateral machine) and to the vehicle with a lateral rotating machine. The causative torques are due to imperfections in the separation spring systems, the spin-up torque system, and PKM thruster misalignments.

2. Angular offsets during PKM burns are the same for the basic GPS and the vehicle with a lateral rotating machine. The averaging effect of the thrusting PKMs on the spinning vehicle is imperfect only due to the change in thrust levels during PKM burns. These changes are due to start-up transients, imperfect "constant " thrust levels, and burn-out transients. The net torque resultant of the nonuniform thrusting over a single spin cycle precesses the system angular momentum vector and causes angular offs ets between the system's angular momentum vectors existing before and after that cycle. Thus, for equal spin rates and similar PKM thrust histories, one can expect incremental vehicle offsets to vary inversely with the total vehicle angular momentum. 
TABLE K-A. 2-1

SUMMARY OF ANGULAR DISTURBANCES (3 $\%)$

BASIC VEHICLE *

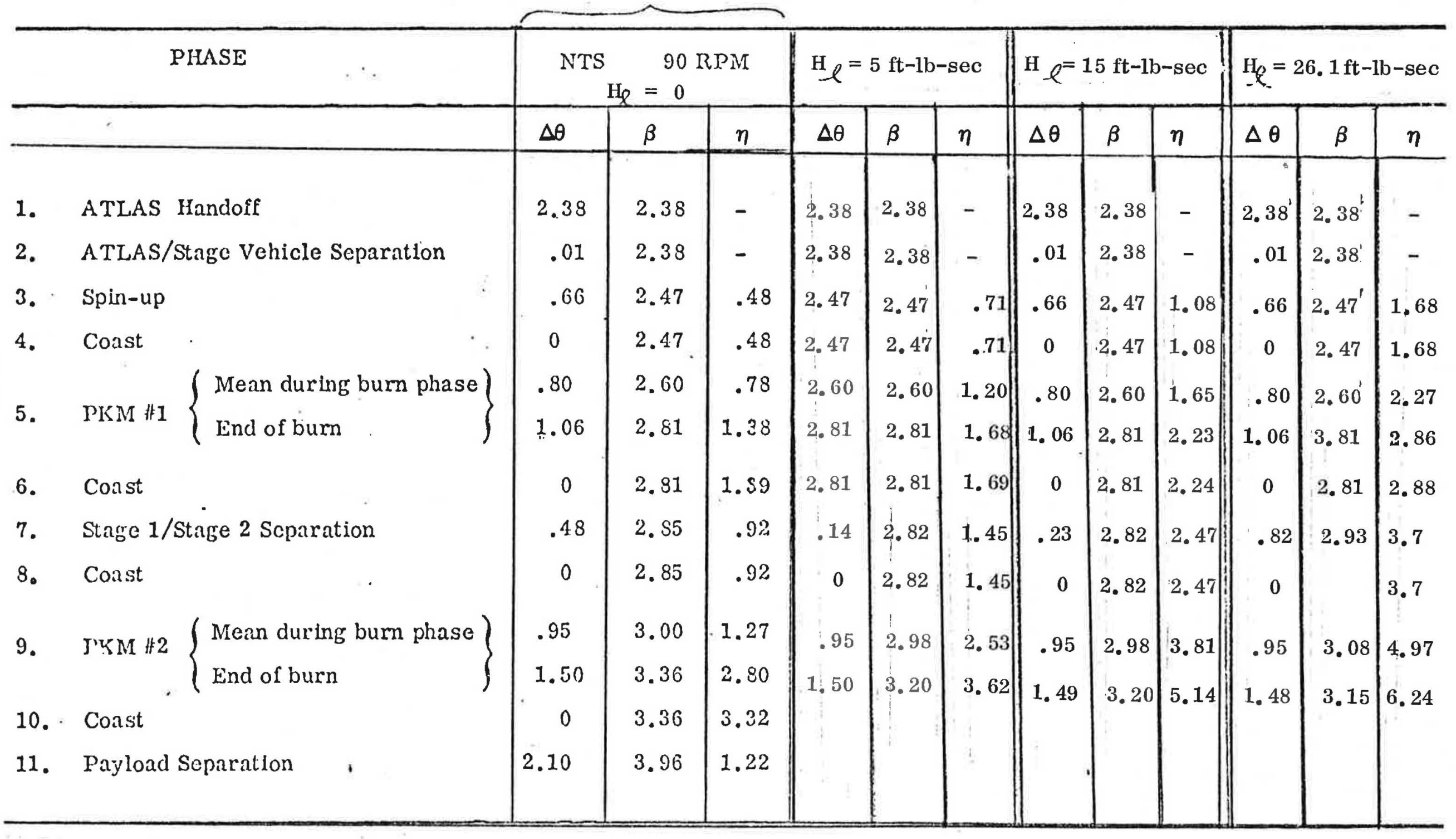

$\Delta \theta$-Increment during phase; $\beta$-Cumulative total error; $\quad \eta$ - nutation $\frac{1}{2}$ cone angle.

* Basic Vehicle values from Reference 1 
A third proposition, based on digital computer simulation results, is also assumed:

3. The total nutation angle of the vehicle, when a laterally-oriented machine exists, is obtained by taking the sum of the nutation half-cone angle of the "basic" vehicle and half the maximum nutation angle of an untorqued vehicle containing the rotating machine.

Using these three propositions and the techniques and results of Reference (1), one can estimate the off set increments $\Delta \theta_{i}^{1}$ and nutation angles $n_{i}^{1}$ at each stage $i$ as a function of lateral axis machine angular momentum:

a. for phases $3,4,5,8,9 *$ (from proposition 2)

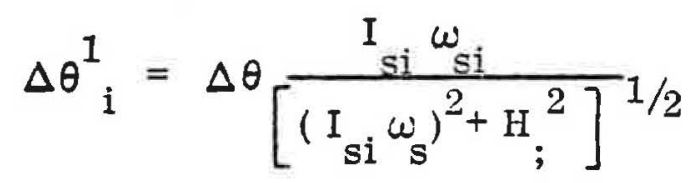

where $\Delta \theta=$ increment during phase for basic vehicle (from Table 2.1 Reference (1) - deg)

$$
\begin{aligned}
& \mathrm{I}_{\mathrm{S}_{\mathrm{i}}}=\text { moment of inertia about nominal spin axis at end of } \\
& \quad \text { phase } \mathrm{i}-\text { slug } \mathrm{ft}^{2} \\
& \omega_{\mathrm{S}}=\text { spin speed }-\mathrm{rad} / \mathrm{sec} \\
& \mathrm{H}_{\mathrm{K}}=\text { angular momentum of lateral axis machine }-\mathrm{ft}-\mathrm{lb}-\mathrm{sec}
\end{aligned}
$$

\footnotetext{
* For phase description see Table D. 2-1.
} 
b. for phase $2 \Delta \theta_{2}^{1}=\Delta \theta$ at Atlas/stage vehicle separation

$$
\eta_{1}^{1}=0
$$

This number is negligible due to the small time that the rates resulting from the small disturbing torques exist.

c. for phase $7 \Delta \theta \frac{1}{7}=\eta_{6}^{1}-\eta_{7}^{1}$ (from analysis in Ref. 1) where $\eta_{6}^{1}, \eta_{7}^{1}$ are half of the nutation angles at end of phases 6,7 as calculated below, for vehicle with lateral angular momentum.

d. for phases 3-9 (from proposition 3)

$$
\left.\eta_{i}^{1}=\left[\frac{I_{i i} \omega_{l i}}{I_{s i} \omega_{s}} \cdot \frac{I_{s i} \omega_{2}}{\left[\left(I_{s i} \omega_{s}\right)^{2}+H_{-l}^{2}\right.}\right]^{1 / 2} \cdot 57.3\right]+\eta_{i h}
$$

where: $\omega_{l i}=$ lateral vehicle rate $-\mathrm{rad} / \mathrm{sec}$

$$
\begin{aligned}
\eta_{i}= & \text { nutation half-cone angle of untorqued vehicle }- \text { deg } \\
& \text { (from Reference } 1 \text { ) } \\
\eta_{i h}= & \text { half maximum nutation angle of untorqued vehicle with } \\
& \text { lateral angular momentum } \mathrm{H}-\mathrm{deg} \text { (from computer } \\
& \text { simulation } \\
\mathrm{I}_{\mathrm{i}}= & \text { moment of inertia about lateral axes at end of phase } \mathrm{i} \\
& -{\text { slug } \mathrm{ft}^{2}}^{2}
\end{aligned}
$$

and for phases $3,4,5,6$

$$
\eta_{\mathrm{i}}=\frac{\mathrm{I}_{\mathrm{i}} \omega_{f}}{\mathrm{I}_{\mathrm{si}} \omega_{\mathrm{s}}} \cdot 57.3
$$

and for phases 7,8

$$
\omega_{l 8} \cong \omega_{l 7} \cong \frac{\eta^{1} 6}{57.3} \cdot \frac{I_{S 6} \omega_{S}}{I_{\ell 6}}
$$


and for phase 9

$$
\omega_{\ell 9}=\left[\left(\frac{I_{88}{ }^{\omega \ell 8}}{I_{\ell 99}}\right)^{2}+\left(\frac{\Delta H}{I_{\ell 9}}\right)^{2}\right]^{1 / 2}
$$

where $\Delta \mathrm{H}=$ incremental lateral angular momentum added during phase $9 \mathrm{ft}-\mathrm{lb}$-sec and since

$$
\begin{aligned}
& \left(\mathrm{I}_{\ell 8} \omega_{\ell 8}\right)^{2}+(\Delta \mathrm{H})^{2}=\left(\mathrm{I}_{\ell 9} \omega_{\ell 9}\right)^{2} \\
& \frac{\Delta \mathrm{H}}{\mathrm{I}_{\mathrm{s}}}=\left[\eta_{9}^{2}-\eta_{8}^{2}\left(\frac{\mathrm{I}_{\mathrm{s} 8}}{\mathrm{I}_{\mathrm{s} 9}}\right)^{2}\right]^{1 / 2} \frac{\omega_{\mathrm{s}}}{57.3}
\end{aligned}
$$

The results of using these equations for lateral angular momenta $\left(\mathrm{H}_{\gamma}\right)$ of 5,15 , and $26.1 \mathrm{ft} / 1 \mathrm{~b} / \mathrm{sec}$ are given in Table $\mathrm{D} .2-1$. The values of $\eta_{\text {ih }}$ were determined by digital computer simulation.

D. 2.2 Trajectory Errors Due To Angular Disturbances

The analysis technique in Reference (2) determines the transfer orbit errors due to:

1. Atlas hand-off errors

2. Atlas/Stage vehicle separation

3. Spin-up

4. Coast

In addition to error sources associated with PKMI burns:

1) PKM Total Impulse Variations

a) $3 \sigma$ variations due to propellant load, propellant performance, manufacturing tolerances, etc., of $0.75 \%$.

b) Variations due to PKM grain temperature variations from the nominal rating temperature of $70^{\circ} \mathrm{F}$.

2) PKM Ignition Time Variations

3) $3 \sigma$ pitch attitude error during burns 
4) 30 yaw attitude error during burns

5) $3 \sigma$ nutation during burns

The results of the analysis is repeated in Table $\mathrm{K}-\mathrm{A} .2 .2$. Nominal three errors are calculated by separately RSS'ing the $3 \sigma$ positive and negative errors.

In this study, the techniques and results of Reference (2) are utilized, together with angular disturbance data from Table D.2-1. Results of this re-application are given in Tables D. 2-3, D. 2-4, and D. 2-5.

Since nutation during PKM burn always shows itself as a reduction in the velocity increment for that burn, and since nutation is an inherent characteristic of the vehicle with lateral angular momentum, it may be concluded that the true 3 apogee error, for apogee high, is less than that indicated in the tables.

$\mathrm{K}-\mathrm{A} \cdot 2.3 \quad$ Conclusions

A comparison of the transfer orbit trajectory errors for the basic GPS vehicle and for vehicles with lateral angular momenta up to at least $26 \mathrm{ft} / \mathrm{lb} / \mathrm{sec}$ shows no major differences.

The maximum difference occurs for $\mathrm{H}_{\ell}=26.1 \mathrm{ft} / \mathrm{lb} / \mathrm{sec}$ in the $3 \sigma$ apogee radial distance. It is only $36 \mathrm{~km}$ less than the basic vehicle's total error of $530 \mathrm{~km}$ (apogee low). The nominal specification value is $525 \mathrm{~km}$. The incremental error is primarily due to inherent loss in along-track velocity increment due to the relatively large nutation angle (4.97 deg) which exists during the PKM 2 burn. Thus, by altering the nominal drift orbit to have a somewhat higher apogee, one can somewhat reduce the true apogee error (apogee low) while still keeping the apogee high error within specification.

It is useful to note, as Reference (2) does, that " Considering only Atlas errors and PKM (which are GFE) impulse variations of $\pm 0.75 \%$, the resulting apogee dispersion is $\pm 514.4 \mathrm{~km}$. . . "If the "true " $3 \sigma$ impulse variation is 0.9 of the nominal then the total apogee altitude error would be only $525 \mathrm{~km}$ (apogee low). 
TABLE K-A. 2-2

PREDICTED $3 \sigma$ TRANSFER ORBIT ERRORS

\begin{tabular}{|c|c|c|c|c|c|}
\hline \multirow[b]{2}{*}{ PIIASE } & \multirow{2}{*}{$\begin{array}{l}\text { ERROR } \\
\text { SOURCE }\end{array}$} & \multirow{2}{*}{$\begin{array}{l}\text { ERROR } \\
\text { MAGNITUDE }\end{array}$} & \multicolumn{3}{|c|}{$` \quad$ ERROR EFFECTS } \\
\hline & & & $\begin{array}{c}\text { APOGEE RADIUS } \\
(\mathrm{kM})\end{array}$ & $\begin{array}{l}\text { PERIGEE RADIUS } \\
(\mathrm{kM})\end{array}$ & $\begin{array}{c}\text { INCLINATION } \\
\text { (DEG) }\end{array}$ \\
\hline ATLAS & $\begin{array}{l}\text { ALTITUDE } \\
\text { VELOCITY MAGNTIUDE } \\
\text { FLIGIIT PATII ANGLE } \\
\text { ORBIT INCLINATION }\end{array}$ & $\begin{array}{l} \pm 2000 \mathrm{FT}) \\
\pm 15 \mathrm{FT} / \mathrm{SEC}) \\
\pm 0.1 \mathrm{DEG} \\
\pm 0.2 \mathrm{DEG}\end{array}$ & $\begin{array}{l} \pm 15.1 \\
\pm 125.3 \\
\mp 18.0 \\
\mp 3.4\end{array}$ & $\begin{array}{r} \pm 0.6 \\
0 \\
\pm 2.2 \\
0\end{array}$ & $\begin{array}{c}0 \\
0 \\
0 \\
\pm 0.189\end{array}$ \\
\hline $\begin{array}{l}\text { PKM } 1 \\
\text { BURN }\end{array}$ & $\begin{array}{l}\text { IMPULSE } \\
\text { GRAIN TEMP VARLATIONS } \\
\text { IGNITION TIME* } \\
\text { PIICI A'T'TITUDE* } \\
\text { YAW A'TTITUDE* } \\
\text { NUTATION }\end{array}$ & $\begin{array}{l} \pm 0.75 \% \\
+10,-15^{\circ} \mathrm{F} \\
\pm 1 \mathrm{SEC} \\
\pm 1.84 \mathrm{DEG} \\
\pm 1.84 \mathrm{DEG} \\
0.78 \mathrm{DEG}\end{array}$ & $\begin{array}{l} \pm 226.7 \\
+4.5,-21.8 \\
\pm \quad 1.4 \\
-90.0 \\
+\quad 29.5 \\
-30.0 \\
-\quad 2.8\end{array}$ & $\begin{array}{r}0 \\
0 \\
\mp 0.2 \\
+4.9 \\
-6.8 \\
0 \\
0\end{array}$ & $\begin{array}{c}0 \\
0 \\
0 \\
0 \\
\pm 0.546 \\
0\end{array}$ \\
\hline $\begin{array}{c}\text { PKM } 2 \\
\text { IURN }\end{array}$ & $\begin{array}{l}\text { IMPULSE } \\
\text { GRAIN TEMP. VARIATIONS } \\
\text { IGNITION TIME } \\
\text { PITCII A'TTITUDE } \\
\text { YAW A'ITITUIDE } \\
\text { NUTATION }\end{array}$ & $\begin{array}{l} \pm 0.75 \% \\
+10,-15^{\circ} \mathrm{F} \\
\pm 4 \mathrm{SEC} \\
\pm 1.07 \mathrm{DEG} \\
\pm 1.07 \mathrm{DEG} \\
1.266 \mathrm{DEG}\end{array}$ & $\begin{array}{l} \pm 443.8 \\
+28.4,-42.6 \\
\mp \quad 7.7 \\
-65.0 \\
+49.0 \\
-\quad 8.0 \\
-14.4 \\
\end{array}$ & $\begin{array}{r}0 \\
0 \\
\mp 0.6 \\
+0.7 \\
-1.0 \\
0 \\
0\end{array}$ & $\begin{array}{r}0 \\
0 \\
0 \\
0 \\
+0.216 \\
0 \\
\end{array}$ \\
\hline & & & $+518.6 / 529.6$ & $+5.5 /-7.3$ & \pm 0.617 \\
\hline
\end{tabular}


TABLE K-A. 2-3;

PREDICTED $3 \sigma$ TRANSFER ORBIT ERRORS

\begin{tabular}{|c|c|c|c|c|c|}
\hline \multirow[b]{2}{*}{ PHASE } & \multirow{2}{*}{$\begin{array}{l}\text { ERROR } \\
\text { SOURCE }\end{array}$} & \multirow{2}{*}{$\begin{array}{l}\text { ERROR } \\
\text { MAGNITUDE }\end{array}$} & \multicolumn{3}{|c|}{$\therefore$ ERROR EFFECTS } \\
\hline & & & $\begin{array}{l}\text { APOGEE RADIUS } \\
(\mathrm{kM})\end{array}$ & $\begin{array}{l}\text { PERIGEE RADIUS } \\
\text { (kM) }\end{array}$ & $\begin{array}{l}\text { INCLANATION } \\
\text { (DNG) }\end{array}$ \\
\hline ATLAS & $\begin{array}{l}\text { ALTIIUDE } \\
\text { VELOCITY MAGNITUDE } \\
\text { FLIGHT PATII ANGLE } \\
\text { ORBIT INCLINATION }\end{array}$ & $\begin{array}{l}(2000 \mathrm{FT}) \\
\pm 15 \mathrm{FT} / \mathrm{SEC}) \\
\pm 0.1 \mathrm{DEG} \\
\pm 0.2 \mathrm{DEG}\end{array}$ & $\begin{array}{l} \pm 15.1 \\
\pm 125.3 \\
\mp 18.0 \\
\mp 3.4\end{array}$ & $\begin{array}{r} \pm 0.5 \\
0 \\
\pm 2.2 \\
0\end{array}$ & $\begin{array}{c}0 \\
0 \\
0 \\
\pm 0.189\end{array}$ \\
\hline $\begin{array}{l}\text { PKM } 1 \\
\text { BURN }\end{array}$ & $\begin{array}{l}\text { IMPULSE } \\
\text { GRAIN TEMP VARIATIONS } \\
\text { IGNITION TIME* } \\
\text { PITCII ATTIIUDE* } \\
\text { 'YAW ATTITUDE* } \\
\text { NUTA'TION }\end{array}$ & $\begin{array}{l} \pm 0.75 \% \\
+10,-15^{\circ} \mathrm{F} \\
\pm 1 \mathrm{SEC} \\
\pm 1.84 \mathrm{DEG} \\
\pm 1.84 \mathrm{DEG} \\
1.2 \mathrm{DEG}\end{array}$ & $\begin{array}{l} \pm 226.7 \\
+ \pm 4.5,-21.8 \\
\pm \quad 1.4 \\
-90.0 \\
+20.5 \\
-30.0 \\
-\quad 6.6 \\
\end{array}$ & $\begin{array}{r}0 \\
\mp 0 \\
\mp 0.2 \\
+4.9 \\
-0.8 \\
0 \\
0\end{array}$ & $\begin{array}{c}0 \\
0 \\
0 \\
0 \\
\pm 0.546 \\
0\end{array}$ \\
\hline $\begin{array}{r}\vdots \\
\text { PKM } 2 \\
\text { 3URN }\end{array}$ & $\begin{array}{l}\text { IMPULSE } \\
\text { GRAIN TEMP. VARIATIONS } \\
\text { IGNTION TIME } \\
\text { PITCII ATTITUDE } \\
\text { YAW AT'TTTUDE } \\
\text { NUTATION }\end{array}$ & $\begin{array}{l} \pm 0.75 \% \\
+10,-15^{\circ} \mathrm{F} \\
\pm 4 \mathrm{SEC} \\
\pm 78 \mathrm{DEG} \\
\pm 73 \mathrm{DEG} \\
2.53 \mathrm{DEG} \\
\end{array}$ & $\begin{array}{l} \pm 443.8 \\
+28.4,-42.6 \\
\mp \quad 7.7 \\
-46 . \\
+38 . \\
-4 \\
-57.7 \\
\end{array}$ & $\begin{array}{r}0 \\
0 \\
\mp 0.6 \\
+0.8 \\
-0.8 \\
0 \\
0 \\
\end{array}$ & $\begin{array}{r}0 \\
0 \\
0 \\
0 . \\
\pm 0.158 \\
0 \\
\end{array}$ \\
\hline IRS TOT & & & $+517 /-530$ & $+5.5 /-7.3$ & \pm .60 \\
\hline
\end{tabular}

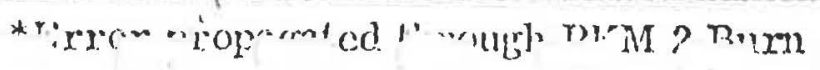


rist.k; K-A. 2-4

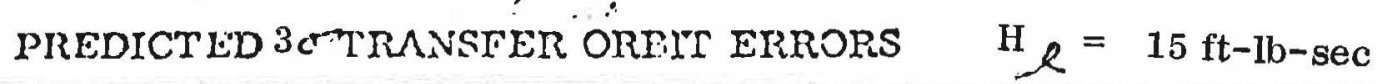

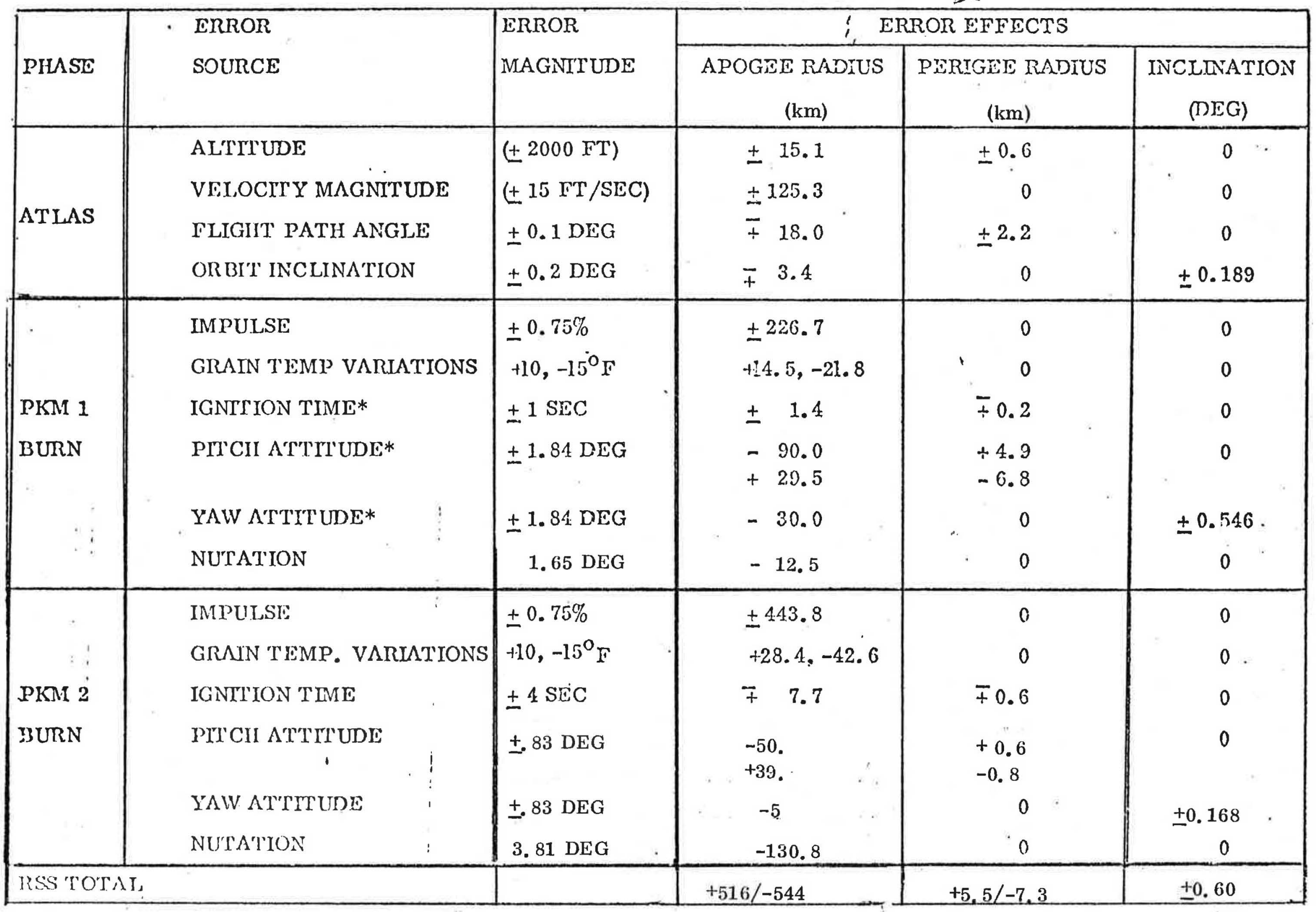

Error propagated through PKM 2 burn. 


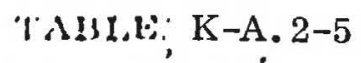

PREDICTED 3o TRANSFER OHRIT ERRORS

${ }^{H} \ell=26.1 \mathrm{ft}-1 \mathrm{~b}-\mathrm{sec}$

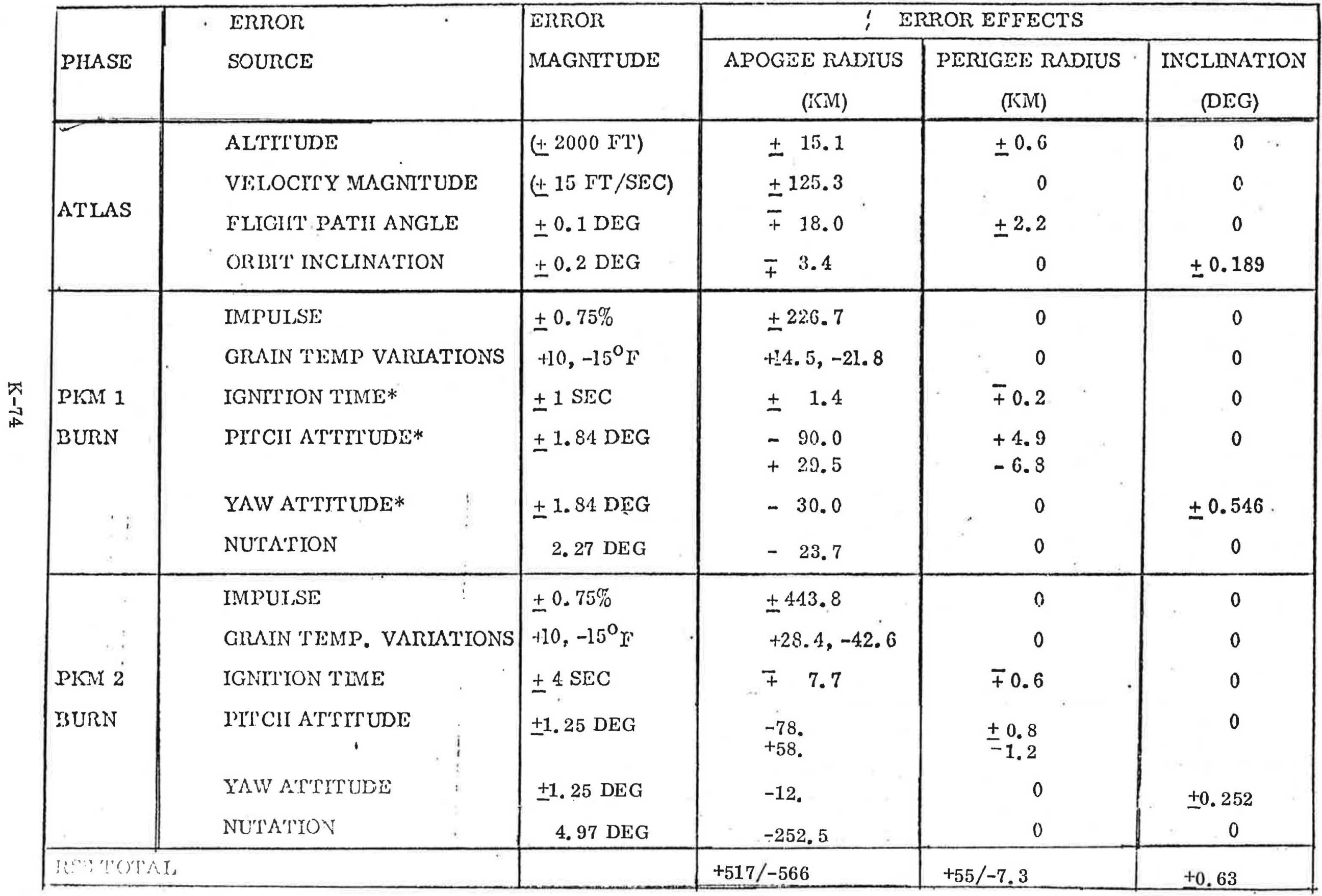

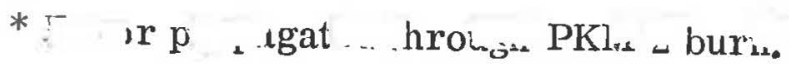


It therefore appears that lateral oriented machines in the GPS do not cause unacceptable orbit trajectory errors.

\section{$\underline{\text { References }}$}

Reference (1) Report No. 309-CD-0501" Stage Vehicle System, Subsystem Design Analysis Report, Stability and Control Analysis CDRL No. A005, dated 28 May 1975, and prepared for SAMSO under Contract Number F04701-75-C-0028, by Fairchild Space and Electronics Company.

Reference (2) Report No. 309 - CD-0601" Stage Vehicle System, Subsystem Design Analysis Report Error Analysis" CDRL No. A006, dated 30 May 1975, and prepared for SAMSO under Contract Number F04701-75-C-0028, by Fairchild Space and Electronics Company.

K-A.3 $\quad$ Reaction Wheel/Propellant Weight Tradeoffs

K-A.3.1 Introduction

A candidate revision of the GPS space vehicle may contain a laterally oriented rotating machine.

At launch into its drift orbit, the vehicle is spun up about its longitudinal axis in an attempt to ameliorate the misalignment effects of the drift orbit insertion engines. It is shown above that at drift orbit apogee the resultant motion of the vehicle, after nutation has damped out, is a rotation at constant speed about a vehicle-fixed axis displaced (by angle $\alpha$ ) from the vehicle longitudinal axis. This displacement causes a loss in velocity increment from the orbit insertion system engine which thrusts along the longitudinal axis.

A trade-off now exists between the propellant weight increment necessary to make-up for the velocity loss and the weight of a reaction wheel, aligned with rotating machine spin, and operating so as to decrease the angle offset $\alpha$.

This study considers this trade-off relation.

$\mathrm{K}-\mathrm{A} \cdot 3.2 \quad$ Analysis

$\mathrm{K}-\mathrm{A} .3 .2 .1 \quad$ Propellant Requirements

Reference (1) evaluates the velocity loss $\Delta V$, in the normalized ratio $\Delta \mathrm{V} / \mathrm{V},(\mathrm{V}=$ total velocity increment), as a function of lateral axis angular momentum 
H $e^{.}$These results are shown in Table K-A.3-1. Here, the value of the total velocity increment is first calculated. Then, the fuel weight necessary to increment the velocity is calculated by using the Rockwell estimate of propellant capability at $3 \mathrm{Ib}$, per $23 \mathrm{ft} / \mathrm{sec}$.

The vis-viva integral for elliptical orbits about the earth is:

$$
v^{2}=\mu\left(\frac{2}{r}-\frac{1}{a}\right)
$$

where $\mathrm{v}=$ velocity in orbit

$$
\begin{aligned}
& \mu=\text { gravitational constant for the earth } \\
& \mathbf{r}=\text { orbit radius } \\
& \mathbf{a}=\text { orbit semi-major axis }
\end{aligned}
$$

Drift orbit perigee $=$ earth radius $+77 \mathrm{~nm}$

$$
\begin{aligned}
r_{p} & =3438+77 \mathrm{~nm} \\
& =3515 \mathrm{~nm}
\end{aligned}
$$

Drift orbit apogee $=$ earth radius $+10818 \mathrm{~nm}$

$$
\begin{aligned}
\mathbf{r}_{\mathrm{a}} & =3438+10818 \mathrm{~nm} \\
& =14,256 \mathrm{~nm}
\end{aligned}
$$

Semi-major axis $=\frac{3515+14256}{2} \mathrm{~nm}$

$$
a=8885 \mathrm{~nm}
$$

transfer orbit apogee velocity $=\frac{\mathrm{V}_{\mathrm{xa}}}{\mathrm{V}_{\mathrm{c}}}$
circular orbit velocity

$$
\begin{aligned}
& =\left[\begin{array}{l}
\mu\left(\frac{2}{r_{a}}-\frac{1}{a}\right) \\
\mu\left(\frac{2}{r_{a}}-\frac{1}{r_{a}}\right)
\end{array}\right]^{1 / 2} \\
& =0.63
\end{aligned}
$$

Therefore, $\Delta \mathrm{V}=0.37 \mathrm{~V}_{\mathrm{c}}$

Also $\mathrm{V}_{\mathrm{c}}=12752 \mathrm{ft} / \mathrm{sec}$.

So that the increment for circularization $\Delta \mathrm{V}$ is $4718 \mathrm{ft} / \mathrm{sec}$.

Table K-A.3-1 contains the numerical values of interest. 


\begin{tabular}{|c|c|c|c|}
\hline \multicolumn{4}{|r|}{ Table $\mathrm{K}-\mathrm{A} .3-1$} \\
\hline $\mathrm{ft} . \mathrm{lb} . \mathrm{sec}$. & $\Delta \mathrm{V} / \mathrm{V}_{\mathrm{c}}$ & $\Delta \mathrm{V}$ & Fuel Weight \\
\hline 1.0 & & $\mathrm{ft} / \mathrm{sec}$. & $1 \mathrm{~b}$. \\
\hline 5.0 & & 0.7 & 0.09 \\
10.0 & .0008 & 3.78 & 0.49 \\
15.0 & .0030 & 14.15 & 1.85 \\
20.0 & .0068 & 32.08 & 4.18 \\
25.0 & .0120 & 56.62 & 7.39 \\
\hline
\end{tabular}

Values of propellant weight versus net angular momentum are plotted in the graph of Figure $\bar{K}-\mathrm{A} .3-1$

K-A.3.2.2 Reaction Wheel

Figure D. 3-2 contains an accumulation of current reaction wheel weight versus angular momentum capability. A curve matching these points is indicated.

Numerical values from Figure K-A.3-1 are repletted in Figure K-A.3-2 so as to show the reduced propellant requirements when a reaction wheel cancels some of the lateral rotating machine's angular momentum (R. M.). For example, if R. M. = $20 \mathrm{ft}$. lb. sec. then $7.4 \mathrm{lb}$. of propellant are required. If a $4 \mathrm{ft}$. lb. sec. reaction wheel weighing $16.2 \mathrm{lbs}$, is added the propellant requirement is reduced to $4.7 \mathrm{lbs}$.

K-A.3 Results

Over the range of net angular momenta shown in Figure K-A.3-2 there is no net weight saving when a reaction wheel is used to cancel some of the rotating machine's angular momentum. An uncompensated rotating machine should be used. If a reaction wheel is required, perhaps for attitude control reasons, it should have the smallest size suitable to meet those needs. Any larger size is unprofitable from a total sy stem weight requirement view-point. 


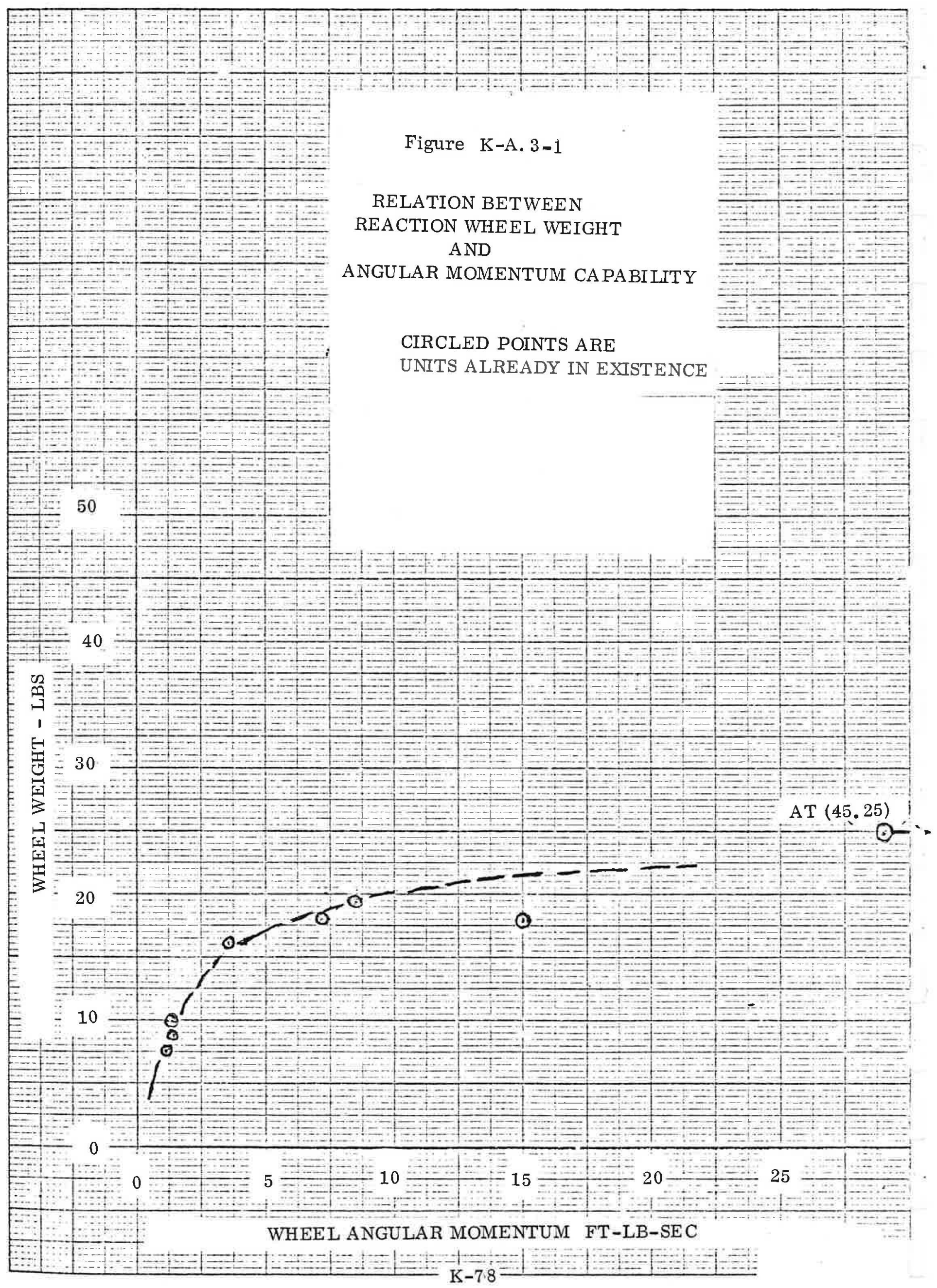


Figure $D_{\text {. 3-2 }}$

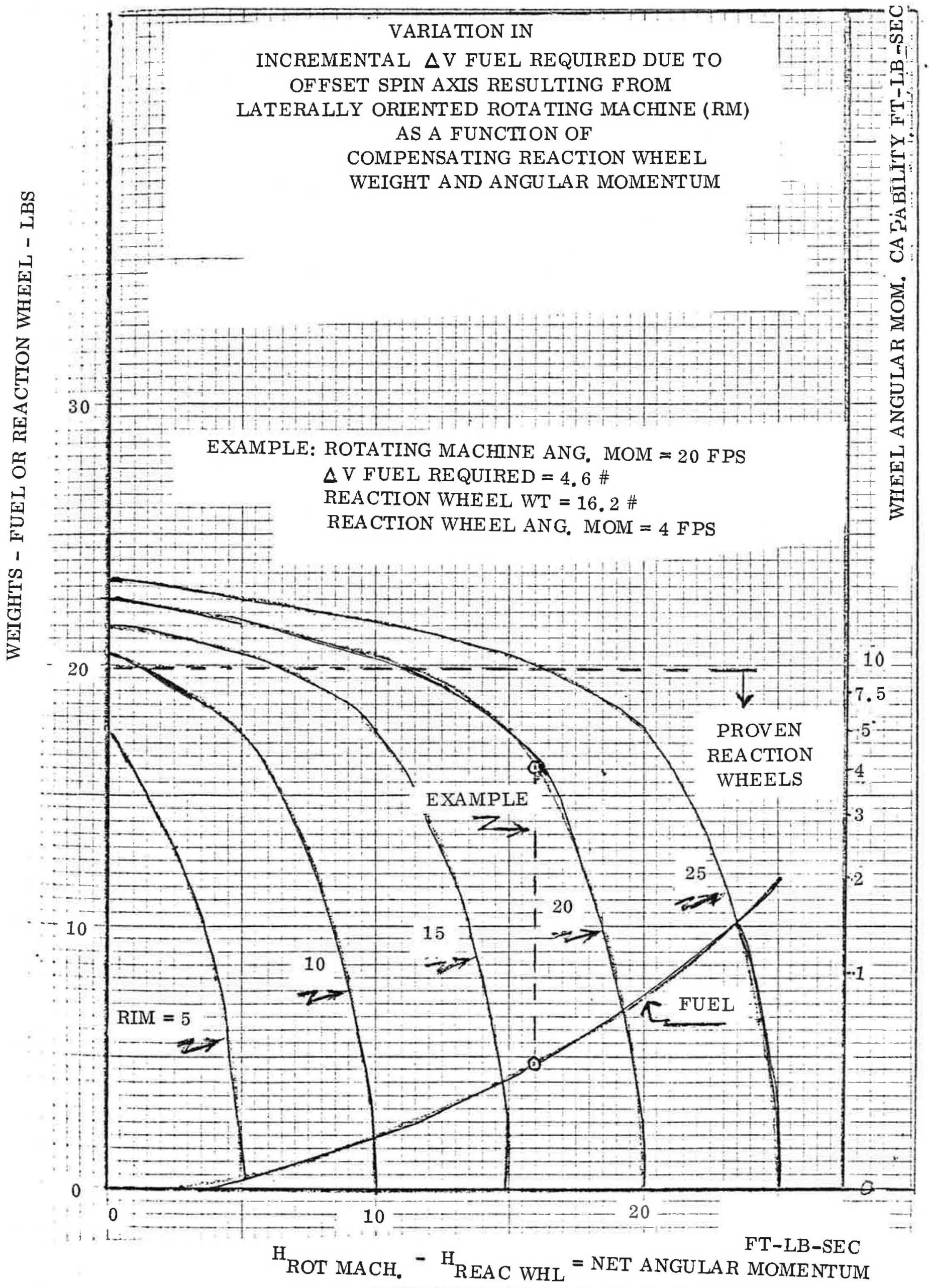


APPENDIX $K-B$

THERMAL ANALYSES 


\section{K-B.1 $\quad$ Radiator Sizing Evaluations}

Analyses pertaining to RTG, ORC and BIPS radiator sizings were performed and Teledyne, Airesearch, and Sunstrand's sizing of RTG, ORC, and BIPS radiators to handle 2400 watts heat source have been verified. Parametric data on radiator designs to handle both 2400 and 3200 watt heat sources are given by figures $-\mathrm{K}-\mathrm{B}-1$ through $\mathrm{K}-\mathrm{B}-5$. Data given is preliminary and is to be consider as "first cut estimates" for radiator sizing.

Teledyne's concept of using heat pipes in the radiators to cool the RTG system were evaluated first. Teledyne proposed using copper/water, grooved heat pipes. The pipes are spaced approximately 2.70 inches apart on a 0.02 aluminum panel. In order to stiffen the radiator panel, tapered ribs (0.02 Alum) are used. The pipes are located at the junction between the ribs and panel. The evaporator length of pipe was designed to eliminate the possibility of nucleate boiling of the water. The fundamental heat flux limit of the evaporator is a maximum of $476,000 \mathrm{BTU} / \mathrm{O}_{\mathrm{R}-\mathrm{ft}}{ }^{2}$ (Reference Dynatherm Heat Pipe Design Handbook). The evaporator heat flux in the proposed design is:

$$
\text { (q) evap. }=170.0 \times 3.414 / \frac{(3 / 16) \Delta \mathrm{T} 3.25}{144}=44,644 \mathrm{BTU} /{ }^{\circ} \mathrm{R} \mathrm{ft}{ }^{2}
$$

The original configuration had a number of bends in the heat pipe and the evaporator section turned in toward the spacecraft's spin axis. During transfer orbit (45 hours), the GPS spins at 100 RPM in order to maintain stabilization. At this spin rate, approximately $10 \mathrm{~g}$ centrifugal acceleration is acting on the water inside the pipes. This high centrifugul acceleration forces the water into the pipe bends and also prevents the liquid from reaching the evaporator. 


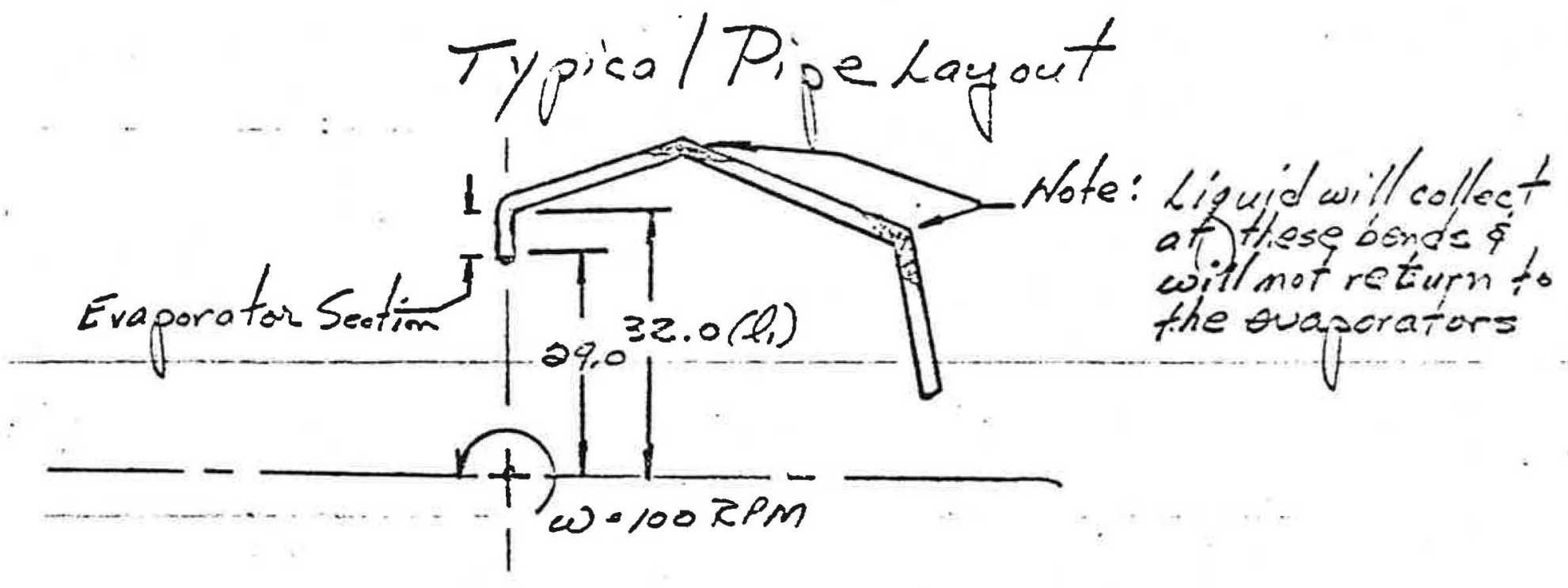

The phenomenon shown above can be demonstrated mathematically. The amount of pressure head needed to pump the water against $10 \mathrm{~g}$ can be computed.

$$
(\Delta P)_{\text {needed }}=\rho_{1} \omega^{2} / 2 g\left(l_{1}^{2}-l_{2}^{2}\right)=60(10.47)^{2} / 2 \times 32.2\left(2.67^{2}-2.4^{2} 2\right)=1310^{\prime}
$$

However, the approximate capillary pressure is:

$(\triangle P)$ available $=2 \sigma \cos \theta / \omega$

$\theta=$ contact angle $=0$

$\mathrm{w}=$ is assumed groove width $(\mathrm{ft})=0.0025$

$\sigma=$ surface tension of $\mathrm{H}_{2} \mathrm{O}=3.4 \times 10^{-3} \mathrm{lbs} / \mathrm{ft}$.

$\therefore(\Delta P)_{\text {available }}=2 \times 3.4 \times 10^{-3} / 0.0025=2.8 \mathrm{lb} / \mathrm{ft}^{2}$

Obviously, the fluid cannot move against such a high acceleration. 
Fairchild proposes that the sharp bends be removed and a constant large radius bend be put into the pipes, and that the evaporator end of the pipes be bent outward. Also, in order to improve the radiator efficiency, the ribs should be moved to the outside of the panel. A thermal math model was constructed that represents the proposed configuration. (See Figure K-B-1. Also, given in Figure K-B-1 are the detailed steady state temperatures $\left({ }^{\circ} \mathrm{F}\right)$ along a typical pipe, rib, and fin composite. Figure K-B-2 shows the temperature gradient along the pipe wall in relation with the vapor temperature. As the assumed radiator and heat pipe radius is increased, the vapor temperature in the pipes decrease. This general relation is shown in Figure E-3 for no sun and full sun conditions and for 2400 and 3200 watt heat sources. In order to minimize weight, the radiator should be sized as to give a steady state vapor temperature of approximately $300^{\circ} \mathrm{F}$. The heat transport factor of water reaches its maximum value at a temperature of $300^{\circ} \mathrm{F}$. (Reference Dynatherm Handbook, Part 1, Page F-33).

Airesearch's concept of a deployable radiator could actually be used for both the BIPS and KIPS. Figures $\mathrm{K}-\mathrm{B}-4$ and $\mathrm{K}-\mathrm{B}-5$ are general working curves for a 72 in. diameter stationary radiator plus deployable radiators of various lengths. The height of stationary and deployable radiators is assumed to be 23.9 inches. Both the stationary and deployable radiators are assumed to be completely covered with Z-93 thermal control paint. In both Figures $\mathrm{K}-\mathrm{B}-4$ and $\mathrm{K}-\mathrm{B}-5$, the radiator temperatures are given as a function of Gross Cycle Efficiency. The Gross Cycle Efficiency for both the BIPS and KIPS varies between 15 and $20 \%$. 


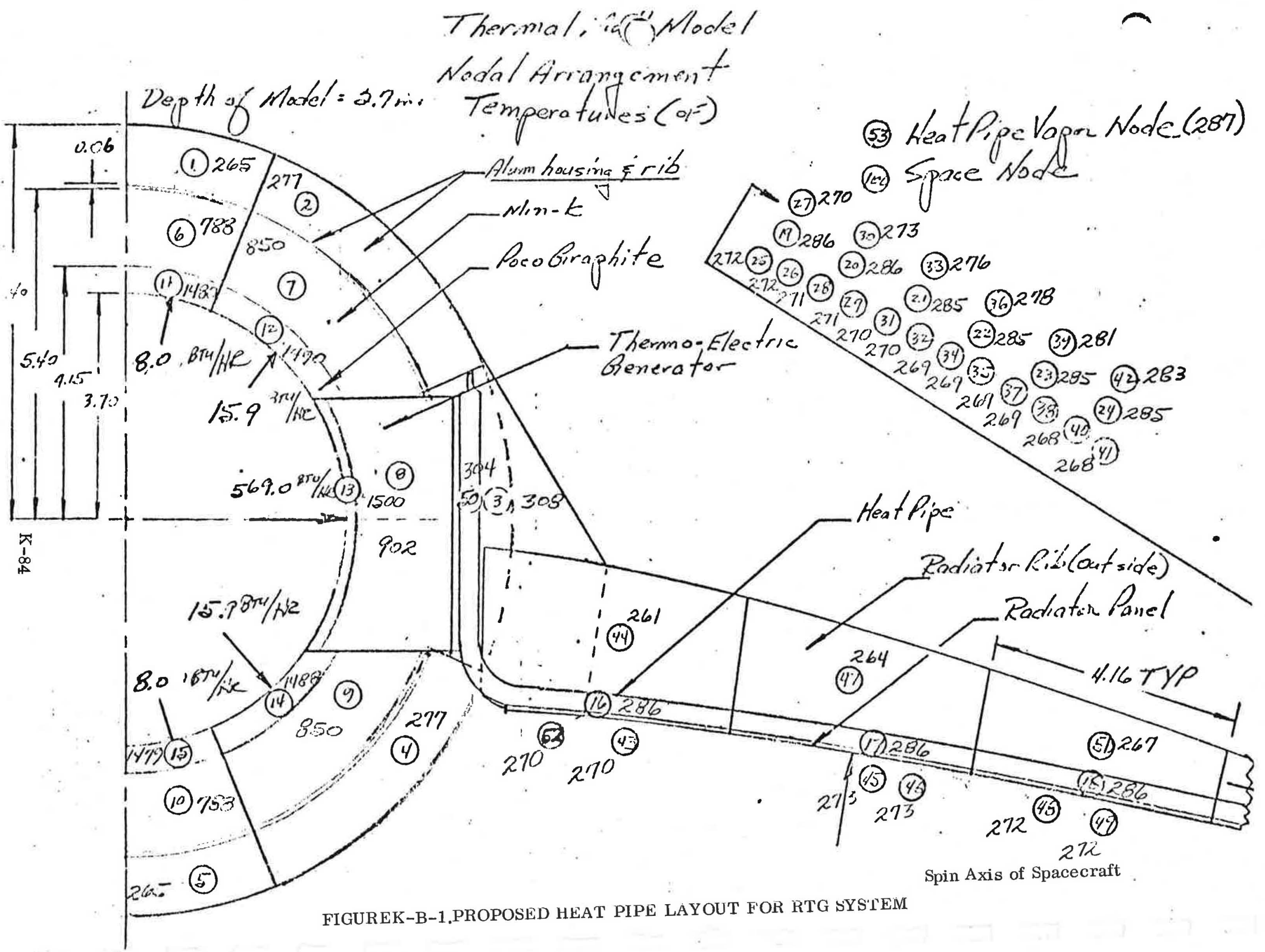


FIGURE K-B-2. TYPICAL TEMPERATURE GRADIENT ALONG RADIATOR HEAT PIPE

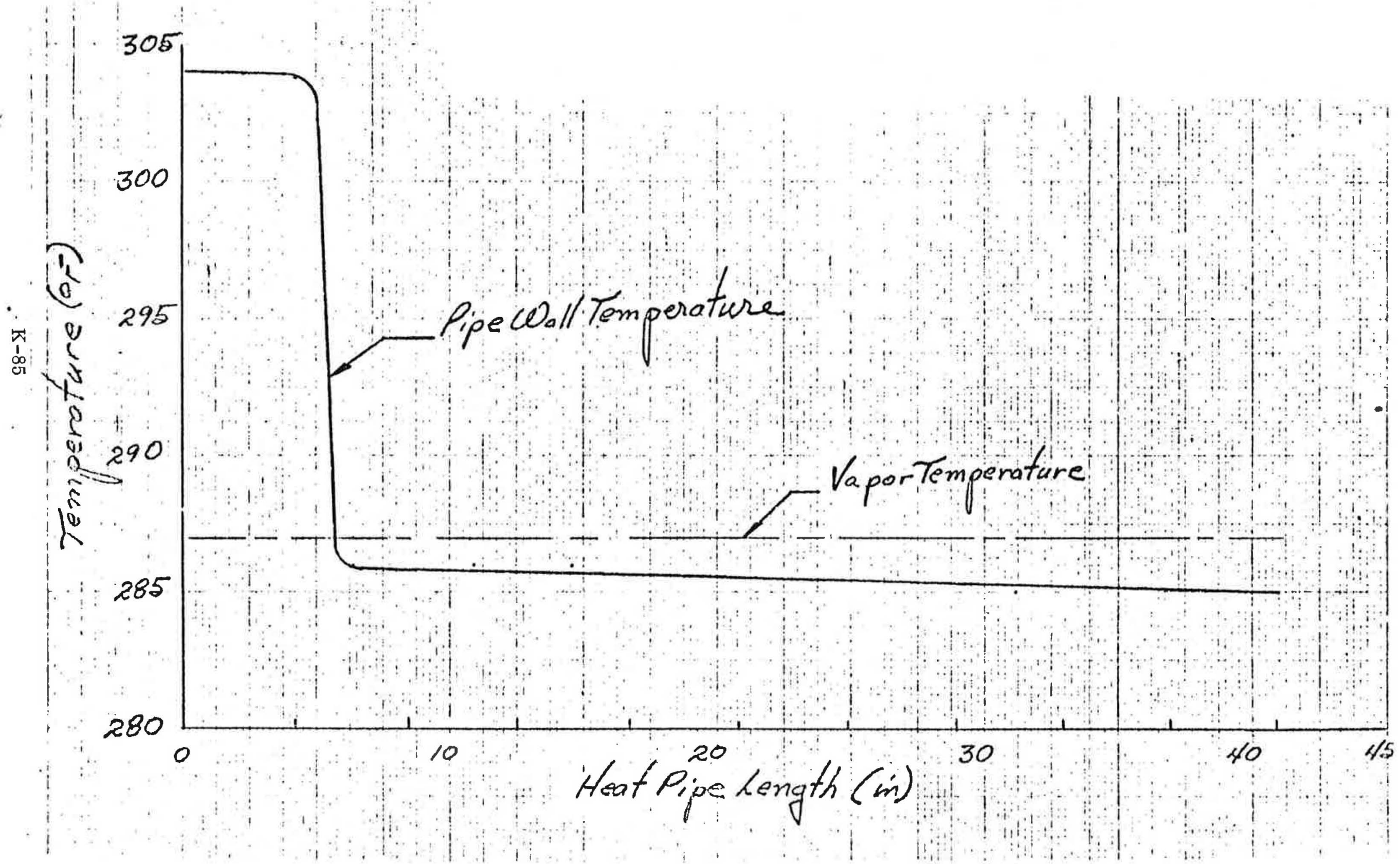


LCHPG INSTALLATION ON GPS

HEAT PIPE VAPOR TEMPERATURE

RADIATOR RADIUS

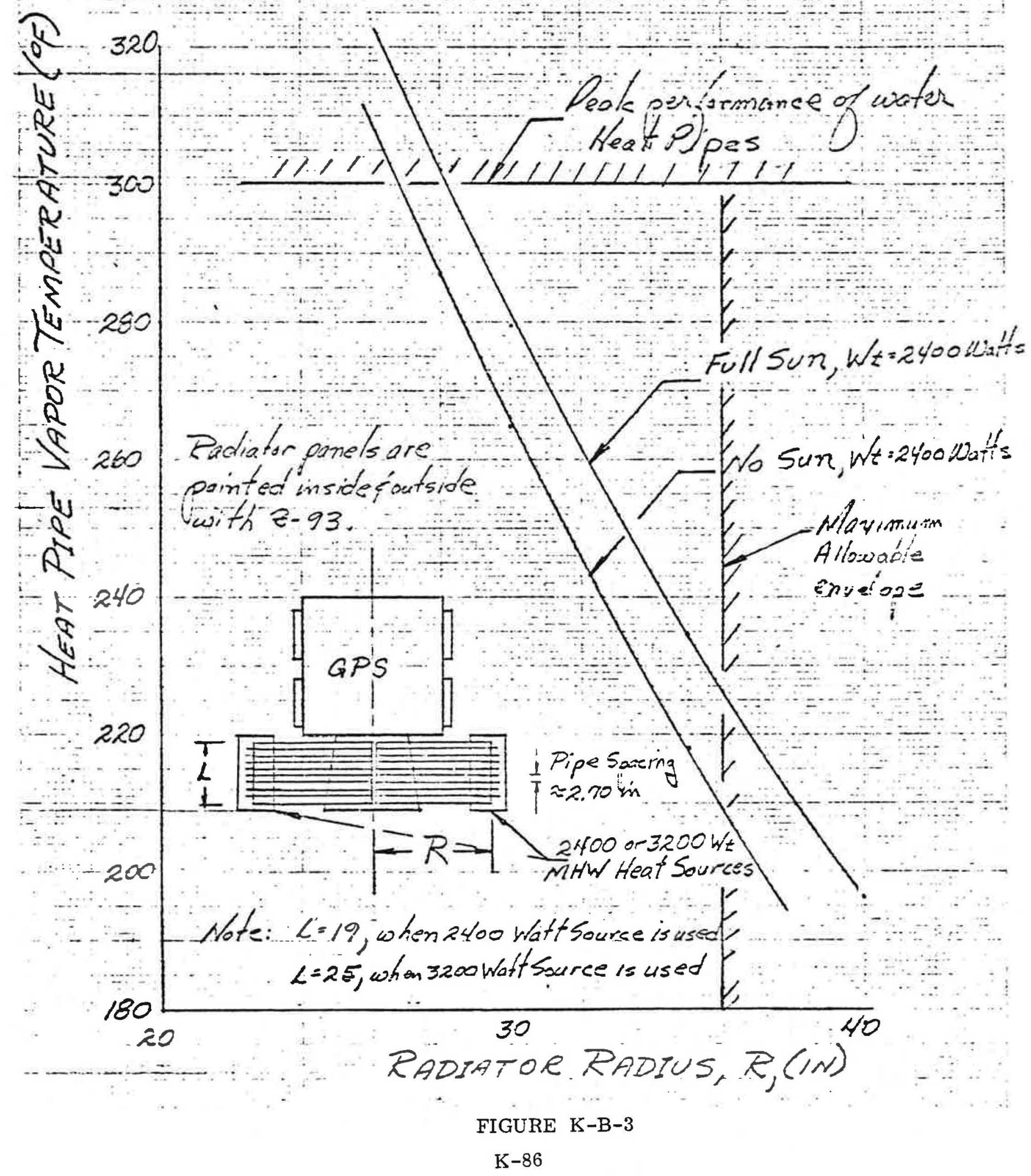


KIPS BIDS STOWED RADIATOR TEMPERATURES

-2400 Watt Heat Sourcellsed. 3200 Walt Heat Source Wised.

Note:

- All radiator surfaces racers with $=-93$

- Radius ofradiaforso 36 mites

Height 8 radiators $=23.9$ niches

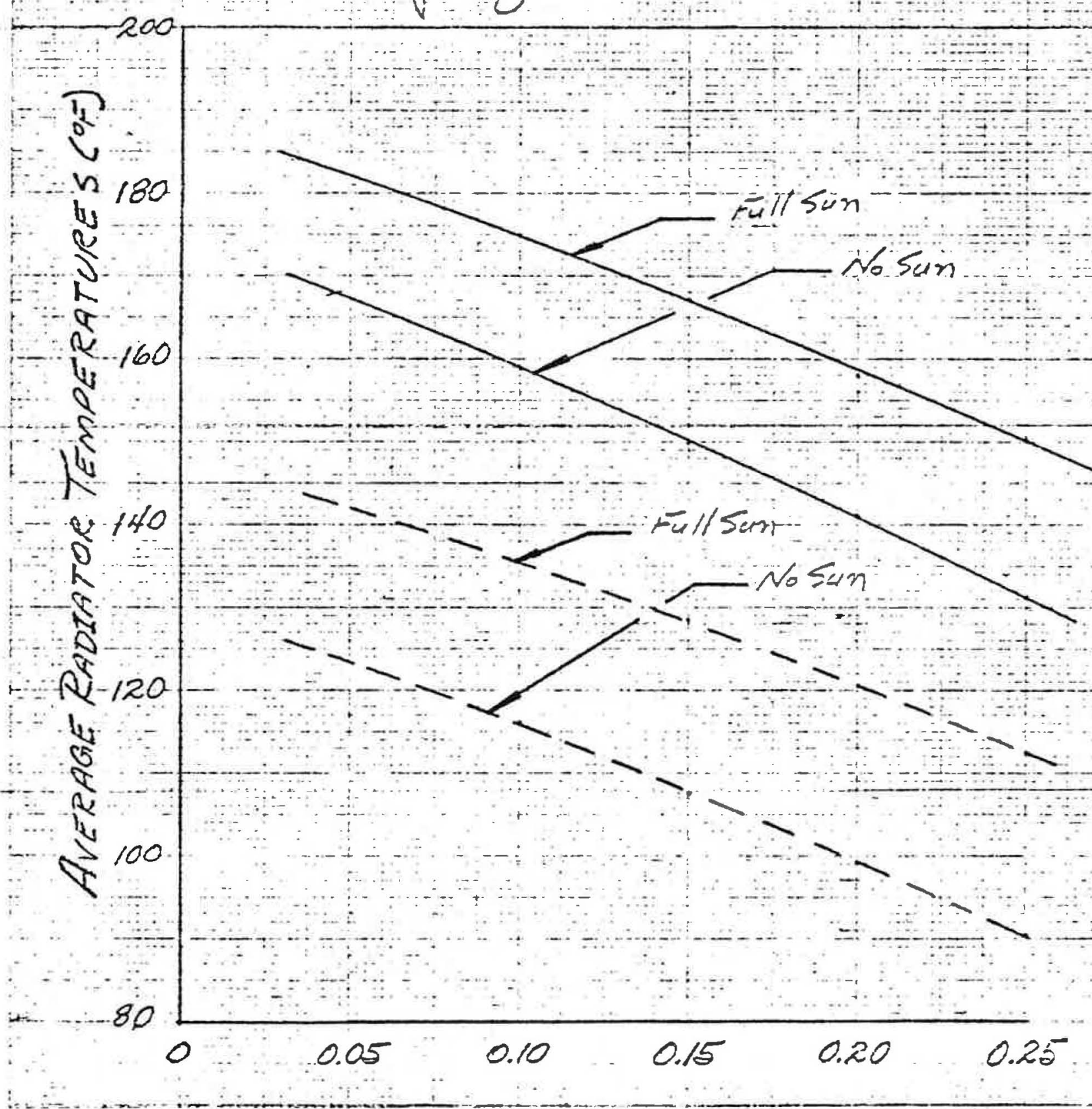

CROSS CYCLE EFFICIENCY

FIGURE K-B-4

$\mathrm{K}-87$ 
KIPSEBIPS DEPLOYED RADIATOR TEMPERATURES

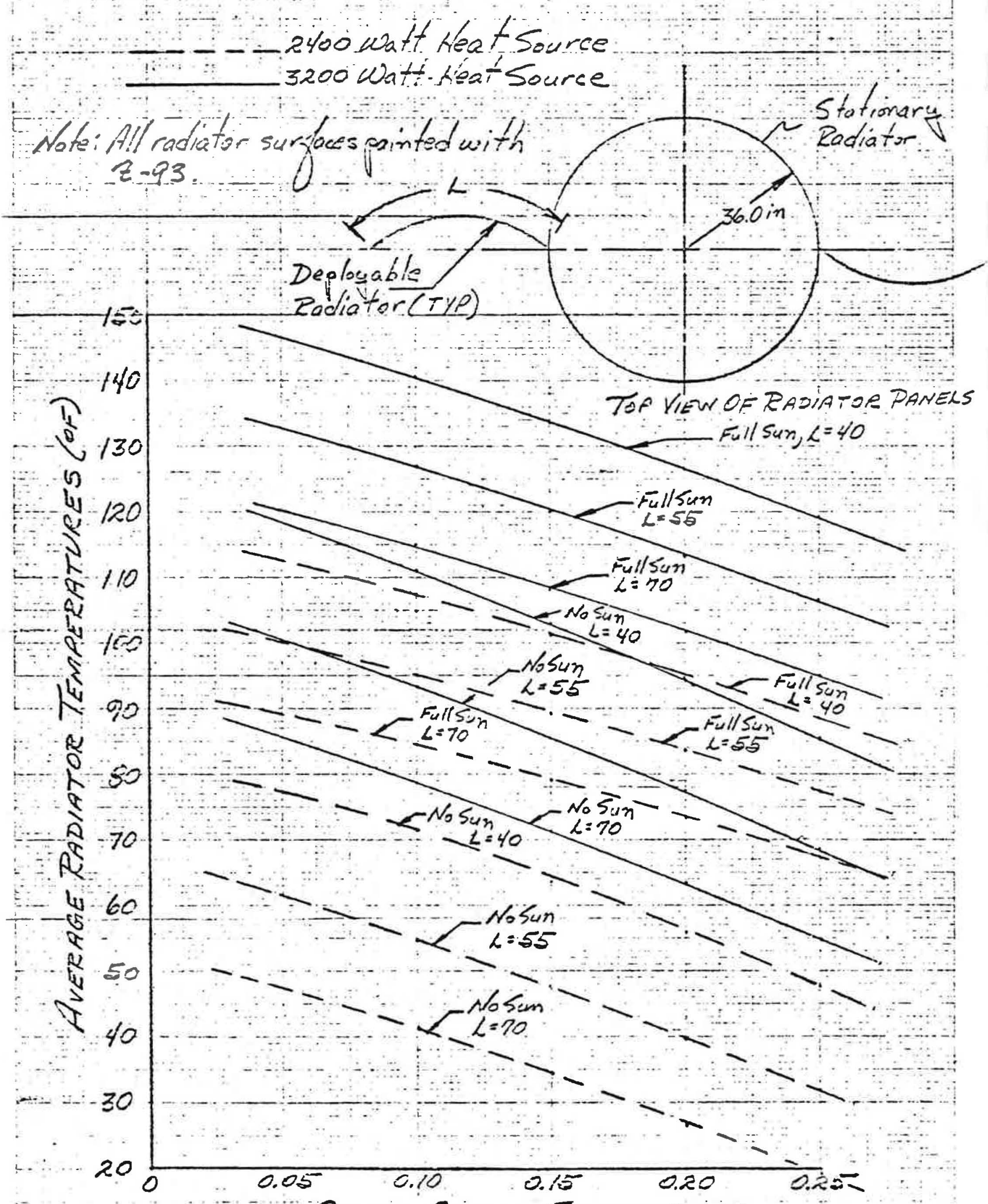

CROSS CYCLE EFFICIENCY

FIG $\underset{\mathrm{K}-88}{\mathrm{R}-8-\mathrm{B}-5}$ 
K-B-2 Changes Required to Current GPS Thermal Control Subsystem

This section presents preliminary findings from investigations which were conducted to evaluate various potential changes to the thermal control subsy stem of the GPS. These changes are being studied as a part of the present study to substitute a nuclear power source for the existing solar powered subsystem. Significant to thermal control of a nuclear powered spacecraft will be the absence of the stowed arrays during transfer orbit, absence of reflected and I. R. inputs from the arrays to the radiator surfaces when the arrays are deployed, and the effect of a relatively constant and predictable heat load in the equipment housing.

Although final design parameters and many details of the GPS configuration are unknown, it is possible to make comparisons and discuss the relative merits of the various alternatives.

Current Design

The GPS louvers are furnished to Rockwell by Fairchild Industries. There are seven louvers per ship; each measures 15.6 × 16.54 inches for a total area of $12.53 \mathrm{ft}^{2}$. Effective emittance in the open position is specified at 0.70 ; in the closed position it is 0.14 . Since these values are with respect to a black baseplate $(\epsilon=0.9)$ and Rockwell is using a silver teflon radiator $\left(\epsilon^{-}=0.76\right)$; the corrected values become

$$
\begin{aligned}
& F_{\epsilon}=0.7 \frac{.76}{.9}=0.591 \quad \text { Open } \\
& F_{\epsilon}=0.14 \frac{.76}{9}=0.12 \quad \text { Closed }
\end{aligned}
$$

The louvers are mounted to the plus and minus $Y$ facing sides which, because of solar syn. yaw control, receive no solar input during orbit. Neglecting inputs from the extended arrays, the maximum heat rejection through the louvers at $90^{\circ} \mathrm{F}$ is 


$$
\mathbf{Q}=0.591 \frac{12.53}{3.413} \sigma \mathrm{T} /^{4}=340 \text { watts }
$$

(In the closed position, 55 watts will suffice to maintain $60^{\circ} \mathrm{F}$, )

\section{Elimination of Yaw Control}

Yaw control is necessary to maximize power output from the solar arrays. It conceivably could be eliminated with the substitution of a nuclear source and an analysis was conducted to evaluate the effect on thermal control of the equipment items inside the spacecraft.

The effective solar absorptance of a typical louver assembly as a function of blade and solar angle may be taken from Figure K-B-6 attached. Assuming a rotisserie mode (full rotation) and hot case with the blades open, the $90^{\circ}$ blade angle curve may be used to obtain an average solar absorptance of 0.169 during a half cycle when the louvers receive sun. Integrated over a full revolution, the absorbed solar flux on the radiator baseplates is 136 watts and the maximum heat rejestion through the louvers is reduced to 204 watts. It is apparent, then, that the present louvers in a rotisserie mode will have their capacity reduced about $40 \%$ and additional louvered and/or passive radiating area must be incorporated to accommodate a rotisserie mode. If, for example, the additional area is all passive, $8.9 \mathrm{ft}^{2}$ would have to be added.

\section{Removal of Louvers}

If the louvers are removed, the present radiators will provide adequate area to reject the component waste heat during a rotisserie mode. Either of two coating systems, the silver teflon presently being employed behind the louvers or the more stable OSR's (Optical Solar Reflectors), might be considered. At $90^{\circ} \mathrm{F}$, the use of the silver teflon will provide a rejection capability of 365.4 watts $(\alpha=0.14$, $\epsilon=0.76)$ whereas the OSRs will reject 386 watts $(\alpha=0.2, \epsilon=.85$ ). 


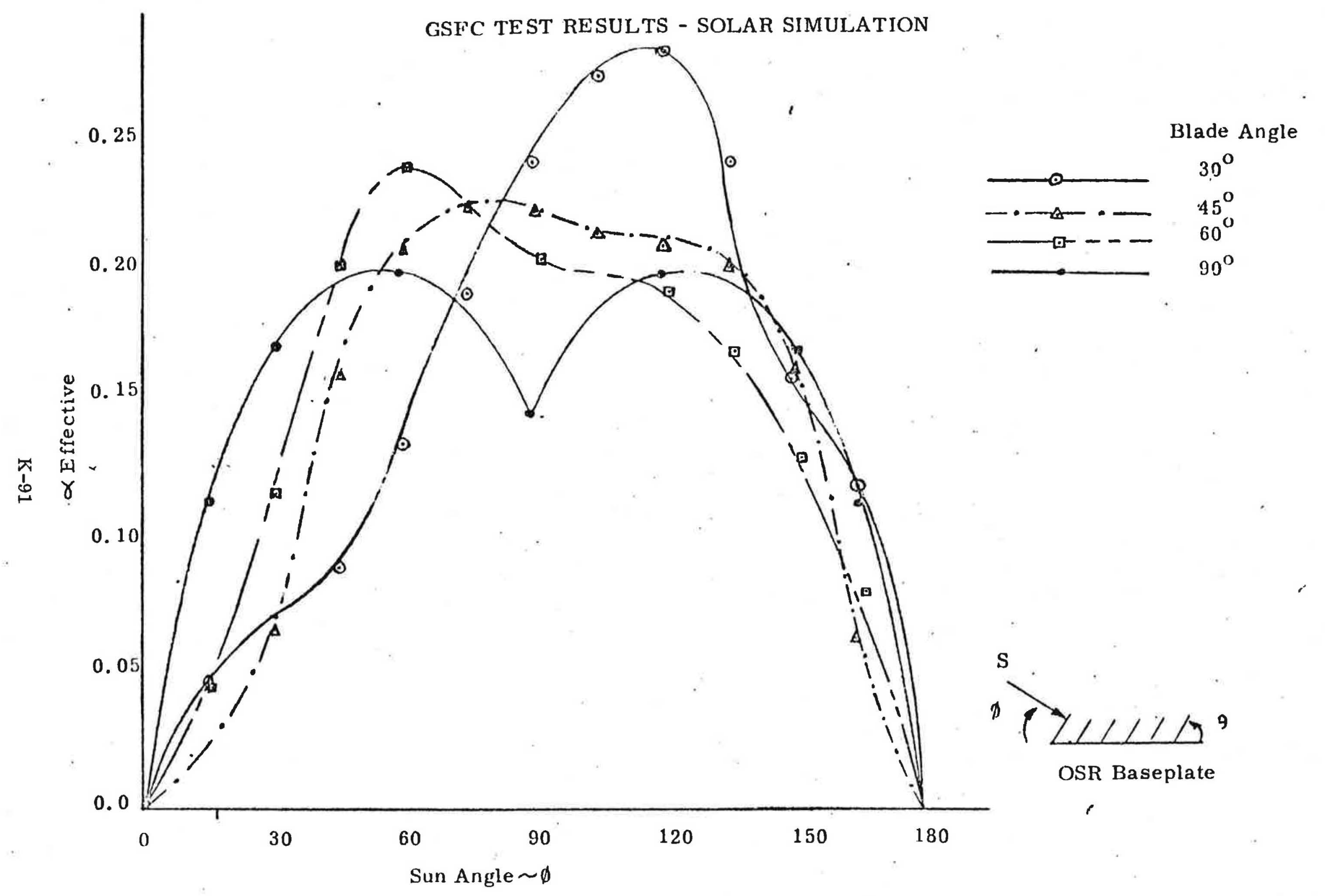

FIGURE K`B-6 
There are several additional considerations which sholid be carefully evaluated if the louvers are to be removed. The first concerns the average baseplate temperature change as a function of heat dissipation. Since the louvers provide a variable radiation resistance as a function of temperature, it is obvious their removal will result in a larger heater power requirement during a given "cold case " or, alternatively, a larger temperature drop per unit change in heat generation as the heat generation varies from the design point. This may be shown graphically by considering two radiators, one covered with louvers and one without, but each designed to maintain $90^{\circ} \mathrm{F}$ at a given dissipation. When the dissipation decreases below the design point, the decrease in temperature per unit change is much greater for the unlouvered radiator. The two curves are shown in Figure $\mathrm{K}-\mathrm{B}-7$.

A second concern is related to the transient behavior of a given radiator (and components thermally coupled thereto) during a given rotation about the yaw axis. Figure $\mathrm{K}-\mathrm{B}-8$. shows the results of an analysis in which a large, 300-lb equivalent mass radiator was assumed to be rotating at one revolution per 12 hours about an axis normal to the sun. The minimum to maximum temperature variation is about $19^{\circ} \mathrm{F}$ and this is probably unacceptable for temperature control of the frequency standard clock. (The clock is thermally coupled to a louvered radiator by means of 0.1 inch doubler strap and the orbital temperature variation of the radiator which, in this case is the heat sink, is very significant to the clock's temperature control). Of course, the curve shown in the figure represents a particular case and additional runs should be made in which the two significant parameters, effective mass and rotational velocity, are varied.

A third consideration relates to the thermal coupling between the components and various radiators on the $\mathrm{Y}$ facing sides. As presently designed, any imbalance in heat distribution will be partially compensated for by the thermal louvers which, by virtue of their independent actuation, can attain different blade positions and vary heat rejection to space in accordance with the temperature of a particular radiator. In the absence of louvers, temperature gradients should be evaluated along and between radiators on both sides. 


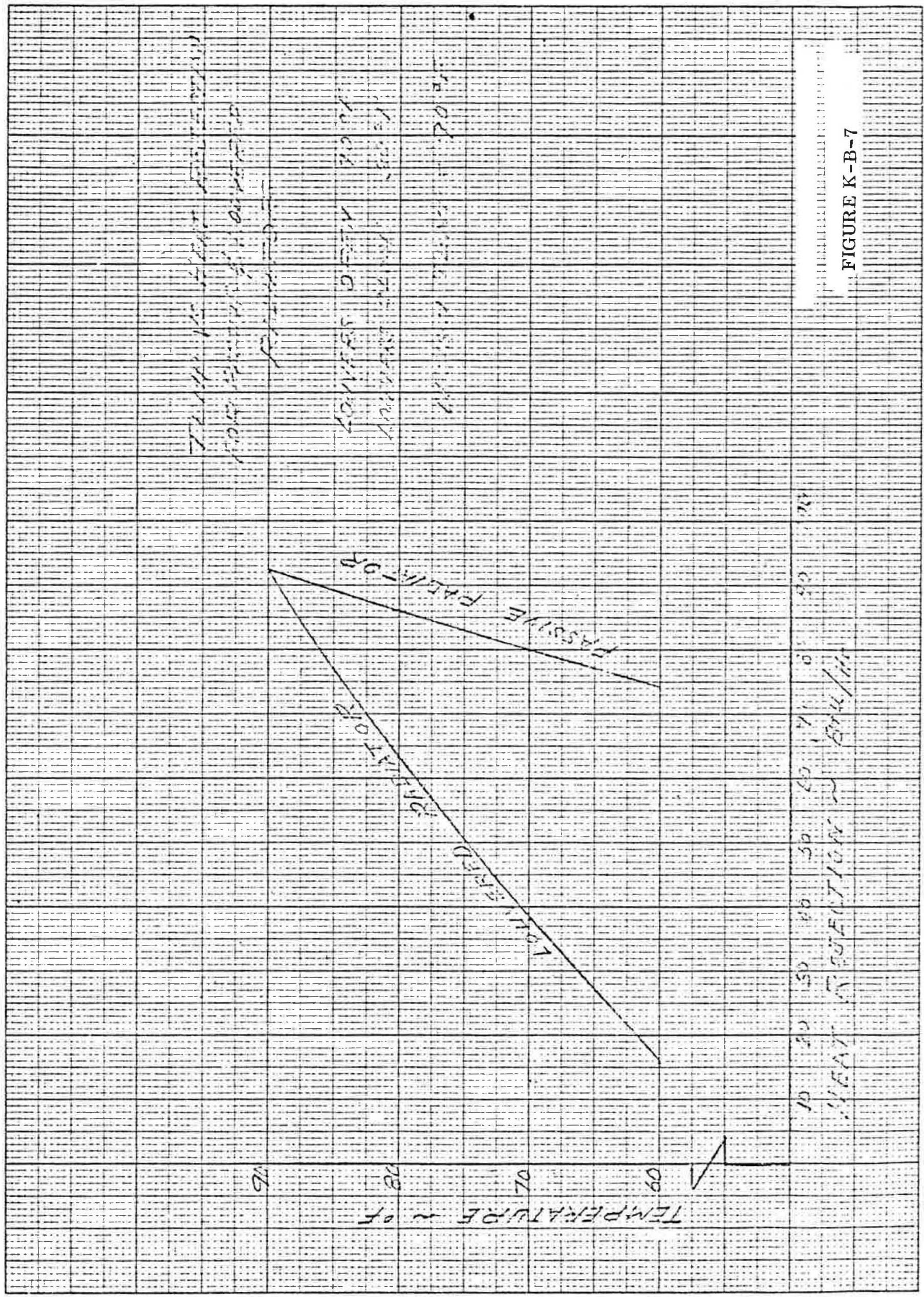




\section{$\underline{\text { Transfer Orbit }}$}

During the transfer orbit, the arrays are stowed so as to nearly surround the louvered radiators and provide an additional radiation resistance to space. This, of course, is necessary for thermal control of the electronics since the power input from the arrays is small and the associated low dissipation mode tends to result in low component temperatures.

Using the current configuration, it is computed that in a cold extreme (spin axis aligned with the sun and louvers closed) 42 watts will be required to maintain a mean radiator temperature above $40^{\circ} \mathrm{F}$. If the louvers and arrays are removed, the same environment will dictate the necessity for providing 334 watts to maintain $40^{\circ} \mathrm{F}$ on the radiators. However, because of the relatively constant output of most nuclear energy sources, it is not anticipated that this will impose a problem.

K-B-3 Heat Soakback Into GPS Structure

Heat soak-back into the GPS basic structure from the RTGs was estimated. Teledyne's design concept was used as a baseline configuration for this study. The assumed 3200 watt RTG attachment arrangement is shown in Figure K-B-9 The upper fitting and lower truss supports the RTG laterally while the top cap fitting carries the vertical loads. In order to minimize the conducted heat input to the structure from the RTGs, epoxy glass isolators should be used between the RTG and attachment fittings. It appears that the most feasible mounting configuration is to attach the upperfittings to the RTG's upper end cap and the lower truss to a support ring. The louver truss cannot readily be attached to the RTG lower end cap due to the RTG's overall length. Both Fairchild and Teledyne agree that approximately 1.6\% of the RTG's total heat is lost through the end caps. Therefore, a total of 25.6 watts/ RTG $(0.016 \times 3200 / Z)$ was assumed to be conducted through the two upper fittings and radiated from the cap to space.

The support ring was assumed to have the same temperature as a typical radial fin $\left(300^{\circ} \mathrm{F}\right)$. The total amount of heat being conducted into the structure was calculated parametrically as a function of structural temperature. Twenty-six watts is conducted into the structure from the RTGs when the structure is approximately sixteen watts as shown by figure $\mathrm{K}-\mathrm{B}-10$. 


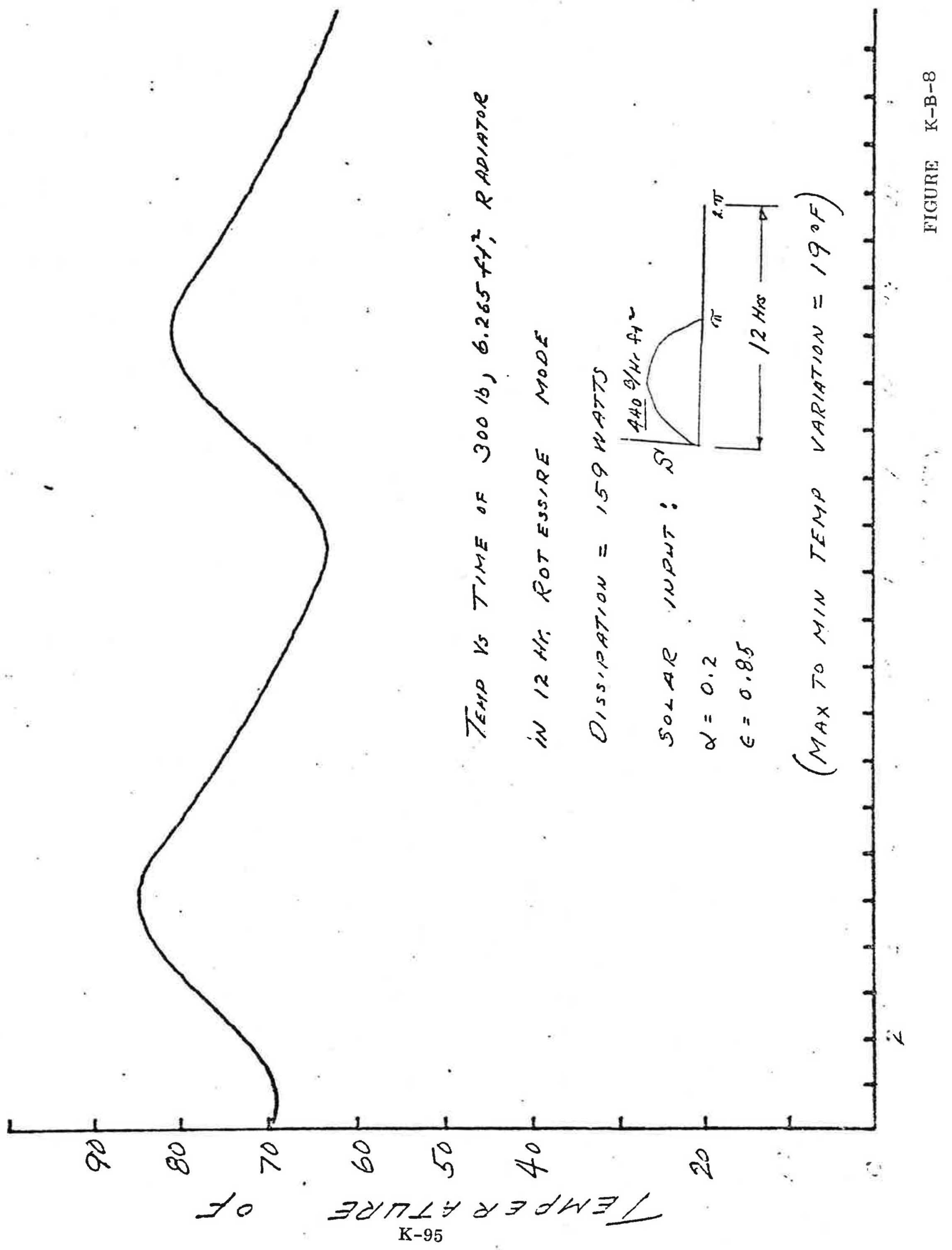


$\psi$

Heat sookbouk nito bipis structure from ieterin

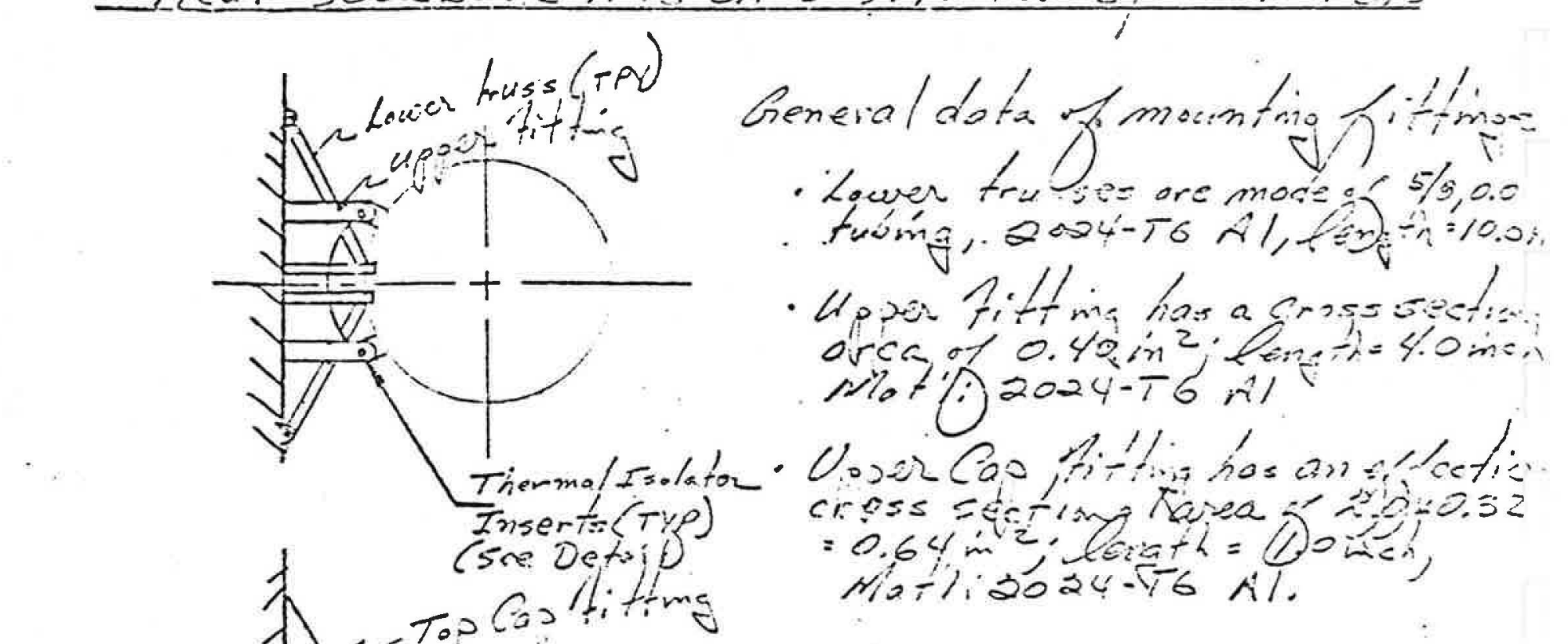

Conciuctance calculations: Upper cap:

GP'S

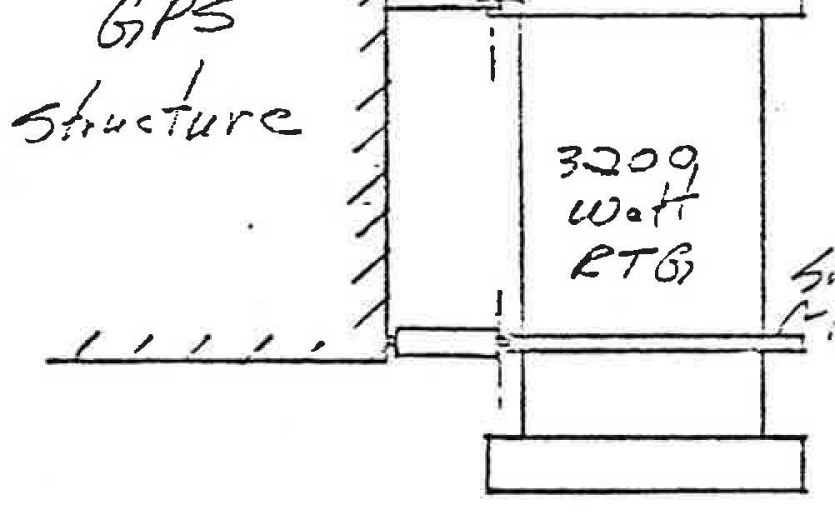

$$
K A / 4 x=109.2 x \cdot 64 / 12 x 1=5.82^{6 \mathrm{~T} u / \mathrm{H}}
$$

Upper Fitimg

$$
k x / \Delta x=109.2 \times .4 / 12<4=0.91
$$

Lower truss

$$
\begin{aligned}
K A / \Delta x & =109.2 x+\pi(10) 0.030 / 12 x \\
& =0.105
\end{aligned}
$$

FIGURE K-B-9 Assumed Aftochiment for

Therma! Folotor Inser-

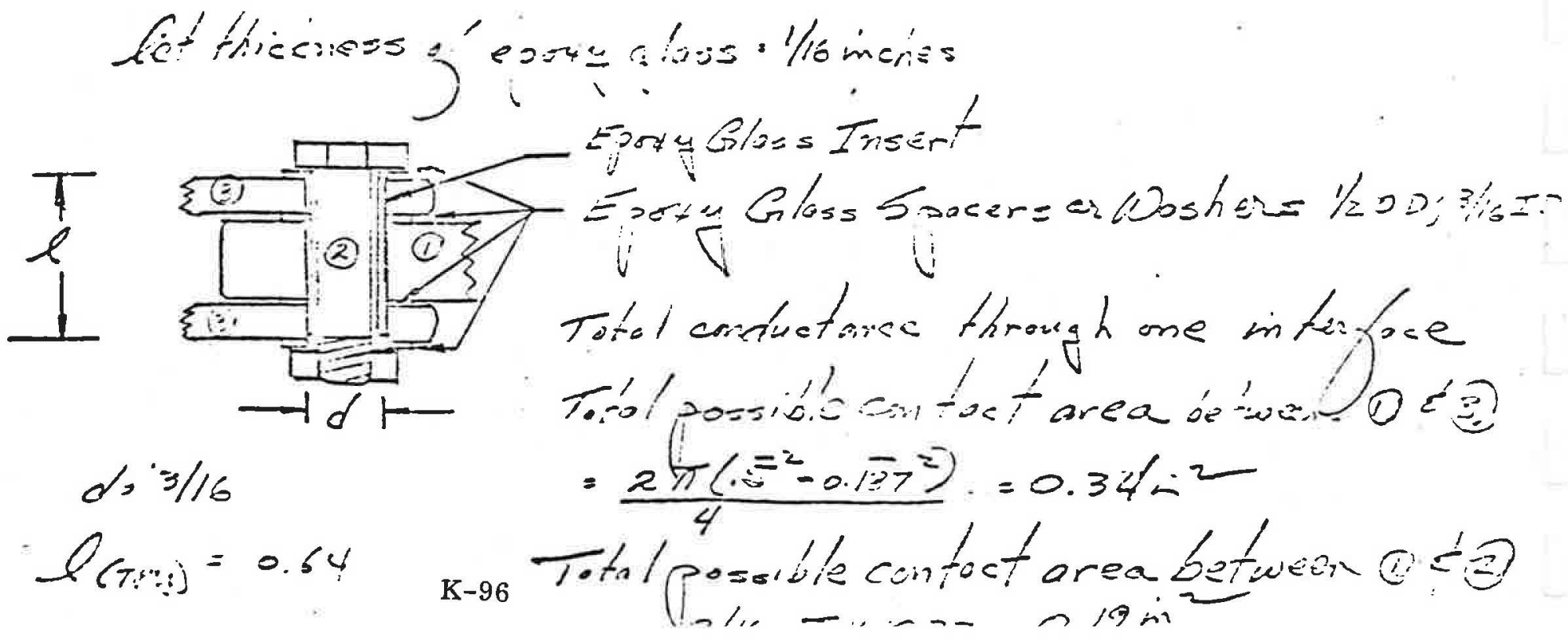


FIGURE $\mathrm{K}-\mathrm{B}-10$

Total heat Conducted si to Gas structure

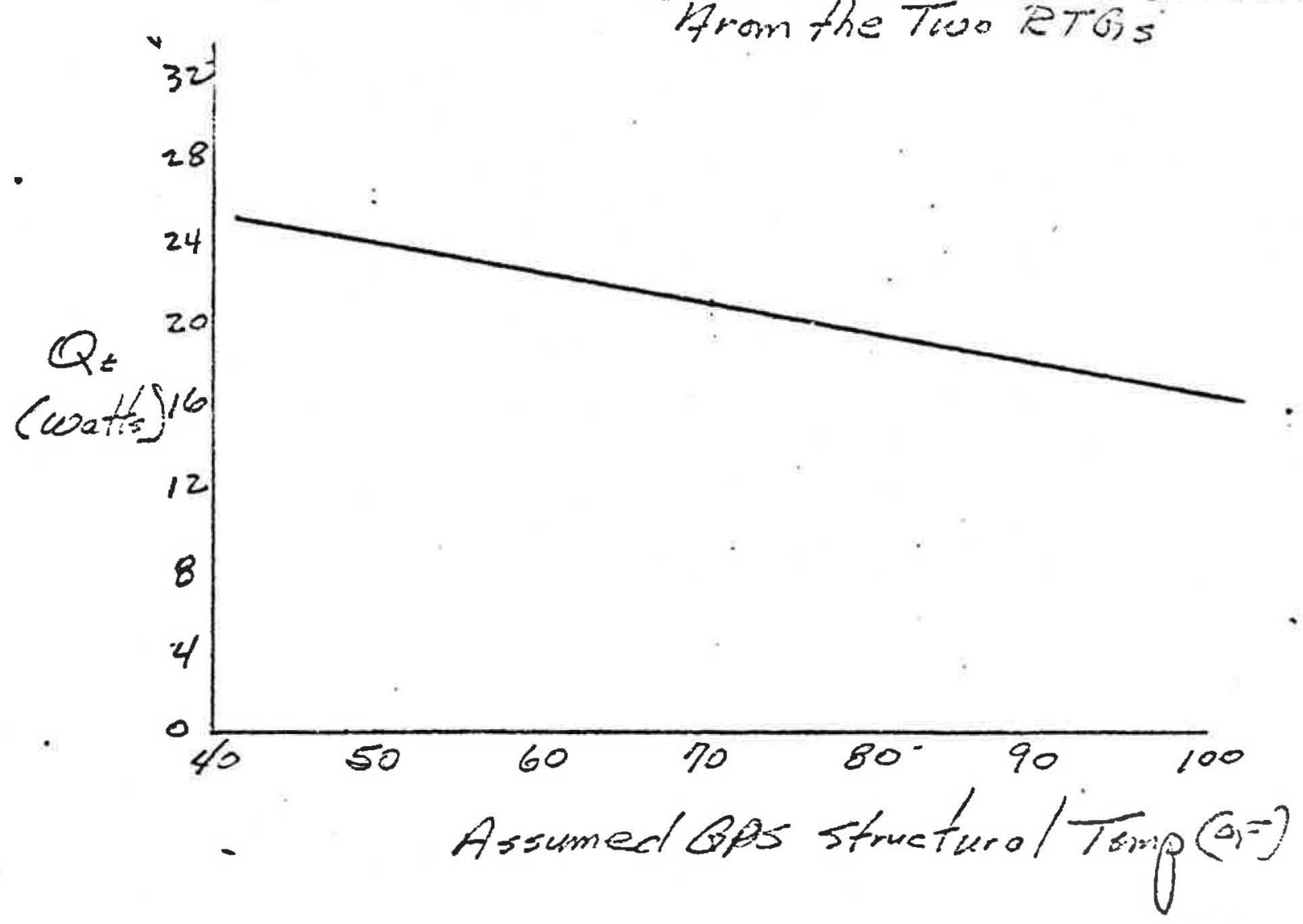

K -97 
The soak-back heat to the structure from the heat sources used in the BIPs and KIPs concepts is less than determined in this analysis. With the solar arrays removed, the present GPS louvered areas $\left(14.2 \mathrm{ft}^{2}\right.$ ) will be able to dump an additional 60.0 watts at $70^{\circ} \mathrm{F}$. Therefore, heat conducted from the RTGs, SIPS heat source, and/or BIPs heat source to the GPS structure is not expected to be a problem.

K-B-4 Frequency Standard Temperature Variation

The Rockwell International GPS spacecraft design has the Frequency Standard mounted directly to a louvered honeycomb panel. The temperature of panel/ Frequency Standard interface varies by approximately $3^{\circ} \mathrm{C}$ during max eclipse orbit. This variation of $3^{\circ} \mathrm{C}$ is within the thermal specification. However, the performance of the GPS Navigation equipment could be improved if this interface temperature variation could be reduced.

The purpose of this memo is to show that the frequency standard mounting surface temperature variation during a max eclipse orbit is caused mainly by radiation coming from the solar array. This variable source of external heat is both IR and reflected solar energy coming from the deployed solar array. Fluctuations in the IR and reflected solar energy levels to the louvered panel is caused by rotation of the solar arrays and by the earth eclipse.

Figure $\mathrm{K}-\mathrm{B}-11$ shows the total solar array radiant heat absorbed by all seven GPS louvered panels over the full range of the louver operating conditions. The absorbed heat is given as a function of panel temperature and time. After the neat heat loss was determined for the louvered panel in which the frequency standards are mounted, the net effective emissivity of the panel was determined. (See Figure K-B-12.) A thermal math model was constructed to represent the frequency standard/louvered panel composite. Assuming a constant thermal dissipation in the Frequency Standard of 18.6 watts and varying the effective thermal mass of the composite parametrically, the orbital temperature of the frequency/standard/panel interface was estimated. As Figure K-B-13 shows, the effective thermal mass ( $\mathrm{mC}_{\mathrm{p}}$ ), has to be approximately $1.8 \mathrm{BTU} /{ }^{\circ} \mathrm{F}$ in order to obtain a mounting face temperature variation of $3.0^{\circ} \mathrm{C}$. When considering the $\mathrm{mC}_{\mathrm{p}}=1.8$, a variation of $\pm 1^{\circ} \mathrm{C}$ can be attributed to the fact that the arrays are rotating and $\mathrm{a} \pm 2{ }^{\circ} \mathrm{C}$ variation can be attri- 
FIGURE K-B-11 Total Absorbed Heat to GPS Louvered Areas

During

Noon Orbit

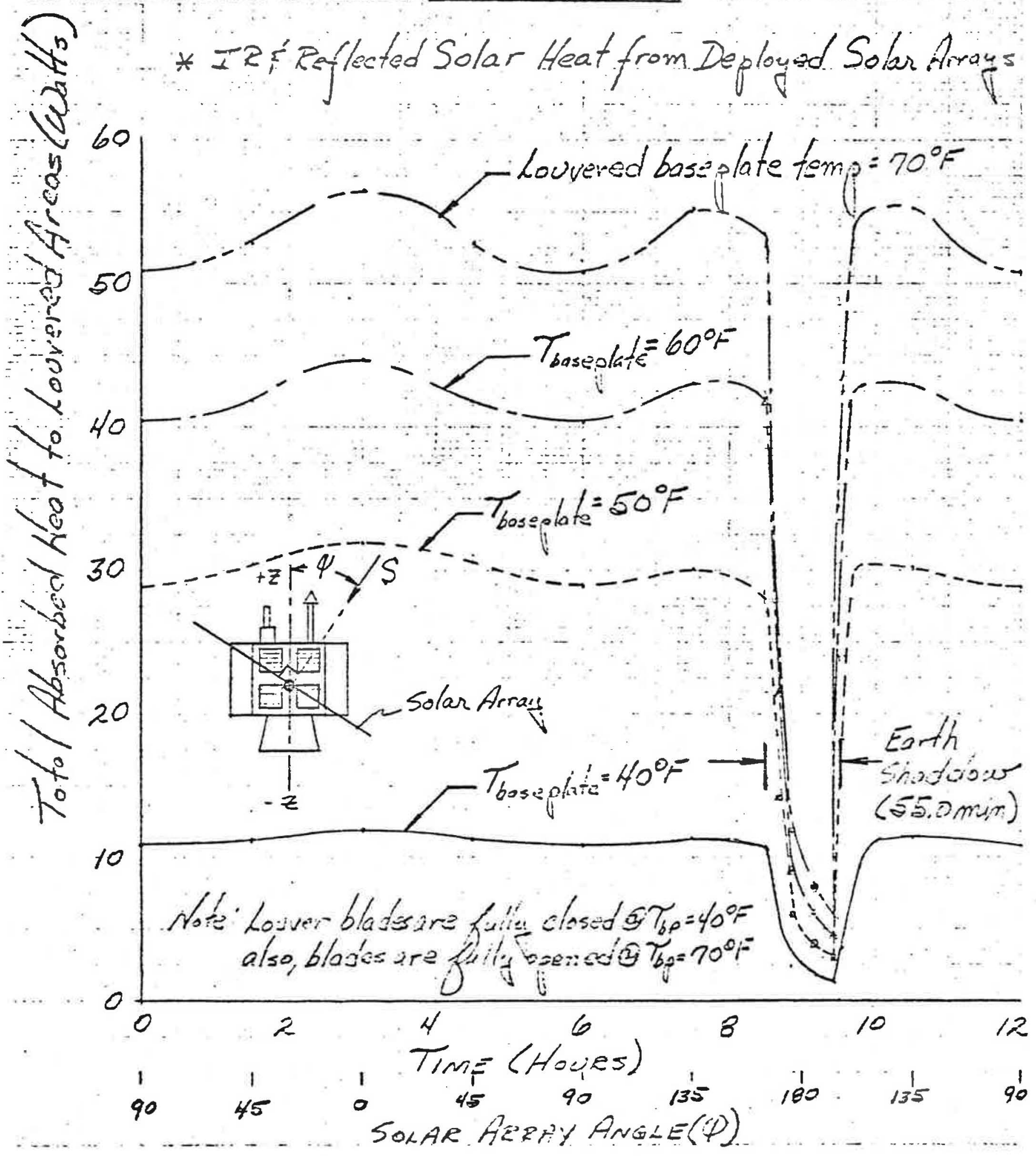

$\mathrm{K}-99$ 
Efiative Emissivitu oflien. Stul houver

- During Moon Afbit.

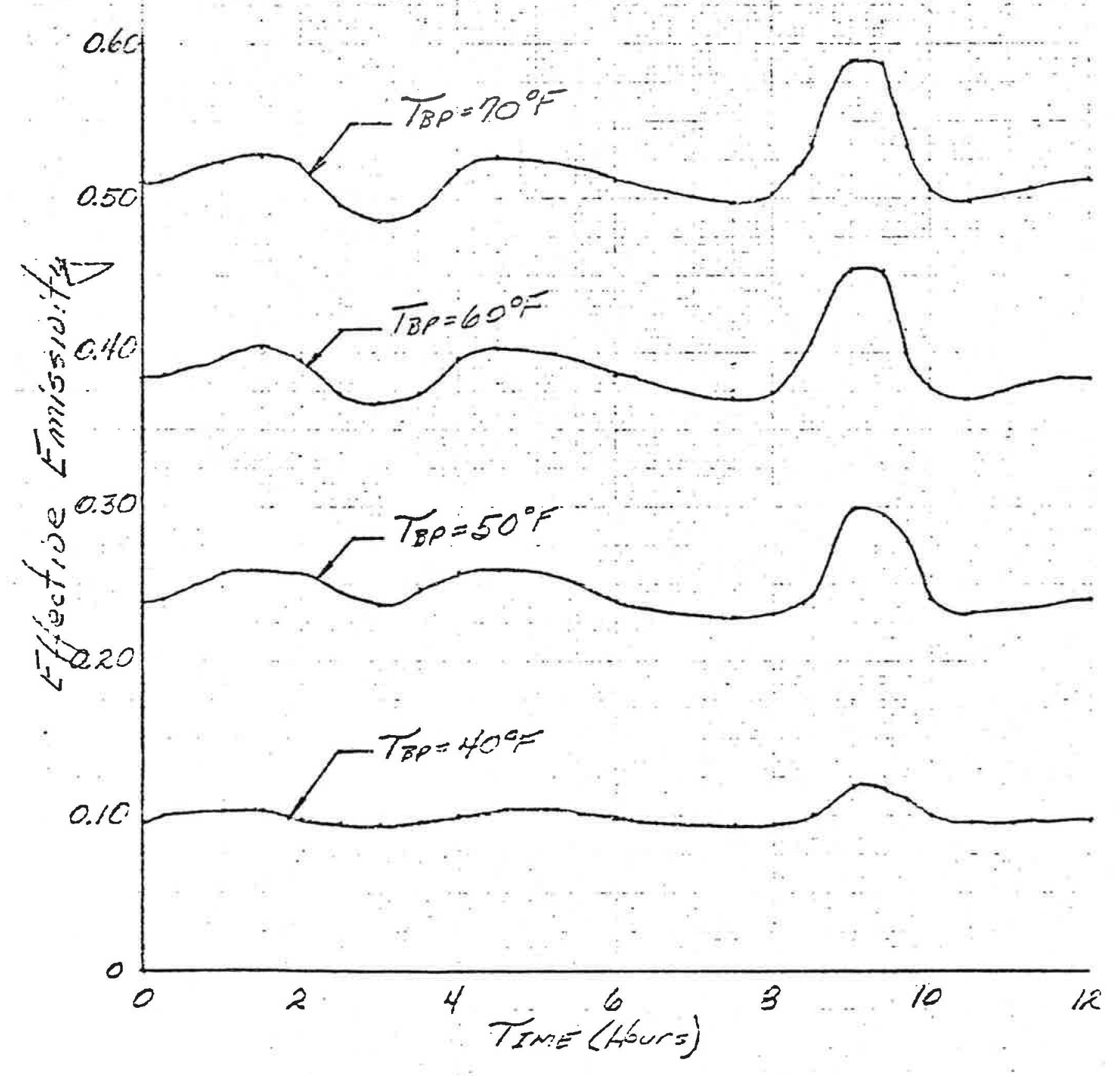


buted to earth shadow. An effective thermal mass of $1.8 \mathrm{BTU} /{ }^{\circ} \mathrm{F}$ is equivalent to $9 \mathrm{lbs}$. of aluminum. The frequency standard unit is designed thermally like three concentric ovens (one inside the other). The weight of the outer oven plus the louvered panel is approximately nine pounds.

This study has shown that the frequency standard mounting base temperature variation $\left( \pm 3^{\circ} \mathrm{C}\right)$ that the Rockwell GPS design experiences during $\max$ eclipse orbit is caused mainly by the solar arrays. The variable external heat input to the louvered panels and therefore the orbital variation of the frequency standard temperature could be dramatically reduced by eliminating the solar arrays and substituting a fixed nuclear power system. 


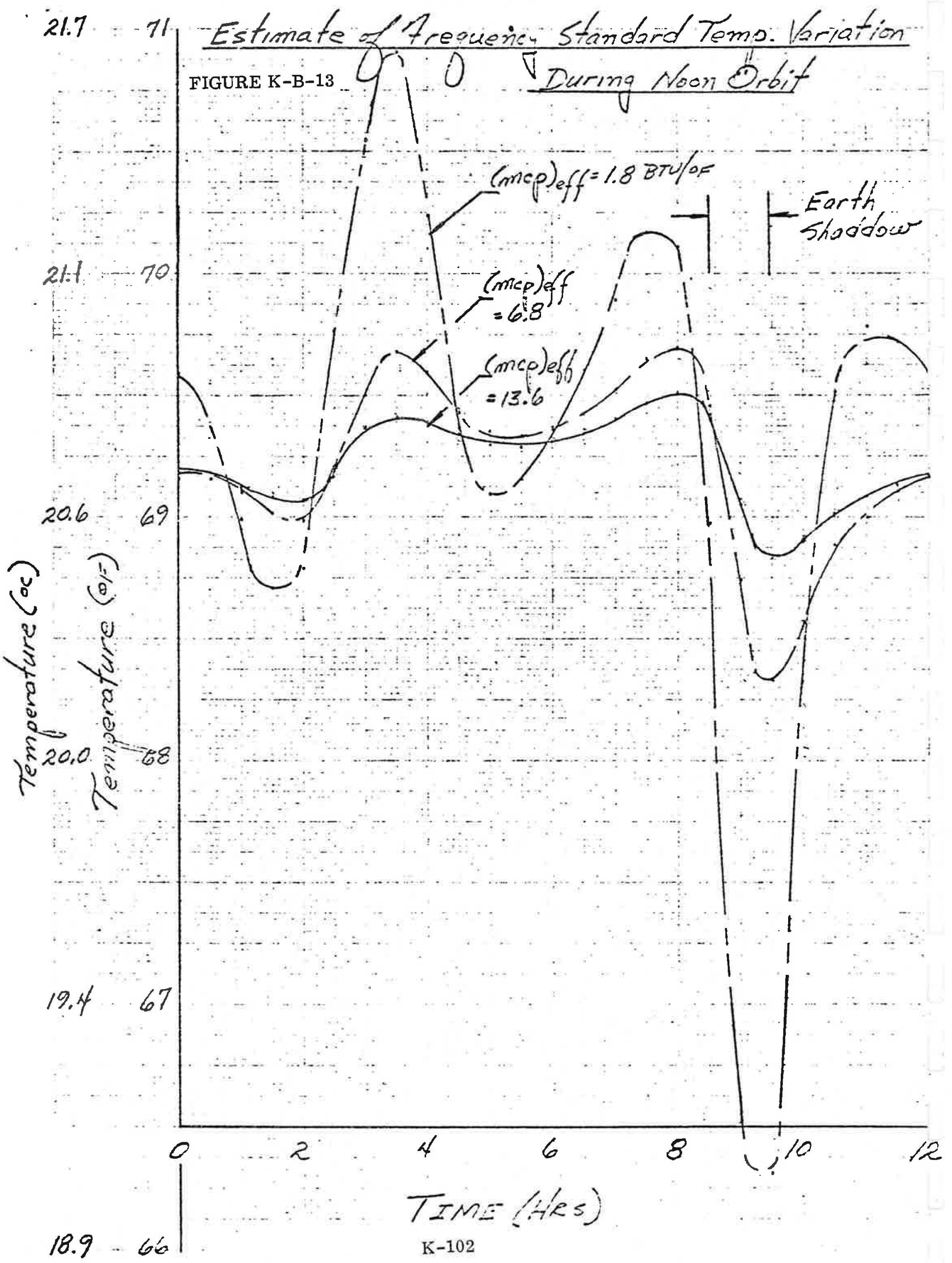

